\title{
One out of Four: Kinetic Resolution of Stereoisomeric Mixtures of Secondary Alcohols with a Quaternary Carbon Atom in the $\beta$-Position by $\mathrm{Cu}-\mathrm{H}$-Catalyzed Enantioselective Silylation
}

Zaneta Papadopulu, Novid Kazeroonian, Elisabeth Irran, and Martin Oestreich*

\author{
Institut für Chemie, Technische Universität Berlin, \\ Straße des 17. Juni 115, D-10623 Berlin, Germany \\ martin.oestreich@tu-berlin.de
}

Supporting Information 


\section{Table of Contents}

$1 \quad$ General Information $\quad$ S3

2 Full Tables $\quad$ S5

2.1 Hydrosilane Screening $\quad$ S5

2.2 Temperature Screening S6

$3 \quad$ General Procedures $\quad$ S7

4 Preparation of Substrates and Reagents S9

$5 \quad$ Procedures and Characterization Data $\quad$ S10

$\begin{array}{ll}5.1 \text { Optimization } & \text { S10 }\end{array}$

5.2 Kinetic Resolution $\quad$ S24

5.2 Preparation of Precursors $\quad$ S49

6 Crystallographic Data $\quad$ S58

6.1 Single Crystal $(2 R, 3 R)-4 a \quad$ S58

6.2 Single Crystal $(2 S, 3 R)-5 \mathbf{a} \quad S 66$

$7 \quad$ HPLC Traces $\quad$ S73

$8 \quad$ NMR Spectra $\quad$ S115

9 References $\quad$ S234 


\section{General Information}

All reactions were performed in flame-dried glassware using conventional Schlenk techniques under a static pressure of nitrogen or in an MBraun glove box $\left(\mathrm{O}_{2}<0.5\right.$ ppm, $\mathrm{H}_{2} \mathrm{O}<5$ ppm) under argon unless otherwise stated. Glassware was dried overnight at $120^{\circ} \mathrm{C}$ or flame dried using a heat gun. All plastic syringes and needles used in the glovebox were dried overnight at $60^{\circ} \mathrm{C}$. Liquids and solutions were transferred with syringes. Technical grade solvents for extraction and chromatography (cyclohexane, ethyl acetate, tert-butyl methyl ether, and $\mathrm{CH}_{2} \mathrm{Cl}_{2}$ ) were distilled prior to use. $\mathrm{Et}_{2} \mathrm{O}, \mathrm{THF}$, and toluene for reactions were dried over sodium/benzophenone and distilled prior to use. $(R, R)$-Ph-BPE was purchased from $A B C R$ and was used as received. Thin-layer column chromatography was performed on Macherey-Nagel Alugram $^{\circledR}$ Xtra SIL G/UV254 silica gel 60 pre-coated aluminum-backed plates $(200 \mu \mathrm{m}$ layer thickness). Product spots were visualized under UV light $\left(\lambda_{\max }=254 \mathrm{~nm}\right)$ and with a ceric ammonium molybdate stain. Column chromatography was performed on Grace 60 (40-63 $\mu \mathrm{m}, 230-400$ mesh, ASTM) silica gel. Automatic column chromatography was performed on a Isolera One ${ }^{\mathrm{TM}}$ (Biotage) using KP Sil columns $(100 \mathrm{~g}, 50 \mathrm{~g}, 25 \mathrm{~g}$, and $10 \mathrm{~g})$. Analytical high performance liquid chromatography (HPLC) was performed on an Agilent Technologies 1290 Infinity instrument equipped with a chiral stationary column (Daice/ Chiralce ${ }^{\circledR} \mathrm{OD}-\mathrm{H}$, Daicel Chiralce ${ }^{\circledR} \mathrm{OJ}-\mathrm{H}$, Daicel Chiralpak ${ }^{\circledR} \mathrm{AD}-\mathrm{H}$, or Daicel Chiralce ${ }^{\circledR} \mathrm{AS}-\mathrm{H}$ ) using $n$-heptane/isopropanol mixtures as the mobile phase. ${ }^{1} \mathrm{H}$ and ${ }^{13} \mathrm{C}$ NMR spectra were recorded in $\mathrm{C}_{6} \mathrm{D}_{6}$ or $\mathrm{CDCl}_{3}$ on Bruker AV 400 and Bruker AV 500 instruments. Chemical shifts are reported in parts per million (ppm) downfield from tetramethylsilane and are referenced to the residual solvent resonance as the internal standard $\left(\mathrm{C}_{6} \mathrm{D}_{5} \mathrm{H}: \delta / \mathrm{ppm}=7.15\right.$ for ${ }^{1} \mathrm{H} N M R$ and $\mathrm{C}_{6} \mathrm{D}_{6}: \delta / \mathrm{ppm}=128.0$ for ${ }^{13} \mathrm{C} \mathrm{NMR}, \mathrm{CHCl}_{3}: \delta / \mathrm{ppm}=7.26$ for ${ }^{1} \mathrm{H} \mathrm{NMR}$ and $\mathrm{CDCl}_{3}: \delta / \mathrm{ppm}$ $=77.16$ for ${ }^{13} \mathrm{C} N M R$ ). Data are reported as follows: chemical shift, multiplicity ( $\mathrm{s}=$ singlet, $\mathrm{d}=$ doublet, $\mathrm{t}=$ triplet, $\mathrm{q}=$ quartet, $\mathrm{m}=$ multiplet), coupling constant $(\mathrm{Hz})$, and integration. Melting points (m.p.) were determined with a Stuart Scientific SMP20 melting point apparatus and are not corrected. Infrared (IR) spectra were recorded from neat samples on an Agilent Technologies Cary 630 FTIR spectrometer equipped with a diamond ATR unit. Selected absorption maxima are reported in wavenumbers $\left(\mathrm{cm}^{-1}\right)$. Optical rotations were measured on a Schmidt \& Haensch Polartronic H532 polarimeter. [a]D values are reported in $10^{-1}\left({ }^{\circ} \times \mathrm{Cm}^{2} \times \mathrm{g}^{-1}\right)$ with $c$ in $\mathrm{g} / 100 \mathrm{~mL}$ and $\lambda=$ 
$589 \mathrm{~nm}$ at room temperature. High resolution mass spectra (HRMS) were recorded on a Thermo Scientific LTQ Orbitrap XL by the Analytical Facility at the Institut für Chemie, Technische Universität Berlin using atmospheric-pressure chemical ionization (APCI), electrospray ionization (ESI) or liquid injection field desorption/ionization (LIFDI) methods. Only the respective molecular-ion peak or a characteristic fragment are reported. 


\section{Full Tables}

\subsection{Hydrosilane Screening}

\section{Table S1. Hydrosilane Screening - Full Table}

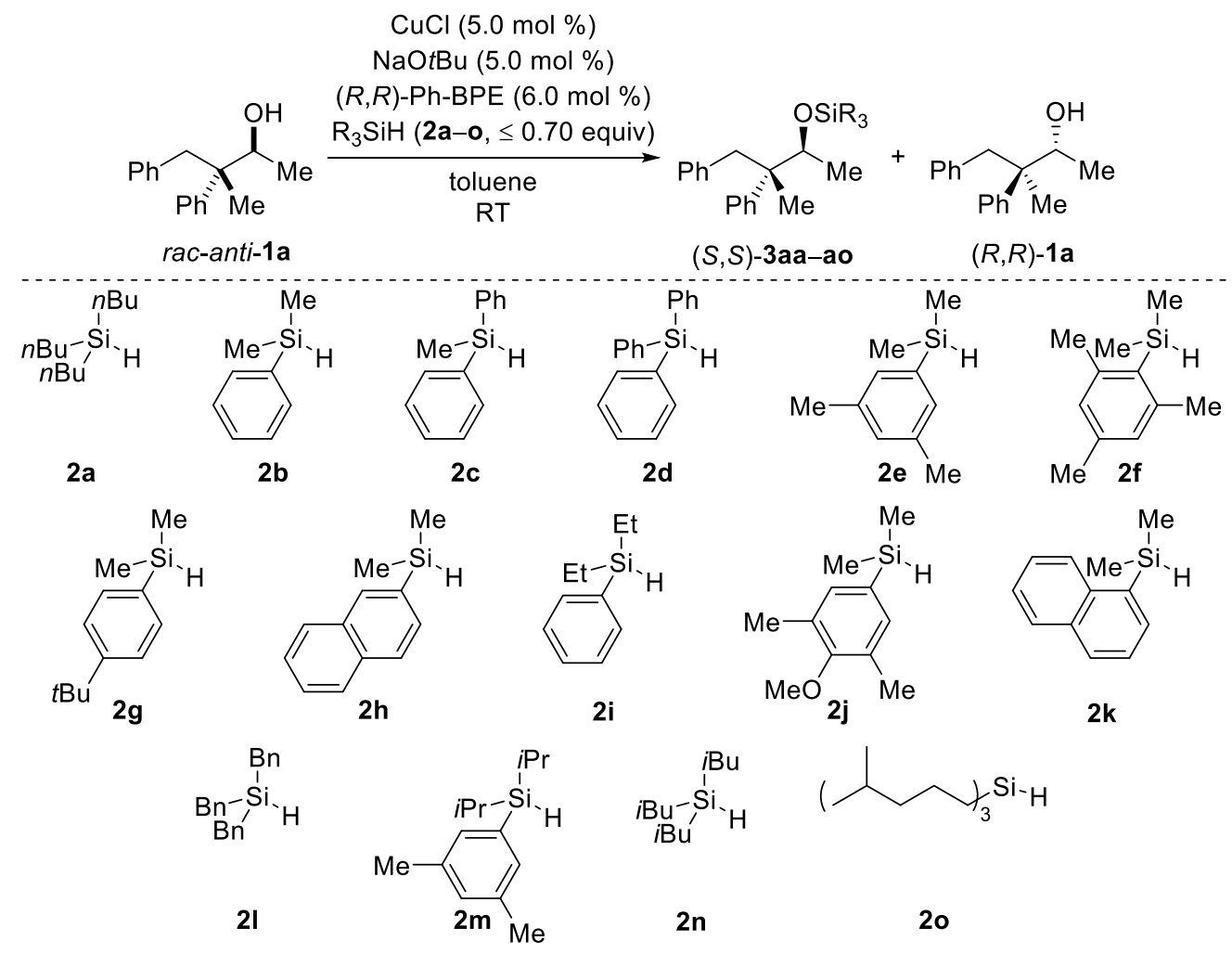

\begin{tabular}{cccccccc} 
entry & hydrosilane & $\begin{array}{l}\text { conv } \\
(\%)\end{array}$ & time & $\begin{array}{l}\text { ee of }(S, S)-3 a \\
(\%)\end{array}$ & $\begin{array}{l}\text { ee of } \\
(\%)\end{array}$ & $\begin{array}{l}(R, R)-1 a \\
\text { (\% }\end{array}$ \\
\hline 1 & $\mathbf{2 a}$ & n.r. & - & - & - & - \\
2 & $\mathbf{2 b}$ & 55 & $18 \mathrm{~h}$ & 66 & 82 & 12 \\
3 & $\mathbf{2 c}$ & 68 & $18 \mathrm{~h}$ & 42 & 90 & 6.8 \\
4 & $\mathbf{2 d}$ & 50 & $18 \mathrm{~h}$ & 17 & 17 & 1.6 \\
5 & $\mathbf{2 e}$ & 46 & $18 \mathrm{~h}$ & 77 & 65 & 15 \\
6 & $\mathbf{2 f}$ & 52 & $42 \mathrm{~h}$ & 32 & 33 & 2.6 \\
7 & $\mathbf{2 g}$ & 48 & $18 \mathrm{~h}$ & 67 & 63 & 9.5 \\
8 & $\mathbf{2 h}$ & 50 & $18 \mathrm{~h}$ & 73 & 73 & 14 \\
9 & $\mathbf{2 i}$ & 50 & $18 \mathrm{~h}$ & 42 & 42 & 3.7 \\
10 & $\mathbf{2 j}$ & 51 & $18 \mathrm{~h}$ & 73 & 76 & 15 \\
11 & $\mathbf{2 k}$ & 53 & $18 \mathrm{~h}$ & 42 & 47 & 3.8 \\
12 & $\mathbf{2 l}$ & 49 & $18 \mathrm{~h}$ & 18 & 18 & 1.7 \\
13 & $\mathbf{2 m}$ & n.r. & - & - & - & - \\
14 & $\mathbf{2 n}$ & n.r. & - & - & - & - \\
15 & $\mathbf{2 0}$ & n.r. & - & - & - & -
\end{tabular}




\subsection{Temperature Screening}

\section{Table S2. Temperature Screening - Full Table}

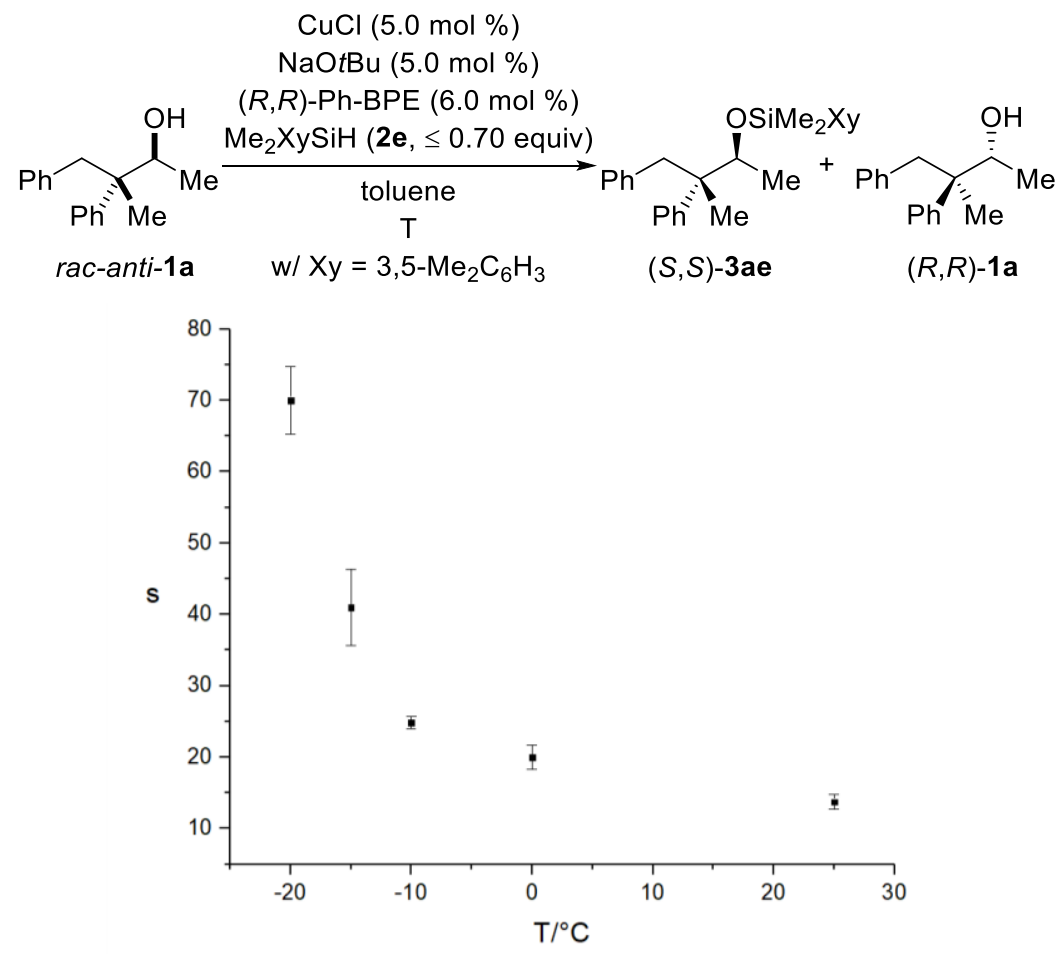

$\begin{array}{ccccccc}\text { entry } & \mathrm{T}\left({ }^{\circ} \mathrm{C}\right) & \text { time } & \begin{array}{c}\text { conv } \\ (\%)\end{array} & \begin{array}{c}\text { ee of }(S, S)- \\ \text { 3ae }(\%)\end{array} & \begin{array}{c}\text { ee of }(R, R)-1 \mathbf{a} \\ (\%)\end{array} & \mathrm{s} \\ 1 & -20 & 10 \mathrm{~d} & 50 & 91 & 92 & 65 \\ 2 & -20 & 10 \mathrm{~d} & 48 & 93 & 86 & 75 \\ 3 & -15 & 56 \mathrm{~h} & 46 & 90 & 76 & 45 \\ 4 & -15 & 72 \mathrm{~h} & 49 & 85 & 81 & 30 \\ 5 & -15 & 72 \mathrm{~h} & 47 & 90 & 81 & 48 \\ 6 & -10 & 34 \mathrm{~h} & 53 & 79 & 91 & 26 \\ 7 & -10 & 52 \mathrm{~h} & 50 & 82 & 82 & 26 \\ 8 & -10 & 52 \mathrm{~h} & 51 & 80 & 84 & 23 \\ 9 & 0 & 28 \mathrm{~h} & 51 & 80 & 83 & 23 \\ 10 & 0 & 30 \mathrm{~h} & 40 & 83 & 56 & 18 \\ 11 & 0 & 30 \mathrm{~h} & 47 & 82 & 73 & 22 \\ 12 & \text { RT } & 18 \mathrm{~h} & 45 & 77 & 65 & 15 \\ 13 & \text { RT } & 20 \mathrm{~h} & 44 & 72 & 58 & 11\end{array}$




\section{General Procedures (GPs)}

\subsection{General Procedure for the Kinetic Resolution of Alcohols (GP1)}

Following a modified literature procedure, ${ }^{[\mathrm{S} 1]}$ in an argon-filled glove box a 1.5-mL GLC vial with a magnetic stir bar is charged with $\mathrm{NaOtBu}(1.0 \mathrm{mg}, 10 \mu \mathrm{mol}, 5.0 \mathrm{~mol} \%)$, $\mathrm{CuCl}(1.0 \mathrm{mg}, 10 \mu \mathrm{mol}, 5.0 \mathrm{~mol} \%)$ and $(R, R)$-Ph-BPE (6.1 mg, $12 \mu \mathrm{mol}, 6.0 \mathrm{~mol} \%)$. Toluene $(0.100 \mathrm{~mL})$ is added via syringe, and the resulting mixture is stirred at room temperature for $10 \mathrm{~min}$. After introduction of the hydrosilane (0.55-0.70 equiv), the yellow reaction mixture is maintained at room temperature for another $10 \mathrm{~min}$. A solution of the indicated alcohol $(0.200 \mathrm{mmol})$ in toluene $(0.500 \mathrm{~mL})$ is added. The reaction is maintained at ambient temperature and monitored by NMR spectroscopy until a satisfying conversion is reached. The reaction is quenched by filtration over a plug of silica. Purification by flash column chromatography on silica gel using cyclohexane/ethyl acetate as eluents affords the silyl ethers and the slow-reacting enantioenriched alcohols.

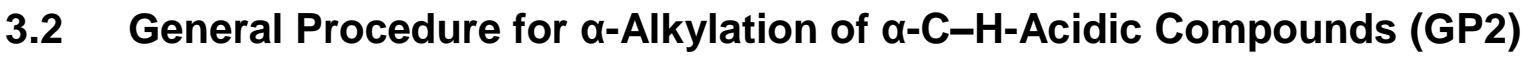

Following a modified literature procedure, ${ }^{[\mathrm{S} 2]}$ to a stirred suspension of $\mathrm{KOH}(6.00$ equiv) in THF or DME (0.1-0.2 M) the indicated aldehyde, nitrile, or ketone (1.00 equiv) is added. The reaction is stirred for $10 \mathrm{~min}$ at room temperature, followed by the addition of the indicated alkyl bromide (1.10-3.00 equiv). The resulting mixture is maintained at room temperature until full conversion of the starting material is monitored by TLC analysis. After filtration, the reaction is diluted with water, the aqueous phase is separated, acidified with aqueous $\mathrm{HCl}$ solution (1 $\mathrm{M})$, and extracted with tert-butyl methyl ether $(3 \mathrm{x})$. The combined organic extracts are dried over anhydrous $\mathrm{Na}_{2} \mathrm{SO}_{4}$, filtered, and concentrated under reduced pressure. Purification by automatic column chromatography using Isolera ${ }^{T M}$ One with cyclohexane/ethyl acetate as eluents affords the desired alkylated product.

\subsection{General Procedure for Deprotection of Silylated Alcohols (GP3)}

Following a literature procedure, ${ }^{[\mathrm{S} 1]}$ to the indicated silyl ether $(5.0-15 \mathrm{mg})$ tetra- $n$ butylammonium fluoride (TBAF, $1.0 \mathrm{~mL}, 1 \mathrm{M}$ in THF) is added at room temperature. The resulting reaction mixture is stirred until full consumption of starting material is indicated by TLC analysis. Upon completion, the reaction is quenched with water and extracted with tert-butyl methyl ether $(3 \mathrm{x})$. The combined organic extracts are dried 
over anhydrous $\mathrm{Na}_{2} \mathrm{SO}_{4}$, filtered and concentrated under reduced pressure. Purification by flash column chromatography on silica gel using cyclohexane/ethyl acetate as eluents affords the deprotected alcohol.

\subsection{General Procedure for Reduction of Nitriles to Aldehydes (GP4)}

Following a modified literature procedure, ${ }^{[\mathrm{S} 3]}$ to a stirred solution of the nitrile $(1.00$ equiv) in toluene $(0.300 \mathrm{M})$ at $-78^{\circ} \mathrm{C}$ a solution of DIBAL (1.20 equiv, $2.00 \mathrm{M}$ in hexane) is added in small portions. The reaction mixture is stirred for $1.5 \mathrm{~h}$ at $-78^{\circ} \mathrm{C}$ or until full consumption of starting material is indicated by TLC analysis. Upon completion, the reaction is quenched with aq. $\mathrm{HCl}(6.00 \mathrm{M})$, stirred for $30 \mathrm{~min}$, and extracted with tertbutyl methyl ether $(3 \mathrm{x})$. The combined organic extracts are washed with brine, dried over anhydrous $\mathrm{Na}_{2} \mathrm{SO}_{4}$, filtered, and concentrated under reduced pressure. Purification by automatic column chromatography using Isolera ${ }^{\mathrm{TM}}$ One with cyclohexane/ ethyl acetate as eluents affords the desired aldehyde.

\subsection{General Procedure for Grignard Addition of Aldehydes (GP5)}

To a stirred solution of the aldehyde (1.00 equiv) in $\operatorname{THF}(0.200 \mathrm{M})$ at $0^{\circ} \mathrm{C}$, a solution of Grignard reagent (3.00 equiv) is added slowly. The reaction is slowly warmed to room temperature and stirred overnight or until full consumption of starting material is indicated by TLC analysis. Upon completion, the reaction is quenched with aq. $\mathrm{NH}_{4} \mathrm{Cl}$ $(1.00 \mathrm{M})$ and water and is then extracted with tert-butyl methyl ether $(3 \mathrm{x})$. The combined organic extracts are dried over anhydrous $\mathrm{Na}_{2} \mathrm{SO}_{4}$, filtered, and concentrated under reduced pressure. Purification by automatic column chromatography on silica gel using Isolera ${ }^{\mathrm{TM}}$ One with cyclohexane/ethyl acetate as eluents affords the racemic alcohol. 


\section{Preparation of Substrates and Reagents}

Tributylhydrosilane (2a, TCI), dimethylphenylhydrosilane (2b, ABCR), methyldiphenylhydrosilane (2c, $\mathrm{TCl}$ ), and triphenylhydrosilane (2d, Sigma Aldrich) are commercially available and were used as received. (3,5-Dimethylphenyl)dimethylsilane (2e) was synthesized according to a literature procedure. ${ }^{[\mathrm{S4}]}$ Diethylphenylhydrosilane (2i), mesityldimethylhydrosilane (2f), dimethyl(1-naphthyl)hydro-silane (2k), dimethyl(2naphthyl)hydrosilane (2h), 4-(tert-butylphenyl)dimethylhydrosilane $(\mathbf{2 g})$, 4-methoxy3,5-dimethylphenylhydrosilane (2j) and tribenzylhydrosilane (2I) were synthesized according to a literature procedure. ${ }^{[\mathrm{S1}]}$ rac-anti-3-Methyl-3,4-diphenylbutan-2-ol (racanti-1a) and rac-syn-3-methyl-3,4-diphenylbutan-2-ol (rac-syn-1a) were prepared from rac-2-methyl-2,3-diphenylpropanal according to GP5. rac-2-Methyl-2,3diphenylpropanal was prepared from 2-phenylpropanal according to GP2. All other reagents were obtained from commercial sources and were used as received. 


\section{Procedures and Characterization Data}

\subsection{Optimization}

$(2 R, 3 R)-3-M e t h y l-3,4-d i p h e n y l b u t a n-2-o l$

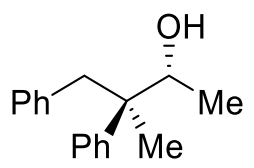

$(2 R, 3 R)-1 \mathbf{a}$

$\mathrm{C}_{17} \mathrm{H}_{20} \mathrm{O}$

$240.35 \mathrm{~g} / \mathrm{mol}$

Prepared according to GP1 from alcohol rac-anti-1a (48.0 mg, $0.200 \mathrm{mmol}, 1.00$ equiv) and (3,5-dimethylphenyl)dimethylsilane (17.8 mg, $0.108 \mathrm{mmol}, 0.542$ equiv). The reaction mixture was stirred for $2 \mathrm{~d}$. Flash column chromatography using cyclohexane/ethyl acetate $(100: 0 \rightarrow 25: 1 \rightarrow 10: 1)$ afforded enantioenriched alcohol $(2 R, 3 R)-1 \mathrm{a}(19.5 \mathrm{mg}, 0.0811 \mathrm{mmol}, 41 \%, 65 \%$ ee) as a colorless oil.

$\mathbf{R}_{\boldsymbol{f}}=0.42$ (cyclohexane/ethyl acetate 10:1). ${ }^{1} \mathbf{H}$ NMR $\left(500 \mathrm{MHz}, \mathrm{CDCl}_{3}\right): \delta / p p m=7.27-$ $7.30(\mathrm{~m}, 2 \mathrm{H}), 7.19-7.22(\mathrm{~m}, 3 \mathrm{H}), 7.05-7.10(\mathrm{~m}, 3 \mathrm{H}), 6.73-6.75(\mathrm{~m}, 2 \mathrm{H}), 4.19\left(\mathrm{q},{ }^{3} \mathrm{~J}=\right.$ $6.2 \mathrm{~Hz}, 1 \mathrm{H}), 3.18\left(\mathrm{~d},{ }^{2} \mathrm{~J}=13.3 \mathrm{~Hz}, 1 \mathrm{H}\right), 3.05\left(\mathrm{~d},{ }^{2} \mathrm{~J}=13.3 \mathrm{~Hz}, 1 \mathrm{H}\right), 1.45\left(\mathrm{~d},{ }^{3} \mathrm{~J}=6.0 \mathrm{~Hz}\right.$, $1 \mathrm{H}), 1.24(\mathrm{~s}, 3 \mathrm{H}), 0.97\left(\mathrm{~d},{ }^{3} \mathrm{~J}=6.4 \mathrm{~Hz}, 3 \mathrm{H}\right) .{ }^{13} \mathrm{C} \mathbf{N M R}\left(126 \mathrm{MHz}, \mathrm{CDCl}_{3}\right): \delta / \mathrm{ppm}=$ 144.7, 138.4, 130.5 (2C), 128.0 (2C), 127.4 (2C), 127.2 (2C), 126.0, 125.8, 73.9, 47.2, 45.6, 18.6, 16.3. HRMS (APCl) m/z: $\left[\mathrm{M}-\mathrm{C}_{2} \mathrm{H}_{5} \mathrm{O}\right]^{+}$Calcd for $\mathrm{C}_{15} \mathrm{H}_{15}$ 195.1174; Found 195.1166. IR (ATR): $\tilde{v} / \mathrm{cm}^{-1}=2975,1598,1494,1446,1373,1250,1073,972,868$, 773, 696. Optical Rotation: $[\alpha]_{D}=-0.92\left(c 0.3 \mathrm{CHCl}_{3}\right)$.

The enantiomeric excess of $(2 R, 3 R)$-1a was determined by HPLC analysis on a chiral stationary phase (Daicel Chiralcel ${ }^{\circledR} \mathrm{OD}-\mathrm{H}$ column, column temperature $20^{\circ} \mathrm{C}$, mobile phase: $n$-heptane:isopropanol $=95: 5$, flow rate: $0.6 \mathrm{~mL} / \mathrm{min}, \lambda=210 \mathrm{~nm}$ ): $\mathrm{t}_{\mathrm{R}}=13.6$ $\min$ for $(2 S, 3 S)-1 a, t_{R}=15.6 \min$ for $(2 R, 3 R)-1 \mathbf{a}$. 
(2S,3S)-(3,5-Dimethylphenyl)dimethyl((3-methyl-3,4-diphenylbutan-2yl)oxy)silane

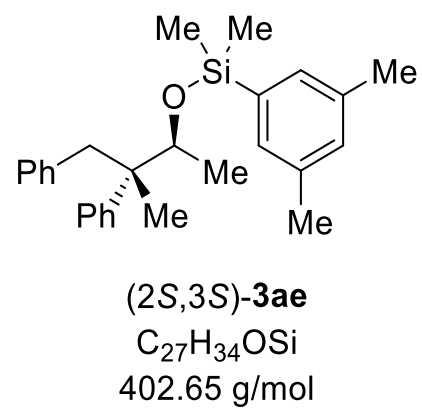

Prepared according to GP1 from alcohol rac-anti-1a (48.0 mg, 0.200 mmol, 1.00 equiv) and (3,5-dimethylphenyl)dimethylsilane (17.8 mg, $0.108 \mathrm{mmol}, 0.542$ equiv). The reaction mixture was stirred for $2 \mathrm{~d}$. Flash column chromatography using cyclohexane/ethyl acetate $(100: 0 \rightarrow 25: 1 \rightarrow 10: 1)$ afforded silyl ether (2S,3S)-3ae (31.4 mg, $0.0780 \mathrm{mmol}, 38 \%, 77 \%$ ee) as a colorless oil.

$\mathbf{R}_{\boldsymbol{f}}=0.52$ (cyclohexane/ethyl acetate 99:1). ${ }^{1} \mathbf{H}$ NMR $\left(500 \mathrm{MHz}, \mathrm{C}_{6} \mathrm{D}_{6}\right): \delta / \mathrm{ppm}=7.44-$ $7.45(\mathrm{~m}, 2 \mathrm{H}), 7.07-7.09(\mathrm{~m}, 4 \mathrm{H}), 7.02-7.06(\mathrm{~m}, 1 \mathrm{H}), 6.94-6.96(\mathrm{~m}, 3 \mathrm{H}), 6.92(\mathrm{~s}, 1 \mathrm{H})$, 6.74-6.76 (m, 2H), $4.31\left(\mathrm{q},{ }^{3} \mathrm{~J}=6.2 \mathrm{~Hz}, 1 \mathrm{H}\right), 3.30\left(\mathrm{~d},{ }^{2} \mathrm{~J}=13.1 \mathrm{~Hz}, 1 \mathrm{H}\right), 3.09\left(\mathrm{~d},{ }^{2} \mathrm{~J}=\right.$ $13.1 \mathrm{~Hz}, 1 \mathrm{H}), 2.21(\mathrm{~s}, 6 \mathrm{H}), 1.31(\mathrm{~s}, 3 \mathrm{H}), 0.91\left(\mathrm{~d},{ }^{3} \mathrm{~J}=6.3 \mathrm{~Hz}, 3 \mathrm{H}\right), 0.48(\mathrm{~s}, 3 \mathrm{H}), 0.46$ (s, 3H). ${ }^{13} \mathrm{C}$ NMR (126 MHz, $\left.\mathrm{C}_{6} \mathrm{D}_{6}\right): \delta / \mathrm{ppm}=145.6,139.1,138.6,137.2,131.8(2 \mathrm{C})$, 131.7, 130.9 (2C), 128.3, 127.7 (2C), 127.7 (2C), 126.1, 126.1, 75.9, 48.2, 46.6, 21.4, 18.9, 15.8, -0.5, -0.6. ${ }^{29}$ Si DEPT NMR (99 MHz, $\left.\mathrm{C}_{6} \mathrm{D}_{6}\right): \delta / \mathrm{ppm}=4.9$. HRMS (APCI) $\mathrm{m} / \mathrm{z}:[\mathrm{M}-\mathrm{H}]^{+}$Calcd for $\mathrm{C}_{27} \mathrm{H}_{33} \mathrm{OSi}$ 401.2301; Found 401.2307. IR (ATR): $\tilde{v} / \mathrm{cm}^{-1}=3425$, 2956, 2918, 1599, 1494, 1253, 1141, 1059, 871, 787, 699. Optical Rotation: $[\alpha]_{\mathrm{D}}=$ $+0.22\left(\right.$ c $\left.0.79, \mathrm{CHCl}_{3}\right)$.

The enantiomeric excess of (2S,3S)-3ae was determined by HPLC analysis on a chiral stationary phase after cleavage of the silyl ether according to GP3 (Daicel Chiralcel ${ }^{\circledR}$ $\mathrm{OD}-\mathrm{H}$ column, column temperature $20^{\circ} \mathrm{C}$, mobile phase: $n$-heptane:isopropanol = 95:5, flow rate: $0.6 \mathrm{~mL} / \mathrm{min}, \lambda=210 \mathrm{~nm}): \mathrm{t}_{\mathrm{R}}=13.6 \mathrm{~min}$ for $(2 S, 3 S)-1 \mathrm{a}, \mathrm{t}_{\mathrm{R}}=15.7 \mathrm{~min}$ for $(2 R, 3 R)-1$ a. 
(2R,3S)-3-Methyl-3,4-diphenylbutan-2-ol

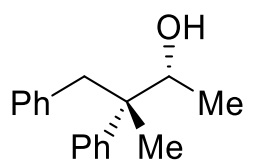

(2R,3S)-1a

$\mathrm{C}_{17} \mathrm{H}_{20} \mathrm{O}$

$240.35 \mathrm{~g} / \mathrm{mol}$

Prepared according to GP1 from alcohol rac-syn-1a (48.0 mg, 0.200 mmol, 1.00 equiv) and (3,5-dimethylphenyl)dimethylsilane (19.1 $\mathrm{mg}, 0.116 \mathrm{mmol}, 0.58$ equiv). The reaction mixture was stirred for $7 \mathrm{~d}$. Flash column chromatography using cyclohexane/ethyl acetate $(100: 0 \rightarrow 25: 1 \rightarrow 10: 1)$ afforded enantioenriched alcohol (2R,3S)-1a (14.9 mg, $0.0620 \mathrm{mmol}, 31 \%, 78 \%$ ee) as a colorless oil.

$\mathbf{R}_{\boldsymbol{f}}=0.50$ (cyclohexane/ethyl acetate 10:1). ${ }^{1} \mathbf{H}$ NMR $\left(500 \mathrm{MHz}, \mathrm{C}_{6} \mathrm{D}_{6}\right): \delta / \mathrm{ppm}=7.42-$ $7.45(\mathrm{~m}, 2 \mathrm{H}), 7.27-7.30(\mathrm{~m}, 3 \mathrm{H}), 7.12-7.23(\mathrm{~m}, 4 \mathrm{H}), 7.02-7.05(\mathrm{~m}, 2 \mathrm{H}), 3.89\left(\mathrm{~m}_{\mathrm{c}},{ }^{3} \mathrm{~J}\right.$ $=4.4 \mathrm{~Hz}, 1 \mathrm{H}), 3.02\left(\mathrm{~m}_{\mathrm{c}}, 2 \mathrm{H}\right), 1.20(\mathrm{~s}, 3 \mathrm{H}), 1.05\left(\mathrm{~d},{ }^{3} \mathrm{~J}=6.3 \mathrm{~Hz}, 3 \mathrm{H}\right) .{ }^{13} \mathrm{C}$ NMR $(126$ $\left.\mathrm{MHz}, \mathrm{C}_{6} \mathrm{D}_{6}\right): \delta / \mathrm{ppm}=144.4,138.8,130.9(2 \mathrm{C}), 128.2(2 \mathrm{C}), 128.2(2 \mathrm{C}), 127.8(2 \mathrm{C})$, 126.4, 126.2, 73.7, 47.2, 45.3, 19.1, 18.2. HRMS (APCI) m/z: $[M-H]^{+}$Calcd for $\mathrm{C}_{17} \mathrm{H}_{19} \mathrm{O}$ 239.1436; Found 239.1431. IR (ATR): $\tilde{v} / \mathrm{cm}^{-1}=3442,2974,2929,1702,1494,1449$, 1265, 1091, 767, 701. Optical Rotation: $[\alpha]_{\mathrm{D}}=+0.26\left(c 1.5, \mathrm{CH}_{2} \mathrm{Cl}_{2}\right)$.

The enantiomeric excess of (2R,3S)-1a was determined by HPLC analysis on a chiral stationary phase (Daicel Chiralcel ${ }^{\circledR} \mathrm{OD}-\mathrm{H}$ column, column temperature $20^{\circ} \mathrm{C}$, mobile phase: $n$-heptane:isopropanol $=95: 5$, flow rate: $0.6 \mathrm{~mL} / \mathrm{min}, \lambda=210 \mathrm{~nm}): \mathrm{t}_{\mathrm{R}}=15.4$ $\min$ for $(2 S, 3 R)-1 \mathbf{a}, \mathrm{t}_{\mathrm{R}}=18.4 \mathrm{~min}$ for $(2 R, 3 S)-\mathbf{1 a}$. 
(2S,3R)-(3,5-Dimethylphenyl)dimethyl((3-methyl-3,4-diphenylbutan-2yl)oxy)silane

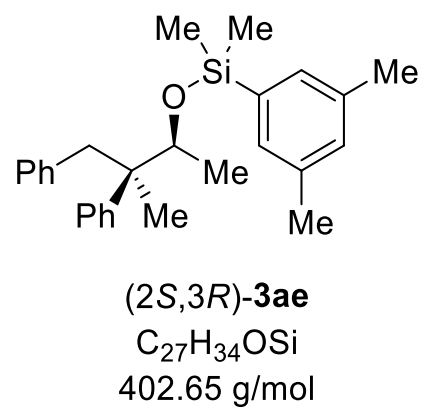

Prepared according to GP1 from alcohol rac-syn-1a (48.0 mg, 0.200 mmol, 1.00 equiv) and (3,5-dimethylphenyl)dimethylsilane (19.1 $\mathrm{mg}, 0.116 \mathrm{mmol}, 0.58$ equiv). The reaction mixture was stirred for $2 \mathrm{~d}$. Flash column chromatography using cyclohexane/ethyl acetate $(100: 0 \rightarrow 25: 1 \rightarrow 10: 1)$ afforded silyl ether $(2 S, 3 R)$-3ae (31.4 mg, $0.0780 \mathrm{mmol}, 42 \%, 77 \%$ ee) as a colorless oil.

$\mathbf{R}_{\boldsymbol{f}}=0.52$ (cyclohexane/ethyl acetate 99:1). ${ }^{1} \mathbf{H}$ NMR $\left(500 \mathrm{MHz}, \mathrm{C}_{6} \mathrm{D}_{6}\right): \delta / \mathrm{ppm}=7.41-$ $7.43(\mathrm{~m}, 2 \mathrm{H}), 7.30-7.30(\mathrm{~m}, 2 \mathrm{H}), 7.16-7.20(\mathrm{~m}, 2 \mathrm{H}), 7.08-7.12(\mathrm{~m}, 1 \mathrm{H})$, 6.95-6.98 (m, $3 \mathrm{H}), 6.88-6.89(\mathrm{~m}, 1 \mathrm{H}), 6.81-6.83(\mathrm{~m}, 2 \mathrm{H}), 3.97\left(\mathrm{q},{ }^{3} \mathrm{~J}=6.2 \mathrm{~Hz}, 1 \mathrm{H}\right), 3.37\left(\mathrm{~d},{ }^{2} \mathrm{~J}=\right.$ 13.3 Hz, 1H), $2.73\left(\mathrm{~d},{ }^{2} \mathrm{~J}=13.3 \mathrm{~Hz}, 1 \mathrm{H}\right), 2.19(\mathrm{~s}, 6 \mathrm{H}), 1.11(\mathrm{~s}, 3 \mathrm{H}), 0.99\left(\mathrm{~d},{ }^{3} J=6.2 \mathrm{~Hz}\right.$, $3 \mathrm{H}), 0.32(\mathrm{~s}, 3 \mathrm{H}), 0.30(\mathrm{~s}, 3 \mathrm{H}) \cdot{ }^{13} \mathrm{C}$ NMR (126 MHz, $\left.\mathrm{C}_{6} \mathrm{D}_{6}\right): \delta / \mathrm{ppm}=144.9,139.2$, 138.6, 137.1, 131.7 (2C), 131.5, 130.8 (2C), 128.8 (2C), 128.3 (2C), 128.1, 127.9, 127.7 (2C), 126.2, 126.1, 77.2, 47.6, 45.8, 21.4, 19.9, 19.4, -0.5, -1.0. ${ }^{29}$ Si DEPT NMR (99 MHz, $\left.\mathrm{C}_{6} \mathrm{D}_{6}\right): \delta / \mathrm{ppm}=4.0$. HRMS $(\mathrm{APCl}) \mathrm{m} / \mathrm{z}:\left[\mathrm{M}-\mathrm{C}_{10} \mathrm{H}_{15} \mathrm{OSi}\right]^{+}$Calcd for $\mathrm{C}_{17} \mathrm{H}_{19}$ 223.1487; Found 223.1481. IR (ATR): $\tilde{v} / \mathrm{cm}^{-1}=3023,2969,1599,1494,1447$, 1251, 1096, 870, 782, 699. Optical Rotation: $[\alpha]_{\mathrm{D}}=-0.18\left(c 1.5, \mathrm{CH}_{2} \mathrm{Cl}_{2}\right)$.

The enantiomeric excess of $(2 S, 3 R)$-3ae was determined by HPLC analysis on a chiral stationary phase after cleavage of the silyl ether according to GP3 (Daicel Chiralcel ${ }^{\circledR}$ $\mathrm{OD}-\mathrm{H}$ column, column temperature $20^{\circ} \mathrm{C}$, mobile phase: $n$-heptane:isopropanol = 95:5, flow rate: $0.6 \mathrm{~mL} / \mathrm{min}, \lambda=210 \mathrm{~nm}): \mathrm{t}_{\mathrm{R}}=15.4 \mathrm{~min}$ for $(2 S, 3 R)-1 \mathrm{a}, \mathrm{t}_{\mathrm{R}}=18.4 \mathrm{~min}$ for $(2 R, 3 S)-1$ a. 
(2S,3S)-Dimethyl((3-methyl-3,4-diphenylbutan-2-yl)oxy)phenylsilane<smiles>CC(O[Si](C)(C)c1ccccc1)C(C)(Cc1ccccc1)c1ccccc1</smiles>

$(2 S, 3 S)-3 a b$
$\mathrm{C}_{25} \mathrm{H}_{30} \mathrm{OSi}$
$374.60 \mathrm{~g} / \mathrm{mol}$

Prepared according to GP1 from alcohol rac-1a (48.0 mg, $0.200 \mathrm{mmol}, 1.00$ equiv) and dimethylphenylhydrosilane (15.4 $\mathrm{mg}, 0.113 \mathrm{mmol}, 0.570$ equiv). The reaction mixture was stirred for $18 \mathrm{~h}$. Flash column chromatography using cyclohexane/ethyl acetate $(100: 0 \rightarrow 25: 1 \rightarrow 10: 1)$ afforded enantioenriched silyl ether (2S,3S)-3ab (36.0 $\mathrm{mg}, 0.0961 \mathrm{mmol}, 48 \%, 66 \%$ ee) as a colorless oil.

$\mathbf{R}_{\boldsymbol{f}}=0.45$ (cyclohexane/ethyl acetate 99:1). ${ }^{1} \mathbf{H}$ NMR $\left(500 \mathrm{MHz}, \mathrm{C}_{6} \mathrm{D}_{6}\right): \delta / \mathrm{ppm}=7.67-$ $7.69(\mathrm{~m}, 2 \mathrm{H}), 7.22-7.27(\mathrm{~m}, 3 \mathrm{H}), 7.01-7.09(\mathrm{~m}, 5 \mathrm{H}), 6.94-6.95(\mathrm{~m}, 3 \mathrm{H}), 6.71-6.73(\mathrm{~m}$, $2 \mathrm{H}), 4.26\left(\mathrm{q},{ }^{3} \mathrm{~J}=6.3 \mathrm{~Hz}, 1 \mathrm{H}\right), 3.24\left(\mathrm{~d},{ }^{2} \mathrm{~J}=13.1 \mathrm{~Hz}, 1 \mathrm{H}\right), 3.03\left(\mathrm{~d},{ }^{2} \mathrm{~J}=13.1 \mathrm{~Hz}, 1 \mathrm{H}\right)$, $1.28(\mathrm{~s}, 3 \mathrm{H}), 0.86\left(\mathrm{~d},{ }^{3} \mathrm{~J}=6.3 \mathrm{~Hz}, 3 \mathrm{H}\right), 0.42(\mathrm{~s}, 3 \mathrm{H}), 0.41(\mathrm{~s}, 3 \mathrm{H}) .{ }^{13} \mathrm{C}$ NMR $(126 \mathrm{MHz}$, $\left.\mathrm{C}_{6} \mathrm{D}_{6}\right): \delta / p p m=145.5,139.0,138.7,133.9(2 \mathrm{C}), 130.9$ (2C), 129.9, 128.3, 128.1, 127.9, 127.7 (2C), 127.7 (2C), 126.1, 126.1, 75.9, 48.2, 46.6, 18.9, 15.6, -0.7, -0.7. ${ }^{29} \mathrm{Si}$ DEPT NMR (99 MHz, $\left.\mathrm{C}_{6} \mathrm{D}_{6}\right): \delta / \mathrm{ppm}=4.7$. HRMS $(\mathrm{APCl}) \mathrm{m} / \mathrm{z}:[\mathrm{M}-\mathrm{H}]^{+}$Calcd for $\mathrm{C}_{25} \mathrm{H}_{29} \mathrm{OSi}$ 373.1988; Found 373.1989. IR (ATR): $\tilde{v} / \mathrm{cm}^{-1}=3026,2975,1494,1252$, 1117, 1074, 976, 828, 689. Optical Rotation: $[\alpha]_{\mathrm{D}}=+1.3\left(c 1.0, \mathrm{C}_{6} \mathrm{H}_{6}\right)$.

The enantiomeric excess of (2S,3S)-3ab was determined by HPLC analysis on a chiral stationary phase after cleavage of the silyl ether according to GP3 (Daicel Chiralcel ${ }^{\circledR}$ OD-H column, column temperature $20^{\circ} \mathrm{C}$, mobile phase: $n$-heptane:isopropanol = 95:5, flow rate: $0.6 \mathrm{~mL} / \mathrm{min}, \lambda=210 \mathrm{~nm}): \mathrm{t}_{\mathrm{R}}=13.0 \mathrm{~min}$ for $(2 S, 3 S)-1 \mathrm{a}, \mathrm{t}_{\mathrm{R}}=15.0 \mathrm{~min}$ for $(2 R, 3 R)-1$ a. 
(2S,3S)-Methyl((3-methyl-3,4-diphenylbutan-2-yl)oxy)diphenylsilane<smiles>CC(O[Si](C)(c1ccccc1)c1ccccc1)C(C)(Cc1ccccc1)c1ccccc1</smiles>

$(2 S, 3 S)-3 a c$

$\mathrm{C}_{30} \mathrm{H}_{32} \mathrm{OSi}$

$436.67 \mathrm{~g} / \mathrm{mol}$

Prepared according to GP1 from alcohol rac-1a (48.0 mg, $0.200 \mathrm{mmol}, 1.00$ equiv) and methyldiphenylhydrosilane (26.0 $\mathrm{mg}, 0.131 \mathrm{mmol}, 0.655$ equiv). The reaction mixture was stirred for $18 \mathrm{~h}$. Flash column chromatography using cyclohexane/ethyl acetate $(100: 0 \rightarrow 25: 1 \rightarrow 10: 1)$ afforded enantioenriched silyl ether (2S,3S)-3ac (45.2 $\mathrm{mg}, 0.104 \mathrm{mmol}, 52 \%, 42 \%$ ee) as a colorless oil.

$\mathbf{R}_{\boldsymbol{f}}=0.39$ (cyclohexane/ethyl acetate 99:1). ${ }^{1} \mathbf{H}$ NMR $\left(500 \mathrm{MHz}, \mathrm{C}_{6} \mathrm{D}_{6}\right): \delta / \mathrm{ppm}=7.73-$ $7.76(\mathrm{~m}, 4 \mathrm{H}), 7.19-7.23(\mathrm{~m}, 6 \mathrm{H}), 6.99-7.06(\mathrm{~m}, 5 \mathrm{H}), 6.93-6.95(\mathrm{~m}, 3 \mathrm{H}), 6.70-6.72(\mathrm{~m}$, $2 \mathrm{H}), 4.39\left(\mathrm{q},{ }^{3} \mathrm{~J}=6.3 \mathrm{~Hz}, 1 \mathrm{H}\right), 3.34\left(\mathrm{~d},{ }^{2} \mathrm{~J}=13.1 \mathrm{~Hz}, 1 \mathrm{H}\right), 3.03\left(\mathrm{~d},{ }^{2} J=13.1 \mathrm{~Hz}, 1 \mathrm{H}\right)$, $1.35(\mathrm{~s}, 3 \mathrm{H}), 0.90\left(\mathrm{~d},{ }^{3} \mathrm{~J}=6.3 \mathrm{~Hz}, 3 \mathrm{H}\right), 0.67(\mathrm{~s}, 3 \mathrm{H}) .{ }^{13} \mathrm{C} \mathrm{NMR}\left(126 \mathrm{MHz}, \mathrm{C}_{6} \mathrm{D}_{6}\right): \delta / p p m$ = 245.4, 139.0, 137.3, 137.1, 134.9 (2C), 134.8 (2C), 134.4, 130.8 (2C), 130.4, 130.1, 128.2 (4C), 127.9, 127.7 (4C), 126.1, 126.1, 76.3, 48.3, 46.7, 18.9, 15.7, -1.9. ${ }^{29} \mathrm{Si}$ DEPT NMR (99 MHz, $\left.\mathrm{C}_{6} \mathrm{D}_{6}\right): \delta / p p m=5.2$. HRMS (APCI) m/z: $[\mathrm{M}-\mathrm{H}]^{+}$Calcd for $\mathrm{C}_{30} \mathrm{H}_{31} \mathrm{OSi}$ 435.2144; Found 435.2136. IR (ATR): $\tilde{v} / \mathrm{cm}^{-1}=3024,2976,1427,1254$, 1111, 1074, 975, 789, 737, 698. Optical Rotation: $[\alpha]_{D}=+0.43\left(c 2.7, \mathrm{C}_{6} \mathrm{H}_{6}\right)$.

The enantiomeric excess of (2S,3S)-3ac was determined by HPLC analysis on a chiral stationary phase after cleavage of the silyl ether according to GP3 (Daicel Chiralcel ${ }^{\circledR}$ OD-H column, column temperature $20^{\circ} \mathrm{C}$, mobile phase: $n$-heptane:isopropanol = 95:5, flow rate: $0.6 \mathrm{~mL} / \mathrm{min}, \lambda=210 \mathrm{~nm}): \mathrm{t}_{\mathrm{R}}=13.0 \mathrm{~min}$ for $(2 S, 3 S)-1 \mathrm{a}, \mathrm{t}_{\mathrm{R}}=15.0 \mathrm{~min}$ for $(2 R, 3 R)-1 a$. 
(2S,3S)-((3-Methyl-3,4-diphenylbutan-2-yl)oxy)triphenylsilane

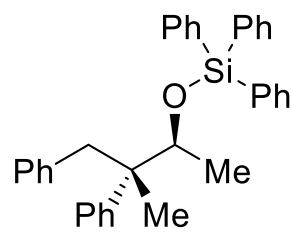

(2S,3S)-3ad

$\mathrm{C}_{35} \mathrm{H}_{34} \mathrm{OSi}$

$498.74 \mathrm{~g} / \mathrm{mol}$

Prepared according to GP1 from alcohol rac-1a (48.0 mg, $0.200 \mathrm{mmol}, 1.00$ equiv) and triphenylhydrosilane (28.3 $\mathrm{mg}, 0.109 \mathrm{mmol}, 0.545$ equiv). The reaction mixture was stirred for $18 \mathrm{~h}$. Flash column chromatography using cyclohexane/ethyl acetate $(100: 0 \rightarrow 25: 1 \rightarrow 10: 1)$ afforded silyl ether (2S,3S)-3ad (48.3 mg, $0.0968 \mathrm{mmol}, 48 \%$, $17 \%$ ee) as a colorless oil.

$\mathbf{R}_{\boldsymbol{f}}=0.35$ (cyclohexane/ethyl acetate 99:1). ${ }^{\mathbf{H}} \mathbf{N M R}\left(500 \mathrm{MHz}, \mathrm{C}_{6} \mathrm{D}_{6}\right): \delta / \mathrm{ppm}=7.85-$ $7.87(\mathrm{~m}, 6 \mathrm{H}), 7.15-7.23(\mathrm{~m}, 9 \mathrm{H}), 6.97-7.00(\mathrm{~m}, 5 \mathrm{H}), 6.92-6.94(\mathrm{~m}, 3 \mathrm{H}), 6.72-6.73(\mathrm{~m}$, $2 \mathrm{H}), 4.58\left(\mathrm{q},{ }^{3} \mathrm{~J}=6.3 \mathrm{~Hz}, 1 \mathrm{H}\right), 3.45\left(\mathrm{~d},{ }^{2} \mathrm{~J}=13.1 \mathrm{~Hz}, 1 \mathrm{H}\right), 2.98\left(\mathrm{~d},{ }^{2} \mathrm{~J}=13.1 \mathrm{~Hz}, 1 \mathrm{H}\right)$, $1.45(\mathrm{~s}, 3 \mathrm{H}), 1.00\left(\mathrm{~d},{ }^{3} \mathrm{~J}=6.3 \mathrm{~Hz}, 3 \mathrm{H}\right) .{ }^{13} \mathrm{C}$ NMR $\left(126 \mathrm{MHz}, \mathrm{C}_{6} \mathrm{D}_{6}\right): \delta / \mathrm{ppm}=145.3$, 139.0, 136.0 (6C), 135.9, 135.5, 130.8 (2C), 130.3 (2C), 128.2 (6C), 127.7 (2C), 127.6 (2C), 126.1, 126.1, 76.9, 48.5, 46.8, 19.1, 15.8. ${ }^{29}$ Si DEPT NMR (99 MHz, C6 $\left.\mathrm{D}_{6}\right)$ : $\delta / p p m=-14.0$. HRMS (APCI) $\mathrm{m} / \mathrm{z}$ : [M- $\left.\mathrm{C}_{18} \mathrm{H}_{15} \mathrm{Si}\right]^{+}$Calcd for $\mathrm{C}_{17} \mathrm{H}_{19} \mathrm{O}$ 239.1436; Found 239.1430. IR (ATR): $\tilde{v} / \mathrm{cm}^{-1}=3024,2976,1600,17,1113,1084,975,740,706$. Optical Rotation: $[\alpha]_{D}=+0.057\left(c 3.9, \mathrm{C}_{6} \mathrm{H}_{6}\right)$.

The enantiomeric excess of (2S,3S)-3ad was determined by HPLC analysis on a chiral stationary phase after cleavage of the silyl ether according to GP3 (Daicel Chiralcel ${ }^{\circledR}$ OD-H column, column temperature $20^{\circ} \mathrm{C}$, mobile phase: $n$-heptane:isopropanol = 95:5, flow rate: $0.6 \mathrm{~mL} / \mathrm{min}, \lambda=210 \mathrm{~nm}): \mathrm{t}_{\mathrm{R}}=13.0 \mathrm{~min}$ for $(2 S, 3 S)-1 \mathrm{a}, \mathrm{t}_{\mathrm{R}}=15.0 \mathrm{~min}$ for $(2 R, 3 R)-1$ a. 
(2S,3S)-Diethyl((3-methyl-3,4-diphenylbutan-2-yl)oxy)(phenyl)silane

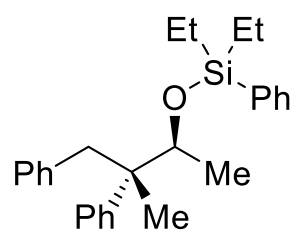

(2S,3S)-3ai

$\mathrm{C}_{27} \mathrm{H}_{34} \mathrm{OSi}$

$402.65 \mathrm{~g} / \mathrm{mol}$

Prepared according to GP1 from alcohol rac-1a (48.0 mg, $0.200 \mathrm{mmol}, 1.00$ equiv) and diethylphenylhydrosilane (18.8 $\mathrm{mg}, 0.115 \mathrm{mmol}, 0.550$ equiv). The reaction mixture was stirred for $18 \mathrm{~h}$. Flash column chromatography using cyclohexane/ethyl acetate $(100: 0 \rightarrow 25: 1 \rightarrow 10: 1)$ afforded silyl ether (2S,3S)-3ai $(44.0 \mathrm{mg}, 0.109 \mathrm{mmol}$, $55 \%, 42 \%$ ee) as a colorless oil.

$\mathbf{R}_{\boldsymbol{f}}=0.58$ (cyclohexane/ethyl acetate 99:1). ${ }^{1} \mathbf{H}$ NMR $\left(500 \mathrm{MHz}, \mathrm{C}_{6} \mathrm{D}_{6}\right): \delta / p p m=7.67-$ $7.69(\mathrm{~m}, 2 \mathrm{H}), 7.21-7.28(\mathrm{~m}, 3 \mathrm{H}), 7.00-7.07(\mathrm{~m}, 5 \mathrm{H}), 6.94-6.97(\mathrm{~m}, 3 \mathrm{H}), 6.74-6.76(\mathrm{~m}$, 2H), $4.32\left(\mathrm{q},{ }^{3} J=6.3 \mathrm{~Hz}, 1 \mathrm{H}\right), 3.33\left(\mathrm{~d},{ }^{2} \mathrm{~J}=13.0 \mathrm{~Hz}, 1 \mathrm{H}\right), 3.06\left(\mathrm{~d},{ }^{2} J=13.0 \mathrm{~Hz}, 1 \mathrm{H}\right)$, $1.30(\mathrm{~s}, 3 \mathrm{H}), 1.09-1.12(\mathrm{~m}, 6 \mathrm{H}), 0.91-0.98(\mathrm{~m}, 4 \mathrm{H}), 0.87\left(\mathrm{~d},{ }^{3} \mathrm{~J}=6.3 \mathrm{~Hz}, 3 \mathrm{H}\right) .{ }^{13} \mathrm{C} \mathrm{NMR}$ $\left(126 \mathrm{MHz}, \mathrm{C}_{6} \mathrm{D}_{6}\right): \delta / \mathrm{ppm}=145.5,139.1,136.8,134.4(2 \mathrm{C}), 130.9$ (2C), 129.8, 128.2 (2C), 128.1, 127.9, 127.7 (2C), 127.7 (2C), 126.1, 126.1, 75.9, 48.4, 46.7, 18.9, 15.5, $7.3(2 \mathrm{C}), 6.1,5.8 .{ }^{29} \mathrm{Si}$ DEPT NMR $\left(99 \mathrm{MHz}, \mathrm{C}_{6} \mathrm{D}_{6}\right): \delta / \mathrm{ppm}=5.2$. HRMS (APCI) m/z: $[\mathrm{M}-\mathrm{H}]^{+}$Calcd for $\mathrm{C}_{27} \mathrm{H}_{33} \mathrm{OSi}$ 401.2301; Found 401.2296. IR (ATR): $\tilde{v} / \mathrm{cm}^{-1}=2955$, $2875,1494,1454,1110,1085,1010,975,725,699$. Optical Rotation: $[\alpha] \mathrm{D}=+0.23$ (c 2.3, $\left.\mathrm{C}_{6} \mathrm{H}_{6}\right)$.

The enantiomeric excess of $(2 S, 3 S)$-3ai was determined by HPLC analysis on a chiral stationary phase after cleavage of the silyl ether according to GP3 (Daicel Chiralcel ${ }^{\circledR}$ OD-H column, column temperature $20^{\circ} \mathrm{C}$, mobile phase: $n$-heptane:isopropanol = 95:5, flow rate: $0.6 \mathrm{~mL} / \mathrm{min}, \lambda=210 \mathrm{~nm}): \mathrm{t}_{\mathrm{R}}=13.6 \mathrm{~min}$ for $(2 S, 3 S)-1 \mathrm{a}, \mathrm{t}_{\mathrm{R}}=15.6 \mathrm{~min}$ for $(2 R, 3 R)-1$ a. 
(2S,3S)-Mesityldimethyl((3-methyl-3,4-diphenylbutan-2-yl)oxy)silane

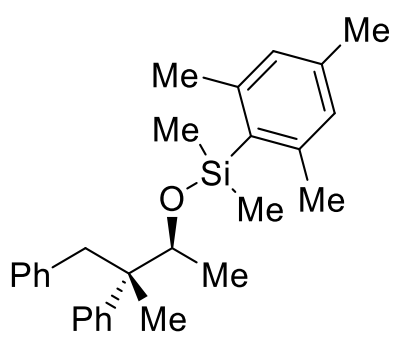

$$
\begin{gathered}
(2 \mathrm{~S}, 3 \mathrm{~S})-3 \mathrm{af} \\
\mathrm{C}_{28} \mathrm{H}_{36} \mathrm{OSi} \\
416.68 \mathrm{~g} / \mathrm{mol}
\end{gathered}
$$

Prepared according to GP1 from alcohol rac-1a (48.0 mg, $0.200 \mathrm{mmol}, 1.00$ equiv) and mesityldimethylhydrosilane (19.6 mg, $0.110 \mathrm{mmol}, 0.550$ equiv). The reaction mixture was stirred for $42 \mathrm{~h}$. Flash column chromatography using cyclohexane/ethyl acetate $(100: 0 \rightarrow 25: 1 \rightarrow 10: 1)$ afforded silyl ether (2S,3S)-3af (31.8 mg, $0.0763 \mathrm{mmol}$, $38 \%, 31 \%$ ee) as a colorless oil.

$\boldsymbol{R}_{\boldsymbol{f}}=0.45$ (cyclohexane/ethyl acetate 99:1). ${ }^{1} \mathbf{H}$ NMR $\left(500 \mathrm{MHz}, \mathrm{C}_{6} \mathrm{D}_{6}\right): \delta / \mathrm{ppm}=7.04-$ $7.10(\mathrm{~m}, 5 \mathrm{H}), 6.93-6.95(\mathrm{~m}, 3 \mathrm{H}), 6.79(\mathrm{~s}, 2 \mathrm{H}), 6.69-6.71(\mathrm{~m}, 2 \mathrm{H}), 4.31\left(\mathrm{q},{ }^{3} \mathrm{~J}=6.2 \mathrm{~Hz}\right.$, $1 \mathrm{H}), 3.25\left(\mathrm{~d},{ }^{2} \mathrm{~J}=13.2 \mathrm{~Hz}, 1 \mathrm{H}\right), 3.08\left(\mathrm{~d},{ }^{2} \mathrm{~J}=13.1 \mathrm{~Hz}, 1 \mathrm{H}\right), 2.56(\mathrm{~s}, 6 \mathrm{H}), 2.12(\mathrm{~s}, 3 \mathrm{H})$, $1.28(\mathrm{~s}, 3 \mathrm{H}), 0.86\left(\mathrm{~d},{ }^{3} \mathrm{~J}=6.2 \mathrm{~Hz}, 3 \mathrm{H}\right), 0.55(\mathrm{~s}, 3 \mathrm{H}), 0.51(\mathrm{~s}, 3 \mathrm{H}) .{ }^{13} \mathrm{C}$ NMR $(126 \mathrm{MHz}$, $\left.\mathrm{C}_{6} \mathrm{D}_{6}\right): \delta / \mathrm{ppm}=145.5,143.9,139.0,138.8,133.0,130.9(2 \mathrm{C}), 129.6,129.5,128.5$, 128.3 (2C), 128.1 (2C), 127.9 (2C), 127.7 (2C), $127.6(2 \mathrm{C}), 126.1,73.3,47.5,46.3$, 30.1, 24.6, 24.5, 21.0, 18.7, 16.0, 5.5. $\left.{ }^{29} \mathrm{Si} \mathrm{DEPT} \mathrm{NMR} \mathrm{(99} \mathrm{MHz,} \mathrm{C}_{6} \mathrm{D}_{6}\right): \delta / \mathrm{ppm}=-1.4$. HRMS (APCI) m/z: [M-H] $]^{+}$Calcd for $\mathrm{C}_{28} \mathrm{H}_{35} \mathrm{OSi}$ 415.2457; Found 415.2463. IR (ATR): $\tilde{v} / \mathrm{cm}^{-1}=2973,2922,1603,1494,1450,1257,1072,1032,797,700$. Optical Rotation: $[\alpha]_{D}=+0.13\left(c 1.5, \mathrm{C}_{6} \mathrm{H}_{6}\right)$.

The enantiomeric excess of (2S,3S)-3af was determined by HPLC analysis on a chiral stationary phase after cleavage of the silyl ether according to GP3 (Daicel Chiralcel ${ }^{\circledR}$ OD-H column, column temperature $20^{\circ} \mathrm{C}$, mobile phase: $n$-heptane:isopropanol = 95:5, flow rate: $0.6 \mathrm{~mL} / \mathrm{min}, \lambda=210 \mathrm{~nm}): \mathrm{t}_{\mathrm{R}}=13.6 \mathrm{~min}$ for $(2 S, 3 S)-1 \mathrm{a}, \mathrm{t}_{\mathrm{R}}=15.6 \mathrm{~min}$ for $(2 R, 3 R)-1$ a. 
(2S,3S)-Dimethyl((3-methyl-3,4-diphenylbutan-2-yl)oxy)(naphthalen-1-yl)silane<smiles>C[C@H](O[Si](C)(C)c1cccc2ccccc12)[C@H](C)c1ccccc1</smiles>

$$
\begin{gathered}
(2 \mathrm{~S}, 3 \mathrm{~S})-3 \mathrm{ak} \\
\mathrm{C}_{29} \mathrm{H}_{32} \mathrm{OSi} \\
424.66 \mathrm{~g} / \mathrm{mol}
\end{gathered}
$$

Prepared according to GP1 from alcohol rac-1a $(48.0 \mathrm{mg}, 0.200 \mathrm{mmol}, 1.00$ equiv) and dimethyl(1-naphthyl)hydrosilane (20.5 mg, $0.110 \mathrm{mmol}, 0.550$ equiv). The reaction mixture was stirred for $18 \mathrm{~h}$. Flash column chromatography using cyclohexane/ethyl acetate $(100: 0 \rightarrow 25: 1 \rightarrow 10: 1)$ afforded silyl ether (2S,3S)-3ak (42.8 mg, $0.101 \mathrm{mmol}, 51 \%, 42 \%$ ee) as a colorless oil.

$\mathbf{R}_{\boldsymbol{f}}=0.26$ (cyclohexane). ${ }^{1} \mathrm{H}$ NMR $\left(500 \mathrm{MHz}, \mathrm{C}_{6} \mathrm{D}_{6}\right): \delta / p p m=8.65\left(\mathrm{~d},{ }^{2} \mathrm{~J}=8.4 \mathrm{~Hz}, 1 \mathrm{H}\right)$, 7.82-7.84 (m, 1H), 7.67-7.71 (m, 2H), 7.41-7.46 (m, 1H), 7.28-7.34 (m, 2H), 6.99$7.07(\mathrm{~m}, 5 \mathrm{H}), 6.92-6.95(\mathrm{~m}, 3 \mathrm{H}), 6.67-6.69(\mathrm{~m}, 2 \mathrm{H}), 4.34\left(\mathrm{q},{ }^{3} \mathrm{~J}=6.2 \mathrm{~Hz}, 1 \mathrm{H}\right), 3.29$ (d, $\left.{ }^{2} J=13.0 \mathrm{~Hz}, 1 \mathrm{H}\right), 3.08\left(\mathrm{~d},{ }^{2} \mathrm{~J}=13.0 \mathrm{~Hz}, 1 \mathrm{H}\right), 1.31(\mathrm{~s}, 3 \mathrm{H}), 0.84\left(\mathrm{~d},{ }^{3} \mathrm{~J}=6.2 \mathrm{~Hz}, 3 \mathrm{H}\right)$, $0.58(\mathrm{~s}, 3 \mathrm{H}), 0.56(\mathrm{~s}, 3 \mathrm{H}) \cdot{ }^{13} \mathrm{C}$ NMR (126 MHz, $\left.\mathrm{C}_{6} \mathrm{D}_{6}\right): \delta / \mathrm{ppm}=145.4,139.0,137.5$, 137.2, 134.1, 133.7, 130.8, 130.8 (2C), 129.4, 129.2, 128.3 (2C), 127.7 (2C), 127.6 (2C), 126.1, 126.1, 126.1, 125.9, 125.3, 76.5, 48.2, 46.5, 18.8, 15.8, 0.5, 0.5. ${ }^{29} \mathrm{Si}$ DEPT NMR (99 MHz, $\left.\mathrm{C}_{6} \mathrm{D}_{6}\right): \delta / p p m=6.0$. HRMS $(\mathrm{APCl}) \mathrm{m} / \mathrm{z}:[\mathrm{M}-\mathrm{H}]^{+}$Calcd for $\mathrm{C}_{29} \mathrm{H}_{31} \mathrm{OSi}$ 423.2144; Found 423.2134. IR (ATR): $\tilde{v} / \mathrm{cm}^{-1}=3028,2975,1496,1254$, 1104, 1085, 837, 783, 700. Optical Rotation: $[\alpha]_{D}=+0.12\left(c 2.9, \mathrm{C}_{6} \mathrm{H}_{6}\right)$.

The enantiomeric excess of $(2 S, 3 S)$-3ak was determined by HPLC analysis on a chiral stationary phase after cleavage of the silyl ether according to GP3 (Daicel Chiralcel ${ }^{\circledR}$ OD-H column, column temperature $20^{\circ} \mathrm{C}$, mobile phase: $n$-heptane:isopropanol = 95:5, flow rate: $0.6 \mathrm{~mL} / \mathrm{min}, \lambda=210 \mathrm{~nm}): \mathrm{t}_{\mathrm{R}}=13.6 \mathrm{~min}$ for $(2 S, 3 S)-1 \mathrm{a}, \mathrm{t}_{\mathrm{R}}=15.6 \mathrm{~min}$ for $(2 R, 3 R)-1$ a. 
(2S,3S)-Dimethyl((3-methyl-3,4-diphenylbutan-2-yl)oxy)(naphthalen-2-yl)silane<smiles>CC(O[Si](C)(C)c1ccc2ccccc2c1)C(C)(Cc1ccccc1)c1ccccc1</smiles>

$$
\begin{gathered}
(2 S, 3 S)-3 a h \\
\mathrm{C}_{29} \mathrm{H}_{32} \mathrm{OSi} \\
424.66 \mathrm{~g} / \mathrm{mol}
\end{gathered}
$$

Prepared according to GP1 from alcohol rac-1a (48.0 mg, $0.200 \mathrm{mmol}, 1.00$ equiv) and dimethyl(2-naphthyl)hydrosilane (20.5 mg, $0.110 \mathrm{mmol}, 0.550$ equiv). The reaction mixture was stirred for $18 \mathrm{~h}$. Flash column chromatography using cyclohexane/ethyl acetate $(100: 0 \rightarrow 25: 1 \rightarrow 10: 1)$ afforded silyl ether (2S,3S)-3ah (35.3 mg, $0.0831 \mathrm{mmol}, 42 \%, 73 \%$ ee) as a colorless oil.

$\mathbf{R}_{\boldsymbol{f}}=0.36$ (cyclohexane/ethyl acetate 99:1). ${ }^{1} \mathbf{H}$ NMR $\left(500 \mathrm{MHz}, \mathrm{C}_{6} \mathrm{D}_{6}\right): \delta / p p m=8.23$ (s, 1H), 7.78-7.80 (m, 1H), 7.73-7.75 (m, 2H), 7.64-7.66 (m, 1H), 7.27-7.29 (m, 2H), 7.06-7.07 (m, 4H), 7.00-7.05 (m, 1H), 6.93-6.96 (m, 3H), 6.74-6.76 (m, 2H), 4.32 (q, $\left.{ }^{3} J=6.2 \mathrm{~Hz}, 1 \mathrm{H}\right), 3.31\left(\mathrm{~d},{ }^{2} \mathrm{~J}=13.1 \mathrm{~Hz}, 1 \mathrm{H}\right), 3.10\left(\mathrm{~d},{ }^{2} \mathrm{~J}=13.1 \mathrm{~Hz}, 1 \mathrm{H}\right), 1.32(\mathrm{~s}, 3 \mathrm{H})$, $0.90\left(\mathrm{~d},{ }^{3} \mathrm{~J}=6.2 \mathrm{~Hz}, 3 \mathrm{H}\right), 0.51(\mathrm{~s}, 3 \mathrm{H}), 0.50(\mathrm{~s}, 3 \mathrm{H}) .{ }^{13} \mathrm{C} \mathrm{NMR}\left(126 \mathrm{MHz}, \mathrm{C}_{6} \mathrm{D}_{6}\right): \delta / p p m$ $=145.4,139.0$ (2C), 136.3, 134.8, 134.6, 133.6, 130.9, 130.1, 128.6, 128.3, 128.1 (2C), 127.7 (2C), 127.7 (2C), 127.6, 126.8, 126.3, 126.1, 126.1, 76.0, 48.2, 46.6, 18.9, 15.8, -0.6, -0.6. ${ }^{29}$ Si DEPT NMR (99 MHz, $\left.\mathrm{C}_{6} \mathrm{D}_{6}\right): \delta / \mathrm{ppm}=4.9$. HRMS (LIFDI) m/z: $\left[\mathrm{M}-\mathrm{C}_{15} \mathrm{H}_{15}\right]^{+}$Calcd for $\mathrm{C}_{14} \mathrm{H}_{17} \mathrm{OSi} 229.1049$; Found 229.1045. IR (ATR): $\tilde{v} / \mathrm{cm}^{-1}=2975$, 2918, 1495, 1253, 1086, 976, 859, 815, 783, 700. Optical Rotation: $[\alpha]_{\mathrm{D}}=+0.42(\mathrm{c}$ $\left.1.1, \mathrm{C}_{6} \mathrm{H}_{6}\right)$.

The enantiomeric excess of $(2 S, 3 S)$-3ah was determined by HPLC analysis on a chiral stationary phase after cleavage of the silyl ether according to GP3 (Daicel Chiralcel ${ }^{\circledR}$ $\mathrm{OD}-\mathrm{H}$ column, column temperature $20^{\circ} \mathrm{C}$, mobile phase: $n$-heptane:isopropanol = 95:5, flow rate: $0.6 \mathrm{~mL} / \mathrm{min}, \lambda=210 \mathrm{~nm}): \mathrm{t}_{\mathrm{R}}=13.6 \mathrm{~min}$ for $(2 S, 3 S)-1 \mathrm{a}, \mathrm{t}_{\mathrm{R}}=15.6 \mathrm{~min}$ for $(2 R, 3 R)-1$ a. 
(2S,3S)-(4-(tert-Butyl)phenyl)dimethyl((3-methyl-3,4-diphenylbutan-2yl)oxy)silane<smiles>CC(O[Si](C)(C)c1ccc(C(C)(C)C)cc1)C(C)(Cc1ccccc1)c1ccccc1</smiles>

$$
\begin{gathered}
(2 S, 3 S)-3 a g \\
\mathrm{C}_{29} \mathrm{H}_{38} \mathrm{OSi} \\
430.71 \mathrm{~g} / \mathrm{mol}
\end{gathered}
$$

Prepared according to GP1 from alcohol rac-1a (48.0 mg, $0.200 \mathrm{mmol}, 1.00$ equiv) and 4-(tert-butylphenyl)dimethylhydrosilane $(21.1 \mathrm{mg}, 0.110 \mathrm{mmol}, 0.550$ equiv). The reaction mixture was stirred for $42 \mathrm{~h}$. Flash column chromatography using cyclohexane/ethyl acetate $(100: 0 \rightarrow 25: 1 \rightarrow 10: 1)$ afforded silyl ether (2S,3S)-3ag (33.9 mg, $0.0787 \mathrm{mmol}, 39 \%, 67 \%$ ee) as a colorless oil.

$\mathbf{R}_{\boldsymbol{f}}=0.39$ (cyclohexane/ethyl acetate 99:1). ${ }^{1} \mathbf{H}$ NMR $\left(500 \mathrm{MHz}, \mathrm{C}_{6} \mathrm{D}_{6}\right): \delta / \mathrm{ppm}=7.70$ $7.72(\mathrm{~m}, 2 \mathrm{H}), 7.39-7.40(\mathrm{~m}, 2 \mathrm{H}), 7.04-7.07(\mathrm{~m}, 4 \mathrm{H}), 7.00-7.04(\mathrm{~m}, 1 \mathrm{H})$, 6.94-6.95 (m, $3 \mathrm{H}), 6.72-6.74(\mathrm{~m}, 2 \mathrm{H}), 4.31\left(\mathrm{q},{ }^{3} \mathrm{~J}=6.2 \mathrm{~Hz}, 1 \mathrm{H}\right), 3.29\left(\mathrm{~d},{ }^{2} \mathrm{~J}=13.1 \mathrm{~Hz}, 1 \mathrm{H}\right), 3.07$ (d, $2 J=13.1 \mathrm{~Hz}, 1 \mathrm{H}), 1.30(\mathrm{~s}, 3 \mathrm{H}), 1.23(\mathrm{~s}, 9 \mathrm{H}), 0.90\left(\mathrm{~d},{ }^{3} \mathrm{~J}=6.2 \mathrm{~Hz}, 3 \mathrm{H}\right), 0.47(\mathrm{~s}, 3 \mathrm{H})$, $0.46(\mathrm{~s}, 3 \mathrm{H}) .{ }^{13} \mathrm{C}$ NMR (126 MHz, $\left.\mathrm{C}_{6} \mathrm{D}_{6}\right): \delta / \mathrm{ppm}=152.6,145.5,139.1,135.4,134.0$ (2C), 130.9 (2C), 128.3 (2C), 127.7 (2C), 127.6 (2C), 126.1, 126.1, 125.2 (2C), 75.9, 48.2, 46.7, 34.7, 31.3 (3C), 18.9, 15.6, -0.5, -0.5. ${ }^{29}$ Si DEPT NMR (99 MHz, C6 $\mathrm{D}_{6}$ ): $\delta / p p m=-1.4$. HRMS $(\mathrm{APCl}) \mathrm{m} / \mathrm{z}:[\mathrm{M}-\mathrm{H}]^{+}$Calcd for $\mathrm{C}_{29} \mathrm{H}_{37} \mathrm{OSi}$ 429.2614; Found 429.2605. IR (ATR): $\tilde{v} / \mathrm{cm}^{-1}=3029,2961,1600,1494,1454,1253,1087,827,788$, 678. Optical Rotation: $[\alpha]_{D}=+0.57\left(c 1.3, \mathrm{C}_{6} \mathrm{H}_{6}\right)$.

The enantiomeric excess of (2S,3S)-3ag was determined by HPLC analysis on a chiral stationary phase after cleavage of the silyl ether according to GP3 (Daicel Chiralcel ${ }^{\circledR}$ $\mathrm{OD}-\mathrm{H}$ column, column temperature $20^{\circ} \mathrm{C}$, mobile phase: $n$-heptane:isopropanol = 95:5, flow rate: $0.6 \mathrm{~mL} / \mathrm{min}, \lambda=210 \mathrm{~nm}): \mathrm{t}_{\mathrm{R}}=14.0 \mathrm{~min}$ for $(2 S, 3 S)-1 \mathrm{a}, \mathrm{t}_{\mathrm{R}}=16.1 \mathrm{~min}$ for $(2 R, 3 R)-1 \mathbf{a}$. 
(2S,3S)-(4-Methoxy-3,5-dimethylphenyl)dimethyl((3-methyl-3,4-diphenylbutan-

\section{2-yl)oxy)silane}

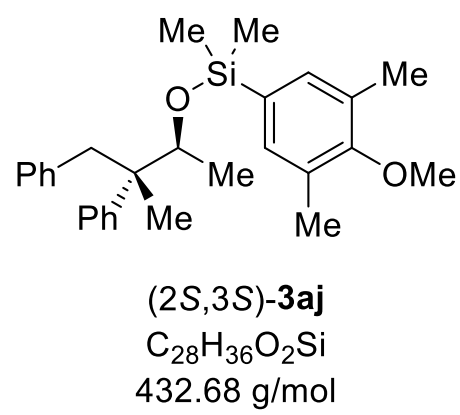

Prepared according to GP1 from alcohol rac-1a (48.0 mg, $0.200 \mathrm{mmol}, 1.00$ equiv) and 4-methoxy-3,5-dimethylphenylhydrosilane (21.4 mg, $0.110 \mathrm{mmol}, 0.550$ equiv). The reaction mixture was stirred for $18 \mathrm{~h}$. Flash column chromatography using cyclohexane/ethyl acetate $(100: 0 \rightarrow 25: 1 \rightarrow 10: 1)$ afforded silyl ether (2S,3S)-3aj (44.1 $\mathrm{mg}, 0.101 \mathrm{mmol}, 51 \%, 73 \%$ ee) as a colorless oil.

$\mathbf{R}_{\boldsymbol{f}}=0.10$ (cyclohexane). ${ }^{1} \mathrm{H}$ NMR $\left(500 \mathrm{MHz}, \mathrm{C}_{6} \mathrm{D}_{6}\right): \delta / \mathrm{ppm}=7.37$ (s, 2H), 7.09-7.10 $(\mathrm{m}, 2 \mathrm{H}), 6.95-7.05(\mathrm{~m}, 6 \mathrm{H}), 6.75-6.77(\mathrm{~m}, 2 \mathrm{H}), 3.92\left(\mathrm{q},{ }^{3} \mathrm{~J}=6.4 \mathrm{~Hz}, 1 \mathrm{H}\right), 3.38(\mathrm{~s}, 3 \mathrm{H})$, $3.08\left(\mathrm{~d},{ }^{2} \mathrm{~J}=13.1 \mathrm{~Hz}, 1 \mathrm{H}\right), 3.04\left(\mathrm{~d},{ }^{2} \mathrm{~J}=13.1 \mathrm{~Hz}, 1 \mathrm{H}\right), 2.23(\mathrm{~s}, 6 \mathrm{H}), 1.16(\mathrm{~s}, 3 \mathrm{H}), 0.74$ $\left(\mathrm{d},{ }^{3} \mathrm{~J}=6.4 \mathrm{~Hz}, 3 \mathrm{H}\right), 0.42(\mathrm{~s}, 6 \mathrm{H}) .{ }^{13} \mathrm{C}$ NMR (126 MHz, $\left.\mathrm{C}_{6} \mathrm{D}_{6}\right): \delta / \mathrm{ppm}=159.0,145.4$, 139.0, 135.0, 134.4 (2C), 130.9 (2C), 130.3, 128.3 (2C), 128.2, 127.9, 127.7 (2C), 127.6 (2C), 126.2, 73.3, 59.1, 47.5, 46.3, 18.7, 16.2, 16.0 (2C), 1.2 (2C). ${ }^{29}$ Si DEPT NMR (99 MHz, $\left.\mathrm{C}_{6} \mathrm{D}_{6}\right): \delta / p p m=-1.5$. HRMS $(\mathrm{APCl}) \mathrm{m} / \mathrm{z}:[\mathrm{M}-\mathrm{H}]^{+}$Calcd for $\mathrm{C}_{28} \mathrm{H}_{35} \mathrm{OSi}$ 431.2406; Found 431.2396. IR (ATR): $\tilde{v} / \mathrm{cm}^{-1}=3465,2953,1449,1253,1122,1057$, 1015, 903, 820, 789, 701. Optical Rotation: $[\alpha]_{\mathrm{D}}=+0.42\left(\right.$ c 1.7, $\left.\mathrm{C}_{6} \mathrm{H}_{6}\right)$.

The enantiomeric excess of (2S,3S)-3aj was determined by HPLC analysis on a chiral stationary phase after cleavage of the silyl ether according to GP3 (Daicel Chiralcel ${ }^{\circledR}$ OD-H column, column temperature $20^{\circ} \mathrm{C}$, mobile phase: $n$-heptane:isopropanol = 95:5, flow rate: $0.6 \mathrm{~mL} / \mathrm{min}, \lambda=210 \mathrm{~nm}): \mathrm{t}_{\mathrm{R}}=14.0 \mathrm{~min}$ for $(2 S, 3 S)-1 \mathrm{a}, \mathrm{t}_{\mathrm{R}}=16.1 \mathrm{~min}$ for $(2 R, 3 R)-1 a$. 
(2S,3S)-Tribenzyl((3-methyl-3,4-diphenylbutan-2-yl)oxy)silane<smiles>C[C@H](O[Si](Cc1ccccc1)(Cc1ccccc1)Cc1ccccc1)C(C)(c1ccccc1)c1ccccc1</smiles>

(2S,3S)-3al

$\mathrm{C}_{38} \mathrm{H}_{40} \mathrm{OSi}$

$540.82 \mathrm{~g} / \mathrm{mol}$

Prepared according to GP1 from alcohol rac-1a (48.0 mg, $0.200 \mathrm{mmol}, 1.00$ equiv) and tribenzylhydrosilane (33.2 $\mathrm{mg}, 0.110 \mathrm{mmol}, 0.550$ equiv). The reaction mixture was stirred for $90 \mathrm{~h}$. Flash column chromatography using cyclohexane/ethyl acetate $(100: 0 \rightarrow 25: 1 \rightarrow 10: 1)$ afforded silyl ether (2S,3S)-3al (54.9 mg, $0.102 \mathrm{mmol}, 51 \%$, $18 \%$ ee) as a colorless oil.

$\boldsymbol{R}_{\boldsymbol{f}}=0.13$ (cyclohexane). ${ }^{1} \mathrm{H}$ NMR $\left(500 \mathrm{MHz}, \mathrm{C}_{6} \mathrm{D}_{6}\right): \delta / \mathrm{ppm}=7.32-7.35(\mathrm{~m}, 9 \mathrm{H}), 7.28-$ $7.31(\mathrm{~m}, 3 \mathrm{H}), 7.20-7.26(\mathrm{~m}, 11 \mathrm{H}), 7.14-7.18(\mathrm{~m}, 2 \mathrm{H}), 4.40\left(\mathrm{q},{ }^{3} \mathrm{~J}=6.3 \mathrm{~Hz}, 1 \mathrm{H}\right), 3.16$ $\left(\mathrm{d},{ }^{2} \mathrm{~J}=13.0 \mathrm{~Hz}, 1 \mathrm{H}\right), 2.98\left(\mathrm{~d},{ }^{2} \mathrm{~J}=13.0 \mathrm{~Hz}, 1 \mathrm{H}\right), 2.45(\mathrm{~s}, 6 \mathrm{H}), 1.36(\mathrm{~s}, 3 \mathrm{H}), 0.92$ (d, ${ }^{3} \mathrm{~J}$ $=6.3 \mathrm{~Hz}, 3 \mathrm{H}) \cdot{ }^{13} \mathrm{C}$ NMR $\left(126 \mathrm{MHz}, \mathrm{C}_{6} \mathrm{D}_{6}\right): \delta / \mathrm{ppm}=145.4,139.0,138.8(2 \mathrm{C}), 130.9$ (2C), 129.4 (6C), 128.7 (6C), 127.7 (2C), 127.6 (2C), 126.2, 126.1, 125.0 (3C), 76.3, 48.3, 46.1, 24.5 (3C), 19.0, 15.2. ${ }^{29}$ Si DEPT NMR (99 MHz, $\left.\mathrm{C}_{6} \mathrm{D}_{6}\right): \delta / \mathrm{ppm}=1.8$. HRMS (APCl) m/z: [M-H] $]^{+}$Calcd for $\mathrm{C}_{38} \mathrm{H}_{39} \mathrm{OSi} 539.2770$; Found 539.2764. IR (ATR): $\tilde{v} / \mathrm{cm}^{-}$ $1=3058,3024,1599,1492,1451,1208,1074,976,820,775$. Optical Rotation: $[\alpha]_{D}$ $=+0.12\left(c 1.4, \mathrm{C}_{6} \mathrm{H}_{6}\right)$.

The enantiomeric excess of (2S,3S)-3al was determined by HPLC analysis on a chiral stationary phase after cleavage of the silyl ether according to GP3 (Daicel Chiralcel ${ }^{\circledR}$ OD-H column, column temperature $20^{\circ} \mathrm{C}$, mobile phase: $n$-heptane:isopropanol = 95:5, flow rate: $0.6 \mathrm{~mL} / \mathrm{min}, \lambda=210 \mathrm{~nm}): \mathrm{t}_{\mathrm{R}}=14.0 \mathrm{~min}$ for $(2 S, 3 S)-1 \mathrm{a}, \mathrm{t}_{\mathrm{R}}=16.1 \mathrm{~min}$ for $(2 R, 3 R)-1$ a. 


\subsection{Kinetic Resolution}

rac-anti/syn-3-Methyl-3,4-diphenylbutan-2-ol

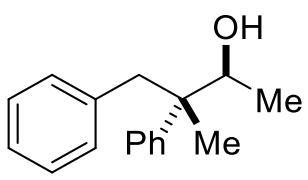

rac-anti-1a

$\mathrm{C}_{17} \mathrm{H}_{20} \mathrm{O}$

$\mathrm{M}=240.35 \mathrm{~g} / \mathrm{mol}$<smiles>C[C@H](O)[C@](C)(Cc1ccccc1)c1ccccc1</smiles>

rac-syn-1a

$\mathrm{C}_{17} \mathrm{H}_{20} \mathrm{O}$

$\mathrm{M}=240.35 \mathrm{~g} / \mathrm{mol}$

Prepared according to GP1 from 2-methyl-2,3-diphenylpropanal (S2, 3.80 g, 17.0 mmol, 1.00 equiv.) using MeMgCl (20\% in THF, $3.76 \mathrm{~mL}, 17.0 \mathrm{mmol}, 1.00$ equiv.). The reaction mixture was stirred for $2 \mathrm{~h}$. Automatic flash column chromatography using cyclohexane/ethyl acetate $(100: 0 \rightarrow 0: 100)$ afforded a mixture of the diastereomers rac-anti-1a and rac-syn-1a (3.10 g, $12.9 \mathrm{mmol}, 76 \%$, d.r. = 82:18) as a colorless oil.

$\mathbf{R}_{\boldsymbol{f}}=0.42$ for rac-anti-1a, 0.50 for rac-syn-1a (cyclohexane/ethyl acetate 10:1). ${ }^{1} \mathbf{H}$ NMR $\left(500 \mathrm{MHz}, \mathrm{C}_{6} \mathrm{D}_{6}\right): \delta / \mathrm{ppm}=7.38-7.42(\mathrm{~m}, 2 \mathrm{H}$, rac-syn-1a), 7.30-7.35 (m, 2H, rac-syn1a), 7.26-7.31 (m, 2H, rac-anti-1a), 7.21-7.23 (m, 2H, rac-anti-1a), 7.19-7.21 (m, 1H, rac-anti-1a), 7.18-7.19 (m, 1H, rac-syn-1a), 7.09-7.10 (m, 2H, rac-syn-1a), 7.07-7.09 ( $\mathrm{m}, 2 \mathrm{H}$, rac-anti-1a), 7.03-7.07 ( $\mathrm{m}, 1 \mathrm{H}$, rac-anti-1a), 6.80-6.85 (m, 2H, rac-syn-1a), 6.70-6.75 (m, 2H, rac-anti-1a), $4.18\left(\mathrm{q},{ }^{3} \mathrm{~J}=6.3 \mathrm{~Hz}, 1 \mathrm{H}\right.$, rac-anti-1a), $4.06\left(\mathrm{q},{ }^{3} \mathrm{~J}=6.3\right.$ $\mathrm{Hz}, 1 \mathrm{H}$, rac-syn-1a), 3.18 (d, ${ }^{2} \mathrm{~J}=13.6 \mathrm{~Hz}, 1 \mathrm{H}$, rac-anti-1a), 3.08 (d, ${ }^{2} \mathrm{~J}=13.6 \mathrm{~Hz}, 1 \mathrm{H}$, rac-syn-1a), 3.05 (d, ${ }^{2} \mathrm{~J}=13.6 \mathrm{~Hz}, 1 \mathrm{H}$, rac-anti-1a), $2.98\left(\mathrm{~d},{ }^{2} \mathrm{~J}=13.6 \mathrm{~Hz}, 1 \mathrm{H}\right.$, rac-syn1a), 1.53 (s, 1H, OH), 1.23 (s, 3H, rac-anti-1a), 1.19 (s, 3H, rac-syn-1a), 1.16 (d, $3 \mathrm{~J}=$ $6.3 \mathrm{~Hz}, 3 \mathrm{H}$, rac-syn-1a), 0.97 (d, ${ }^{3} \mathrm{~J}=6.3 \mathrm{~Hz}, 3 \mathrm{H}, \mathrm{H}-1$, rac-anti-1a). ${ }^{13} \mathrm{C}$ NMR (126 MHz, $\left.\mathrm{CDCl}_{3}\right): \delta / p p m=144.8$ (rac-anti-1a), 143.8 (rac-syn-1a), 138.6 (rac-anti-1a), 138.3 (rac-syn-1a), 130.6 (2C, rac-syn-1a), 130.5 (2C, rac-anti-1a), 128.3 (2C, rac-syn-1a), 128.1 (2C, rac-anti-1a), 127.8 (2C, rac-syn-1a), 127.5 (2C, rac-anti-1a), 127.6 (2C, rac-syn-1a), 127.4 (2C, rac-anti-1a), 126.5 (rac-syn-1a), 126.2 (rac-anti-1a), 126.0 (rac-syn-1a), 125.9 (rac-anti-1a), 74.1 (rac-anti-1a), 73.9 (rac-syn-1a), 47.3, 45.8 (racanti-1a), 45.1 (rac-syn-1a), 18.8 (rac-anti-1a), 18.3 (rac-syn-1a), 17.9 (rac-syn-1a), 16.4 (rac-anti-1a). HRMS (APCI) m/z: [M-OH] ${ }^{+}$Calcd for $\mathrm{C}_{17} \mathrm{H}_{19}$ 223.1487; Found 223.1480 . 
(2S,3S)-(3,5-Dimethylphenyl)dimethyl((3-methyl-3,4-diphenylbutan-2yl)oxy)silane

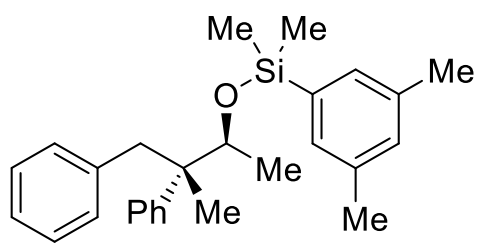

$$
\begin{gathered}
(2 \mathrm{~S}, 3 \mathrm{~S})-3 \mathrm{ae} \\
\mathrm{C}_{27} \mathrm{H}_{34} \mathrm{OSi} \\
\mathrm{M}=402.65 \mathrm{~g} / \mathrm{mol}
\end{gathered}
$$

Prepared according to GP1 from rac-anti/syn-1a (48.1 mg, $0.200 \mathrm{mmol}, 1.00$ equiv.) and (3,5-dimethylphenyl)dimethylsilane (13.5 $\mathrm{mg}, 0.0800 \mathrm{mmol}, 0.400$ equiv.). The reaction mixture was stirred at $-10^{\circ} \mathrm{C}$ for $42 \mathrm{~h}$. Flash column chromatography using cyclohexane/ethyl acetate $(30: 1 \rightarrow 7: 1)$ afforded silyl ether $(2 S, 3 S)$-3ae $(26.0 \mathrm{mg}$, $0.0640 \mathrm{mmol}, 32 \%$, d.r. $=97: 3,85 \%$ ee) as a yellow oil.

$\mathbf{R}_{\boldsymbol{f}}=0.92$ (cyclohexane/ethyl acetate 10:1). ${ }^{1} \mathrm{H}$ NMR (500 MHz, $\left.\mathrm{C}_{6} \mathrm{D}_{6}\right): \delta / p p m=7.45$ $\left(\mathrm{m}_{\mathrm{c}}, 2 \mathrm{H}\right), 7.08-7.09(\mathrm{~m}, 4 \mathrm{H}), 7.02-7.07(\mathrm{~m}, 1 \mathrm{H}), 6.95-6.97(\mathrm{~m}, 3 \mathrm{H}), 6.89-6.91(\mathrm{~m}$, $1 \mathrm{H}), 6.75-6.77(\mathrm{~m}, 2 \mathrm{H}), 4.39\left(\mathrm{q},{ }^{3} \mathrm{~J}=6.2 \mathrm{~Hz}, 1 \mathrm{H}\right), 3.38\left(\mathrm{~d},{ }^{2} \mathrm{~J}=13.1 \mathrm{~Hz}, 1 \mathrm{H}\right), 3.17(\mathrm{~d}$, $2 J=13.1 \mathrm{~Hz}, 1 \mathrm{H}), 2.30(\mathrm{~s}, 6 \mathrm{H}), 1.39(\mathrm{~s}, 3 \mathrm{H}), 0.99\left(\mathrm{~d},{ }^{3} \mathrm{~J}=6.2 \mathrm{~Hz}, 3 \mathrm{H}\right), 0.56(\mathrm{~s}, 3 \mathrm{H})$, $0.55(\mathrm{~s}, 3 \mathrm{H}) .{ }^{13} \mathrm{C}$ NMR $\left(126 \mathrm{MHz}, \mathrm{C}_{6} \mathrm{D}_{6}\right): \delta / p p m=145.6,139.2,138.6,137.3(2 \mathrm{C})$, 131.8 (2C), 131.7, 130.9 (2C), 128.3 (2C), 128.0 (2C), 127.8, 127.7, 126.2, 126.1, 75.9, 48.3, 46.6, 21.5 (2C), 19.0, 15.8, -0.5, -0.5. ${ }^{29} \mathrm{Si}$ DEPT NMR (99 MHz, $6 \mathrm{C}_{6}$ ): $\delta / p p m=4.8$. HRMS (APCl) m/z: $\left[\mathrm{M}-\mathrm{C}_{17} \mathrm{H}_{19}\right]^{+}$Calcd for $\mathrm{C}_{10} \mathrm{H}_{15} \mathrm{OSi}$ 179.0892; Found 179.0889. IR (ATR): v/cm ${ }^{-1}=3023,2974,2268,2083,1495,1447,1374,1251,1103$, 1085, 834, 780, 698. Optical Rotation: $[\alpha]_{D}=+0.78\left(c 1.3, \mathrm{C}_{6} \mathrm{D}_{6}\right)$.

The enantiomeric excess of (2S,3S)-3ae was determined by HPLC analysis on a chiral stationary phase after cleavage of the silyl ether according to GP3 (Daicel Chiralcel ${ }^{\circledR}$ OD-H column, column temperature $20^{\circ} \mathrm{C}$, mobile phase: $n$-heptane:isopropanol = 95:5, flow rate: $0.8 \mathrm{~mL} / \mathrm{min}, \lambda=210 \mathrm{~nm}): \mathrm{t}_{\mathrm{R}}=37.9 \mathrm{~min}$ for $(2 S, 3 S)-1 \mathrm{a}, \mathrm{t}_{\mathrm{R}}=41.6 \mathrm{~min}$ for $\left(2 S^{\star}, 3 R^{\star}\right)-1 \mathrm{a}, \mathrm{t}_{\mathrm{R}}=43.5 \mathrm{~min}$ for $(2 R, 3 R)-1 \mathrm{a}$, and $\mathrm{t}_{\mathrm{R}}=47.2 \mathrm{~min}$ for $\left(2 R^{\star}, 3 S^{*}\right)-1 \mathrm{a}$. 
rac-anti/syn-3-Benzyl-3-phenylpentan-2-ol
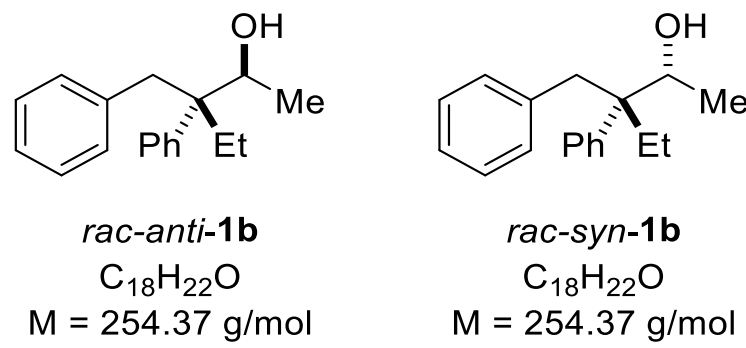

Prepared according to GP1 from 2-Benzyl-2-phenylbutanal (rac-S4, 330 mg, 1.38 mmol, 1.00 equiv.) using MeMgCl (20\% in THF, 0.306 mL, 1.38 mmol, 1.00 equiv.). The reaction mixture was stirred for $24 \mathrm{~h}$. Automatic flash column chromatography using cyclohexane/ethyl acetate $(100: 0 \rightarrow 0: 100)$ afforded a mixture of the diastereomers rac-anti-1b and rac-syn-1b (170 mg, $0.668 \mathrm{mmol}, 48 \%$, d.r. = 67:33) as a colorless oil.

$\mathbf{R}_{\boldsymbol{f}}=0.48$ for rac-anti-1b, 0.52 for rac-syn-1b (cyclohexane/ethyl acetate 10:1). ${ }^{\mathbf{1}} \mathbf{H}$ NMR (500 MHz, CDCl 3 ): $/ \mathrm{ppm}=7.42-7.44(\mathrm{~m}, 1 \mathrm{H}$, rac-anti-1b), 7.38-7.39 (m, 1H, rac-syn-1b), 7.40-7.42 (m, 2H, rac-anti-1b), 7.36-7.38 (m, 2H, rac-syn-1b), 7.33-7.36 (m, 2H, rac-syn-1b), 7.26-7.29 (m, 2H, rac-anti-1b), 7.20-7.22 (m, 2H, rac-syn-1b), 7.18-7.20 (m, 2H, rac-anti-1b), 7.22-7.25 (m, 1H, rac-syn-1b), 7.16-7.18 (m, 1H, racanti-1b), 7.08-7.12 (m, 2H, rac-syn-1b), 7.04-7.07 (m, 2H, rac-anti-1b), 3.97 (q, ${ }^{3} \mathrm{~J}=$ $6.4 \mathrm{~Hz}, 1 \mathrm{H}$, rac-anti-1b), $3.92\left(\mathrm{q},{ }^{3} \mathrm{~J}=6.4 \mathrm{~Hz}, 1 \mathrm{H}\right.$, rac-syn-1b), 3.42 (d, ${ }^{2} \mathrm{~J}=14.1 \mathrm{~Hz}$, $1 \mathrm{H}$, rac-syn-1b), $3.18\left(\mathrm{~d},{ }^{2} \mathrm{~J}=14.1 \mathrm{~Hz}, 1 \mathrm{H}\right.$, rac-anti-1b), $3.15\left(\mathrm{~d},{ }^{2} \mathrm{~J}=14.1 \mathrm{~Hz}, 1 \mathrm{H}\right.$, racanti-1b), 3.00 (d, ${ }^{2} \mathrm{~J}=14.1 \mathrm{~Hz}, 1 \mathrm{H}$, rac-syn-1b), 1.81 ( $\mathrm{m}_{\mathrm{c}}, 2 \mathrm{H}$, rac-anti-1b), 1.59 (mc, 2H, rac-syn-1b), 1.53 (s, 1H,), 1.09 (d, ${ }^{3} \mathrm{~J}=6.4 \mathrm{~Hz}, 3 \mathrm{H}$, rac-anti-1b), 1.04 (d, ${ }^{3} \mathrm{~J}=6.4$ $\mathrm{Hz}, 3 \mathrm{H}$, rac-syn-1b), 0.97 (t, ${ }^{3} \mathrm{~J}=7.5 \mathrm{~Hz}, 3 \mathrm{H}$, rac-syn-1b), 0.89 (t, ${ }^{3} \mathrm{~J}=7.5 \mathrm{~Hz}, 3 \mathrm{H}$, racanti-1b). ${ }^{13} \mathrm{C}$ NMR (126 MHz, $\mathrm{CDCl}_{3}$ ): $\delta / p p m=142.9$ (rac-anti-1b), 138.8 (rac-syn-1b), 138.7 (rac-anti-1b), 130.8 (2C, rac-syn-1b), 130.5 (2C, rac-anti-1b), 128.7 (2C, rac-syn-1b), 128.4 (2C, rac-anti-1b), 128.2 (2C, rac-syn-1b), 128.1 (2C, rac-anti1b), 128.0 (2C, rac-syn-1b), 126.2, 127.9 (2C, rac-anti-1b), 126.4 (rac-syn-1b), 126.3 (rac-anti-1b), 126.1 (rac-syn-1b), 72.0 (rac-anti-1b), 71.3 (rac-syn-1b), 50.3 (rac-syn1b), 49.8 (rac-anti-1b), 39.5 (rac-syn-1b), 39.1 (rac-anti-1b), 24.9 (rac-anti-1b), 24.1 (rac-syn-1b), 19.2 (rac-anti-1b), 18.5 (rac-syn-1b), 9.1 (C-5, rac-syn-1b), 9.0 (rac-anti1b). HRMS (APCl) m/z: [M-OH] ${ }^{+}$Calcd for $\mathrm{C}_{18} \mathrm{H}_{21}$ 237.1643; Found 237.1637. 
(2S,3S)-(3,5-Dimethylphenyl)dimethyl((3-methyl-3,4-diphenylbutan-2yl)oxy)silane<smiles>CC[C@](Cc1ccccc1)(c1ccccc1)[C@@H](C)O[Si](C)(C)c1cc(C)cc(C)c1</smiles>

$$
\begin{gathered}
(2 \mathrm{~S}, 3 \mathrm{~S})-3 \mathrm{be} \\
\mathrm{C}_{28} \mathrm{H}_{36} \mathrm{OSi} \\
\mathrm{M}=416.68 \mathrm{~g} / \mathrm{mol}
\end{gathered}
$$

Prepared according to GP1 from rac-anti/syn-1b (50.9 mg, 0.200 mmol, 1.00 equiv.) and (3,5-dimethylphenyl)dimethylsilane $(11.0 \mathrm{mg}, 0.0660 \mathrm{mmol}, 0.330$ equiv.). The reaction mixture was stirred at $-10^{\circ} \mathrm{C}$ for $192 \mathrm{~h}$. Flash column chromatography using cyclohexane/ethyl acetate $(30: 1 \rightarrow 7: 1)$ afforded silyl ether $(2 S, 3 S)$-3be (18.0 mg, $0.0430 \mathrm{mmol}, 21 \%$, d.r. $=88: 12,79 \%$ ee) as a yellow oil.

$\boldsymbol{R}_{\boldsymbol{f}}=0.89$ (pentane/ethyl acetate 15:1). ${ }^{1} \mathrm{H}$ NMR (500 MHz, $\left.\mathrm{C}_{6} \mathrm{D}_{6}\right): \delta / p p m=7.42-7.43$ $(\mathrm{m}, 2 \mathrm{H}), 7.31-7.31\left(\mathrm{~m}_{\mathrm{c}}, 2 \mathrm{H}\right), 7.20-7.23(\mathrm{~m}, 2 \mathrm{H}), 7.11-7.13(\mathrm{~m}, 1 \mathrm{H}), 7.04-7.07(\mathrm{~m}$, 2H), 7.00-7.02 (m, 2H), 6.88-6.89 (m, 2H), $4.16\left(\mathrm{q},{ }^{3} \mathrm{~J}=6.3 \mathrm{~Hz}, 1 \mathrm{H}\right), 3.16\left(\mathrm{~d},{ }^{2} \mathrm{~J}=13.8\right.$ $\mathrm{Hz}, 1 \mathrm{H}), 3.07\left(\mathrm{~d},{ }^{2} \mathrm{~J}=13.8 \mathrm{~Hz}, 1 \mathrm{H}\right), 2.18(\mathrm{~s}, 6 \mathrm{H}), 1.72-1.81(\mathrm{~m}, 2 \mathrm{H}), 0.98\left(\mathrm{~d},{ }^{3} J=6.3\right.$ $\mathrm{Hz}, 3 \mathrm{H}), 0.40-0.42(\mathrm{~m}, 3 \mathrm{H}), 0.34(\mathrm{~s}, 3 \mathrm{H}), 0.33$ (s, 3H). ${ }^{13} \mathrm{C}$ NMR (126 MHz, $\left.\mathrm{C}_{6} \mathrm{D}_{6}\right)$ : $\delta / p p m=144.8,139.3,138.4,137.1(2 \mathrm{C}), 131.7(2 \mathrm{C}), 131.6(2 \mathrm{C}), 131.4(2 \mathrm{C}), 130.8$ (2C), 128.6 (2C), 127.4, 126.3, 126.1, 74.0, 48.9, 39.3, 25.3, 21.5 (2C), 19.5, 1.2, 0.7, -0.9. ${ }^{29} \mathrm{Si}$ DEPT NMR (99 $\left.\mathrm{MHz}, \mathrm{C}_{6} \mathrm{D}_{6}\right): \delta / \mathrm{ppm}=4.6$. HRMS (APCI) m/z: [M$\left.\mathrm{C}_{17} \mathrm{H}_{19}\right]^{+}$Calcd for $\mathrm{C}_{10} \mathrm{H}_{15} \mathrm{OSi}$ 179.0892; Found 179.0889. IR (ATR): v/cm $\mathrm{cm}^{-1}=2965$, 1598, 1497, 1374, 1251, 1070, 993, 869, 791, 699. Optical Rotation: $[\alpha]_{D}=+1.23$ (c 1.3, $\left.\mathrm{C}_{6} \mathrm{D}_{6}\right)$.

The enantiomeric excess of (2S,3S)-3be was determined by HPLC analysis on a chiral stationary phase after cleavage of the silyl ether according to GP3 (Daicel Chiralcel ${ }^{\circledR}$ AS-H column, column temperature $20^{\circ} \mathrm{C}$, mobile phase: $n$-heptane:isopropanol = 99:1, flow rate: $0.6 \mathrm{~mL} / \mathrm{min}, \lambda=210 \mathrm{~nm}): t_{R}=12.3 \mathrm{~min}$ for $\left(2 S^{*}, 3 R^{*}\right)-1 \mathbf{b}, t_{R}=13.3 \mathrm{~min}$ for $\left(2 R^{*}, 3 S^{*}\right)-\mathbf{1 b}, \mathrm{t}_{\mathrm{R}}=16.9 \mathrm{~min}$ for $(2 R, 3 R)-\mathbf{1 b}$ and $\mathrm{t}_{\mathrm{R}}=19.1 \mathrm{~min}$ for $(2 S, 3 S)-\mathbf{1 b}$. 
rac-anti/syn-3-Benzyl-3-methylpentan-2-ol

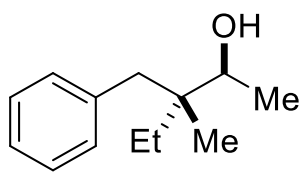

rac-anti-1c $\mathrm{C}_{13} \mathrm{H}_{20} \mathrm{O}$ $M=192.30 \mathrm{~g} / \mathrm{mol}$<smiles>CCC(C)(Cc1ccccc1)[C@@H](C)O</smiles>

rac-syn-1c $\mathrm{C}_{13} \mathrm{H}_{20} \mathrm{O}$

$M=192.30 \mathrm{~g} / \mathrm{mol}$

Prepared according to GP1 from 2-benzyl-2-methylbutanal (rac-S15, $300 \mathrm{mg}, 1.70$ mmol, 1.00 equiv.) using MeMgCl (20\% in THF, $0.380 \mathrm{~mL}, 1.70 \mathrm{mmol}, 1.00$ equiv.). The reaction mixture was stirred for $48 \mathrm{~h}$. Automatic flash column chromatography using cyclohexane/ethyl acetate $(100: 0 \rightarrow 0: 100)$ afforded a mixture of the diastereomers rac-anti-1c and rac-syn-1c (135 mg, $0.702 \mathrm{mmol}, 41 \%$, d.r. = 54:46) as a colorless oil.

$\mathbf{R}_{\boldsymbol{f}}=0.50$ for rac-anti-1c, 0.52 for rac-syn-1c (cyclohexane/ethyl acetate 10:1). ${ }^{\mathbf{1}} \mathbf{H}$ NMR $\left(400 \mathrm{MHz}, \mathrm{CDCl}_{3}\right): \delta / \mathrm{ppm}=0.79(\mathrm{~s}, 3 \mathrm{H}$, rac-syn-1c), $0.82(\mathrm{~s}, 3 \mathrm{H}$, rac-anti-1c), $0.90(\mathrm{t}$, ${ }^{3} \mathrm{~J}=7.4 \mathrm{~Hz}, 3 \mathrm{H}$, rac-anti-1c), 0.92 (t, ${ }^{3} \mathrm{~J}=7.4 \mathrm{~Hz}, 3 \mathrm{H}$, rac-syn-1c), 1.17 (d, ${ }^{3} \mathrm{~J}=8.4 \mathrm{~Hz}$, $3 \mathrm{H}), 1.30-1.35$ (m, 2H, rac-syn-1c), 1.35-1.41 (m, 2H, rac-anti-1c), $2.52\left(\mathrm{~d},{ }^{2} \mathrm{~J}=13.3\right.$ $\mathrm{Hz}, 1 \mathrm{H}$, rac-syn-1c), 2.64 (d, ${ }^{2} \mathrm{~J}=13.3 \mathrm{~Hz}, 1 \mathrm{H}$, rac-anti-1c), $2.71\left(\mathrm{~d},{ }^{2} \mathrm{~J}=13.3 \mathrm{~Hz}, 1 \mathrm{H}\right)$, $3.97\left(\mathrm{q},{ }^{3} \mathrm{~J}=6.4 \mathrm{~Hz}, 1 \mathrm{H},\right), 7.17-7.19(\mathrm{~m}, 2 \mathrm{H}$, rac-syn-1c), 7.19-7.20 (m, $2 \mathrm{H}$, rac-anti-1c), 7.20-7.22 (m, 1H, rac-anti-1c), 7.24-7.25 (m, 2H, rac-syn-1c), 7.26-7.27 (m, 2H, rac-anti-1c), 7.27-7.29 (m, 1H). ${ }^{13} \mathrm{C}$ NMR (126 MHz, CDCl 3 ): $\delta / p p m=8.2$ (rac-syn-1c), 8.3 (rac-anti-1c), 17.8 (rac-syn-1c), 17.9 (rac-anti-1c), 19.5 (rac-anti-1c), 20.7 (rac-syn-1c), 27.4 (rac-syn-1c), 27.8 (rac-anti-1c), 40.9 (rac-anti-1c), 41.0 (rac-syn-1c), 41.1 (rac-syn-1c), 41.2 (rac-anti-1c), 71.8 (rac-anti-1c), 72.1 (rac-syn-1c), 126.0, 127.9 (2C), 130.9 (2C), 142.6. HRMS (APCI) m/z: [M-OH] Calcd for $\mathrm{C}_{13} \mathrm{H}_{19} \mathrm{O}$ 175.1487; Found 175.1478. 
$(2 S, 3 S) /(2 R, 3 S)-((3-B e n z y l-3-m e t h y l p e n t a n-2-y l) o x y)(3,5-$ dimethylphenyl)dimethylsilane

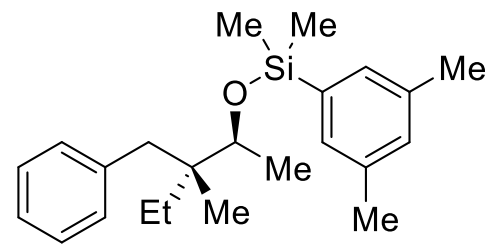

$(2 S, 3 R)-3 \mathrm{ce}$

$\mathrm{C}_{28} \mathrm{H}_{36} \mathrm{OSi}$

$\mathrm{M}=416.68 \mathrm{~g} / \mathrm{mol}$

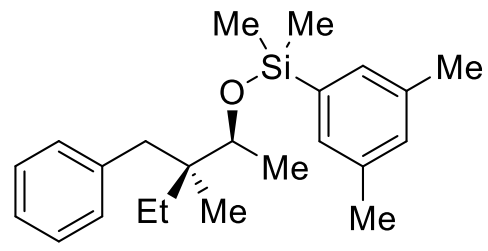

$(2 S, 3 S)-3 c e$

$\mathrm{C}_{28} \mathrm{H}_{36} \mathrm{OSi}$ $\mathrm{M}=416.68 \mathrm{~g} / \mathrm{mol}$

Prepared according to GP1 from rac-anti/syn-1c (38.5 mg, 0.200 mmol, 1.00 equiv.) and (3,5-dimethylphenyl)dimethylsilane (10.0 mg, $0.05400 \mathrm{mmol}, 0.270$ equiv.). The reaction mixture was stirred at $-10^{\circ} \mathrm{C}$ for $120 \mathrm{~h}$. Flash column chromatography using cyclohexane/ethyl acetate $(30: 1 \rightarrow 7: 1)$ afforded a mixture of silyl ethers $(2 S, 3 S)$-3ce and (2R,3S)-3ce (14.0 mg, $0.0390 \mathrm{mmol}, 20 \%$, d.r. $=54: 46,74 \%$ ee) as a yellow oil.

$\boldsymbol{R}_{\boldsymbol{f}}=0.83$ (cyclohexane). ${ }^{1} \mathrm{H}$ NMR $\left(500 \mathrm{MHz}, \mathrm{C}_{6} \mathrm{D}_{6}\right): \delta / p p m=7.35-7.36(\mathrm{~m}, 2 \mathrm{H}$, $\left.\left(2 S^{\star}, 3 S^{\star}\right)-3 \mathrm{ce}\right), 7.35-7.36\left(\mathrm{~m}, 2 \mathrm{H},\left(2 R^{*}, 3 S^{\star}\right)-3 \mathrm{ce}\right), 7.29-7.30(\mathrm{~m}, 1 \mathrm{H}), 7.07-7.15(\mathrm{~m}$, $9 \mathrm{H}), 6.89-6.89(\mathrm{~m}, 2 \mathrm{H}), 3.77\left(\mathrm{~m}_{\mathrm{c}},{ }^{3} \mathrm{~J}=6.4 \mathrm{~Hz}, 1 \mathrm{H},\left(2 \mathrm{~S}^{*}, 3 \mathrm{~S}^{*}\right)-3 \mathrm{ce}\right), 3.77\left(\mathrm{~m}_{\mathrm{c}},{ }^{3} \mathrm{~J}=6.4\right.$ $\left.\mathrm{Hz}, 1 \mathrm{H},\left(2 R^{*}, 3 S^{*}\right)-3 \mathrm{ce}\right), 2.81\left(\mathrm{~d},{ }^{2} \mathrm{~J}=13.3 \mathrm{~Hz}, 1 \mathrm{H},\left(2 R^{*}, 3 S^{*}\right)-3 \mathrm{ce}\right), 2.76\left(\mathrm{~d},{ }^{2} \mathrm{~J}=13.3 \mathrm{~Hz}\right.$, $\left.1 \mathrm{H},\left(2 S^{*}, 3 S^{*}\right)-3 \mathrm{ce}\right), 2.70\left(\mathrm{~d},{ }^{2} \mathrm{~J}=13.3 \mathrm{~Hz}, 1 \mathrm{H},\left(2 S^{*}, 3 S^{*}\right)-3 \mathrm{ce}\right), 2.53\left(\mathrm{~d},{ }^{2} \mathrm{~J}=13.3 \mathrm{~Hz}, 1 \mathrm{H}\right.$, $\left.\left(2 R^{*}, 3 S^{*}\right)-3 \mathrm{ce}\right), 2.20\left(\mathrm{~s}, 6 \mathrm{H},\left(2 S^{*}, 3 S^{*}\right)-3 \mathrm{ce}\right), 2.20$ (s, 6H, $\left.\left(2 R^{*}, 3 S^{*}\right)-3 \mathrm{ce}\right), 1.41-1.49(\mathrm{~m}$, $\left.2 \mathrm{H},\left(2 R^{*}, 3 S^{*}\right)-3 \mathrm{ce}\right), 1.24-1.32\left(\mathrm{~m}, 2 \mathrm{H},\left(2 S^{*}, 3 S^{*}\right)-3 \mathrm{ce}\right), 1.23\left(\mathrm{~s}, 3 \mathrm{H},\left(2 R^{*}, 3 S^{*}\right)-3 \mathrm{ce}\right), 1.09$ $\left(\mathrm{t},{ }^{3} \mathrm{~J}=7.3 \mathrm{~Hz}, 3 \mathrm{H},\left(2 S^{*}, 3 S^{*}\right)-3 \mathrm{ce}\right), 1.09\left(\mathrm{t}, 3 \mathrm{~J}=7.3 \mathrm{~Hz}, 3 \mathrm{H},\left(2 R^{*}, 3 S^{*}\right)-3 \mathrm{ce}\right), 0.80(\mathrm{~m}$, $\left.3 \mathrm{H},\left(2 S^{*}, 3 S^{*}\right)-3 \mathrm{ce}\right), 0.80\left(\mathrm{~m}, 3 \mathrm{H},\left(2 R^{*}, 3 S^{*}\right)-3 \mathrm{ce}\right), 0.44$ (s, 6H, $\left.\left(2 R^{*}, 3 S^{*}\right)-3 \mathrm{ce}\right), 0.44$ (s,

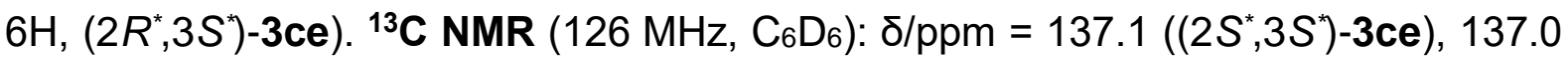
$\left(\left(2 R^{*}, 3 S^{*}\right)-3 \mathrm{ce}\right), 131.7\left(2 \mathrm{C},\left(2 S^{*}, 3 S^{*}\right)-3 \mathrm{ce}\right), 131.5\left(\left(2 R^{*}, 3 S^{*}\right)-3 \mathrm{ce}\right), 131.3\left(2 \mathrm{C},\left(2 R^{*}, 3 S^{*}\right)-\right.$

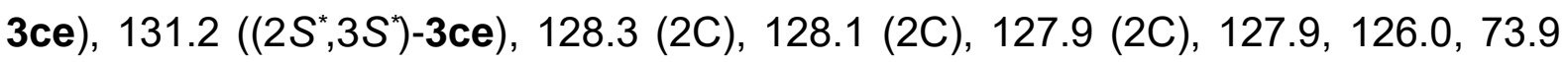

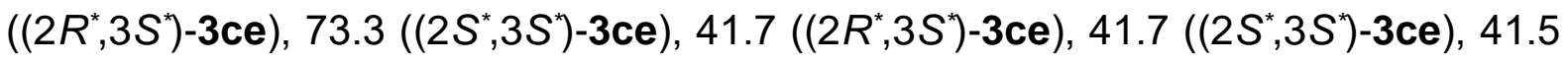

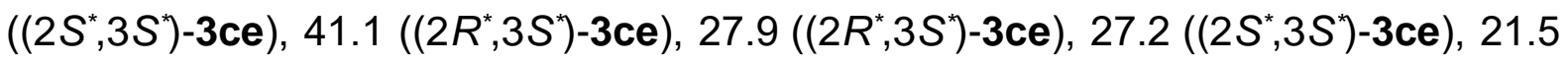
(2C, $\left.\left(2 S^{*}, 3 S^{*}\right)-3 \mathrm{ce}\right), 21.4\left(2 \mathrm{C},\left(2 R^{*}, 3 S^{*}\right)-3 \mathrm{ce}\right), 20.1\left(\left(2 S^{*}, 3 S^{*}\right)-3 \mathrm{ce}\right), 18.3\left(\left(2 R^{*}, 3 S^{*}\right)-\right.$ 3ce $), 18.2\left(\left(2 S^{*}, 3 S^{*}\right)-3 \mathrm{ce}\right), 8.4\left(\left(2 R^{*}, 3 S^{*}\right)-3 \mathrm{ce}\right), 8.2\left(\left(2 S^{*}, 3 S^{*}\right)-3 \mathrm{ce}\right), 1.7\left(2 \mathrm{C},\left(2 R^{*}, 3 S^{*}\right)-\right.$ 3ce $), 1.2\left(2 \mathrm{C},\left(2 S^{*}, 3 S^{*}\right)-3 \mathrm{ce}\right) .{ }^{29} \mathrm{Si}$ DEPT NMR (99 MHz, $\left.\mathrm{C}_{6} \mathrm{D}_{6}\right): \delta / p p m=3.4,3.6$. IR (ATR): v/cm ${ }^{-1}=3022,2963,2278,1453,1376,1251,1195,1087,967,869,701$. 
HRMS (APCl) m/z: [M- $\left.{ }_{6} \mathrm{H}_{5}\right]^{+}$Calcd for $\mathrm{C}_{17} \mathrm{H}_{29} \mathrm{OSi} 277.1988$; Found 277.1979. Optical Rotation: $[\alpha]_{D}=+0.09\left(c 1.3, \mathrm{C}_{6} \mathrm{D}_{6}\right)$.

The enantiomeric excess of (2S,3S)-3ce was determined by HPLC analysis on a chiral stationary phase after cleavage of the silyl ether according to GP3 (Daicel Chiralcel ${ }^{\circledR}$ OD-H column, column temperature $20^{\circ} \mathrm{C}$, mobile phase: $n$-heptane:isopropanol = 97:3, flow rate: $0.6 \mathrm{~mL} / \mathrm{min}, \lambda=210 \mathrm{~nm}): \mathrm{t}_{\mathrm{R}}=18.6 \mathrm{~min}$ for $\left(2 S^{*}, 3 R^{*}\right)-1 \mathrm{c}, \mathrm{t}_{\mathrm{R}}=20.1 \mathrm{~min}$ for $\left(2 R^{\star}, 3 S^{\star}\right)-1 \mathrm{c}, \mathrm{t}_{\mathrm{R}}=22.7 \mathrm{~min}$ for $(2 S, 3 S)-1 \mathrm{c}$ and $\mathrm{t}_{\mathrm{R}}=25.5 \mathrm{~min}$ for $(2 R, 3 R)-1 \mathrm{c}$. 
rac-anti/syn-4-(4-Fluorophenyl)-3-methyl-3-phenylbutan-2-ol<smiles>CC(O)[C@](C)(Cc1ccc(F)cc1)c1ccccc1</smiles>

rac-anti-1d

$\mathrm{C}_{17} \mathrm{H}_{19} \mathrm{FO}$

$\mathrm{M}=258.34 \mathrm{~g} / \mathrm{mol}$<smiles>C[C@H](O)[C@](C)(Cc1ccc(F)cc1)c1ccccc1</smiles>

rac-syn-1d

$\mathrm{C}_{17} \mathrm{H}_{19} \mathrm{FO}$

$\mathrm{M}=258.34 \mathrm{~g} / \mathrm{mol}$

Prepared according to GP1 from 3-(4-fluorophenyl)-2-methyl-2-phenylpropanal (racS5, $604 \mathrm{mg}, 2.69 \mathrm{mmol}, 1.00$ equiv.) using MeMgCl (20\% in THF, $0.596 \mathrm{~mL}, 2.69$ mmol, 1.00 equiv.). The reaction mixture was stirred for $48 \mathrm{~h}$. Flash column chromatography using cyclohexane/ethyl acetate (10:1) afforded a mixture of the diastereomers rac-anti-1d and rac-syn-1d (150 mg, $0.581 \mathrm{mmol}, 21 \%$, d.r. = 83:17) as a yellow oil.

$\mathbf{R}_{\boldsymbol{f}}=0.21$ for rac-anti-1d, 0.32 for rac-syn-1d (cyclohexane/ethyl acetate 10:1). ${ }^{1} \mathrm{H}$ NMR $\left(400 \mathrm{MHz}, \mathrm{CDCl}_{3}\right): \delta / p p m=7.36-7.41$ (m, 2H, rac-syn-1d), 7.31-7.36 (m, $2 \mathrm{H}$, rac-syn-1d), 7.26-7.31 (m, 2H, rac-anti-1d), 7.23-7.25 (m, 1H, rac-syn-1d), 7.20$7.23(\mathrm{~m}, 1 \mathrm{H}$, rac-anti-1d), 7.16-7.20 (m, 2H, rac-anti-1d), 6.78-6.85 (m, 2H, rac-syn-1d), 6.71-6.78 (m, 2H, rac-anti-1d), 6.65-6.68 (m, 2H, rac-syn-1d), 6.62-6.65 (m, 2H, rac-anti-1d), $4.18\left(\mathrm{q},{ }^{3} \mathrm{~J}=6.4 \mathrm{~Hz}, 1 \mathrm{H}\right.$, rac-anti-1d), $4.04\left(\mathrm{q},{ }^{3} \mathrm{~J}=6.4 \mathrm{~Hz}, 1 \mathrm{H}\right.$, rac-syn-1d), $3.13\left(\mathrm{~d},{ }^{2} \mathrm{~J}=13.3 \mathrm{~Hz}, 1 \mathrm{H}\right.$, rac-anti-1d), $3.05\left(\mathrm{~d},{ }^{2} \mathrm{~J}=13.3 \mathrm{~Hz}, 1 \mathrm{H}\right.$, rac-syn-1d), $3.02\left(\mathrm{~d},{ }^{2} \mathrm{~J}=13.3 \mathrm{~Hz}, 1 \mathrm{H}\right.$, rac-anti-1d), $2.94\left(\mathrm{~d},{ }^{2} \mathrm{~J}=13.3 \mathrm{~Hz}, 1 \mathrm{H}\right.$, rac-syn-1d), $1.56(\mathrm{~s}, 1 \mathrm{H}), 1.20(\mathrm{~s}, 3 \mathrm{H}$, rac-anti-1d), 1.17 (s, 3H, rac-syn-1d), 1.16 $\left(\mathrm{d},{ }^{3} \mathrm{~J}=6.4 \mathrm{~Hz}, 3 \mathrm{H}\right.$, rac-syn-1d), $0.97\left(\mathrm{~d},{ }^{3} \mathrm{~J}=6.4 \mathrm{~Hz}, 3 \mathrm{H}\right.$, rac-anti-1d). ${ }^{13} \mathrm{C}$ NMR $\left(126 \mathrm{MHz}_{\mathrm{CDCl}}\right.$ ): $\mathrm{\delta} / \mathrm{ppm}=162.8$ (rac-syn-1d), 162.8 (rac-anti-1d), 144.6, 136.7 (rac-anti-1d), 136.9 (rac-syn-1d), 131.9 (2C, rac-syn-1d), 131.8 (2C, rac-anti-1d), 128.3 (2C, rac-syn-1d), 128.1 (2C, rac-anti-1d), 127.7 (2C, rac-syn-1d), 127.3 (2C, rac-anti-1d), 126.2 (rac-syn-1d), 126.2 (rac-anti-1d), 114.3 (2C, rac-syn-1d), 114.1 (2C, rac-anti-1d), 74.0 (rac-syn-1d), 73.9 (rac-anti-1d), 47.3 (rac-syn-1d), 47.2 (rac-anti-1d), 45.0, 18.8, 16.2 (rac-anti-1d), 16.1 (rac-syn-1d). HRMS (APCI) m/z: [M$\left.\mathrm{C}_{10} \mathrm{H}_{13} \mathrm{O}\right]^{+}$Calcd for $\mathrm{C}_{10} \mathrm{H}_{15} \mathrm{OSi} 109.0454$; Found 109.0449. 
(2S,3S)-(3,5-Dimethylphenyl)(( 4-(4-fluorophenyl)-3-methyl-3-phenylbutan-2yl)oxy)dimethylsilane

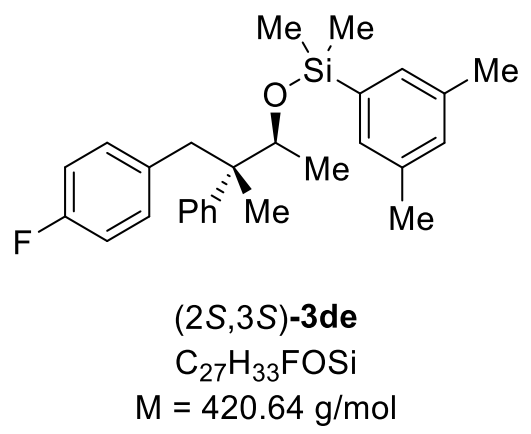

Prepared according to GP1 from rac-anti/syn-1d (49.0 mg, 0.200 mmol, 1.00 equiv.) and (3,5-dimethylphenyl)dimethylsilane (13.5 $\mathrm{mg}, 0.0800 \mathrm{mmol}, 0.400$ equiv.). The reaction mixture was stirred at $-10^{\circ} \mathrm{C}$ for $168 \mathrm{~h}$. Flash column chromatography using cyclohexane/ethyl acetate $(30: 1 \rightarrow 7: 1)$ afforded silyl ether (2S,3S)-3de (10 mg, 0.023 mmol, $11 \%$, d.r. > 99:1, 88\% ee) as a yellow oil.

$\boldsymbol{R}_{\boldsymbol{f}}=0.80$ (cyclohexane). ${ }^{1} \mathrm{H}$ NMR $\left(500 \mathrm{MHz}, \mathrm{C}_{6} \mathrm{D}_{6}\right): \delta / p p m=7.44\left(\mathrm{~m}_{\mathrm{c}}, 2 \mathrm{H}\right), 7.04-7.10$ $(\mathrm{m}, 2 \mathrm{H}), 7.01-7.04(\mathrm{~m}, 2 \mathrm{H}), 6.88-6.93(\mathrm{~m}, 2 \mathrm{H}), 6.59-6.62(\mathrm{~m}, 1 \mathrm{H}), 6.56-6.59(\mathrm{~m}, 1 \mathrm{H})$, 6.48-6.53 (m, 2H), $4.28\left(\mathrm{q},{ }^{3} \mathrm{~J}=6.2 \mathrm{~Hz}, 1 \mathrm{H}\right), 3.18\left(\mathrm{~d},{ }^{2} \mathrm{~J}=13.1 \mathrm{~Hz}, 1 \mathrm{H}\right), 2.95\left(\mathrm{~d},{ }^{2} \mathrm{~J}=\right.$ $13.1 \mathrm{~Hz}, 1 \mathrm{H}), 2.22(\mathrm{~s}, 6 \mathrm{H}), 1.24(\mathrm{~s}, 3 \mathrm{H}), 0.91\left(\mathrm{~d},{ }^{3} \mathrm{~J}=6.2 \mathrm{~Hz}, 3 \mathrm{H}\right), 0.49(\mathrm{~s}, 3 \mathrm{H}), 0.47$ (s, 3H). ${ }^{13} \mathrm{C}$ NMR (126 MHz, C6 6 ): $\delta / p p m=163.1,156.4,145.3,138.5,137.3$ (2C), 132.2 (2C), 132.1 (2C), 131.8 (2C), 131.7 (2C), 127.6 (2C), 126.3, 114.3, 75.8, 48.2, 45.7, 32.3, 21.5, 19.0, 15.6, -0.5, -0.6. ${ }^{29}$ Si DEPT NMR $\left(99 \mathrm{MHz}, \mathrm{C}_{6} \mathrm{D}_{6}\right): \delta / p p m=4.9$. HRMS (APCl) $\mathrm{m} / \mathrm{z}$ : [M- $\left.\mathrm{C}_{10} \mathrm{H}_{15} \mathrm{OSi}\right]^{+}$Calcd for $\mathrm{C}_{17} \mathrm{H}_{18} \mathrm{~F}$ 241.1393; Found 241.1386. IR (ATR): $\mathrm{v} / \mathrm{cm}^{-1}=2974,2277,1600,1446,1375,1251,1093,976,869,841$, 699. Optical Rotation: $[\alpha]_{D}=+0.34\left(c 1.3, \mathrm{C}_{6} \mathrm{D}_{6}\right)$.

The enantiomeric excess of (2S,3S)-3de was determined by HPLC analysis on a chiral stationary phase after cleavage of the silyl ether according to GP3 (Daicel Chiralcel ${ }^{\circledR}$ IA column, column temperature $20^{\circ} \mathrm{C}$, mobile phase: $n$-heptane:isopropanol = 99.3:0.7, flow rate: $0.6 \mathrm{~mL} / \mathrm{min}, \lambda=210 \mathrm{~nm}): \mathrm{t}_{\mathrm{R}}=38.4 \mathrm{~min}$ for $\left(2 S^{*}, 3 R^{*}\right)-1 \mathrm{~d}$, $\mathrm{t}_{\mathrm{R}}=42.3$ $\min$ for $\left(2 R^{\star}, 3 S^{\star}\right)-\mathbf{1 d}, \mathrm{t}_{\mathrm{R}}=47.5 \mathrm{~min}$ for $(2 S, 3 S)-\mathbf{1 d}$ and $\mathrm{t}_{\mathrm{R}}=52.6 \mathrm{~min}$ for $(2 R, 3 R)-\mathbf{1 d}$. 
rac-anti/syn-3-Methyl-3-phenyl-4-(p-tolyl)butan-2-ol<smiles>Cc1ccc(C[C@@](C)(c2ccccc2)C(C)O)cc1</smiles>

rac-anti-1i

$\mathrm{C}_{18} \mathrm{H}_{22} \mathrm{O}$

$\mathrm{M}=254.37 \mathrm{~g} / \mathrm{mol}$<smiles>Cc1ccc(C[C@@](C)(c2ccccc2)[C@H](C)O)cc1</smiles>

rac-syn-1i

$\mathrm{C}_{18} \mathrm{H}_{22} \mathrm{O}$

$\mathrm{M}=254.37 \mathrm{~g} / \mathrm{mol}$

Prepared according to GP1 from 2-methyl-2-phenyl-3-( $p$-tolyl)propanal (rac-S6, 500 mg, 2.10 mmol, 1.00 equiv.) using $\mathrm{MeMgCl}(20 \%$ in THF, 0.476 mL, $2.10 \mathrm{mmol}, 1.00$ equiv.). The reaction mixture was stirred for $120 \mathrm{~h}$. Automatic flash column chromatography using cyclohexane/ethyl acetate $(0: 100 \rightarrow 100: 1)$ afforded a mixture of the diastereomers rac-anti-1i and rac-syn-1i (290 mg, $1.14 \mathrm{mmol}, 54 \%$, d.r. = 90:10) as a yellow oil.

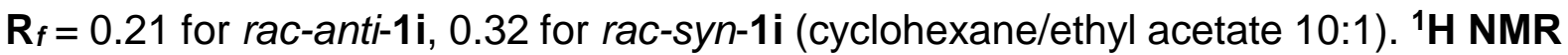
(400 MHz, CDCl $): \delta / p p m=7.38-7.43(\mathrm{~m}, 1 \mathrm{H}$, rac-syn-1i), 7.33-7.36 $(\mathrm{m}, 1 \mathrm{H}$, rac-anti1i), 7.29-7.31 (m, 2H, rac-syn-1i), 7.27-7.30 (m, 2H, rac-anti-1i), 7.21-7.24 (m, 2H, rac-anti-1i), 7.17-7.21 (m, 2H, rac-syn-1i), 6.85-6.89 (m, 2H, rac-anti-1i), 6.75-6.79 $(\mathrm{m}, 2 \mathrm{H}$, rac-syn-1i), 6.70-6.73 $(\mathrm{m}, 2 \mathrm{H}$, rac-syn-1i), 6.60-6.65 $(\mathrm{m}, 2 \mathrm{H}$, rac-anti-1i), 4.12 (q, ${ }^{3} \mathrm{~J}=6.3 \mathrm{~Hz}, 1 \mathrm{H}$, rac-anti-1i), 4.04 (q, ${ }^{3} \mathrm{~J}=6.3 \mathrm{~Hz}, 1 \mathrm{H}$, rac-syn-1i), 3.84 (d, ${ }^{2} \mathrm{~J}=$ $13.3 \mathrm{~Hz}, 1 \mathrm{H}$, rac-syn-1i), 3.64 (d, ${ }^{2} \mathrm{~J}=13.3 \mathrm{~Hz}, 1 \mathrm{H}$, rac-syn-1i), 3.14 (d, ${ }^{2} \mathrm{~J}=13.3 \mathrm{~Hz}$, $1 \mathrm{H}$, rac-anti-1i), 3.01 (d, ${ }^{2} \mathrm{~J}=13.3 \mathrm{~Hz}, 1 \mathrm{H}$, rac-anti-1i), 2.24 (s, 3H, rac-syn-1i), 2.23 (s, 3H, rac-anti-1i), 1.54 (s, 1H), 1.23 (s, 3H, rac-anti-1i), 1.18 (s, 3H, rac-syn-1i), 1.15 $\left(\mathrm{d},{ }^{3} \mathrm{~J}=6.3 \mathrm{~Hz}, 3 \mathrm{H}\right.$, rac-syn-1i), $0.96\left(\mathrm{~d},{ }^{3} \mathrm{~J}=6.3 \mathrm{~Hz}, 3 \mathrm{H}\right.$, rac-anti-1i). ${ }^{13} \mathrm{C}$ NMR (126 MHz, $\left.\mathrm{CDCl}_{3}\right): \delta / p p m=144.9,135.5$ (rac-syn-1i), 135.4 (rac-anti-1i), 130.5 (2C, rac-syn-1i), 130.4 (2C, rac-anti-1i), 128.4 (2C, rac-syn-1i), 128.3 (2C, rac-anti-1i), 128.2 (2C, rac-syn-1i), 128.1 (2C, rac-anti-1i), 127.4 (2C), 126.2 (rac-anti-1i), 126.1 (rac-syn-1i), 125.4, 74.2 (rac-anti-1i), 74.1 (rac-syn-1i), 47.4 (rac-anti-1i), 47.3 (rac-syn-1i), 45.4 (rac-anti-1i), 45.2 (rac-syn-1i), 21.1 (rac-anti-1i), 21.0 (rac-syn-1i), 18.8 (rac-anti-1i), 18.7 (rac-syn-1i), 16.5. HRMS (APCl) m/z: [M- $\left.\mathrm{C}_{8} \mathrm{H}_{9}\right]^{+}$Calcd for $\mathrm{C}_{10} \mathrm{H}_{13} \mathrm{O}$ 149.0966; Found 149.0963. 
(2S,3S)-(3,5-Dimethylphenyl)dimethyl((3-methyl-3-phenyl-4-(p-tolyl)butan-2yl)oxy)silane

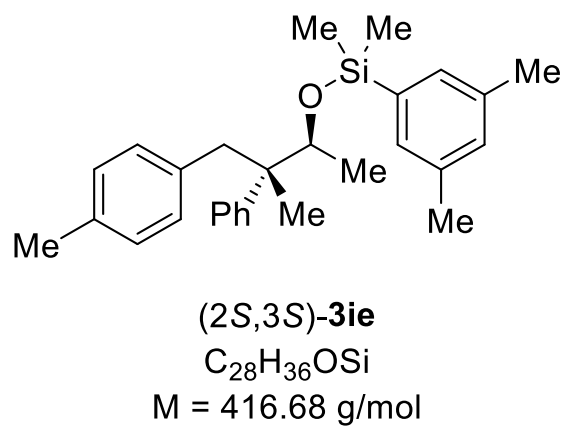

Prepared according to GP1 from rac-anti/syn-1i (50.9 mg, 0.200 mmol, 1.00 equiv.) and (3,5-dimethylphenyl)dimethylsilane (14.8 $\mathrm{mg}, 0.0900 \mathrm{mmol}, 0.450$ equiv.). The reaction mixture was stirred at $-10^{\circ} \mathrm{C}$ for $144 \mathrm{~h}$. Flash column chromatography using $n$-pentane/ethyl acetate $(30: 1 \rightarrow 7: 1)$ afforded silyl ether (2S,3S)-3ie (27 mg, 0.064 mmol, 32\%, d.r. = 99:1, 90\% ee) as a yellow oil.

$\boldsymbol{R}_{\boldsymbol{f}}=0.80$ (cyclohexane). ${ }^{1} \mathrm{H}$ NMR (500 MHz, $\left.\mathrm{C}_{6} \mathrm{D}_{6}\right): \delta / p p m=7.45-7.45(\mathrm{~m}, 2 \mathrm{H}), 7.07-$ $7.12(\mathrm{~m}, 4 \mathrm{H}), 7.03-7.05(\mathrm{~m}, 1 \mathrm{H}), 6.90-6.90(\mathrm{~m}, 1 \mathrm{H}), 6.78-6.80(\mathrm{~m}, 2 \mathrm{H}), 6.69-6.70(\mathrm{~m}$, $2 \mathrm{H}), 4.33\left(\mathrm{q},{ }^{3} \mathrm{~J}=6.2 \mathrm{~Hz}, 1 \mathrm{H}\right), 3.32\left(\mathrm{~d},{ }^{2} \mathrm{~J}=13.1 \mathrm{~Hz}, 1 \mathrm{H}\right), 3.10\left(\mathrm{~d},{ }^{2} J=13.1 \mathrm{~Hz}, 1 \mathrm{H}\right)$, $2.22(\mathrm{~s}, 6 \mathrm{H}), 2.03(\mathrm{~s}, 3 \mathrm{H}), 1.35(\mathrm{~s}, 3 \mathrm{H}), 0.93$ (d, $\left.{ }^{3} \mathrm{~J}=6.2 \mathrm{~Hz}, 3 \mathrm{H}\right), 0.49$ (s, 3H), 0.48 (s, $3 \mathrm{H}) .{ }^{13} \mathrm{C}$ NMR (126 MHz, $\left.\mathrm{C}_{6} \mathrm{D}_{6}\right): \delta / \mathrm{ppm}=145.7,138.6,137.3(2 \mathrm{C}), 136.0,135.2,131.8$ (2C), 131.7, 130.8 (2C), 128.5 (2C), 128.2 (2C), 127.9 (2C), 126.1, 75.9, 48.2, 46.2, 21.5 (2C), 21.0, 19.0, 15.8, -0.5, -0.5. $\left.{ }^{29} \mathrm{Si} \mathrm{DEPT} \mathrm{NMR} \mathrm{(99} \mathrm{MHz,} \mathrm{C}_{6} \mathrm{D}_{6}\right): \delta / p p m=4.7$. HRMS (APCl) m/z: [M-C $\left.{ }_{18} \mathrm{H}_{21}\right]^{+}$Calcd for $\mathrm{C}_{10} \mathrm{H}_{15} \mathrm{OSi} 179.0892$; Found 179.0888. IR (ATR): v/cm ${ }^{-1}=3019,2974,1513,1446,1251,1030,975,840,801,698$. Optical Rotation: $[\alpha]_{D}=+0.86\left(c 1.3, \mathrm{C}_{6} \mathrm{D}_{6}\right)$.

The enantiomeric excess of (2S,3S)-3ie was determined by HPLC analysis on a chiral stationary phase after cleavage of the silyl ether according to GP3 (Daicel Chiralcel ${ }^{\circledR}$ IA column, column temperature $20^{\circ} \mathrm{C}$, mobile phase: $n$-heptane:isopropanol = 99.3:0.7, flow rate: $0.6 \mathrm{~mL} / \mathrm{min}, \lambda=210 \mathrm{~nm}): \mathrm{t}_{\mathrm{R}}=31.6 \mathrm{~min}$ for $\left(2 R^{*}, 3 S^{*}\right)-1 \mathrm{i}, \mathrm{t}_{\mathrm{R}}=40.0$ $\min$ for $\left(2 R^{\star}, 3 S^{\star}\right)-1 \mathbf{i}, t_{R}=44.0 \mathrm{~min}$ for $(2 S, 3 S)-1 \mathbf{i}$ and $46.8 \mathrm{~min}$ for $(2 R, 3 R)-\mathbf{1} \mathbf{i}$. 
rac-anti/syn-4-(3,5-Dimethylphenyl)-3-methyl-3-phenylbutan-2-ol

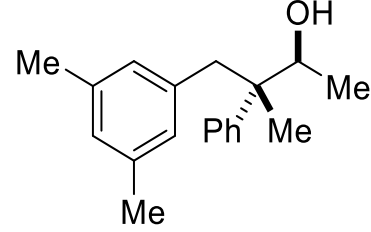

rac-anti-1j

$\mathrm{C}_{19} \mathrm{H}_{24} \mathrm{O}$

$\mathrm{M}=268.40 \mathrm{~g} / \mathrm{mol}$<smiles>Cc1cc(C)cc(C[C@](C)(c2ccccc2)[C@H](C)O)c1</smiles>

rac-syn-1j

$\mathrm{C}_{19} \mathrm{H}_{24} \mathrm{O}$

$\mathrm{M}=268.40 \mathrm{~g} / \mathrm{mol}$

Prepared according to GP1 from 3-(3,5-Dimethylphenyl)-2-methyl-2-phenylpropanal (rac-S7, 500 mg, 1.98 mmol, 1.00 equiv.) using MeMgCl (20\% in THF, 0.438 mL, 1.98 mmol, 1.00 equiv.). The reaction mixture was stirred for $96 \mathrm{~h}$. Automatic flash column chromatography using cyclohexane/ethyl acetate $(0: 100 \rightarrow 100: 1)$ afforded a mixture of the diastereomers rac-anti-1j and rac-syn-1j $(228 \mathrm{mg}, 0.849 \mathrm{mmol}, 43 \%$, d.r. = $80: 20)$ as a yellow oil.

$\mathbf{R}_{\boldsymbol{f}}=0.39$ for rac-anti-1j, 0.46 for rac-syn-1j (cyclohexane/ethyl acetate 10:1). ${ }^{\mathbf{1}} \mathbf{H} \mathbf{N M R}$ $\left(400 \mathrm{MHz}, \mathrm{CDCl}_{3}\right): \delta / p p m=7.46-7.49(\mathrm{~m}, 1 \mathrm{H}$, rac-syn-1j), 7.37-7.41 (m, 2H, rac-syn1j), 7.26-7.28 (m, 2H, rac-anti-1j), 7.21-7.23 (m, 2H, rac-anti-1j), 7.17-7.21 (m, 1H, rac-anti-1j), 6.83-6.90 (m, 1H, rac-anti-1j), 6.67-6.75 (m, 1H, rac-anti-1j), $6.31\left(\mathrm{~m}_{\mathrm{c}}\right.$, $2 \mathrm{H}$, rac-syn-1j), 6.19 (mc, 2H, rac-anti-1j), 4.24 (q, ${ }^{3} \mathrm{~J}=6.4 \mathrm{~Hz}, 1 \mathrm{H}$, rac-anti-1j), 4.12 (q, ${ }^{3} \mathrm{~J}=6.4 \mathrm{~Hz}, 1 \mathrm{H}$, rac-syn-1j), $3.13\left(\mathrm{~d},{ }^{2} \mathrm{~J}=13.3 \mathrm{~Hz}, 1 \mathrm{H}\right.$, rac-anti-1j), $3.04\left(\mathrm{~d},{ }^{2} \mathrm{~J}=13.3\right.$ $\mathrm{Hz}, 1 \mathrm{H}$, rac-anti-1j), 2.20 (s, 6H, rac-syn-1j), 2.18 (S, 6H, rac-anti-1j), 1.29 (s, 3H, racanti-1j), 1.22 (d, ${ }^{3} \mathrm{~J}=6.4 \mathrm{~Hz}, 1 \mathrm{H}$, rac-anti-1j), 1.20 (d, ${ }^{3} \mathrm{~J}=6.4 \mathrm{~Hz}, 1 \mathrm{H}$, rac-syn-1j), 1.03 (d, ${ }^{3} \mathrm{~J}=6.4 \mathrm{~Hz}, 3 \mathrm{H}$, rac-syn-1j), $1.02\left(\mathrm{~d},{ }^{3} \mathrm{~J}=6.4 \mathrm{~Hz}, 3 \mathrm{H}\right.$, rac-anti-1j). ${ }^{13} \mathrm{C}$ NMR $\left(126 \mathrm{MHz}, \mathrm{CDCl}_{3}\right): \delta / p p m=138.4$ (rac-anti-1j), 136.9 (2C, rac-syn-1j), 136.8 (2C, rac-anti-1j), 136.7 (rac-anti-1j), 128.6 (2C, rac-syn-1j), 128.5 (2C, rac-anti-1j), 128.2 (rac-anti-1j), 128.1 (2C, rac-syn-1j), 128.0 (2C, rac-anti-1j), 127.5 (2C, rac-syn-1j), 127.4 (2C, rac-anti-1j), 126.2 (rac-syn-1j), 126.1 (rac-anti-1j), 74.4 (rac-syn-1j), 74.0 (rac-anti-1j), 46.3 (rac-syn-1j), 46.2 (rac-anti-1j), 45.9 (rac-anti-1j), 45.8 (rac-syn-1j), 21.3 (rac-anti-1j), 18.8 (rac-anti-1j), 16.5 (rac-syn-1j), 16.4 (C-5, rac-anti-1j). IR (ATR): $\mathrm{v} / \mathrm{cm}^{-1}=2974,2917,1602,1447,1375,1156,1061,923,760,700$. HRMS (APCl) $\mathrm{m} / \mathrm{z}:\left[\mathrm{M}-\mathrm{C}_{9} \mathrm{H}_{11}\right]^{+}$Calcd for $\mathrm{C}_{10} \mathrm{H}_{13} \mathrm{O}$ 149.0966; Found 149.0963. 
(2S,3S)-(3,5-Dimethylphenyl)(( 4-(3,5-dimethylphenyl)-3-methyl-3-phenylbutan2-yl)oxy)dimethylsilane

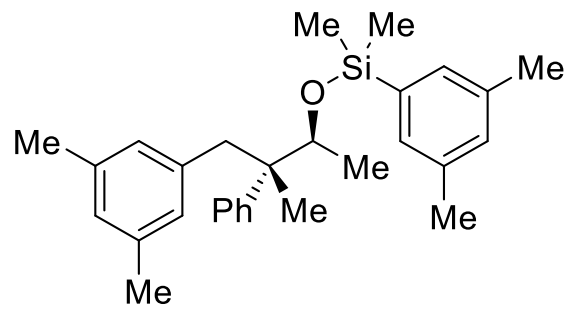

$$
\begin{gathered}
(2 \mathrm{~S}, 3 \mathrm{~S})-3 \mathbf{j e} \\
\mathrm{C}_{29} \mathrm{H}_{38} \mathrm{OSi} \\
M=430.71 \mathrm{~g} / \mathrm{mol}
\end{gathered}
$$

Prepared according to GP1 from rac-anti/syn-1j (53.7 mg, $0.200 \mathrm{mmol}, 1.00$ equiv.) and (3,5-dimethylphenyl)dimethylsilane (13.2 $\mathrm{mg}, 0.0800 \mathrm{mmol}, 0.400$ equiv.). The reaction mixture was stirred at $-10^{\circ} \mathrm{C}$ for $144 \mathrm{~h}$. Flash column chromatography using $n$-pentane/ethyl acetate (30:1 $\rightarrow$ 7:1) afforded silyl ether (2S,3S)-3je (31 mg, 0.072 mmol, 36\%, d.r. = 99:1, 43\% ee) as a yellow oil.

${ }^{1} \mathrm{H}$ NMR $\left(500 \mathrm{MHz}, \mathrm{C}_{6} \mathrm{D}_{6}\right): \delta / p p m=7.47(\mathrm{~s}, 2 \mathrm{H}), 7.11-7.14(\mathrm{~m}, 2 \mathrm{H}), 7.02-7.04(\mathrm{~m}$, $1 \mathrm{H}), 6.89-6.92(\mathrm{~m}, 1 \mathrm{H}), 6.61-6.67(\mathrm{~m}, 2 \mathrm{H}), 6.41-6.45(\mathrm{~m}, 2 \mathrm{H}), 6.33\left(\mathrm{~m}_{\mathrm{c}}, 1 \mathrm{H}\right), 4.37$ (q, $\left.{ }^{3} J=6.1 \mathrm{~Hz}, 1 \mathrm{H}\right), 3.30\left(\mathrm{~d},{ }^{2} J=13.1 \mathrm{~Hz}, 1 \mathrm{H}\right), 3.09\left(\mathrm{~d},{ }^{2} \mathrm{~J}=13.1 \mathrm{~Hz}, 1 \mathrm{H}\right), 2.22(\mathrm{~s}, 6 \mathrm{H})$, $2.05(\mathrm{~s}, 6 \mathrm{H}), 1.40(\mathrm{~s}, 3 \mathrm{H}), 0.95\left(\mathrm{~d},{ }^{3} \mathrm{~J}=6.3 \mathrm{~Hz}, 3 \mathrm{H}\right), 0.50(\mathrm{~s}, 3 \mathrm{H}), 0.49(\mathrm{~s}, 3 \mathrm{H}) .{ }^{13} \mathrm{C}$ NMR (126 MHz, $\left.\mathrm{C}_{6} \mathrm{D}_{6}\right): \delta / p p m=145.9,144.4,138.9,138.7,137.8,137.3,136.6,131.8(2 \mathrm{C})$, 131.7, 129.0 (2C), 129.0 (2C), 127.9 (2C), 126.4, 126.0, 75.8, 48.2, 46.7, 21.5 (2C), $21.3(2 \mathrm{C}), 19.0,15.8,-0.36,-0.51 .{ }^{29} \mathrm{Si} \mathrm{DEPT} \mathrm{NMR}\left(99 \mathrm{MHz}, \mathrm{C}_{6} \mathrm{D}_{6}\right): \delta / p p m=4.7$. HRMS (APCl) m/z: [M-C $\left.{ }_{19} \mathrm{H}_{23}\right]^{+}$Calcd for $\mathrm{C}_{10} \mathrm{H}_{15} \mathrm{OSi}$ 179.0892; Found 179.0882. IR (ATR): v/cm ${ }^{-1}=2975,2359,1944,1738,1447,1373,1247,1102,869,781,699$. Optical Rotation: $[\alpha]_{D}=+0.010\left(\right.$ c 7.5, $\left.\mathrm{CDCl}_{3}\right)$.

The enantiomeric excess of (2S,3S)-3je was determined by HPLC analysis on a chiral stationary phase after cleavage of the silyl ether according to GP3 (Daicel Chiralcel ${ }^{\circledR}$ $\mathrm{OD}-\mathrm{RH}$ column, column temperature $20^{\circ} \mathrm{C}$, mobile phase: acetonitrile: water $=40: 60$, flow rate: $0.5 \mathrm{~mL} / \mathrm{min}, \Lambda=210 \mathrm{~nm}): \mathrm{t}_{\mathrm{R}}=88.9 \mathrm{~min}$ for $\left(2 S^{*}, 3 R^{*}\right)-1 \mathrm{j}, \mathrm{t}_{\mathrm{R}}=96.4 \mathrm{~min}$ for $\left(2 R^{*}, 3 S^{*}\right)-\mathbf{1} \mathbf{j}, t_{R}=109.1 \mathrm{~min}$ for $(2 S, 3 S)-\mathbf{1} \mathbf{j}$ and $118.0 \mathrm{~min}$ for $(2 R, 3 R)-\mathbf{1} \mathbf{j}$. 
rac-anti/syn-4-(3,5-Dimethoxyphenyl)-3-methyl-3-phenylbutan-2-ol<smiles>COc1cc(C[C@@](C)(c2ccccc2)C(C)O)cc(OC)c1</smiles>

rac-anti-1I $\mathrm{C}_{19} \mathrm{H}_{24} \mathrm{O}_{3}$ $\mathrm{M}=300.40 \mathrm{~g} / \mathrm{mol}$<smiles>COc1cc(C[C@@](C)(c2ccccc2)[C@H](C)O)cc(OC)c1</smiles>

rac-syn-1I $\mathrm{C}_{19} \mathrm{H}_{24} \mathrm{O}_{3}$ $\mathrm{M}=300.40 \mathrm{~g} / \mathrm{mol}$

Prepared according to GP1 from 3-(3,5-dimethoxyphenyl)-2-methyl-2-phenylpropanal (rac-113, 130 mg, 0.459 mmol, 1.00 equiv.) using $\mathrm{MeMgCl}$ (20\% in THF, $0.102 \mathrm{~mL}$, $0.459 \mathrm{mmol}, 1.00$ equiv.). The reaction mixture was stirred for $96 \mathrm{~h}$. Automatic flash column chromatography using cyclohexane/ethyl acetate $(0: 100 \rightarrow 100: 1)$ afforded a mixture of the diastereomers rac-anti-1I and rac-syn-1I $(120 \mathrm{mg}, 0.400 \mathrm{mmol}, 87 \%$, d.r. = 79:21) as a yellow oil.

$\mathbf{R}_{\boldsymbol{f}}=0.17$ for rac-anti-1I, 0.22 for rac-syn-1I (cyclohexane/ethyl acetate 10:1). ${ }^{\mathbf{1}} \mathbf{H}$ NMR $\left(400 \mathrm{MHz}, \mathrm{CDCl}_{3}\right): \delta / p p m=7.39-7.44(\mathrm{~m}, 1 \mathrm{H}$, rac-anti-1I $), 7.33-7.36(\mathrm{~m}, 1 \mathrm{H}$, rac-syn-1I), 7.30-7.33 ( $\mathrm{m}, 2 \mathrm{H}$, rac-syn-1I), 7.27-7.30 (m, 2H, rac-anti-1I), 7.22-7.25 (m, 2H, rac-anti-1I), 7.17-7.22 ( $\mathrm{m}, 2 \mathrm{H}$, rac-syn-1I), 6.20-6.22 ( $\mathrm{m}, 1 \mathrm{H}$, rac-syn-1I), 6.19-6.20 (m, 1H, rac-anti-1I), 5.92-5.93 (m, 2H, rac-syn-1I), 5.83-5.87 (m, $2 \mathrm{H}$, rac-anti-1I), 4.20 (q, ${ }^{3} \mathrm{~J}=6.3 \mathrm{~Hz}, 1 \mathrm{H}$, rac-anti-1I), 4.10 (q, ${ }^{3} \mathrm{~J}=6.3 \mathrm{~Hz}, 1 \mathrm{H}$, rac-syn-1I), 3.56 (s, 6H, rac-syn-1I), 3.53 (s, 6H, rac-anti-1I), 3.12 (d, ${ }^{2} \mathrm{~J}=12.7 \mathrm{~Hz}, 1 \mathrm{H}$, rac-anti-1I), $3.07\left(\mathrm{~d},{ }^{2} \mathrm{~J}=12.7 \mathrm{~Hz}, 1 \mathrm{H}\right.$, rac-syn-1I), 2.98 (d, ${ }^{2} \mathrm{~J}=12.7 \mathrm{~Hz}, 1 \mathrm{H}$, rac-anti-1I), 2.83 (d, ${ }^{2} \mathrm{~J}$ $=12.7 \mathrm{~Hz}, 1 \mathrm{H}$, rac-syn-1I), $1.54(\mathrm{~s}, 1 \mathrm{H}), 1.22(\mathrm{~s}, 3 \mathrm{H}$, rac-anti-1I), $1.19(\mathrm{~s}, 3 \mathrm{H}$, rac-syn-1I), 1.17 (d, ${ }^{3} \mathrm{~J}=6.3 \mathrm{~Hz}, 3 \mathrm{H}$, rac-syn-1I), 0.97 (d, ${ }^{3} \mathrm{~J}=6.3 \mathrm{~Hz}, 3 \mathrm{H}$, rac-anti-1I). ${ }^{13} \mathrm{C}$ NMR (126 MHz, CDCl 3 ): $/ \mathrm{ppm}=145.1,140.9$ (rac-syn-1I), 140.8 (rac-anti-1I), 128.3 (2C, rac-syn-1I), 128.2 (2C, rac-anti-1I), 127.5 (2C, rac-anti-1I), 127.4 (2C, rac-syn-1I), 126.1 (2C), 122.1 (rac-syn-1I), 122.0 (rac-anti-1I), 108.5 (2C, rac-syn-1I), 108.4 (2C, rac-anti-1I), 98.6 (rac-syn-1I), 98.5 (rac-anti-1I), 74.1 (rac-anti-1I), 74.0 (rac-syn-1I), 55.1, 47.4 (rac-syn-1I), 47.3 (rac-anti-1I), 46.2, 18.8, 16.0. HRMS (APCI) $\mathrm{m} / \mathrm{z}:[\mathrm{M}-\mathrm{OH}]^{+}$Calcd for $\mathrm{C}_{19} \mathrm{H}_{23} \mathrm{O}_{2}$ 283.1698; Found 283.1693. 
(2S,3S)-((4-(3,5-Dimethoxyphenyl)-3-methyl-3-phenylbutan-2-yl)oxy)(3,5dimethylphenyl)dimethylsilane

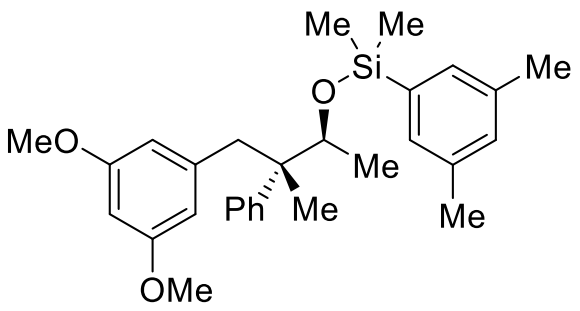

$(2 S, 3 S)-3 l e$

$\mathrm{C}_{29} \mathrm{H}_{38} \mathrm{O}_{3} \mathrm{Si}$

$\mathrm{M}=462.71 \mathrm{~g} / \mathrm{mol}$

Prepared according to GP1 from rac-anti/syn-1I (60.1 mg, $0.200 \mathrm{mmol}, 1.00$ equiv.) and (3,5-dimethylphenyl)dimethylsilane (13.2 $\mathrm{mg}, 0.0800 \mathrm{mmol}, 0.400$ equiv.). The reaction mixture was stirred at $-10^{\circ} \mathrm{C}$ for $336 \mathrm{~h}$. Flash column chromatography using $n$-pentane/ethyl acetate $(30: 1 \rightarrow 7: 1)$ afforded silyl ether (2S,3S)-3le (22 mg, 0.047 mmol, $24 \%$, d.r. $=97: 3,86 \%$ ee) as a yellow oil.

$\mathbf{R}_{\boldsymbol{f}}=0.83$ (cyclohexane/ethyl acetate 10:1). ${ }^{1} \mathbf{H}$ NMR $\left(500 \mathrm{MHz}, \mathrm{C}_{6} \mathrm{D}_{6}\right): \delta / p p m=7.44-$ $7.46(\mathrm{~m}, 2 \mathrm{H}), 7.09-7.13(\mathrm{~m}, 2 \mathrm{H}), 7.04-7.09(\mathrm{~m}, 2 \mathrm{H}), 6.97-7.03(\mathrm{~m}, 1 \mathrm{H}), 6.89-6.91(\mathrm{~m}$, $1 \mathrm{H}), 6.39-6.43(\mathrm{~m}, 1 \mathrm{H}), 6.05-6.08(\mathrm{~m}, 2 \mathrm{H}), 4.35\left(\mathrm{q},{ }^{3} \mathrm{~J}=6.2 \mathrm{~Hz}, 1 \mathrm{H}\right), 3.31\left(\mathrm{~d},{ }^{2} \mathrm{~J}=13.1\right.$ $\mathrm{Hz}, 1 \mathrm{H}), 3.22(\mathrm{~s}, 6 \mathrm{H}), 3.10\left(\mathrm{~d},{ }^{2} \mathrm{~J}=13.1 \mathrm{~Hz}, 1 \mathrm{H}\right), 2.22(\mathrm{~s}, 6 \mathrm{H}), 1.38(\mathrm{~s}, 3 \mathrm{H}), 0.93$ (d, $\left.{ }^{3} J=6.2 \mathrm{~Hz}, 3 \mathrm{H}\right), 0.49$ (s, 3H), 0.48 (s, 3H). ${ }^{13} \mathrm{C}$ NMR (126 MHz, $\left.\mathrm{C}_{6} \mathrm{D}_{6}\right): \delta / p p m=160.6$ (2C), 146.0, 141.3, 138.6, 137.3 (2C), 131.8 (2C), 131.7, 128.2 (2C), 127.9 (2C), 126.0, 109.0 (2C), 99.0, 75.8, 54.6 (2C), 48.3, 47.1, 21.5 (2C), 18.9, 15.7, -0.4, -0.6. ${ }^{29} \mathrm{Si}$ DEPT NMR (99 MHz, $\left.\mathrm{C}_{6} \mathrm{D}_{6}\right): \delta / p p m=4.9$. HRMS $(\mathrm{APCl}) \mathrm{m} / \mathrm{z}:\left[\mathrm{M}-\mathrm{C}_{20} \mathrm{H}_{27} \mathrm{OSi}\right]^{+}$ Calcd for $\mathrm{C}_{9} \mathrm{H}_{19} \mathrm{O}_{2}$ 151.0759; Found 151.0759. IR (ATR): $\mathrm{v} / \mathrm{cm}^{-1}=2953,2090,1887$, $1461,1251,1151,977,832,781,699$. Optical Rotation: $[\alpha]_{D}=+0.060\left(c 1.1, C_{6} D_{6}\right)$.

The enantiomeric excess of (2S,3S)-3le was determined by HPLC analysis on a chiral stationary phase after cleavage of the silyl ether according to GP3 (Daicel Chiralcel ${ }^{\circledR}$ OD-H column, column temperature $20^{\circ} \mathrm{C}$, mobile phase: $n$-heptane:isopropanol = 98:2, flow rate: $0.6 \mathrm{~mL} / \mathrm{min}, \lambda=210 \mathrm{~nm}): \mathrm{t}_{\mathrm{R}}=51.6 \mathrm{~min}$ for $\left(2 S^{*}, 3 R^{*}\right)-11$, t $\mathrm{t}=55.0 \mathrm{~min}$ for $(2 S, 3 S)-11, t_{R}=64.0 \mathrm{~min}$ for $\left(2 R^{*}, 3 S^{*}\right)-11$, and $74.7 \mathrm{~min}$ for $(2 R, 3 R)-11$. 
rac-anti/syn-4-(4-Bromophenyl)-3-methyl-3-phenylbutan-2-ol<smiles>CC(O)C(C)(Cc1ccc(Br)cc1)c1ccccc1</smiles>

rac-anti-1f $\mathrm{C}_{17} \mathrm{H}_{19} \mathrm{BrO}$ $M=319.24 \mathrm{~g} / \mathrm{mol}$<smiles>C[C@H](O)[C@](C)(Cc1ccc(Br)cc1)c1ccccc1</smiles>

rac-syn-1f $\mathrm{C}_{17} \mathrm{H}_{19} \mathrm{BrO}$ $M=319.24 \mathrm{~g} / \mathrm{mol}$

Prepared according to GP1 from 3-(4-bromophenyl)-2-methyl-2-phenylpropanal (racS9, 400 mg, 1.32 mmol, 1.00 equiv.) using MeMgCl (20\% in THF, 0.300 mL, 1.32 mmol, 1.00 equiv.). The reaction mixture was stirred for $120 \mathrm{~h}$. Automatic flash column chromatography using cyclohexane/ethyl acetate $(0: 100 \rightarrow 100: 1)$ afforded a mixture of the diastereomers rac-anti-1f and rac-syn-1f (234 mg, $0.733 \mathrm{mmol}, 56 \%$, d.r. = 91:9) as a yellow oil.

$\mathbf{R}_{\boldsymbol{f}}=0.25$ for rac-anti-1f, 0.36 for rac-syn-1f (cyclohexane/ethyl acetate 10:1). ${ }^{\mathbf{1}} \mathbf{H}$ NMR $\left(400 \mathrm{MHz}, \mathrm{CDCl}_{3}\right): \delta / \mathrm{ppm}=7.37-7.40(\mathrm{~m}, 2 \mathrm{H}$, rac-syn-1f), 7.33-7.37 $(\mathrm{m}, 2 \mathrm{H}$, rac-syn-1f), 7.29-7.33 (m, 2H, rac-anti-1f), 7.26-7.28 (m, 2H, rac-anti-1f), 7.23-7.25 (m, 1H, rac-syn-1f), 7.19-7.23 (m, 1H, rac-anti-1f), 7.17-7.19 (m, 2H, rac-anti-1f), 7.14-7.16 (m, 2H, rac-syn-1f), 6.67-6.74 (m, 2H, rac-syn-1f), 6.54-6.59 (m, $2 \mathrm{H}$, rac-anti-1f), 4.17 (q, ${ }^{3} \mathrm{~J}=6.4 \mathrm{~Hz}, 1 \mathrm{H}$, rac-anti-1f), $4.03\left(\mathrm{q},{ }^{3} \mathrm{~J}=6.4 \mathrm{~Hz}, 1 \mathrm{H}\right.$, rac-syn-1f), $3.11\left(\mathrm{~d},{ }^{2} \mathrm{~J}=13.3 \mathrm{~Hz}, 1 \mathrm{H}\right.$, rac-anti-1f), $3.07\left(\mathrm{~d},{ }^{2} \mathrm{~J}=6.6 \mathrm{~Hz}, 1 \mathrm{H}\right.$, rac-syn-1f), 3.00 (d, ${ }^{2} J$ $=13.3 \mathrm{~Hz}, 1 \mathrm{H}$, rac-anti-1f), 2.97 (d, ${ }^{2} \mathrm{~J}=6.6 \mathrm{~Hz}, 1 \mathrm{H}$, rac-syn-1f), $1.48(\mathrm{~s}, 1 \mathrm{H}), 1.27$ (s, $3 \mathrm{H}$, rac-syn-1f), 1.20 (s, 3H, rac-anti-1f), 1.15 (d, ${ }^{3} \mathrm{~J}=6.4 \mathrm{~Hz}, 3 \mathrm{H}$, rac-syn-1f), 0.96 (d, ${ }^{3} \mathrm{~J}=6.4 \mathrm{~Hz}, 3 \mathrm{H}$, rac-anti-1f). ${ }^{13} \mathrm{C}$ NMR (126 MHz, CDCl 3 ): $\delta / p p m=144.6,137.5,132.3$ (2C), 130.6 (2C), 128.6, 128.3 (2C), 127.3 (2C, rac-anti-1f), 126.3 (2C, rac-syn-1f), 125.1, 73.9, 47.2 (rac-syn-1f), 45.2 (rac-anti-1f), 34.2 (rac-anti-1f), 33.9 (rac-syn-1f), 22.5 (rac-anti-1f), 18.8 (rac-syn-1f), 16.1 (rac-syn-1f), 14.2 (rac-anti-1f). HRMS (APCI) $\mathrm{m} / \mathrm{z}:\left[\mathrm{M}-\mathrm{C}_{10} \mathrm{H}_{13} \mathrm{O}\right]^{+}$Calcd for $\mathrm{C}_{7} \mathrm{H}_{6} \mathrm{Br}$ 168.9653; Found 168.9643. 
(2S,3S)-((4-(4-Bromophenyl)-3-methyl-3-phenylbutan-2-yl)oxy)(3,5dimethylphenyl)dimethylsilane

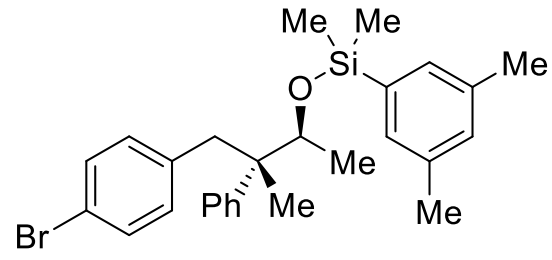

$$
\begin{gathered}
(2 \mathrm{~S}, 3 \mathrm{~S})-3 \mathrm{fe} \\
\mathrm{C}_{27} \mathrm{H}_{33} \mathrm{BrOSi} \\
M=481.55 \mathrm{~g} / \mathrm{mol}
\end{gathered}
$$

Prepared according to GP1 from rac-anti/syn-1f (63.8 mg, $0.200 \mathrm{mmol}, 1.00$ equiv.) and (3,5-dimethylphenyl)dimethylsilane (13.2 $\mathrm{mg}, 0.0800 \mathrm{mmol}, 0.400$ equiv.). The reaction mixture was stirred at $-10^{\circ} \mathrm{C}$ for $216 \mathrm{~h}$. Flash column chromatography using $n$-pentane/ethyl acetate $(30: 1 \rightarrow 7: 1)$ afforded silyl ether $(2 S, 3 S)-3$ fe $(28 \mathrm{mg}, 0.058$ mmol, 29\%, d.r. > 99:1, 86\% ee) as a yellow oil.

$\mathbf{R}_{\boldsymbol{f}}=0.86$ (cyclohexane/ethyl acetate 10:1). ${ }^{1} \mathbf{H}$ NMR $\left(500 \mathrm{MHz}, \mathrm{C}_{6} \mathrm{D}_{6}\right): \delta / p p m=$ 7.41-7.43 (m, 2H), 7.10-7.12 (m, 3H), 7.03-7.05 (m, 2H), 6.98-7.00 (m, 2H), 6.54$6.56(\mathrm{~m}, 1 \mathrm{H}), 6.38-6.39(\mathrm{~m}, 2 \mathrm{H}), 4.25\left(\mathrm{q},{ }^{3} \mathrm{~J}=6.2 \mathrm{~Hz}, 1 \mathrm{H}\right), 3.12\left(\mathrm{~d},{ }^{2} \mathrm{~J}=13.1 \mathrm{~Hz}, 1 \mathrm{H},\right)$, $2.88\left(\mathrm{~d},{ }^{2} \mathrm{~J}=13.1 \mathrm{~Hz}, 1 \mathrm{H}\right), 2.22(\mathrm{~s}, 6 \mathrm{H}), 1.24(\mathrm{~s}, 3 \mathrm{H}), 0.88\left(\mathrm{~d},{ }^{3} \mathrm{~J}=6.2 \mathrm{~Hz}, 3 \mathrm{H}\right), 0.47$ $(\mathrm{s}, 3 \mathrm{H}), 0.46(\mathrm{~s}, 3 \mathrm{H}) .{ }^{13} \mathrm{C}$ NMR $\left(126 \mathrm{MHz}, \mathrm{C}_{6} \mathrm{D}_{6}\right): \delta / \mathrm{ppm}=145.1,138.0,137.4$, 132.6 (2C), 131.8 (2C), 131.6, 130.8 (2C), 127.6 (2C), 126.3, 120.0, 75.7, 70.5, 48.1, 46.0, $21.5(2 \mathrm{C}), 18.9,15.6,-0.46,-0.58 .{ }^{29} \mathrm{Si} \mathrm{DEPT}$ NMR $\left(99 \mathrm{MHz}, \mathrm{C}_{6} \mathrm{D}_{6}\right): \delta / \mathrm{ppm}=$ 5.0. IR (ATR): v/cm ${ }^{-1}=2957,2920,1595,1487,1446,1251,1097,976,871,782$, 698. HRMS (APCl) m/z: [M- $\left.\mathrm{C}_{10} \mathrm{H}_{15} \mathrm{OSi}\right]^{+}$Calcd for $\mathrm{C}_{17} \mathrm{H}_{18} \mathrm{Br} 301.0592$; Found 301.0592. Optical Rotation: $[\alpha]_{D}=+0.50\left(c 1.3, \mathrm{C}_{6} \mathrm{D}_{6}\right)$.

The enantiomeric excess of (2S,3S)-3fe was determined by HPLC analysis on a chiral stationary phase after cleavage of the silyl ether according to GP3 (Daicel Chiralcel ${ }^{\circledR}$ IA column, column temperature $20^{\circ} \mathrm{C}$, mobile phase: $n$-heptane:isopropanol = 99.7:0.3, flow rate: $0.6 \mathrm{~mL} / \mathrm{min}, \lambda=210 \mathrm{~nm}): \mathrm{t}_{\mathrm{R}}=56.4 \mathrm{~min}$ for $(2 S, 3 S)-1 \mathrm{f}$ and $\mathrm{t}_{\mathrm{R}}=59.9$ min for $(2 R, 3 R)-\mathbf{1 f}$. 
rac-anti/syn-4-(4-Chlorophenyl)-3-methyl-3-phenylbutan-2-ol
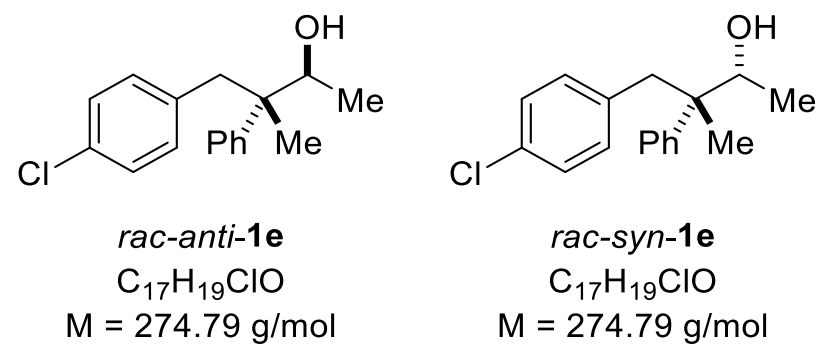

Prepared according to GP1 from 3-(4-chlorophenyl)-2-methyl-2-phenylpropanal (racS10, $440 \mathrm{mg}, 1.70 \mathrm{mmol}, 1.00$ equiv.) using $\mathrm{MeMgCl}$ (20\% in THF, $0.400 \mathrm{~mL}, 1.70$ $\mathrm{mmol}, 1.00$ equiv.). The reaction mixture was stirred for $48 \mathrm{~h}$. Automatic flash column chromatography using cyclohexane/ethyl acetate $(0: 100 \rightarrow 100: 1)$ afforded a mixture of the diastereomers rac-anti-1e and rac-syn-1e $(350 \mathrm{mg}, 1.27 \mathrm{mmol}, 75 \%$, d.r. $=$ $84: 16)$ as a yellow oil.

$\mathbf{R}_{\boldsymbol{f}}=\mathbf{0 . 2 4}$ for rac-anti-1e, 0.36 for rac-syn-1e (cyclohexane/ethyl acetate 10:1). ${ }^{1} \mathbf{H}$ NMR $\left(400 \mathrm{MHz}, \mathrm{CDCl}_{3}\right): \delta / \mathrm{ppm}=7.31-7.36(\mathrm{~m}, 1 \mathrm{H}$, rac-syn-1e $), 7.31-7.36(\mathrm{~m}, 1 \mathrm{H}$, rac-anti-1e), 7.29-7.30 (m, 2H, rac-syn-1e), 7.26-7.29 (m, 2H, rac-anti-1e), 7.19-7.23 ( $\mathrm{m}, 1 \mathrm{H}$, rac-syn-1e), 7.15-7.19 ( $\mathrm{m}, 2 \mathrm{H}$, rac-anti-1e), 7.04-7.07 ( $\mathrm{m}, 2 \mathrm{H}$, rac-syn-1e), 6.99-7.03 $(\mathrm{m}, 2 \mathrm{H}$, rac-anti-1e $), 6.73-6.76(\mathrm{~m}, 2 \mathrm{H}$, rac-syn-1e $), 6.60-6.64(\mathrm{~m}, 2 \mathrm{H}$, rac-anti-1e), $4.17\left(\mathrm{q},{ }^{3} \mathrm{~J}=6.4 \mathrm{~Hz}, 1 \mathrm{H}\right.$, rac-anti-1e $), 4.03\left(\mathrm{q},{ }^{3} \mathrm{~J}=6.4 \mathrm{~Hz}, 1 \mathrm{H}\right.$, rac-syn-1e), $3.13\left(\mathrm{~d},{ }^{2} \mathrm{~J}=13.3 \mathrm{~Hz}, 1 \mathrm{H}\right.$, rac-anti-1e $), 3.06\left(\mathrm{~d},{ }^{2} \mathrm{~J}=13.3 \mathrm{~Hz}, 1 \mathrm{H}\right.$, rac-syn-1e), $3.02\left(\mathrm{~d},{ }^{2} \mathrm{~J}=13.3 \mathrm{~Hz}, 1 \mathrm{H}\right.$, rac-anti-1e), $2.93\left(\mathrm{~d},{ }^{2} \mathrm{~J}=13.3 \mathrm{~Hz}, 1 \mathrm{H}\right.$, rac-syn-1e), $1.49(\mathrm{~s}, 1 \mathrm{H}), 1.20(\mathrm{~s}, 3 \mathrm{H}$, rac-anti-1e), $1.17(\mathrm{~s}, 3 \mathrm{H}$, rac-syn-1e), 1.15 (d, ${ }^{3} \mathrm{~J}=6.4 \mathrm{~Hz}, 3 \mathrm{H}$, rac-syn-1e), $0.96\left(\mathrm{~d},{ }^{3} \mathrm{~J}=6.4 \mathrm{~Hz}, 3 \mathrm{H}\right.$, rac-anti-1e). ${ }^{13} \mathrm{C}$ NMR $\left(126 \mathrm{MHz}, \mathrm{CDCl}_{3}\right): \delta / \mathrm{ppm}=164.0,144.5$ (rac-syn-1e), 144.4 (rac-anti-1e), 137.1 (rac-syn-1e), 137.0 (rac-anti-1e), 131.8 (2C), 128.4 (2C, rac-syn-1e), 128.2 (2C, rac-anti-1e), 127.8 (2C, rac-syn-1e), 127.7 (2C, rac-syn-1e), 127.6 (2C, rac-anti-1e), 127.3 (2C, rac-anti-1e), 126.3, 74.0, 47.3, 45.3, 27.0, 18.8 (rac-anti-1e), 16.1 (rac-syn-1e). HRMS (APCl) m/z: [M- $\left.\mathrm{C}_{10} \mathrm{H}_{13} \mathrm{O}\right]^{+}$Calcd for $\mathrm{C}_{17} \mathrm{H}_{6} \mathrm{Cl}$ 125.0158; Found 125.0151. 
(2S,3S)-((4-(4-Bromophenyl)-3-methyl-3-phenylbutan-2-yl)oxy)(3,5dimethylphenyl)dimethylsilane

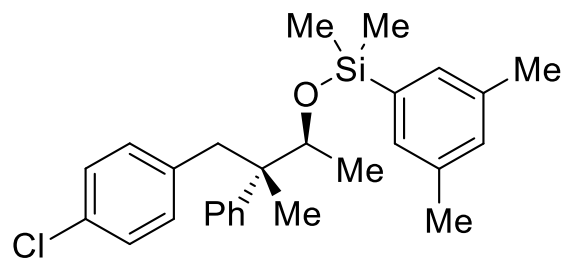

$(2 S, 3 S)-3$ ee

$\mathrm{C}_{27} \mathrm{H}_{33} \mathrm{ClOSi}$

$\mathrm{M}=437.10 \mathrm{~g} / \mathrm{mol}$

Prepared according to GP1 from rac-anti/syn-1e (55.0 mg, 0.200 mmol, 1.00 equiv.) and (3,5-dimethylphenyl)dimethylsilane (13.2 $\mathrm{mg}, 0.0800 \mathrm{mmol}, 0.400$ equiv.). The reaction mixture was stirred at $-10^{\circ} \mathrm{C}$ for $168 \mathrm{~h}$. Flash column chromatography using $n$-pentane/ethyl acetate $(30: 1 \rightarrow 7: 1)$ afforded silyl ether $(2 S, 3 S)$-3ee (26 mg, 0.059 mmol, $30 \%$, d.r. $=97: 3,87 \%$ ee) as a yellow oil.

$\mathbf{R}_{\boldsymbol{f}}=0.70$ (pentane/ethyl acetate 30:1). ${ }^{1} \mathrm{H}$ NMR (500 MHz, $\left.\mathrm{C}_{6} \mathrm{D}_{6}\right): \delta / p p m=7.42-7.44$ $(\mathrm{m}, 2 \mathrm{H}), 7.09-7.14(\mathrm{~m}, 1 \mathrm{H}), 7.03-7.06(\mathrm{~m}, 2 \mathrm{H}), 6.98-7.00(\mathrm{~m}, 2 \mathrm{H}), 6.90-6.92(\mathrm{~m}, 1 \mathrm{H})$, 6.87-6.91 (m, 2H), 6.45-6.47 (m, 2H), $4.26\left(\mathrm{q},{ }^{3} \mathrm{~J}=6.1 \mathrm{~Hz}, 1 \mathrm{H}\right), 3.14\left(\mathrm{~d},{ }^{2} \mathrm{~J}=13.1 \mathrm{~Hz}\right.$, $1 \mathrm{H}), 2.91\left(\mathrm{~d},{ }^{2} \mathrm{~J}=13.1 \mathrm{~Hz}, 1 \mathrm{H}\right), 2.22(\mathrm{~s}, 6 \mathrm{H}), 1.21(\mathrm{~s}, 3 \mathrm{H}), 0.89\left(\mathrm{~d},{ }^{3} J=6.3 \mathrm{~Hz}, 3 \mathrm{H}\right)$, $0.48(\mathrm{~s}, 3 \mathrm{H}), 0.46(\mathrm{~s}, 3 \mathrm{H}) .{ }^{13} \mathrm{C}$ NMR (126 MHz, $\left.\mathrm{C}_{6} \mathrm{D}_{6}\right): \delta / \mathrm{ppm}=145.1,138.5$, 137.6, $137.3(2 \mathrm{C}), 132.2(2 \mathrm{C}), 131.8,128.3(2 \mathrm{C}), 128.2(2 \mathrm{C}), 128.0(2 \mathrm{C}), 127.6(2 \mathrm{C})$, 126.3, 122.4 , 75.7, 48.1, 46.0, 21.5 (2C), 18.9, 15.7, -0.5, -0.5. ${ }^{29}$ Si DEPT NMR (99 $\left.\mathrm{MHz}, \mathrm{C}_{6} \mathrm{D}_{6}\right): \delta / \mathrm{ppm}=4.8$. IR $(\mathrm{ATR}): \mathrm{v} / \mathrm{cm}^{-1}=2975,2096,1596,1490,1251,1140$, 1096, 975, 870, 802, 699. HRMS (APCl) m/z: [M- $\left.\mathrm{C}_{10} \mathrm{H}_{15} \mathrm{OSi}\right]^{+}$Calcd for $\mathrm{C}_{17} \mathrm{H}_{18} \mathrm{Cl}$ 257.1097; Found 257.1097. Optical Rotation: $[\alpha]_{D}=+0.49\left(c 1.3, C_{6} D_{6}\right)$.

The enantiomeric excess of $(2 S, 3 S)$-3ee was determined by HPLC analysis on a chiral stationary phase after cleavage of the silyl ether according to GP3 (Daicel Chiralcel ${ }^{\circledR}$ IA column, column temperature $20^{\circ} \mathrm{C}$, mobile phase: $n$-heptane:isopropanol = 99.7:0.3, flow rate: $0.6 \mathrm{~mL} / \mathrm{min}, \lambda=210 \mathrm{~nm}): \mathrm{t}_{\mathrm{R}}=36.6 \mathrm{~min}$ for $\left(2 S^{*}, 3 R^{*}\right)-1 \mathrm{e}, \mathrm{t}_{\mathrm{R}}=40.5$ $\min$ for $\left(2 R^{*}, 3 S^{*}\right)-1 \mathrm{e}, \mathrm{t}_{\mathrm{R}}=51.4 \mathrm{~min}$ for $(2 S, 3 S)-1 \mathrm{e}$ and $\mathrm{t}_{\mathrm{R}}=55.1 \mathrm{~min}$ für $(2 R, 3 R)-1 \mathrm{e}$. 
rac-anti/syn-4-(4-Methoxyphenyl)-3-methyl-3-phenylbutan-2-ol<smiles>COc1ccc(CC(C)([C@H](C)O)[C@@H](C)O)cc1</smiles>

rac-anti-1k

$\mathrm{C}_{18} \mathrm{H}_{22} \mathrm{O}_{2}$

$\mathrm{M}=270.37 \mathrm{~g} / \mathrm{mol}$<smiles>COc1ccc(C[C@@](C)(c2ccccc2)[C@@H](C)O)cc1</smiles>

rac-syn-1k

$\mathrm{C}_{18} \mathrm{H}_{22} \mathrm{O}_{2}$

$\mathrm{M}=270.37 \mathrm{~g} / \mathrm{mol}$

Prepared according to GP1 from 3-(4-methoxyphenyl)-2-methyl-2-phenylpropanal (rac-S12, 770 mg, 3.03 mmol, 1.00 equiv.) using $\mathrm{MeMgCl}$ (20\% in THF, $0.400 \mathrm{~mL}$, $1.70 \mathrm{mmol}, 1.00$ equiv.). The reaction mixture was stirred for $24 \mathrm{~h}$. Automatic flash column chromatography using cyclohexane/ethyl acetate $(0: 100 \rightarrow 100: 1)$ afforded a mixture of the diastereomers rac-anti-1k and rac-syn-1k $(350 \mathrm{mg}, 1.27 \mathrm{mmol}, 42 \%$, d.r. $=90: 10)$ as a yellow oil.

$\mathbf{R}_{\boldsymbol{f}}=0.19$ for rac-anti-1k, 0.29 for rac-syn-1k (cyclohexane/ethyl acetate 10:1). ${ }^{\mathbf{1}} \mathbf{H}$ NMR $\left(500 \mathrm{MHz}, \mathrm{CDCl}_{3}\right): \delta / p p m=7.38-7.42(\mathrm{~m}, 1 \mathrm{H}$, rac-syn-1k $), 7.30-7.35(\mathrm{~m}, 1 \mathrm{H}$, rac-anti-1k), 7.29-7.30 (m, 2H, rac-syn-1k), 7.26-7.29 (m, 2H, rac-anti-1k), 7.19-7.22 ( $\mathrm{m}, 2 \mathrm{H}$, rac-anti-1k), 7.17-7.19 (m, 2H, rac-syn-1k), 6.73-6.80 (m, 2H, rac-syn-1k), 6.66-6.73 (m, 2H, rac-syn-1k), 6.62-6.66 (m, 2H, rac-anti-1k), 6.59-6.62 (m, 2H, rac-anti-1k), $4.16\left(\mathrm{q},{ }^{3} \mathrm{~J}=7.3 \mathrm{~Hz}, 1 \mathrm{H}, \quad\right.$ rac-anti-1k), $4.04 \quad\left(\mathrm{q},{ }^{3} \mathrm{~J}=7.3 \mathrm{~Hz}, 1 \mathrm{H}\right.$, rac-syn-1k), 3.75 (s, 3H, rac-syn-1k), 3.71 (s, 3H, rac-anti-1k), 3.11 (d, ${ }^{2} J=13.3 \mathrm{~Hz}$, $1 \mathrm{H}, \mathrm{H}-4 \beta$ ), 2.98 (d, $\left.{ }^{2} \mathrm{~J}=13.3 \mathrm{~Hz}, 1 \mathrm{H}\right), 1.46$. (s, 1H), 1.26 (s, 3H, rac-syn-1k), 1.21 (s, $3 \mathrm{H}$, rac-anti-1k), $1.15\left(\mathrm{~d},{ }^{3} \mathrm{~J}=6.3 \mathrm{~Hz}, 3 \mathrm{H}\right.$, rac-syn-1k), $0.96\left(\mathrm{~d},{ }^{3} \mathrm{~J}=6.3 \mathrm{~Hz}, 3 \mathrm{H}\right.$, rac-anti-1k). ${ }^{13} \mathrm{C} \quad \mathrm{NMR}\left(126 \mathrm{MHz}, \quad \mathrm{CDCl}_{3}\right): \delta / \mathrm{ppm}=157.9,144.9,131.5$ (2C, rac-syn-1k), 131.4 (2C, rac-anti-1k), 130.4, 128.1 (2C, rac-anti-1k), 127.9 (2C, rac-syn-1k), 127.4 (2C), 126.5 (rac-syn-1k), 126.1 (rac-anti-1k), 113.2 (2C, rac-syn-1k), 113.0 (2C, rac-anti-1k), 74.0, 60.5 (rac-syn-1k), 55.2 (rac-anti-1k), 47.3, 44.9 (rac-anti-1k), 44.8 (rac-syn-1k), 19.0 (rac-syn-1k), 18.8 (rac-anti-1k), 16.5 (rac-anti-1k), 14.3 (rac-syn-1k). HRMS (APCI) m/z: [M- $\left.\mathrm{C}_{10} \mathrm{H}_{13} \mathrm{O}\right]^{+}$Calcd for $\mathrm{C}_{8} \mathrm{H}_{9} \mathrm{O}$ 121.0653; Found 121.0648. 
(2S,3S)-(3,5-Dimethylphenyl)((4-(4-methoxyphenyl)-3-methyl-3-phenylbutan-2yl)oxy)dimethylsilane<smiles>COc1ccc(C[C@H](C)[C@H](C)O[Si](C)(O[Si](C)(C)c2ccc(C)cc2)c2cc(C)cc(C)c2)cc1</smiles>

$$
\begin{gathered}
(2 \mathrm{~S}, 3 \mathrm{~S})-3 \mathrm{ke} \\
\mathrm{C}_{28} \mathrm{H}_{36} \mathrm{O}_{2} \mathrm{Si} \\
M=432.68 \mathrm{~g} / \mathrm{mol}
\end{gathered}
$$

Prepared according to GP1 from rac-anti/syn-1k (54.1 mg, 0.200 mmol, 1.00 equiv.) and (3,5-dimethylphenyl)dimethylsilane (13.2 $\mathrm{mg}, 0.0800 \mathrm{mmol}, 0.400$ equiv.). The reaction mixture was stirred at $-10^{\circ} \mathrm{C}$ for $144 \mathrm{~h}$. Flash column chromatography using $n$-pentane/ethyl acetate (30:1 $\rightarrow$ 7:1) afforded silyl ethers (2S,3S)-3ke (19 mg, 0.0440 mmol, $22 \%$, d.r. > 99:1, 91\% ee) as a yellow oil.

${ }^{1} \mathrm{H}$ NMR $\left(500 \mathrm{MHz}, \mathrm{C}_{6} \mathrm{D}_{6}\right): \mathrm{\delta} / \mathrm{ppm}=7.46-7.47(\mathrm{~m}, 2 \mathrm{H}), 7.11-7.12(\mathrm{~m}, 4 \mathrm{H}), 6.90-6.92$ $(\mathrm{m}, 2 \mathrm{H}), 6.65-6.70(\mathrm{~m}, 2 \mathrm{H}), 6.57-6.59(\mathrm{~m}, 2 \mathrm{H}), 4.34\left(\mathrm{q},{ }^{3} \mathrm{~J}=6.2 \mathrm{~Hz}, 1 \mathrm{H}\right), 3.29\left(\mathrm{~d},{ }^{2} \mathrm{~J}=\right.$ $13.3 \mathrm{~Hz}, 1 \mathrm{H}), 3.22(\mathrm{~s}, 3 \mathrm{H}), 3.08\left(\mathrm{~d},{ }^{2} \mathrm{~J}=13.1 \mathrm{~Hz}, 1 \mathrm{H}\right), 2.22(\mathrm{~s}, 6 \mathrm{H}), 1.34(\mathrm{~s}, 3 \mathrm{H}), 0.94$ $\left(\mathrm{d},{ }^{3} \mathrm{~J}=6.2 \mathrm{~Hz}, 3 \mathrm{H}\right), 0.50(\mathrm{~s}, 3 \mathrm{H}), 0.49(\mathrm{~s}, 3 \mathrm{H}),{ }^{13} \mathrm{C}$ NMR $\left(126 \mathrm{MHz}, \mathrm{C}_{6} \mathrm{D}_{6}\right): \delta / p p m=$ $158.5,145.7,137.2,131.7$ (2C), 131.7, 131.0, 128.3 (2C), 128.1 (2C), 127.9 (2C), 127.8 (2C), 126.1, 113.4, 113.3 (2C), 75.8, 54.5, 48.3, 45.8, 21.4, 19.0, 15.8, -0.5, 0.5. ${ }^{29}$ Si DEPT NMR (99 MHz, C6 6 ) : $\delta / p p m=4.7$. IR (ATR): v/cm ${ }^{-1}=2957,1610$, 1462, 1248, 1178, 1140, 1099, 976, 870, 782, 698. HRMS (APCI) m/z: [M-C $\left.{ }_{10} \mathrm{H}_{15} \mathrm{OSi}\right]^{+}$ Calcd for $\mathrm{C}_{18} \mathrm{H}_{21} \mathrm{O}$ 258.1592; Found 258.1588. Optical Rotation: $[\alpha] \mathrm{D}=+0.78$ (c 1.3, $\left.\mathrm{C}_{6} \mathrm{D}_{6}\right)$.

The enantiomeric excess of (2S,3S)-3ke was determined by HPLC analysis on a chiral stationary phase after cleavage of the silyl ether according to GP3 (Daicel Chiralcel ${ }^{\circledR}$ $\mathrm{AD}-\mathrm{H}$ column, column temperature $20^{\circ} \mathrm{C}$, mobile phase: $n$-heptane:isopropanol = 97:3, flow rate: $0.6 \mathrm{~mL} / \mathrm{min}, \lambda=210 \mathrm{~nm}): \mathrm{t}_{\mathrm{R}}=28.9 \mathrm{~min}$ for $(2 S, 3 S)-1 \mathrm{k}, \mathrm{t}_{\mathrm{R}}=33.8$ (2R,3R)-1k. 
rac-anti/syn-4-(2-Bromophenyl)-2-methyl-2-phenylpropan-1-ol<smiles>CC(O)C(C)(Cc1ccccc1Br)c1ccccc1</smiles>

rac-anti-1h $\mathrm{C}_{16} \mathrm{H}_{17} \mathrm{BrO}$ $M=319.24 \mathrm{~g} / \mathrm{mol}$<smiles>CC(O)[C@](C)(Cc1ccccc1Br)c1ccccc1</smiles>

rac-syn-1h $\mathrm{C}_{16} \mathrm{H}_{17} \mathrm{BrO}$ $M=319.24 \mathrm{~g} / \mathrm{mol}$

Prepared according to GP1 from 3-(2-bromophenyl)-2-methyl-2-phenylpropanal (racS13, $440 \mathrm{mg}, 1.45 \mathrm{mmol}, 1.00$ equiv.) using $\mathrm{MeMgCl}$ (20\% in THF, $0.320 \mathrm{~mL}, 1.45$ mmol, 1.00 equiv.). The reaction mixture was stirred for $42 \mathrm{~h}$. Automatic flash column chromatography using cyclohexane/ethyl acetate $(0: 100 \rightarrow 100: 1)$ afforded a mixture of the diastereomers rac-anti-1h and rac-syn-1h $(280 \mathrm{mg}, 0.877 \mathrm{mmol}, 60 \%$, d.r. = 78:22) as a yellow oil.

$\mathbf{R}_{\boldsymbol{f}}=\mathbf{0 . 1 6}$ for rac-anti-1h, 0.25 for rac-syn-1h (cyclohexane/ethyl acetate 10:1). ${ }^{1} \mathrm{H}$ NMR $\left(500 \mathrm{MHz}, \mathrm{CDCl}_{3}\right): \delta / p p m=7.48-7.51$ (m, 1H, rac-syn-1h), 7.45-7.48 (m, $1 \mathrm{H}$, rac-anti-1h), 7.40-7.45 (m, 1H, rac-syn-1h), 7.33-7.38 (m, 1H, rac-anti-1h), 7.26$7.32(\mathrm{~m}, 2 \mathrm{H}), 7.22-7.25(\mathrm{~m}, 2 \mathrm{H}$, rac-anti-1h), 7.20-7.22 (m, 2H, rac-syn-1h), 6.92$6.97(\mathrm{~m}, 1 \mathrm{H}), 6.86-6.91(\mathrm{~m}, 1 \mathrm{H}), 6.51\left(\mathrm{~m}_{\mathrm{c}}, 1 \mathrm{H}\right.$, rac-syn-1h), $6.42\left(\mathrm{~m}_{\mathrm{c}}, 1 \mathrm{H}\right.$, rac-anti-1h), 4.22 (q, ${ }^{3} \mathrm{~J}=6.4 \mathrm{~Hz}, 1 \mathrm{H}$, rac-anti-1h), 4.13 (q, ${ }^{3} \mathrm{~J}=6.4 \mathrm{~Hz}, 1 \mathrm{H}$, rac-syn-1h), 3.60 (d, ${ }^{2} \mathrm{~J}$ $=13.3 \mathrm{~Hz}, 1 \mathrm{H}$, rac-anti-1h), $3.44\left(\mathrm{~d},{ }^{2} \mathrm{~J}=13.3 \mathrm{~Hz}, 1 \mathrm{H}\right.$, rac-syn-1h), 3.14 (d, ${ }^{2} \mathrm{~J}=13.3$ $\mathrm{Hz}, 1 \mathrm{H}), 1.53$ (s, 1H), 1.27 (s, 3H, rac-anti-1h), 1.23 (s, 3H, rac-syn-1h), 1.20 (d, ${ }^{3} \mathrm{~J}=$ $6.4 \mathrm{~Hz}, 3 \mathrm{H}$, rac-syn-1h), 0.97 (d, ${ }^{3} \mathrm{~J}=6.4 \mathrm{~Hz}, 3 \mathrm{H}$, rac-anti-1h). ${ }^{13} \mathrm{C}$ NMR $(126 \mathrm{MHz}$, $\left.\mathrm{CDCl}_{3}\right): \delta / p p m=148.2$ (rac-anti-1h), 147.3 (rac-syn-1h), 144.9 (rac-anti-1h), 144.1 (rac-syn-1h), 138.3 (rac-anti-1h), 138.1 (rac-syn-1h), 132.8 (rac-syn-1h), 132.7 (rac-anti-1h), 131.6 (rac-anti-1h), 131.4 (rac-syn-1h), 128.5 (2C, rac-syn-1h), 128.3 (2C, rac-anti-1h), 127.9 (2C, rac-syn-1h), 127.7 (rac-syn-1h), 127.6 (rac-anti-1h), 127.4 (2C, rac-anti-1h), 126.8 (rac-syn-1h), 126.7 (rac-syn-1h), 126.5 (rac-anti-1h), 126.3 (rac-anti-1h), 74.2 (rac-syn-1h), 74.1 (rac-anti-1h), 48.2 (rac-syn-1h), 48.1 (rac-anti-1h), 43.6 (rac-anti-1h), 43.0 (rac-anti-1h), 18.6 (rac-anti-1h), 17.9 (rac-syn-1h), 17.0 (rac-syn-1h), 15.6 (rac-anti-1h). HRMS (APCI) m/z: [M-C $\left.{ }_{10} \mathrm{H}_{13} \mathrm{O}\right]^{+}$ Calcd for $\mathrm{C}_{7} \mathrm{H}_{6} \mathrm{Br}$ 319.0619; Found 319.0629. 
(2S,3S)-((4-(2-Bromophenyl)-3-methyl-3-phenylbutan-2-yl)oxy)(3,5dimethylphenyl)dimethylsilane

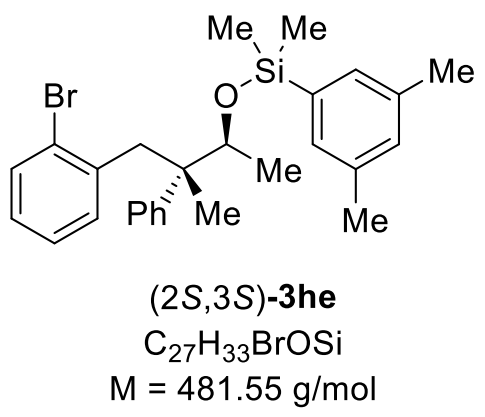

Prepared according to GP1 from rac-anti/syn-1h (53.9 mg, 0.200 mmol, 1.00 equiv.) and (3,5-dimethylphenyl)dimethylsilane (13.5 $\mathrm{mg}, 0.0800 \mathrm{mmol}, 0.400$ equiv.). The reaction mixture was stirred at $-10^{\circ} \mathrm{C}$ for $120 \mathrm{~h}$. Flash column chromatography using $n$-pentane/ethyl acetate $(30: 1 \rightarrow 7: 1)$ afforded silyl ether (2S,3S)-3he (30 mg, 0.062 mmol, 31\%, d.r. $=96: 4,84 \%$ ee) as a yellow oil.

$\mathbf{R}_{\boldsymbol{f}}=0.81$ (cyclohexane/ethyl acetate 10:1). ${ }^{1} \mathbf{H}$ NMR $\left(500 \mathrm{MHz}, \mathrm{C}_{6} \mathrm{D}_{6}\right): \delta / p p m=7.49$ $7.53(\mathrm{~m}, 2 \mathrm{H}), 7.33-7.44(\mathrm{~m}, 1 \mathrm{H}), 7.07-7.10(\mathrm{~m}, 2 \mathrm{H}), 7.06-7.07(\mathrm{~m}, 2 \mathrm{H}), 7.00-7.06(\mathrm{~m}$, $1 \mathrm{H})$, 6.87-6.92 (m, 1H), 6.55-6.58 (m, 1H), 6.52-6.55 (m, 1H), 6.27-6.33 $(\mathrm{m}, 1 \mathrm{H})$, $4.32\left(\mathrm{q},{ }^{3} \mathrm{~J}=6.1 \mathrm{~Hz}, 1 \mathrm{H}\right), 4.07\left(\mathrm{~d},{ }^{2} \mathrm{~J}=13.1 \mathrm{~Hz}, 1 \mathrm{H}\right), 2.96\left(\mathrm{~d},{ }^{2} \mathrm{~J}=13.1 \mathrm{~Hz}, 1 \mathrm{H}\right), 2.23$ (s, 6H), $1.38(\mathrm{~s}, 3 \mathrm{H}), 0.90\left(\mathrm{~d},{ }^{3} \mathrm{~J}=6.3 \mathrm{~Hz}, 3 \mathrm{H}\right), 0.54(\mathrm{~s}, 3 \mathrm{H}), 0.53(\mathrm{~s}, 3 \mathrm{H}) .{ }^{13} \mathrm{C}$ NMR $\left(126 \mathrm{MHz}, \mathrm{C}_{6} \mathrm{D}_{6}\right): \delta / \mathrm{ppm}=145.8,139.1,138.4,137.2,133.0,131.9$ (2C), 131.8, 131.7, 128.4 (2C), 128.2, 127.9 (2C), 127.7, 127.3, 126.5, 126.3, 75.8, 49.2, 44.4, 21.5 (2C), 18.7, 14.7, -0.4, -0.5. ${ }^{29}$ Si DEPT NMR (99 MHz, C6 $\left.\mathrm{D}_{6}\right): \delta / p p m=5.1$. IR (ATR): v/cm$1=2974,2124,1596,1374,1251,1078$, 975, 870, 780, 699. HRMS (APCI) m/z: [M$\left.\mathrm{C}_{10} \mathrm{H}_{15} \mathrm{OSi}\right]^{+}$Calcd for $\mathrm{C}_{17} \mathrm{H}_{18} \mathrm{Br} 301.0592$; Found 301.0590. Optical Rotation: $[\alpha]_{\mathrm{D}}=$ $+0.37\left(c 1.2, \mathrm{C}_{6} \mathrm{D}_{6}\right)$.

The enantiomeric excess of (2S,3S)-3he was determined by HPLC analysis on a chiral stationary phase after cleavage of the silyl ether according to GP3 (Daicel Chiralcel ${ }^{\circledR}$ $\mathrm{OD}-\mathrm{H}$ column, column temperature $20^{\circ} \mathrm{C}$, mobile phase: $n$-heptane:isopropanol = 98:2, flow rate: $0.6 \mathrm{~mL} / \mathrm{min}, \lambda=210 \mathrm{~nm}): \mathrm{tR}_{\mathrm{R}}=24.4 \mathrm{~min}$ for $\left(2 S^{*}, 3 R^{*}\right)-1 \mathrm{~h}$, tR $=30.5 \mathrm{~min}$ for $(2 R, 3 R)-\mathbf{1 h}, \mathrm{t}_{\mathrm{R}}=34.5 \mathrm{~min}$ for $\left(2 R^{*}, 3 S^{*}\right)-\mathbf{1 h}$, and $\mathrm{t}_{\mathrm{R}}=36.6 \mathrm{~min}$ for $(2 S, 3 S)-\mathbf{1 h}$. 
rac-anti/syn-4-(4-Iodophenyl)-3-methyl-3-phenylbutan-2-ol
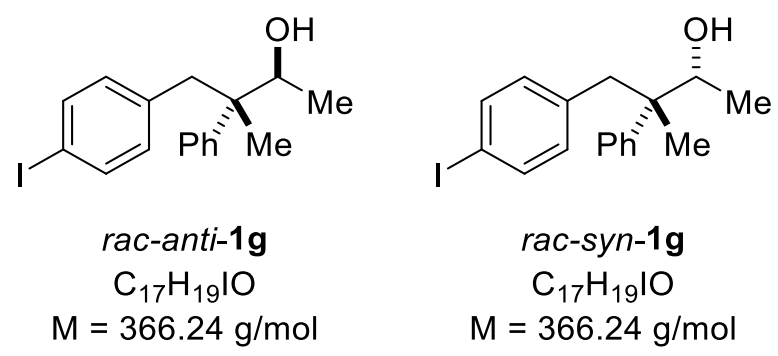

Prepared according to GP1 from 3-(2-bromophenyl)-2-methyl-2-phenylpropanal (racS14, $600 \mathrm{mg}, 1.71 \mathrm{mmol}, 1.00$ equiv.) using $\mathrm{MeMgCl}$ (20\% in THF, $0.378 \mathrm{~mL}, 1.71$ mmol, 1.00 equiv.). The reaction mixture was stirred for $70 \mathrm{~h}$. Automatic flash column chromatography using cyclohexane/ethyl acetate $(0: 100 \rightarrow 100: 1)$ afforded a mixture of the diastereomers rac-anti-1 $\mathbf{g}$ and rac-syn-19 (455 mg, $1.24 \mathrm{mmol}, 72 \%$, d.r. = 91:9) as a yellow oil.

$\mathbf{R}_{\boldsymbol{f}}=\mathbf{0 . 1 8}$ for rac-anti-1g, 0.27 for rac-syn-1g (cyclohexane/ethyl acetate 10:1). ${ }^{1} \mathrm{H}$ NMR $\left(400 \mathrm{MHz}, \mathrm{CDCl}_{3}\right): \delta / p p m=7.40-7.42(\mathrm{~m}, 2 \mathrm{H}$, rac-syn-1 g), 7.36-7.37 (m, $2 \mathrm{H}$, rac-anti-1 g), 7.27-7.30 (m, 1H, rac-anti-1g), 7.26-7.29 (m, 2H, rac-anti-1 g), 7.21-7.22 (m, 2H, rac-syn-1g), 7.20-7.22 (m, 1H), 7.17-7.19 (m, 2H, rac-anti-1g), 6.56-6.58 (m, 2H, rac-syn-1 g), 6.44-6.45 (m, 2H, rac-anti-1g), 4.17 (q, ${ }^{3} \mathrm{~J}=6.4 \mathrm{~Hz}$, $1 \mathrm{H}$, rac-anti-1g), $4.12\left(\mathrm{q},{ }^{3} \mathrm{~J}=7.2 \mathrm{~Hz}, 1 \mathrm{H}\right.$, rac-syn-1g), $3.81\left(\mathrm{~d},{ }^{2} \mathrm{~J}=10.8 \mathrm{~Hz}, 1 \mathrm{H}\right.$, rac-syn-1g), $3.65\left(\mathrm{~d},{ }^{2} \mathrm{~J}=10.8 \mathrm{~Hz}, 1 \mathrm{H}\right.$, rac-syn-1g), $3.11\left(\mathrm{~d},{ }^{2} \mathrm{~J}=13.2 \mathrm{~Hz}, 1 \mathrm{H}\right.$, rac-anti-1g), $2.99\left(\mathrm{~d},{ }^{2} \mathrm{~J}=13.2 \mathrm{~Hz}, 1 \mathrm{H}\right.$, rac-anti-1g), $1.20(\mathrm{~s}, 3 \mathrm{H}$, rac-anti-1g), 1.17 (s, $3 \mathrm{H}$, rac-syn-1g), 1.14 (d, ${ }^{3} \mathrm{~J}=6.3 \mathrm{~Hz}, 3 \mathrm{H}, \mathrm{H}-1$, rac-syn-1g), $0.96\left(\mathrm{~d},{ }^{3} \mathrm{~J}_{1,2}=6.4 \mathrm{~Hz}, 3 \mathrm{H}\right.$, rac-anti-1 g). ${ }^{13} \mathrm{C}$ NMR $\left(126 \mathrm{MHz}, \mathrm{CDCl}_{3}\right): \delta / \mathrm{ppm}=145.1,144.4,138.3,136.7(2 \mathrm{C}$, rac-syn-1g), 136.6 (2C, rac-anti-1g), 132.8 (2C, rac-syn-1g), 132.6 (2C, rac-anti-1g), 128.4 (2C, rac-syn-1g), 128.3 (2C, rac-anti-1g), 127.3 (2C), 126.4, 74.0, 47.3, 45.3

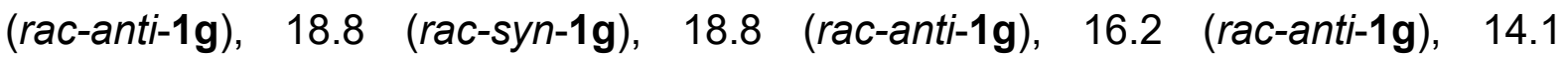
(rac-syn-1g). HRMS (APCl) m/z: [M- $\left.\mathrm{C}_{10} \mathrm{H}_{13} \mathrm{O}\right]^{+}$Calcd for $\mathrm{C}_{7} \mathrm{H}_{6} \mathrm{l}$ 216.9514; Found 216.9511. 
(2S,3S)-(3,5-Dimethylphenyl)(( 4-(4-iodophenyl)-3-methyl-3-phenylbutan-2yl)oxy)dimethylsilane

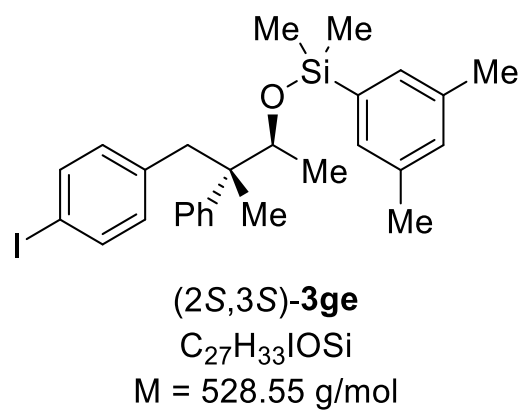

Prepared according to GP1 from rac-anti/syn-1g (73.3 mg, 0.200 mmol, 1.00 equiv.) and (3,5-dimethylphenyl)dimethylsilane (14.8 $\mathrm{mg}, 0.0900 \mathrm{mmol}, 0.450$ equiv.). The reaction mixture was stirred at $-10^{\circ} \mathrm{C}$ for $120 \mathrm{~h}$. Flash column chromatography using $n$-pentane/ethyl acetate $(30: 1 \rightarrow 7: 1)$ afforded silyl ether (2S,3S)-3ge (39 mg, 0.074 mmol, 37\%, d.r. $=96: 4,90 \%$ ee) as a yellow oil.

$\mathbf{R}_{\boldsymbol{f}}=0.82$ (cyclohexane/ethyl acetate 10:1). ${ }^{1} \mathbf{H}$ NMR $\left(500 \mathrm{MHz}, \mathrm{C}_{6} \mathrm{D}_{6}\right): \delta / p p m=7.41-$ $7.43(\mathrm{~m}, 2 \mathrm{H}), 7.21-7.25(\mathrm{~m}, 2 \mathrm{H}), 7.09-7.12(\mathrm{~m}, 1 \mathrm{H}), 7.00-7.03(\mathrm{~m}, 2 \mathrm{H}), 6.95-7.00$ $(\mathrm{m}, 2 \mathrm{H}), 6.86-6.93(\mathrm{~m}, 1 \mathrm{H}), 6.24-6.29(\mathrm{~m}, 2 \mathrm{H}), 4.25\left(\mathrm{q},{ }^{3} \mathrm{~J}=6.1 \mathrm{~Hz}, 1 \mathrm{H}\right), 3.09\left(\mathrm{~d},{ }^{2} \mathrm{~J}=\right.$ $13.1 \mathrm{~Hz}, 1 \mathrm{H}), 2.88\left(\mathrm{~d},{ }^{2} \mathrm{~J}=13.1 \mathrm{~Hz}, 1 \mathrm{H}\right), 2.21(\mathrm{~s}, 6 \mathrm{H}), 1.19(\mathrm{~s}, 3 \mathrm{H}), 0.88\left(\mathrm{~d},{ }^{3} J=6.3 \mathrm{~Hz}\right.$, $3 \mathrm{H}), 0.47$ (s, 3H), 0.46 (s, 3H). ${ }^{13} \mathrm{C}$ NMR (126 MHz, $\left.\mathrm{C}_{6} \mathrm{D}_{6}\right): \delta / \mathrm{ppm}=145.0,138.7$, 137.3, 136.8 (2C), 132.9 (2C), 131.8, 131.8 (2C), 128.2 (2C), 127.9 (2C), 127.6 (2C), 126.3, 91.6, 75.7, 48.0, 46.0, 21.5 (2C), 18.9, 15.6, -0.6 (2C). ${ }^{29}$ Si DEPT NMR $\left(99 \mathrm{MHz}, \mathrm{C}_{6} \mathrm{D}_{6}\right): \delta / p p m=4.9$. IR $($ ATR $): \mathrm{v} / \mathrm{cm}^{-1}=2973,2277,1483,1399,1251,1097$, 976, 870, 800, 699. HRMS (APCI) m/z: [M- $\left.\mathrm{C}_{10} \mathrm{H}_{15} \mathrm{OSi}\right]^{+}$Calcd for $\mathrm{C}_{17} \mathrm{H}_{18} \mathrm{l}$ 349.0453; Found 349.0451. Optical Rotation: $[\alpha]_{D}=+0.10\left(c 1.3, \mathrm{C}_{6} \mathrm{D}_{6}\right)$.

The enantiomeric excess of (2S,3S)-3ge was determined by HPLC analysis on a chiral stationary phase after cleavage of the silyl ether according to GP3 (Daicel Chiralcel ${ }^{\circledR}$ IA column, column temperature $20^{\circ} \mathrm{C}$, mobile phase: $n$-heptane:isopropanol $=99: 1$, flow rate: $0.6 \mathrm{~mL} / \mathrm{min}, \lambda=210 \mathrm{~nm}): \mathrm{t}_{\mathrm{R}}=36.6 \mathrm{~min}$ for $\left(2 S^{*}, 3 R^{*}\right)-1 \mathrm{~g}, \mathrm{t}_{\mathrm{R}}=43.8 \mathrm{~min}$ for $\left(2 R^{*}, 3 S^{*}\right)-\mathbf{1 g}, \mathrm{t}_{\mathrm{R}}=46.1 \mathrm{~min}$ for $(2 S, 3 S)-\mathbf{1 g}$ and $\mathrm{t}_{\mathrm{R}}=49.3 \mathrm{~min}$ for $(2 R, 3 R) \mathbf{- 1} \mathbf{g}$. 


\subsection{Preparation of Precursors}

rac-2-Methyl-2,3-diphenylpropanal

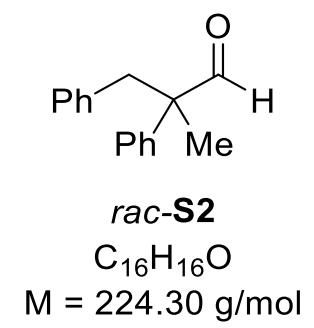

Prepared according to GP2 from 2-phenylpropanal (4.00 g, $29.8 \mathrm{mmol}, 1.00$ equiv.) and benzyl bromide (10.6 mL, $89.4 \mathrm{mmol}, 3.00$ equiv.) with potassium hydroxide (9.58 $\mathrm{g}, 179 \mathrm{mmol}, 6.00$ equiv.) in DME (230 mL). The reaction mixture was stirred at rt for $48 \mathrm{~h}$. Automatic flash column chromatography using cyclohexane/ethyl acetate (100:0 $\rightarrow$ 95:5) afforded aldehyde rac-S2 (1.71 g, $7.63 \mathrm{mmol}, 26 \%)$ as a yellow oil.

$\mathbf{R}_{\boldsymbol{f}}=0.76$ (cyclohexane/ethyl acetate 10:1). ${ }^{1} \mathbf{H}$ NMR (400 MHz, $\mathrm{CDCl}_{3}$ ): $\delta / p p m=9.64$ $(\mathrm{s}, 1 \mathrm{H}), 7.35-7.39(\mathrm{~m}, 2 \mathrm{H}), 7.29-7.33(\mathrm{~m}, 1 \mathrm{H}), 7.17-7.20(\mathrm{~m}, 2 \mathrm{H}), 7.13-7.16(\mathrm{~m}, 3 \mathrm{H})$, 6.79-6.81 (m, 2H), $3.23\left(\mathrm{~d},{ }^{2} \mathrm{~J}=13.6 \mathrm{~Hz}, 1 \mathrm{H}\right), 3.18\left(\mathrm{~d},{ }^{2} \mathrm{~J}=13.6 \mathrm{~Hz}, 1 \mathrm{H}\right), 1.38(\mathrm{~s}$, $3 \mathrm{H}) .{ }^{13} \mathrm{C}$ NMR $\left(126 \mathrm{MHz} \mathrm{CDCl}_{3}\right): \delta / \mathrm{ppm}=202.1,139.5,137.0,130.6(2 \mathrm{C}), 128.9(2 \mathrm{C})$, $128.0(2 \mathrm{C}), 127.8(2 \mathrm{C}), 127.6,126.6,55.2,42.9,18.4$.

The analytic data are in accordance with those reported in the literature. [S5] 
rac-2-Benzyl-2-phenylbutanenitrile

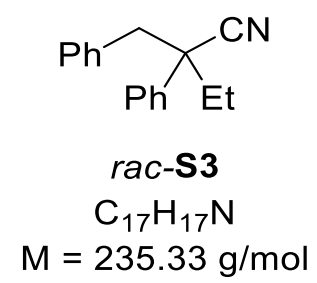

Prepared according to GP2 from 2-phenylbutanenitrile $(2.00 \mathrm{~g}, 13.7 \mathrm{mmol}$, 1.00 equiv.) and benzyl bromide (1.95 mL, $16.4 \mathrm{mmol}, 1.20$ equiv.) with potassium hydroxide (4.61 g, $82.2 \mathrm{mmol}, 6.00$ equiv.) in THF (137 mL). The reaction mixture was stirred at rt for $2 \mathrm{~h}$. Automatic flash column chromatography using cyclohexane/ethyl acetate $(100: 0 \rightarrow 1: 100)$ afforded nitrile rac-S3 $(2.66 \mathrm{~g}, 11.3 \mathrm{mmol}, 82 \%)$ as a colorless oil.

$\mathbf{R}_{\boldsymbol{f}}=0.48$ (cyclohexane/ethyl acetate 30:1). ${ }^{1} \mathrm{H}$ NMR $\left(400 \mathrm{MHz}, \mathrm{CDCl}_{3}\right): \delta / p p m=7.39$ $7.42(\mathrm{~m}, 1 \mathrm{H}), 7.38-7.39(\mathrm{~m}, 2 \mathrm{H}), 7.34-7.38(\mathrm{~m}, 2 \mathrm{H}), 7.31-7.34(\mathrm{~m}, 1 \mathrm{H}), 7.21-7.25(\mathrm{~m}$, 2H), 7.01-7.06 (m, 2H), $3.26\left(\mathrm{~d},{ }^{2} \mathrm{~J}=13.3 \mathrm{~Hz}, 1 \mathrm{H}\right), 3.18\left(\mathrm{~d},{ }^{2} \mathrm{~J}=13.3 \mathrm{~Hz}, 1 \mathrm{H}\right), 2.16$ (q, $\left.{ }^{3} J=7.6 \mathrm{~Hz}, 2 \mathrm{H}\right), 1.00\left(\mathrm{t},{ }^{3} J=7.6 \mathrm{~Hz}, 3 \mathrm{H}\right) .{ }^{13} \mathrm{C} \mathrm{NMR}\left(126 \mathrm{MHz}, \mathrm{CDCl}_{3}\right): \delta / p p m=137.7$, 135.3, 130.5 (2C), 129.0 (2C), 128.2 (2C), 127.9, 127.4, 126.6 (2C), 122.1, 50.7, 47.6, 32.8, 9.9. IR (ATR): $\mathrm{v} / \mathrm{cm}^{-1}=2970,2234,1494,1383,1079,1031,767,698$. HRMS (APCl) m/z: [M-CN] ${ }^{+}$Calcd for $\mathrm{C}_{16} \mathrm{H}_{17}$ 209.1330; Found 209.1327.

rac-2-Benzyl-2-phenylbutanal

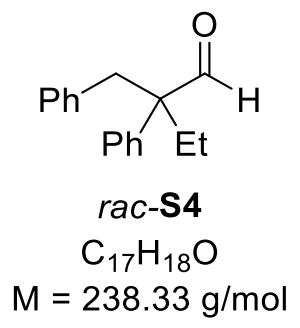

Prepared according to GP4 from rac-S3 (939 mg, $3.99 \mathrm{mmol}, 1.00$ equiv.) with DIBAL (2.00 $\mathrm{M}$ in pentane, $2.00 \mathrm{~mL}, 3.99 \mathrm{mmol}, 1.00$ equiv.) in THF (10.5 mL). The reaction mixture was stirred at $0{ }^{\circ} \mathrm{C}$ for $2 \mathrm{~h}$ and at $\mathrm{rt}$ for $18 \mathrm{~h}$. Due to fast decomposition the desired aldehyde rac-S4 (350 mg, $1.47 \mathrm{mmol}, 37 \%$ ) was used without further purification for the following step. 
$\mathbf{R}_{\boldsymbol{f}}=0.85$ (cyclohexane/ethyl acetate 10:1). ${ }^{1} \mathbf{H} \mathbf{~ N M R}\left(400 \mathrm{MHz}, \mathrm{CDCl}_{3}\right): \delta / p p m=9.69$ (s, 1H), 7.34-7.37 (m, 2H), 7.28-7.31 (m, 1H), 7.10-7.14 (m, 5H), 6.75-6.77 (m, 2H), $3.28\left(\mathrm{~d},{ }^{2} \mathrm{~J}=14.1 \mathrm{~Hz}, 1 \mathrm{H}\right), 3.20\left(\mathrm{~d},{ }^{2} \mathrm{~J}=14.1 \mathrm{~Hz}, 1 \mathrm{H}\right), 1.95\left(\mathrm{~m}_{\mathrm{c}}, 2 \mathrm{H}\right), 0.95\left(\mathrm{t},{ }^{3} \mathrm{~J}=7.6\right.$ $\mathrm{Hz}, 3 \mathrm{H})$.

rac-3-(4-Fluorophenyl)-2-methyl-2-phenylpropanal

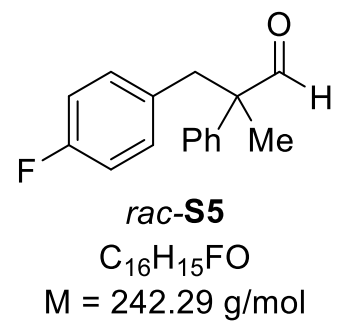

Prepared according to GP2 from 2-phenylpropanal (500 mg, 3.73 mmol, 1.00 equiv.) and 1-(bromomethyl)-4-fluorobenzene (704 mg, $3.73 \mathrm{mmol}, 1.00$ equiv.) with potassium hydroxide (1.25 g, $22.4 \mathrm{mmol}, 6.00$ equiv.) in THF (40 mL). The reaction mixture was stirred under reflux for $3 \mathrm{~h}$. Due to fast decomposition the desired aldehyde rac-S5 (702 mg, $2.90 \mathrm{mmol}, 77 \%$ ) was used without further purification for the following step.

$\mathbf{R}_{\boldsymbol{f}}=0.85$ (cyclohexane/ethyl acetate 10:1). ${ }^{1} \mathbf{H}$ NMR $\left(400 \mathrm{MHz}, \mathrm{CDCl}_{3}\right): \delta / p p m=9.60$ (s, 1H), 7.33-7.38 (m, 2H), 7.26-7.33 (m, 2H), 7.13-7.17 (m, 2H), 6.79-6.83 (m, 1H), 6.69-6.75 (m, 2H), $2.89\left(\mathrm{~d},{ }^{2} \mathrm{~J}=13.6 \mathrm{~Hz}, 1 \mathrm{H}\right), 2.77\left(\mathrm{~d},{ }^{2} \mathrm{~J}=13.6 \mathrm{~Hz}, 1 \mathrm{H}\right), 1.37(\mathrm{~s}, 3 \mathrm{H})$.

rac-2-Methyl-2-phenyl-3-(p-tolyl)propanal

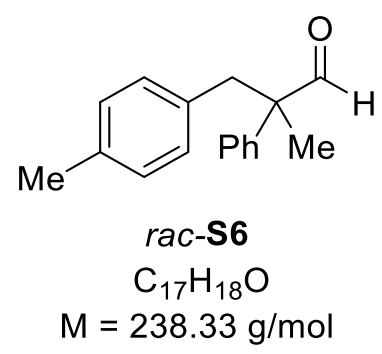

Prepared according to GP2 from 2-phenylpropanal (1.00 g, $7.45 \mathrm{mmol}, 1.00$ equiv.) and 4-methylbenzyl bromide (1.65 g, $8.94 \mathrm{mmol}, 1.20$ equiv.) with potassium hydroxide (2.51 g, $44.7 \mathrm{mmol}, 6.00$ equiv.) in THF (74 mL). The reaction mixture was stirred at $40{ }^{\circ} \mathrm{C}$ for $15 \mathrm{~min}$ and at rt for $24 \mathrm{~h}$. Due to fast decomposition the desired 
aldehyde rac-S6 (563 mg, $2.36 \mathrm{mmol}, 31 \%$ ) was used without further purification for the following step.

$\mathbf{R}_{\boldsymbol{f}}=0.90$ (cyclohexane/ethyl acetate 10:1). ${ }^{1} \mathbf{H}$ NMR $\left(400 \mathrm{MHz}, \mathrm{CDCl}_{3}\right): \delta / p p m=9.64$ (s, 1H), 7.35-7.39 (m, 2H), 7.29-7.33 (m, 1H), 7.18-20 (m, 2H), 6.94-6.96 (m, 2H), 6.69-6.70 (m, 2H), $3.18\left(\mathrm{~d},{ }^{2} \mathrm{~J}=13.6 \mathrm{~Hz}, 1 \mathrm{H}\right), 2.94\left(\mathrm{~d},{ }^{2} \mathrm{~J}=13.6 \mathrm{~Hz}, 1 \mathrm{H}\right), 2.17(\mathrm{~s}, 3 \mathrm{H})$, $1.37(\mathrm{~s}, 3 \mathrm{H})$.

\section{rac-3-(3,5-Dimethylphenyl)-2-methyl-2-phenylpropanal}

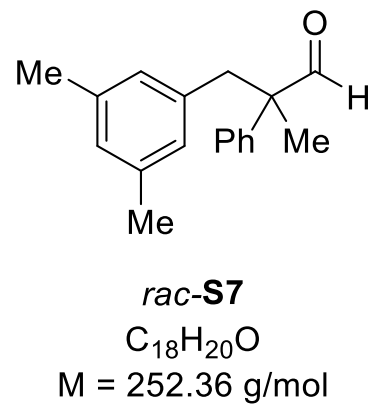

Prepared according to GP2 from 2-phenylpropanal (500 mg, $3.73 \mathrm{mmol}, 1.00$ equiv.) and 1-(bromomethyl)-3,5-dimethylbenzene (890 mg, $4.47 \mathrm{mmol}, 1.20$ Äquiv.) with potassium hydroxide (1.25 g, $22.4 \mathrm{mmol}, 6.00$ equiv.) in THF (40 mL). The reaction mixture was stirred at $40^{\circ} \mathrm{C}$ for $15 \mathrm{~min}$ and for $96 \mathrm{~h}$ at rt. Due to fast decomposition the desired aldehyde rac-S7 (702 $\mathrm{mg}, 2.90 \mathrm{mmol}, 77 \%)$ was used without further purification for the following step.

$\boldsymbol{R}_{\boldsymbol{f}}=0.82$ (cyclohexane/ethyl acetate 10:1). ${ }^{1} \mathbf{H}$ NMR $\left(400 \mathrm{MHz}, \mathrm{CDCl}_{3}\right): \delta / p p m=9.65$ (s, 1H), 7.38-7.43 (m, 1H), 7.35-7.38 (m, 1H), 7.29-7.34 (m, 2H), 7.16-7.21 (m, 2H), 6.39-6.44 (m, 2H), $3.18\left(\mathrm{~d},{ }^{2} \mathrm{~J}=13.6 \mathrm{~Hz}, 1 \mathrm{H}\right), 2.94\left(\mathrm{~d},{ }^{2} \mathrm{~J}=13.6 \mathrm{~Hz}, 1 \mathrm{H}\right), 2.17$ (s, $6 \mathrm{H}), 1.37(\mathrm{~s}, 3 \mathrm{H})$. 
rac-3-(3,5-Dimethoxyphenyl)-2-methyl-2-phenylpropanal

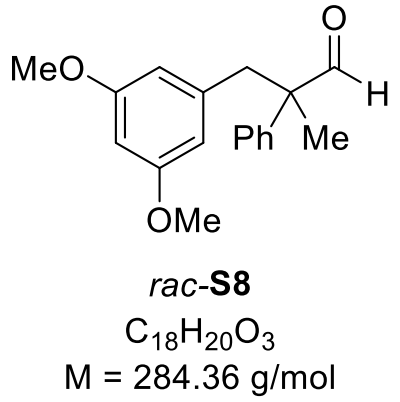

Prepared according to GP2 from 2-phenylpropanal (500 mg, $3.73 \mathrm{mmol}, 1.00$ equiv.) and 3,5-dimethoxylbenzyl bromide (1.03 g, $4.47 \mathrm{mmol}, 1.20$ equiv.) with potassium hydroxide (1.25 g, $22.4 \mathrm{mmol}, 6.00$ equiv.) in THF (35 mL). The reaction mixture was stirred at $40{ }^{\circ} \mathrm{C}$ for $15 \mathrm{~min}$ and at rt for $24 \mathrm{~h}$. Due to fast decomposition the desired aldehyde rac-S8 (149 mg, $0.524 \mathrm{mmol}, 14 \%$ ) was used without further purification for the following step.

$\mathbf{R}_{\boldsymbol{f}}=0.66$ (cyclohexane/ethyl acetate 10:1). ${ }^{1} \mathbf{H} \mathbf{N M R}\left(400 \mathrm{MHz}, \mathrm{CDCl}_{3}\right): \delta / p p m=9.62$ (s, $1 \mathrm{H}), 7.33-7.40(\mathrm{~m}, 2 \mathrm{H}), 7.28-7.32(\mathrm{~m}, 1 \mathrm{H}), 7.17-7.22(\mathrm{~m}, 2 \mathrm{H}), 6.23-6.28(\mathrm{~m}, 1 \mathrm{H})$, 5.89-5.92 (m, 2H), $3.60(\mathrm{~s}, 6 \mathrm{H}), 3.17\left(\mathrm{~d},{ }^{2} \mathrm{~J}=13.6 \mathrm{~Hz}, 1 \mathrm{H}\right), 3.08\left(\mathrm{~d},{ }^{2} \mathrm{~J}=13.6 \mathrm{~Hz}, 1 \mathrm{H}\right)$, $1.40(\mathrm{~s}, 3 \mathrm{H})$.

rac-3-(4-Bromophenyl)-2-methyl-2-phenylpropanal

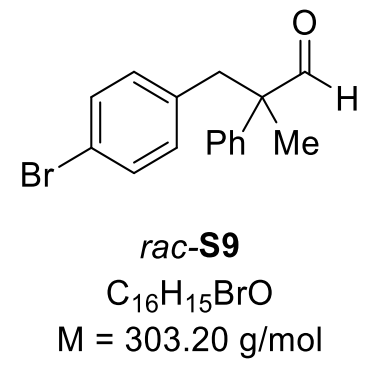

Prepared according to GP2 from 2-phenylpropanal (500 mg, $3.73 \mathrm{mmol}, 1.00$ equiv.) and 4-bromobenzyl bromide (1.12 g, $4.47 \mathrm{mmol}, 1.20$ equiv.) with potassium hydroxide (1.25 g, $22.4 \mathrm{mmol}, 6.00$ equiv.) in THF (40 mL). The reaction mixture was stirred at $40{ }^{\circ} \mathrm{C}$ for $15 \mathrm{~min}$ and at rt for $24 \mathrm{~h}$. Due to fast decomposition the desired aldehyde rac-S9 (415 mg, $1.37 \mathrm{mmol}, 37 \%$ ) was used without further purification for the following step. 
$\mathbf{R}_{\boldsymbol{f}}=0.67$ (cyclohexane/ethyl acetate 10:1). ${ }^{1} \mathbf{H} \mathbf{~ N M R}\left(400 \mathrm{MHz}, \mathrm{CDCl}_{3}\right): \delta / p p m=9.62$ (s, 1H), 7.34-7.38 (m, 2H), 7.30-7.34 (m, 2H), 7.22-7.24 (m, 1H), 7.12-7.15 (m, 2H), 6.59-6.65 (m, 2H), $3.06\left(\mathrm{~d},{ }^{2} \mathrm{~J}=13.6 \mathrm{~Hz}, 1 \mathrm{H}\right), 2.97\left(\mathrm{~d},{ }^{2} \mathrm{~J}=13.6 \mathrm{~Hz}, 1 \mathrm{H}\right), 1.36(\mathrm{~s}, 3 \mathrm{H})$.

rac-3-(4-Chlorophenyl)-2-methyl-2-phenylpropanal<smiles>CC(Cc1ccc(Cl)cc1)C(C)(C)C=O</smiles>

Prepared according to GP2 from 2-phenylpropanal (500 mg, $3.73 \mathrm{mmol}, 1.00$ equiv.) and 4-chlorobenzyl bromide (919 $\mathrm{mg}, 4.47 \mathrm{mmol}, 1.20$ equiv.) with potassium hydroxide (1.25 g, $22.4 \mathrm{mmol}, 6.00$ equiv.) in THF (40 mL). The reaction mixture was stirred at $40{ }^{\circ} \mathrm{C}$ for $15 \mathrm{~min}$ and at rt for $16 \mathrm{~h}$. Due to fast decomposition the desired aldehyde rac-S10 (800 mg, $3.09 \mathrm{mmol}, 82 \%)$ was used without further purification for the following step.

$\mathbf{R}_{\boldsymbol{f}}=0.80$ (cyclohexane/ethyl acetate 10:1). ${ }^{1} \mathbf{H}$ NMR $\left(400 \mathrm{MHz}, \mathrm{CDCl}_{3}\right): \delta / p p m=9.59$ (s, 1H), 7.34-7.39 (m, 2H), 7.30-7.34 (m, 1H), 7.12-7.16 (m, 2H), 7.06-7.11 (m, 2H), 6.66-6.71 (m, 2H), $3.19\left(\mathrm{~d},{ }^{2} J=13.6 \mathrm{~Hz}, 1 \mathrm{H}\right), 3.11\left(\mathrm{~d},{ }^{2} \mathrm{~J}=13.6 \mathrm{~Hz}, 1 \mathrm{H}\right), 1.37(\mathrm{~s}, 3 \mathrm{H})$. 


\section{4-Methoxybenzylbromid}

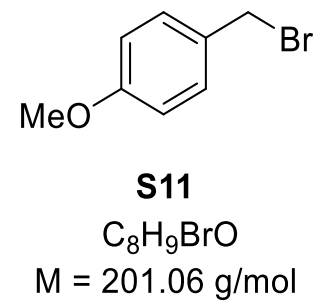

Following a modified literature procedure, ${ }^{[\mathrm{S} 6]}$ to a solution of 4-methoxybenzyl alcohol ( $1.00 \mathrm{~g}, 7.24 \mathrm{mmol}, 2.00$ equiv.) in diethyl ether $(8 \mathrm{~mL})$ phosphorus tribromide (979.2 $\mathrm{mg}, 3.618 \mathrm{mmol}, 1.000$ equiv.) was added dropwise at $0^{\circ} \mathrm{C}$. The resulting solution was stirred at rt for $2 \mathrm{~h}$ and quenched with aq. $\mathrm{NaHCO}_{3}(10 \mathrm{~mL})$ and ice $(2 \mathrm{~g})$. The organic phase was washed with aq. $\mathrm{NaHCO}_{3}(3 \times 10 \mathrm{~mL})$, the combined organic extracts were dried over anhydrous $\mathrm{MgSO}_{4}$, filtered, and concentrated under reduced pressure. The desired alcohol $\mathbf{S 1 1}(964 \mathrm{mg}, 4.79 \mathrm{mmol}, 66 \%)$ was received as a colorless oil and used without further purification for the next step.

${ }^{1} \mathrm{H}$ NMR $\left(500 \mathrm{MHz}, \mathrm{CDCl}_{3}\right): \delta / p p m=7.30-7.34(\mathrm{~m}, 2 \mathrm{H}), 6.84-6.88(\mathrm{~m}, 2 \mathrm{H}), 4.50$ (s, 2H), $3.81(\mathrm{~S}, 3 \mathrm{H})$.

The analytic data are in accordance with those reported in the literature. ${ }^{[S 6]}$

rac-3-(4-Methoxyphenyl)-2-methyl-2-phenylpropanal

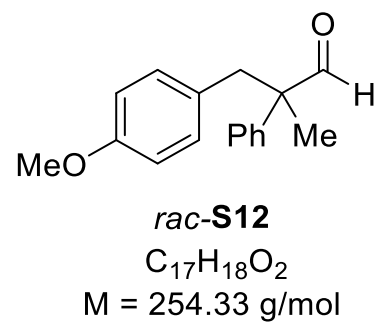

Prepared according to GP2 from 2-phenylpropanal (500 mg, $3.73 \mathrm{mmol}, 1.00$ equiv.) and 4-methoxybenzyl bromide (S11, $900 \mathrm{mg}, 4.47 \mathrm{mmol}, 1.20$ equiv.) with potassium hydroxide (1.25 g, $22.4 \mathrm{mmol}, 6.00$ equiv.) in THF (37 mL). The reaction mixture was stirred at $40{ }^{\circ} \mathrm{C}$ for $15 \mathrm{~min}$ and at $\mathrm{rt}$ for $16 \mathrm{~h}$. Due to fast decomposition the desired aldehyde rac-S12 (900 mg, $3.54 \mathrm{mmol}, 95 \%)$ was used without further purification for the following step. 
$\mathbf{R}_{\boldsymbol{f}}=0.76$ (cyclohexane/ethyl acetate 10:1). ${ }^{1} \mathbf{H} \mathbf{~ N M R}\left(400 \mathrm{MHz} \mathrm{CDCl}_{3}\right): \delta / p p m=9.88$ (s, 1H), 7.32-7.35 (m, 2H), 7.22-7.24 (m, 1H), 6.99-7.02 (m, 2H), 6.88-6.91 (m, 2H), 6.75-6.77 (m, 2H), $3.88(\mathrm{~s}, 3 \mathrm{H}), 3.08\left(\mathrm{~d},{ }^{2} \mathrm{~J}=13.6 \mathrm{~Hz}, 1 \mathrm{H}\right), 2.97\left(\mathrm{~d},{ }^{2} \mathrm{~J}=13.6 \mathrm{~Hz}, 1 \mathrm{H}\right)$, $1.24(\mathrm{~s}, 3 \mathrm{H})$.

rac-3-(2-Bromophenyl)-2-methyl-2-phenylpropanal<smiles>CC(C=O)(Cc1ccccc1)Cc1ccccc1Br</smiles>

rac-S13

$\mathrm{C}_{16} \mathrm{H}_{15} \mathrm{BrO}$

$M=303.20 \mathrm{~g} / \mathrm{mol}$

Prepared according to GP2 from 2-phenylpropanal (500 mg, $3.73 \mathrm{mmol}, 1.00$ equiv.) and 2-bromobenzyl bromide (1.12 g, $4.47 \mathrm{mmol}, 1.20$ equiv.) with potassium hydroxide (1.25 g, $22.4 \mathrm{mmol}, 6.00$ equiv.) in THF (39 mL). The reaction mixture was stirred at $40{ }^{\circ} \mathrm{C}$ for $15 \mathrm{~min}$ and at $\mathrm{rt}$ for $24 \mathrm{~h}$. Due to fast decomposition the desired aldehyde rac-S13 (447 mg, $1.47 \mathrm{mmol}, 39 \%$ ) was used without further purification for the following step.

$\mathbf{R}_{\boldsymbol{f}}=0.36$ (cyclohexane/ethyl acetate 30:1). ${ }^{1} \mathrm{H}$ NMR $\left(400 \mathrm{MHz}, \mathrm{CDCl}_{3}\right): \delta / p p m=9.64$ (s, 1H), 7.48-7.52 (m, 2H), 7.37-7.39 (m, 1H), 7.31-7.33 (m, 2H), 7.19-7.23 (m, 1H), 7.02-7.05 (m, 1H), 7.00-7.02 (m, 1H), 6.66-6.69 (m, 1H), $3.64\left(\mathrm{~d},{ }^{2} \mathrm{~J}=13.6 \mathrm{~Hz}, 1 \mathrm{H}\right)$, $3.17\left(\mathrm{~d},{ }^{2} \mathrm{~J}=13.6 \mathrm{~Hz}, 1 \mathrm{H}\right), 1.44(\mathrm{~s}, 3 \mathrm{H})$.

rac-3-(4-lodophenyl)-2-methyl-2-phenylpropanal

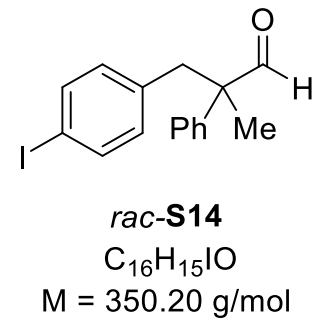

Prepared according to GP2 from 2-phenylpropanal (500 mg, 3.73 mmol, 1.00 equiv.) and 2-iodobenzyl bromide (1.33 g, $4.47 \mathrm{mmol}, 1.20$ equiv.) with potassium hydroxide 
(1.25 g, $22.4 \mathrm{mmol}, 6.00$ equiv.) in THF (39 mL). The reaction mixture was stirred at $40{ }^{\circ} \mathrm{C}$ for $10 \mathrm{~min}$ and at $\mathrm{rt}$ for $24 \mathrm{~h}$. Due to fast decomposition the desired aldehyde rac-S14 (648 mg, $1.85 \mathrm{mmol}, 49 \%$ ) was used without further purification for the following step.

$\mathbf{R}_{\boldsymbol{f}}=0.45$ (cyclohexane/ethyl acetate 10:1). ${ }^{1} \mathbf{H}$ NMR $\left(400 \mathrm{MHz}, \mathrm{CDCl}_{3}\right): \delta / p p m=9.58$ (s, 1H), 7.42-7.46 (m, 2H), 7.34-7.39 (m, 2H), 7.30-7.33 (m, 1H), 7.10-7.16 (m, 2H), 6.48-6.52 (m, 2H), $3.16\left(\mathrm{~d},{ }^{2} J=13.6 \mathrm{~Hz}, 1 \mathrm{H}\right), 3.08\left(\mathrm{~d},{ }^{2} \mathrm{~J}=13.6 \mathrm{~Hz}, 1 \mathrm{H}\right), 1.37(\mathrm{~s}, 3 \mathrm{H})$.

rac-2-Benzyl-2-methylbutanal

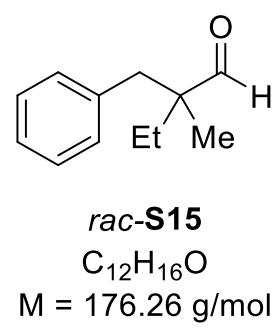

Prepared according to GP2 from 2-methylbutyraldehyde (500 mg, $5.81 \mathrm{mmol}, 1.00$ equiv.) and benzyl bromide (1.19 g, $6.97 \mathrm{mmol}, 1.20$ equiv.) with potassium hydroxide (1.95 g, $34.8 \mathrm{mmol}, 6.00$ equiv.) in THF (61 mL). The reaction mixture was stirred at $40{ }^{\circ} \mathrm{C}$ for $10 \mathrm{~min}$ and at $\mathrm{rt}$ for $20 \mathrm{~h}$. Due to fast decomposition the desired aldehyde rac-S15 (648 mg, $3.67 \mathrm{mmol}, 63 \%$ ) was used without further purification for the following step.

$\mathbf{R}_{\boldsymbol{f}}=0.90$ (cyclohexane/ethyl acetate 10:1). ${ }^{1} \mathbf{H}$ NMR $\left(400 \mathrm{MHz}, \mathrm{CDCl}_{3}\right): \delta / p p m=9.56$ (s, 1H), 7.34-7.37 (m, 2H), 7.20-7.23 (m, 1H), 7.06-7.11 (m, 2H), $2.87\left(\mathrm{~d},{ }^{2} J=13.6\right.$ $\mathrm{Hz}, 1 \mathrm{H}), 2.72\left(\mathrm{~d},{ }^{2} \mathrm{~J}=13.6 \mathrm{~Hz}, 1 \mathrm{H}\right), 1.41-1.53(\mathrm{~m}, 2 \mathrm{H}), 0.99(\mathrm{~s}, 3 \mathrm{H}), 0.88\left(\mathrm{t},{ }^{3} \mathrm{~J}=7.4\right.$ $\mathrm{Hz}, 3 \mathrm{H})$. 


\section{$6 \quad$ Crystallographic Data}

Data for the single crystal structure determination were collected with an Agilent SuperNova diffractometer equipped with a CCD area Atlas detector and a mirror monochromator by utilizing $\mathrm{Cu}-\mathrm{K}_{\alpha}$ radiation $(\lambda=1.5418 \AA$ ). Software packages used: CrysAlis PRO for data collection, cell refinement and data reduction, ${ }^{[S 7]}$ SHELXS-97 for structure solution, ${ }^{[S 8]}$ SHELXL97 for structure refinement, ${ }^{[S 9]}$ and Mercury 3.9[S10] for graphics.

\subsection{Single Crystal of $(2 R, 3 R)-4 a$}

The single crystal $(2 R, 3 R)-4$ a was obtained by crystallization from cyclohexane/ethyl acetate $(10: 1)$ at room temperature.

Figure S6.1. Molecular structure of $(2 R, 3 R)-4 a$. Thermal ellipsoids are shown at the $50 \%$ probability level. C: gray; $\mathrm{H}$ : white; O: red; Br: brown.

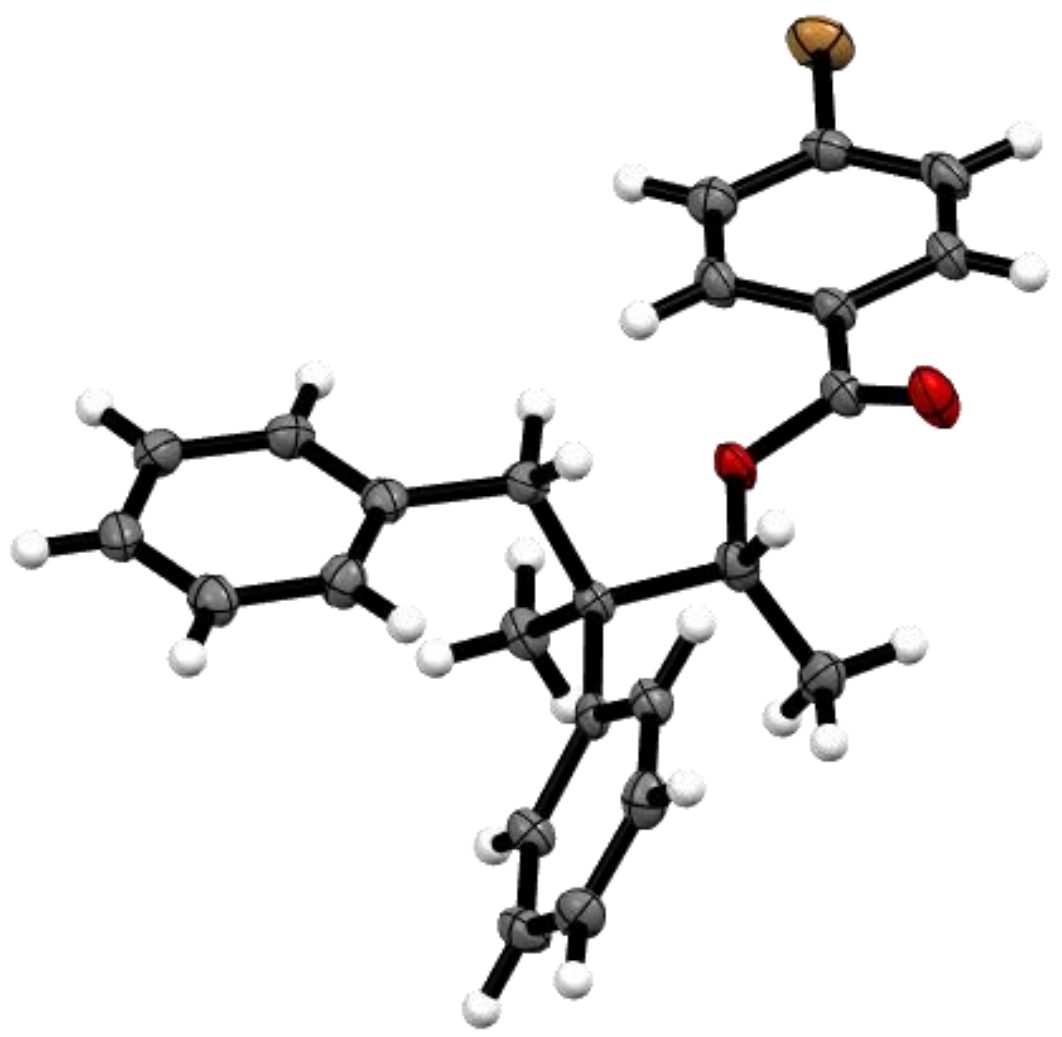

Table S3. Crystal data and structure refinement for $(2 R, 3 R)-4 a$.

Compound (CCDC number)

Empirical formula
$(2 R, 3 R)-4 \mathbf{a}(2103765)$

$\mathrm{C}_{24} \mathrm{H}_{23} \mathrm{BrO}_{2}$ 
Formula weight

Temperature

Wavelength

Crystal system

Space group

Unit cell dimensions

Volume

Z

Density (calculated)

Absorption coefficient

$F(000)$

Crystal size

Theta range for data collection

Index ranges

Reflections collected

Independent reflections

Completeness to theta $=67.48^{\circ}$

Absorption correction

Max. and min. transmission

Refinement method
423.33

$150.00(10) \mathrm{K}$

$1.54184 \AA$

Monoclinic

$\mathrm{P} 21$

$$
\begin{gathered}
a=6.50942(10) \AA \\
\alpha=90^{\circ} .
\end{gathered}
$$$$
\mathrm{b}=7.09005(10) \AA
$$$$
\beta=92.8487(14)^{\circ} \text {. }
$$$$
\mathrm{C}=21.4941(4) \AA
$$

$$
\gamma=90^{\circ} \text {. }
$$

$$
990.77(3) \AA^{3}
$$

\section{2}

$1.419 \mathrm{mg} / \mathrm{m}^{3}$

$2.943 \mathrm{~mm}^{-1}$

436

$0.82 \times 0.25 \times 0.03 \mathrm{~mm}^{3}$

4.12 to $67.46^{\circ}$.

$-7<=\mathrm{h}<=6,-8<=\mathrm{k}<=8,-20<=\mathrm{k}<=25$

6024

$3461[R($ int $)=0.0218]$

$99.9 \%$

Semi-empirical from equivalents

0.9248 and 0.4983

Full-matrix least-squares on $\mathrm{F}^{2}$ 
Data/restraints/parameters

Goodness-of-fit on $\mathrm{F}^{2}$

Final $\mathrm{R}$ indices [l>2sigma(I)]

$R$ indices (all data)

Absolute structure parameter

Largest diff. peak and hole
$3461 / 1 / 246$

1.040

$R 1=0.0345, w R 2=0.0923$

$R 1=0.0354, w R 2=0.0946$

$-0.038(17)$

0.289 and -0.463 e. $\AA^{-3}$ 
Table S4. Atomic coordinates $\left(\times 10^{4}\right)$ and equivalent isotropic displacement parameters $\left(\AA^{2} \times 10^{3}\right)$ for $(2 R, 3 R)-4 \mathrm{a}$. $\mathrm{U}(\mathrm{eq})$ is defined as one third of the trace of the orthogonalized Uij tensor.

\begin{tabular}{|c|c|c|c|c|}
\hline & $x$ & $y$ & z & $\mathrm{U}(\mathrm{eq})$ \\
\hline $\operatorname{Br}(1)$ & $4821(1)$ & $3430(1)$ & $6256(1)$ & $41(1)$ \\
\hline$O(1)$ & $8435(3)$ & $4259(3)$ & $3367(1)$ & $26(1)$ \\
\hline $\mathrm{O}(2)$ & 11523(3) & $4216(4)$ & $3893(1)$ & $40(1)$ \\
\hline$C(1)$ & $9671(4)$ & $4157(4)$ & $3889(1)$ & $26(1)$ \\
\hline$C(2)$ & $8452(4)$ & $3969(4)$ & $4452(1)$ & $25(1)$ \\
\hline$C(3)$ & $9430(4)$ & $4365(4)$ & $5033(1)$ & $28(1)$ \\
\hline$C(4)$ & $8360(5)$ & $4212(5)$ & $5572(1)$ & $31(1)$ \\
\hline$C(5)$ & $6333(4)$ & $3627(5)$ & $5526(1)$ & $28(1)$ \\
\hline$C(6)$ & $5332(4)$ & $3219(5)$ & 4955(1) & $30(1)$ \\
\hline$C(7)$ & $6401(4)$ & $3410(5)$ & $4420(1)$ & $27(1)$ \\
\hline$C(8)$ & $9401(4)$ & $4695(4)$ & $2788(1)$ & $26(1)$ \\
\hline $\mathrm{C}(9)$ & $9958(5)$ & $6779(5)$ & $2786(1)$ & $34(1)$ \\
\hline$C(10)$ & $7850(4)$ & $4082(4)$ & $2255(1)$ & $21(1)$ \\
\hline$C(11)$ & $7680(4)$ & $1883(4)$ & $2271(1)$ & $25(1)$ \\
\hline$C(12)$ & $6203(4)$ & $1041(4)$ & $1782(1)$ & $26(1)$ \\
\hline$C(13)$ & $6641(5)$ & $1007(4)$ & $1151(1)$ & $28(1)$ \\
\hline$C(14)$ & $5261(5)$ & $282(4)$ & 703(2) & $31(1)$ \\
\hline$C(15)$ & $3417(5)$ & $-482(4)$ & $874(2)$ & $32(1)$ \\
\hline$C(16)$ & $2969(4)$ & $-515(4)$ & $1498(2)$ & $32(1)$ \\
\hline$C(17)$ & $4344(5)$ & $257(4)$ & $1946(1)$ & $28(1)$ \\
\hline$C(18)$ & $8715(4)$ & $4702(4)$ & $1638(1)$ & $21(1)$ \\
\hline$C(19)$ & 10649(4) & $4032(4)$ & $1477(1)$ & $25(1)$ \\
\hline$C(20)$ & $11436(4)$ & $4453(5)$ & $907(1)$ & $30(1)$ \\
\hline
\end{tabular}




$\begin{array}{lllll}\mathrm{C}(21) & 10312(5) & 5556(5) & 480(1) & 30(1) \\ \mathrm{C}(22) & 8427(5) & 6267(4) & 640(1) & 30(1) \\ \mathrm{C}(23) & 7637(4) & 5854(4) & 1214(1) & 26(1) \\ \mathrm{C}(24) & 5723(4) & 4929(4) & 2355(1) & 26(1)\end{array}$

Table S5. Bond lengths $[\AA]]$ and angles $\left[^{\circ}\right]$ for $(2 R, 3 R)-4 a$.

\begin{tabular}{|c|c|c|c|}
\hline $\operatorname{Br}(1)-C(5)$ & $1.898(3)$ & $C(15)-C(16)$ & $1.386(5)$ \\
\hline $\mathrm{O}(1)-\mathrm{C}(1)$ & $1.351(3)$ & $C(16)-C(17)$ & $1.394(4)$ \\
\hline $\mathrm{O}(1)-\mathrm{C}(8)$ & $1.455(3)$ & $C(18)-C(23)$ & $1.388(4)$ \\
\hline $\mathrm{O}(2)-\mathrm{C}(1)$ & $1.206(4)$ & $C(18)-C(19)$ & $1.405(4)$ \\
\hline$C(1)-C(2)$ & $1.486(4)$ & $C(19)-C(20)$ & $1.385(4)$ \\
\hline $\mathrm{C}(2)-\mathrm{C}(7)$ & $1.391(4)$ & $C(20)-C(21)$ & $1.387(5)$ \\
\hline $\mathrm{C}(2)-\mathrm{C}(3)$ & $1.401(4)$ & $C(21)-C(22)$ & $1.386(4)$ \\
\hline$C(3)-C(4)$ & $1.386(4)$ & $C(22)-C(23)$ & $1.391(4)$ \\
\hline$C(4)-C(5)$ & $1.382(4)$ & $C(1)-O(1)-C(8)$ & $117.2(2)$ \\
\hline$C(5)-C(6)$ & $1.391(4)$ & $\mathrm{O}(2)-\mathrm{C}(1)-\mathrm{O}(1)$ & $123.9(3)$ \\
\hline$C(6)-C(7)$ & $1.379(4)$ & $\mathrm{O}(2)-\mathrm{C}(1)-\mathrm{C}(2)$ & $124.9(2)$ \\
\hline $\mathrm{C}(8)-\mathrm{C}(9)$ & $1.522(4)$ & $\mathrm{O}(1)-\mathrm{C}(1)-\mathrm{C}(2)$ & 111.2(2) \\
\hline$C(8)-C(10)$ & $1.550(4)$ & $C(7)-C(2)-C(3)$ & 119.6(2) \\
\hline$C(10)-C(18)$ & $1.530(4)$ & $C(7)-C(2)-C(1)$ & $122.4(2)$ \\
\hline$C(10)-C(24)$ & $1.534(3)$ & $C(3)-C(2)-C(1)$ & $118.0(2)$ \\
\hline$C(10)-C(11)$ & $1.564(4)$ & $C(4)-C(3)-C(2)$ & $120.3(2)$ \\
\hline$C(11)-C(12)$ & $1.511(4)$ & $C(5)-C(4)-C(3)$ & $118.8(2)$ \\
\hline$C(12)-C(17)$ & $1.393(4)$ & $C(4)-C(5)-C(6)$ & $122.0(2)$ \\
\hline$C(12)-C(13)$ & $1.402(4)$ & $C(4)-C(5)-B r(1)$ & $119.7(2)$ \\
\hline$C(13)-C(14)$ & $1.382(4)$ & $C(6)-C(5)-B r(1)$ & $118.3(2)$ \\
\hline$C(14)-C(15)$ & $1.383(4)$ & $C(7)-C(6)-C(5)$ & $118.7(2)$ \\
\hline
\end{tabular}




\begin{tabular}{cc||cr}
$C(6)-C(7)-C(2)$ & $120.7(2)$ & $C(14)-C(13)-C(12)$ & $121.6(3)$ \\
$O(1)-C(8)-C(9)$ & $108.8(2)$ & $C(13)-C(14)-C(15)$ & $120.3(3)$ \\
$O(1)-C(8)-C(10)$ & $106.2(2)$ & $C(14)-C(15)-C(16)$ & $119.3(3)$ \\
$C(9)-C(8)-C(10)$ & $114.7(2)$ & $C(15)-C(16)-C(17)$ & $120.2(3)$ \\
$C(18)-C(10)-C(24)$ & $112.5(2)$ & $C(12)-C(17)-C(16)$ & $121.2(3)$ \\
$C(18)-C(10)-C(8)$ & $107.7(2)$ & $C(23)-C(18)-C(19)$ & $117.8(3)$ \\
$C(24)-C(10)-C(8)$ & $110.2(2)$ & $C(23)-C(18)-C(10)$ & $122.9(2)$ \\
$C(18)-C(10)-C(11)$ & $109.6(2)$ & $C(19)-C(18)-C(10)$ & $119.3(2)$ \\
$C(24)-C(10)-C(11)$ & $108.8(2)$ & $C(20)-C(19)-C(18)$ & $121.4(3)$ \\
$C(8)-C(10)-C(11)$ & $107.9(2)$ & $C(19)-C(20)-C(21)$ & $120.1(3)$ \\
$C(12)-C(11)-C(10)$ & $114.9(2)$ & $C(22)-C(21)-C(20)$ & $119.0(3)$ \\
$C(17)-C(12)-C(13)$ & $117.3(3)$ & $C(21)-C(22)-C(23)$ & $121.0(3)$ \\
$C(17)-C(12)-C(11)$ & $120.9(3)$ & $C(18)-C(23)-C(22)$ & $120.6(3)$ \\
$C(13)-C(12)-C(11)$ & $121.8(3)$ & & \\
& &
\end{tabular}

Symmetry transformations used to generate equivalent atoms. 
Table S6. Anisotropic displacement parameters $\left(\AA^{2} \times 10^{3}\right)$ for $(2 R, 3 R)-4 a$. The anisotropic displacement factor exponent takes the form: $-2 \pi^{2}\left[h^{2} a^{* 2} U^{11}+\ldots+2 h k\right.$ $\left.a^{*} b^{*} U^{12}\right]$.

\begin{tabular}{lllllll} 
& $\mathrm{U} 11$ & $\mathrm{U} 22$ & $\mathrm{U} 33$ & $\mathrm{U} 23$ & $\mathrm{U} 13$ & $\mathrm{U} 12$ \\
$\mathrm{Br}(1)$ & $48(1)$ & $53(1)$ & $24(1)$ & $5(1)$ & $9(1)$ & $1(1)$ \\
$\mathrm{O}(1)$ & $24(1)$ & $38(1)$ & $15(1)$ & $2(1)$ & $-3(1)$ & $-2(1)$ \\
$\mathrm{O}(2)$ & $24(1)$ & $66(1)$ & $28(1)$ & $6(1)$ & $-4(1)$ & $-2(1)$ \\
$\mathrm{C}(1)$ & $28(1)$ & $32(1)$ & $20(1)$ & $2(1)$ & $-5(1)$ & $-1(1)$ \\
$\mathrm{C}(2)$ & $29(1)$ & $29(2)$ & $15(1)$ & $1(1)$ & $-2(1)$ & $-1(1)$ \\
$\mathrm{C}(3)$ & $27(1)$ & $33(1)$ & $23(1)$ & $3(1)$ & $-8(1)$ & $0(1)$ \\
$\mathrm{C}(4)$ & $40(2)$ & $34(1)$ & $18(1)$ & $1(1)$ & $-7(1)$ & $2(1)$ \\
$\mathrm{C}(5)$ & $33(1)$ & $29(1)$ & $22(1)$ & $5(1)$ & $1(1)$ & $1(1)$ \\
$\mathrm{C}(6)$ & $29(1)$ & $35(2)$ & $26(1)$ & $2(1)$ & $1(1)$ & $-3(1)$ \\
$\mathrm{C}(7)$ & $29(1)$ & $34(1)$ & $19(1)$ & $-1(2)$ & $-5(1)$ & $-1(1)$ \\
$\mathrm{C}(8)$ & $25(1)$ & $35(2)$ & $17(1)$ & $-1(1)$ & $1(1)$ & $-2(1)$ \\
$\mathrm{C}(9)$ & $44(2)$ & $36(2)$ & $22(1)$ & $-1(1)$ & $-1(1)$ & $-13(1)$ \\
$\mathrm{C}(10)$ & $21(1)$ & $24(1)$ & $17(1)$ & $-1(1)$ & $-2(1)$ & $1(1)$ \\
$\mathrm{C}(11)$ & $27(1)$ & $24(1)$ & $24(1)$ & $2(1)$ & $-1(1)$ & $1(1)$ \\
$\mathrm{C}(12)$ & $29(1)$ & $22(1)$ & $26(1)$ & $0(1)$ & $-2(1)$ & $1(1)$ \\
$\mathrm{C}(13)$ & $30(1)$ & $30(1)$ & $24(1)$ & $-5(1)$ & $3(1)$ & $-3(1)$ \\
$\mathrm{C}(14)$ & $36(2)$ & $30(2)$ & $25(1)$ & $-3(1)$ & $-2(1)$ & $0(1)$ \\
$\mathrm{C}(15)$ & $33(1)$ & $28(1)$ & $34(2)$ & $1(1)$ & $-9(1)$ & $-3(1)$ \\
$\mathrm{C}(16)$ & $29(1)$ & $31(2)$ & $36(2)$ & $8(1)$ & $-1(1)$ & $-5(1)$ \\
$\mathrm{C}(17)$ & $32(1)$ & $28(1)$ & $25(1)$ & $4(1)$ & $2(1)$ & $-3(1)$ \\
$\mathrm{C}(18)$ & $23(1)$ & $23(1)$ & $18(1)$ & $-4(1)$ & $-4(1)$ & $-4(1)$ \\
$\mathrm{C}(19)$ & $24(1)$ & $28(1)$ & $24(1)$ & $0(1)$ & $-3(1)$ & $1(1)$ \\
$\mathrm{C}(20)$ & $27(1)$ & $36(2)$ & $27(1)$ & $-7(1)$ & $3(1)$ & $-3(1)$ \\
& & & & & & \\
\hline
\end{tabular}




$\begin{array}{lllllll}\mathrm{C}(21) & 38(2) & 34(2) & 18(1) & -3(1) & 4(1) & -6(1) \\ \mathrm{C}(22) & 38(2) & 30(1) & 21(1) & 2(1) & -3(1) & 4(1) \\ \mathrm{C}(23) & 27(1) & 29(1) & 21(1) & -1(1) & -1(1) & 3(1) \\ \mathrm{C}(24) & 24(1) & 31(1) & 23(1) & -3(1) & 1(1) & 3(1)\end{array}$




\subsection{Single Crystal of $(2 S, 3 R)-5 a$}

The single crystal (2S,3R)-5a was obtained by crystallization from pentane/dichloromethane (10:1) at room temperature.

Figure S6.2. Molecular structure of $(2 S, 3 R)-5 a$. Thermal ellipsoids are shown at the $50 \%$ probability level. C: gray; H: white; O: red.

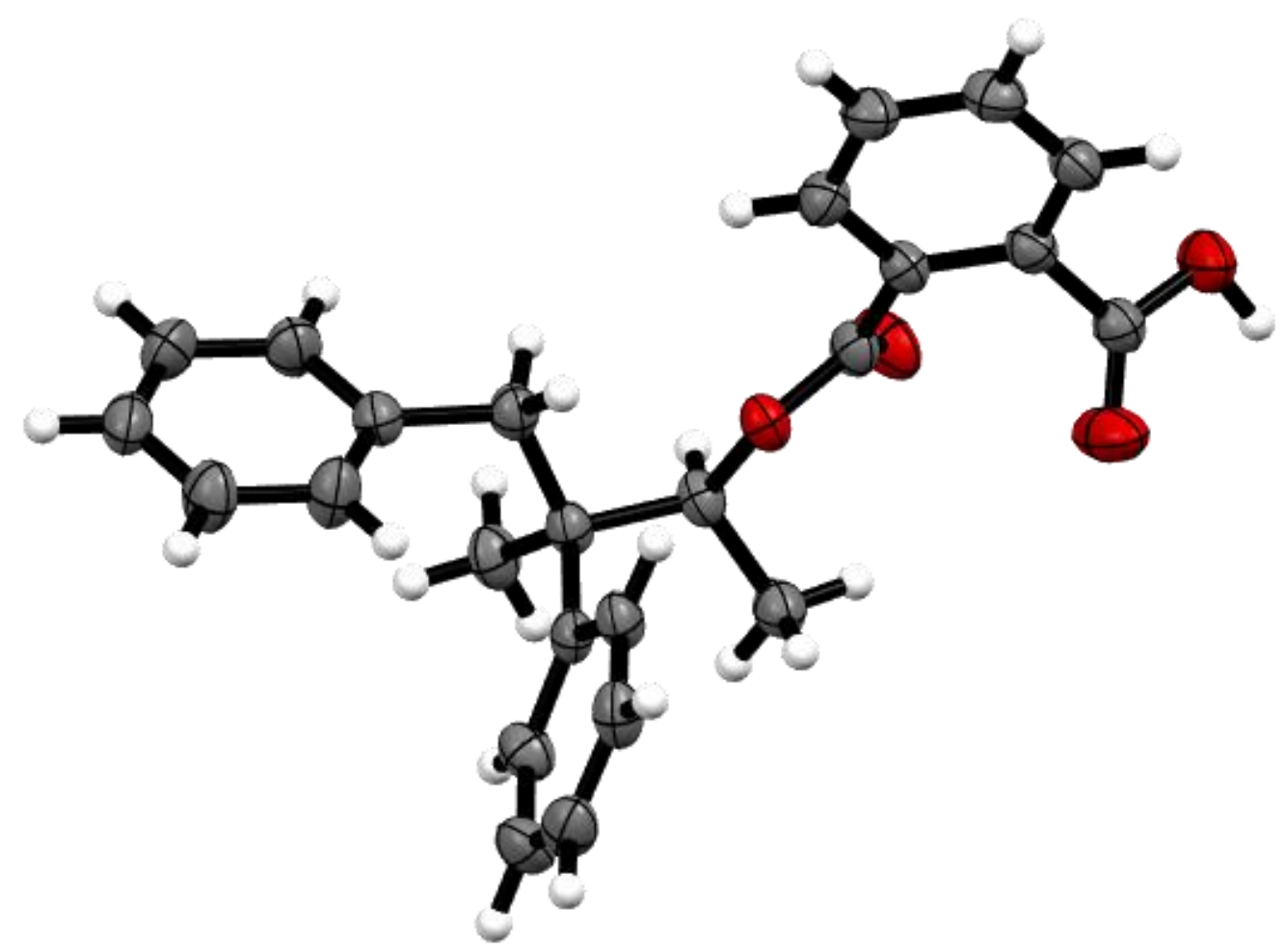

Table S7. Crystal data and structure refinement for $(2 S, 3 R)-5 \mathbf{a}$.

Compound (CCDC number)

Empirical formula

Formula weight

Temperature

Wavelength

Crystal system

Space group

Unit cell dimensions
$(2 S, 3 R)-5 a(2120080)$

$\mathrm{C}_{25} \mathrm{H}_{24} \mathrm{O}_{4}$

388.44

$150.01(10) \mathrm{K}$

$1.54184 \AA$

Orthorhombic

P212121 (No. 19)

$a=6.5680(3) \AA$

$\alpha=90^{\circ}$. 
Volume

Z

Density (calculated)

Absorption coefficient

$\mathrm{F}(000)$

Crystal size

Theta range for data collection

Index ranges

Reflections collected

Independent reflections

Completeness to theta $=67.48^{\circ}$

Absorption correction

Max. and min. transmission

Refinement method

Data/restraints/parameters

Goodness-of-fit on $\mathrm{F}^{2}$

Final $\mathrm{R}$ indices [l>2sigma(l)]

$R$ indices (all data)

Absolute structure parameter

Largest diff. peak and hole

$$
\mathrm{b}=7.7475(4) \AA
$$$$
\beta=90^{\circ} \text {. }
$$$$
\mathrm{C}=39.873(2) \AA
$$$$
\gamma=90^{\circ} \text {. }
$$

2028.95(18) $\AA^{3}$

4

$1.272 \mathrm{mg} / \mathrm{m}^{3}$

$0.686 \mathrm{~mm}^{-1}$

824

$0.21 \times 0.09 \times 0.02 \mathrm{~mm}^{3}$

4.44 to $67.50^{\circ}$.

$-7<=\mathrm{h}<=7,-9<=\mathrm{k}<=8,-47<=\mathrm{|}<=47$

13726

$3642[R($ int $)=0.0585]$

$100.0 \%$

Semi-empirical from equivalents

0.9851 and 0.8705

Full-matrix least-squares on $\mathrm{F}^{2}$

3642 / 0 / 267

1.032

$\mathrm{R} 1=0.0450, \mathrm{wR} 2=0.1029$

$\mathrm{R} 1=0.0635, \mathrm{wR} 2=0.1121$

$-0.4(3)$

0.190 and -0.168 e. $\AA^{-3}$ 
Table S8. Atomic coordinates $\left(\times 10^{4}\right)$ and equivalent isotropic displacement parameters $\left(\AA^{2} \times 10^{3}\right)$ for $(2 S, 3 R)-5 \mathbf{a}$. $U(e q)$ is defined as one third of the trace of the orthogonalized $U^{i j}$ tensor.

\begin{tabular}{|c|c|c|c|c|}
\hline & $x$ & $y$ & z & $\mathrm{U}(\mathrm{eq})$ \\
\hline $\mathrm{O}(1)$ & $4885(3)$ & $4813(2)$ & $929(1)$ & $35(1)$ \\
\hline $\mathrm{O}(2)$ & $4687(3)$ & $4140(3)$ & $382(1)$ & $56(1)$ \\
\hline $\mathrm{O}(3)$ & $9548(4)$ & $4050(3)$ & $-96(1)$ & $59(1)$ \\
\hline $\mathrm{O}(4)$ & $9012(4)$ & 2863(3) & $399(1)$ & $71(1)$ \\
\hline$C(1)$ & $5456(4)$ & 4939(3) & $609(1)$ & $37(1)$ \\
\hline $\mathrm{C}(2)$ & $7085(4)$ & $6247(3)$ & $556(1)$ & $35(1)$ \\
\hline$C(3)$ & $8758(4)$ & 5893(3) & $349(1)$ & $36(1)$ \\
\hline$C(4)$ & $10160(4)$ & 7173(3) & $278(1)$ & $42(1)$ \\
\hline$C(5)$ & $9898(5)$ & $8828(3)$ & $408(1)$ & $45(1)$ \\
\hline$C(6)$ & $8251(4)$ & $9175(3)$ & $610(1)$ & $44(1)$ \\
\hline$C(7)$ & $6838(4)$ & $7906(3)$ & $684(1)$ & $41(1)$ \\
\hline$C(8)$ & $9103(4)$ & $4100(4)$ & $224(1)$ & $39(1)$ \\
\hline $\mathrm{C}(9)$ & $3142(4)$ & $3677(3)$ & 1002(1) & $36(1)$ \\
\hline$C(10)$ & $3860(4)$ & $1830(3)$ & 1011(1) & $44(1)$ \\
\hline$C(11)$ & $2087(4)$ & $4319(3)$ & $1329(1)$ & $34(1)$ \\
\hline$C(12)$ & 1979(4) & $6321(3)$ & $1325(1)$ & $38(1)$ \\
\hline$C(13)$ & $689(4)$ & $7151(3)$ & 1599(1) & $37(1)$ \\
\hline$C(14)$ & $1245(5)$ & $7143(4)$ & 1932(1) & $50(1)$ \\
\hline$C(15)$ & $44(5)$ & $7919(4)$ & $2176(1)$ & $52(1)$ \\
\hline$C(16)$ & $-1732(4)$ & $8754(4)$ & 2086(1) & $48(1)$ \\
\hline$C(17)$ & $-2295(4)$ & $8793(4)$ & $1758(1)$ & $53(1)$ \\
\hline$C(18)$ & $-1103(4)$ & 7994(4) & $1515(1)$ & $48(1)$ \\
\hline$C(19)$ & $3210(4)$ & $3669(3)$ & $1641(1)$ & $32(1)$ \\
\hline
\end{tabular}




$\begin{array}{lllll}\mathrm{C}(20) & 5099(4) & 4370(3) & 1730(1) & 38(1) \\ \mathrm{C}(21) & 6095(4) & 3870(4) & 2019(1) & 44(1) \\ \mathrm{C}(22) & 5257(4) & 2635(4) & 2225(1) & 47(1) \\ \mathrm{C}(23) & 3434(5) & 1885(4) & 2137(1) & 49(1) \\ \mathrm{C}(24) & 2423(4) & 2393(3) & 1850(1) & 41(1) \\ \mathrm{C}(25) & -93(4) & 3622(4) & 1308(1) & 44(1)\end{array}$

Table S9. Bond lengths $[\AA]$ and angles $\left[^{\circ}\right]$ for $(2 S, 3 R)-5$ a.

\begin{tabular}{ll||ll}
$\mathrm{O}(1)-\mathrm{C}(1)$ & $1.334(3)$ & $\mathrm{C}(13)-\mathrm{C}(14)$ & $1.380(4)$ \\
$\mathrm{O}(1)-\mathrm{C}(9)$ & $1.473(3)$ & $\mathrm{C}(13)-\mathrm{C}(18)$ & $1.386(4)$ \\
$\mathrm{O}(2)-\mathrm{C}(1)$ & $1.206(3)$ & $\mathrm{C}(14)-\mathrm{C}(15)$ & $1.387(4)$ \\
$\mathrm{O}(3)-\mathrm{C}(8)$ & $1.311(3)$ & $\mathrm{C}(15)-\mathrm{C}(16)$ & $1.381(4)$ \\
$\mathrm{O}(4)-\mathrm{C}(8)$ & $1.187(3)$ & $\mathrm{C}(16)-\mathrm{C}(17)$ & $1.360(4)$ \\
$\mathrm{C}(1)-\mathrm{C}(2)$ & $1.489(4)$ & $\mathrm{C}(17)-\mathrm{C}(18)$ & $1.391(4)$ \\
$\mathrm{C}(2)-\mathrm{C}(7)$ & $1.393(4)$ & $\mathrm{C}(19)-\mathrm{C}(24)$ & $1.392(4)$ \\
$\mathrm{C}(2)-\mathrm{C}(3)$ & $1.401(4)$ & $\mathrm{C}(19)-\mathrm{C}(20)$ & $1.400(4)$ \\
$\mathrm{C}(3)-\mathrm{C}(4)$ & $1.383(4)$ & $\mathrm{C}(20)-\mathrm{C}(21)$ & $1.380(4)$ \\
$\mathrm{C}(3)-\mathrm{C}(8)$ & $1.492(4)$ & $\mathrm{C}(21)-\mathrm{C}(22)$ & $1.375(4)$ \\
$\mathrm{C}(4)-\mathrm{C}(5)$ & $1.393(4)$ & $\mathrm{C}(22)-\mathrm{C}(23)$ & $1.376(4)$ \\
$\mathrm{C}(5)-\mathrm{C}(6)$ & $1.376(4)$ & $\mathrm{C}(23)-\mathrm{C}(24)$ & $1.381(4)$ \\
$\mathrm{C}(6)-\mathrm{C}(7)$ & $1.384(4)$ & $\mathrm{C}(1)-\mathrm{O}(1)-\mathrm{C}(9)$ & $116.87(19)$ \\
$\mathrm{C}(9)-\mathrm{C}(10)$ & $1.508(4)$ & $\mathrm{O}(2)-\mathrm{C}(1)-\mathrm{O}(1)$ & $124.1(2)$ \\
$\mathrm{C}(9)-\mathrm{C}(11)$ & $1.558(3)$ & $\mathrm{O}(2)-\mathrm{C}(1)-\mathrm{C}(2)$ & $123.0(2)$ \\
$\mathrm{C}(11)-\mathrm{C}(25)$ & $1.532(3)$ & $\mathrm{O}(1)-\mathrm{C}(1)-\mathrm{C}(2)$ & $112.8(2)$ \\
$\mathrm{C}(11)-\mathrm{C}(19)$ & $1.533(4)$ & $\mathrm{C}(7)-\mathrm{C}(2)-\mathrm{C}(3)$ & $119.3(2)$ \\
$\mathrm{C}(11)-\mathrm{C}(12)$ & $1.553(4)$ & $\mathrm{C}(7)-\mathrm{C}(2)-\mathrm{C}(1)$ & $119.5(2)$ \\
$\mathrm{C}(12)-\mathrm{C}(13)$ & $1.525(4)$ & $\mathrm{C}(3)-\mathrm{C}(2)-\mathrm{C}(1)$ & $120.9(2)$ \\
& & & \\
& & & \\
& & &
\end{tabular}




\begin{tabular}{|c|c|c|c|}
\hline$C(4)-C(3)-C(2)$ & $120.2(2)$ & $C(12)-C(11)-C(9)$ & $109.3(2)$ \\
\hline$C(4)-C(3)-C(8)$ & $119.8(2)$ & $C(13)-C(12)-C(11)$ & $116.1(2)$ \\
\hline$C(2)-C(3)-C(8)$ & $119.9(2)$ & $C(14)-C(13)-C(18)$ & $117.3(2)$ \\
\hline$C(3)-C(4)-C(5)$ & $120.1(3)$ & $C(14)-C(13)-C(12)$ & $122.8(2)$ \\
\hline$C(6)-C(5)-C(4)$ & $119.6(3)$ & $C(18)-C(13)-C(12)$ & $119.9(2)$ \\
\hline$C(5)-C(6)-C(7)$ & $121.0(3)$ & $C(13)-C(14)-C(15)$ & $121.5(3)$ \\
\hline$C(6)-C(7)-C(2)$ & $119.9(3)$ & $C(16)-C(15)-C(14)$ & $120.1(3)$ \\
\hline $\mathrm{O}(4)-\mathrm{C}(8)-\mathrm{O}(3)$ & $124.0(3)$ & $C(17)-C(16)-C(15)$ & 119.3(3) \\
\hline $\mathrm{O}(4)-\mathrm{C}(8)-\mathrm{C}(3)$ & $123.2(2)$ & $C(16)-C(17)-C(18)$ & $120.5(3)$ \\
\hline $\mathrm{O}(3)-\mathrm{C}(8)-\mathrm{C}(3)$ & $112.7(2)$ & $C(13)-C(18)-C(17)$ & 121.3(3) \\
\hline $\mathrm{O}(1)-\mathrm{C}(9)-\mathrm{C}(10)$ & $109.2(2)$ & $C(24)-C(19)-C(20)$ & $117.0(2)$ \\
\hline $\mathrm{O}(1)-\mathrm{C}(9)-\mathrm{C}(11)$ & $108.74(19)$ & $C(24)-C(19)-C(11)$ & $122.7(2)$ \\
\hline$C(10)-C(9)-C(11)$ & $115.0(2)$ & $C(20)-C(19)-C(11)$ & $120.3(2)$ \\
\hline$C(25)-C(11)-C(19)$ & $112.2(2)$ & $C(21)-C(20)-C(19)$ & $121.5(3)$ \\
\hline$C(25)-C(11)-C(12)$ & $108.0(2)$ & $C(22)-C(21)-C(20)$ & $120.2(3)$ \\
\hline$C(19)-C(11)-C(12)$ & $111.0(2)$ & $C(21)-C(22)-C(23)$ & 119.4(3) \\
\hline$C(25)-C(11)-C(9)$ & $105.0(2)$ & $C(22)-C(23)-C(24)$ & $120.6(3)$ \\
\hline$C(19)-C(11)-C(9)$ & $111.2(2)$ & $C(23)-C(24)-C(19)$ & $121.3(3)$ \\
\hline
\end{tabular}

Symmetry transformations used to generate equivalent atoms. 
Table S10. Anisotropic displacement parameters $\left(\AA^{2} \times 10^{3}\right)$ for $(2 S, 3 R)-5 \mathbf{a}$. The anisotropic displacement factor exponent takes the form: $-2 \pi^{2}\left[h^{2} a^{* 2} U^{11}+\ldots+2 h k\right.$ $\left.a^{*} b^{*} U^{12}\right]$.

$\begin{array}{lllllll} & \mathrm{U} 11 & \mathrm{U} 22 & \mathrm{U} 33 & \mathrm{U} 23 & \mathrm{U} 13 & \mathrm{U} 12 \\ \mathrm{O}(1) & 33(1) & 43(1) & 30(1) & -2(1) & 2(1) & -3(1) \\ \mathrm{O}(2) & 71(1) & 62(1) & 34(1) & -11(1) & 7(1) & -19(1) \\ \mathrm{O}(3) & 89(2) & 46(1) & 42(1) & -4(1) & 22(1) & 8(1) \\ \mathrm{O}(4) & 127(2) & 39(1) & 48(1) & 3(1) & 15(1) & 7(1) \\ \mathrm{C}(1) & 43(2) & 40(1) & 27(1) & -4(1) & 2(1) & 5(1) \\ \mathrm{C}(2) & 39(1) & 38(1) & 29(1) & 1(1) & 4(1) & 1(1) \\ \mathrm{C}(3) & 43(1) & 36(1) & 29(1) & 2(1) & 2(1) & 0(1) \\ \mathrm{C}(4) & 44(2) & 46(2) & 35(2) & 6(1) & 6(1) & -2(1) \\ \mathrm{C}(5) & 51(2) & 38(1) & 46(2) & 6(1) & 2(1) & -6(2) \\ \mathrm{C}(6) & 56(2) & 35(1) & 40(2) & 2(1) & 6(1) & 0(1) \\ \mathrm{C}(7) & 46(2) & 37(1) & 40(2) & -2(1) & 3(1) & 6(1) \\ \mathrm{C}(8) & 45(1) & 41(2) & 32(2) & 0(1) & 4(1) & 1(1) \\ \mathrm{C}(9) & 32(1) & 41(1) & 35(2) & -1(1) & 6(1) & -4(1) \\ \mathrm{C}(10) & 49(2) & 42(2) & 40(2) & -3(1) & 10(1) & 0(1) \\ \mathrm{C}(11) & 29(1) & 42(1) & 32(1) & -1(1) & 3(1) & 1(1) \\ \mathrm{C}(12) & 37(1) & 45(1) & 33(1) & -2(1) & 3(1) & 4(1) \\ \mathrm{C}(13) & 35(1) & 38(1) & 38(2) & 0(1) & 5(1) & 1(1) \\ \mathrm{C}(14) & 44(2) & 64(2) & 43(2) & -11(2) & -2(1) & 14(2) \\ \mathrm{C}(15) & 54(2) & 62(2) & 40(2) & -13(1) & 1(2) & 11(2) \\ \mathrm{C}(16) & 46(2) & 42(2) & 56(2) & -3(1) & 15(1) & 5(1) \\ \mathrm{C}(17) & 46(2) & 56(2) & 58(2) & 6(2) & 6(2) & 19(2) \\ \mathrm{C}(18) & 45(2) & 58(2) & 39(2) & 5(1) & 1(1) & 13(1) \\ \mathrm{C}(19) & 31(1) & 37(1) & 30(1) & -5(1) & 5(1) & 4(1)\end{array}$




$\begin{array}{llllllr}\mathrm{C}(20) & 30(1) & 48(1) & 36(2) & -1(1) & 5(1) & 1(1) \\ \mathrm{C}(21) & 33(1) & 61(2) & 40(2) & -8(1) & 2(1) & 5(1) \\ \mathrm{C}(22) & 50(2) & 59(2) & 32(2) & -2(1) & -3(1) & 14(2) \\ \mathrm{C}(23) & 60(2) & 52(2) & 35(2) & 7(1) & 7(1) & 1(2) \\ \mathrm{C}(24) & 43(2) & 43(2) & 36(2) & -3(1) & 3(1) & -7(1) \\ \mathrm{C}(25) & 29(1) & 58(2) & 44(2) & -1(1) & 3(1) & -2(1)\end{array}$




\section{HPLC Traces}

\subsection{Hydrosilane Screening}

(2R,3R)-3-Methyl-3,4-diphenylbutan-2-ol [(2R,3R)-1a] and

(2S,3S)-(3,5-Dimethylphenyl)dimethyl((3-methyl-3,4-diphenylbutan-2-

yl)oxy)silane [(2S,3S)-3ae]

Figure 7.1. rac-anti-3-Methyl-3,4-diphenylbutan-2-ol [rac-anti-1a].

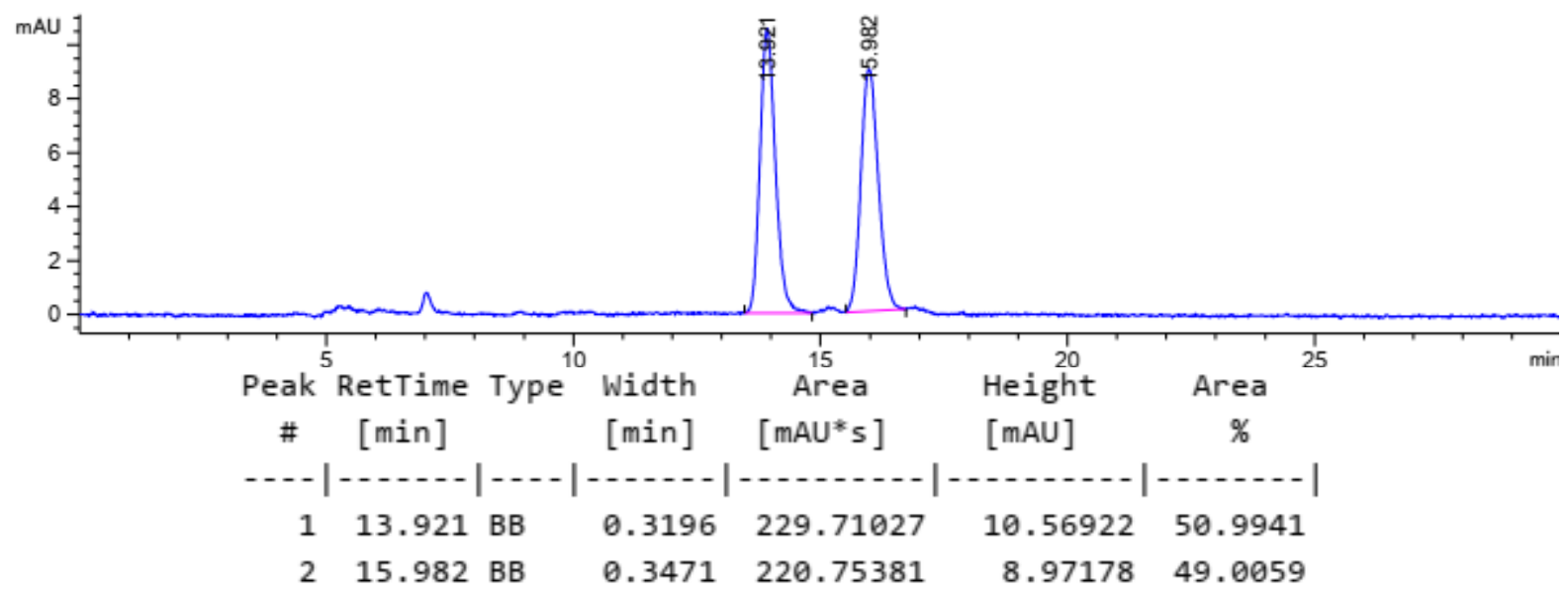

Figure 7.2. (2R,3R)-3-Methyl-3,4-diphenylbutan-2-ol [(2R,3R)-1a, $65 \%$ ee].

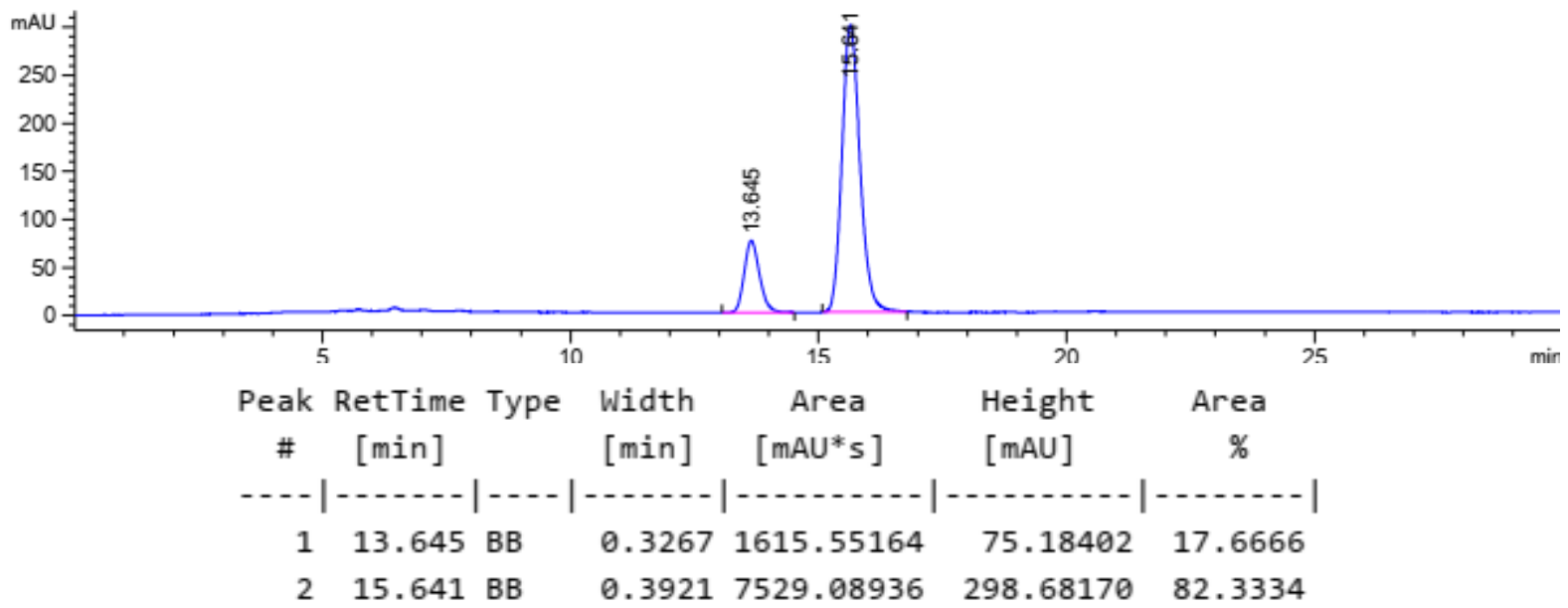


Figure 7.3. (2S,3S)-3-Methyl-3,4-diphenylbutan-2-ol [(2S,3S)-1a, 77\% ee].

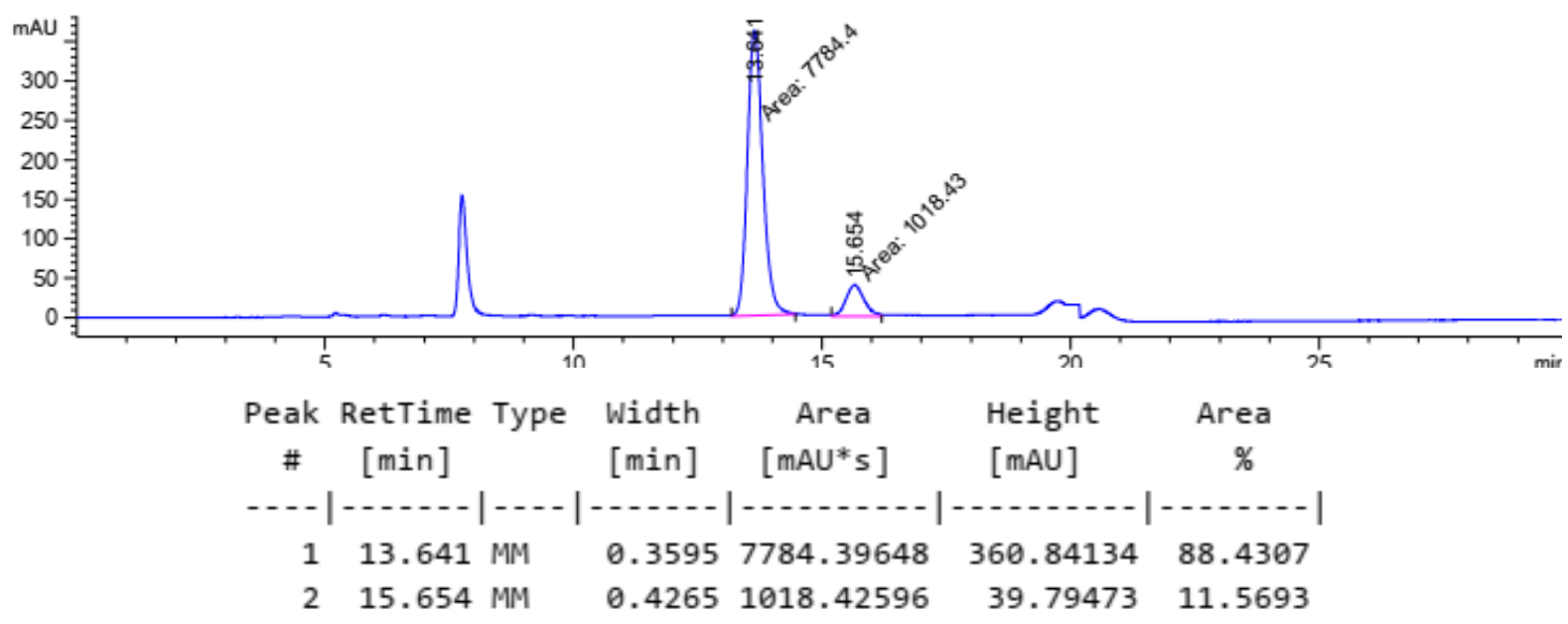

(2R,3S)-3-Methyl-3,4-diphenylbutan-2-ol [(2R,3S)-1a] and (2S,3R)-(3,5-Dimethylphenyl)dimethyl((3-methyl-3,4-diphenylbutan-2yl)oxy)silane [(2S,3R)-3ae]

Figure 7.4. rac-syn-3-Methyl-3,4-diphenylbutan-2-ol [rac-syn-1a].

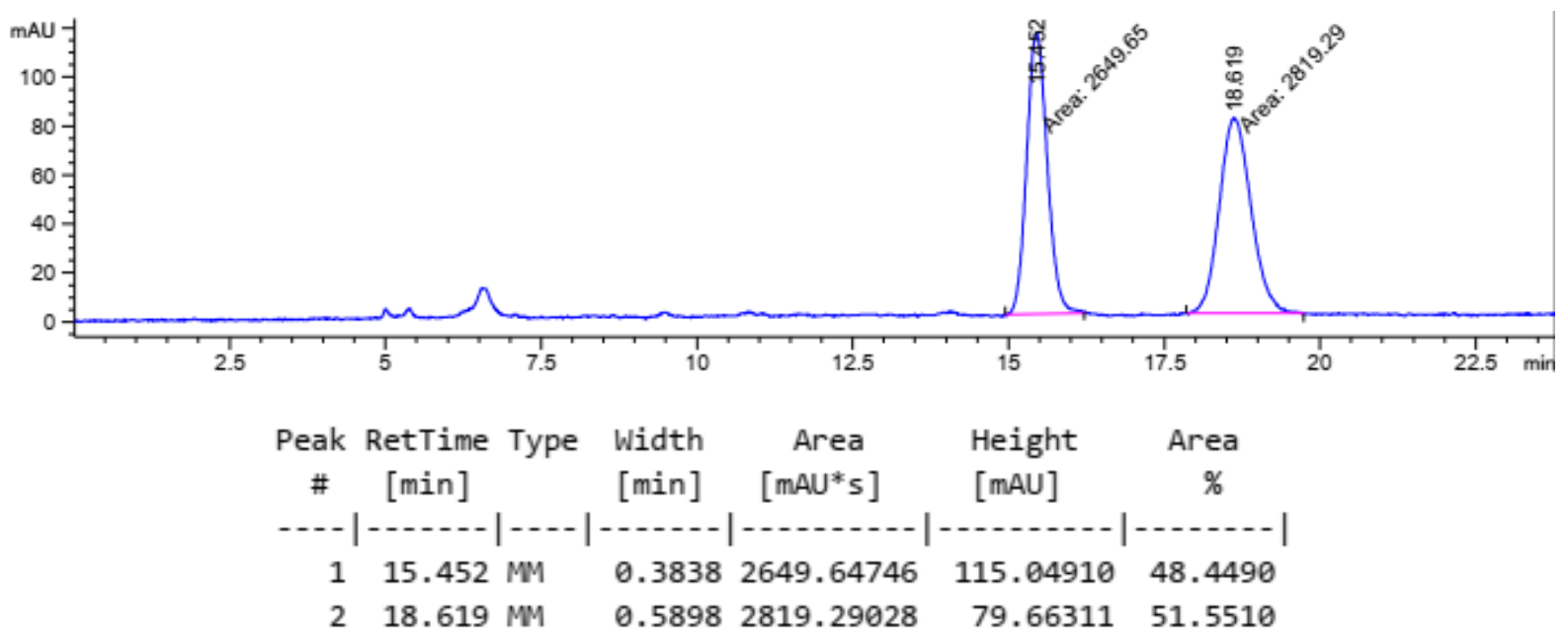


Figure 7.5. (2R,3S)-3-Methyl-3,4-diphenylbutan-2-ol [(2R,3S)-1a, 78\% ee].
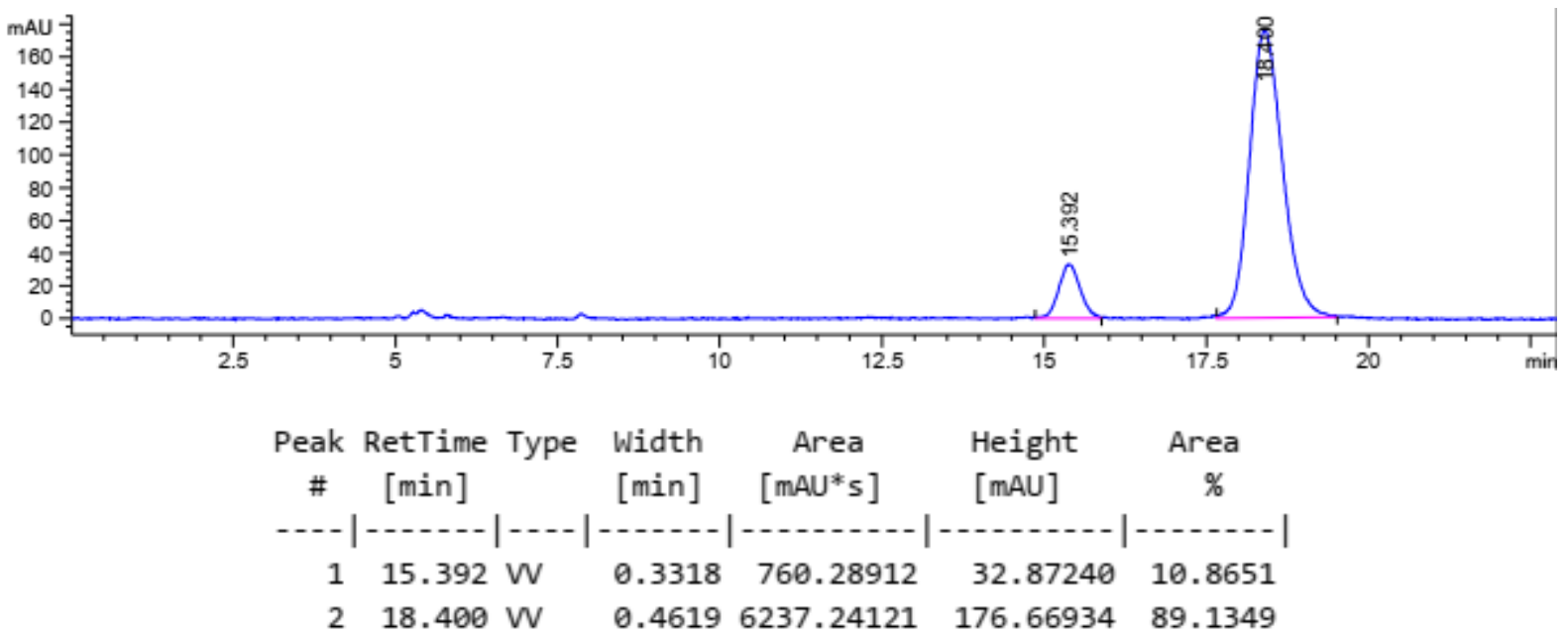

Figure 7.6. (2S,3R)-3-Methyl-3,4-diphenylbutan-2-ol [(2S,3R)-1a, 77\% ee].

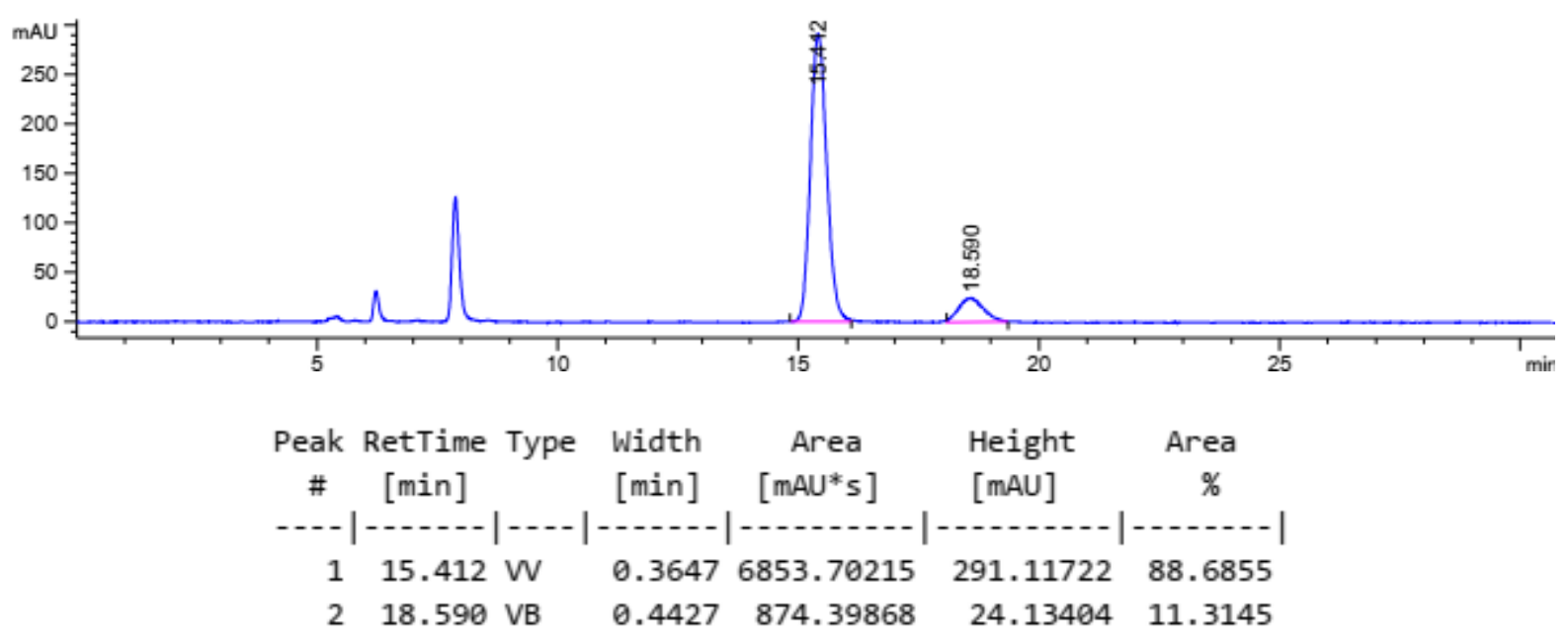


(2R,3R)-3-Methyl-3,4-diphenylbutan-2-ol [(2R,3R)-1a] and (2S,3S)-Dimethyl((3methyl-3,4-diphenylbutan-2-yl)oxy)(phenyl)silane [(2S,3S)-3ab]

Figure 7.7. (2R,3R)-3-Methyl-3,4-diphenylbutan-2-ol [(2R,3R)-1a, $82 \%$ ee].

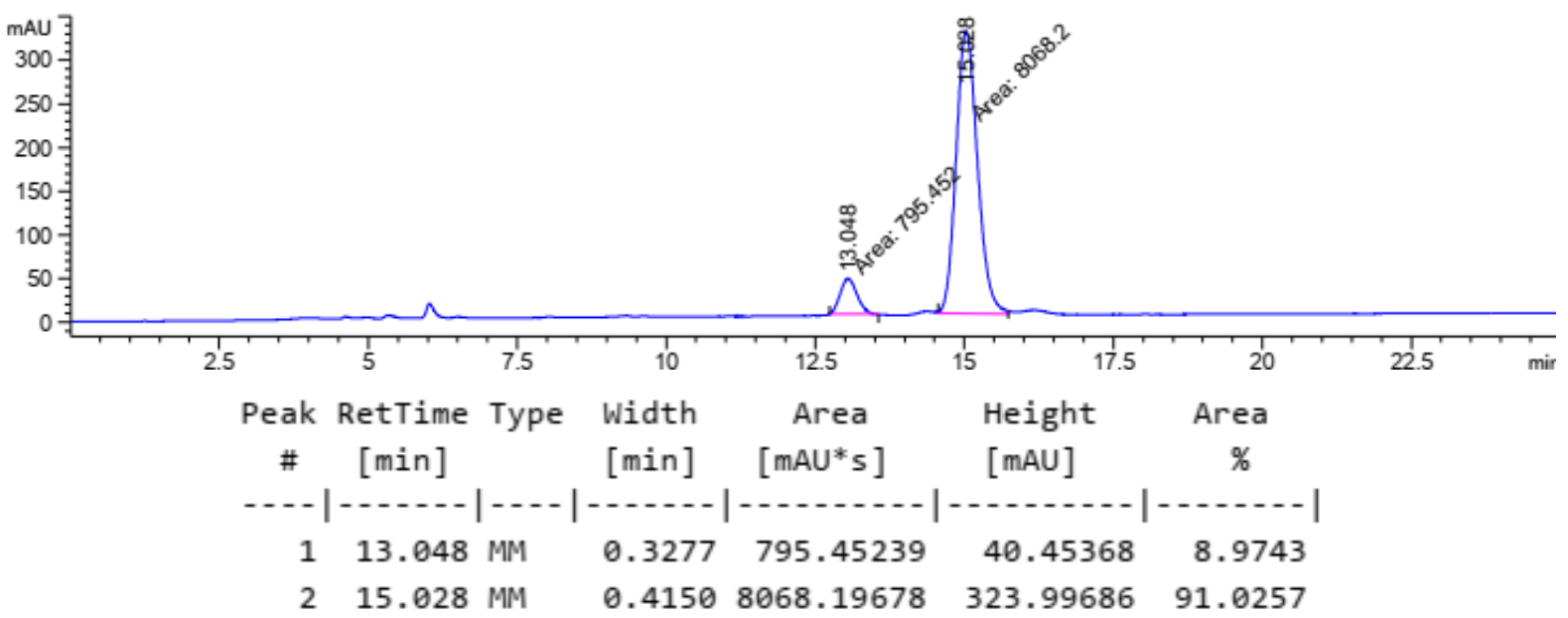

Figure 7.8. (2S,3S)-3-Methyl-3,4-diphenylbutan-2-ol [(2S,3S)-1a, 66\% ee].

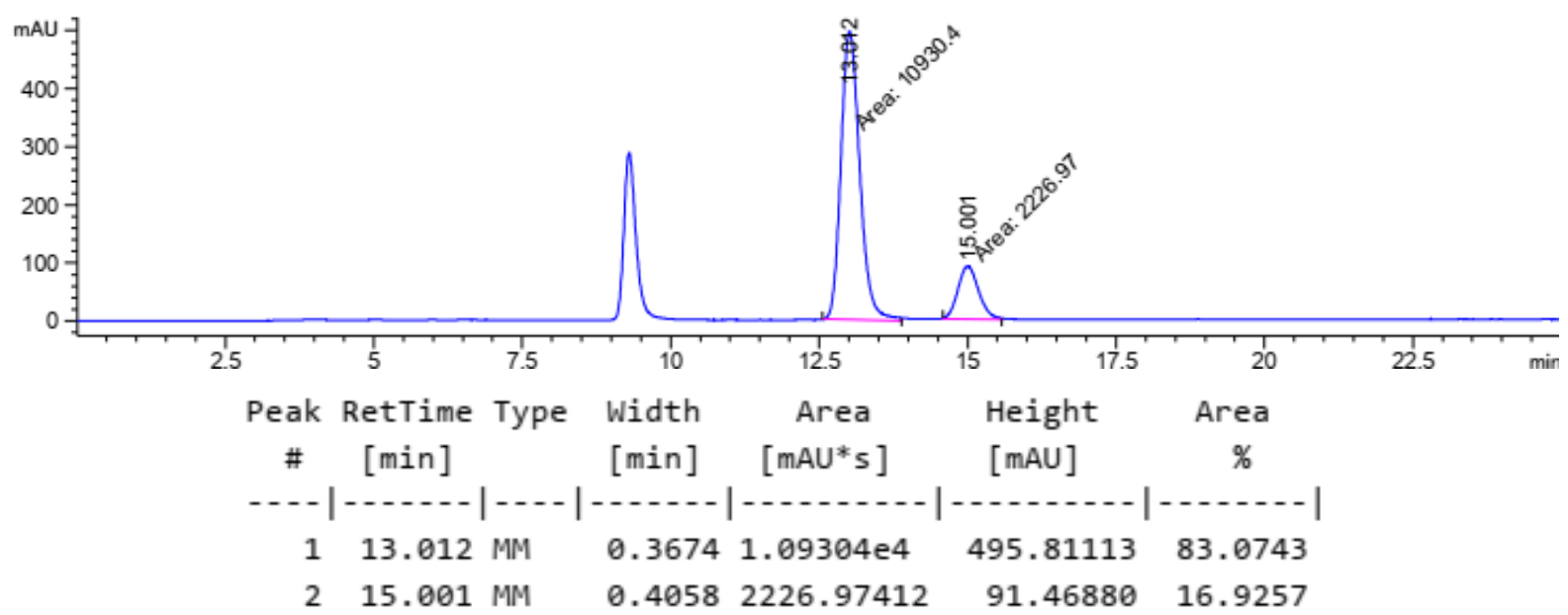


(2R,3R)-3-Methyl-3,4-diphenylbutan-2-ol [(2R,3R)-1a] and (2S,3S)-Methyl((3methyl-3,4-diphenylbutan-2-yl)oxy)diphenylsilane [(2S,3S)-3ac]

Figure 7.9. (2R,3R)-3-Methyl-3,4-diphenylbutan-2-ol [(2R,3R)-1a, 90\% ee].

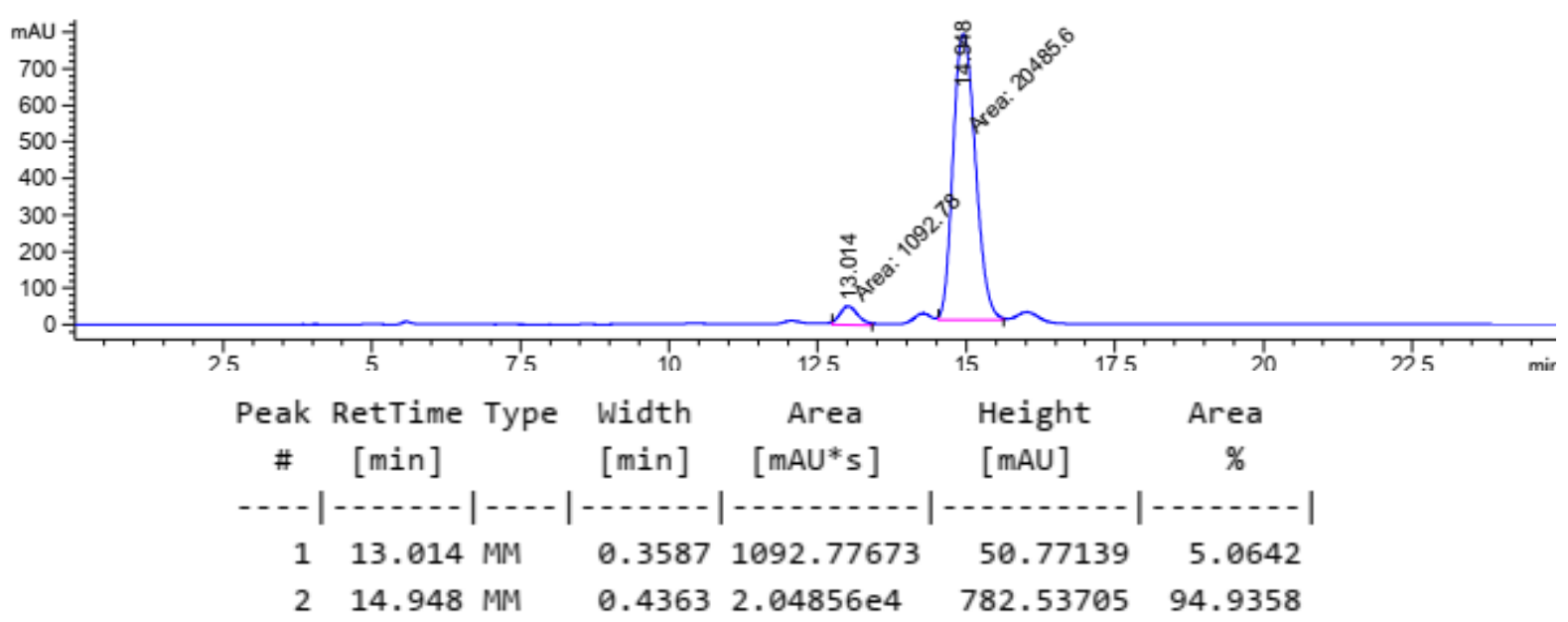

Figure 7.10. (2S,3S)-3-Methyl-3,4-diphenylbutan-2-ol [(2S,3S)-1a, 42\% ee].

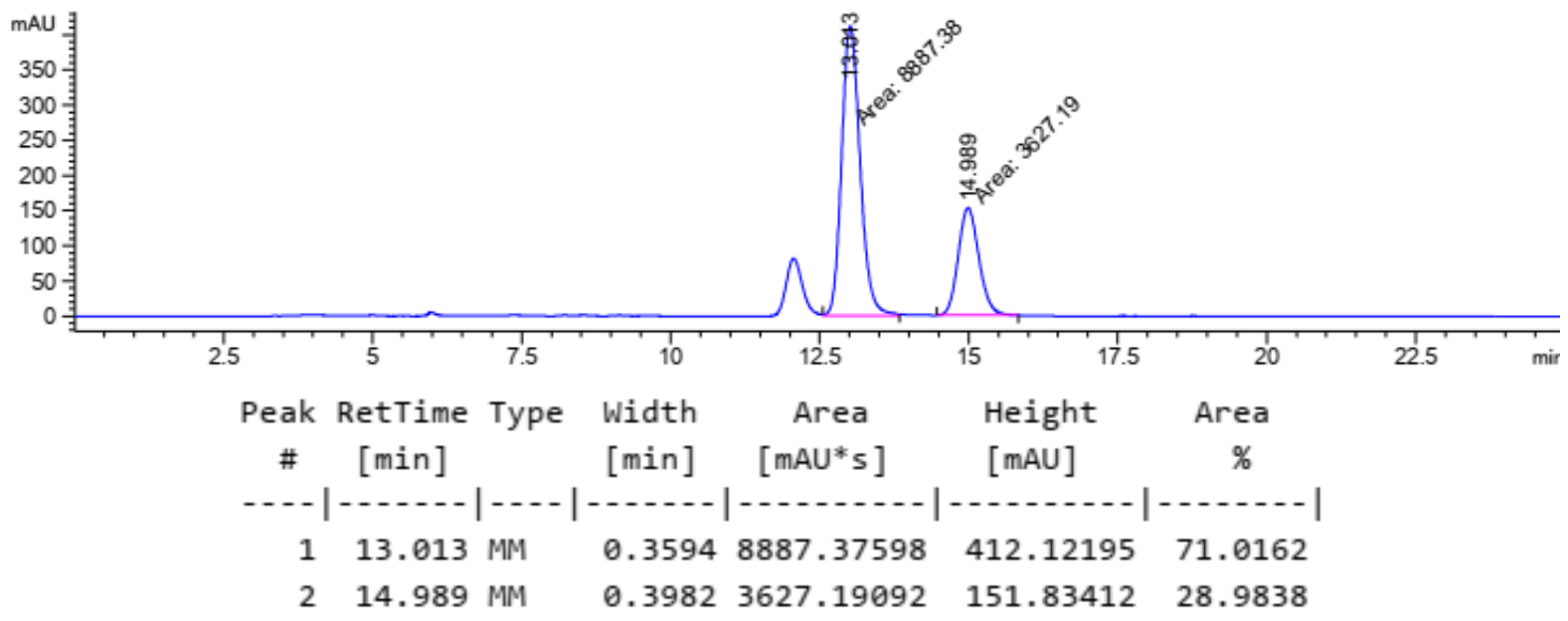


$(2 R, 3 R)-3-M e t h y l-3,4-d i p h e n y l b u t a n-2-o l$ [(2R,3R)-1a] and $(2 S, 3 S)-((3-M e t h y l-$

\section{3,4-diphenylbutan-2-yl)oxy)triphenylsilane [(2S,3S)-3ad]}

Figure 7.11. (2R,3R)-3-Methyl-3,4-diphenylbutan-2-ol [(2R,3R)-1a, 17\% ee].

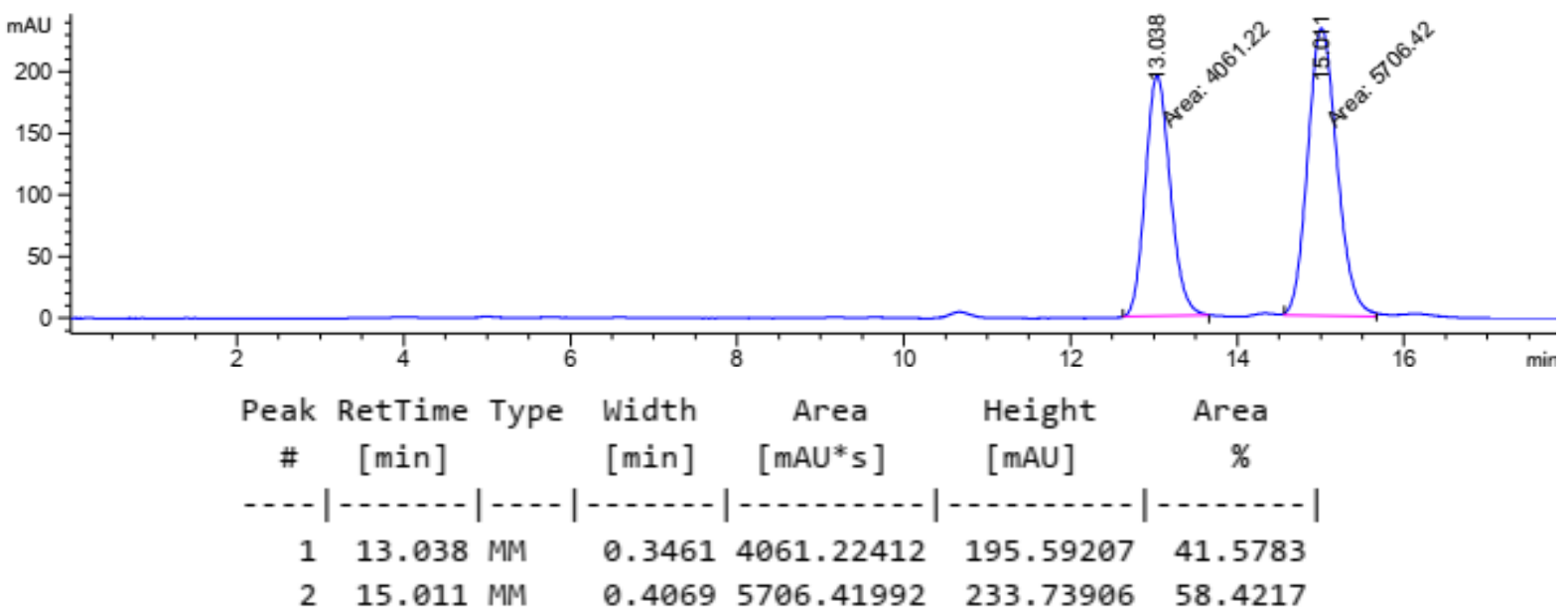

Figure 7.12. (2S,3S)-3-Methyl-3,4-diphenylbutan-2-ol [(2S,3S)-1a, 17\% ee].

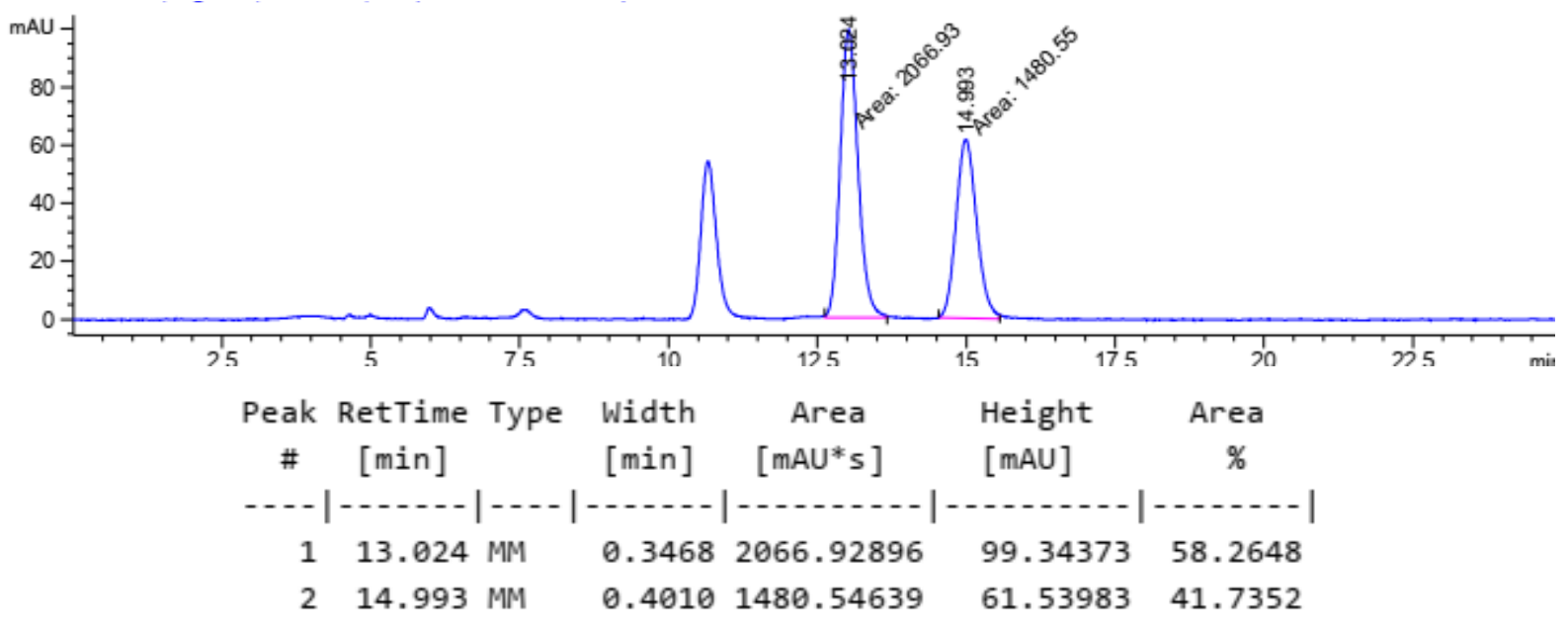


(2R,3R)-3-Methyl-3,4-diphenylbutan-2-ol [(2R,3R)-1a] and (2S,3S)-Diethyl((3methyl-3,4-diphenylbutan-2-yl)oxy)(phenyl)silane [(2S,3S)-3ai]

Figure 7.13. (2R,3R)-3-Methyl-3,4-diphenylbutan-2-ol [(2R,3R)-1a, 42\% ee].

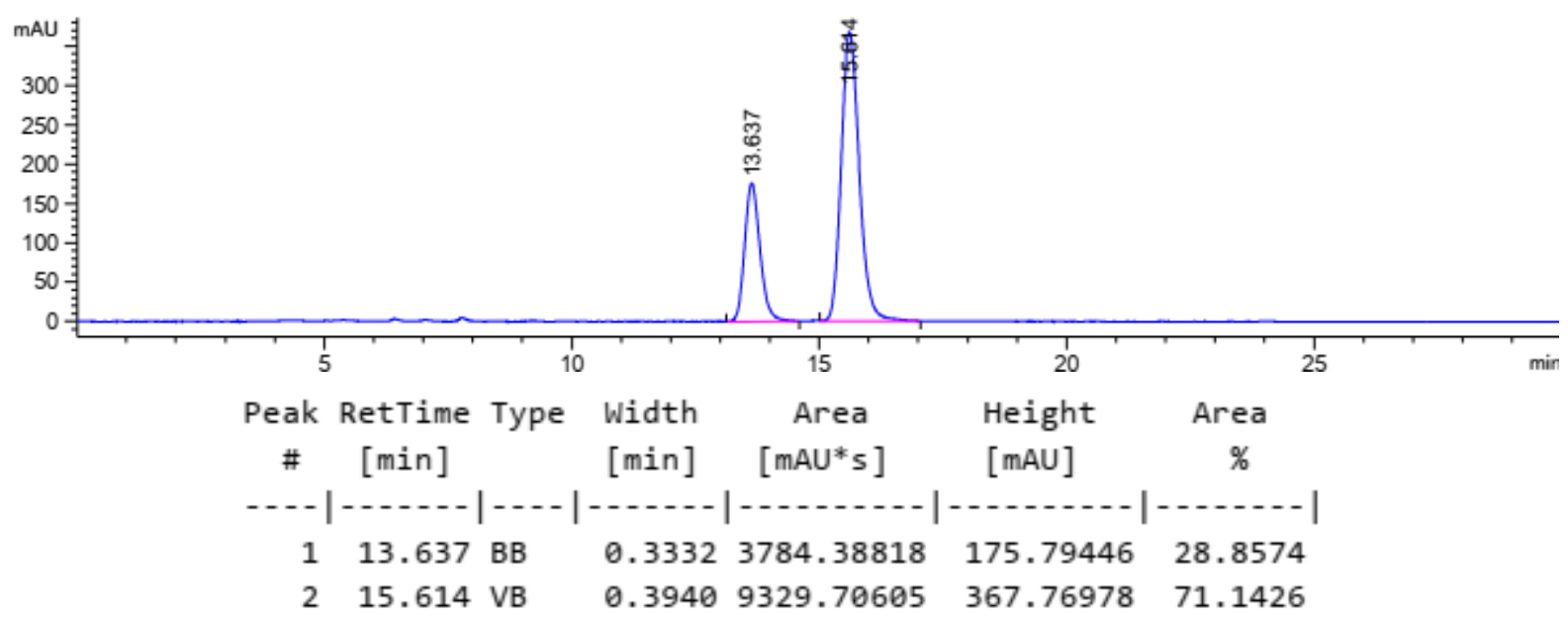

Figure 7.14. (2S,3S)-3-Methyl-3,4-diphenylbutan-2-ol [(2S,3S)-1a, 42\% ee].

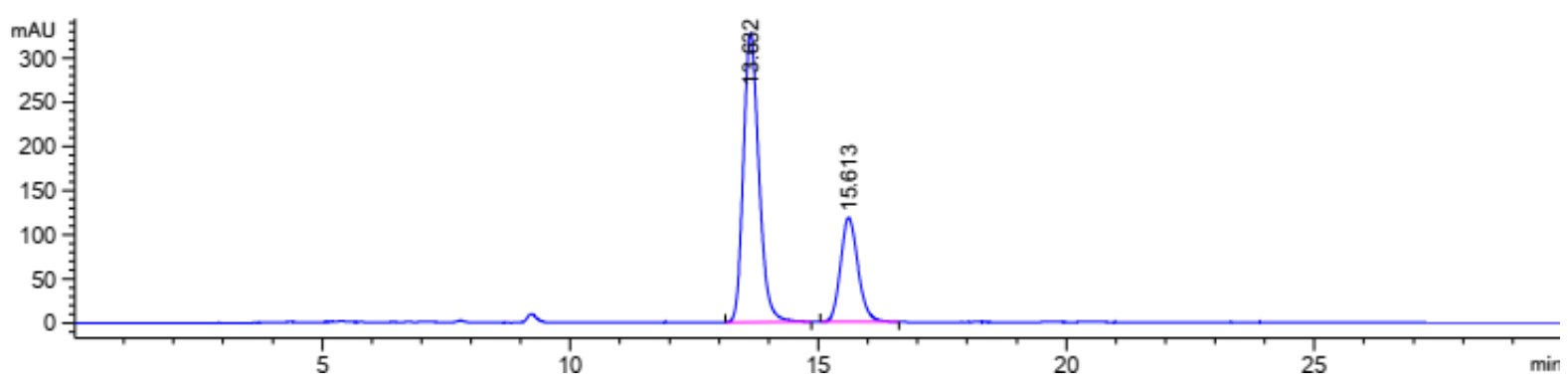

\begin{tabular}{|c|c|c|c|c|c|c|}
\hline $\begin{array}{l}\text { eak } \\
\#\end{array}$ & $\begin{array}{c}\text { RetTime } \\
\text { [min] }\end{array}$ & Type & $\begin{array}{l}\text { Width } \\
\text { [min] }\end{array}$ & $\begin{array}{c}\text { Area } \\
{\left[\mathrm{mAU}^{*} \mathrm{~s}\right]}\end{array}$ & $\begin{array}{l}\text { Height } \\
\text { [mAU] }\end{array}$ & $\begin{array}{c}\text { Area } \\
\%\end{array}$ \\
\hline & & & & & & \\
\hline 1 & & B & & 7134.41260 & 3085 & 151 \\
\hline & 15 & & 6 & 2897.79199 & 250 & 884 \\
\hline
\end{tabular}


(2R,3R)-3-Methyl-3,4-diphenylbutan-2-ol [(2R,3R)-1a] and (2S,3S)-

Mesityldimethyl((3-methyl-3,4-diphenylbutan-2-yl)oxy)silane [(2S,3S)-3af]

Figure 7.15. (2R,3R)-3-Methyl-3,4-diphenylbutan-2-ol [(2R,3R)-1a, 33\% ee].

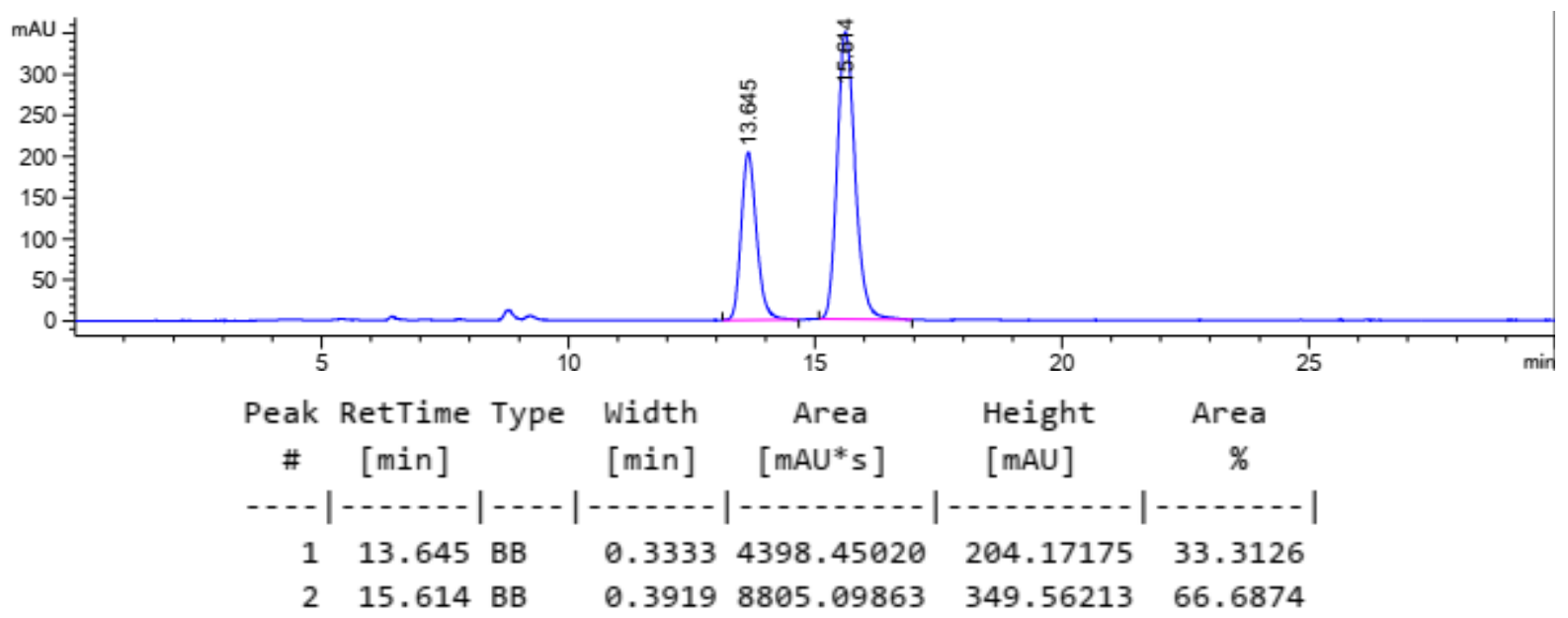

Figure 7.16. (2S,3S)-3-Methyl-3,4-diphenylbutan-2-ol [(2S,3S)-1a, 31\% ee].

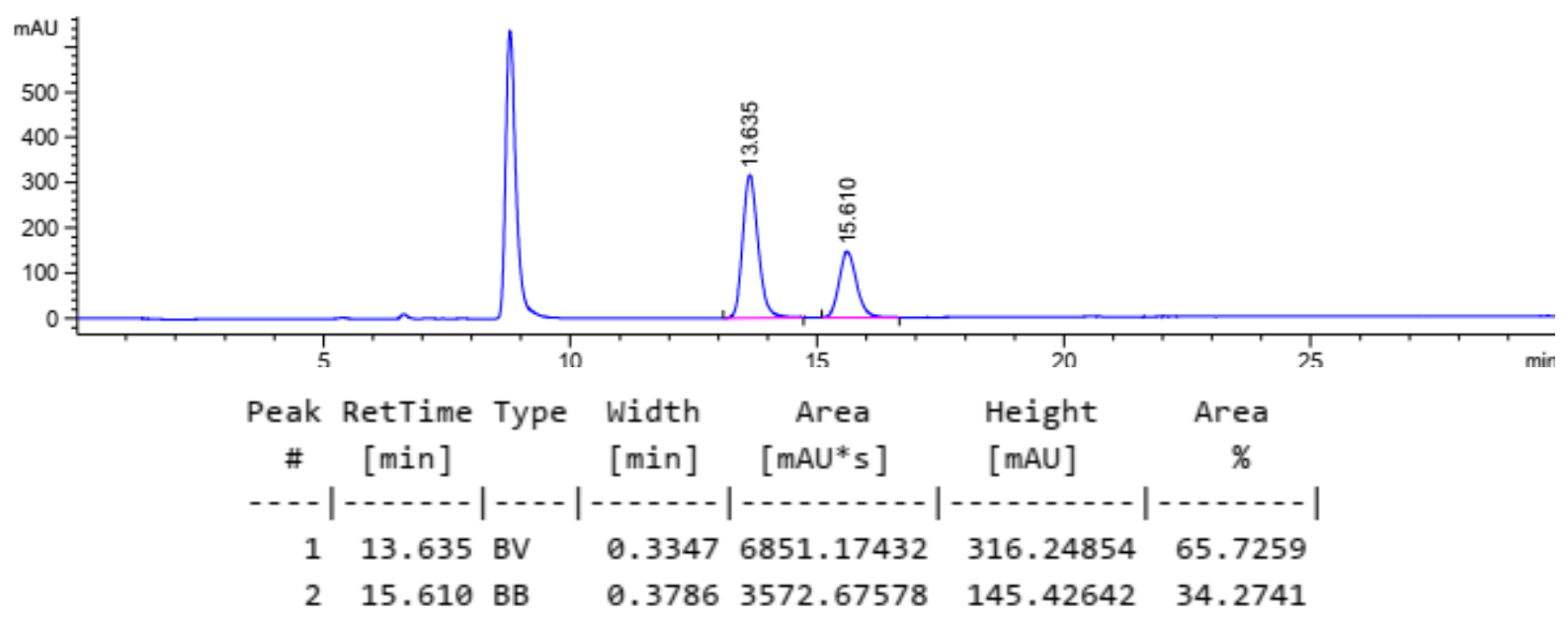


(2R,3R)-3-Methyl-3,4-diphenylbutan-2-ol [(2R,3R)-1a] and (2S,3S)-Dimethyl((3methyl-3,4-diphenylbutan-2-yl)oxy)(naphthalen-1-yl)silane [(2S,3S)-3ak]

Figure 7.17. $(2 R, 3 R)$-3-Methyl-3,4-diphenylbutan-2-ol [(2R,3R)-1a, 47\% ee].

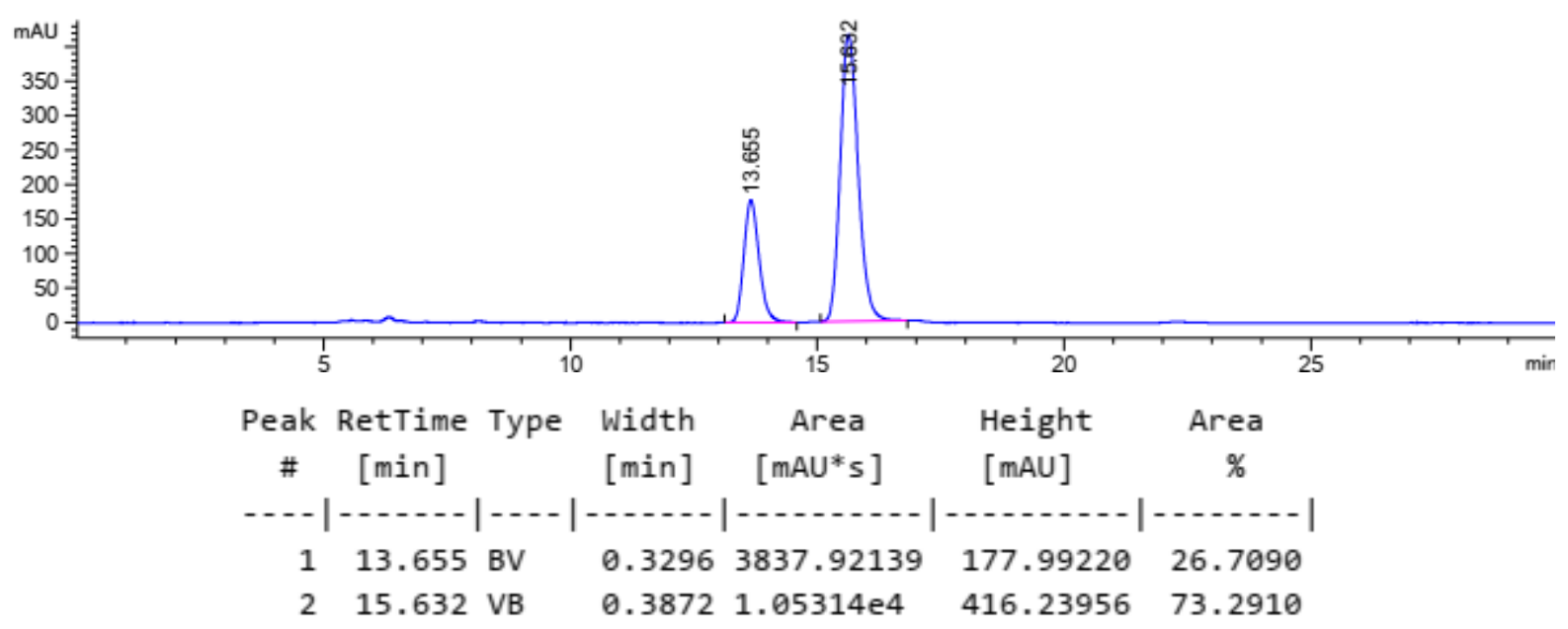

Figure 7.18. (2S,3S)-3-Methyl-3,4-diphenylbutan-2-ol [(2S,3S)-1a, 42\% ee].

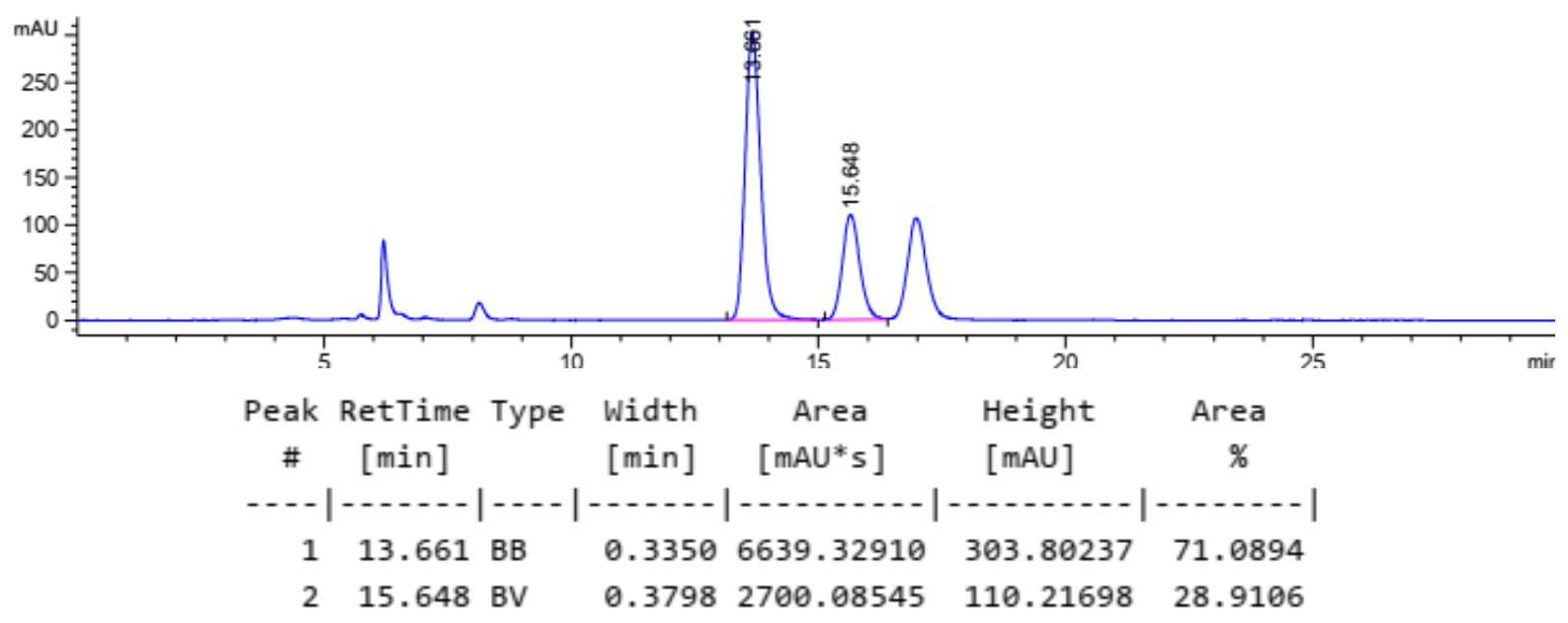


(2R,3R)-3-Methyl-3,4-diphenylbutan-2-ol [(2R,3R)-1a] and (2S,3S)-Dimethyl((3methyl-3,4-diphenylbutan-2-yl)oxy)(naphthalen-2-yl)silane [(2S,3S)-3ah]

Figure 7.19. (2R,3R)-3-Methyl-3,4-diphenylbutan-2-ol [(2R,3R)-1a, 73\% ee].

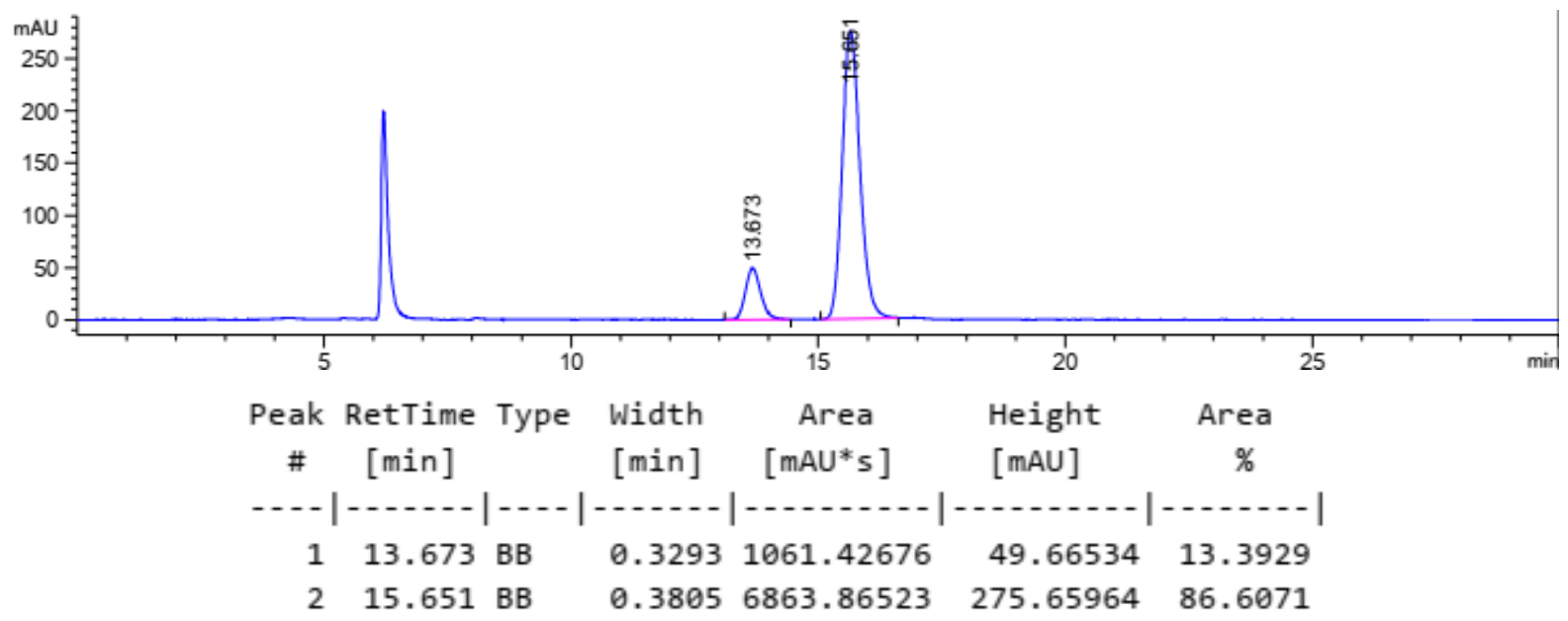

Figure 7.20. (2S,3S)-3-Methyl-3,4-diphenylbutan-2-ol [(2S,3S)-1a, 73\% ee].

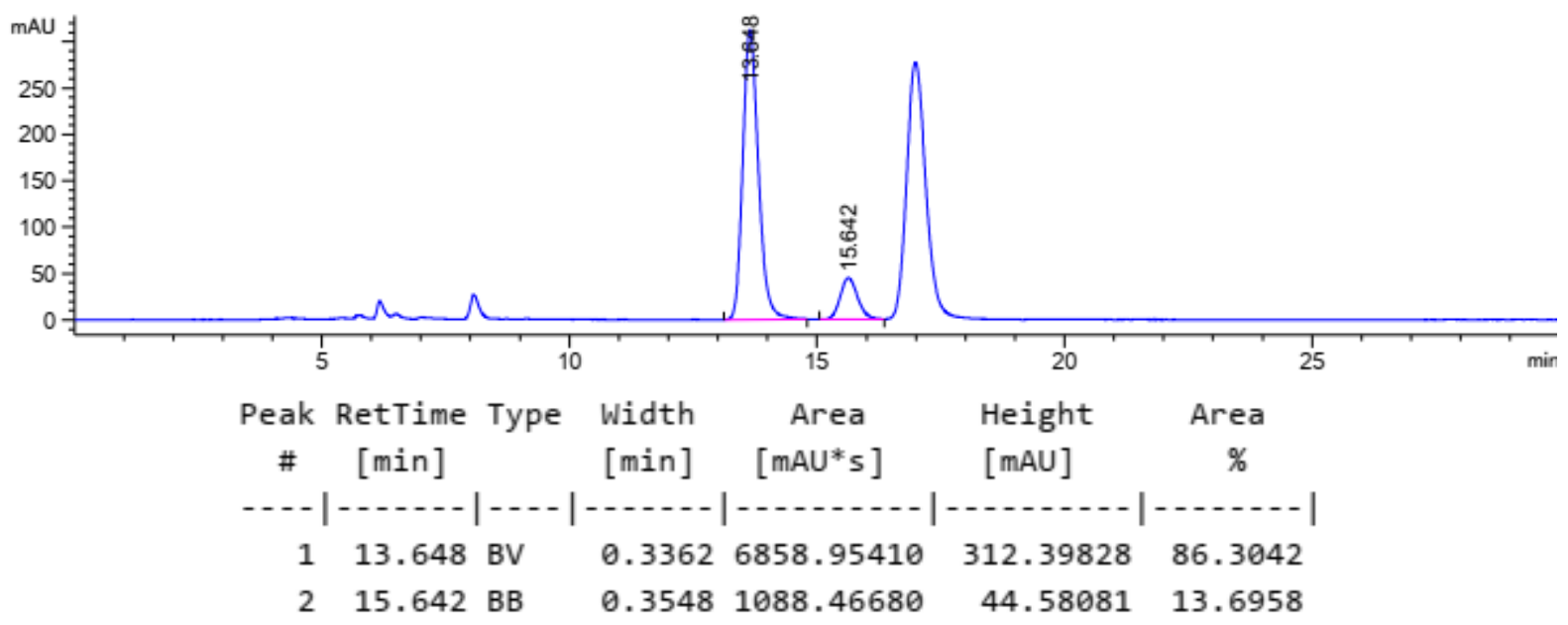


$(2 R, 3 R)-3-M e t h y l-3,4-d i p h e n y l b u t a n-2-o l$ [(2R,3R)-1a] and (2S,3S)-(4-(tertButyl)phenyl)dimethyl((3-methyl-3,4-diphenylbutan-2-yl)oxy)silane [(2S,3S)3ag]

Figure 7.21 (2R,3R)-3-Methyl-3,4-diphenylbutan-2-ol [(2R,3R)-1a, 63\% ee].

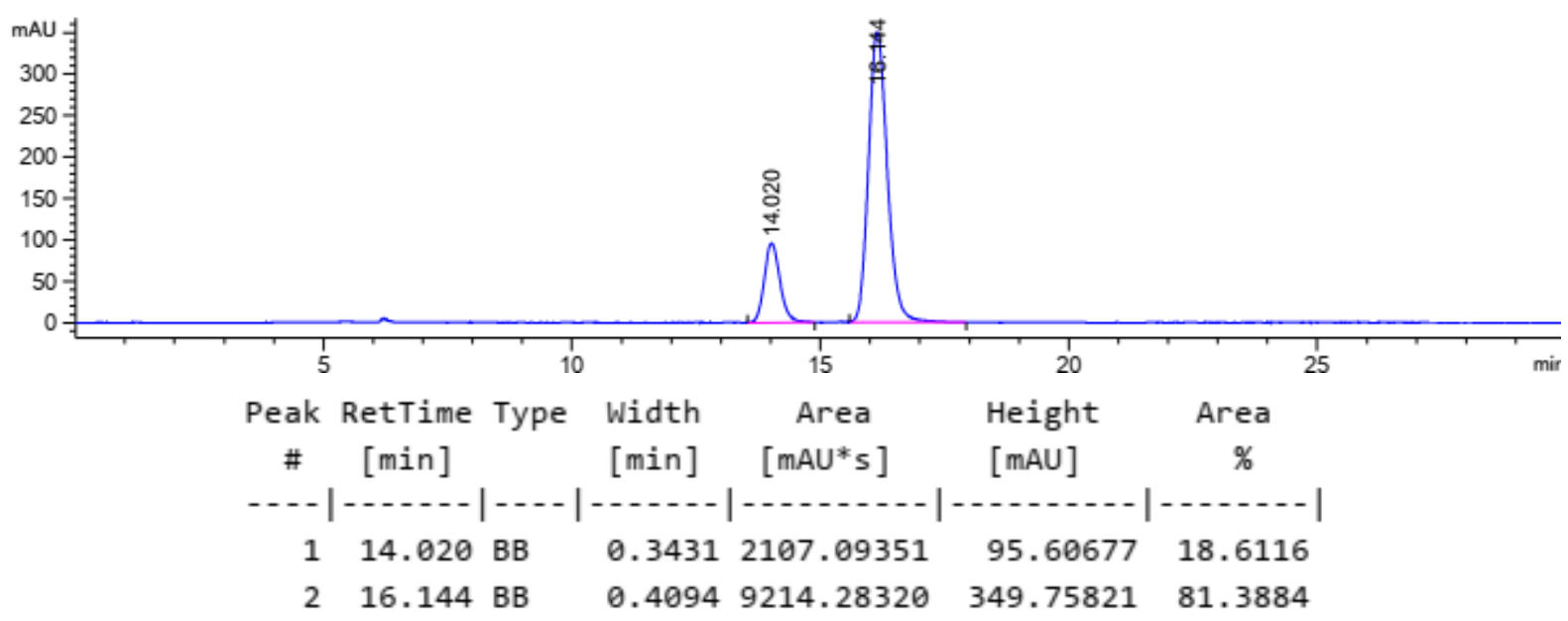

Figure 7.22 (2S,3S)-3-Methyl-3,4-diphenylbutan-2-ol [(2S,3S)-1a, 67\% ee].

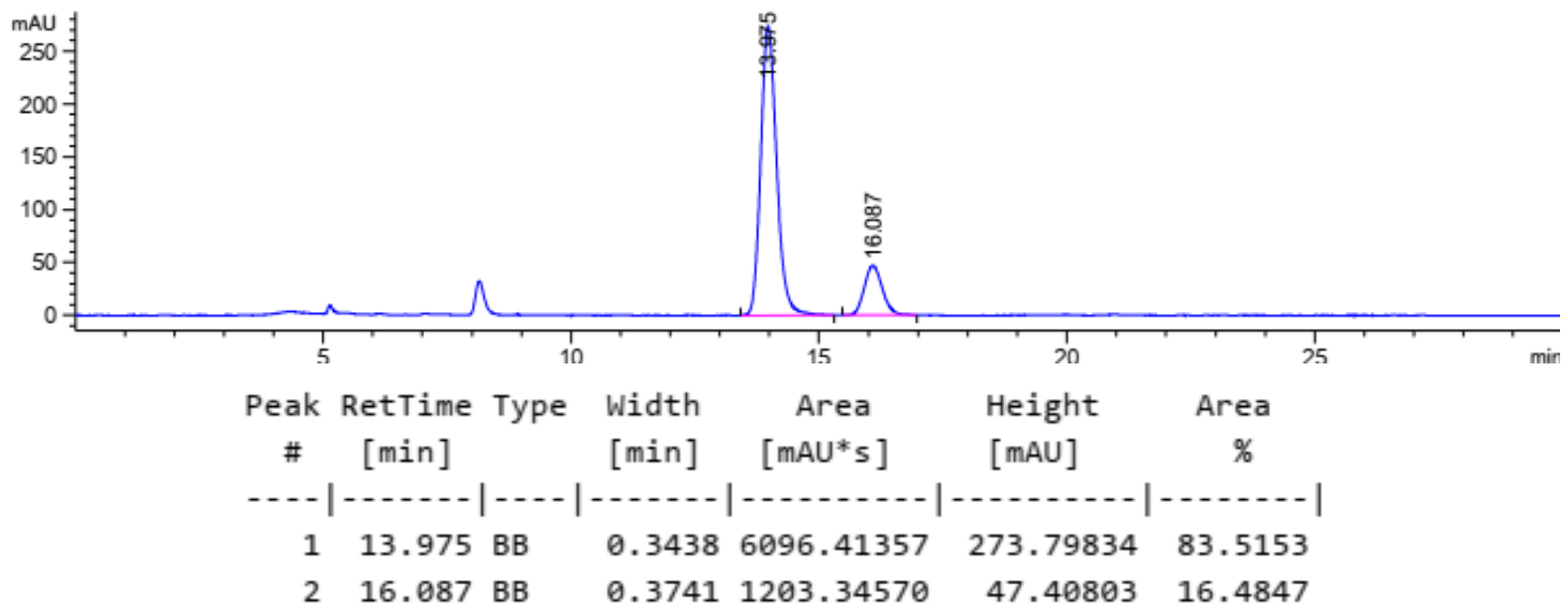


(2R,3R)-3-Methyl-3,4-diphenylbutan-2-ol [(2R,3R)-1a] and (2S,3S)-(4-Methoxy-

\section{3,5-dimethylphenyl)dimethyl((3-methyl-3,4-diphenylbutan-2-yl)oxy)silane}

[(2S,3S)-3aj]

Figure $7.23(2 R, 3 R)$-3-Methyl-3,4-diphenylbutan-2-ol [(2R,3R)-1a, 76\% ee].

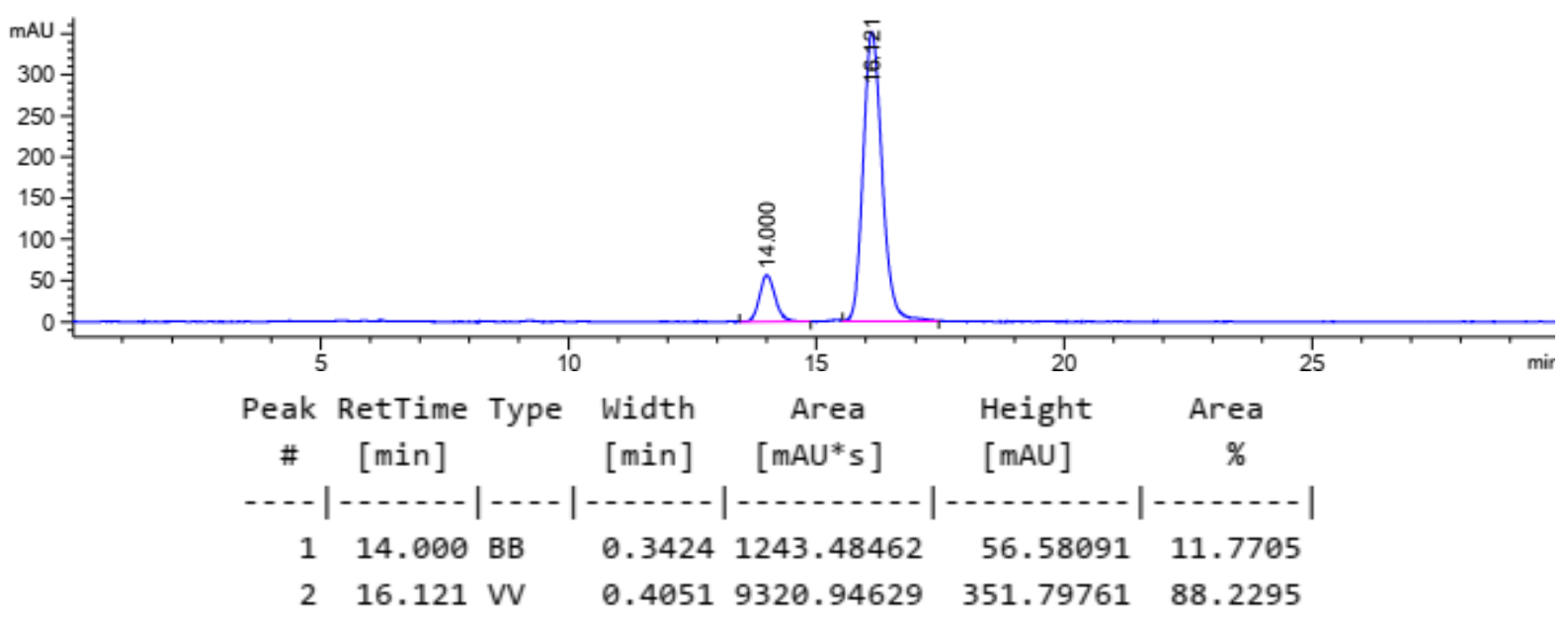

Figure 7.24 (2S,3S)-3-Methyl-3,4-diphenylbutan-2-ol [(2S,3S)-1a, 73\% ee].

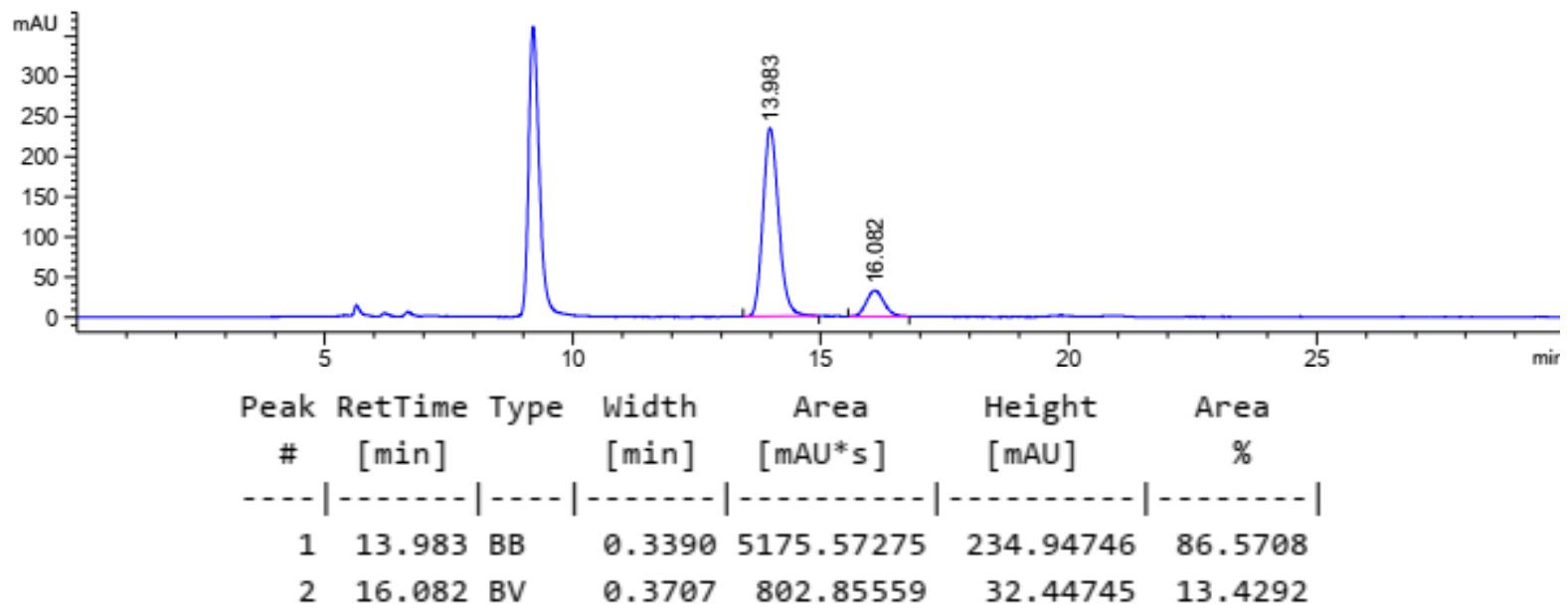


(2R,3R)-3-Methyl-3,4-diphenylbutan-2-ol [(2R,3R)-1a] and (2S,3S)-Tribenzyl((3methyl-3,4-diphenylbutan-2-yl)oxy)silane [(2S,3S)-3al]

Figure 7.25 (2R,3R)-3-Methyl-3,4-diphenylbutan-2-ol [(2R,3R)-1a, 18\% ee].

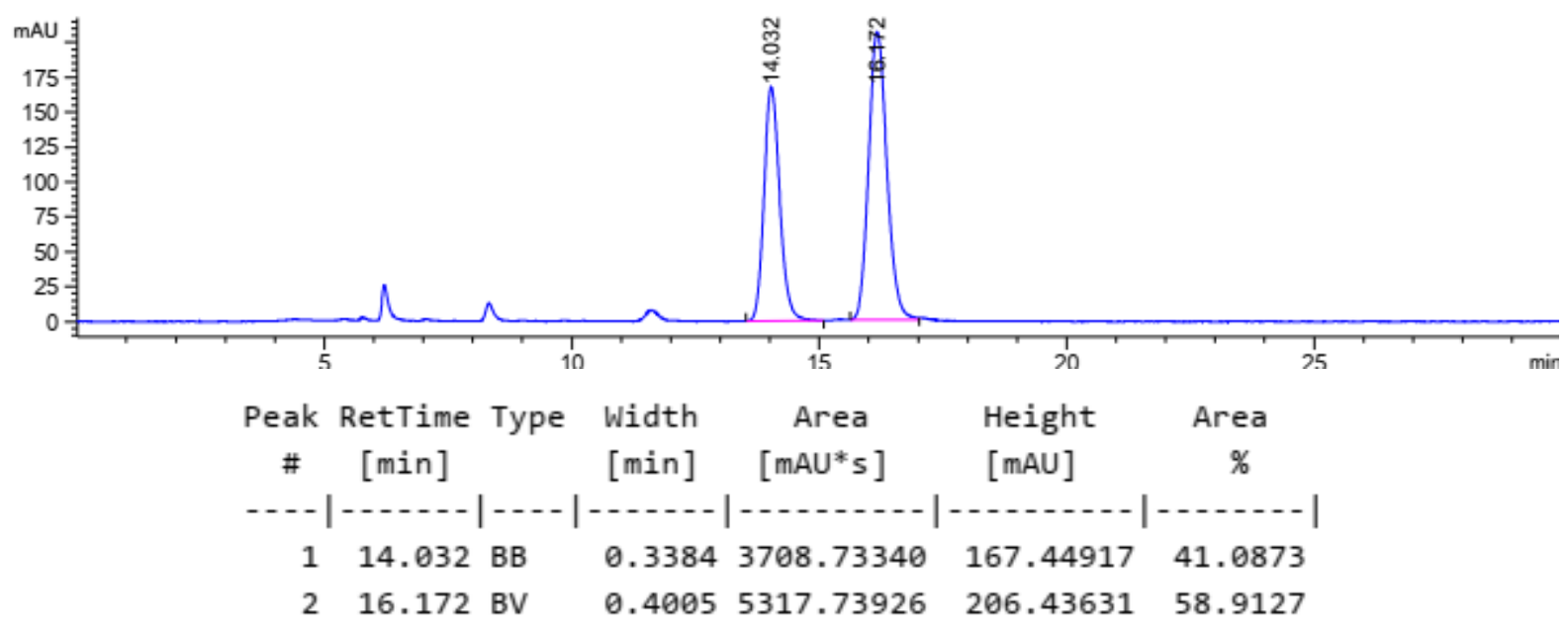

Figure 7.26 (2S,3S)-3-Methyl-3,4-diphenylbutan-2-ol [(2S,3S)-1a, 18\% ee].

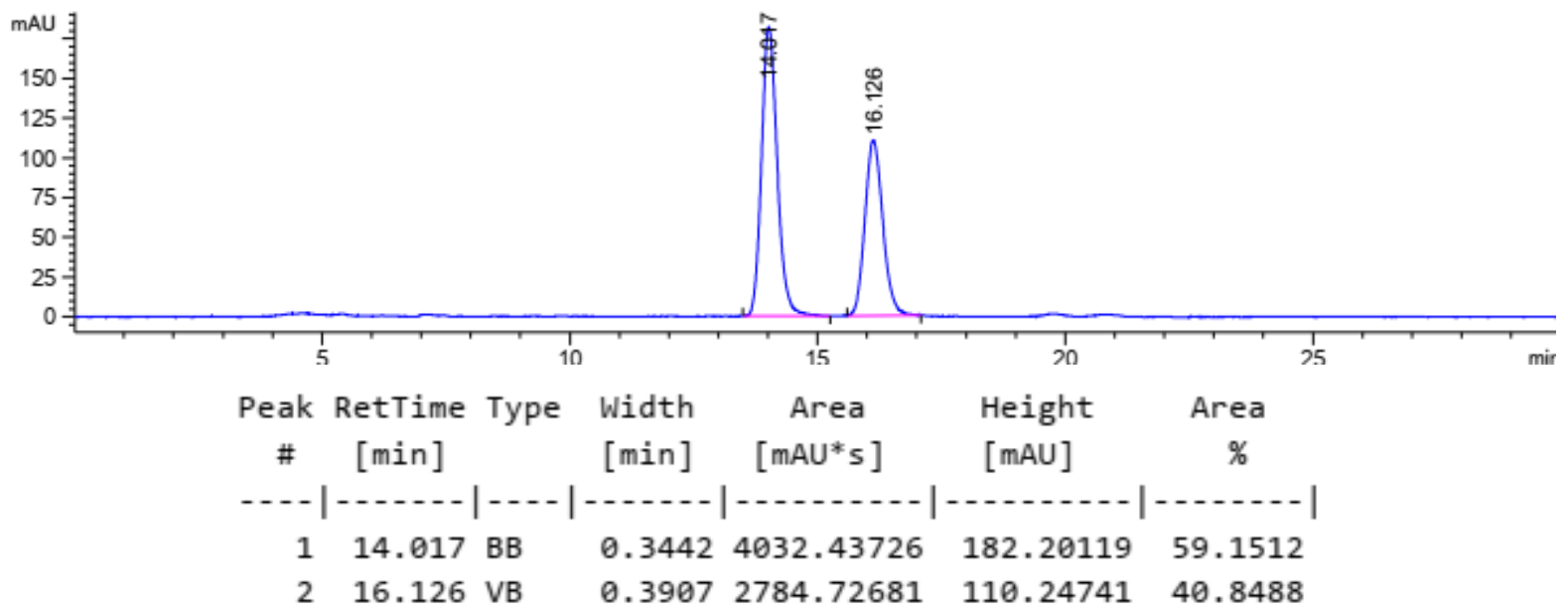




\subsection{Temperature Screening}

\section{rac-anti-3-Methyl-3,4-diphenylbutan-2-ol}

Figure 7.27 rac-anti-3-Methyl-3,4-diphenylbutan-2-ol [rac-anti-1a].

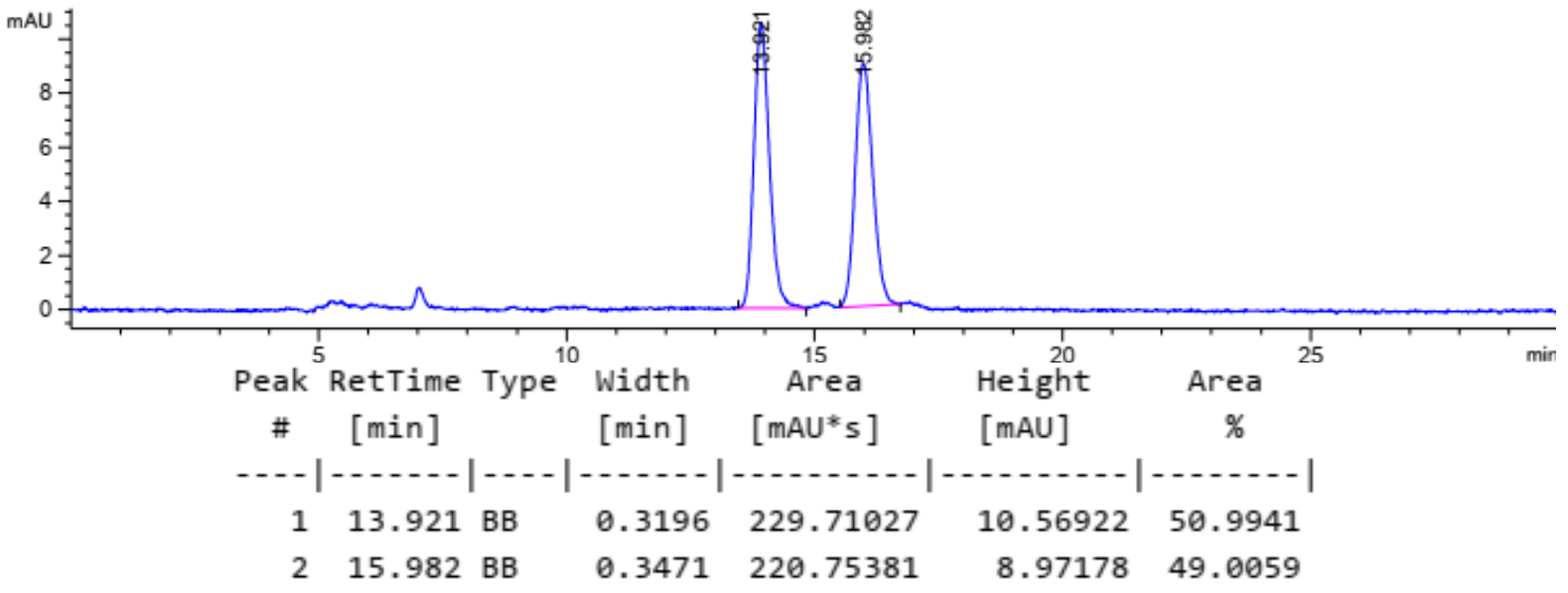

(2R,3R)-3-Methyl-3,4-diphenylbutan-2-ol [(2R,3R)-1a] and

(2S,3S)-(3,5-Dimethylphenyl)dimethyl((3-methyl-3,4-diphenylbutan-2-

yl)oxy)silane [(2S,3S)-3ae] - Temperature: $-20^{\circ} \mathrm{C}$

Figure $7.28(2 R, 3 R)$-3-Methyl-3,4-diphenylbutan-2-ol [(2R,3R)-1a, 92\% ee, Table S2, entry 1].

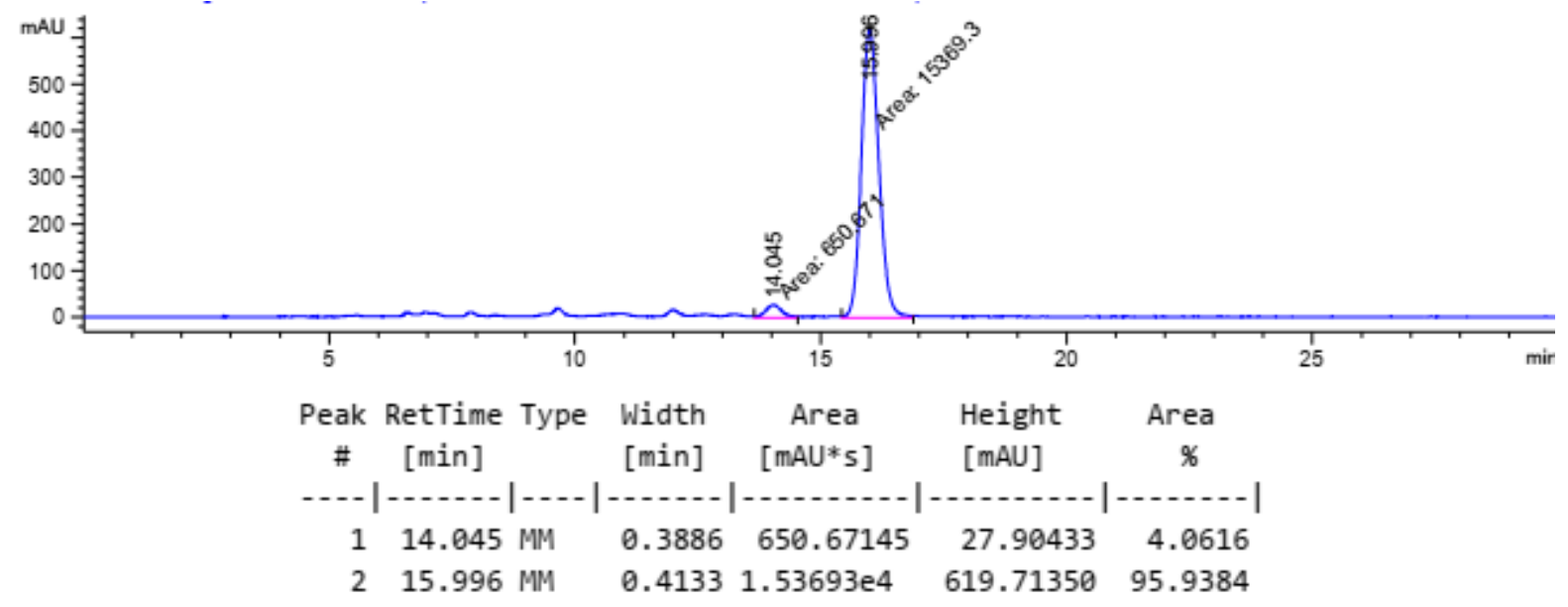


Figure 7.29 (2S,3S)-3-Methyl-3,4-diphenylbutan-2-ol [(2S,3S)-1a, 91\% ee, Table S2, entry 1].

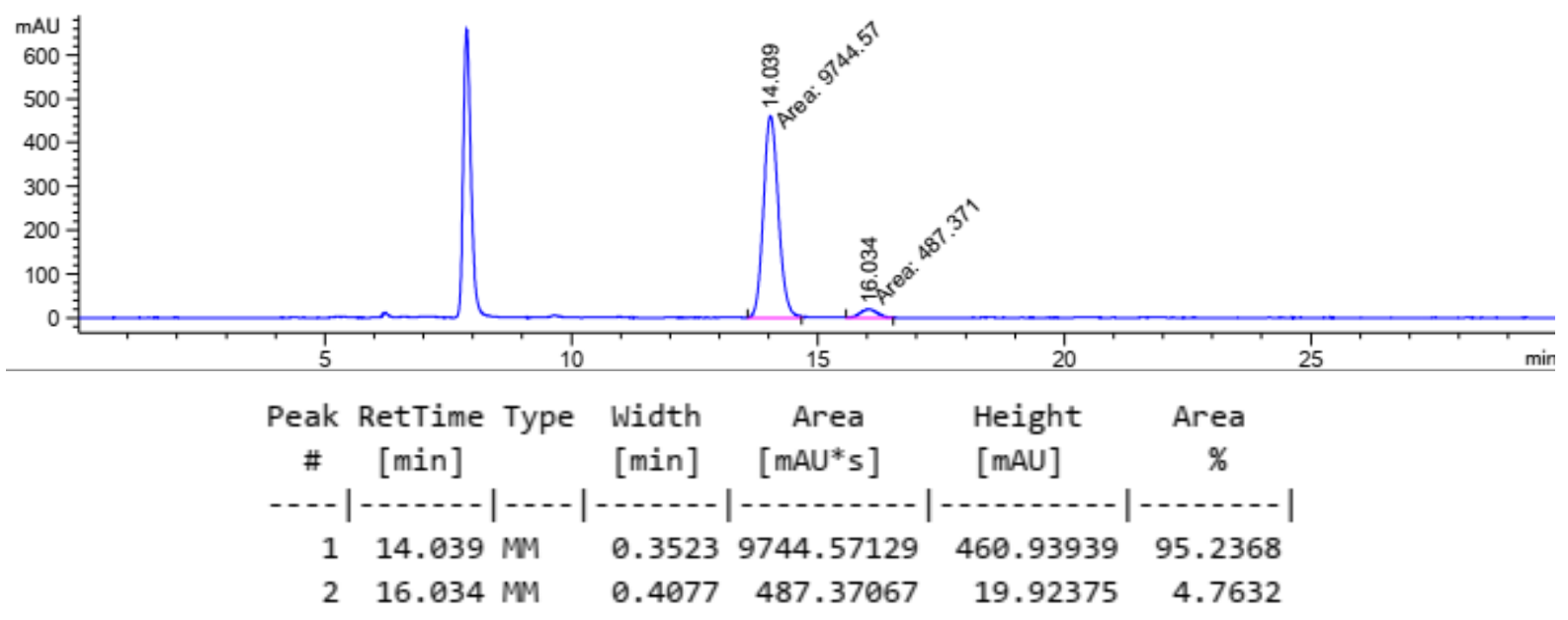

Figure $7.30(2 R, 3 R)$-3-Methyl-3,4-diphenylbutan-2-ol [(2R,3R)-1a, 86\% ee, Table S2, entry 2].

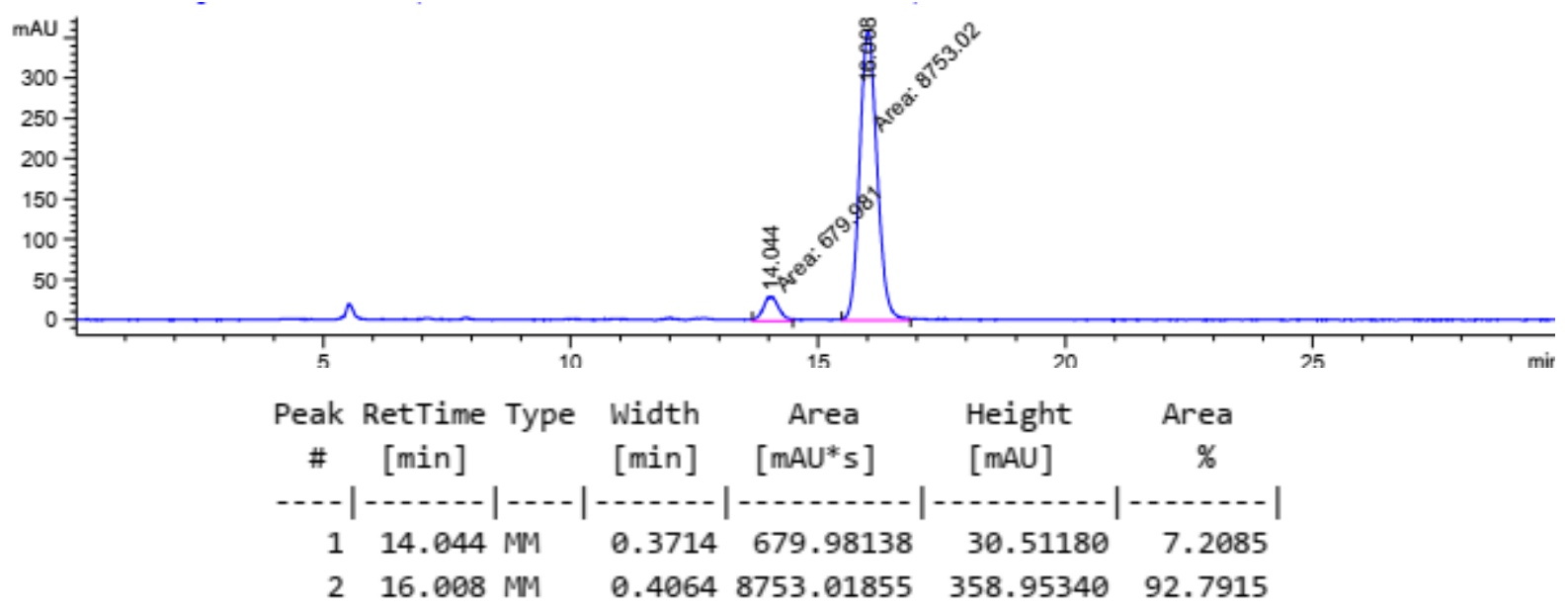

Figure 7.31 (2S,3S)-3-Methyl-3,4-diphenylbutan-2-ol [(2S,3S)-1a, 93\% ee, Table S2, entry 2].

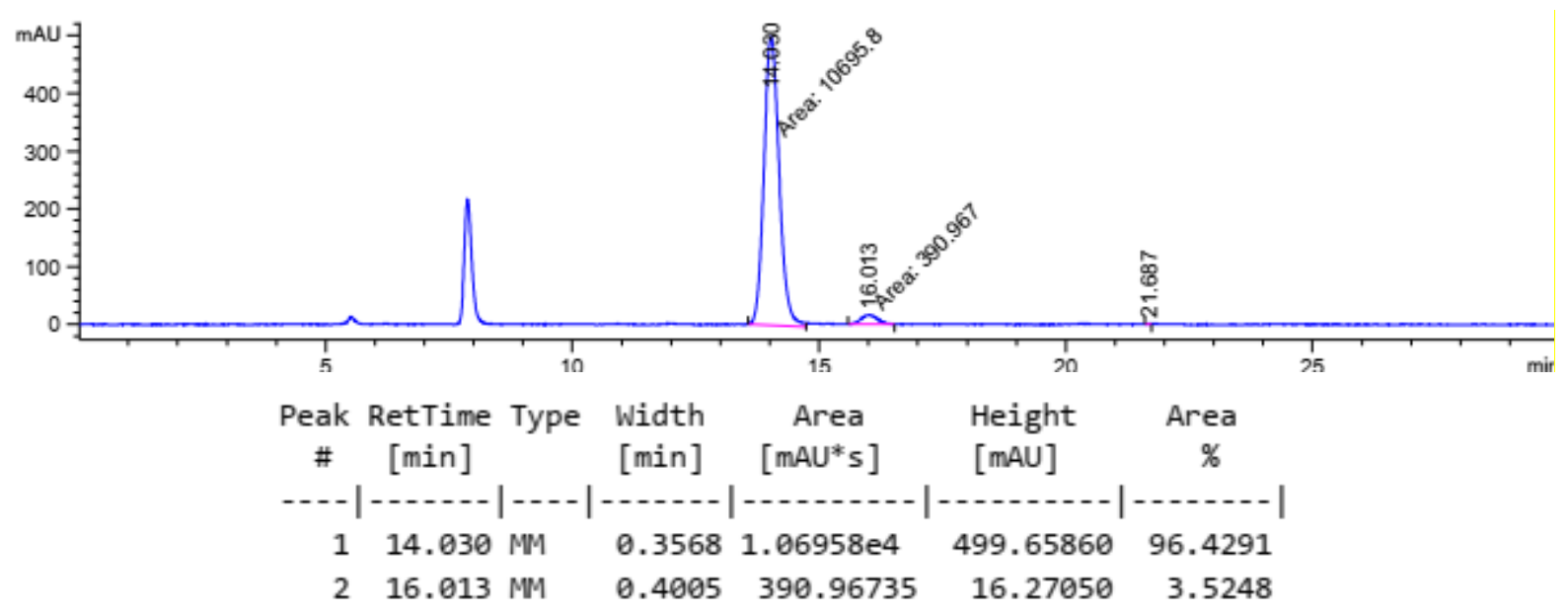


(2R,3R)-3-Methyl-3,4-diphenylbutan-2-ol [(2R,3R)-1a] and

(2S,3S)-(3,5-Dimethylphenyl)dimethyl((3-methyl-3,4-diphenylbutan-2-

yl)oxy)silane [(2S,3S)-3ae] - Temperature: $-15^{\circ} \mathrm{C}$

Figure 7.32 (2R,3R)-3-Methyl-3,4-diphenylbutan-2-ol [(2R,3R)-1a, 81\% ee, Table S2, entry 3].

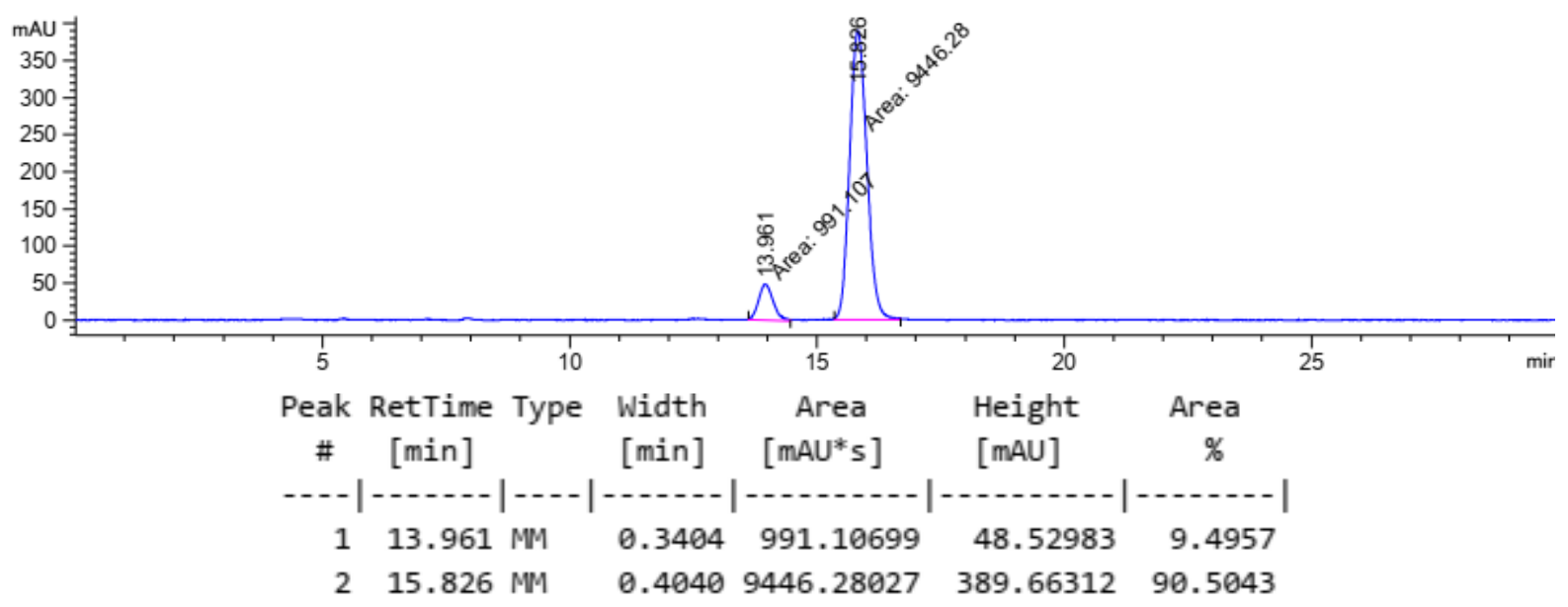

Figure 7.33 (2S,3S)-3-Methyl-3,4-diphenylbutan-2-ol [(2S,3S)-1a, 85\% ee, Table S2, entry 3].

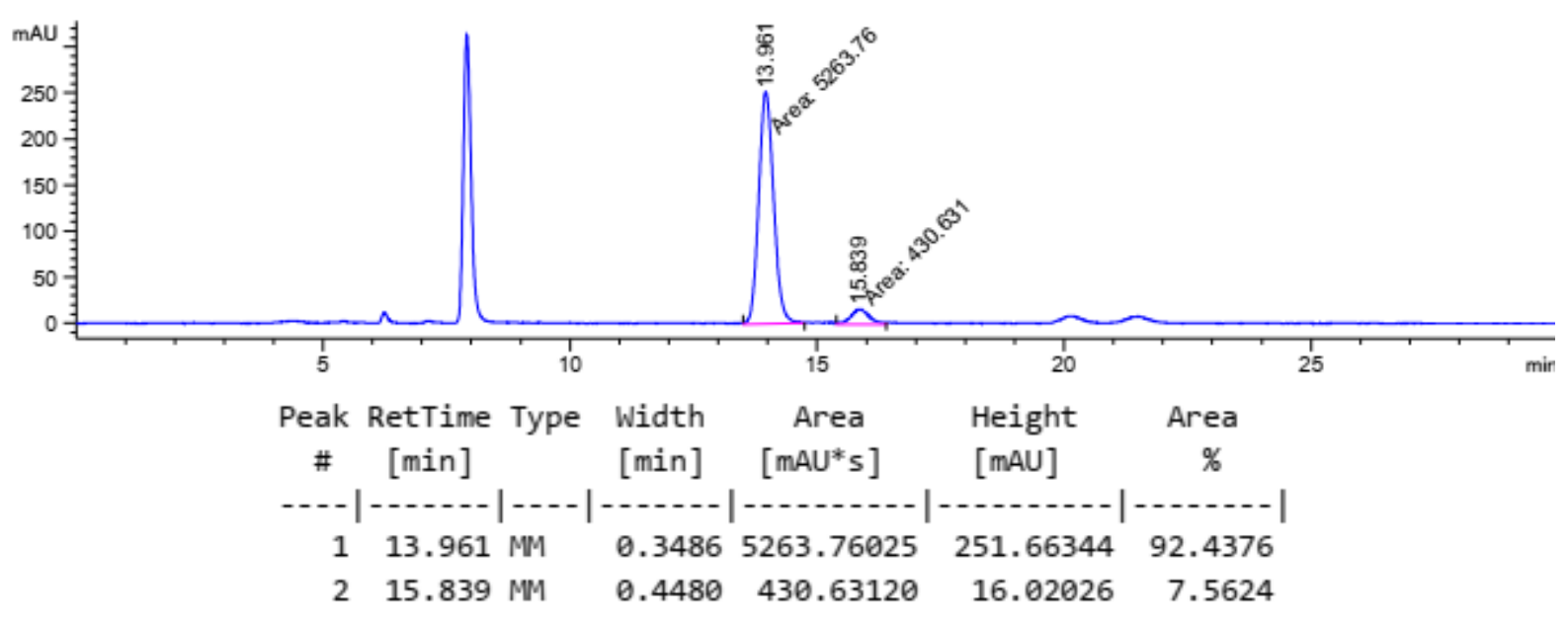


Figure 7.34 (2R,3R)-3-Methyl-3,4-diphenylbutan-2-ol [(2R,3R)-1a, 81\% ee, Table S2, entry 4].

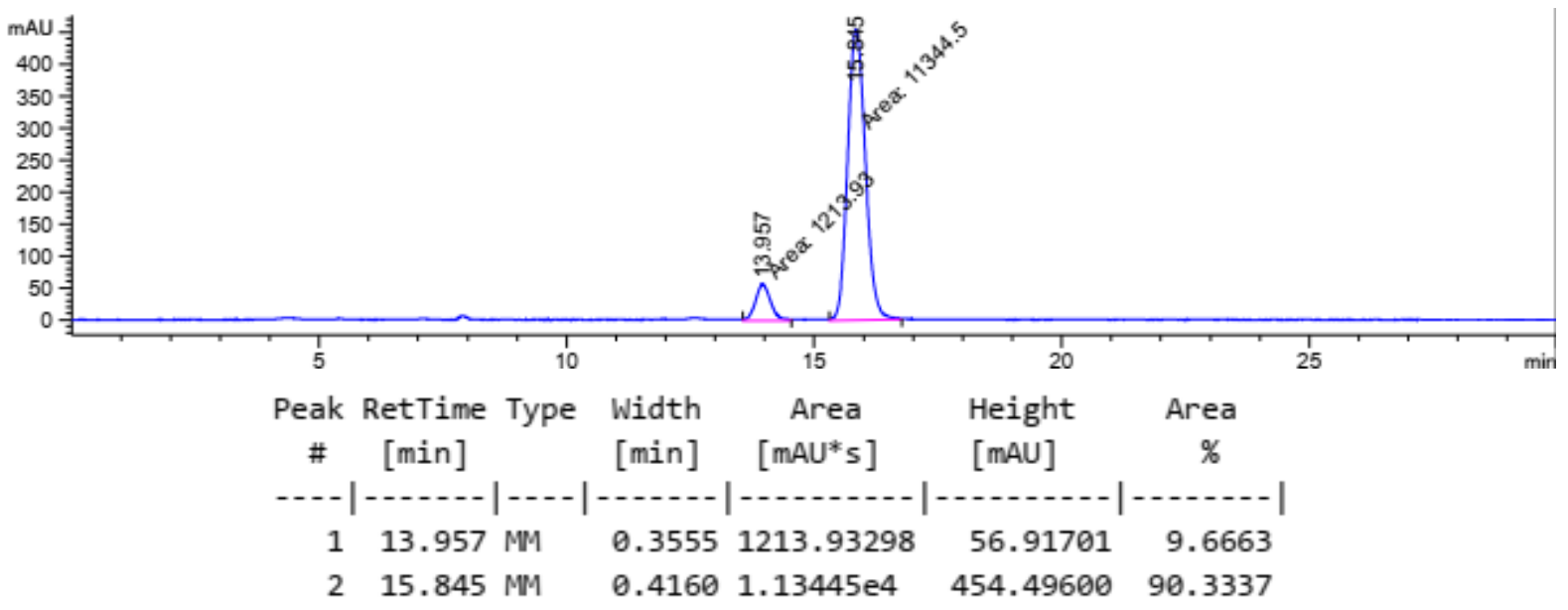

Figure 7.35 (2S,3S)-3-Methyl-3,4-diphenylbutan-2-ol [(2S,3S)-1a, 90\% ee, Table S2, entry 4].

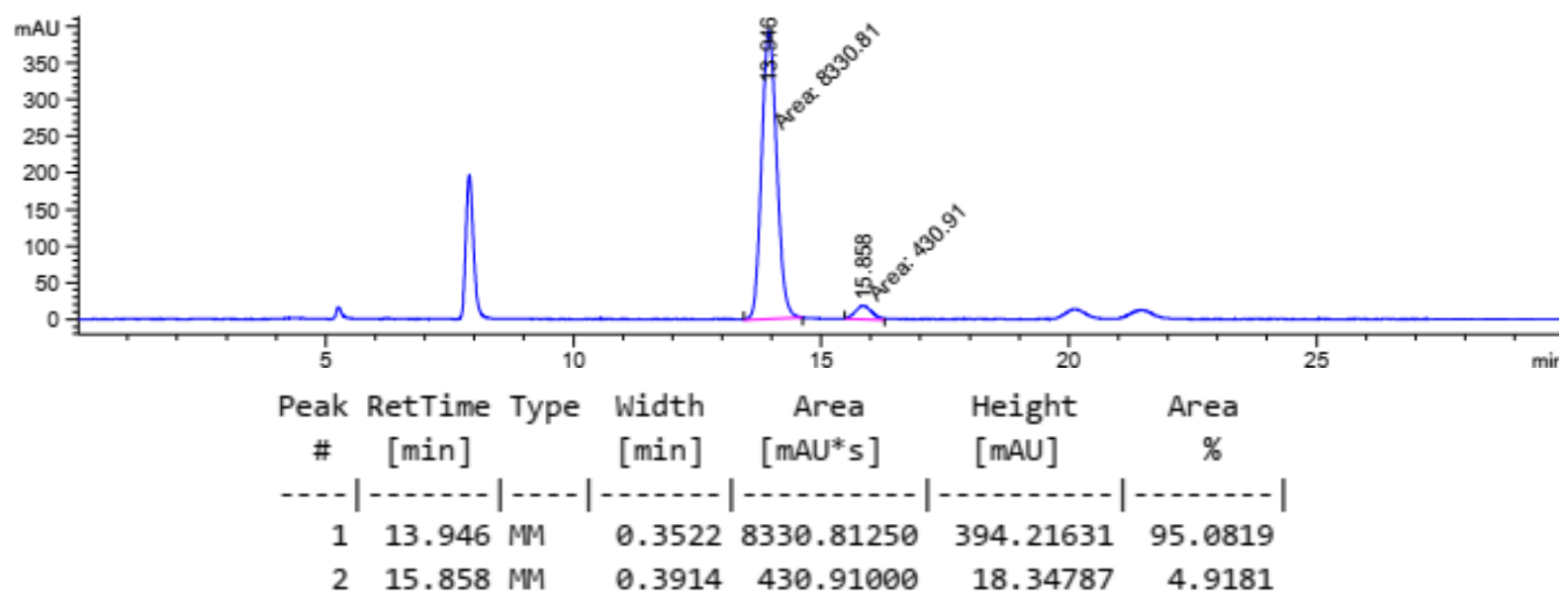


(2R,3R)-3-Methyl-3,4-diphenylbutan-2-ol [(2R,3R)-1a] and

(2S,3S)-(3,5-Dimethylphenyl)dimethyl((3-methyl-3,4-diphenylbutan-2-

yl)oxy)silane [(2S,3S)-3ae] - Temperature: $-10^{\circ} \mathrm{C}$

Figure 7.36 (2R,3R)-3-Methyl-3,4-diphenylbutan-2-ol [(2R,3R)-1a, 91\% ee, Table S2, entry 5].

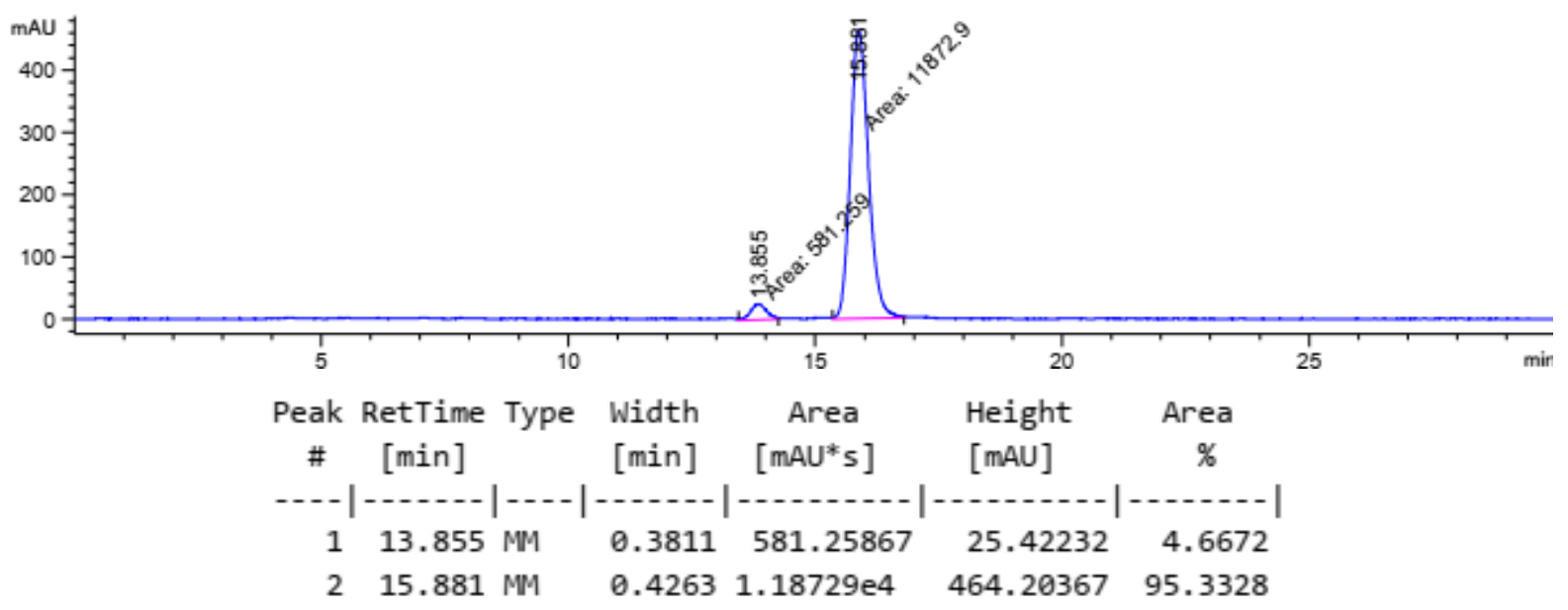

Figure 7.37 (2S,3S)-3-Methyl-3,4-diphenylbutan-2-ol [(2S,3S)-1a, 79\% ee, Table S2, entry 5].

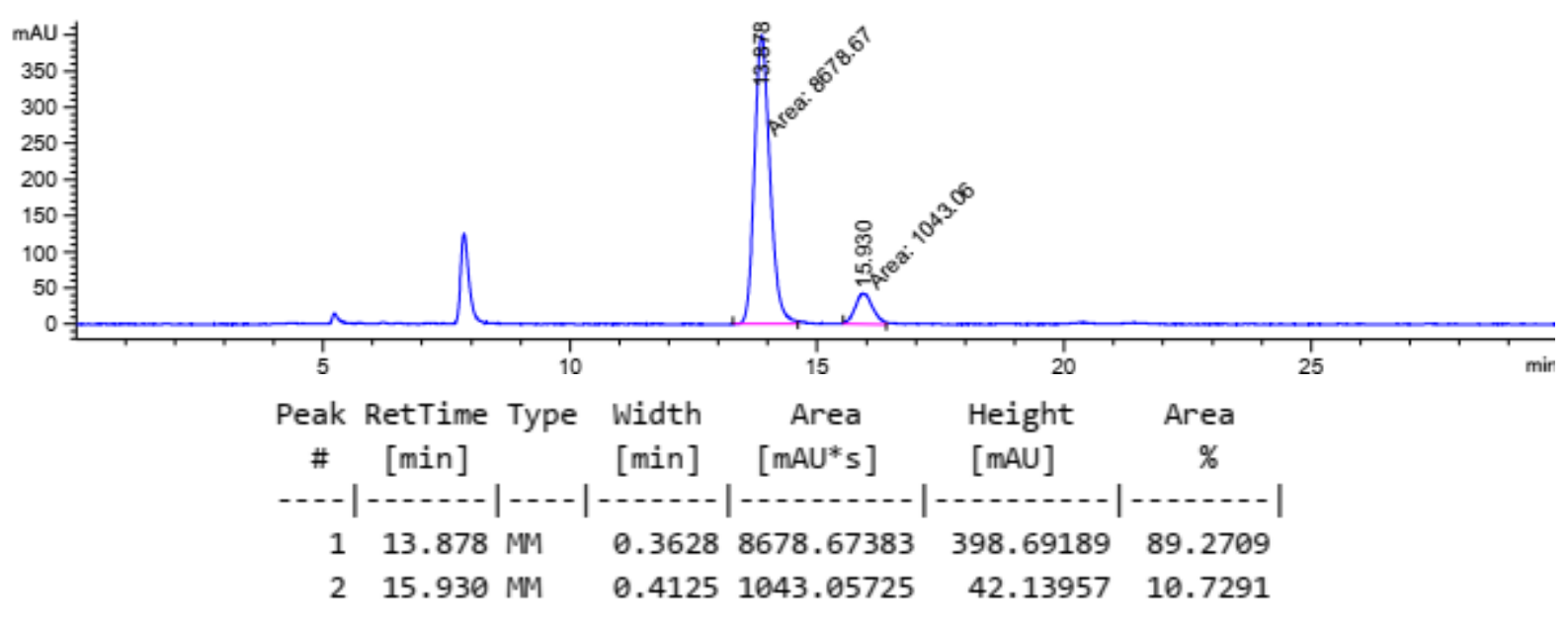


Figure $7.38(2 R, 3 R)$-3-Methyl-3,4-diphenylbutan-2-ol [(2R,3R)-1a, 82\% ee, Table S2, entry 6].

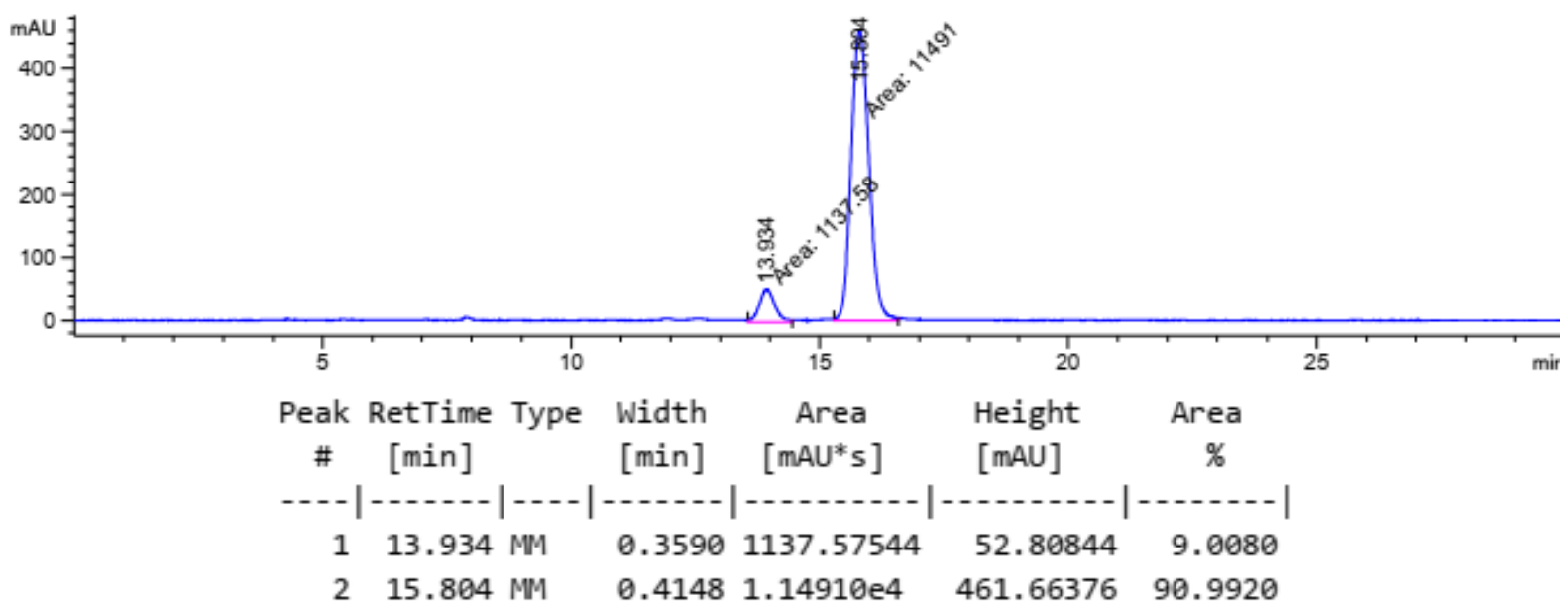

Figure 7.39 (2S,3S)-3-Methyl-3,4-diphenylbutan-2-ol [(2S,3S)-1a, 82\% ee, Table S2, entry 6].

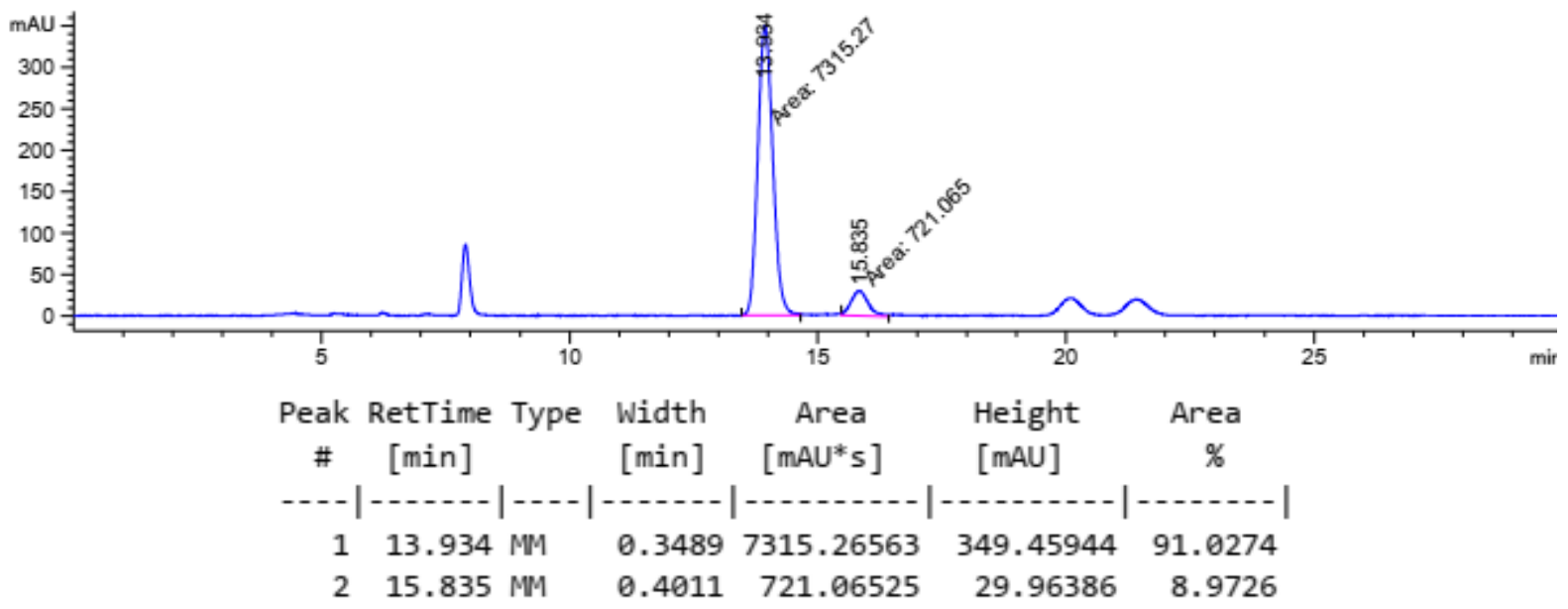

Figure 7.40 (2R,3R)-3-Methyl-3,4-diphenylbutan-2-ol [(2R,3R)-1a, 84\% ee, Table S2, entry 7].

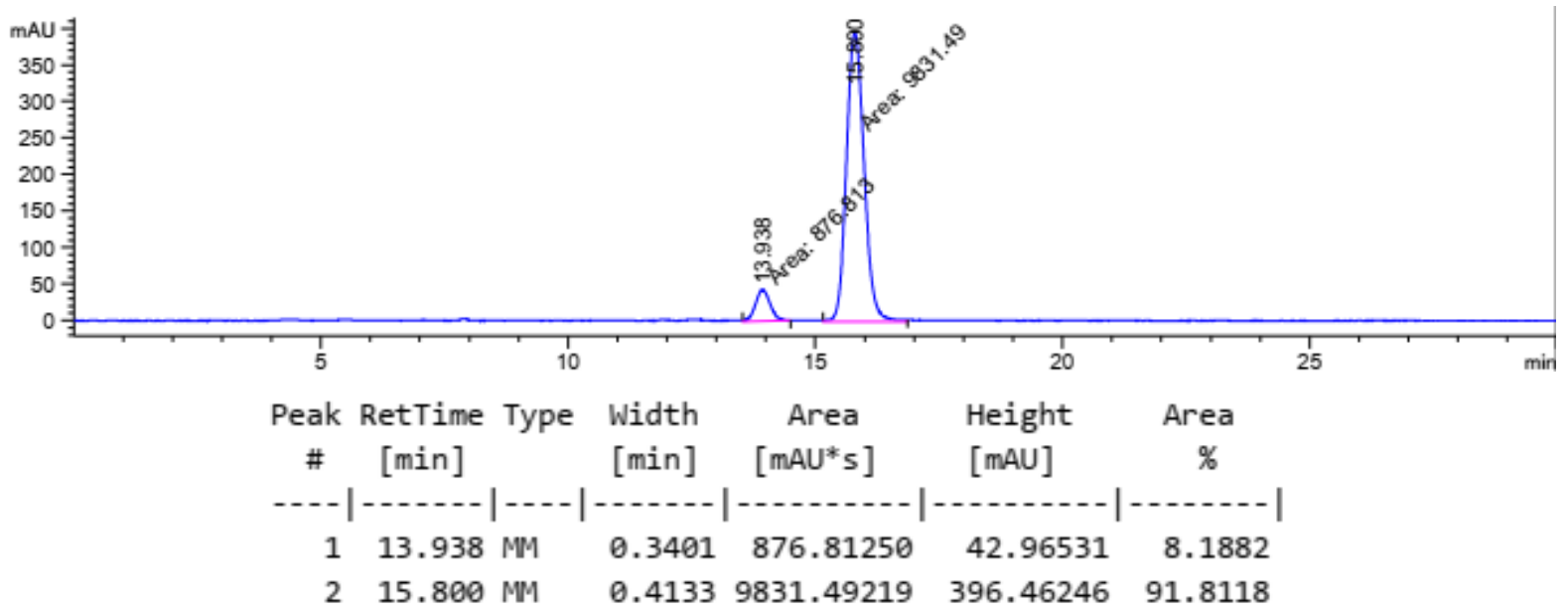


Figure 7.41 (2S,3S)-3-Methyl-3,4-diphenylbutan-2-ol [(2S,3S)-1a, 80\% ee, Table S2, entry 7].

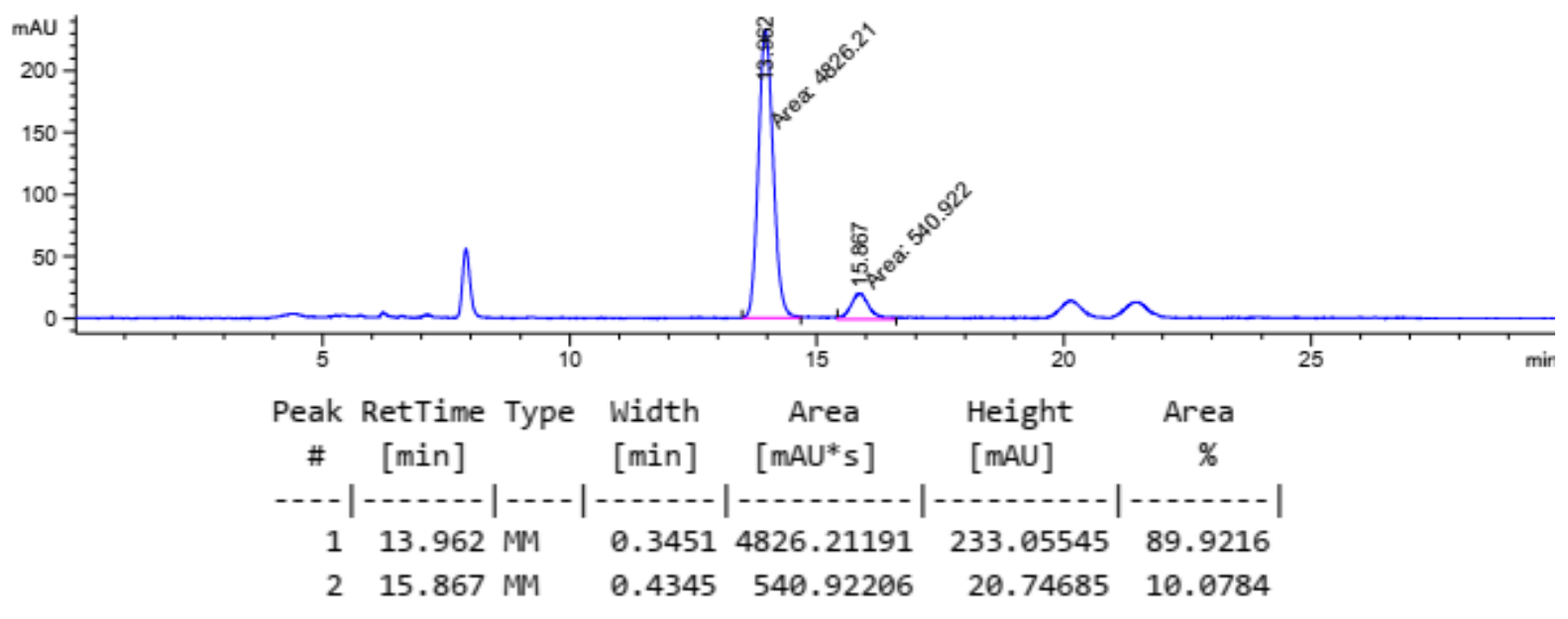

$(2 R, 3 R)$-3-Methyl-3,4-diphenylbutan-2-ol [ $(2 R, 3 R)-1 \mathrm{a}]$ and

(2S,3S)-(3,5-Dimethylphenyl)dimethyl((3-methyl-3,4-diphenylbutan-2-

yl)oxy)silane [(2S,3S)-3ae] - Temperature: $0^{\circ} \mathrm{C}$

Figure $7.42(2 R, 3 R)$-3-Methyl-3,4-diphenylbutan-2-ol [(2R,3R)-1a, 83\% ee, Table S2, entry 8].

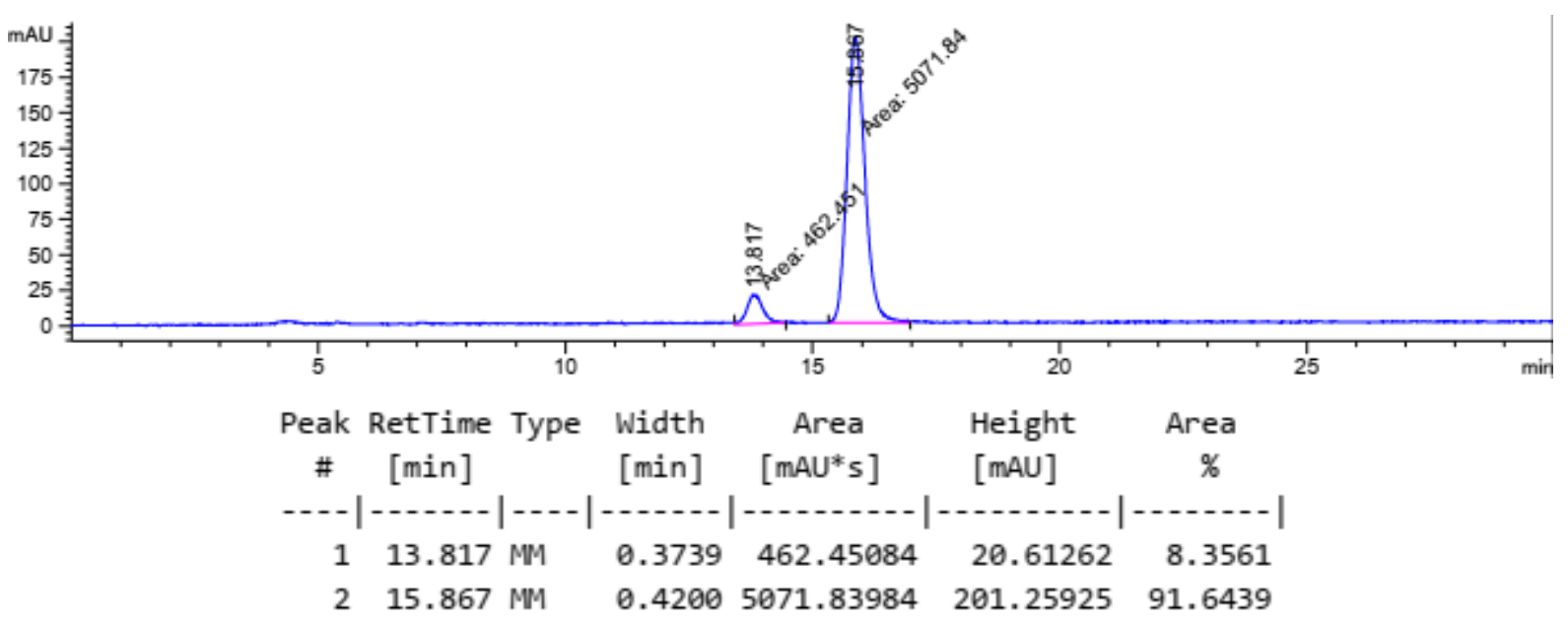


Figure 7.43 (2S,3S)-3-Methyl-3,4-diphenylbutan-2-ol [(2S,3S)-1a, 80\% ee, Table S2, entry 8].

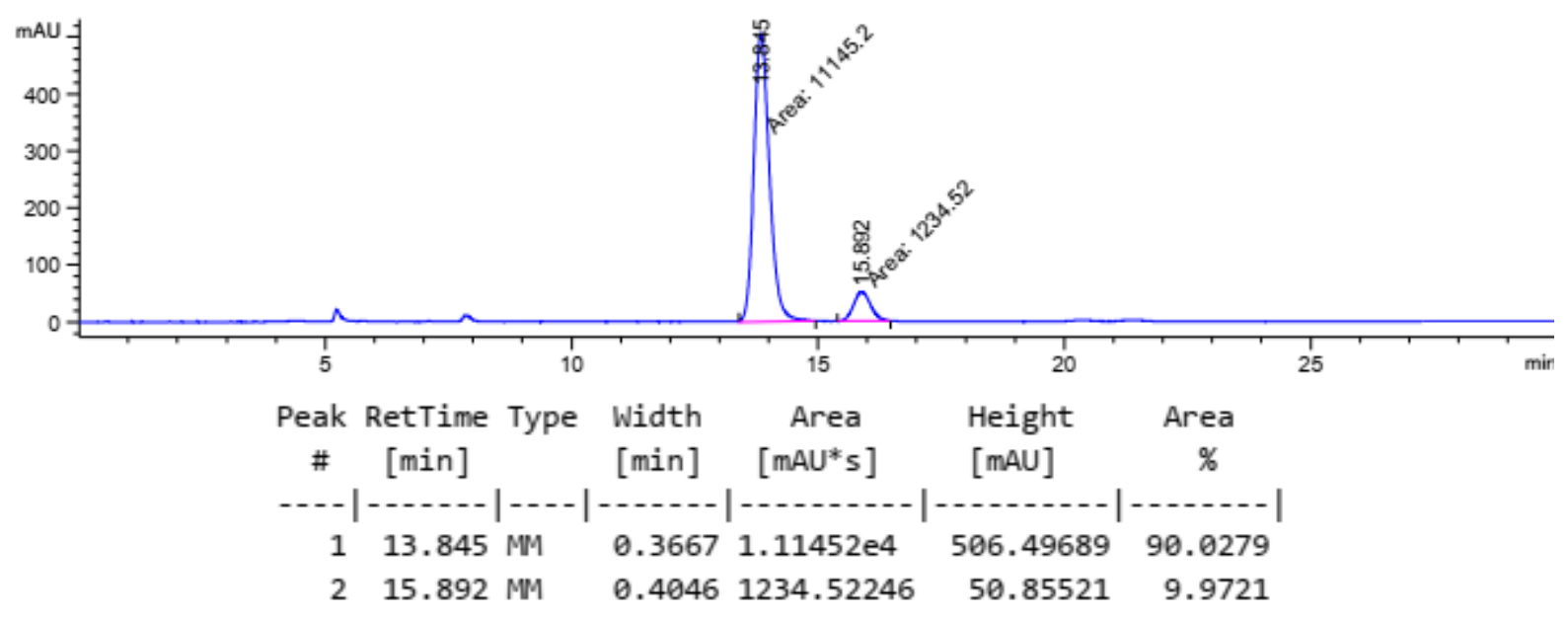

Figure $7.44(2 R, 3 R)$-3-Methyl-3,4-diphenylbutan-2-ol [(2R,3R)-1a, 56\% ee, Table S2, entry 9].

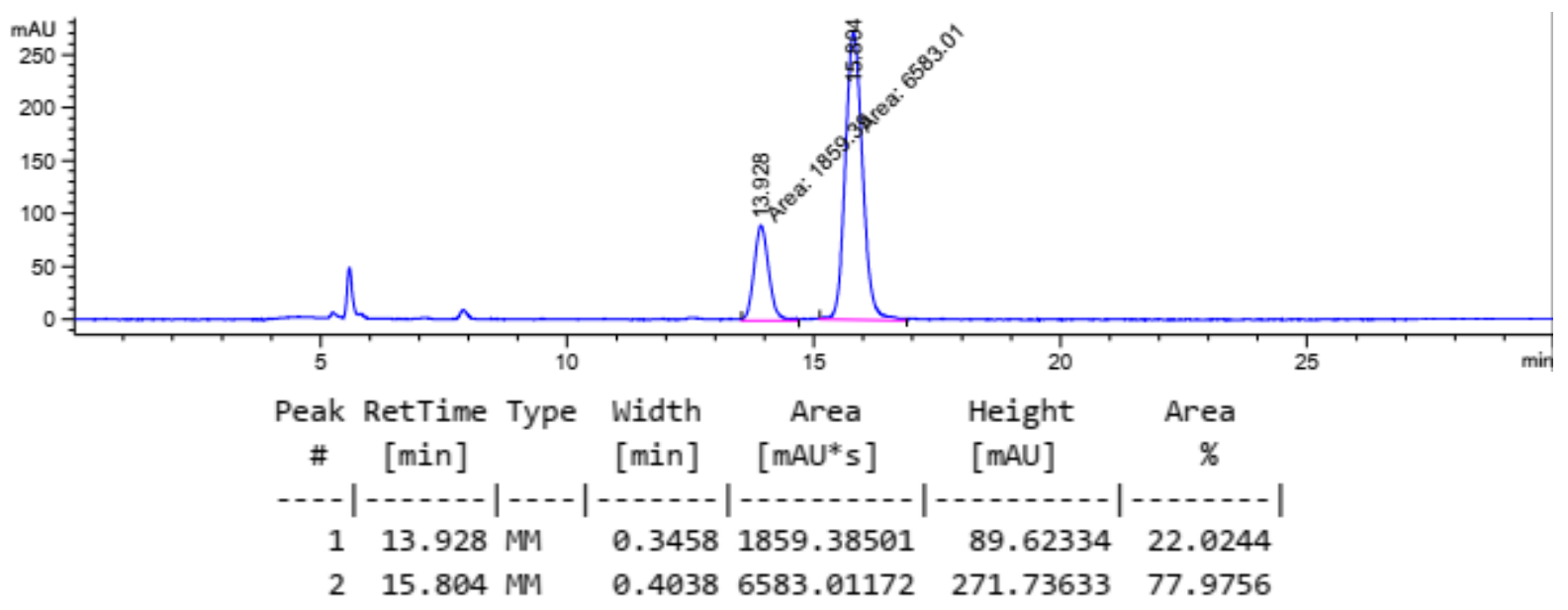

Figure 7.45 (2S,3S)-3-Methyl-3,4-diphenylbutan-2-ol [(2S,3S)-1a, 83\% ee, Table S2, entry 9].

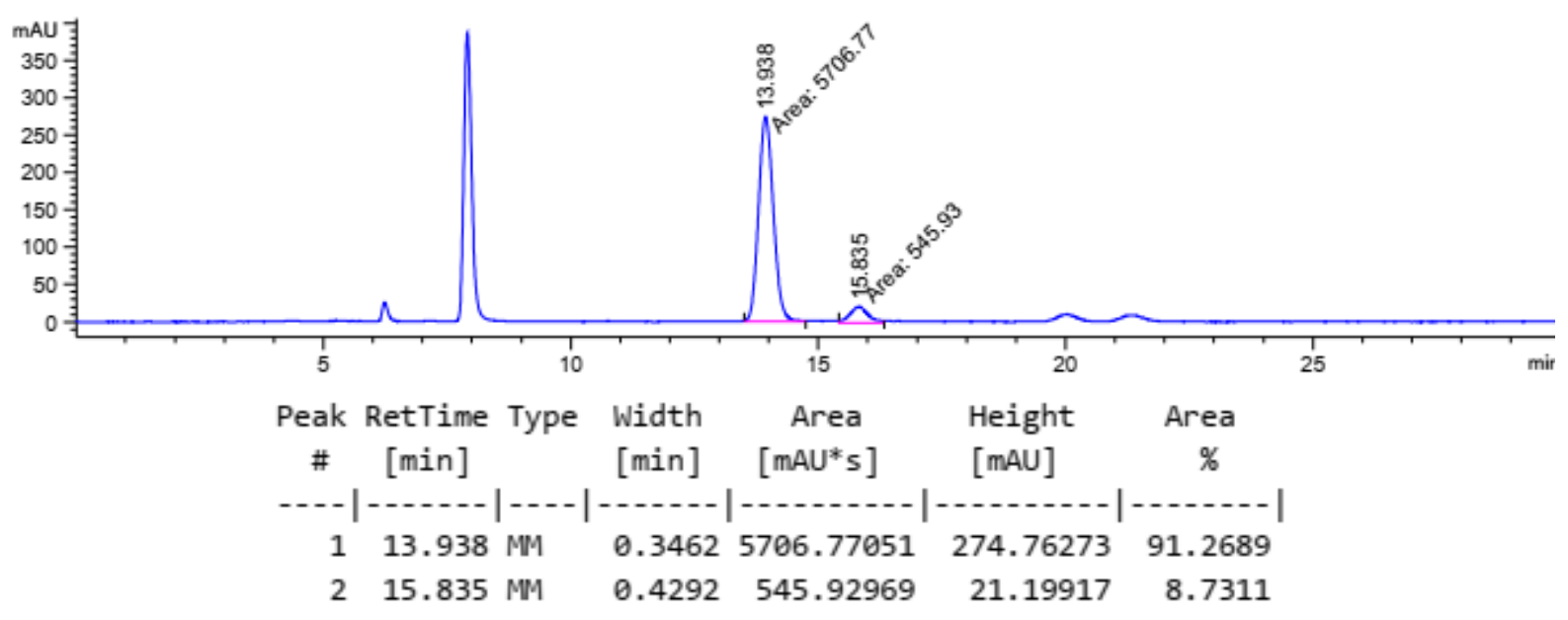


Figure $7.46(2 R, 3 R)$-3-Methyl-3,4-diphenylbutan-2-ol [(2R,3R)-1a, 73\% ee, Table S2, entry 10].

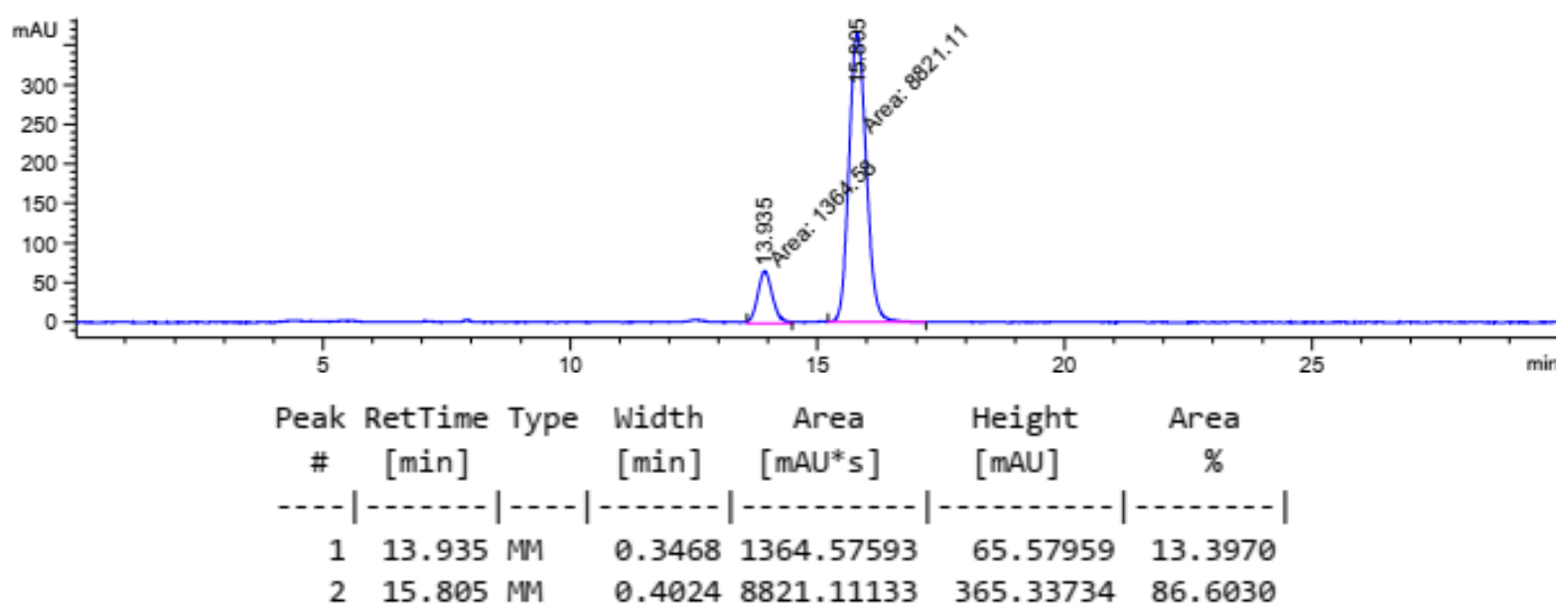

Figure 7.47 (2S,3S)-3-Methyl-3,4-diphenylbutan-2-ol [(2S,3S)-1a, 82\% ee, Table S2, entry 10].

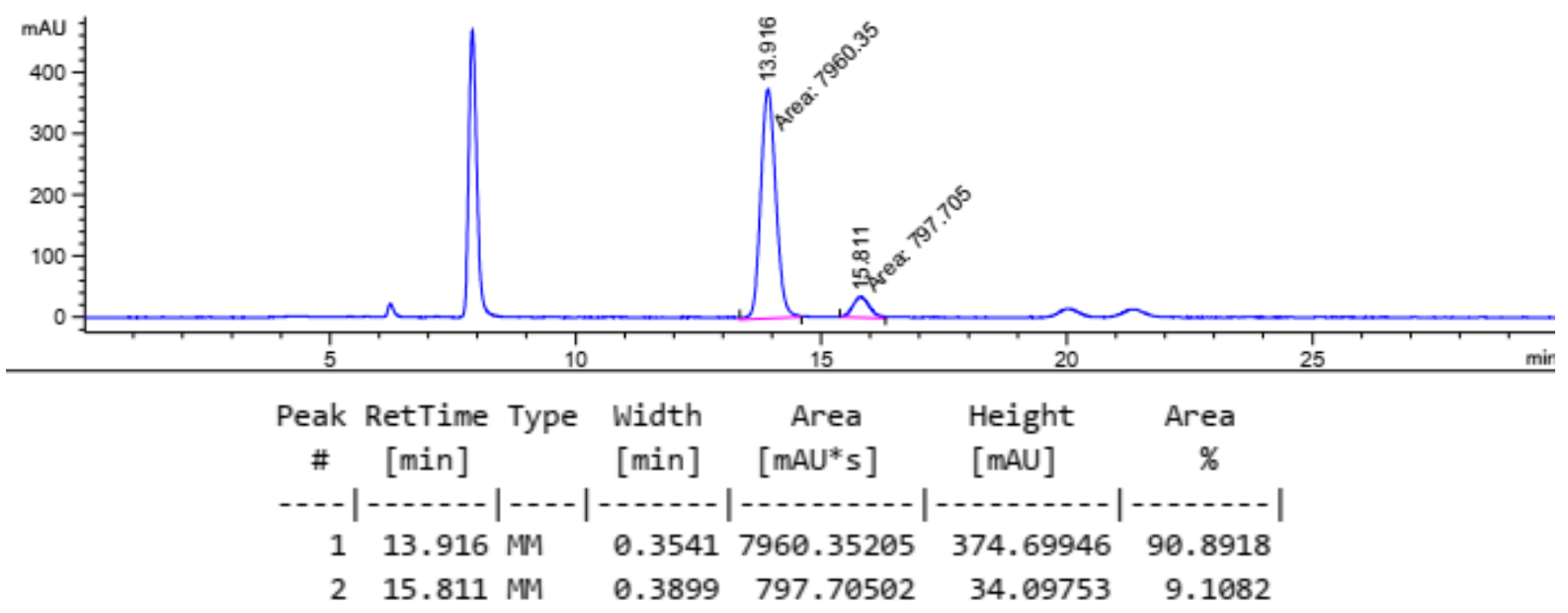


(2R,3R)-3-Methyl-3,4-diphenylbutan-2-ol [(2R,3R)-1a] and

(2S,3S)-(3,5-Dimethylphenyl)dimethyl((3-methyl-3,4-diphenylbutan-2-

yl)oxy)silane [(2S,3S)-3ae] - Temperature: rt

Figure 7.48 (2R,3R)-3-Methyl-3,4-diphenylbutan-2-ol [(2R,3R)-1a, 65\% ee, Table S2, entry 12].

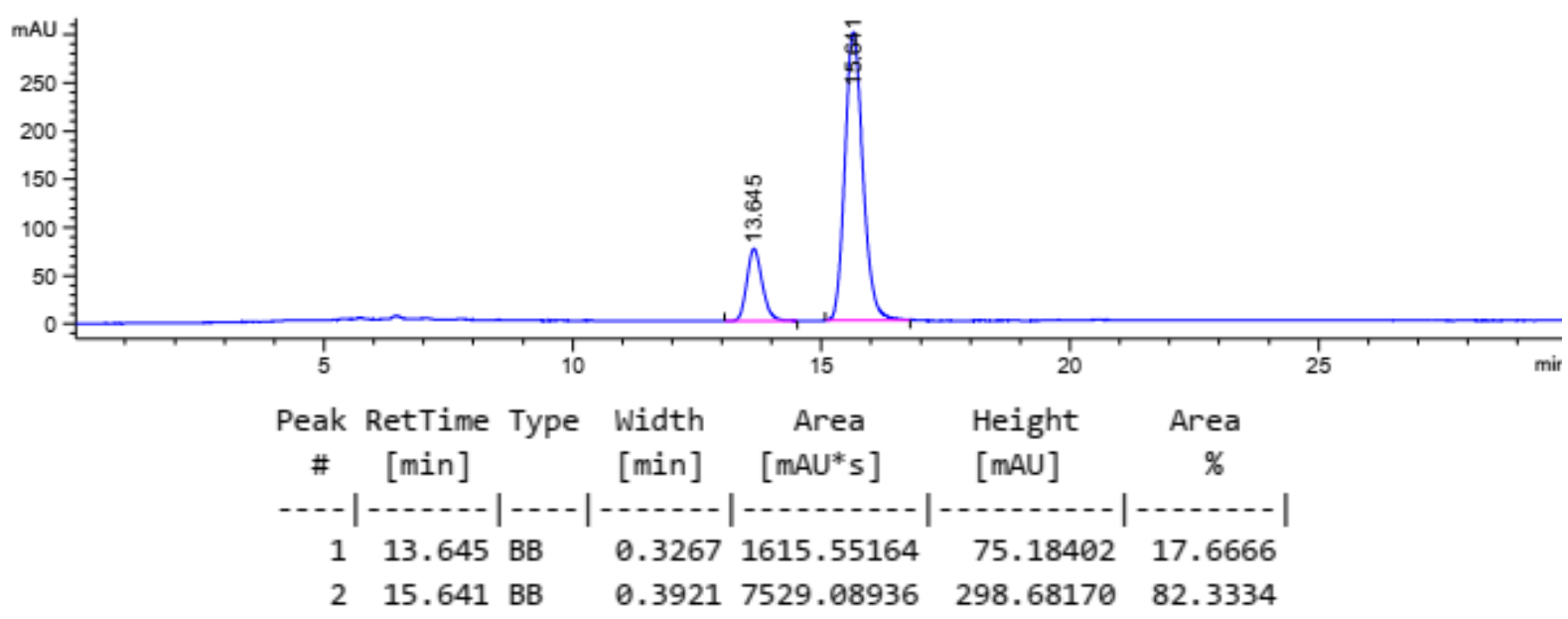

Figure 7.49 (2S,3S)-3-Methyl-3,4-diphenylbutan-2-ol [(2S,3S)-1a, 77\% ee, Table S2, entry 12].

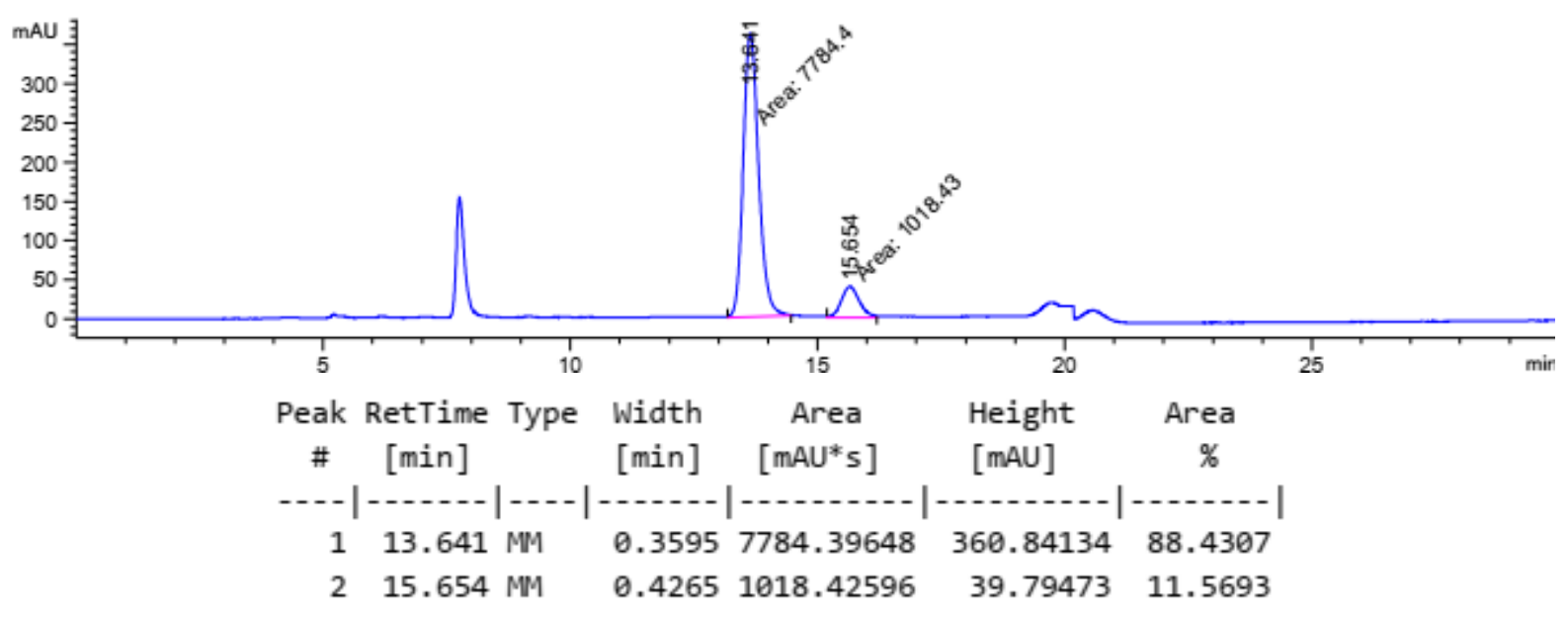


Figure $7.50(2 R, 3 R)$-3-Methyl-3,4-diphenylbutan-2-ol [(2R,3R)-1a, 58\% ee, Table S2, entry 12].

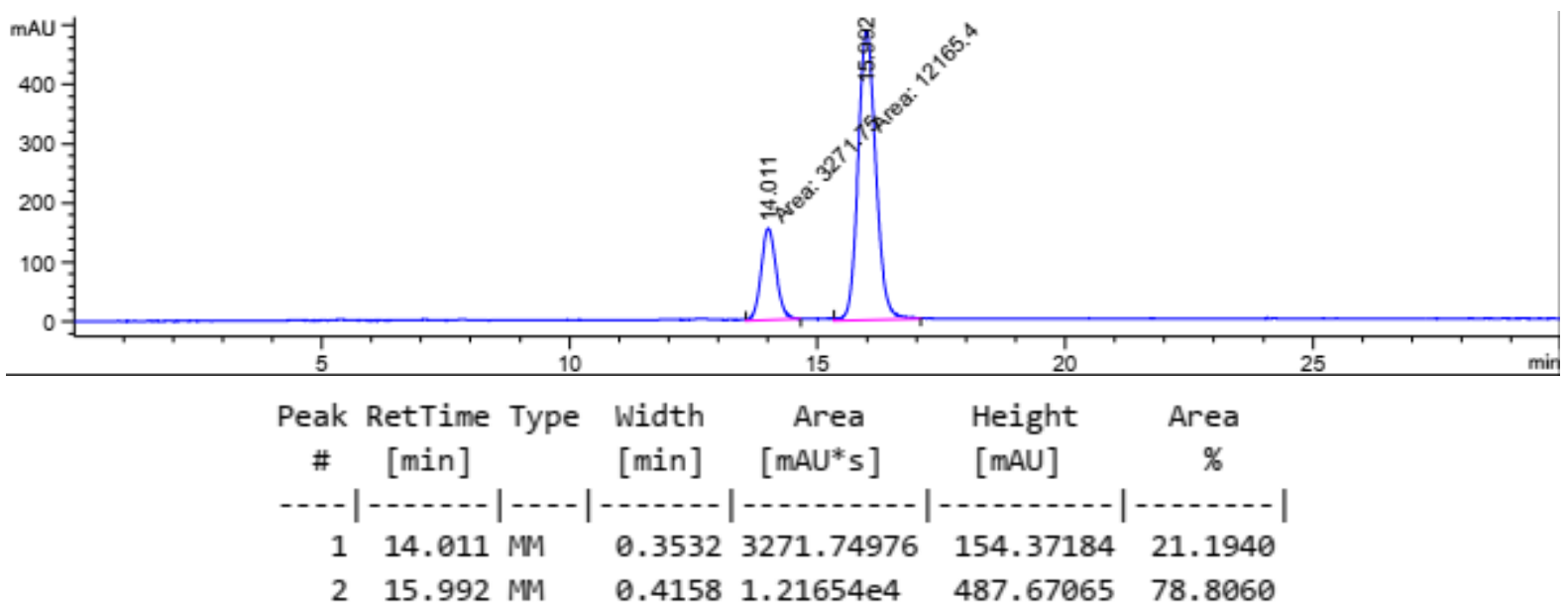

Figure 7.51 (2S,3S)-3-Methyl-3,4-diphenylbutan-2-ol [(2S,3S)-1a, 72\% ee, Table S2, entry 12].

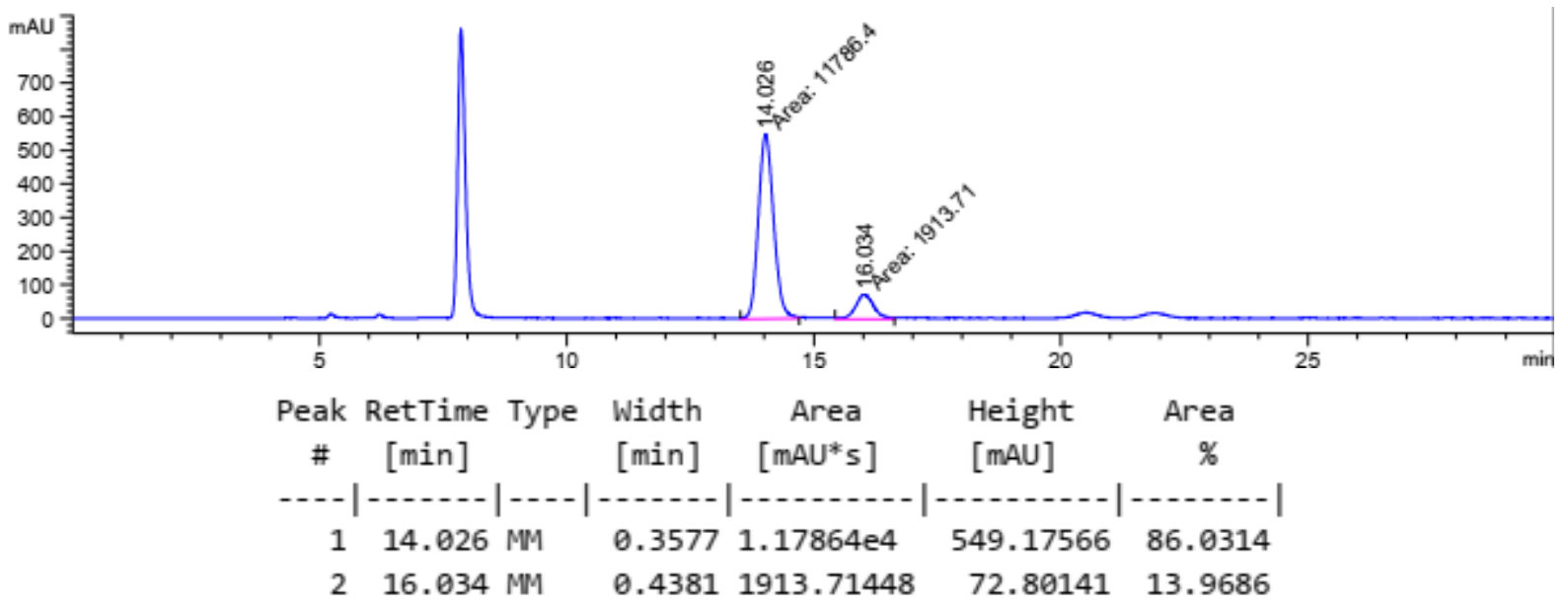




\subsection{Kinetic Resolution}

\section{(2S,3S)-(3,5-Dimethylphenyl)dimethyl((3-methyl-3,4-diphenylbutan-2-}

yl)oxy)silane [(2S,3S)-3ae] and anti/syn-3-Methyl-3,4-diphenylbutan-2-ol [anti/syn-1a]

Figure 7.52 rac-anti/syn-3-Methyl-3,4-diphenylbutan-2-ol [rac-anti/syn-1a].

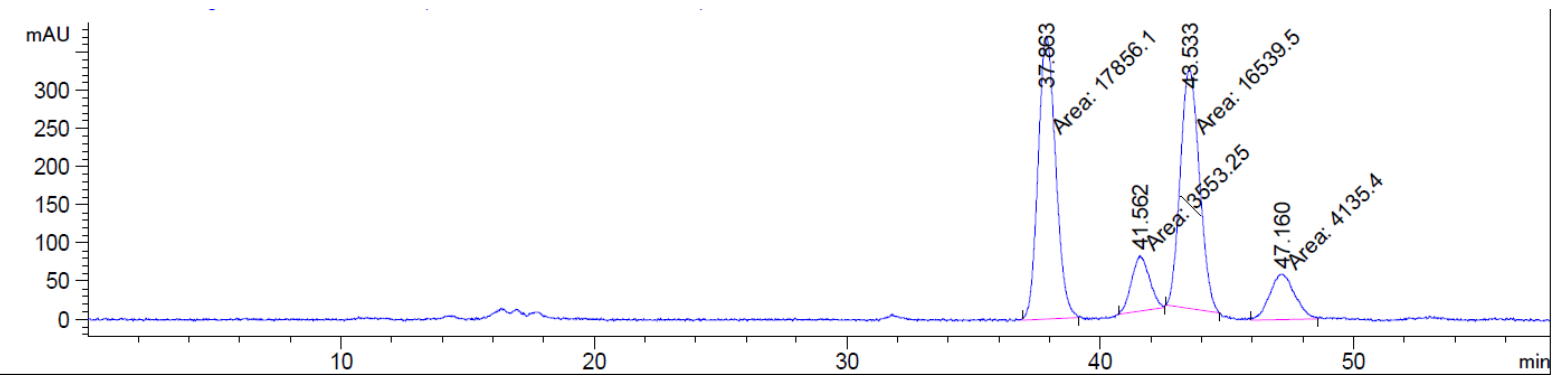

\begin{tabular}{|c|c|c|c|c|c|c|}
\hline $\begin{array}{c}\text { Peak } \\
\#\end{array}$ & $\begin{array}{c}\text { RetTime } \\
\text { [min] }\end{array}$ & Type & $\begin{array}{l}\text { Width } \\
\text { [min] }\end{array}$ & $\begin{array}{c}\text { Area } \\
{\left[\mathrm{mAU}^{*} \mathrm{~s}\right]}\end{array}$ & $\begin{array}{l}\text { Height } \\
{[\mathrm{mAU}]}\end{array}$ & $\begin{array}{c}\text { Area } \\
\%\end{array}$ \\
\hline & & & & -1 & -1 & | \\
\hline 1 & 37.863 & MM & 0.8035 & $1.78561 \mathrm{e} 4$ & 370.39108 & 42.4294 \\
\hline 2 & 41.562 & MM & 0.8140 & 3553.25317 & 72.75130 & 8.4432 \\
\hline 3 & 43.533 & MM & 0.8821 & $1.65395 \mathrm{e} 4$ & 312.50491 & 39.3010 \\
\hline 4 & 47.160 & MM & 1.1495 & 4135.39844 & 59.96147 & 9.8265 \\
\hline
\end{tabular}

Figure 7.53 (2S,3S)-3-Methyl-3,4-diphenylbutan-2-ol [(2S,3S)-1a, 85\% ee].

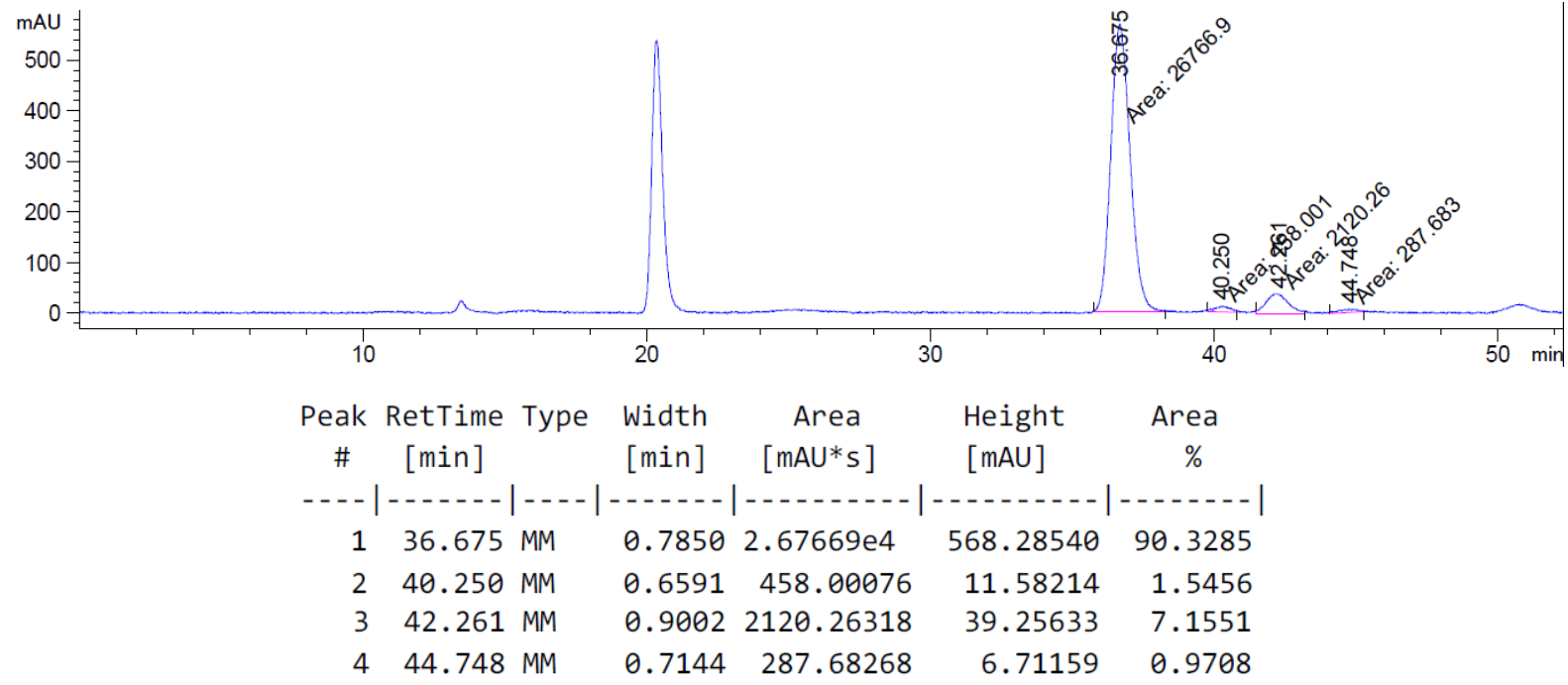


Figure 7.54 anti/syn-3-Methyl-3,4-diphenylbutan-2-ol [anti/syn-1a, 68\% ee for $(2 R, 3 R)-1 \mathrm{a}]$.

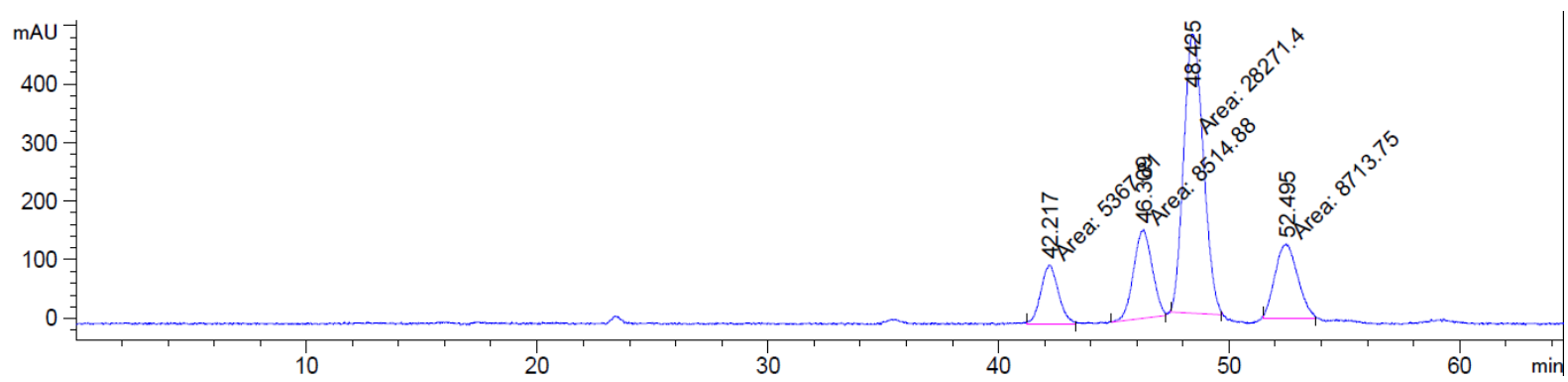

\begin{tabular}{|c|c|c|c|c|c|c|}
\hline $\begin{array}{c}\text { Peak } \\
\#\end{array}$ & $\begin{array}{c}\text { RetTime } \\
\text { [min] }\end{array}$ & Type & $\begin{array}{l}\text { Width } \\
\text { [min] }\end{array}$ & $\begin{array}{c}\text { Area } \\
{\left[\mathrm{mAU}^{*} \mathrm{~s}\right]}\end{array}$ & $\begin{array}{l}\text { Height } \\
{[\mathrm{mAU}]}\end{array}$ & $\begin{array}{c}\text { Area } \\
\%\end{array}$ \\
\hline & & & & & & \\
\hline 1 & 42.217 & MM & 0.8807 & 5367.81006 & 101.57687 & 10.5525 \\
\hline 2 & 46.309 & MM & 0.9398 & 8514.87793 & 151.01294 & 16.7392 \\
\hline 3 & 48.425 & MM & 0.9884 & $2.82714 \mathrm{e} 4$ & 476.73178 & 55.5781 \\
\hline 4 & 52.495 & MM & 1.1426 & 8713.75391 & 127.10323 & 17.1302 \\
\hline
\end{tabular}

(2S,3S)-(3,5-Dimethylphenyl)dimethyl((3-ethyl-3,4-diphenylbutan-2-

yl)oxy)silane [(2S,3S)-3be] and anti/syn-3-Benzyl-3-phenylpentan-2-ol [anti/syn1b]

Figure 7.55 rac-anti/syn-3-Benzyl-3-phenylpentan-2-ol [rac-anti/syn-1b].

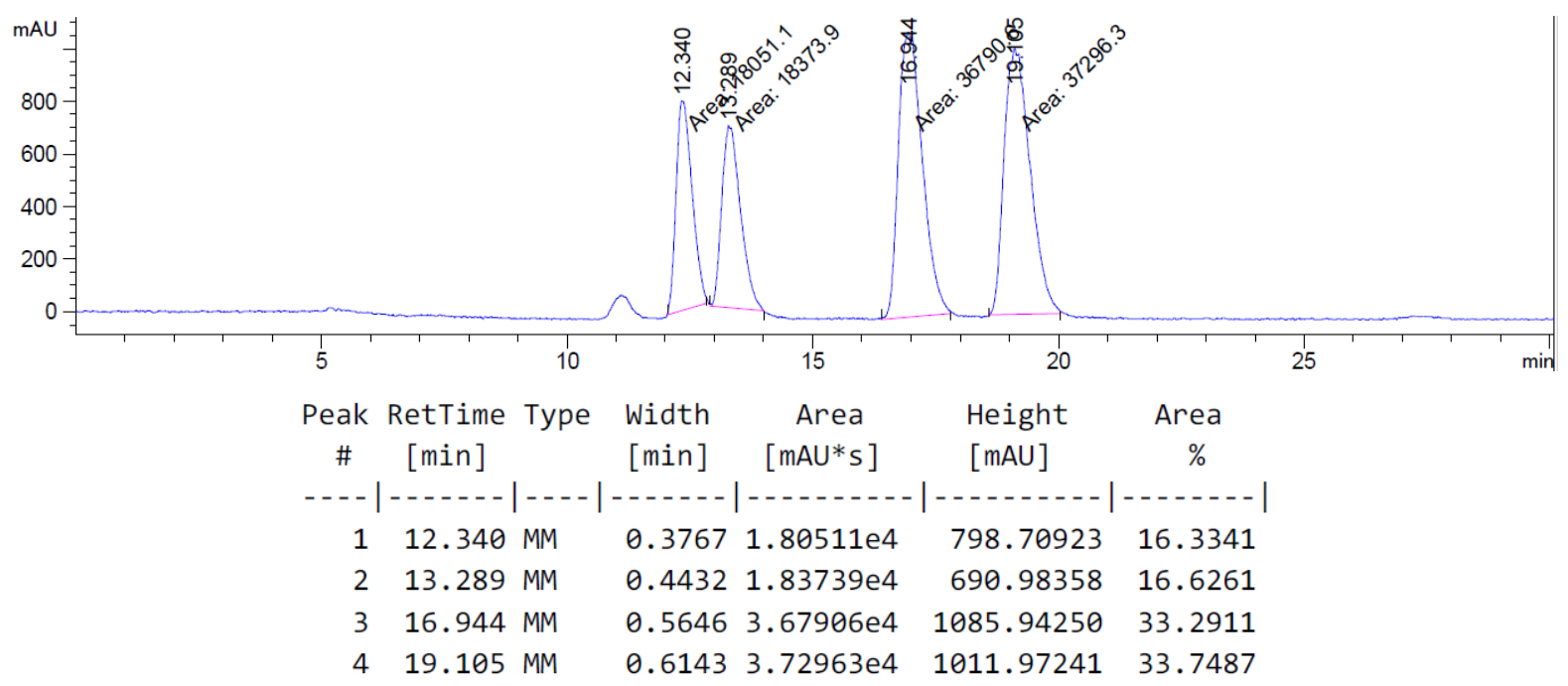


Figure 7.56 (2S,3S)-3-Benzyl-3-phenylpentan-2-ol [(2S,3S)-1b, 79\% ee].

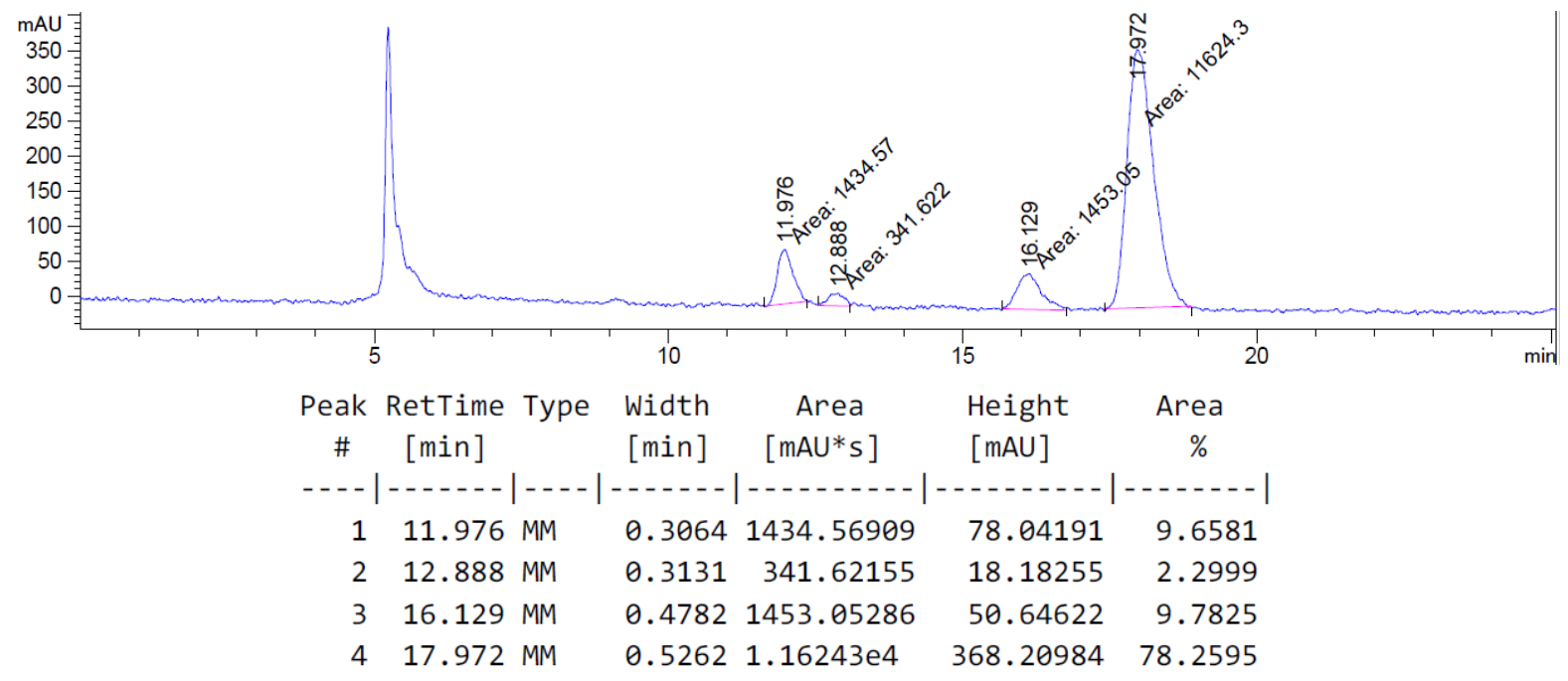

Figure 7.57 anti/syn-3-Benzyl-3-phenylpentan-2-ol [anti/syn-1b, 27\% ee for $(2 R, 3 R)$ 1b].

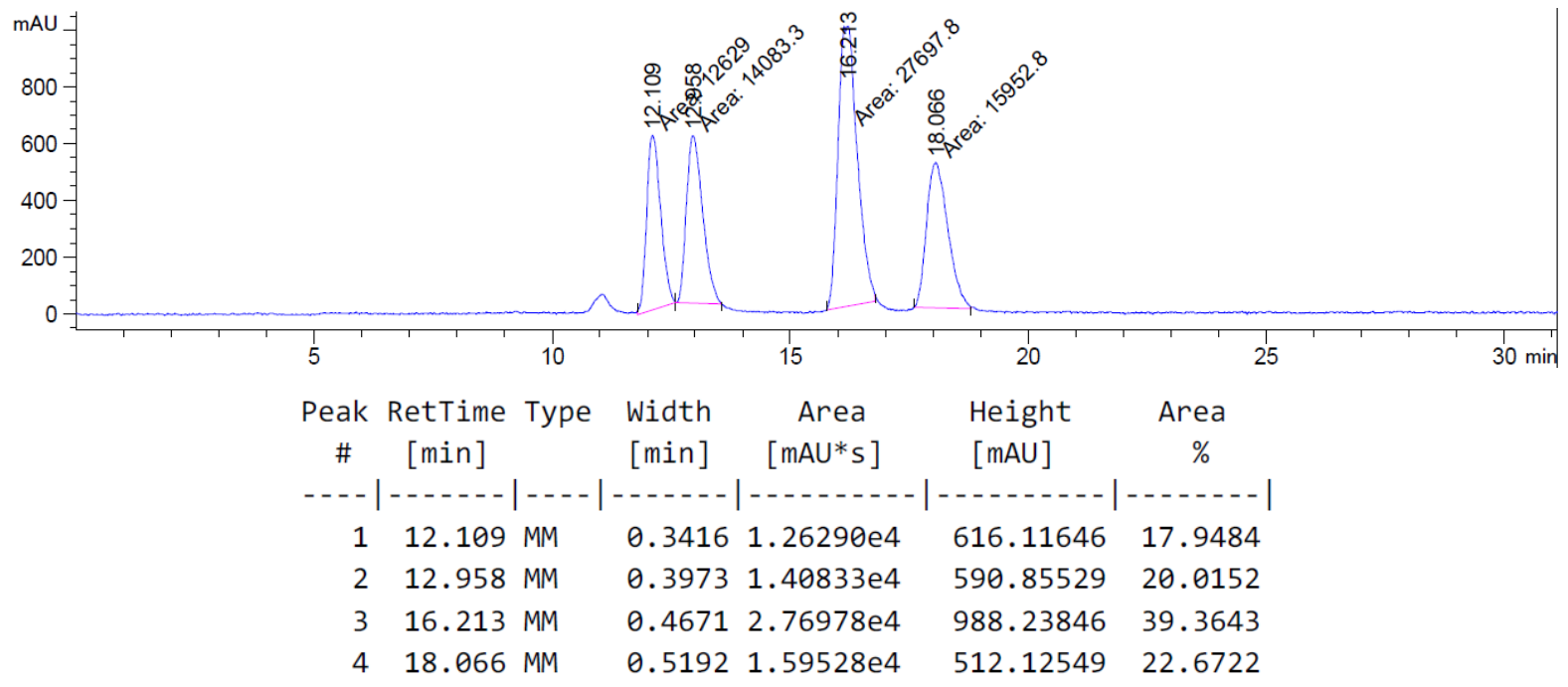




\section{(2S,3S)-(3,5-Dimethylphenyl)((4-(4-fluorophenyl)-3-methyl-3-phenylbutan-2-} yl)oxy)dimethylsilane [(2S,3S)-3de] and anti/syn-4-(4-Fluorophenyl)-3-methyl-3phenylbutan-2-ol [anti/syn-1d]

Figure 7.58 rac-anti/syn-4-(4-Fluorophenyl)-3-methyl-3-phenylbutan-2-ol [racanti/syn 1d].

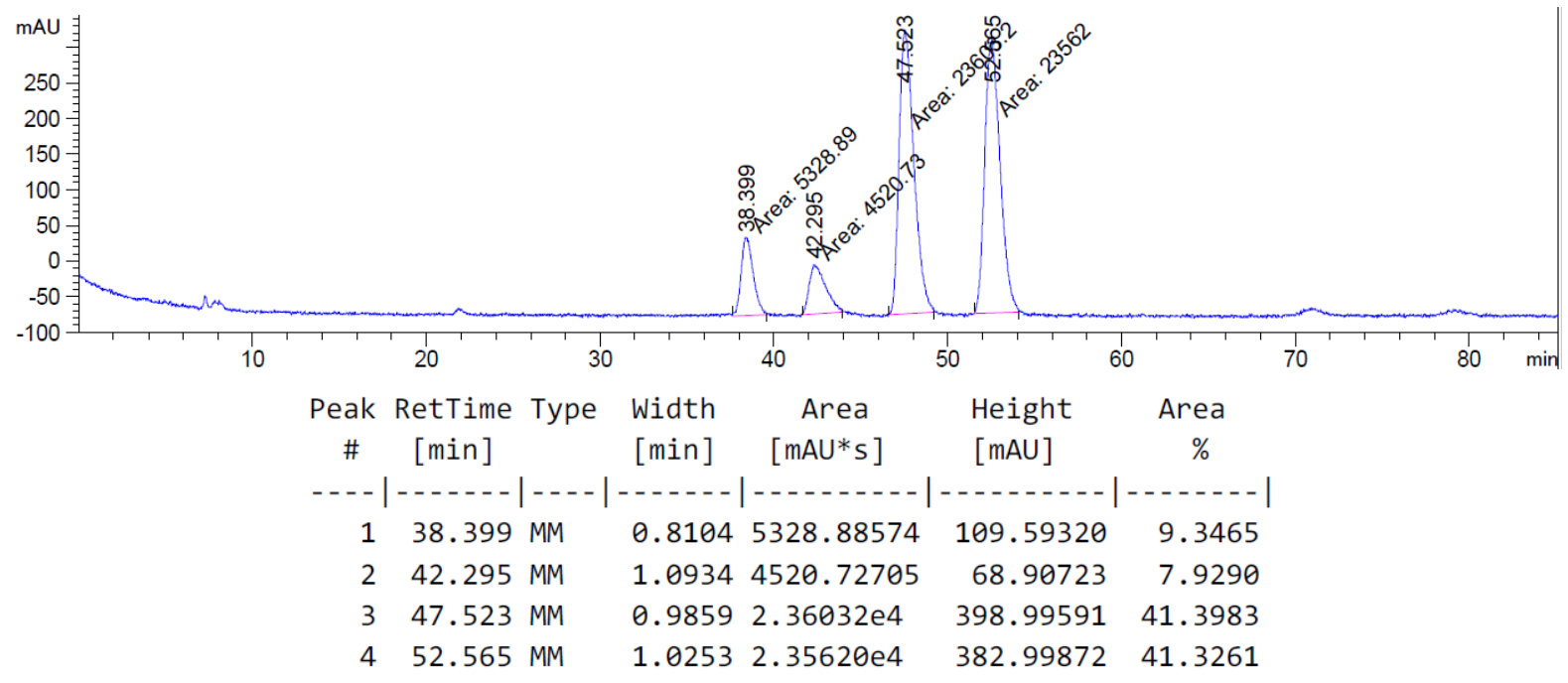

Figure 7.59 (2S,3S)-4-(4-Fluorophenyl)-3-methyl-3-phenylbutan-2-ol [(2S,3S)-1d, $88 \%$ ee].

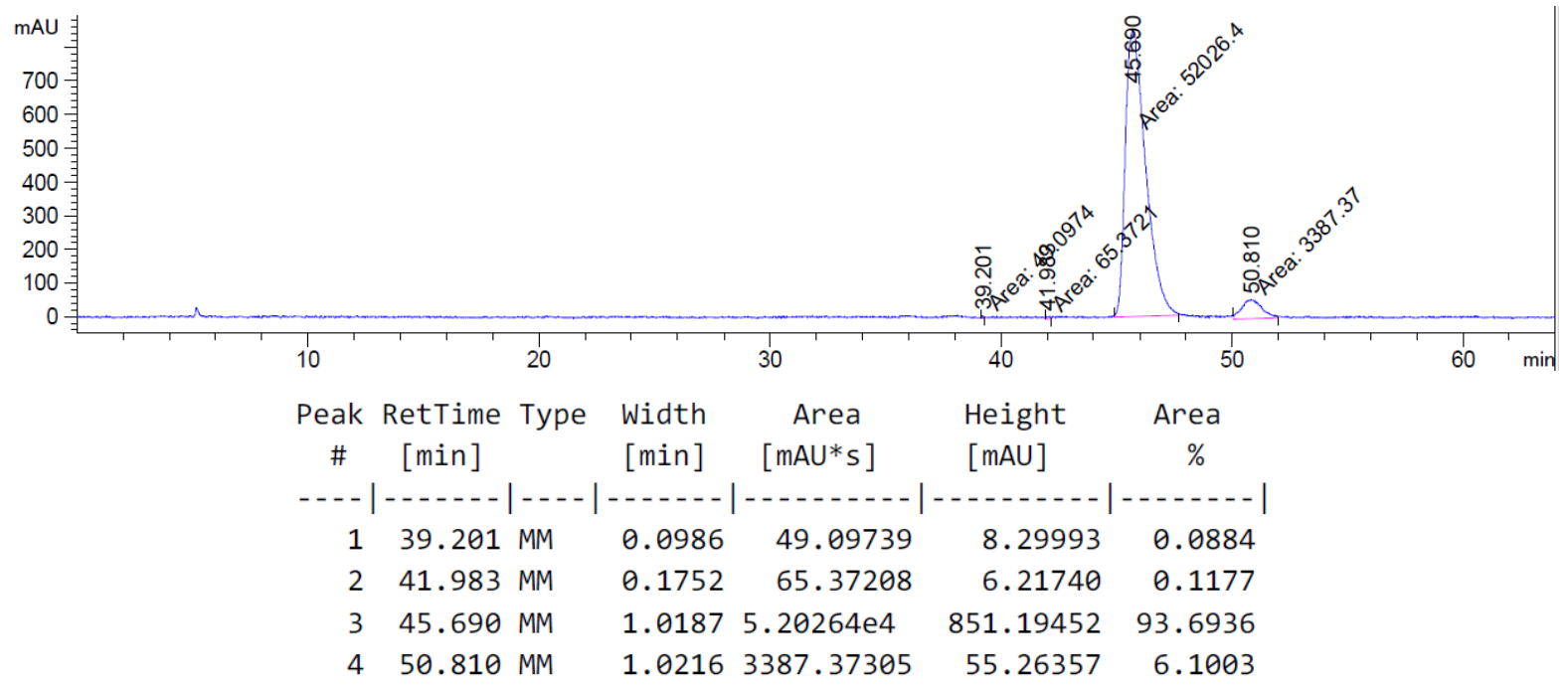


Figure 7.60 anti/syn-4-(4-Fluorophenyl)-3-methyl-3-phenylbutan-2-ol [anti/syn-1d, $43 \%$ ee for $(2 R, 3 R)-1 \mathrm{~d}]$.

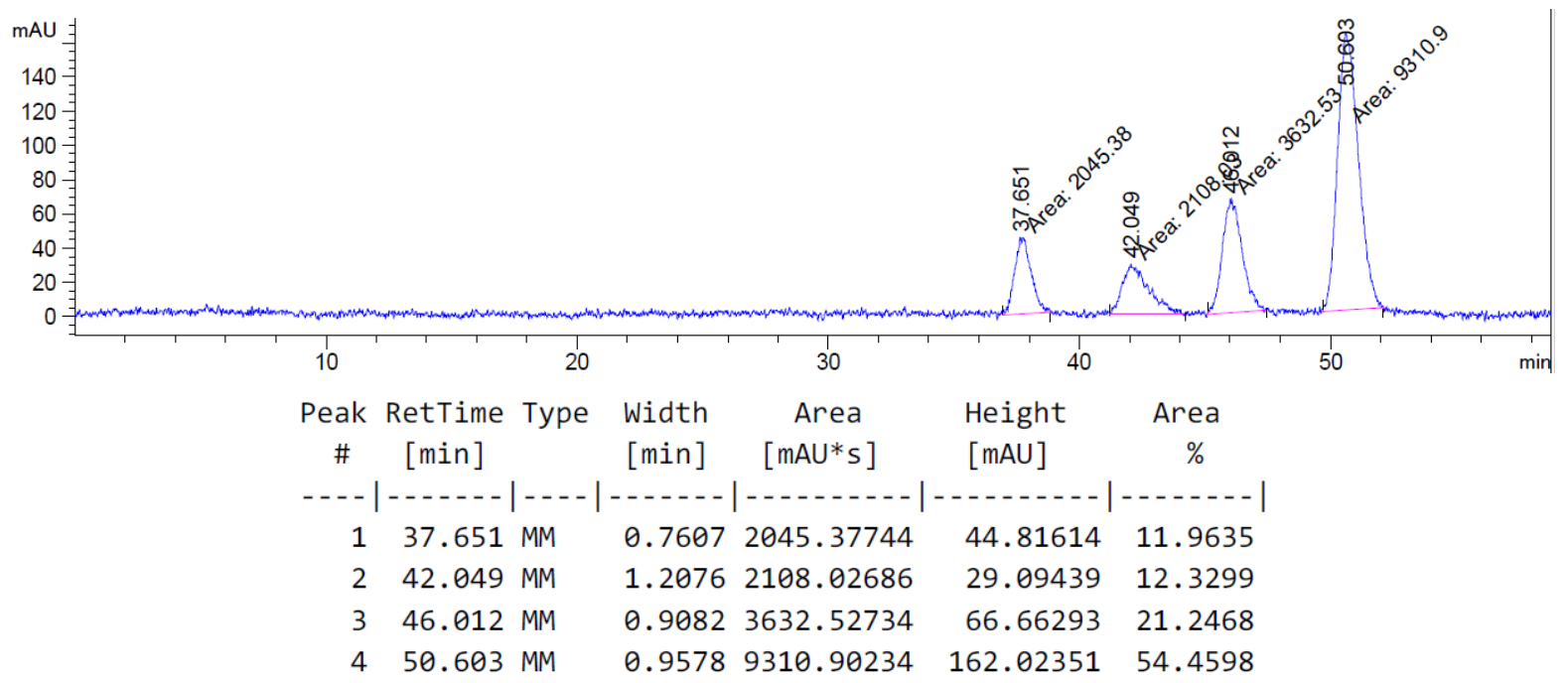

(2S,3S)-(3,5-Dimethylphenyl)dimethyl((3-methyl-3-phenyl-4-( $p$-tolyl)butan-2yl)oxy)silane [(2S,3S)-3ie] and anti/syn-3-Methyl-3-phenyl-4-(p-tolyl)butan-2-ol [anti/syn-1i]

Figure 7.61 rac-anti/syn-3-Methyl-3-phenyl-4-(p-tolyl)butan-2-ol [rac-anti/syn-1i].

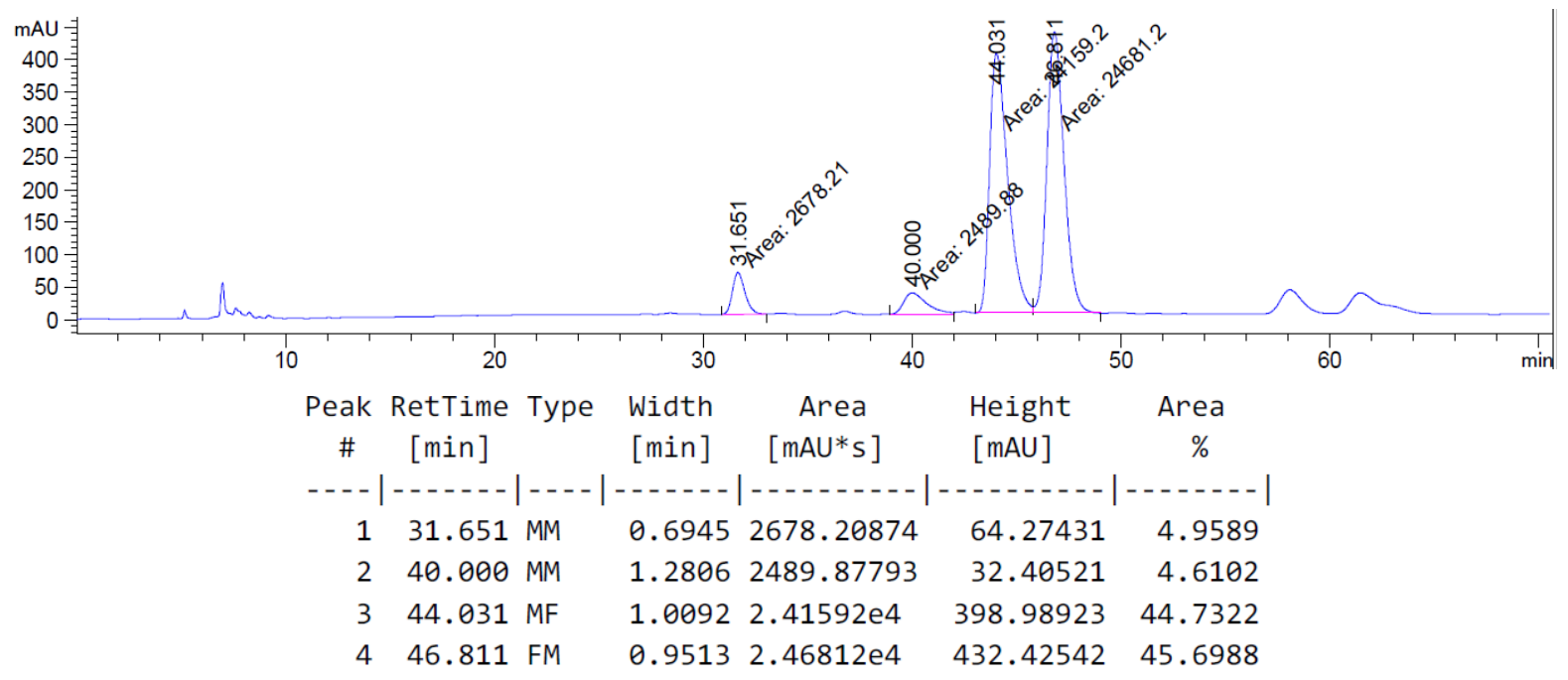


Figure 7.62 (2S,3S)-3-Methyl-3-phenyl-4-(p-tolyl)butan-2-ol [(2S,3S)-1i, 90\% ee].

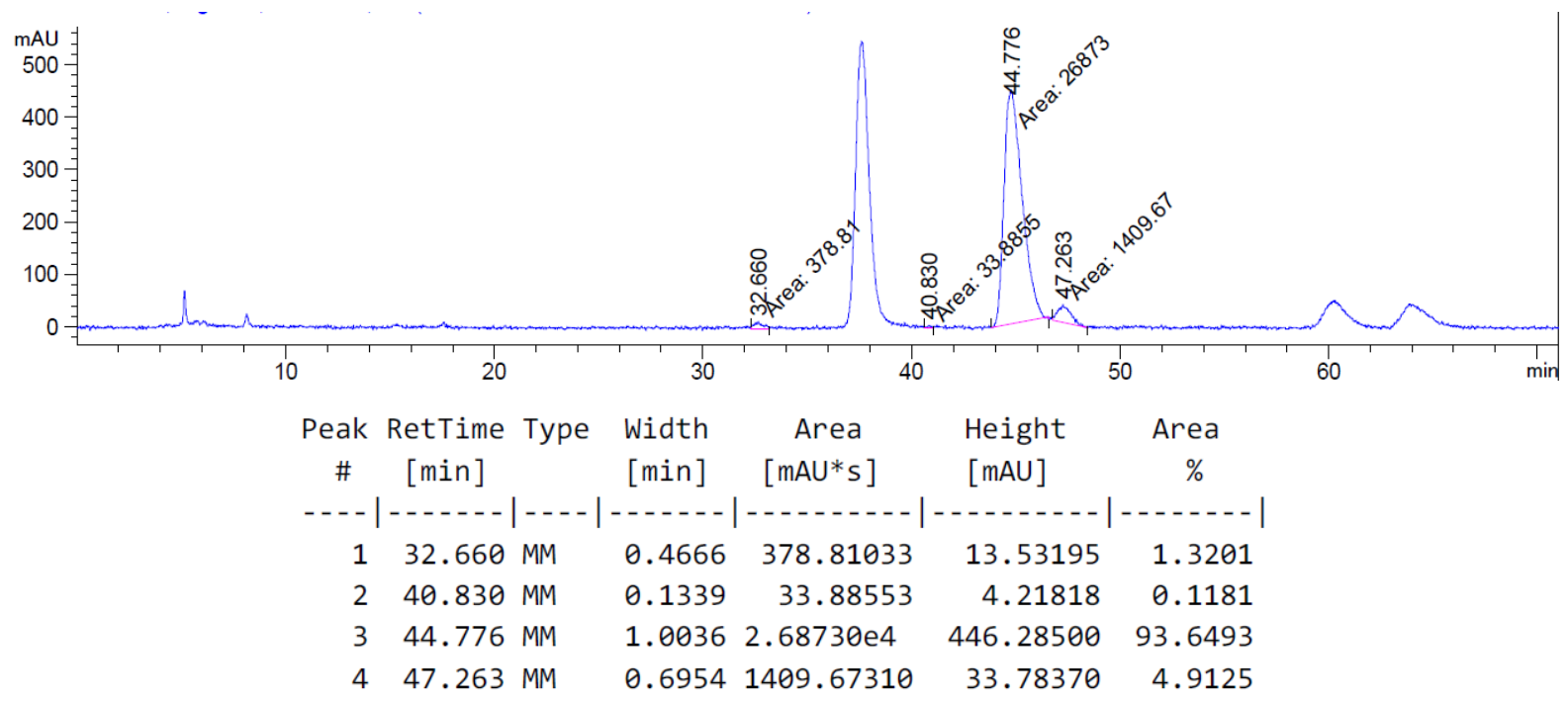

Figure 7.63 anti/syn-3-Methyl-3-phenyl-4-(p-tolyl)butan-2-ol [anti/syn-1i, 60\% ee for $(2 R, 3 R)-1 \mathrm{i}]$.

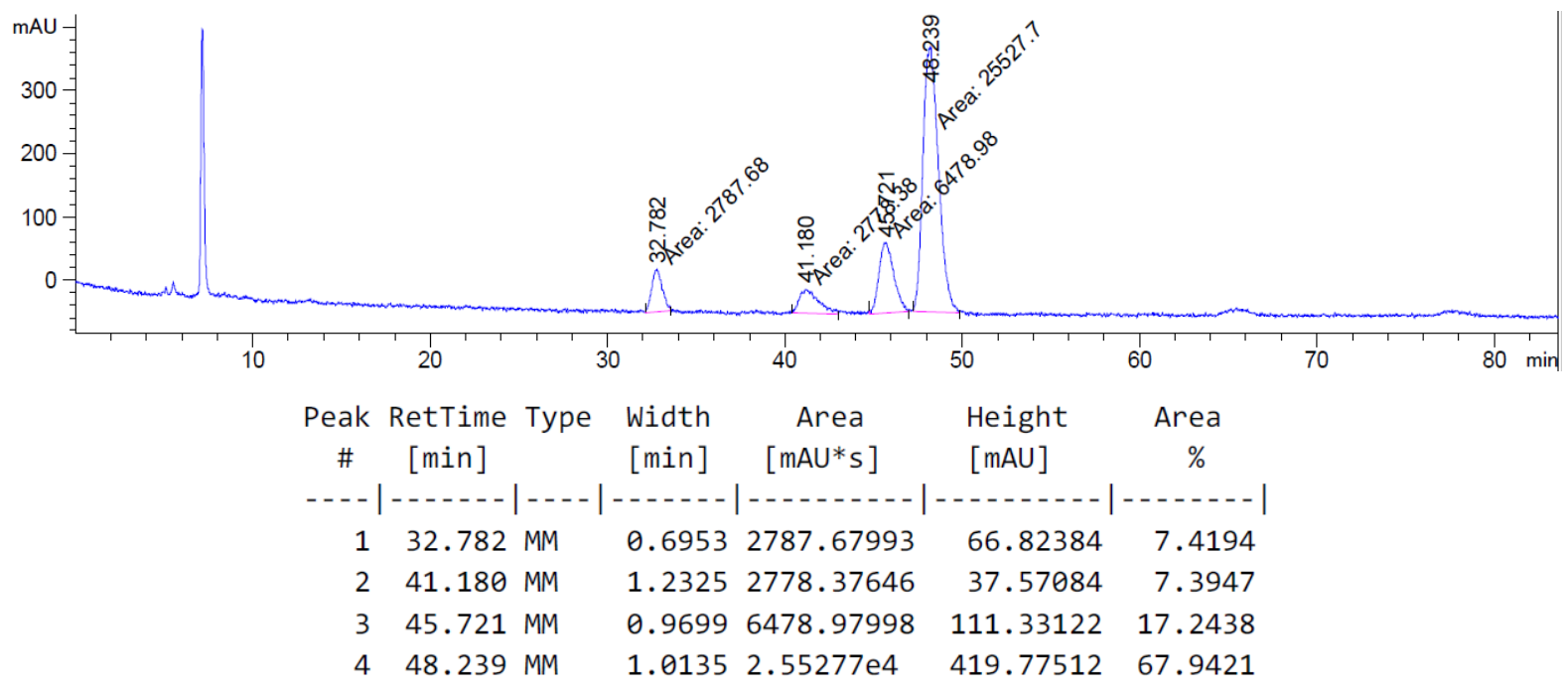


(2S,3S)-(3,5-Dimethylphenyl)((4-(3,5-dimethylphenyl)-3-methyl-3-phenylbutan2-yl)oxy)dimethylsilane [(2S,3S)-3je] and anti/syn-4-(3,5-Dimethylphenyl)-3methyl-3-phenylbutan-2-ol [anti/syn-1j]

Figure 7.64 rac-anti/syn-4-(3,5-Dimethylphenyl)-3-methyl-3-phenylbutan-2-ol

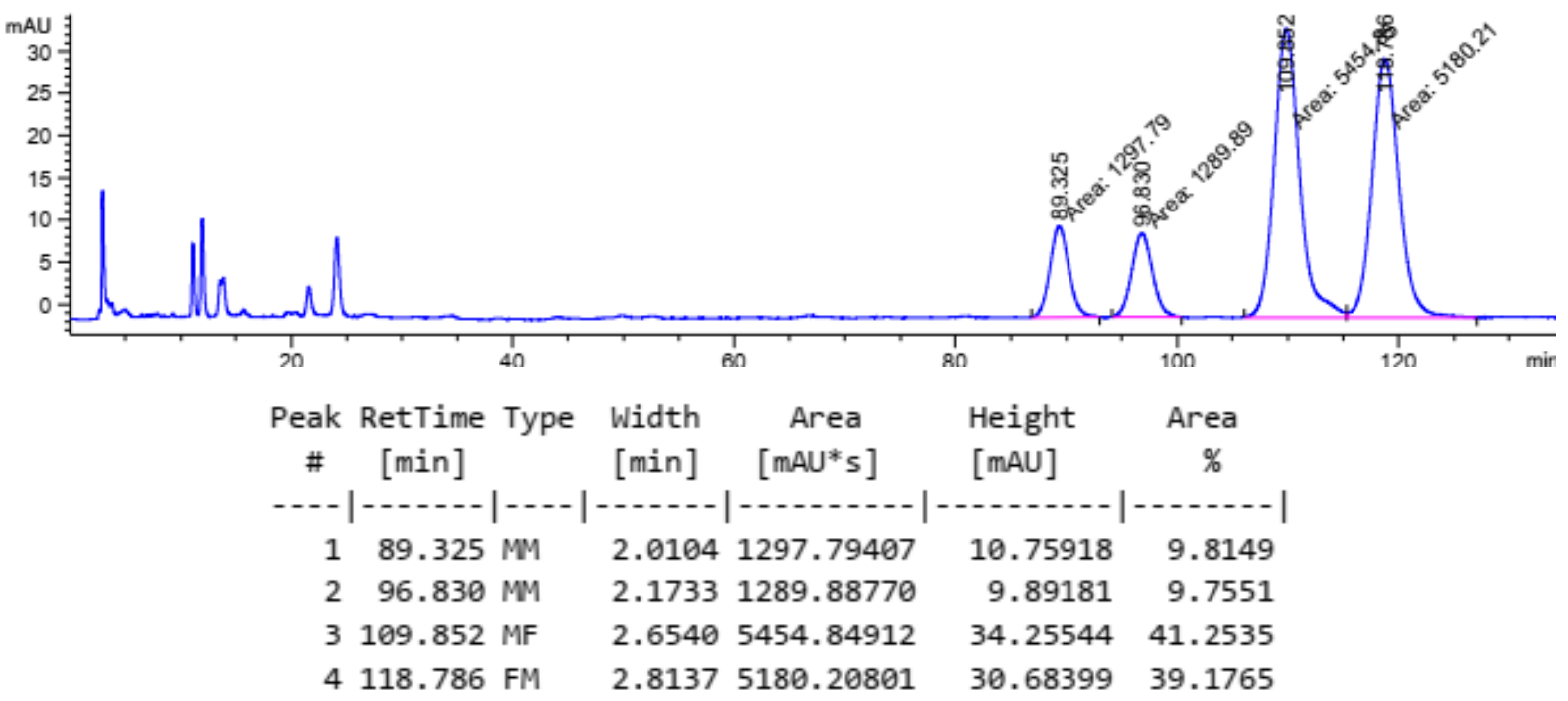

Figure 7.65 (2S,3S)-4-(3,5-Dimethylphenyl)-3-methyl-3-phenylbutan-2-ol [(2S,3S)-

1j, $43 \%$ ee].

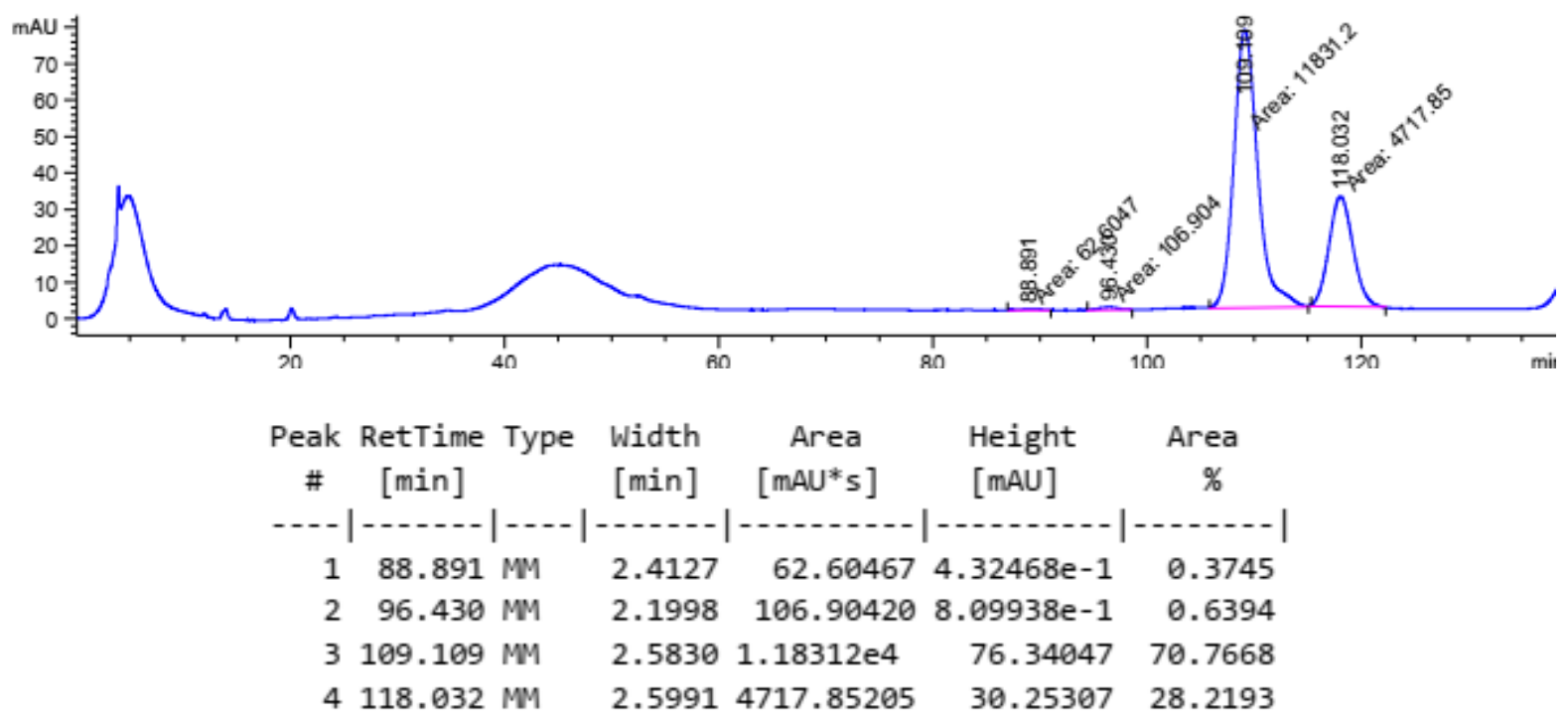


Figure 7.66 anti/syn-4-(3,5-Dimethylphenyl)-3-methyl-3-phenylbutan-2-ol [anti/syn$1 \mathbf{i}, 96 \%$ ee for $(2 R, 3 R)-\mathbf{1 i}]$.

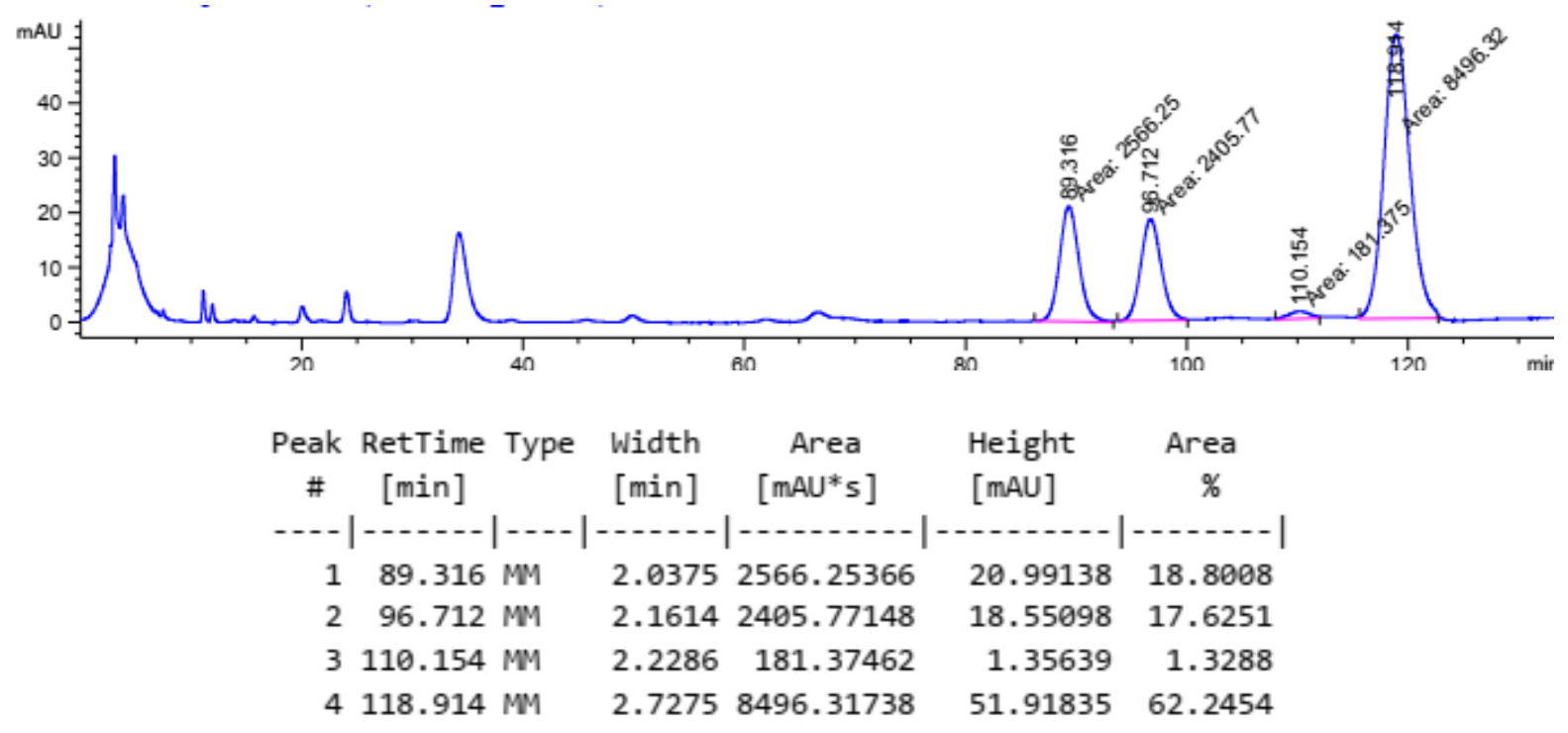

(2S,3S)-((4-(3,5-Dimethoxyphenyl)-3-methyl-3-phenylbutan-2-yl)oxy)(3,5dimethylphenyl)dimethylsilane [(2S,3S)-3le] and anti/syn-4-(3,5Dimethoxyphenyl)-3-methyl-3-phenylbutan-2-ol [anti/syn-1I]

Figure 7.67 rac-anti/syn-4-(3,5-Dimethoxyphenyl)-3-methyl-3-phenylbutan-2-ol [racanti/syn-11].

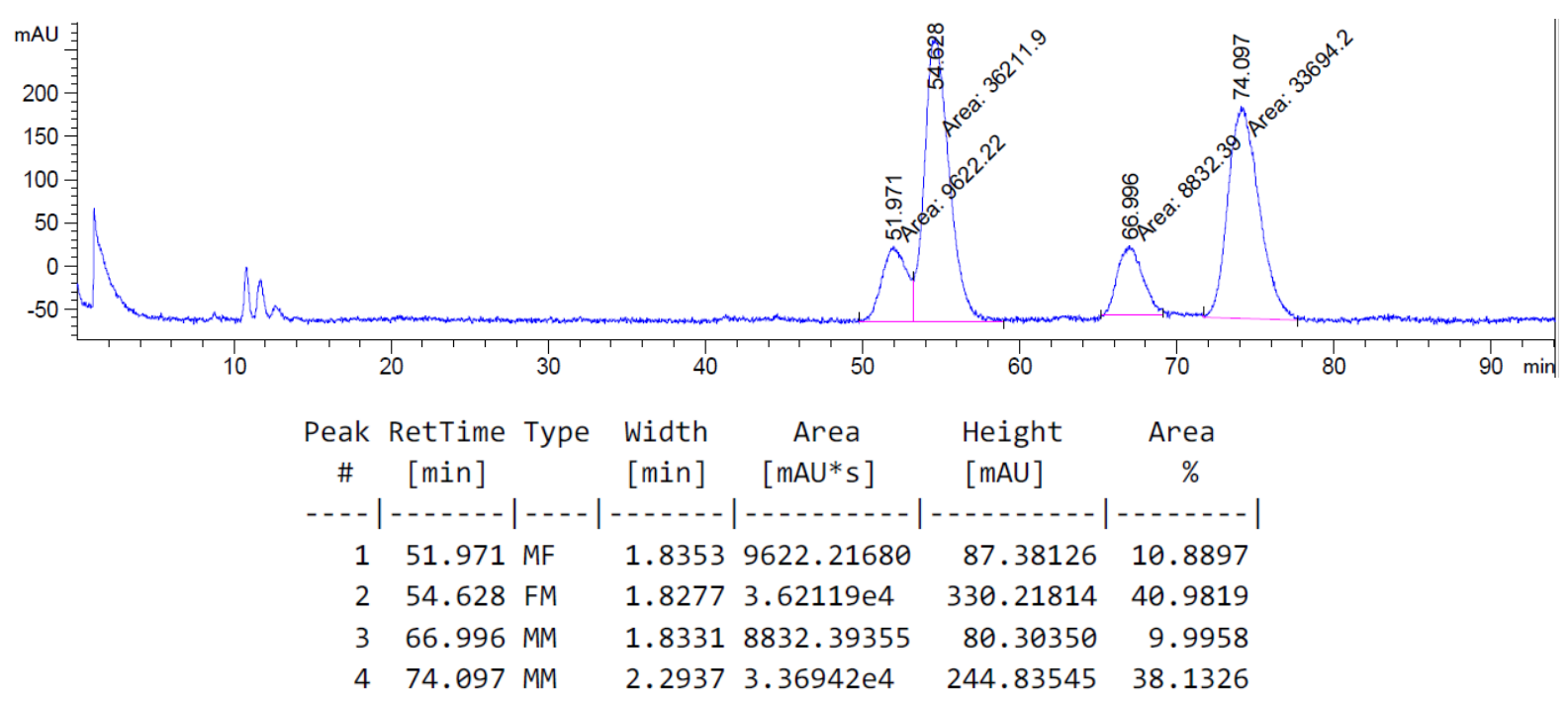


Figure 7.68 (2S,3S)-4-(3,5-Dimethoxyphenyl)-3-methyl-3-phenylbutan-2-ol [(2S,3S)$11,86 \%$ ee].

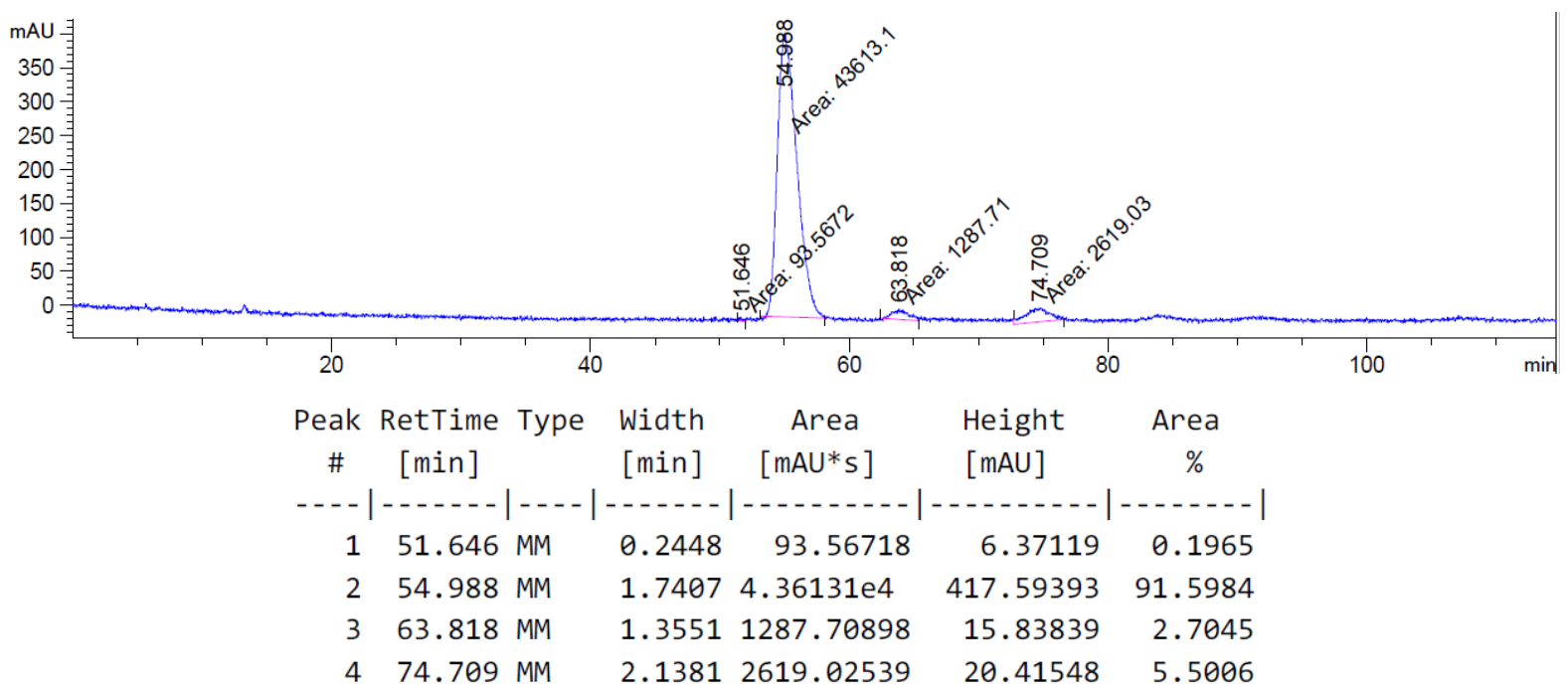

Figure 7.69 anti/syn-4-(3,5-Dimethoxyphenyl)-3-methyl-3-phenylbutan-2-ol [anti/syn$11,52 \%$ ee for $(2 R, 3 R)-11]$.

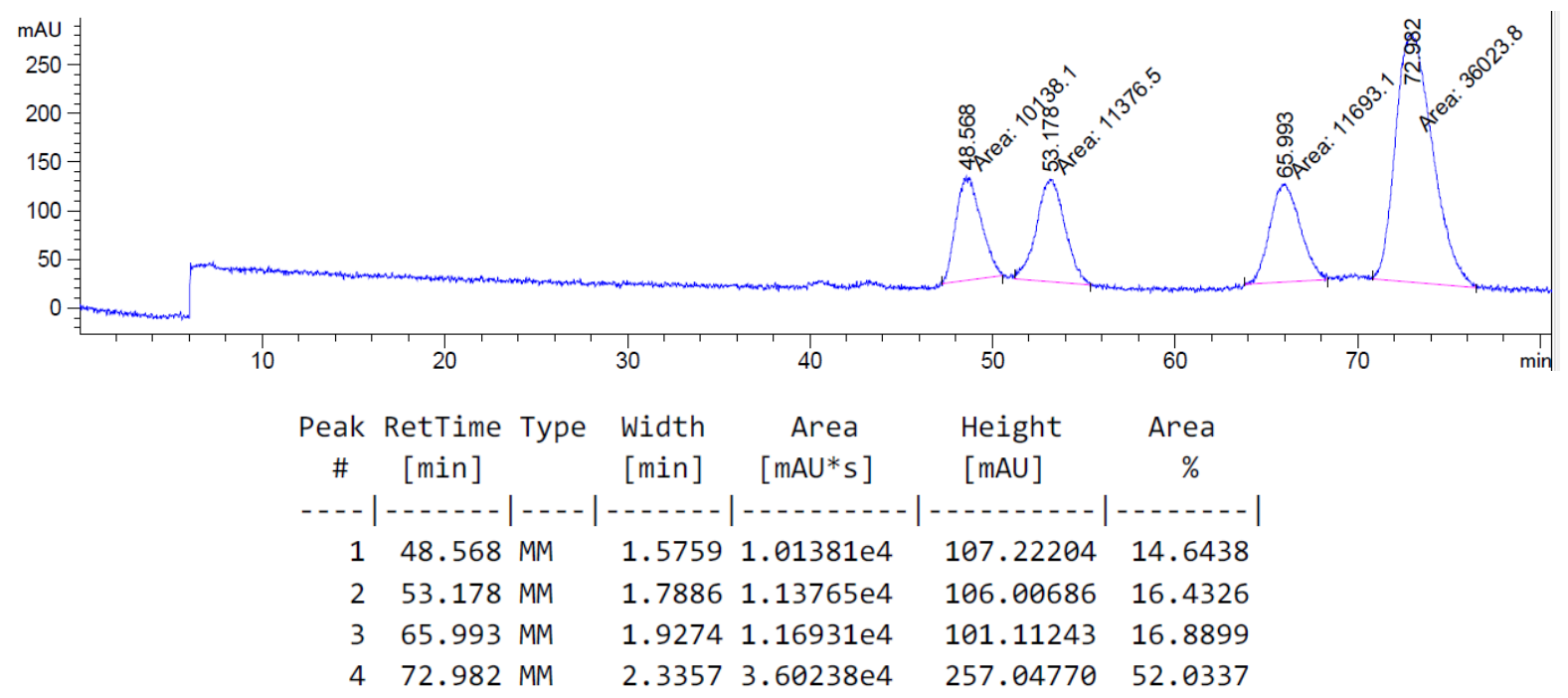


(2S,3S)-((4-(4-Bromophenyl)-3-methyl-3-phenylbutan-2-yl)oxy)(3,5-

dimethylphenyl)dimethylsilane [(2S,3S)-3fe] and anti/syn-4-(4-Bromophenyl)-3methyl-3-phenylbutan-2-ol [anti/syn-1f]

Figure 7.70 rac-anti/syn-4-(4-Bromophenyl)-3-methyl-3-phenylbutan-2-ol [racanti/syn-1f].

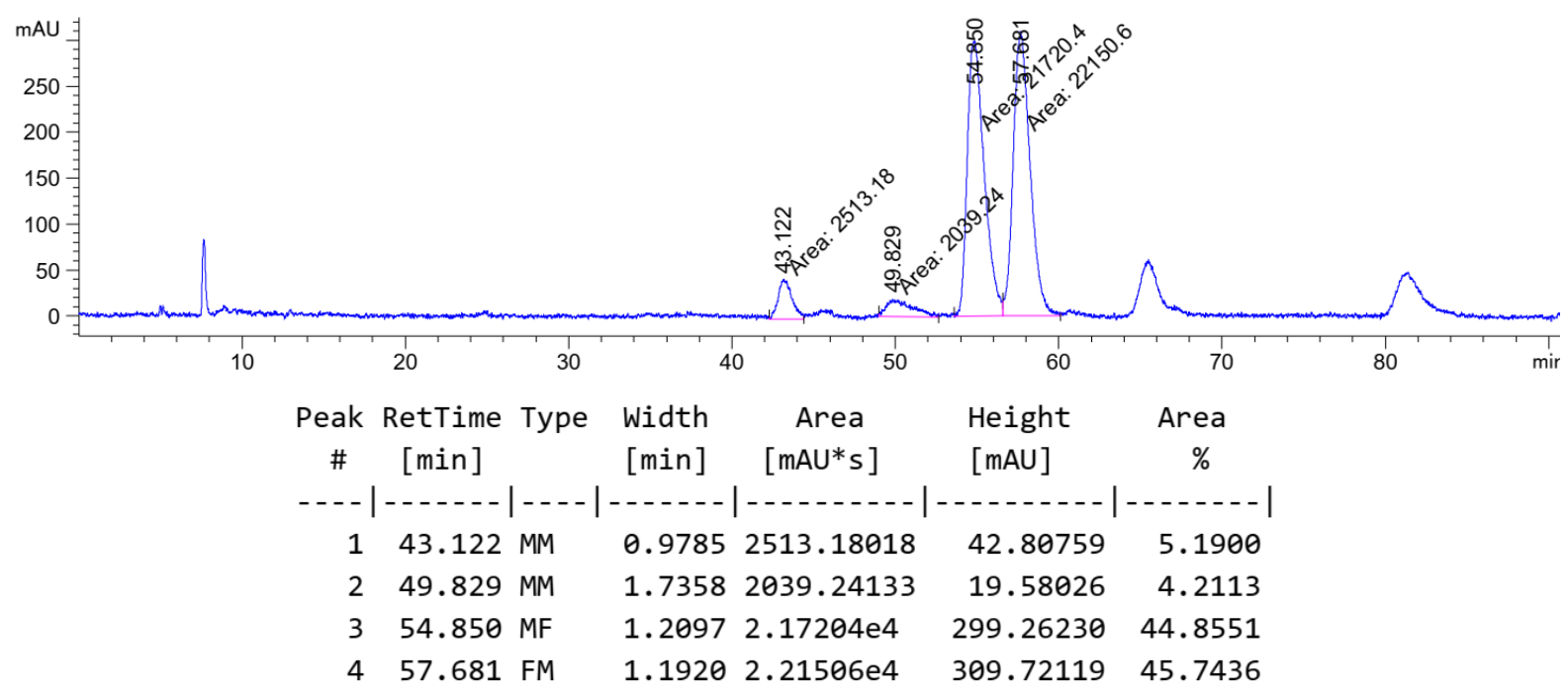

Figure 7.71 (2S,3S)-4-(4-Bromophenyl)-3-methyl-3-phenylbutan-2-ol [(2S,3S)-1f, $86 \%$ ee].

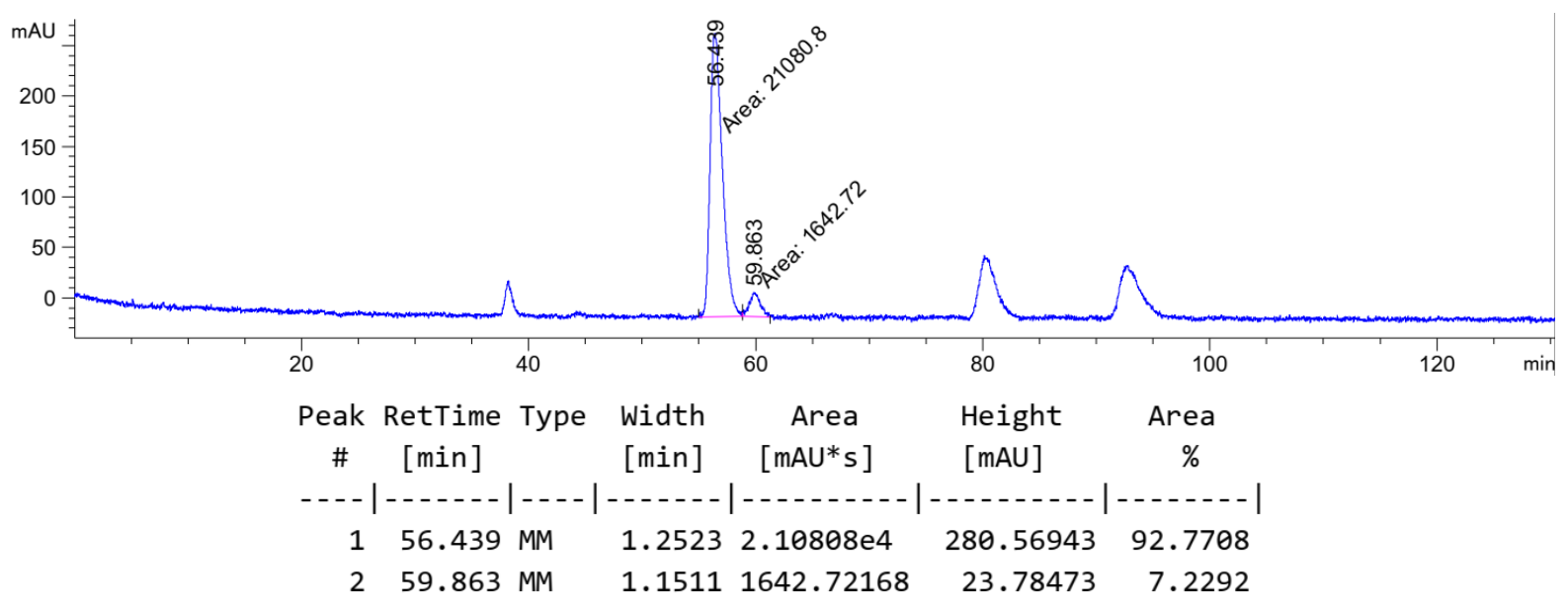


Figure 7.72 anti/syn-4-(4-Bromophenyl)-3-methyl-3-phenylbutan-2-ol [anti/syn-1f, $45 \%$ ee for $(2 R, 3 R)-1 \mathrm{f}]$.

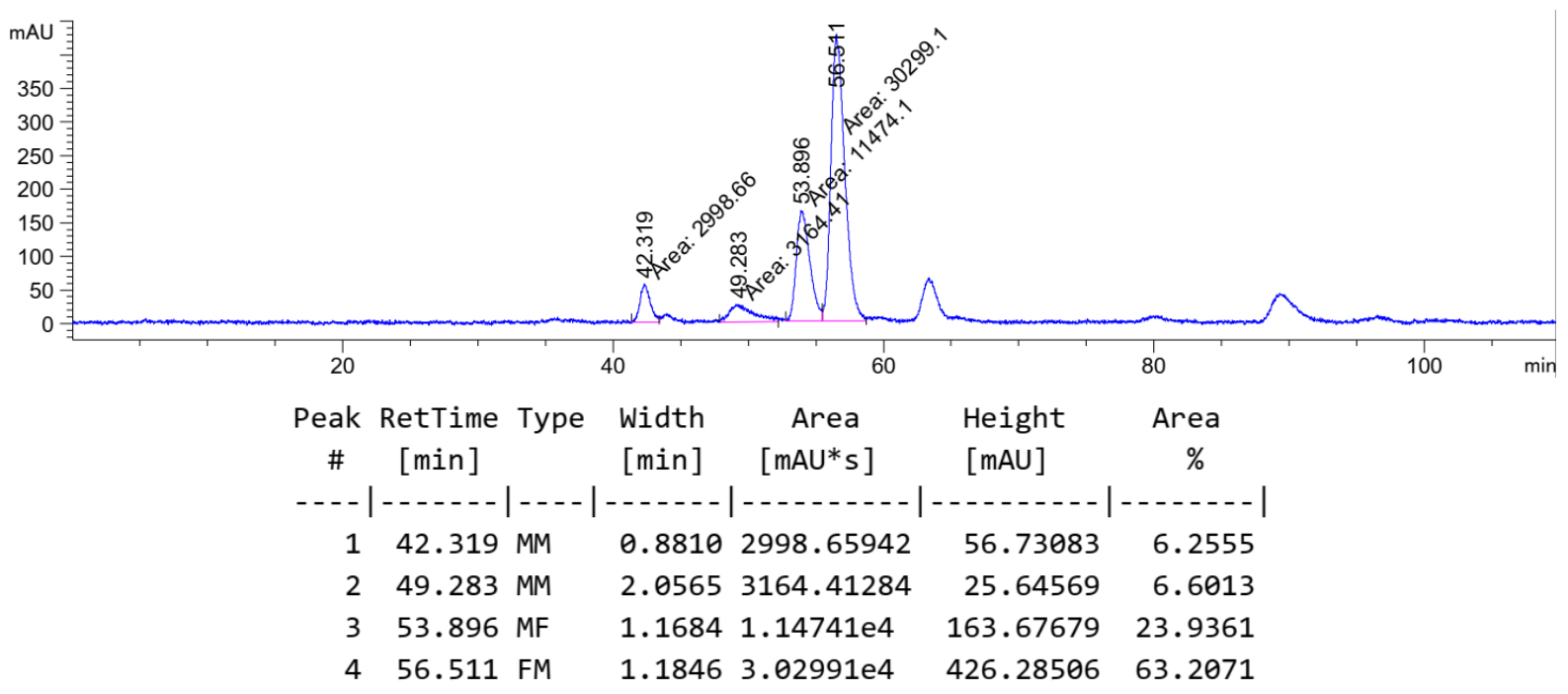

(2S,3S)-((4-(4-Chlorophenyl)-3-methyl-3-phenylbutan-2-yl)oxy)(3,5-

dimethylphenyl)dimethylsilane [(2S,3S)-3ee] and anti/syn-4-(4-Chlorophenyl)-3methyl-3-phenylbutan-2-ol [anti/syn-1e]

Figure 7.73 rac-anti/syn-4-(4-Chlorophenyl)-3-methyl-3-phenylbutan-2-ol [racanti/syn-1e].

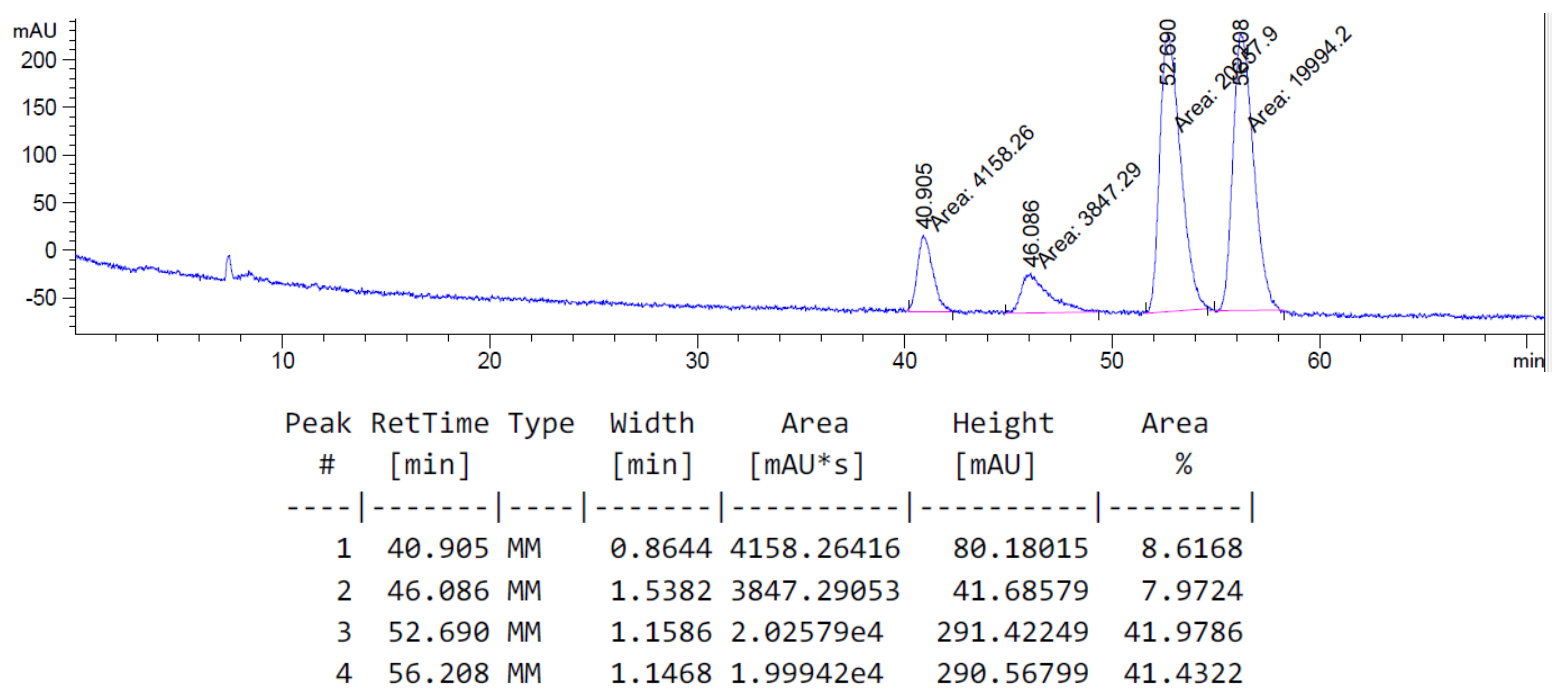


Figure 7.74 (2S,3S)-4-(4-Chlorophenyl)-3-methyl-3-phenylbutan-2-ol [(2S,3S)-1e, $87 \%$ ee].

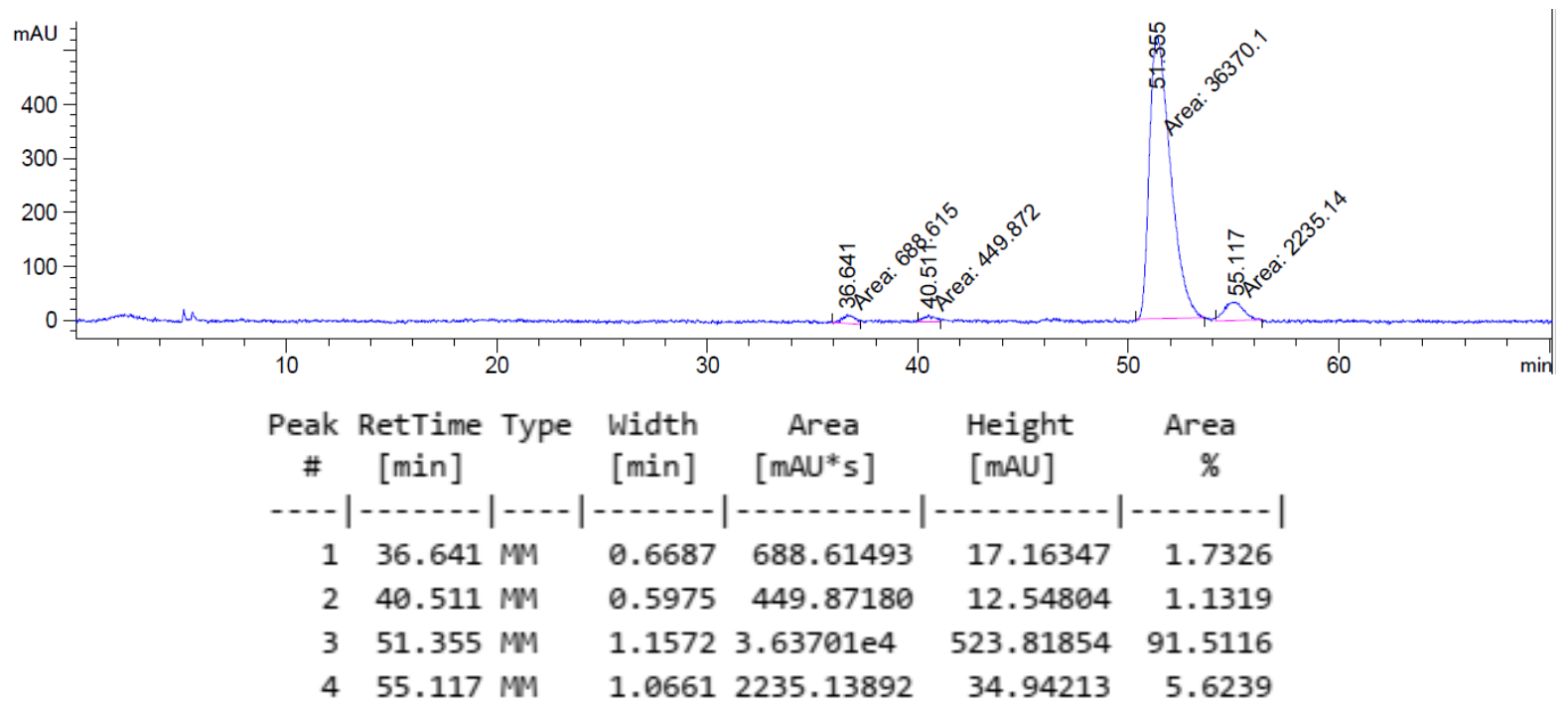

Figure 7.75 anti/syn-4-(4-Chlorophenyl)-3-methyl-3-phenylbutan-2-ol [anti/syn-1e, $56 \%$ ee for $(2 R, 3 R)-1 e]$.

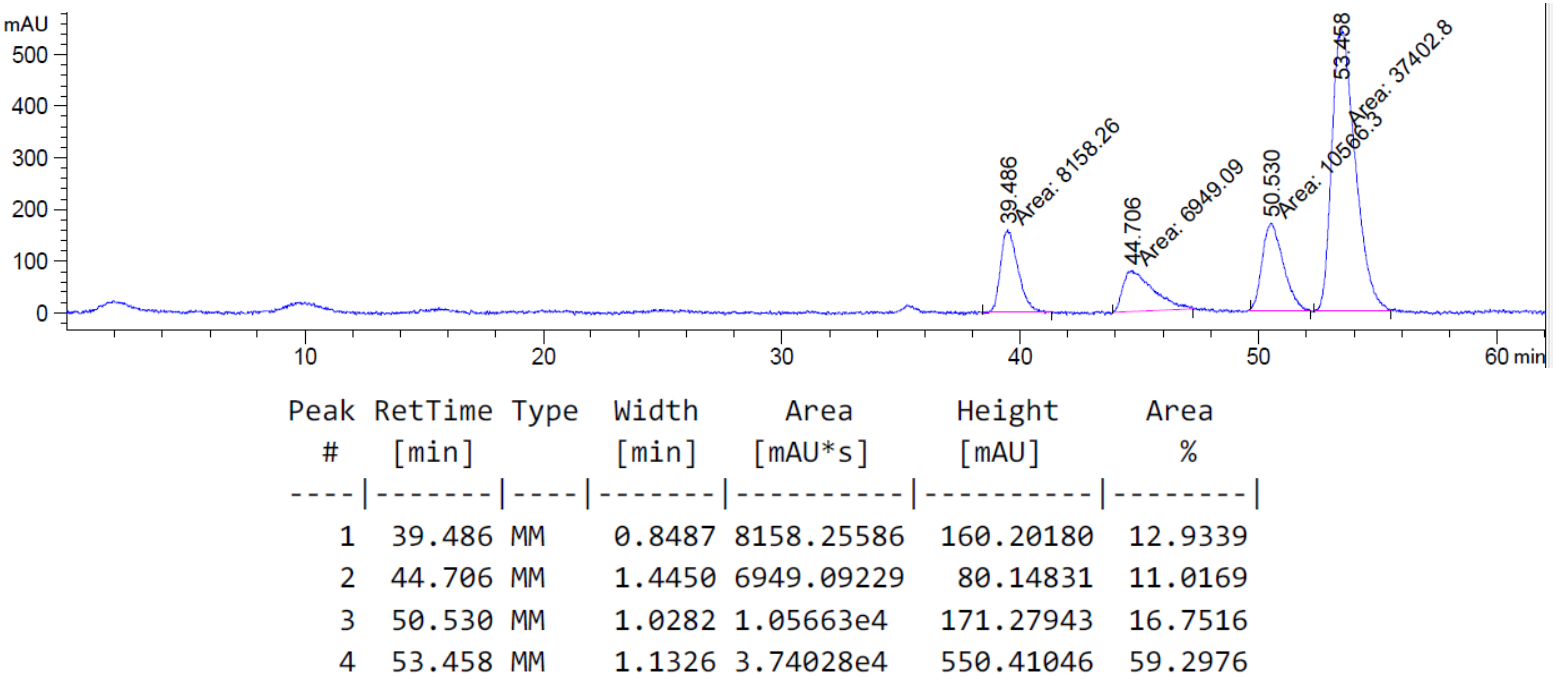


(2S,3S)-((4-(2-Bromophenyl)-3-methyl-3-phenylbutan-2-yl)oxy)(3,5-

dimethylphenyl)dimethylsilane [(2S,3S)-3he] and anti/syn-4-(2-Bromophenyl)-2methyl-2-phenylpropan-1-ol [anti/syn-1h]

Figure 7.76 rac-anti/syn-4-(2-Bromophenyl)-2-methyl-2-phenylpropan-1-ol [racanti/syn-1h].

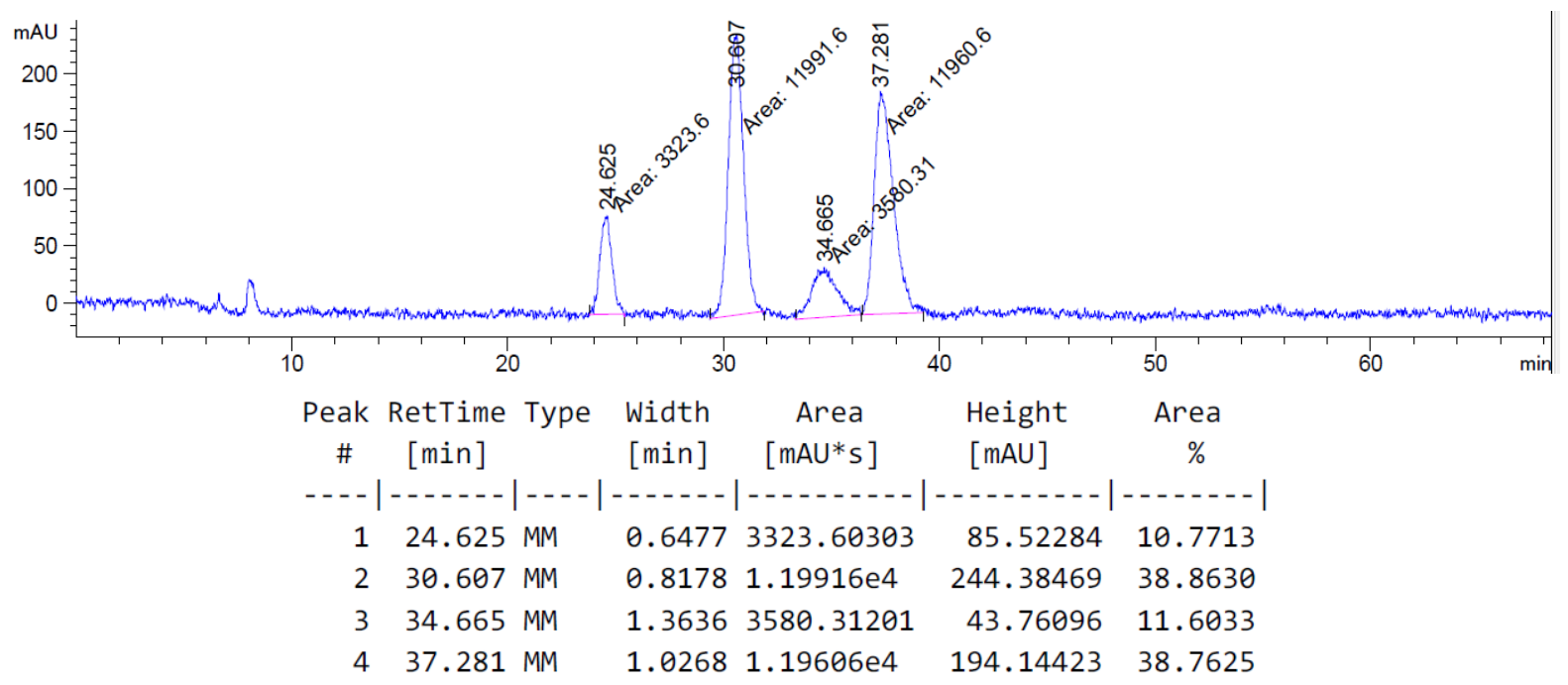

Figure 7.77 (2S,3S)-4-(2-Bromophenyl)-2-methyl-2-phenylpropan-1-ol [(2S,3S)-1h, $84 \%$ ee].

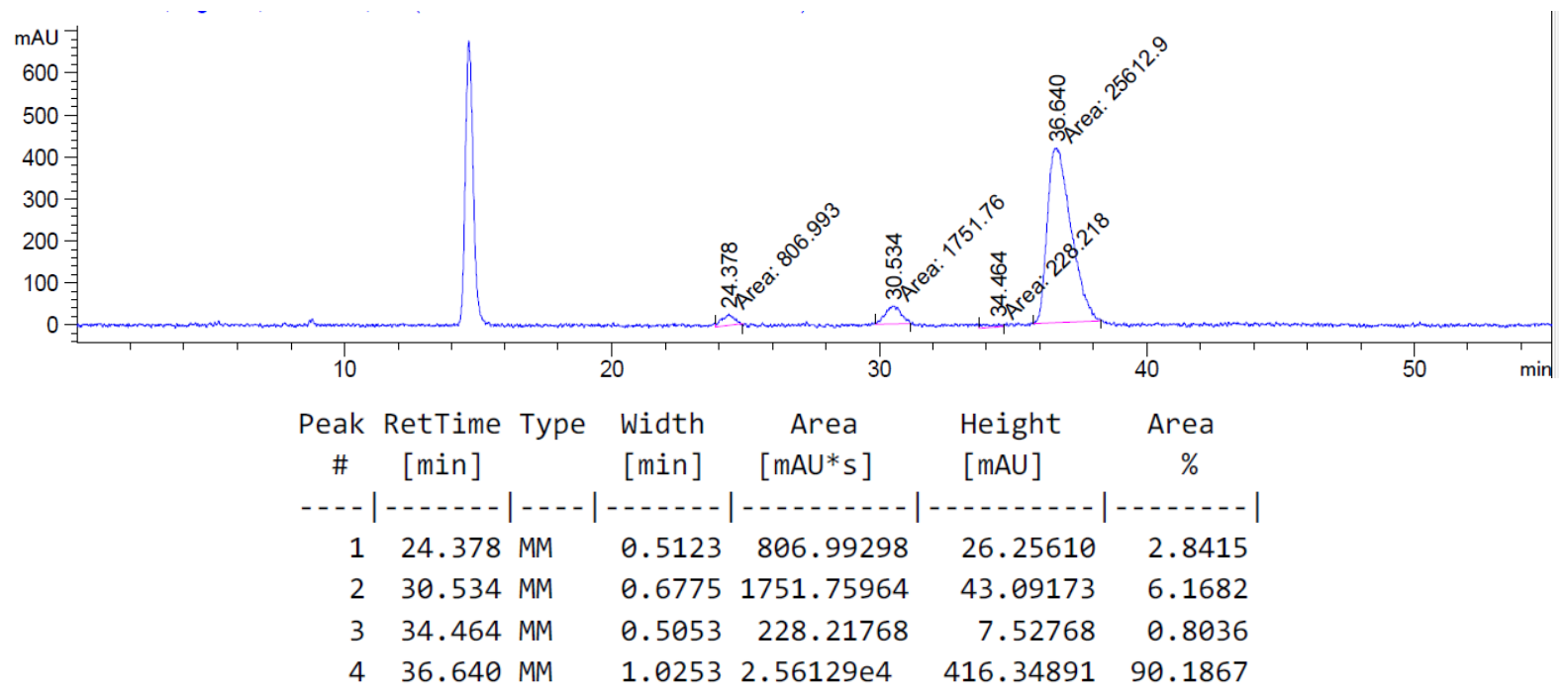


Figure 7.78 anti/syn-4-(2-Bromophenyl)-2-methyl-2-phenylpropan-1-ol [anti/syn-1h, $65 \%$ ee for $(2 R, 3 R)-1 \mathrm{~h}]$.

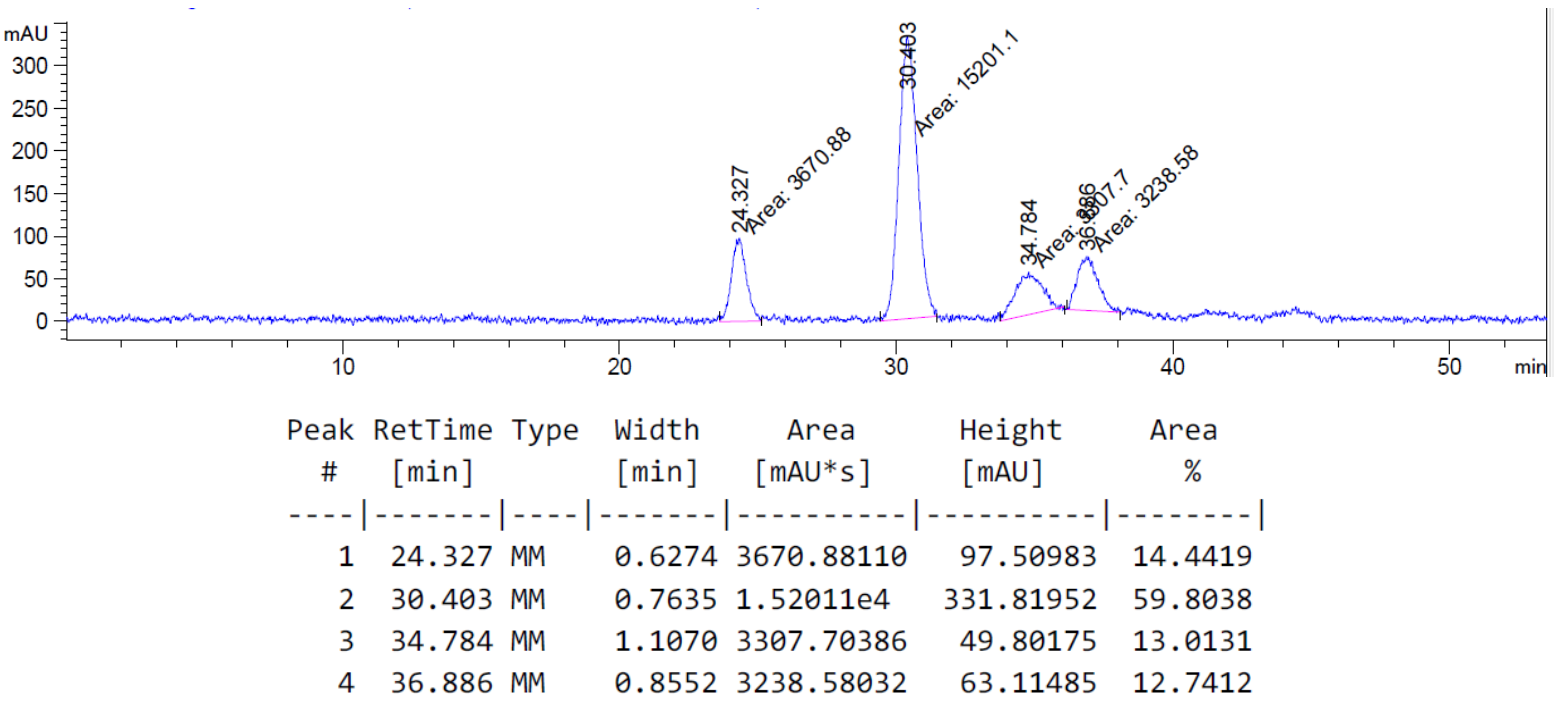

(2S,3S)-(3,5-Dimethylphenyl)((4-(4-iodophenyl)-3-methyl-3-phenylbutan-2-

yl)oxy)dimethylsilane [(2S,3S)-3ge] and anti/syn-4-(4-lodophenyl)-3-methyl-3phenylbutan-2-ol [anti/syn-1g]

Figure 7.79 rac-anti/syn-4-(4-lodophenyl)-3-methyl-3-phenylbutan-2-ol [rac-anti/syn1g].

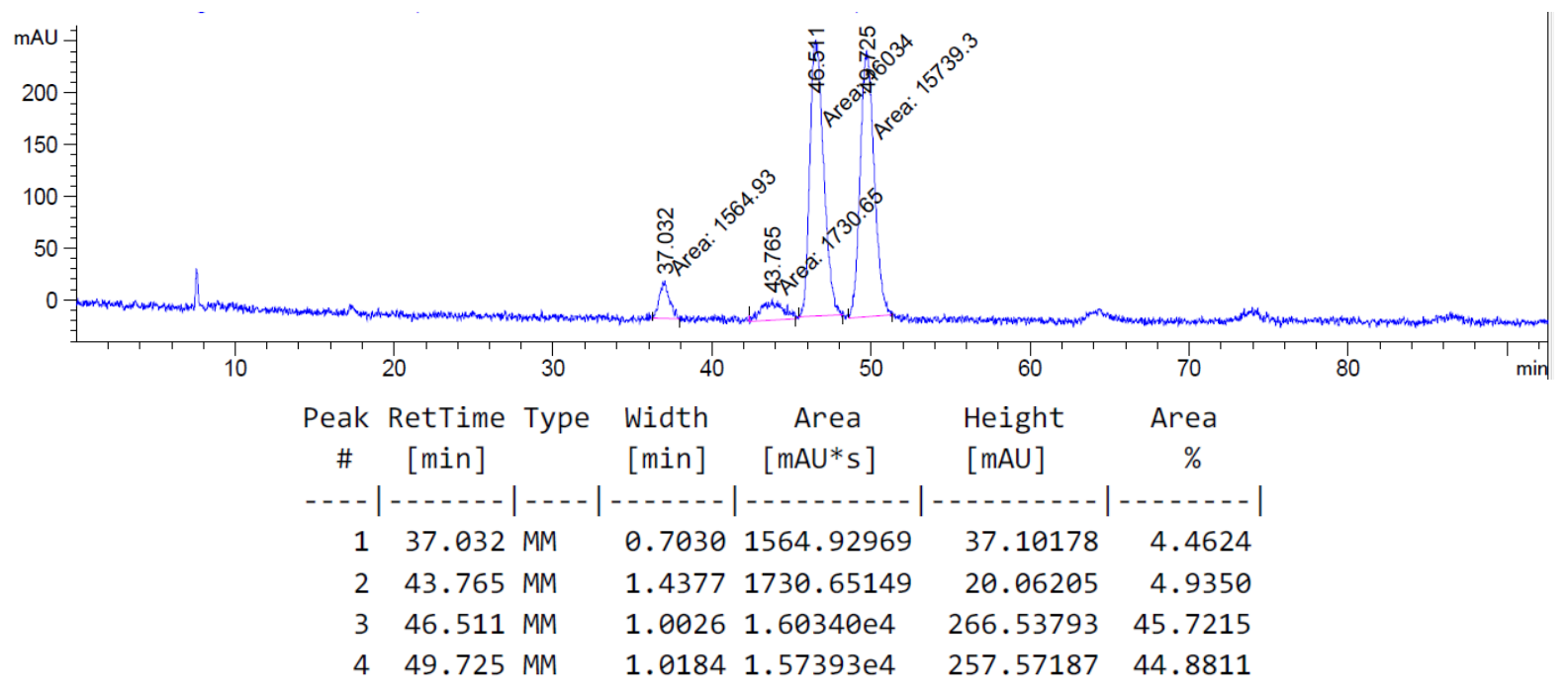


Figure 7.80 (2S,3S)-4-(4-lodophenyl)-3-methyl-3-phenylbutan-2-ol [(2S,3S)-1g, 90\% ee].

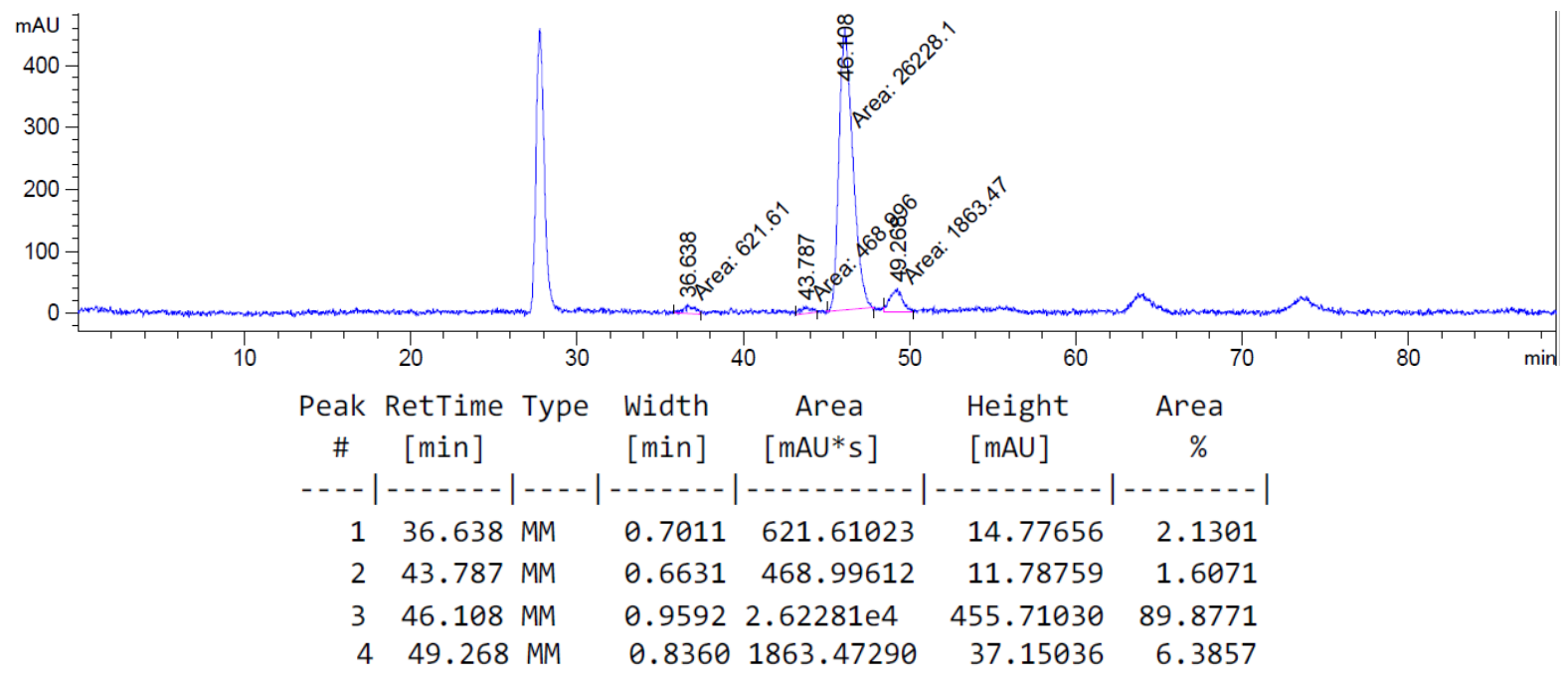

Figure 7.81 anti/syn-4-(4-lodophenyl)-3-methyl-3-phenylbutan-2-ol [anti/syn-1g, 63\% ee for $(2 R, 3 R)-\mathbf{1 g}$.

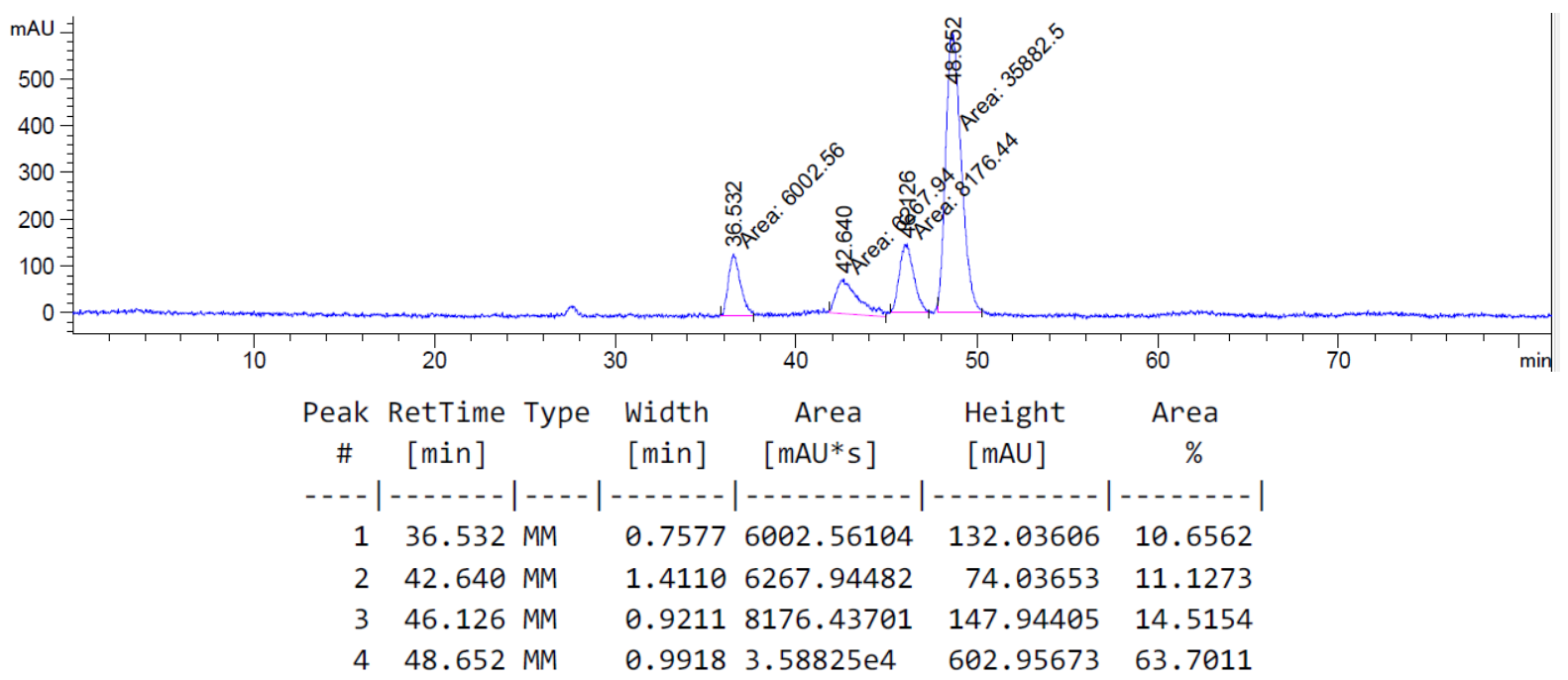


(2S,3R)-((3-Benzyl-3-methylpentan-2-yl)oxy)(3,5-dimethylphenyl)dimethylsilane [(2S,3S)-3ce] and anti/syn-3-Benzyl-3-methylpentan-2-ol [anti/syn-1c]

Figure 7.82 rac-anti/syn-3-Benzyl-3-methylpentan-2-ol [rac-anti/syn-1c]

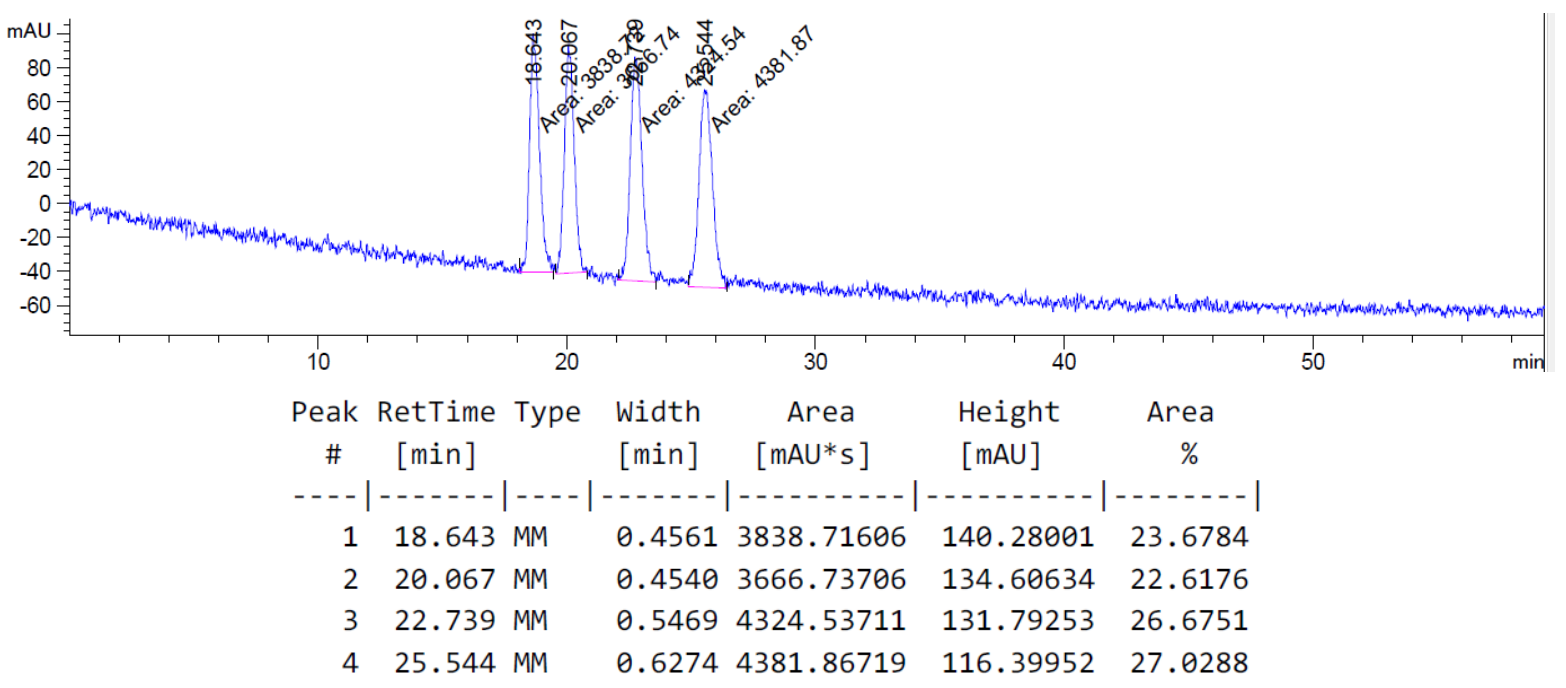

Figure 7.83 (2S,3S)-3-Benzyl-3-methylpentan-2-ol [(2S,3S)-1c, 74\% ee].

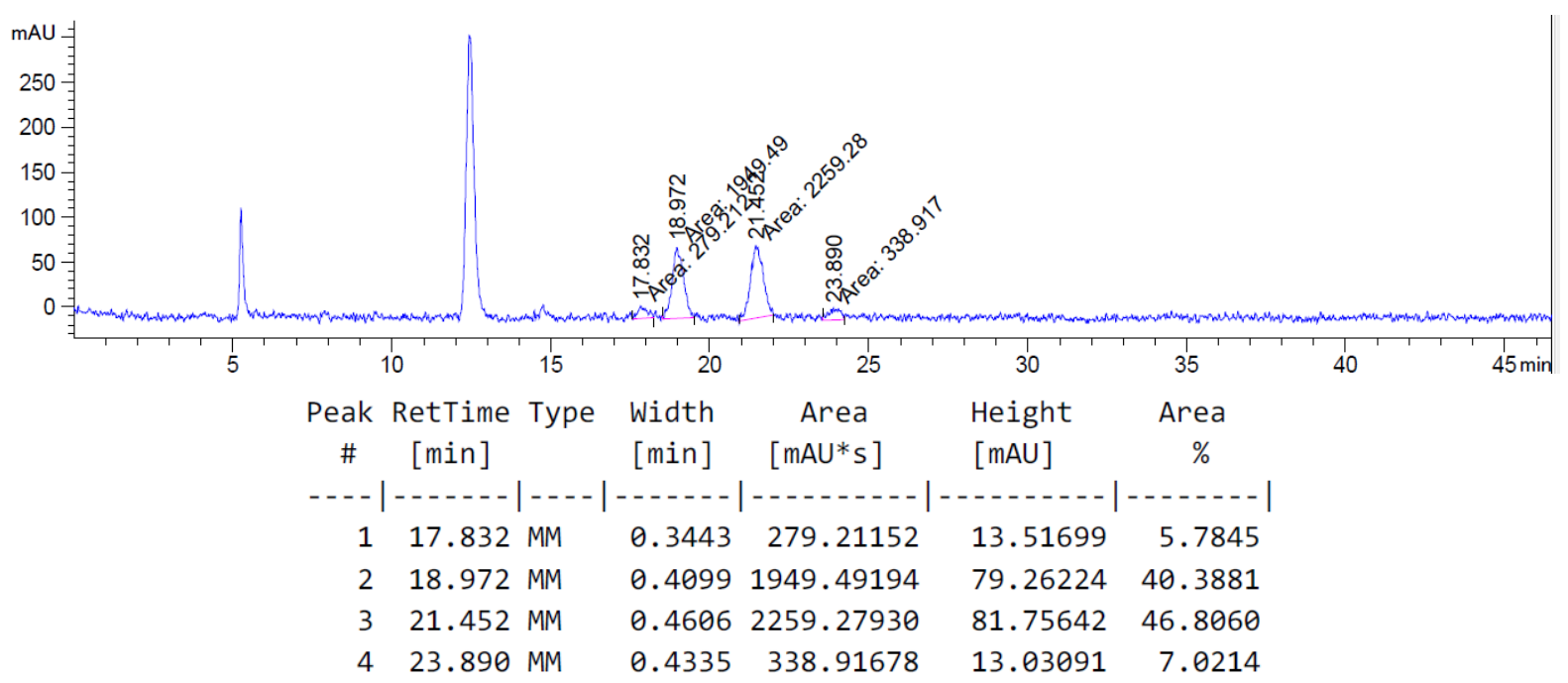


Figure 7.84 anti/syn-3-Benzyl-3-methylpentan-2-ol [anti/syn-1c, 14\% ee for $(2 R, 3 R)$ 1c].

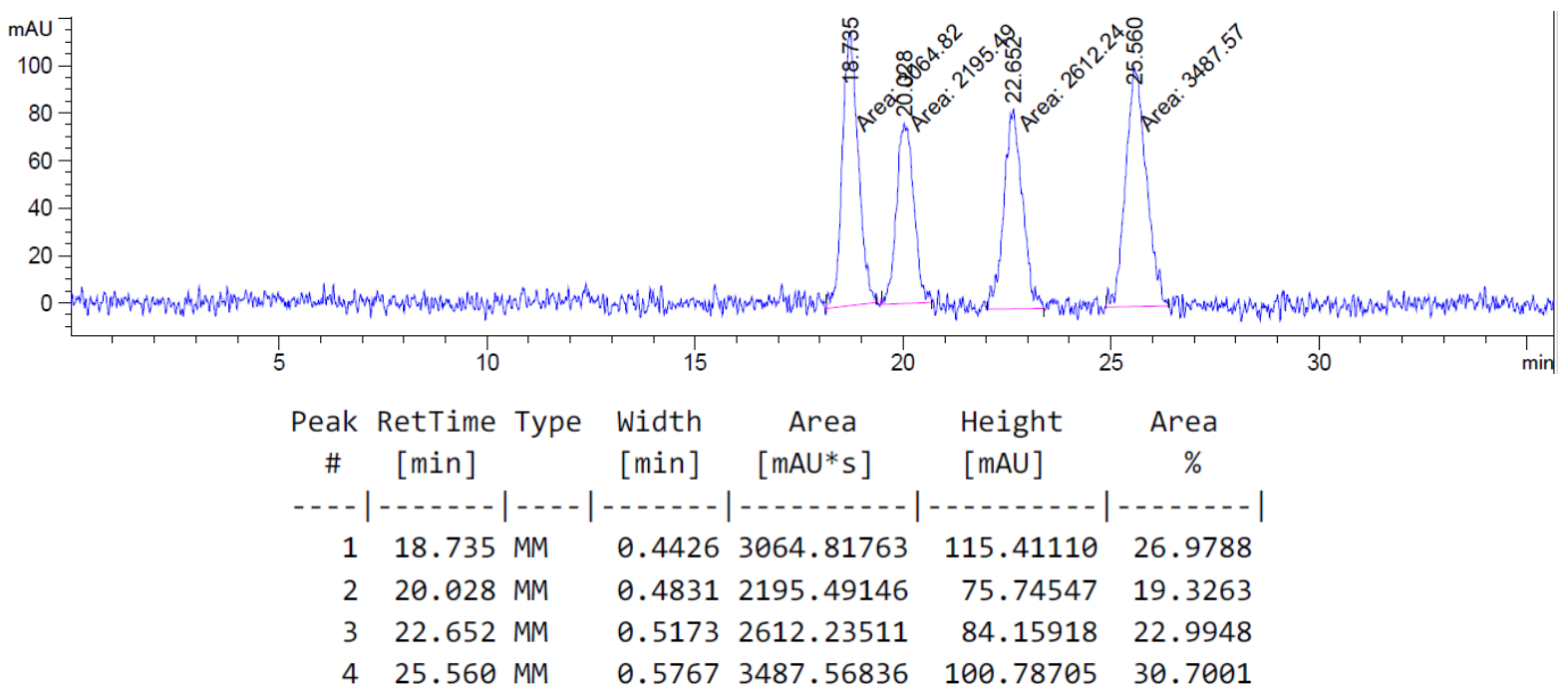

(2S,3S)-(3,5-Dimethylphenyl)((4-(4-methoxyphenyl)-3-methyl-3-phenylbutan-2yl)oxy)dimethylsilane [(2S,3S)-3ke] and anti/syn-4-(4-Methoxyphenyl)-3-methyl3-phenylbutan-2-ol [anti/syn-1k]

Figure 7.85 rac-anti/syn-4-(4-Methoxyphenyl)-3-methyl-3-phenylbutan-2-ol [racanti/syn-1k].

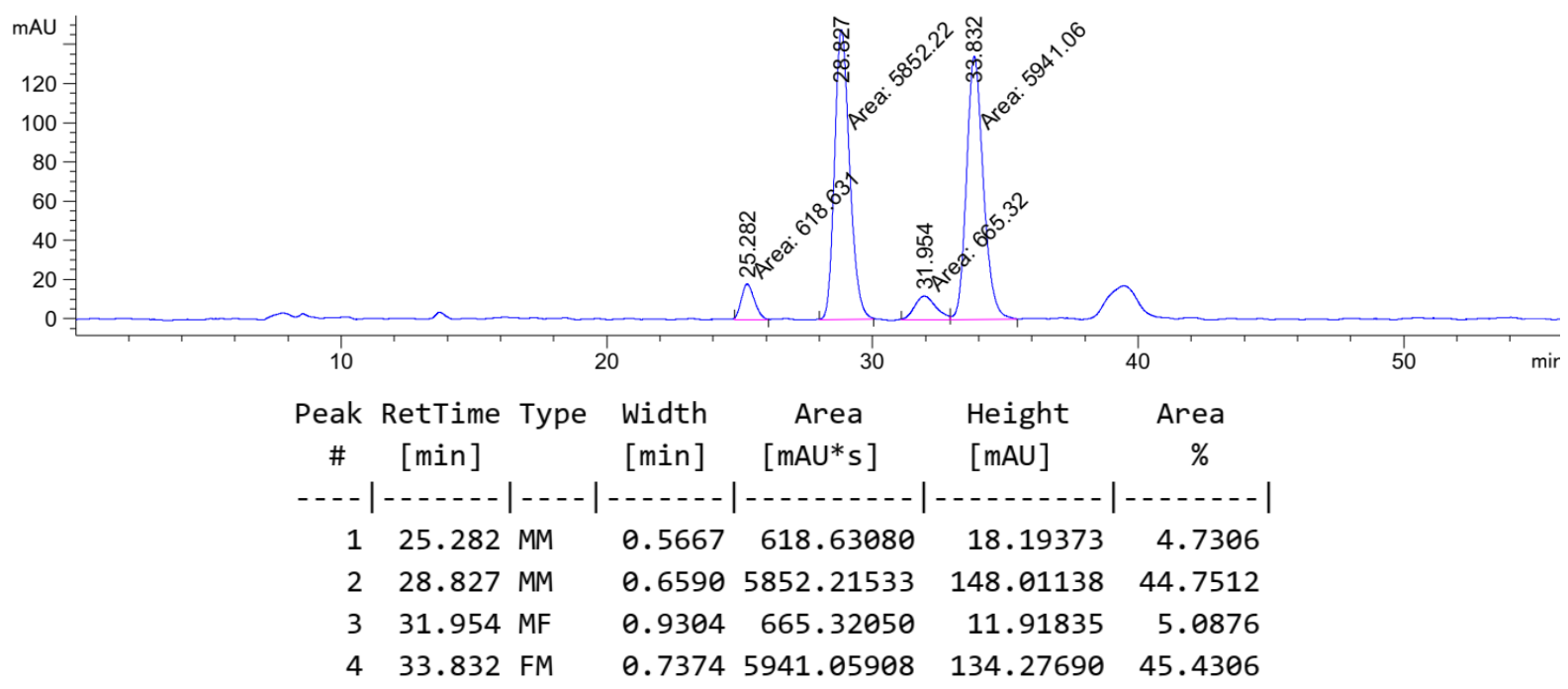


Figure 7.86 (2S,3S)-4-(4-Methoxyphenyl)-3-methyl-3-phenylbutan-2-ol [(2S,3S)-1k, $91 \%$ ee].

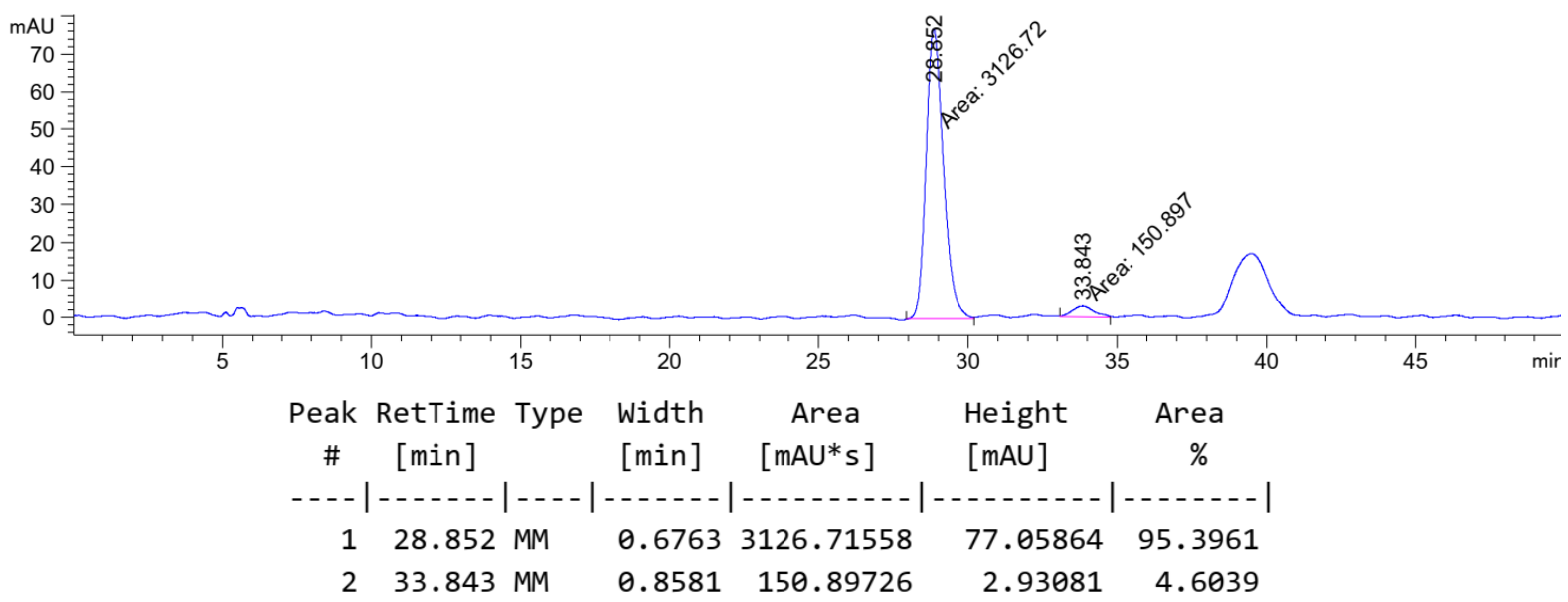

Figure 7.87 anti/syn-4-(4-Methoxyphenyl)-3-methyl-3-phenylbutan-2-ol [anti/syn-1k, $13 \%$ ee for $(2 R, 3 R)-1 \mathbf{k}]$.

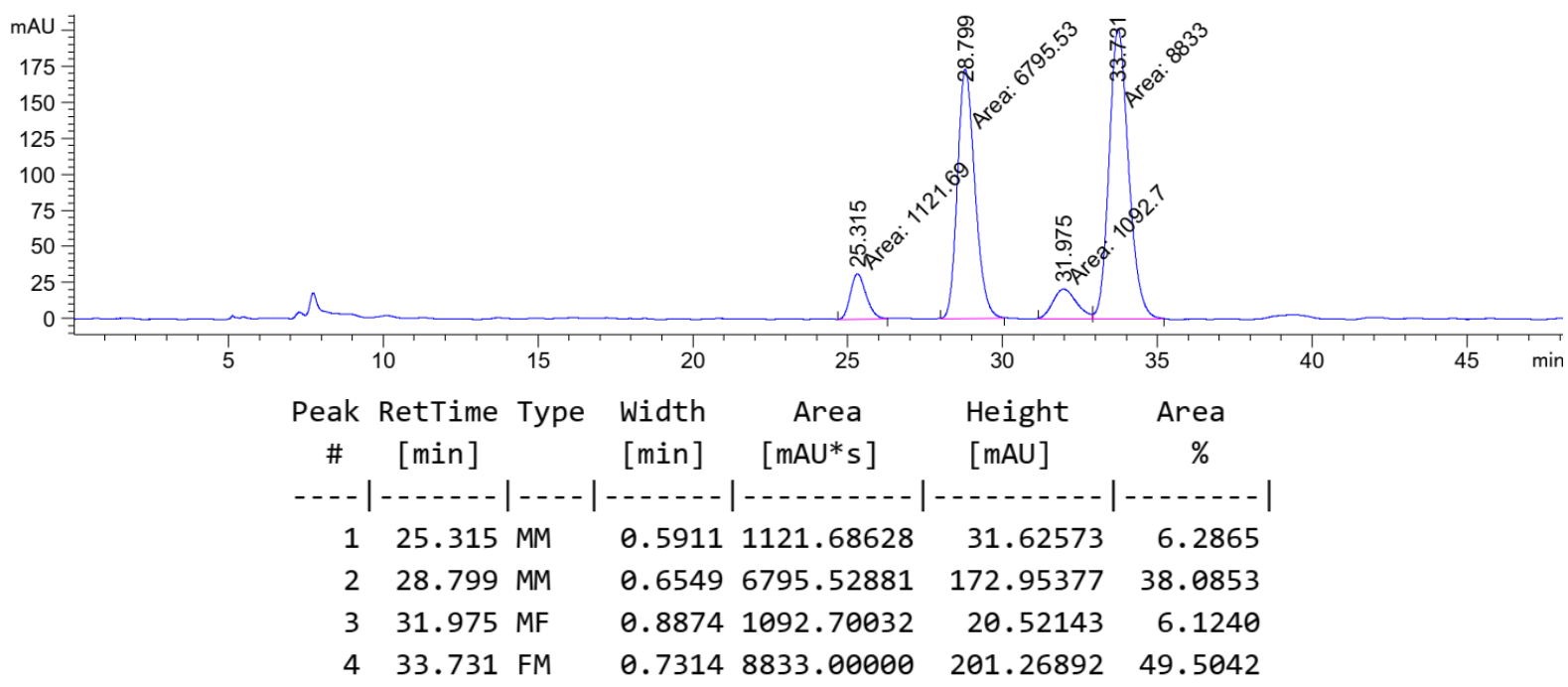




\section{NMR Spectra}

Figure $8.1^{1} \mathrm{H}$ NMR $\left(500 \mathrm{MHz}, \mathrm{CDCl}_{3}, 298 \mathrm{~K}\right)$ of $(2 R, 3 R)$-3-Methyl-3,4-diphenylbutan-2-ol [(2R,3R)-1a].
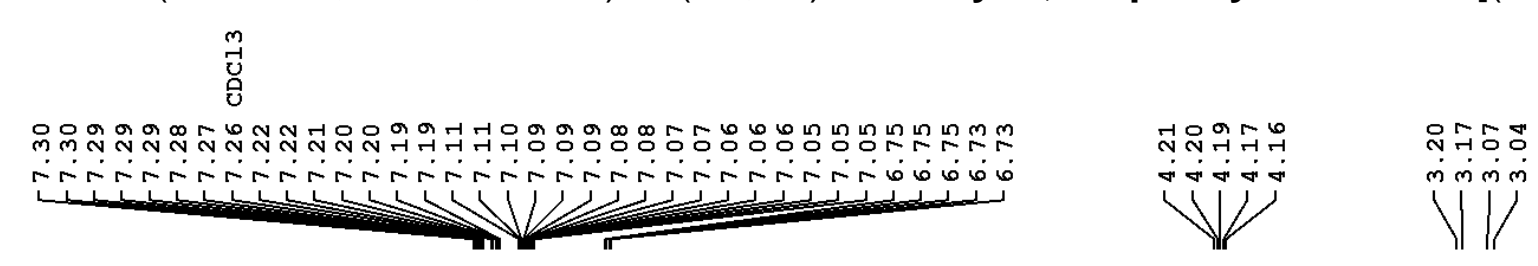

윰

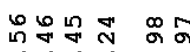

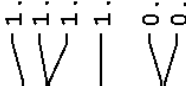
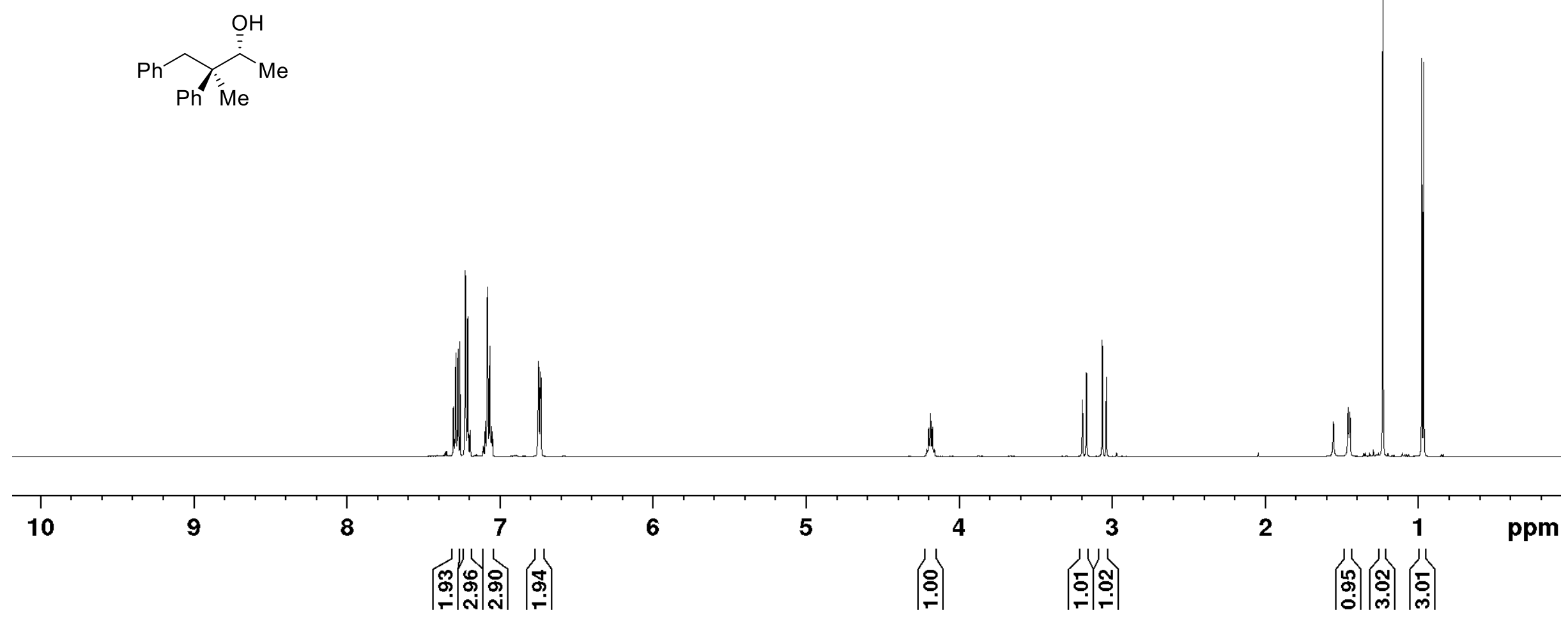
Figure $8.2{ }^{13} \mathrm{C} \mathrm{NMR}\left(126 \mathrm{MHz}, \mathrm{CDCl}_{3}, 298 \mathrm{~K}\right)$ of $(2 R, 3 R)$-3-Methyl-3,4-diphenylbutan-2-ol [(2R,3R)-1a].

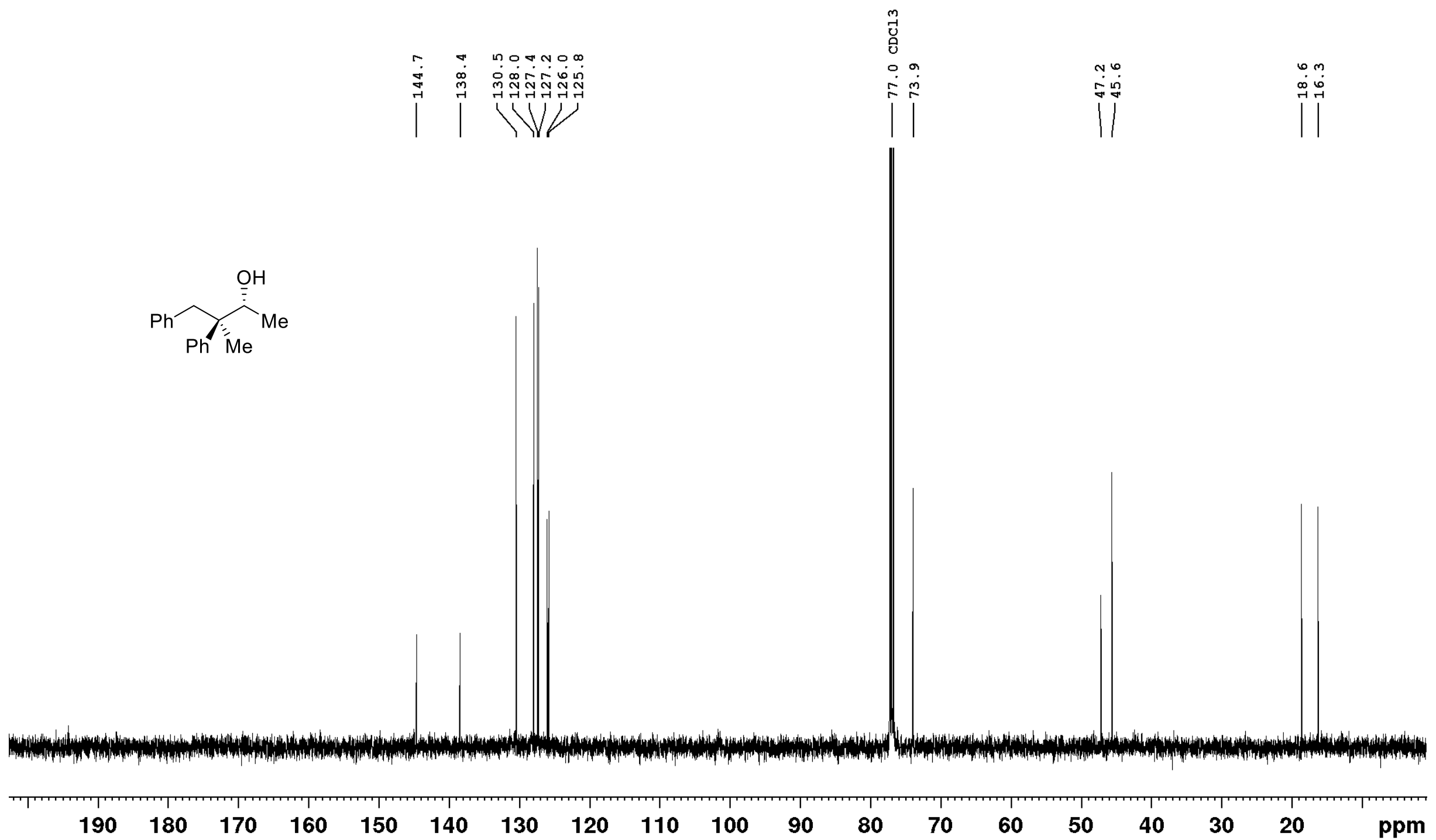


Figure 8.3 ${ }^{1} \mathrm{H} \quad \mathrm{NMR}\left(500 \mathrm{MHz}, \mathrm{C}_{6} \mathrm{D}_{6}, 298 \mathrm{~K}\right)$ of $(2 S, 3 S)$-(3,5-Dimethylphenyl)dimethyl((3-methyl-3,4-diphenylbutan-2yl)oxy)silane $[(2 S, 3 S)-3 a e]$.
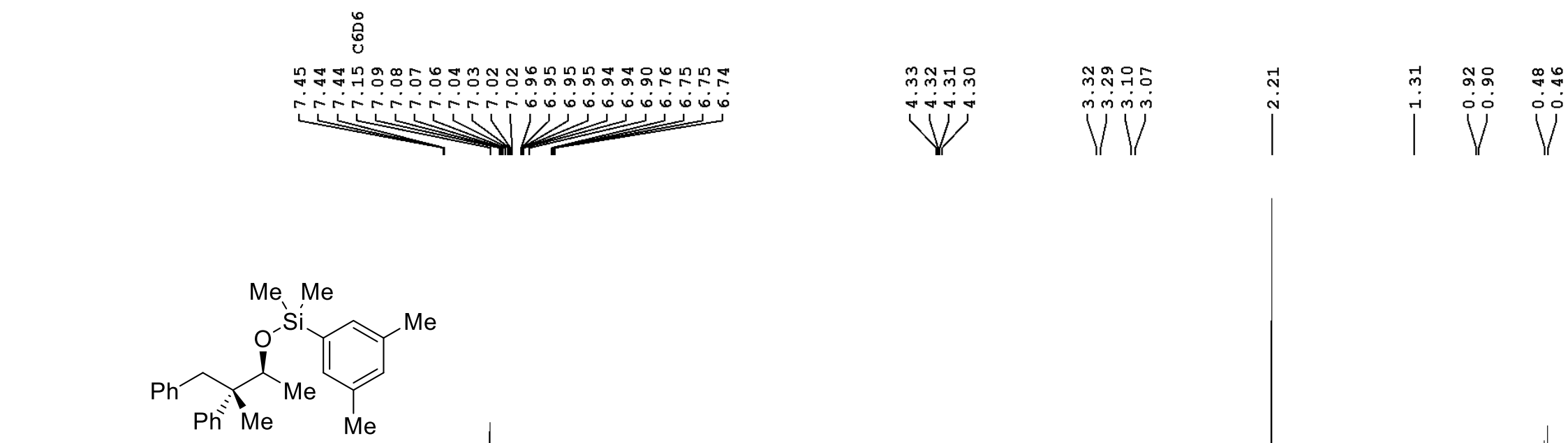

8

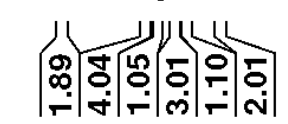

6

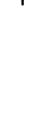

r

5

\%

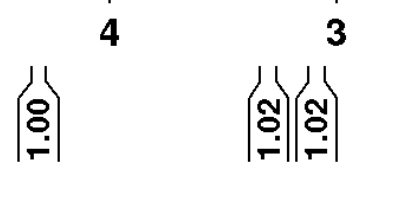

2

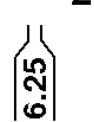

|⿵人一

ppm 
Figure 8.4 ${ }^{13} \mathrm{C}$ NMR (126 MHz, $\left.\mathrm{C}_{6} \mathrm{D}_{6}, 298 \mathrm{~K}\right)$ of $(2 S, 3 S)-(3,5-$ Dimethylphenyl)dimethyl((3-methyl-3,4-diphenylbutan-2yl)oxy)silane $[(2 S, 3 S)-3 a e]$.

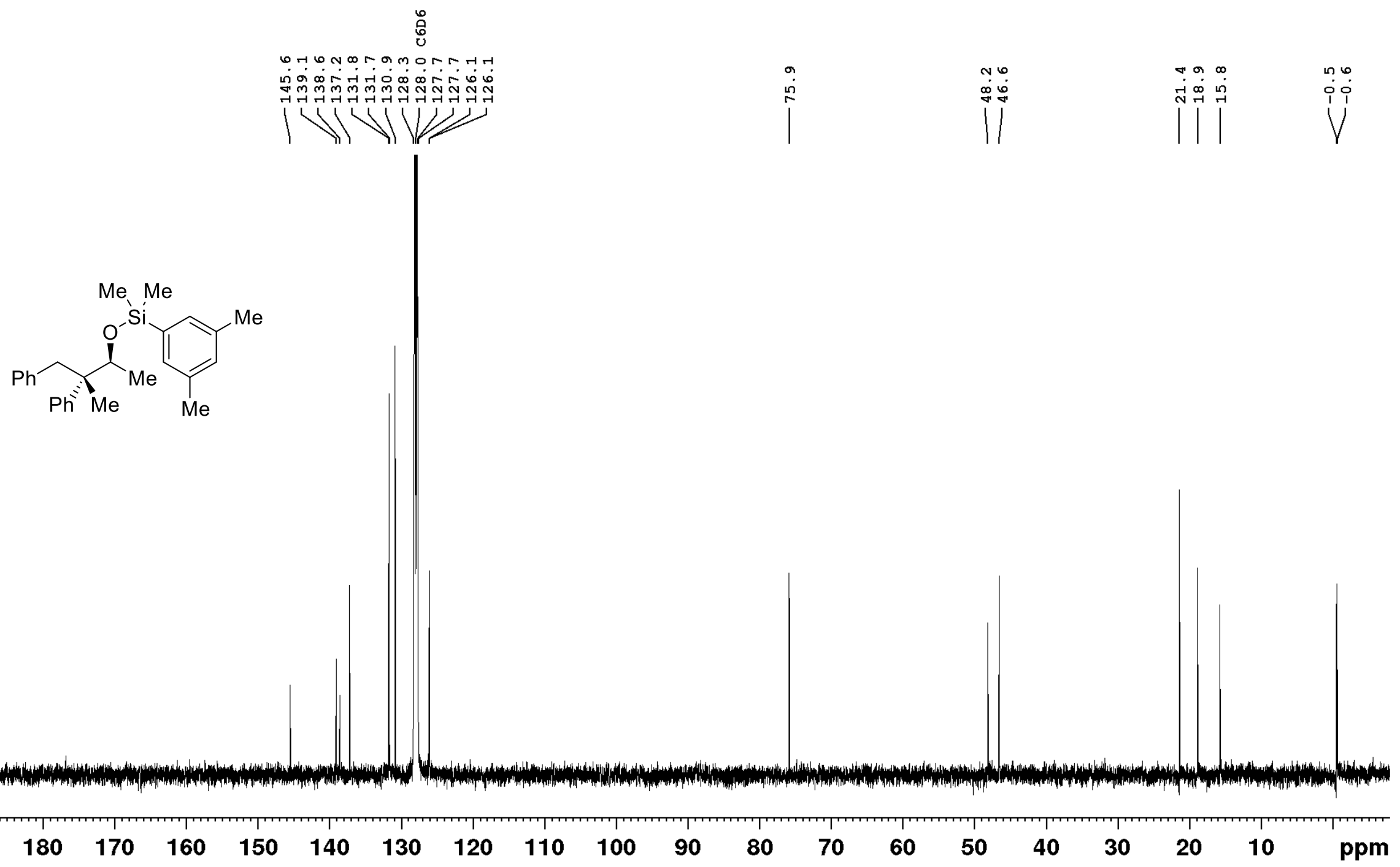


Figure 8.5 ${ }^{29} \mathrm{Si}$ DEPT NMR (99 MHz, $\left.\mathrm{CDCl}_{3}, 298 \mathrm{~K}\right)$ of $(2 S, 3 S)-(3,5-$ Dimethylphenyl)dimethyl((3-methyl-3,4-diphenylbutan-2yl)oxy)silane $[(2 S, 3 S)-3 a e]$.

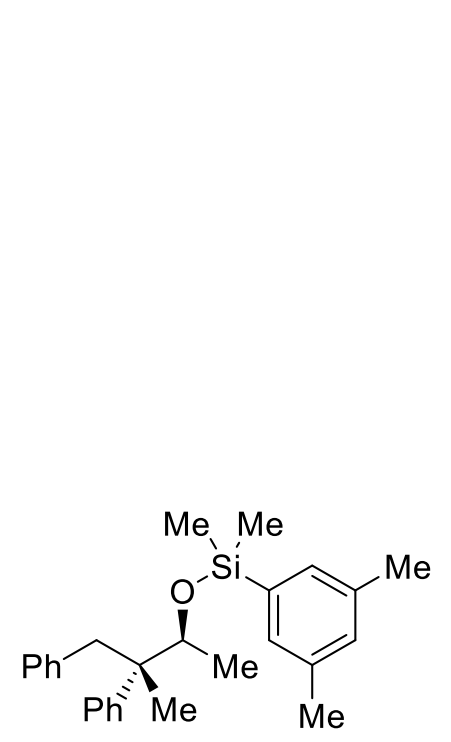

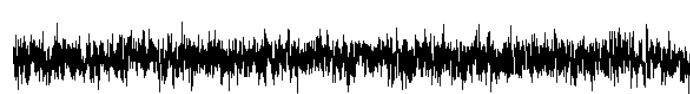

$\begin{array}{llllllllllllllllllll}100 & 90 & 80 & 70 & 60 & 50 & 40 & 30 & 20 & 10 & 0 & -10 & -20 & -30 & -40 & -50 & -60 & -70 & -80 & p p m\end{array}$


Figure 8.6 ${ }^{1} \mathrm{H} \mathrm{NMR}\left(500 \mathrm{MHz}, \mathrm{CDCl}_{3}, 298 \mathrm{~K}\right)$ of (2R,3S)-3-Methyl-3,4-diphenylbutan-2-ol [(2R,3S)-1a].
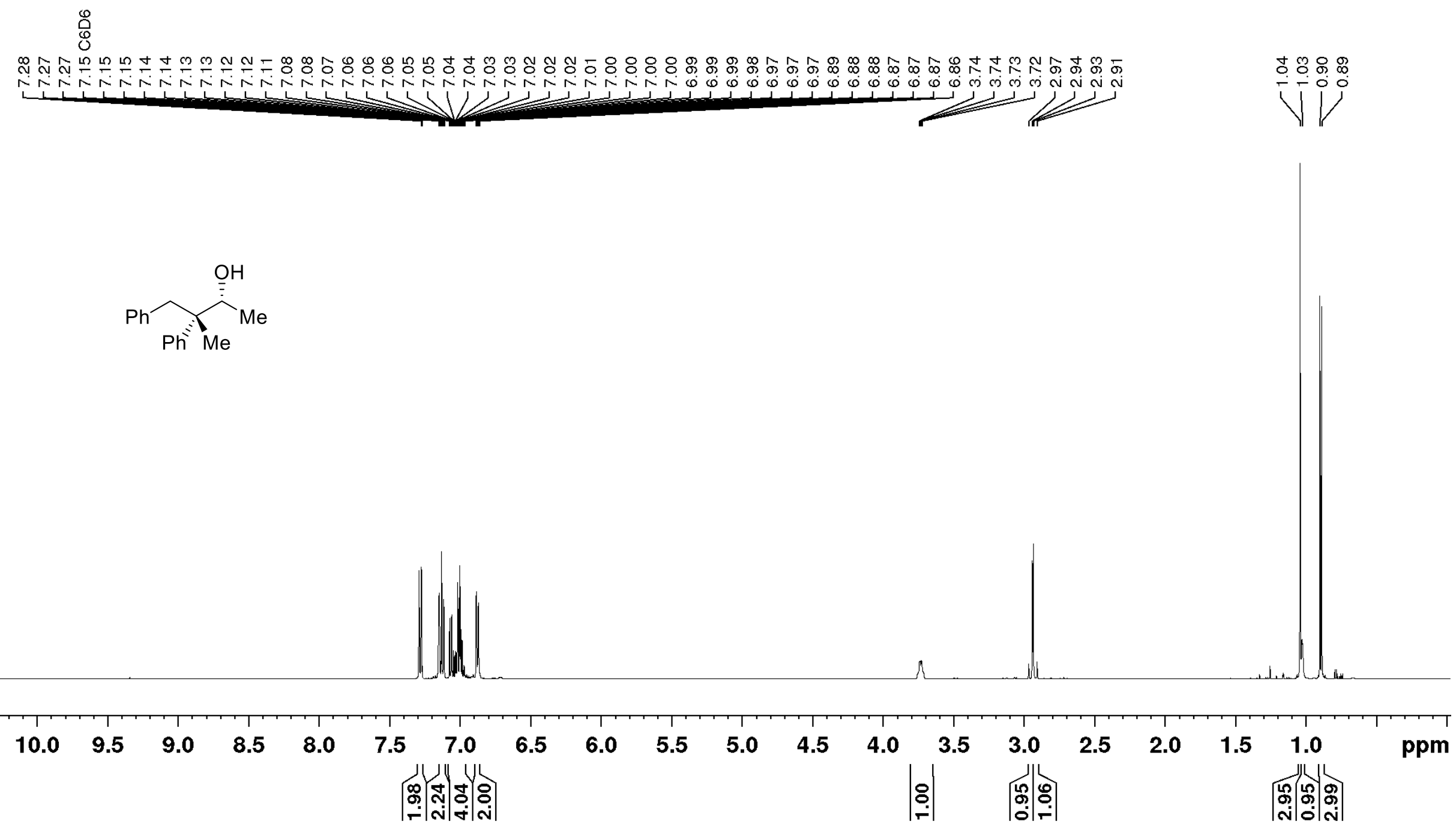
Figure $8.7{ }^{13} \mathrm{C} \mathrm{NMR}\left(126 \mathrm{MHz}, \mathrm{CDCl}_{3}, 298 \mathrm{~K}\right)$ of (2R,3S)-3-Methyl-3,4-diphenylbutan-2-ol [(2R,3S)-1a].

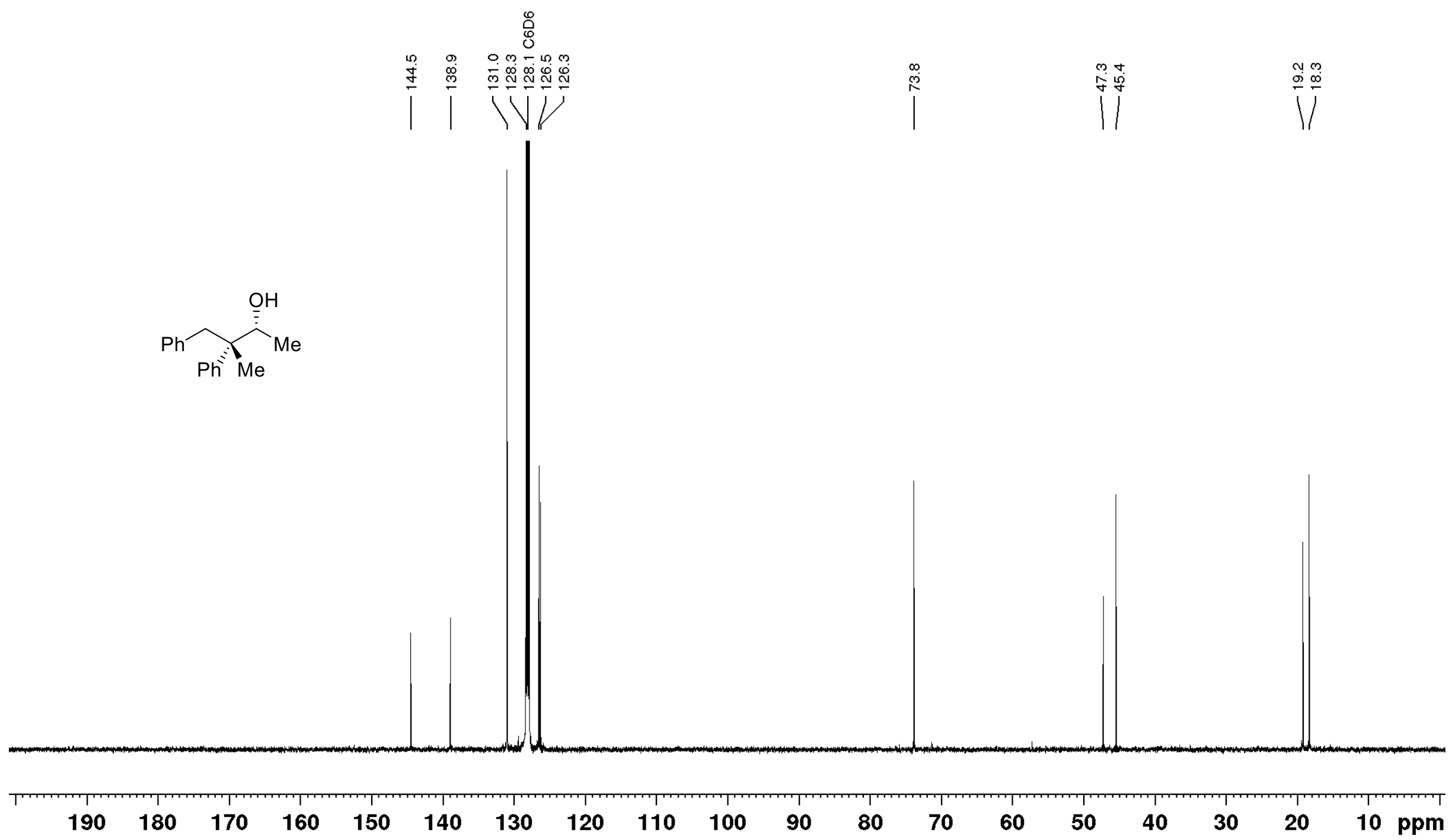


Figure 8.8 ${ }^{1} \mathrm{H}$ NMR $\left(500 \mathrm{MHz}, \mathrm{C}_{6} \mathrm{D}_{6}, 298 \mathrm{~K}\right)$ of (2S,3R)-(3,5-Dimethylphenyl)dimethyl((3-methyl-3,4-diphenylbutan-2yl)oxy)silane $[(2 S, 3 R)-3 a e]$.
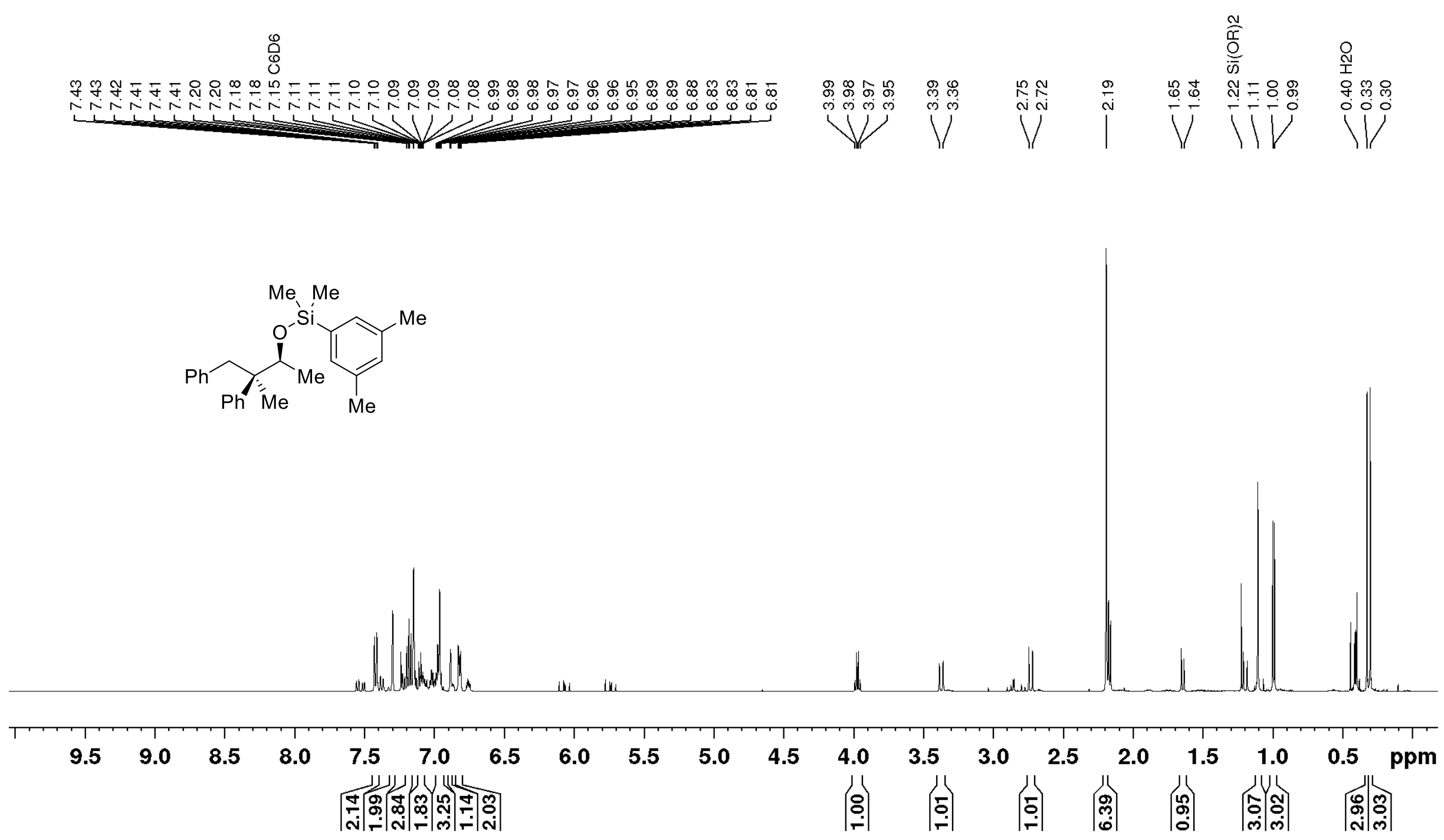
Figure 8.9 ${ }^{13} \mathrm{C}$ NMR $\left(126 \mathrm{MHz}, \mathrm{C}_{6} \mathrm{D}_{6}, 298 \mathrm{~K}\right)$ of $(2 S, 3 R)$-(3,5-Dimethylphenyl)dimethyl((3-methyl-3,4-diphenylbutan-2yl)oxy)silane $[(2 S, 3 R)-3 a e]$.

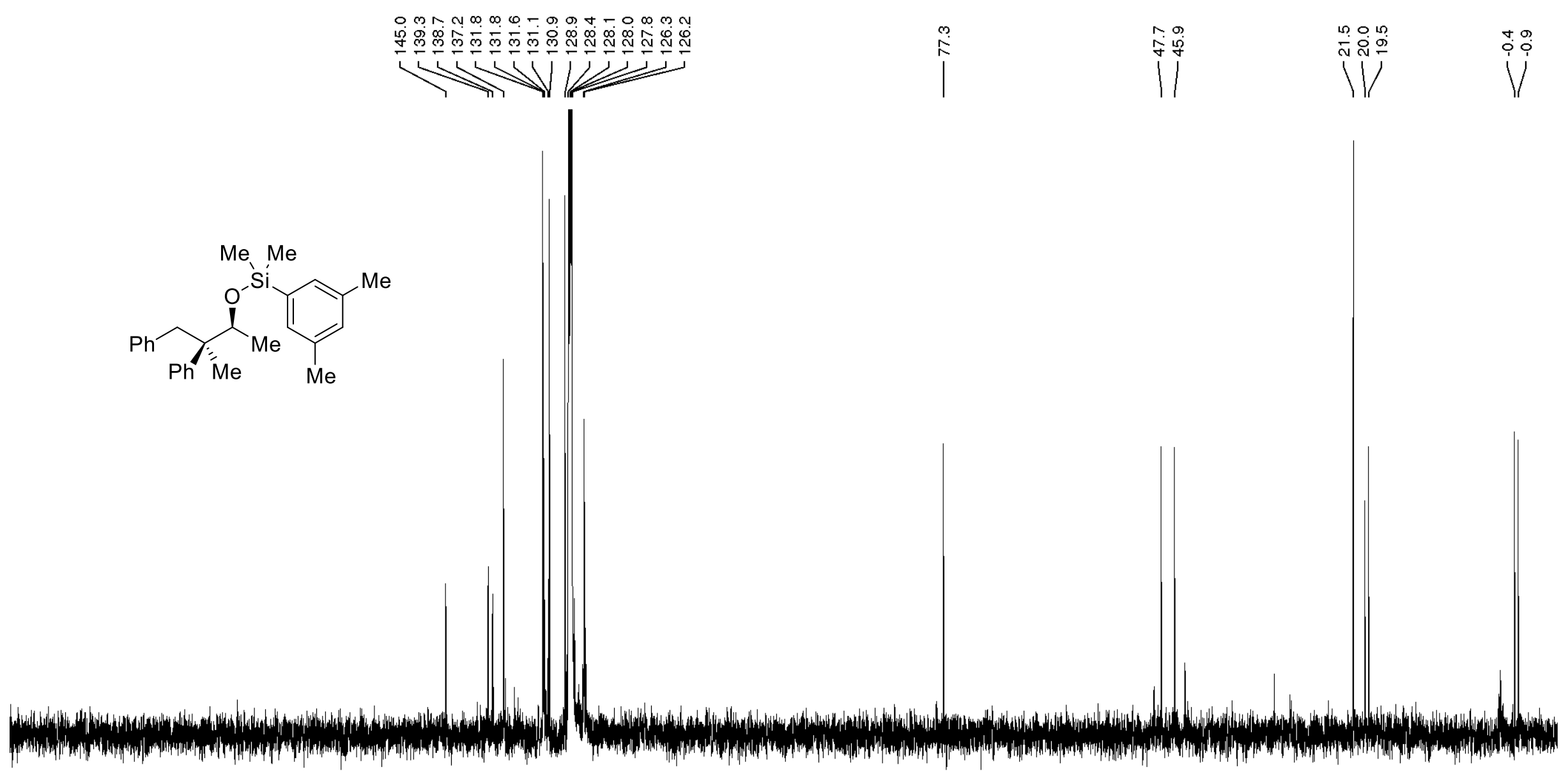

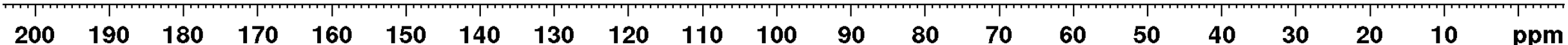


Figure 8.10 ${ }^{29} \mathrm{Si}$ DEPT NMR (99 MHz, $\left.\mathrm{C}_{6} \mathrm{D}_{6}, 298 \mathrm{~K}\right)$ of $(2 S, 3 R)-($ 3,5-Dimethylphenyl)dimethyl((3-methyl-3,4-diphenylbutan-2yl)oxy)silane $[(2 S, 3 R)-3 a e]$.

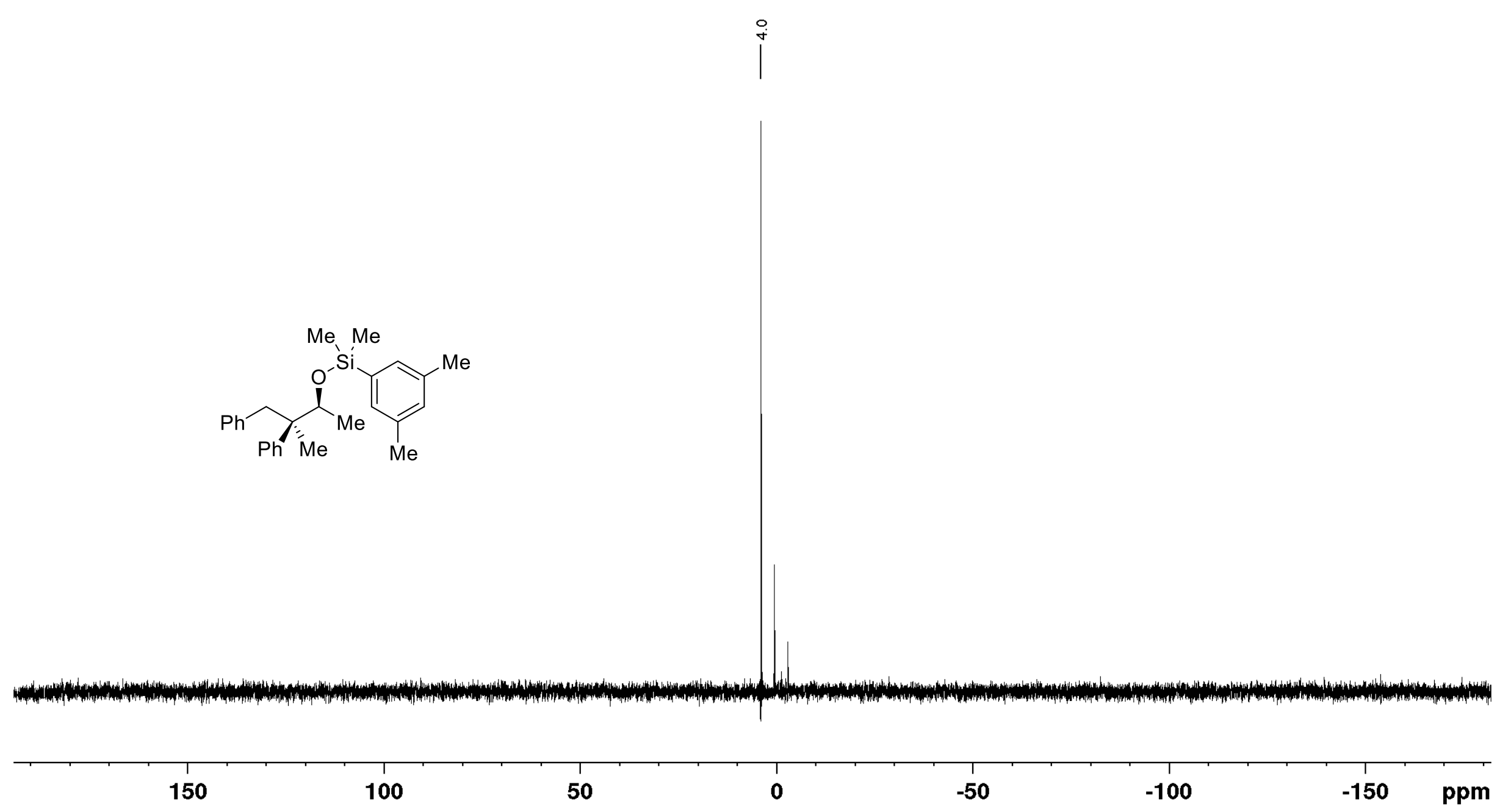


Figure 8.11 ${ }^{1} \mathrm{H}$ NMR (500 MHz, $\left.\mathrm{C}_{6} \mathrm{D}_{6}, 298 \mathrm{~K}\right)$ of (2S,3S)-Dimethyl((3-methyl-3,4-diphenylbutan-2-yl)oxy)(phenyl)silane [(2S,3S)$3 a b]$.

$$
\text { ○. }
$$

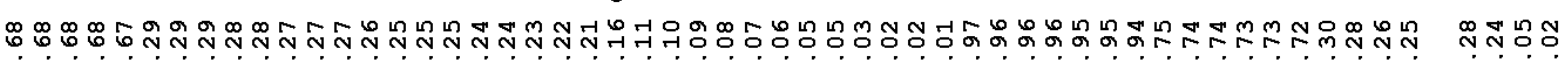

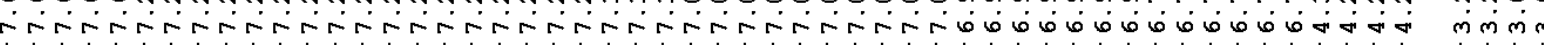

(1)
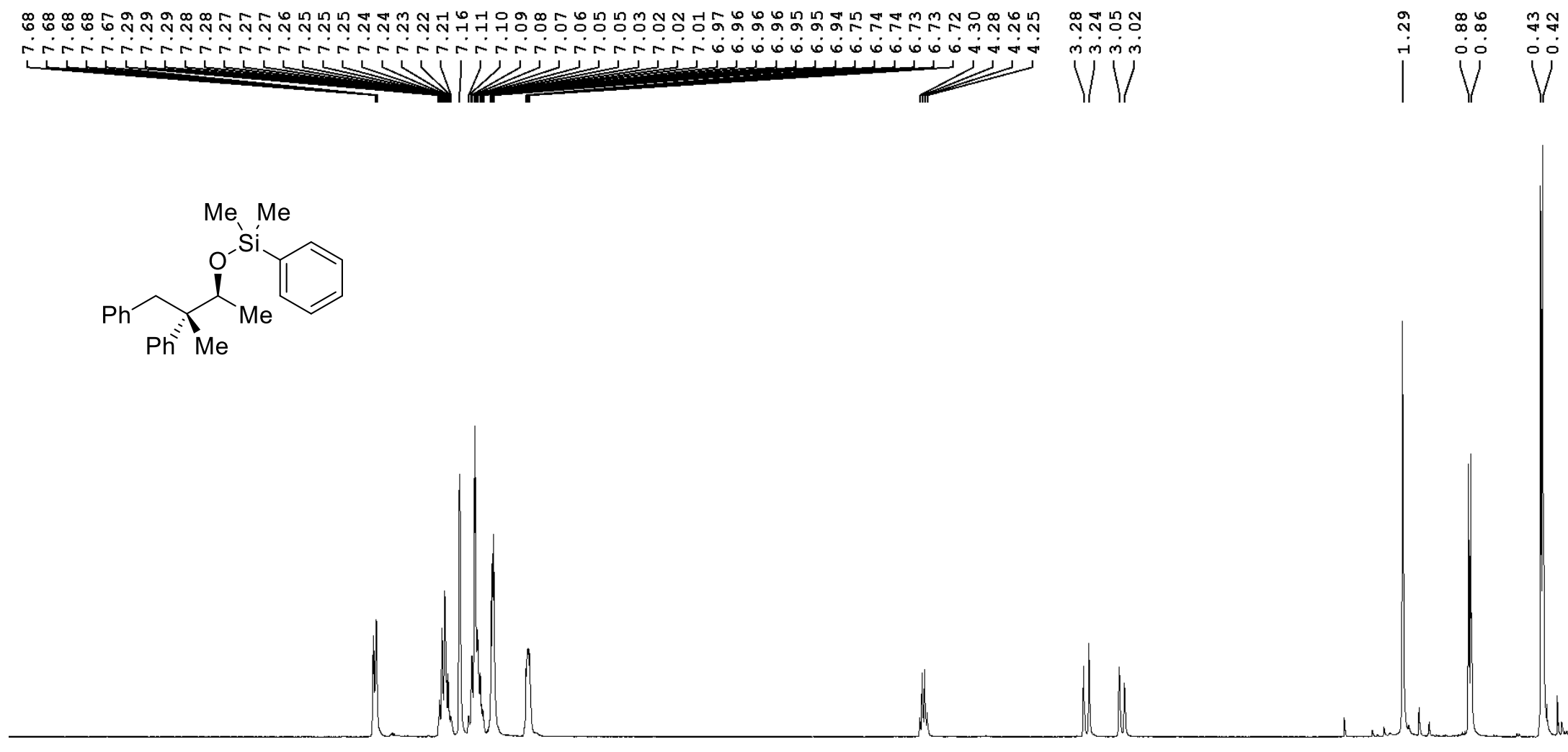

,

8

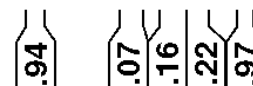

5

$\mid$
4

:
2

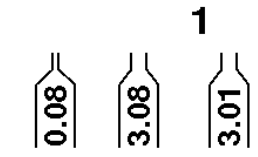

ppm

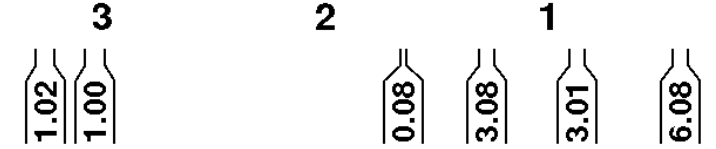


Figure 8.12 ${ }^{13} \mathrm{C}$ NMR (126 MHz, $\left.\mathrm{C}_{6} \mathrm{D}_{6}, 298 \mathrm{~K}\right)$ of (2S,3S)-Dimethyl((3-methyl-3,4-diphenylbutan-2-yl)oxy)(phenyl)silane [(2S,3S)$3 \mathrm{ab}]$.

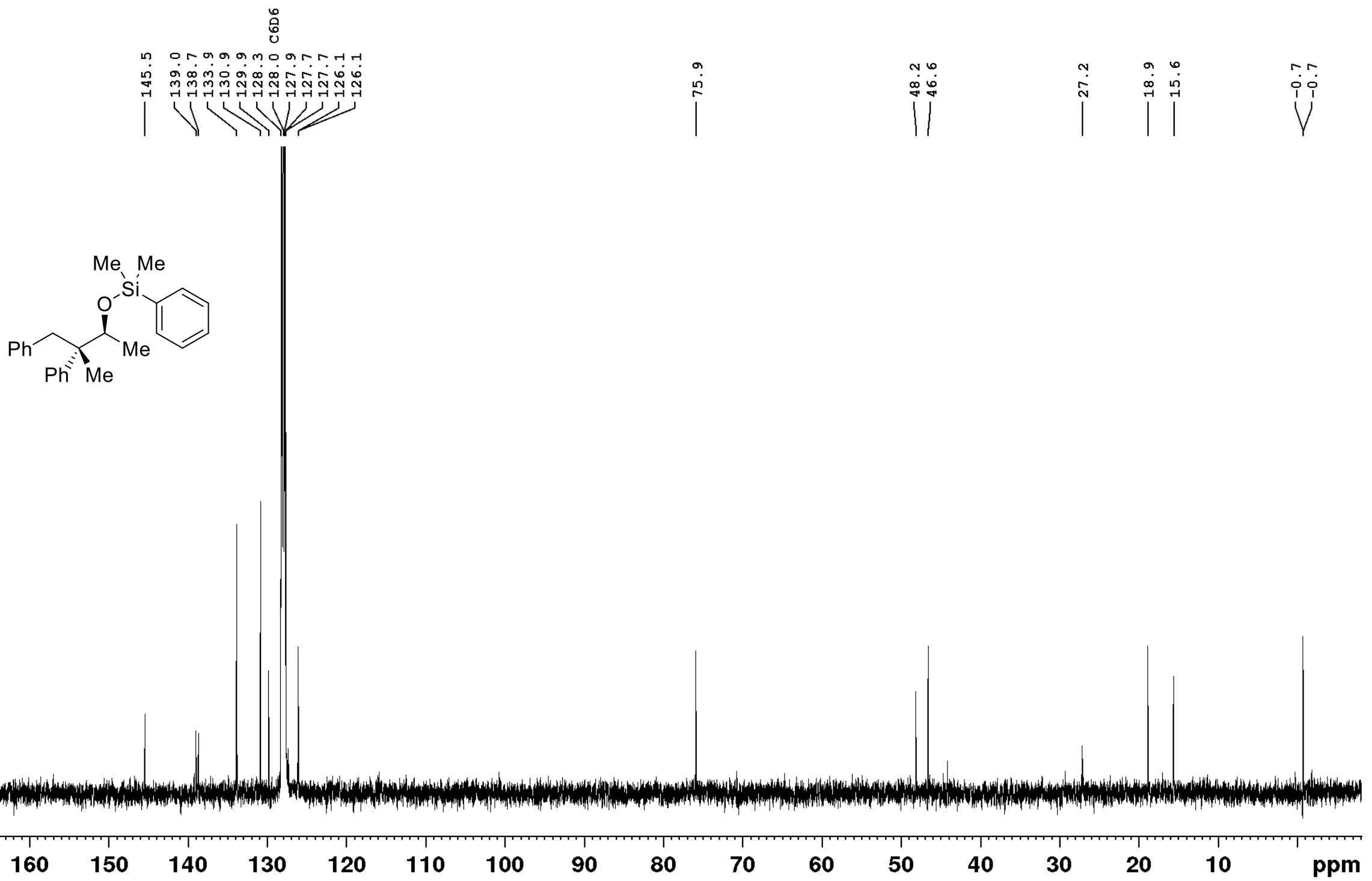


Figure 8.13 ${ }^{29} \mathrm{Si}$ DEPT NMR (99 MHz, $\left.\mathrm{C}_{6} \mathrm{D}_{6}, 298 \mathrm{~K}\right)$ of (2S,3S)-Dimethyl((3-methyl-3,4-diphenylbutan-2-yl)oxy)(phenyl)silane $[(2 S, 3 S)-3 a b]$.

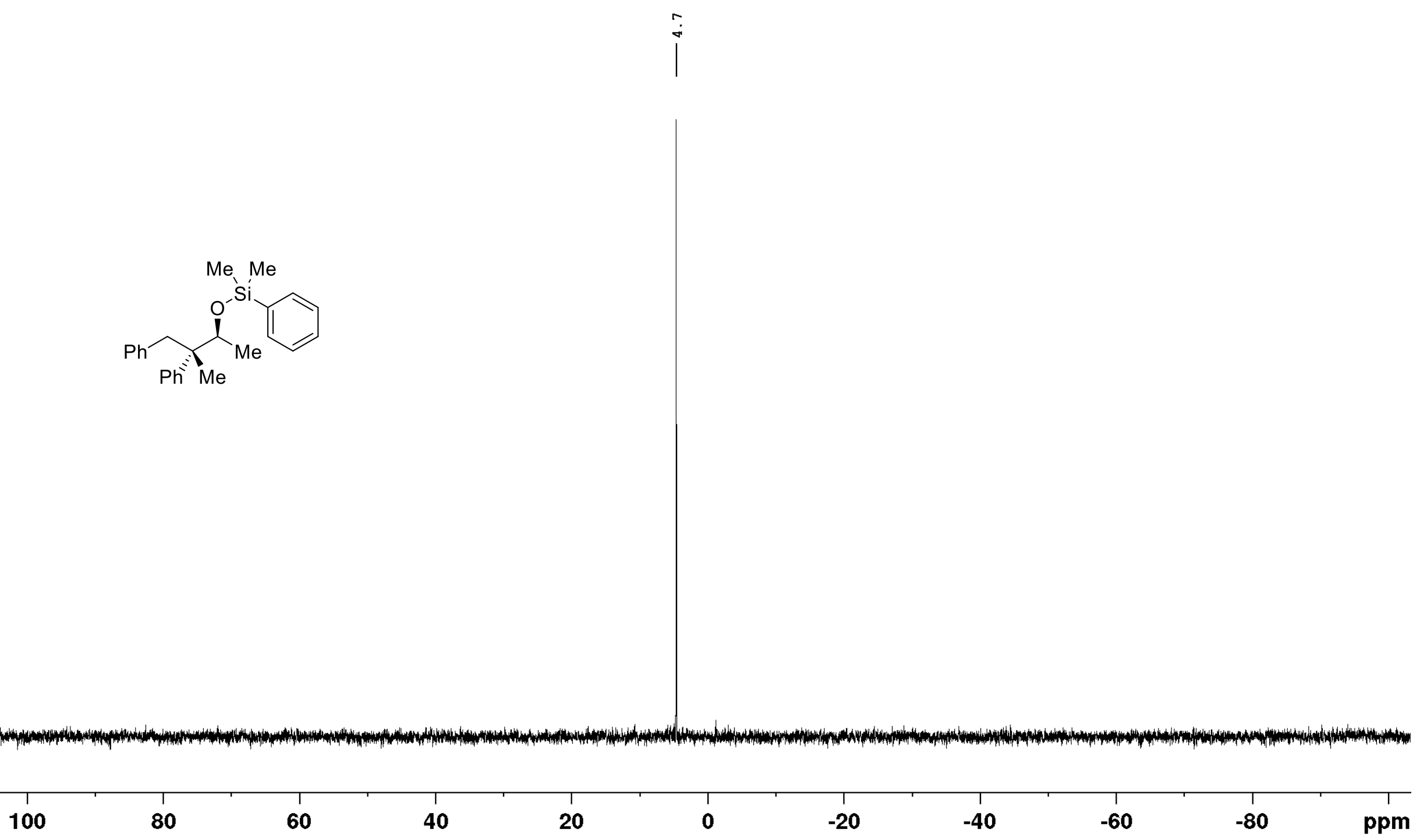


Figure 8.14 ${ }^{1} \mathrm{H}$ NMR (500 MHz, $\left.\mathrm{C}_{6} \mathrm{D}_{6}, 298 \mathrm{~K}\right)$ of (2S,3S)-Methyl((3-methyl-3,4-diphenylbutan-2-yl)oxy)diphenylsilane [(2S,3S)3ac].
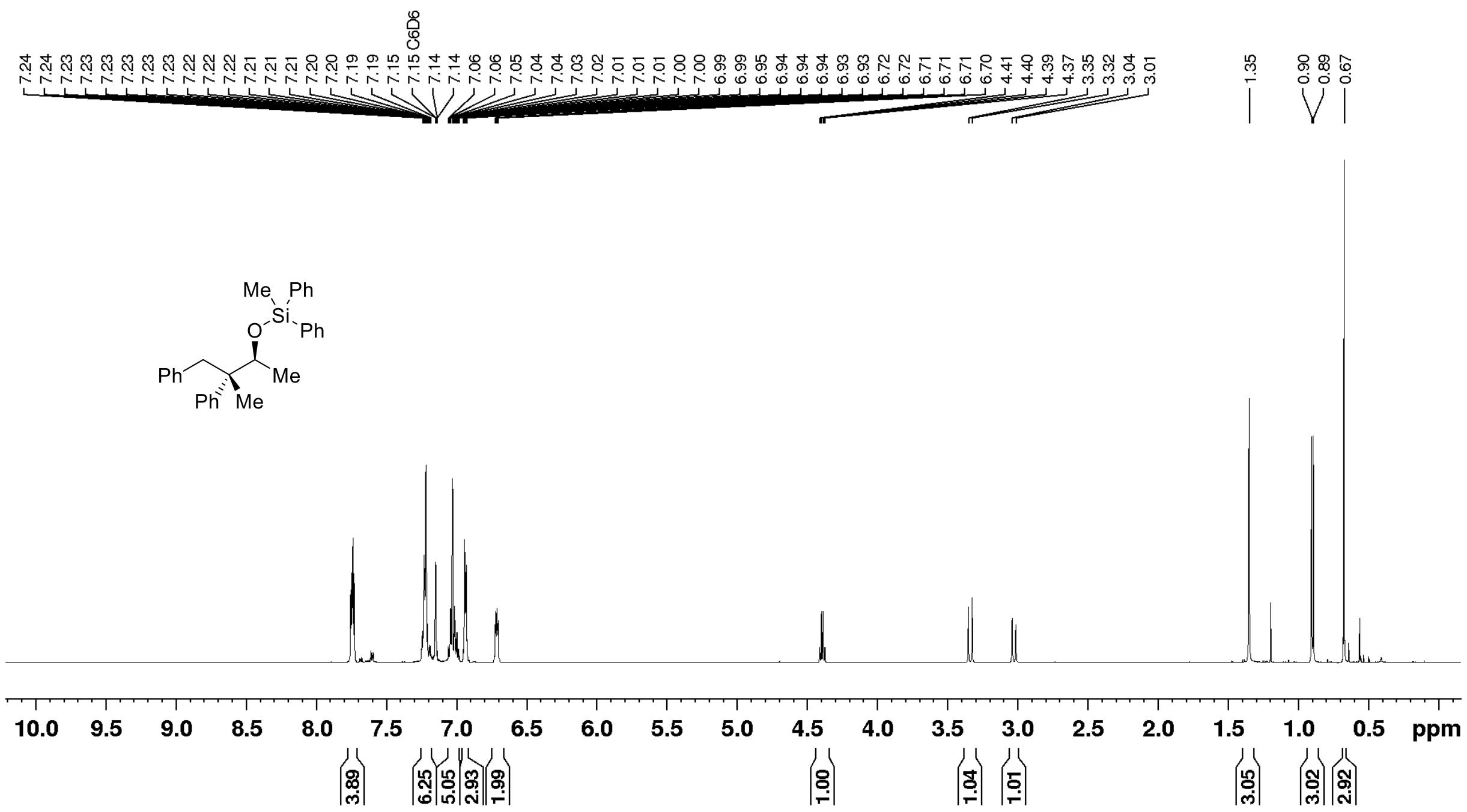
Figure 8.15 ${ }^{13} \mathrm{C}$ NMR (126 MHz, $\left.\mathrm{C}_{6} \mathrm{D}_{6}, 298 \mathrm{~K}\right)$ of (2S,3S)-Methyl((3-methyl-3,4-diphenylbutan-2-yl)oxy)diphenylsilane [(2S,3S)3ac].

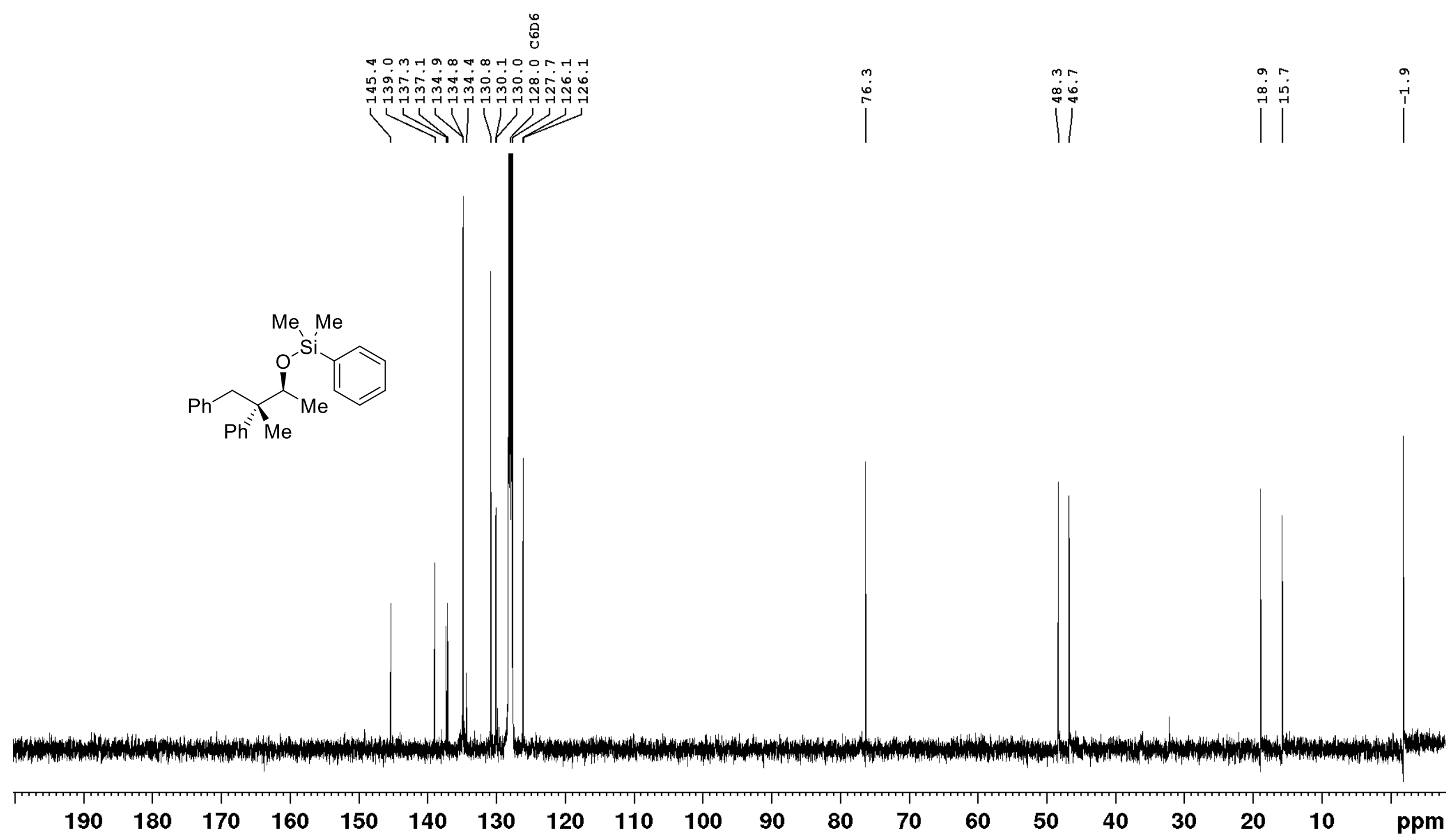


Figure 8.16 ${ }^{29} \mathrm{Si}$ DEPT NMR (99 MHz, CDCl $\left.3,298 \mathrm{~K}\right)$ of $(2 S, 3 S)$-Methyl((3-methyl-3,4-diphenylbutan-2-yl)oxy)diphenylsilane $[(2 S, 3 S)-3 a c]$.

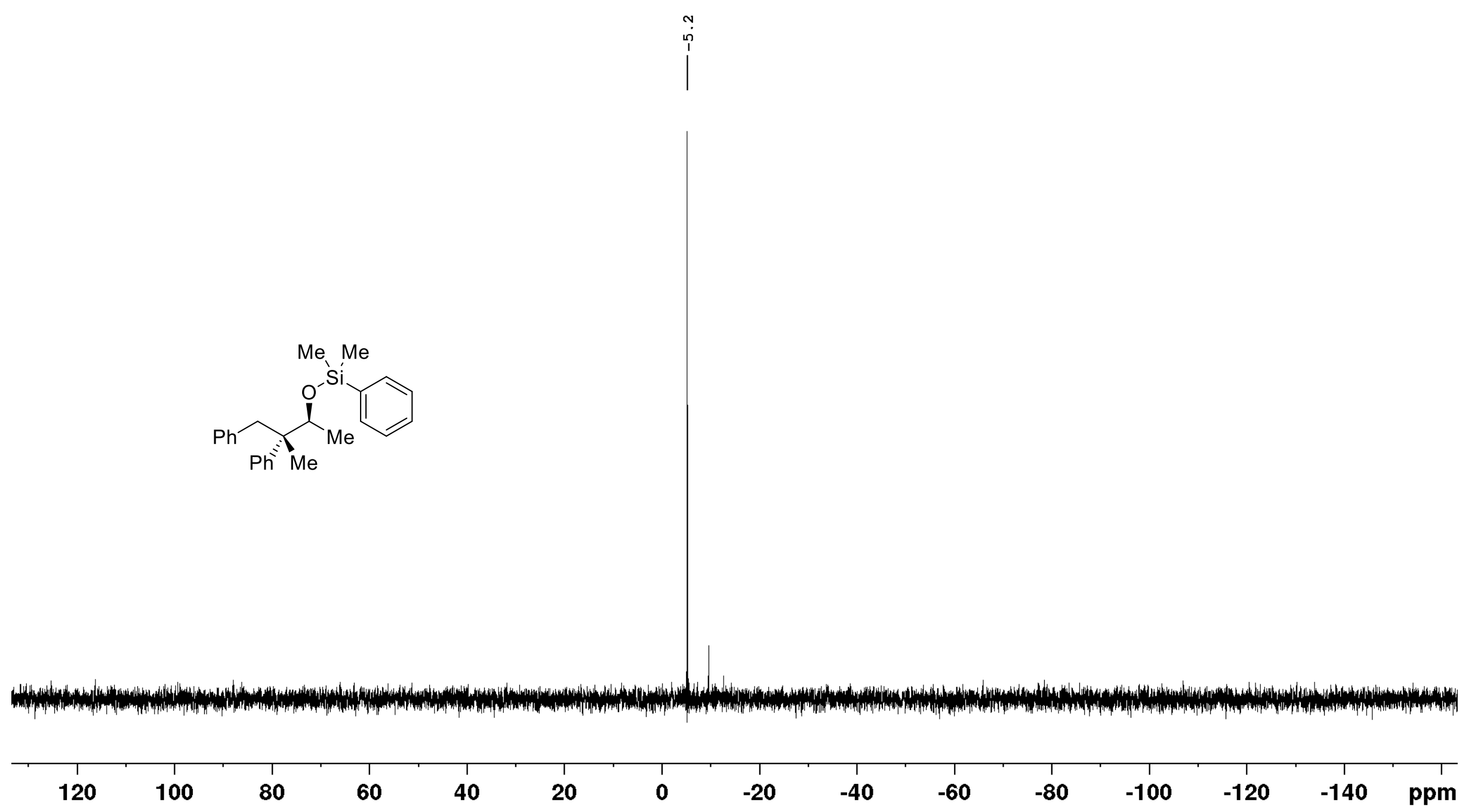


Figure 8.17 ${ }^{1} \mathrm{H}$ NMR (500 MHz, $\left.\mathrm{C}_{6} \mathrm{D}_{6}, 298 \mathrm{~K}\right)$ of (2S,3S)-((3-Methyl-3,4-diphenylbutan-2-yl)oxy)triphenylsilane [(2S,3S)-3ad].

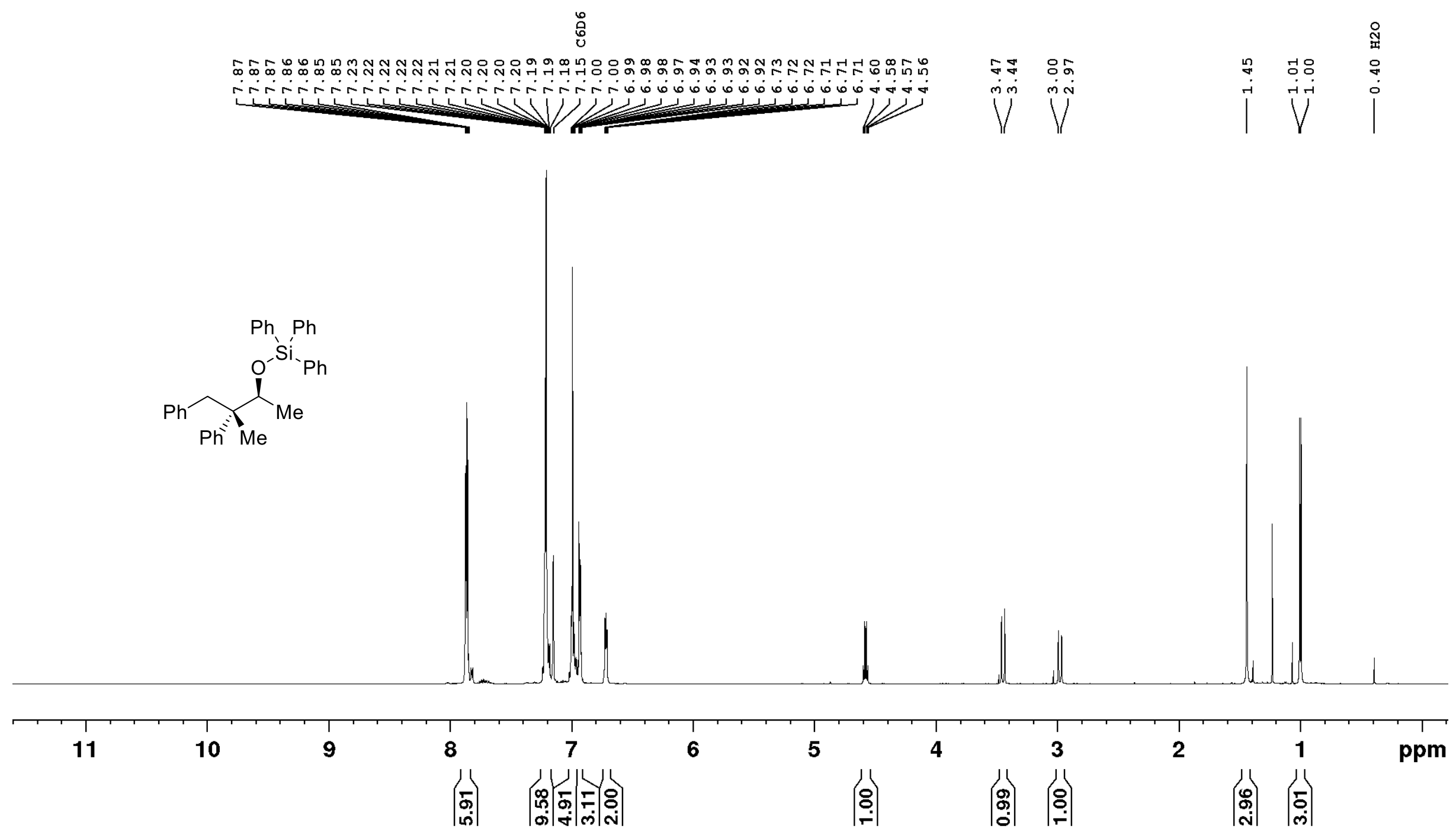


Figure 8.18 ${ }^{13} \mathrm{C}$ NMR (126 MHz, $\left.\mathrm{C}_{6} \mathrm{D}_{6}, 298 \mathrm{~K}\right)$ of (2S,3S)-((3-Methyl-3,4-diphenylbutan-2-yl)oxy)triphenylsilane [(2S,3S)-3ad].

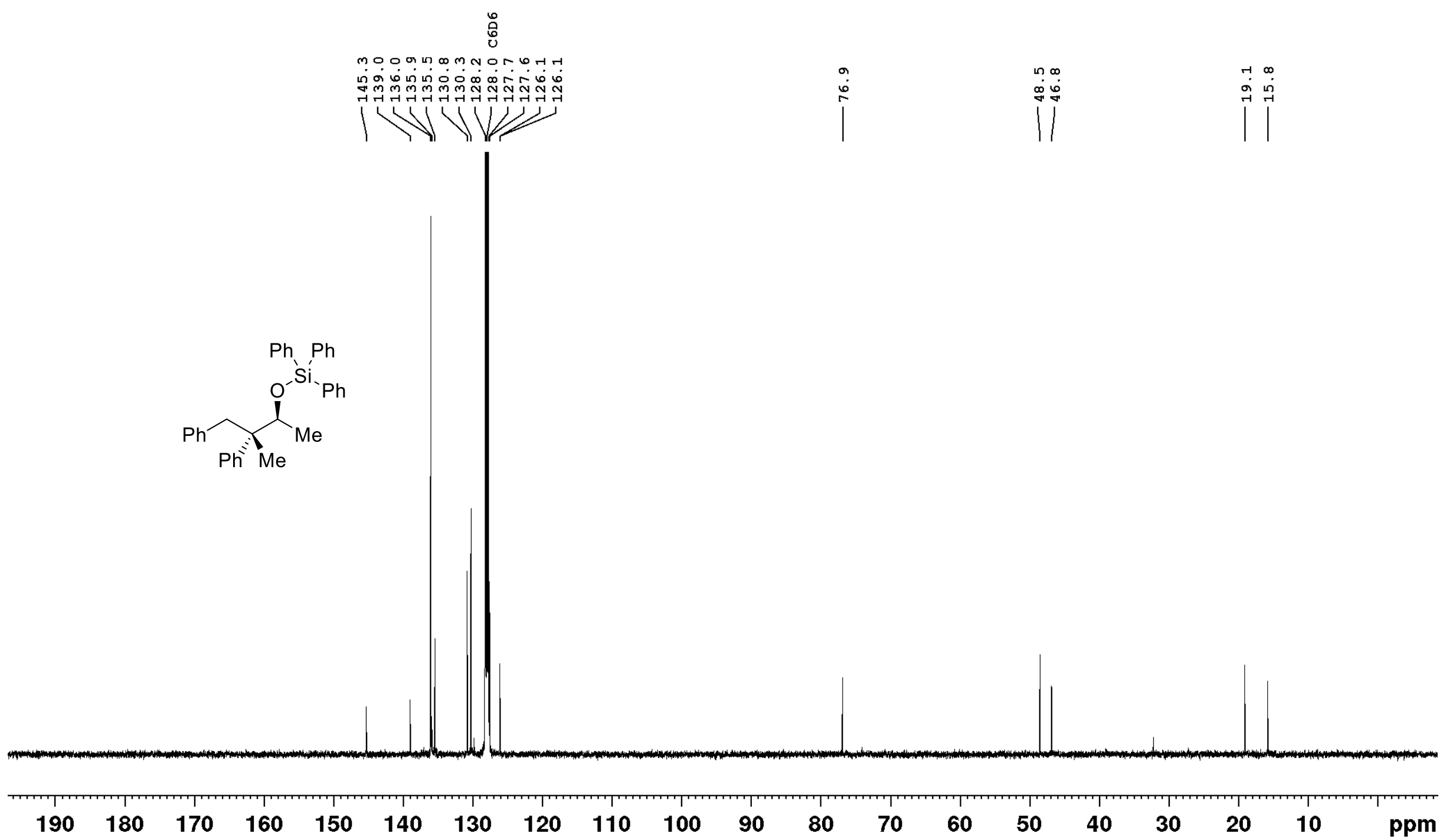


Figure 8.19 ${ }^{29} \mathrm{Si} \mathrm{DEPT} \mathrm{NMR}\left(99 \mathrm{MHz}, \mathrm{CDCl}_{3}, 298 \mathrm{~K}\right)$ of $(2 S, 3 S)-((3-M e t h y l-3,4-$ diphenylbutan-2-yl)oxy)triphenylsilane [(2S,3S)3ad].
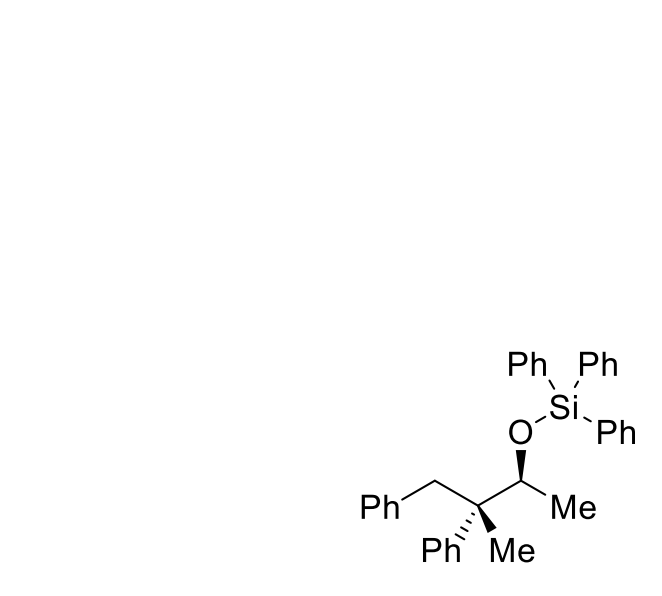

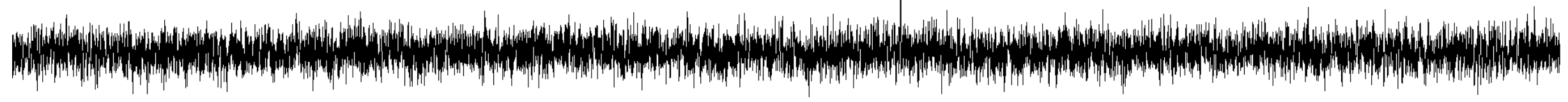

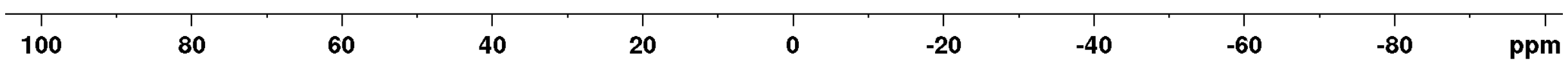


Figure 8.20 ${ }^{1} \mathrm{H}$ NMR (500 MHz, $\left.\mathrm{C}_{6} \mathrm{D}_{6}, 298 \mathrm{~K}\right)$ of (2S,3S)-Diethyl((3-methyl-3,4-diphenylbutan-2-yl)oxy)(phenyl)silane [(2S,3S)3ai].

$$
\stackrel{\circ}{0}
$$

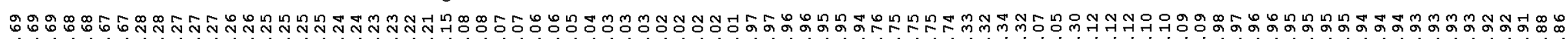

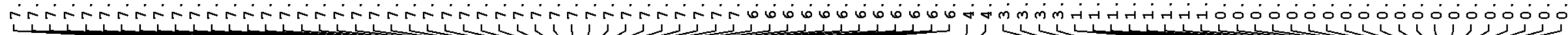
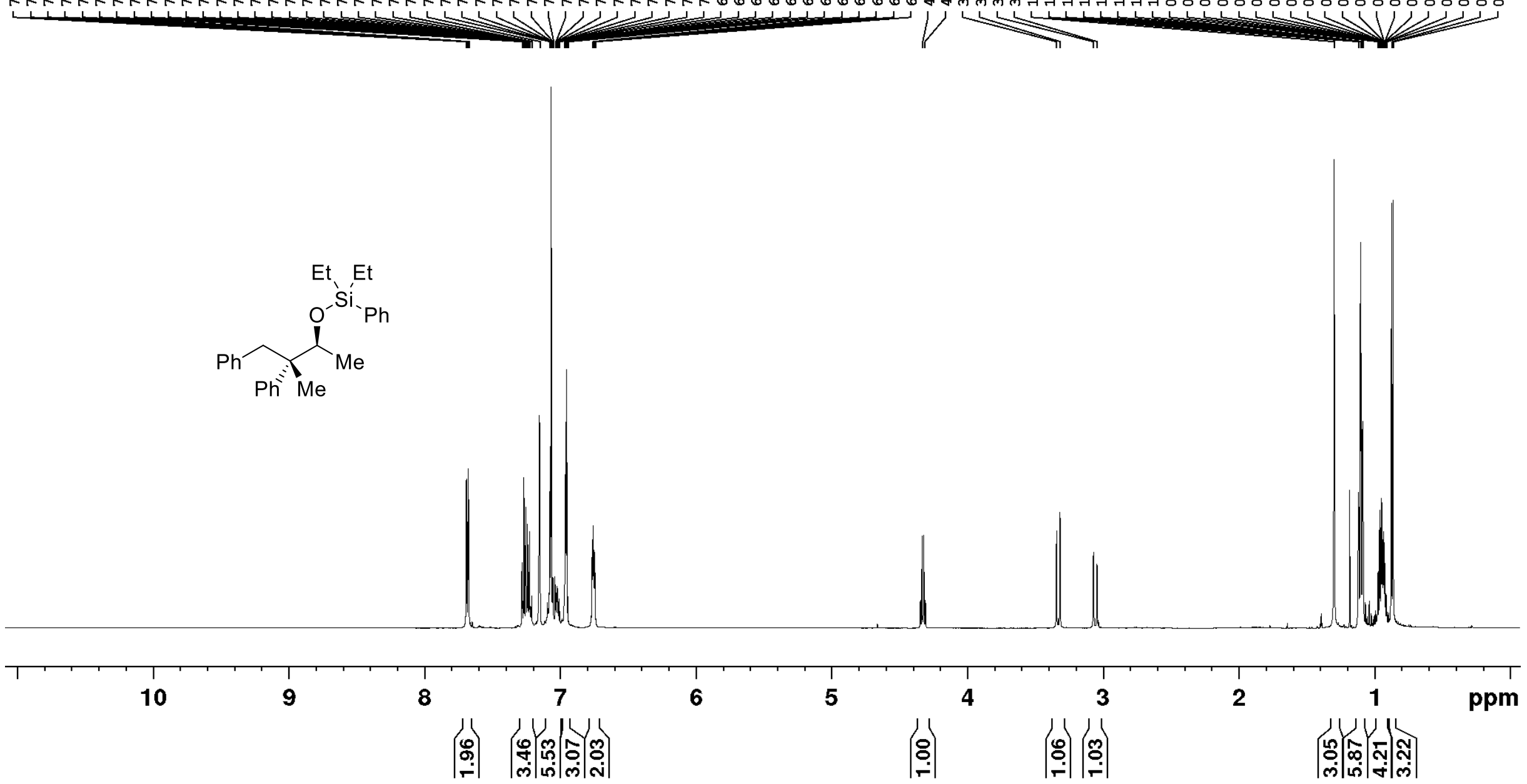
Figure 8.21 ${ }^{13} \mathrm{C}$ NMR (126 MHz, $\left.\mathrm{C}_{6} \mathrm{D}_{6}, 298 \mathrm{~K}\right)$ of (2S,3S)-Diethyl((3-methyl-3,4-diphenylbutan-2-yl)oxy)(phenyl)silane [(2S,3S)3ai].

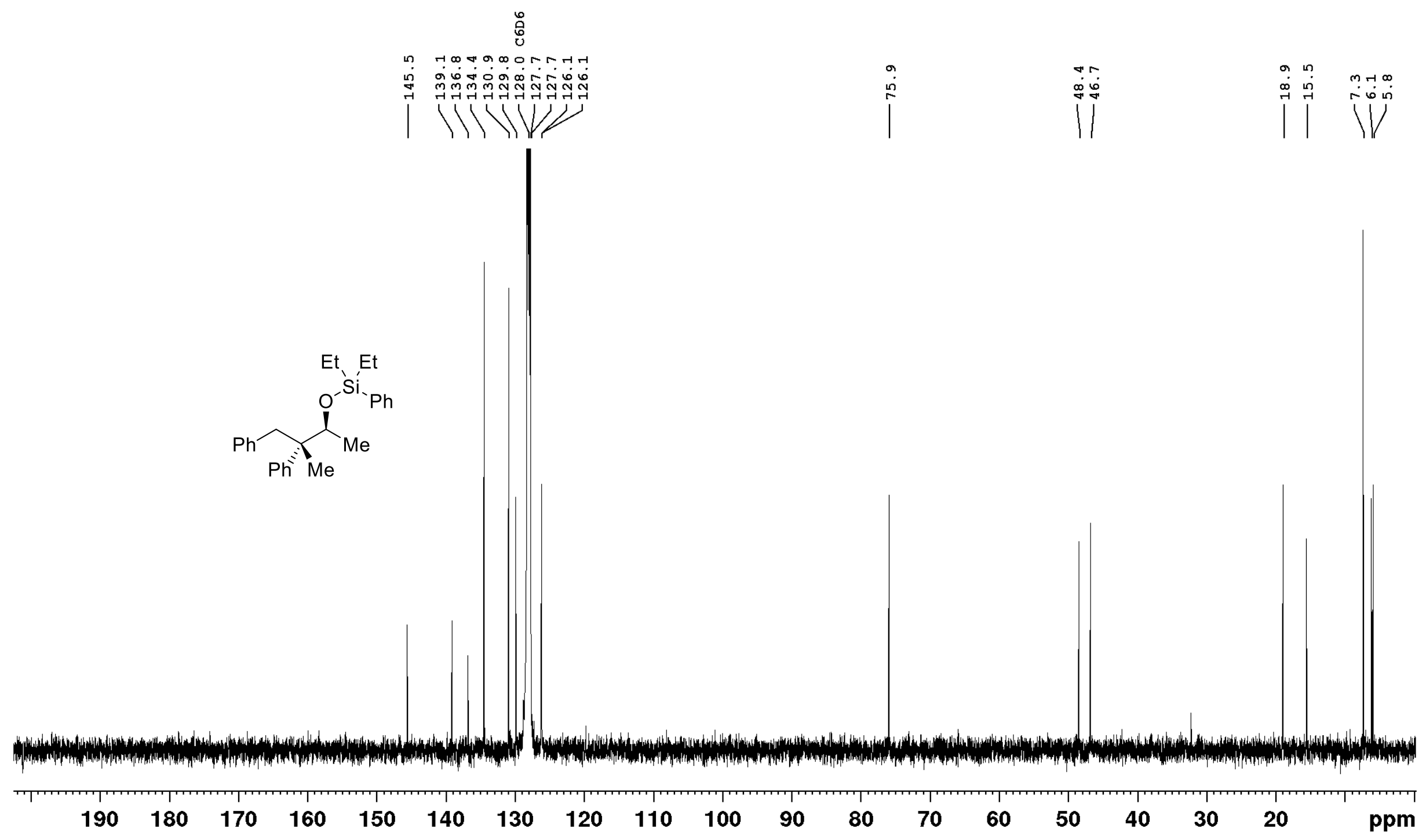


Figure 8.22 ${ }^{29} \mathrm{Si}$ DEPT NMR (99 MHz, $\left.\mathrm{CDCl}_{3}, 298 \mathrm{~K}\right)$ of (2S,3S)-Diethyl((3-methyl-3,4-diphenylbutan-2-yl)oxy)(phenyl)silane [(2S,3S)-3ai].

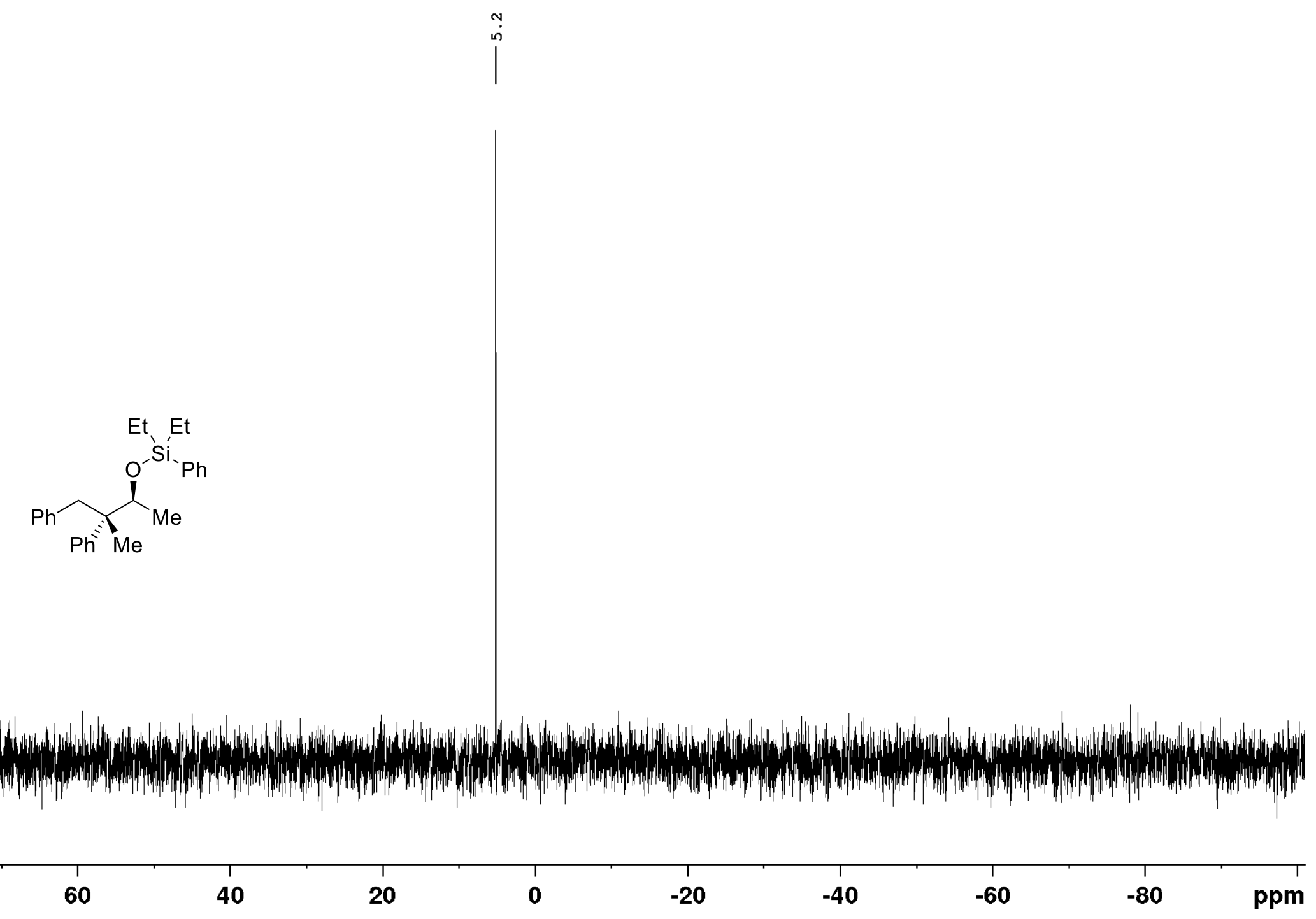


Figure 8.23 ${ }^{1} \mathrm{H}$ NMR (500 MHz, $\left.\mathrm{C}_{6} \mathrm{D}_{6}, 298 \mathrm{~K}\right)$ of (2S,3S)-Mesityldimethyl((3-methyl-3,4-diphenylbutan-2-yl)oxy)silane [(2S,3S)3af].

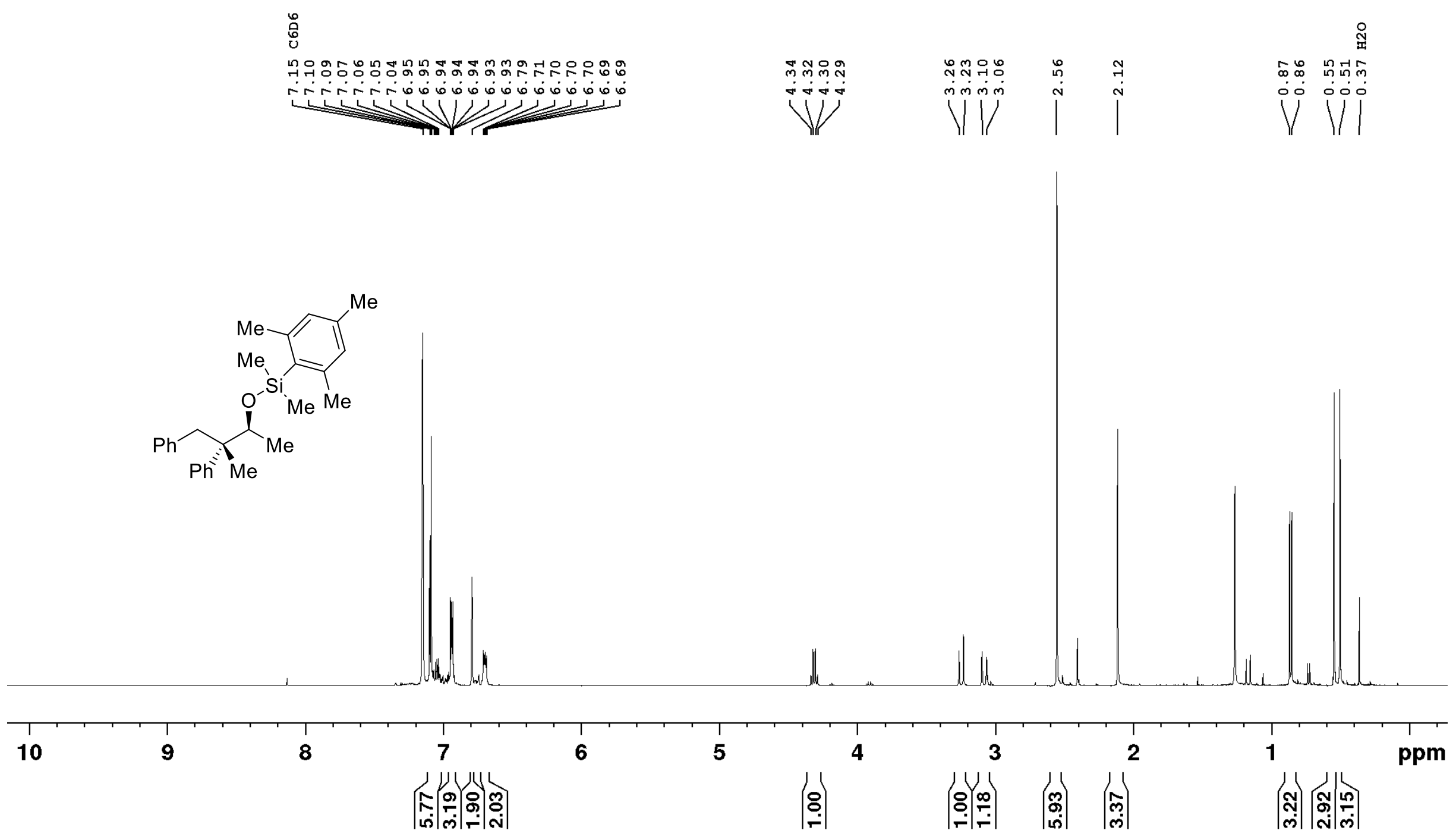


Figure 8.24 ${ }^{13} \mathrm{C}$ NMR (126 MHz, $\left.\mathrm{C}_{6} \mathrm{D}_{6}, 298 \mathrm{~K}\right)$ of (2S,3S)-Mesityldimethyl((3-methyl-3,4-diphenylbutan-2-yl)oxy)silane [(2S,3S)3af].

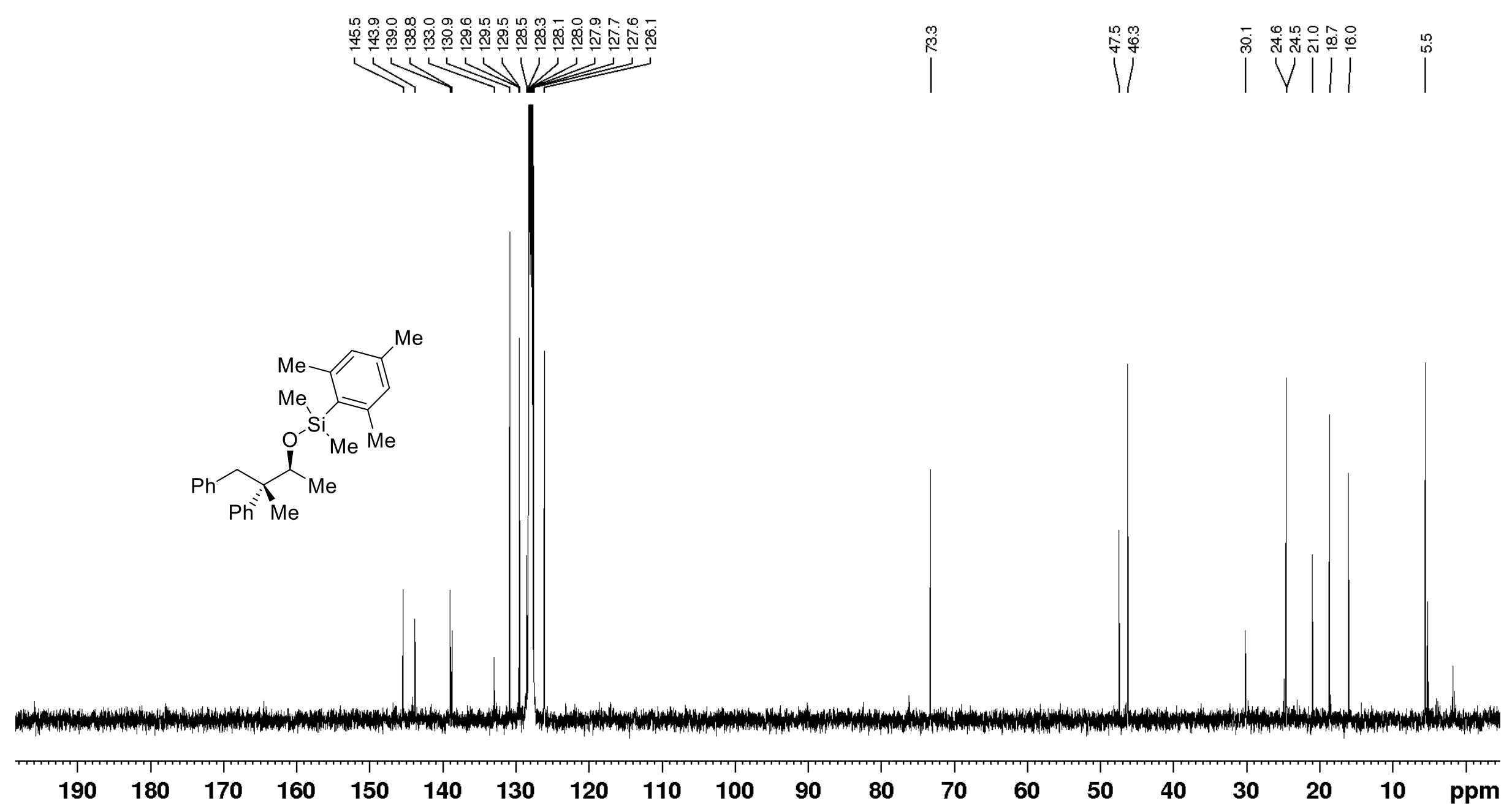


Figure 8.25 ${ }^{29} \mathrm{Si}$ DEPT NMR (99 MHz, $\left.\mathrm{CDCl}_{3}, 298 \mathrm{~K}\right)$ of (2S,3S)-Diethyl((3-methyl-3,4-diphenylbutan-2-yl)oxy)(phenyl)silane [(2S,3S)-3af].

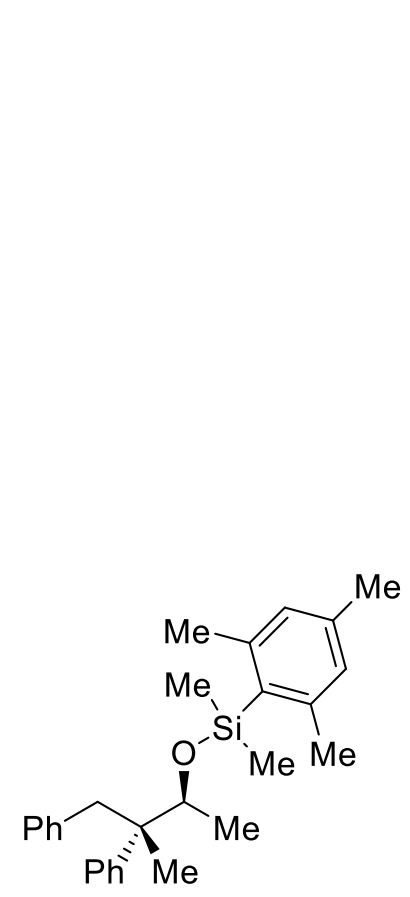

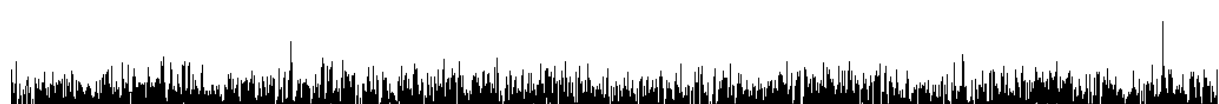

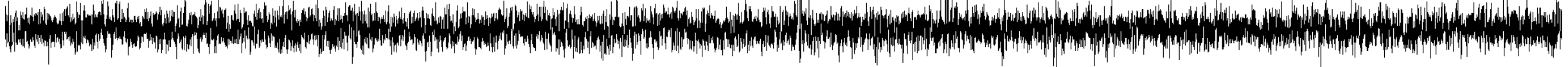

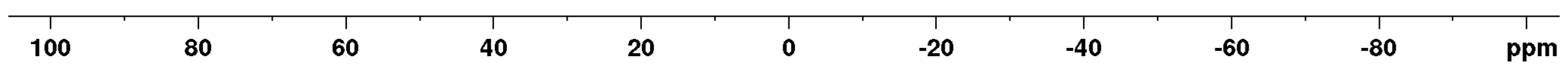


Figure 8.26 ${ }^{1} \mathrm{H}$ NMR (500 MHz, $\left.\mathrm{C}_{6} \mathrm{D}_{6}, 298 \mathrm{~K}\right)$ of (2S,3S)-Dimethyl((3-methyl-3,4-diphenylbutan-2-yl)oxy)(naphthalen-1-yl)silane $[(2 S, 3 S)-3 a k]$.

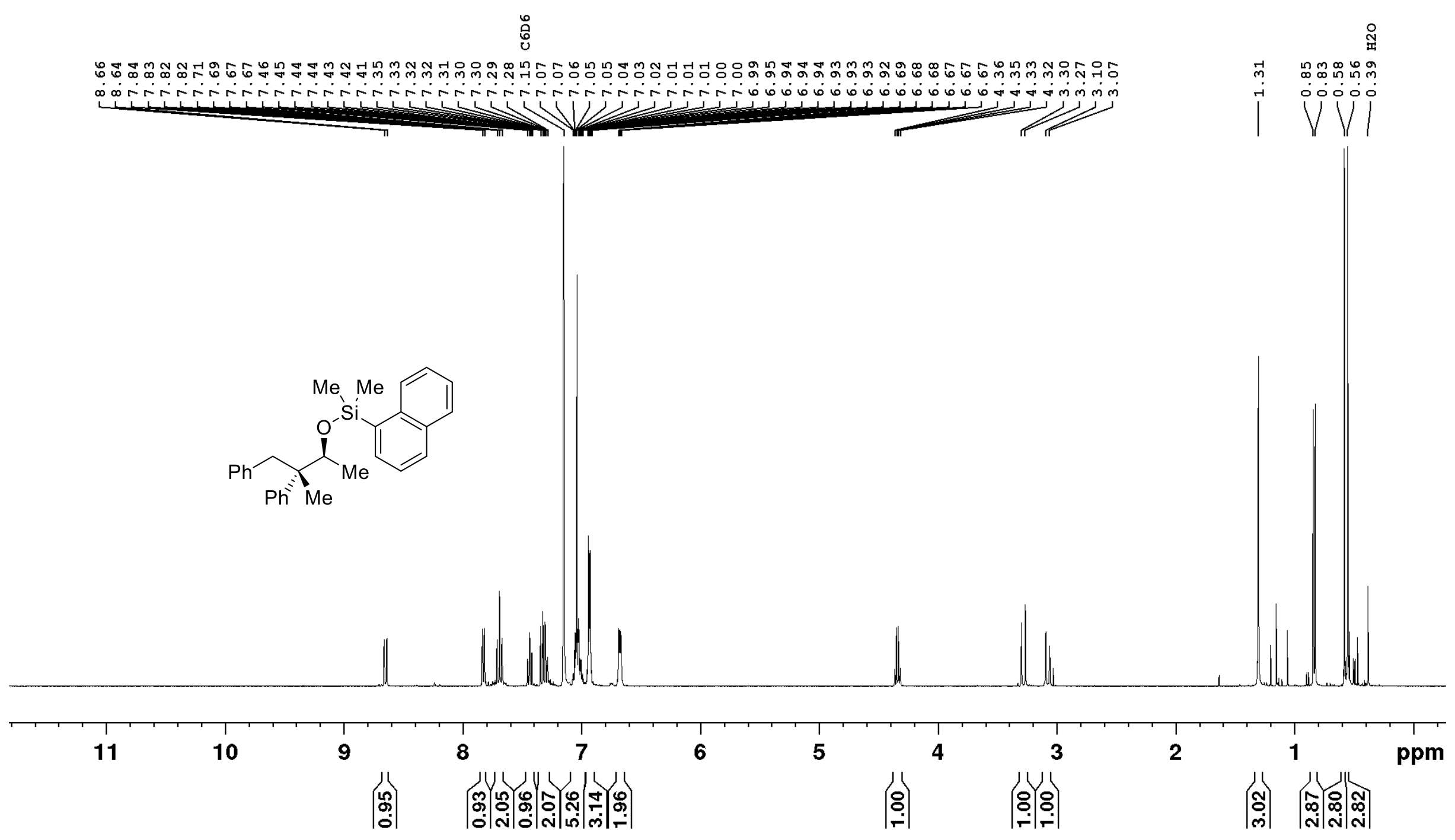


Figure 8.27 ${ }^{13} \mathrm{C}$ NMR (126 MHz, $\left.\mathrm{C}_{6} \mathrm{D}_{6}, 298 \mathrm{~K}\right)$ of (2S,3S)-Dimethyl((3-methyl-3,4-diphenylbutan-2-yl)oxy)(naphthalen-1-yl)silane $[(2 S, 3 S)-3 a k]$.

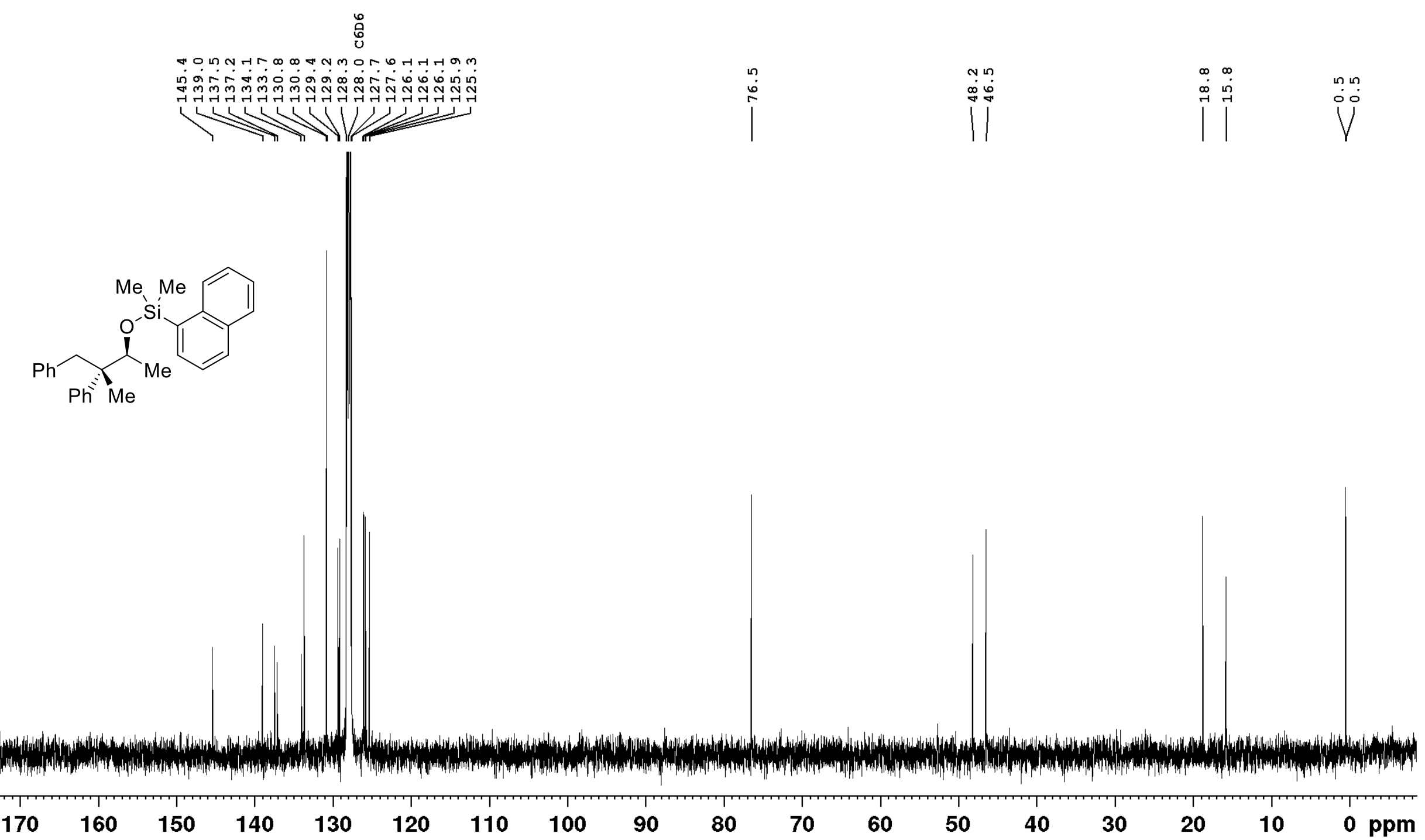


Figure 8.28 $\left.{ }^{29} \mathrm{Si} \mathrm{DEPT} \mathrm{NMR} \mathrm{(99} \mathrm{MHz,} \mathrm{CDCl} 3,298 \mathrm{~K}\right)$ of (2S,3S)-Dimethyl((3-methyl-3,4-diphenylbutan-2-yl)oxy)(naphthalen-1yl)silane $[(2 S, 3 S)-3 a k]$.

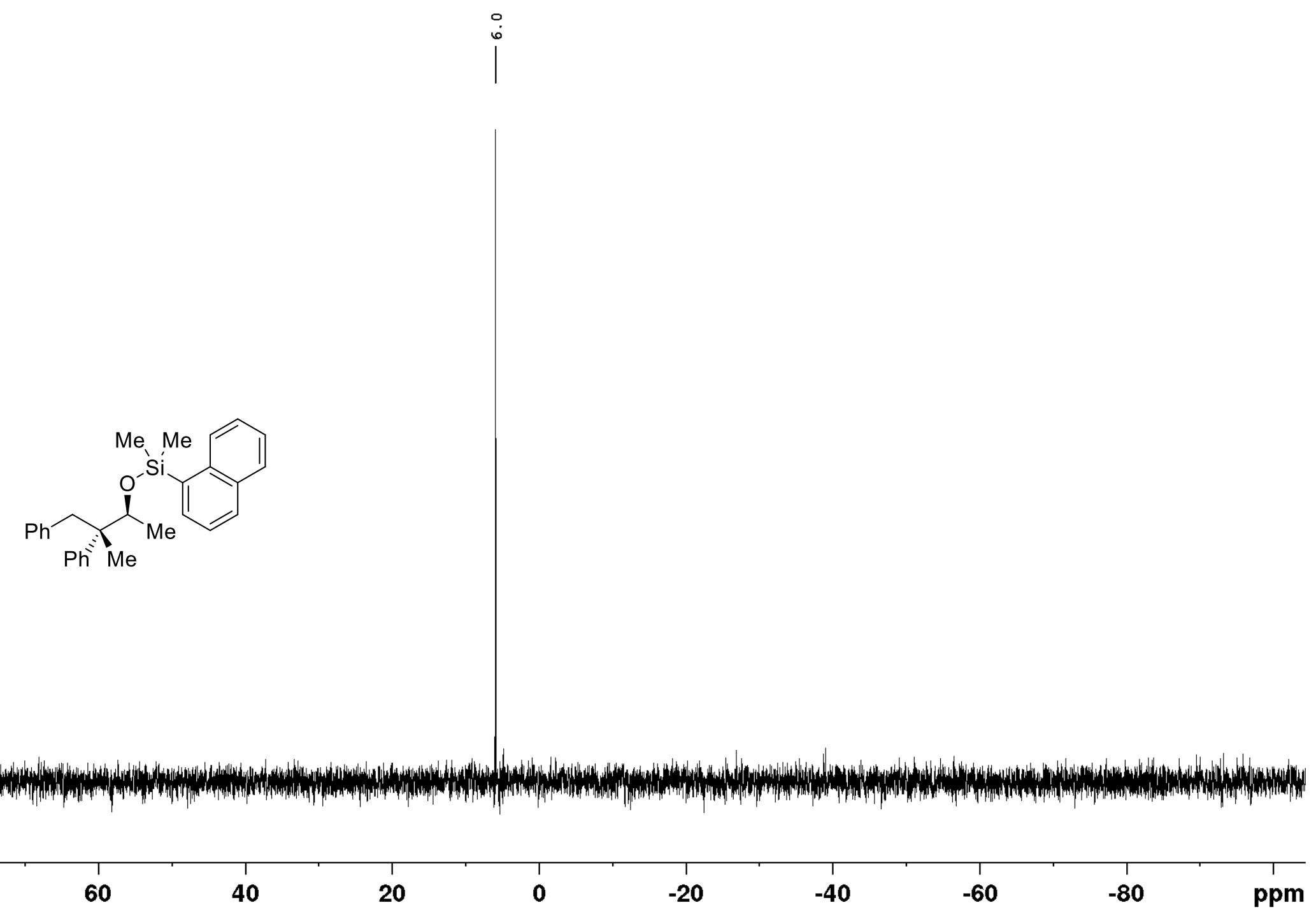

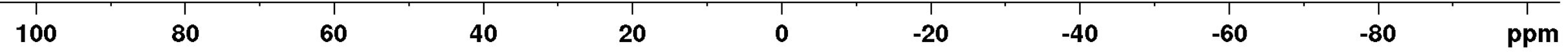


Figure 8.29 ${ }^{1} \mathrm{H}$ NMR (500 MHz, $\left.\mathrm{C}_{6} \mathrm{D}_{6}, 298 \mathrm{~K}\right)$ of (2S,3S)-Dimethyl((3-methyl-3,4-diphenylbutan-2-yl)oxy)(naphthalen-2-yl)silane $[(2 S, 3 S)-3 a h]$.

$\stackrel{\circ}{0}$

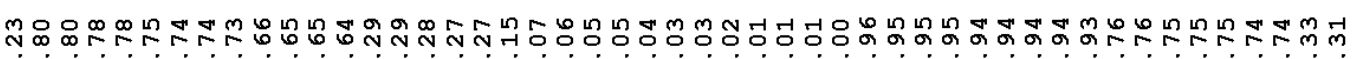

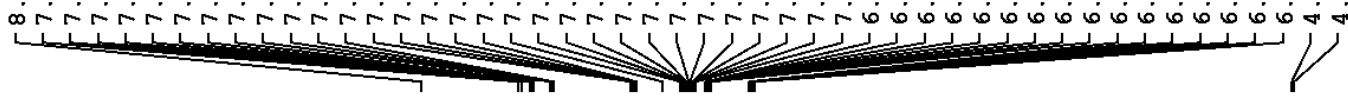

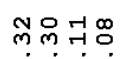

$\operatorname{linj}^{2}$

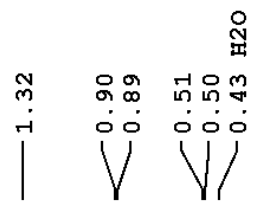

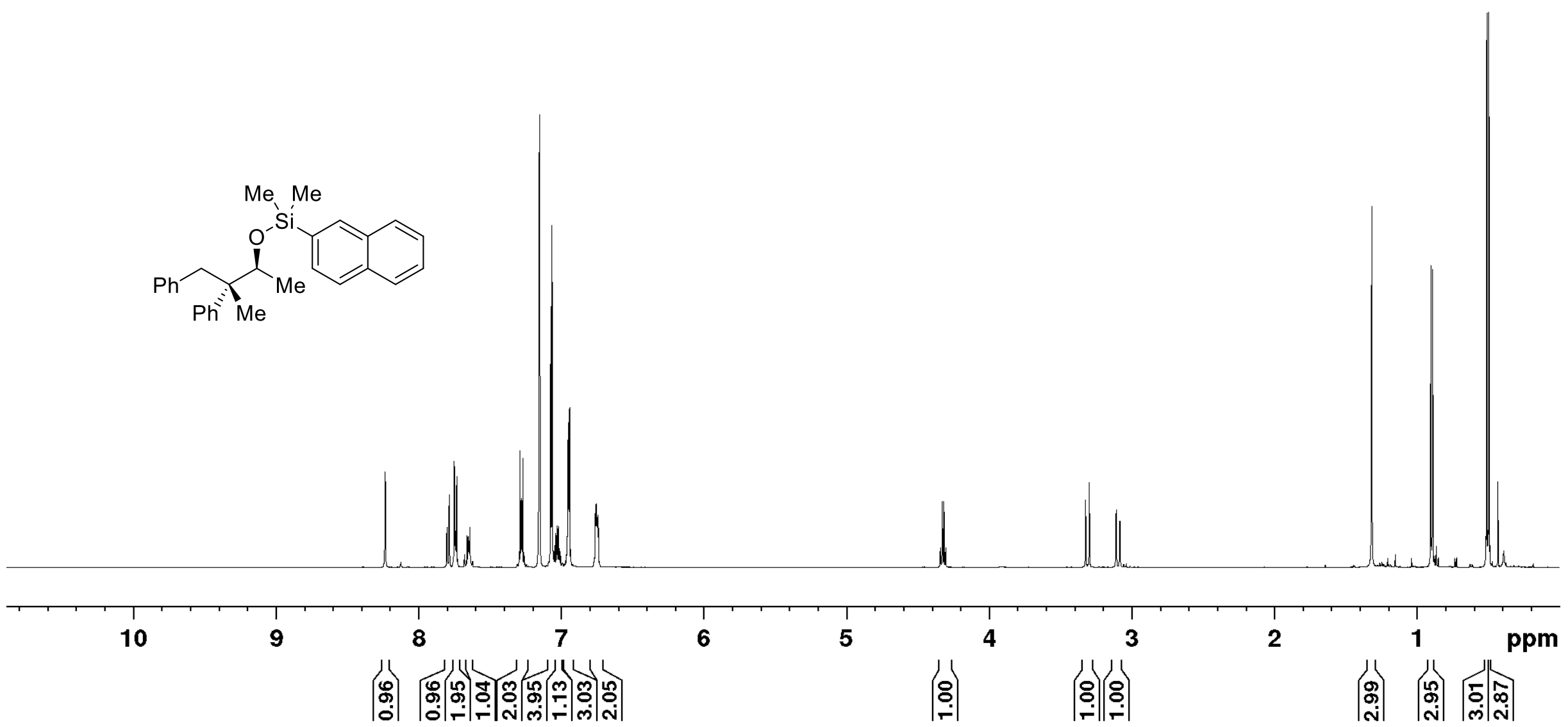


Figure 8.30 ${ }^{13} \mathrm{C}$ NMR (126 MHz, $\left.\mathrm{C}_{6} \mathrm{D}_{6}, 298 \mathrm{~K}\right)$ of (2S,3S)-Dimethyl((3-methyl-3,4-diphenylbutan-2-yl)oxy)(naphthalen-2-yl)silane $[(2 S, 3 S)-3 a h]$.

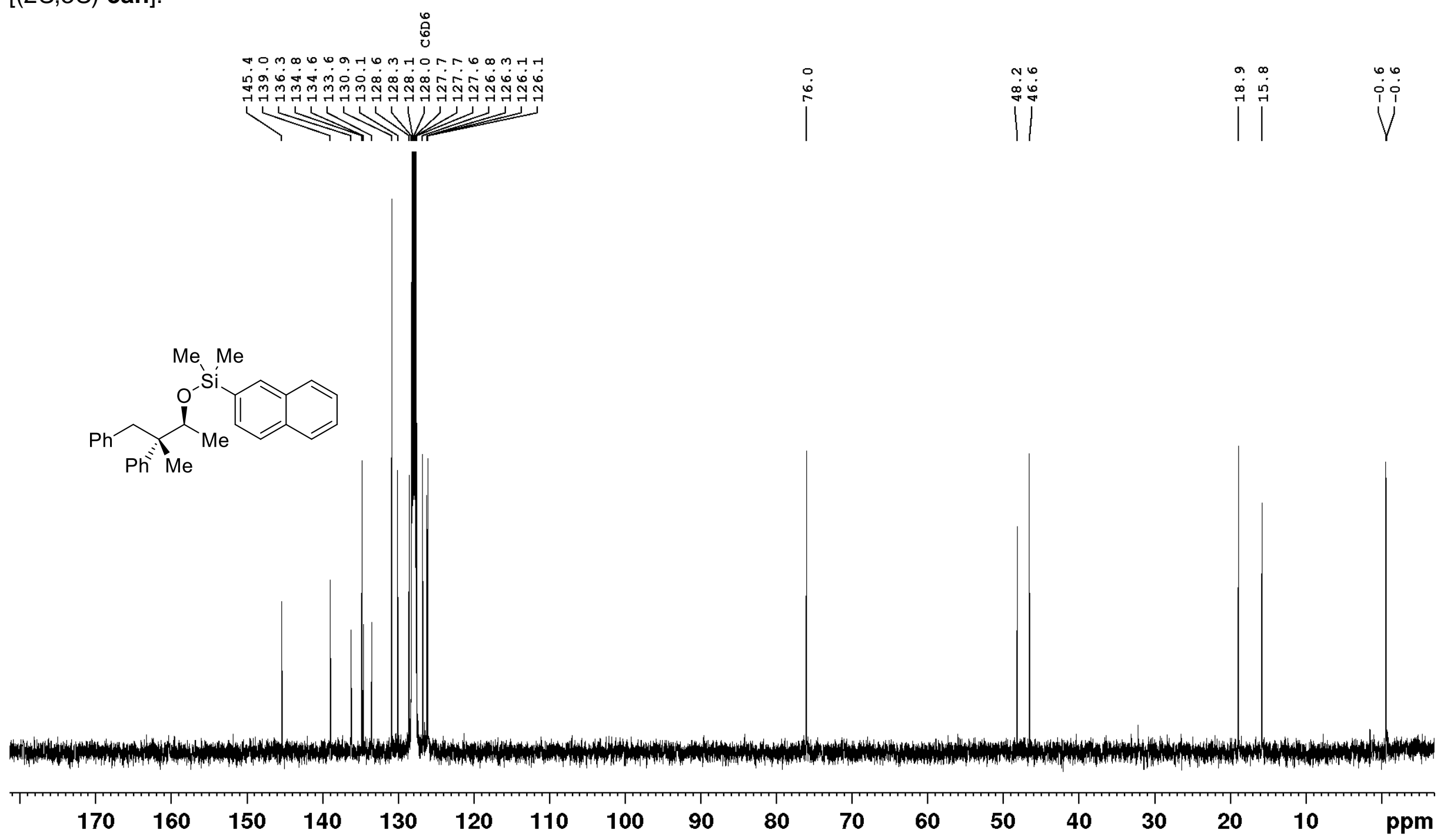


Figure 8.31 ${ }^{29} \mathrm{Si}$ DEPT NMR (99 MHz, $\left.\mathrm{CDCl}_{3}, 298 \mathrm{~K}\right)$ of (2S,3S)-Dimethyl((3-methyl-3,4-diphenylbutan-2-yl)oxy)(naphthalen-2yl)silane $[(2 S, 3 S)-3 a h]$.

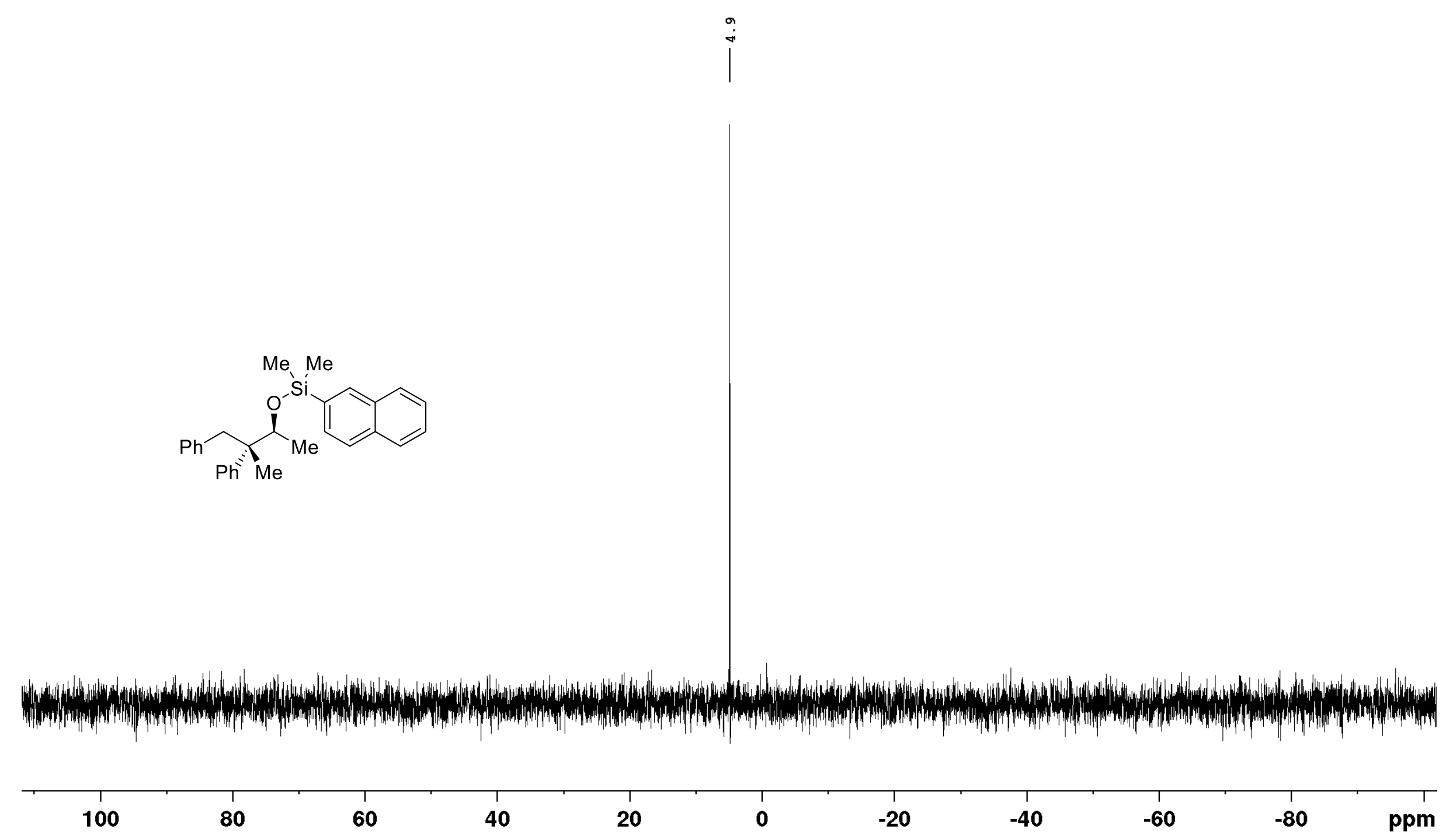


Figure 8.32 ${ }^{1} \mathrm{H}$ NMR (500 MHz, $\left.\mathrm{C}_{6} \mathrm{D}_{6}, 298 \mathrm{~K}\right)$ of $(2 S, 3 S)$-(4-(tert-Butyl)phenyl)dimethyl((3-methyl-3,4-diphenylbutan-2yl)oxy)silane $[(2 S, 3 S)-3 a g]$.
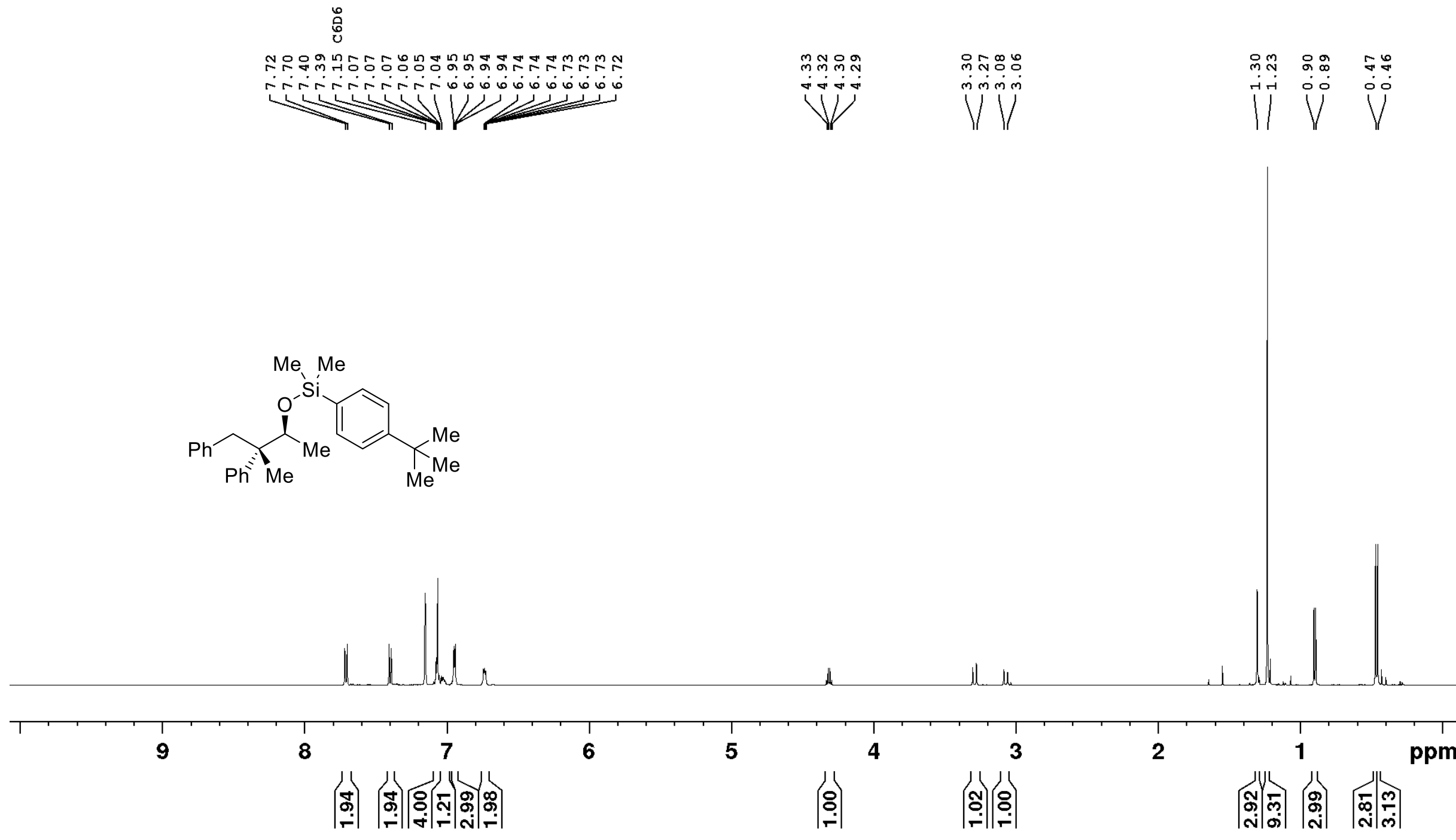
Figure 8.33 ${ }^{13} \mathrm{C}$ NMR (126 MHz, $\left.\mathrm{C}_{6} \mathrm{D}_{6}, 298 \mathrm{~K}\right)$ of $(2 S, 3 S)$-(4-(tert-Butyl)phenyl)dimethyl((3-methyl-3,4-diphenylbutan-2yl)oxy)silane $[(2 S, 3 S)-3 a g]$.

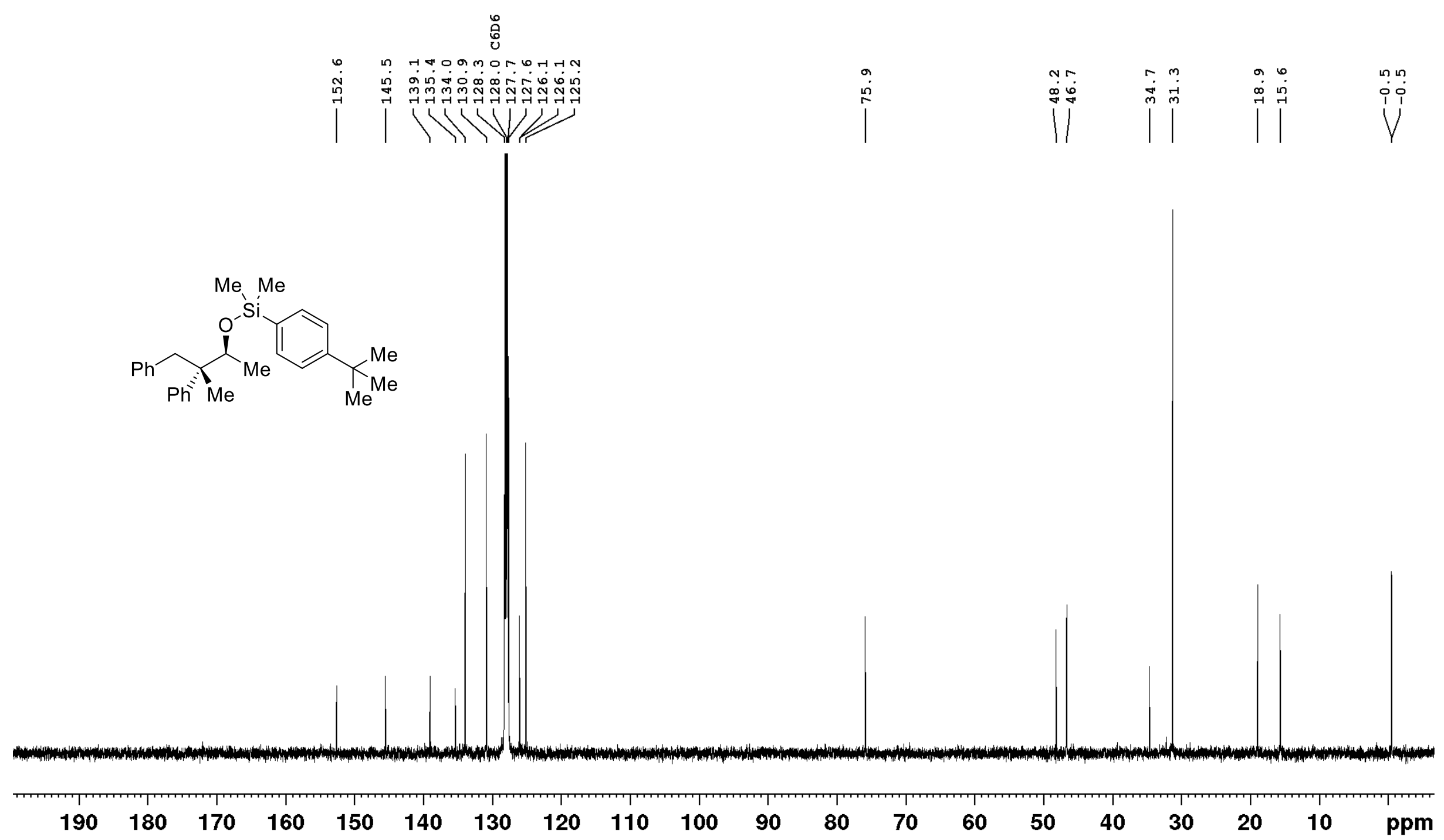


Figure 8.34 ${ }^{29} \mathrm{Si}$ DEPT NMR (99 MHz, $\left.\mathrm{C}_{6} \mathrm{D}_{6}, 298 \mathrm{~K}\right)$ of $(2 S, 3 S)$-(4-(tert-Butyl)phenyl)dimethyl((3-methyl-3,4-diphenylbutan-2yl)oxy)silane $[(2 S, 3 S)-3 a g]$.

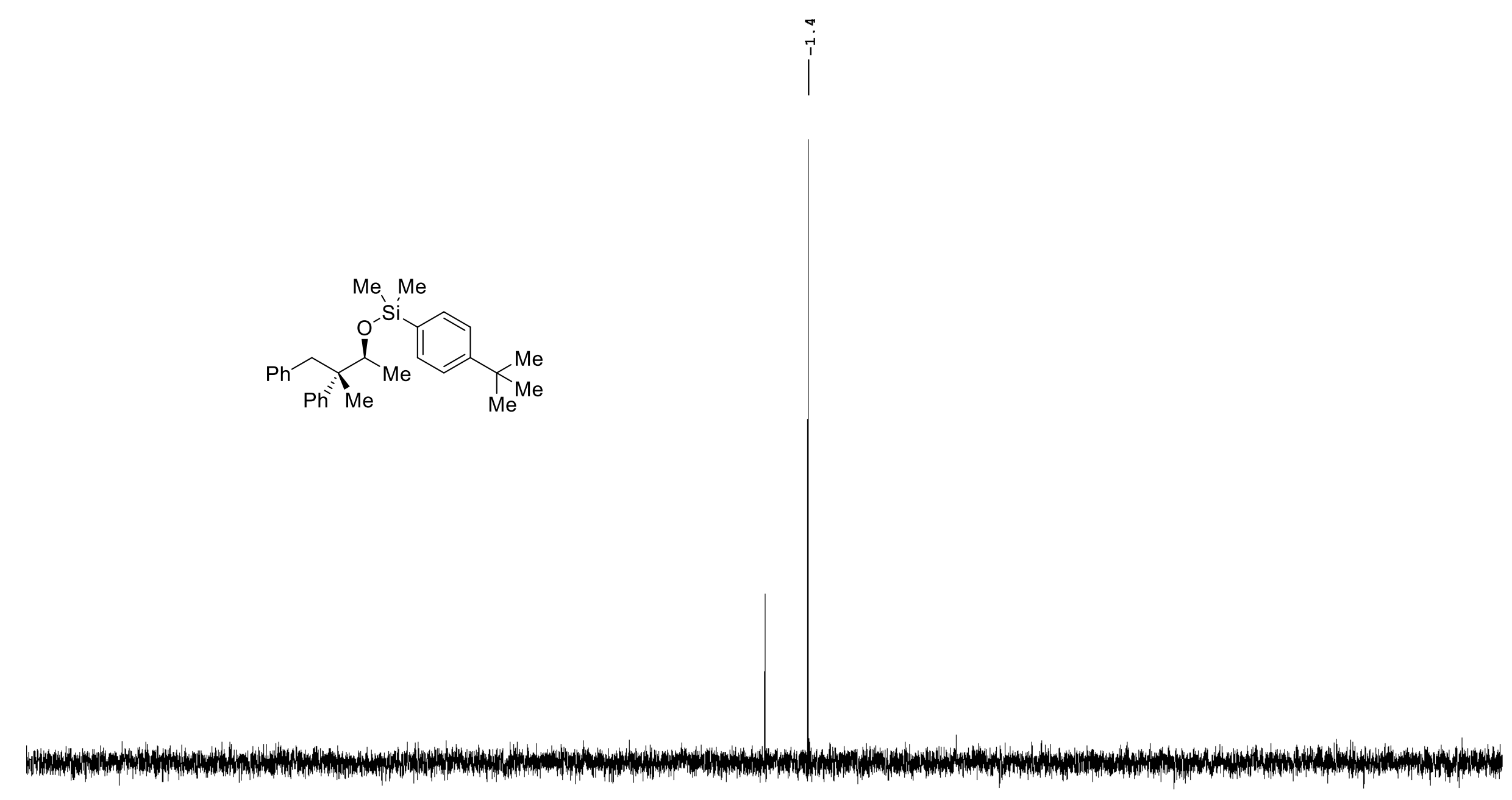

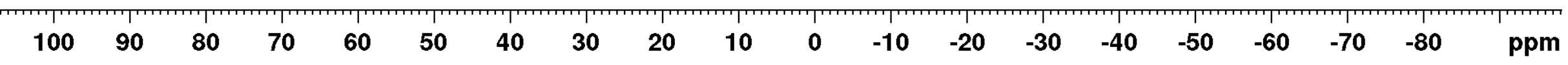


Figure 8.35 ${ }^{1} \mathrm{H}$ NMR (500 MHz, $\left.\mathrm{C}_{6} \mathrm{D}_{6}, 298 \mathrm{~K}\right)$ of (2S,3S)-(4-Methoxy-3,5-dimethylphenyl)dimethyl((3-methyl-3,4-diphenylbutan2-yl)oxy)silane [(2S,3S)-3aj].
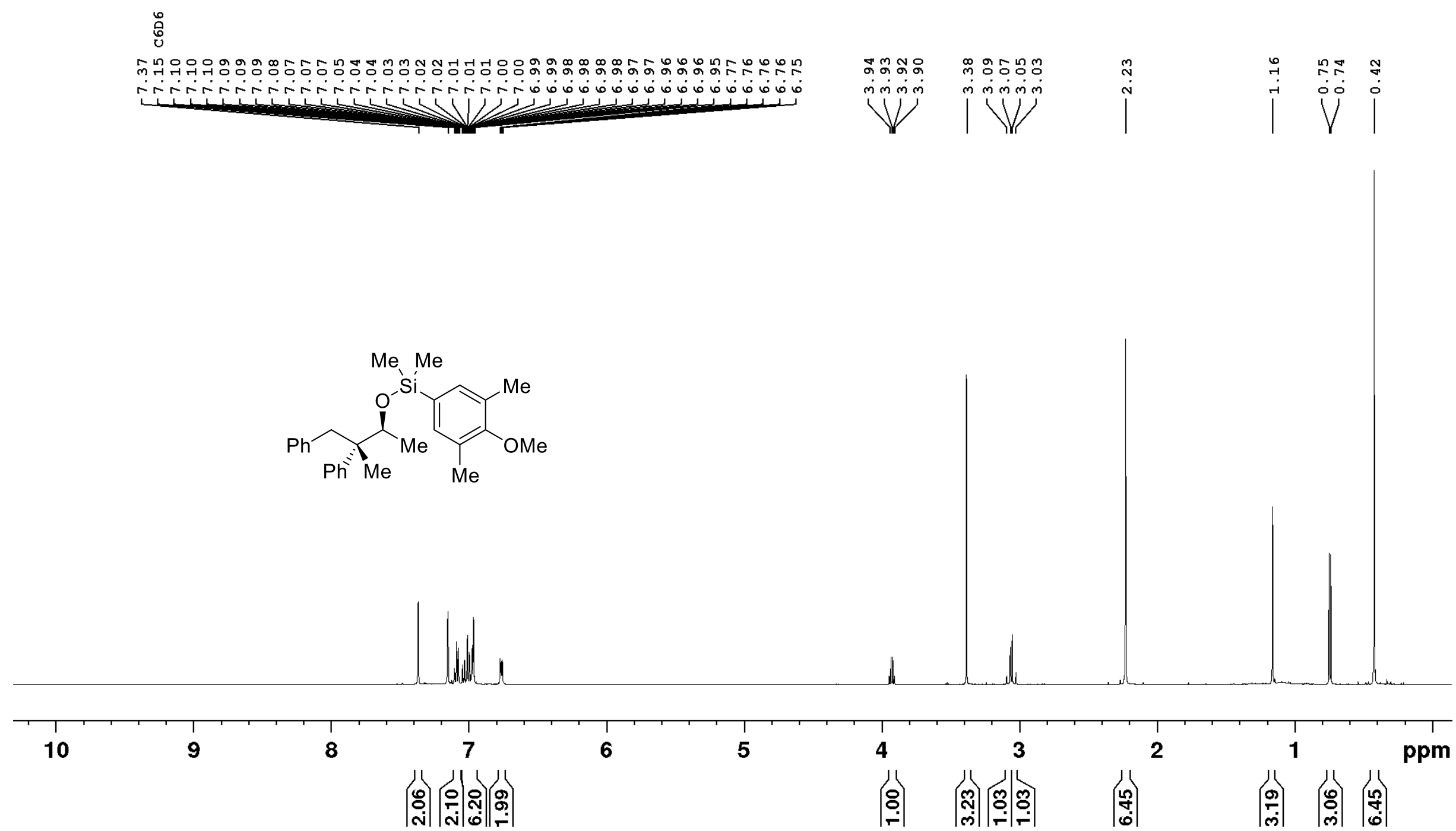
Figure 8.36 ${ }^{13} \mathrm{C}$ NMR (126 MHz, $\left.\mathrm{C}_{6} \mathrm{D}_{6}, 298 \mathrm{~K}\right)$ of (2S,3S)-(4-Methoxy-3,5-dimethylphenyl)dimethyl((3-methyl-3,4-diphenylbutan2-yl)oxy)silane [(2S,3S)-3aj].

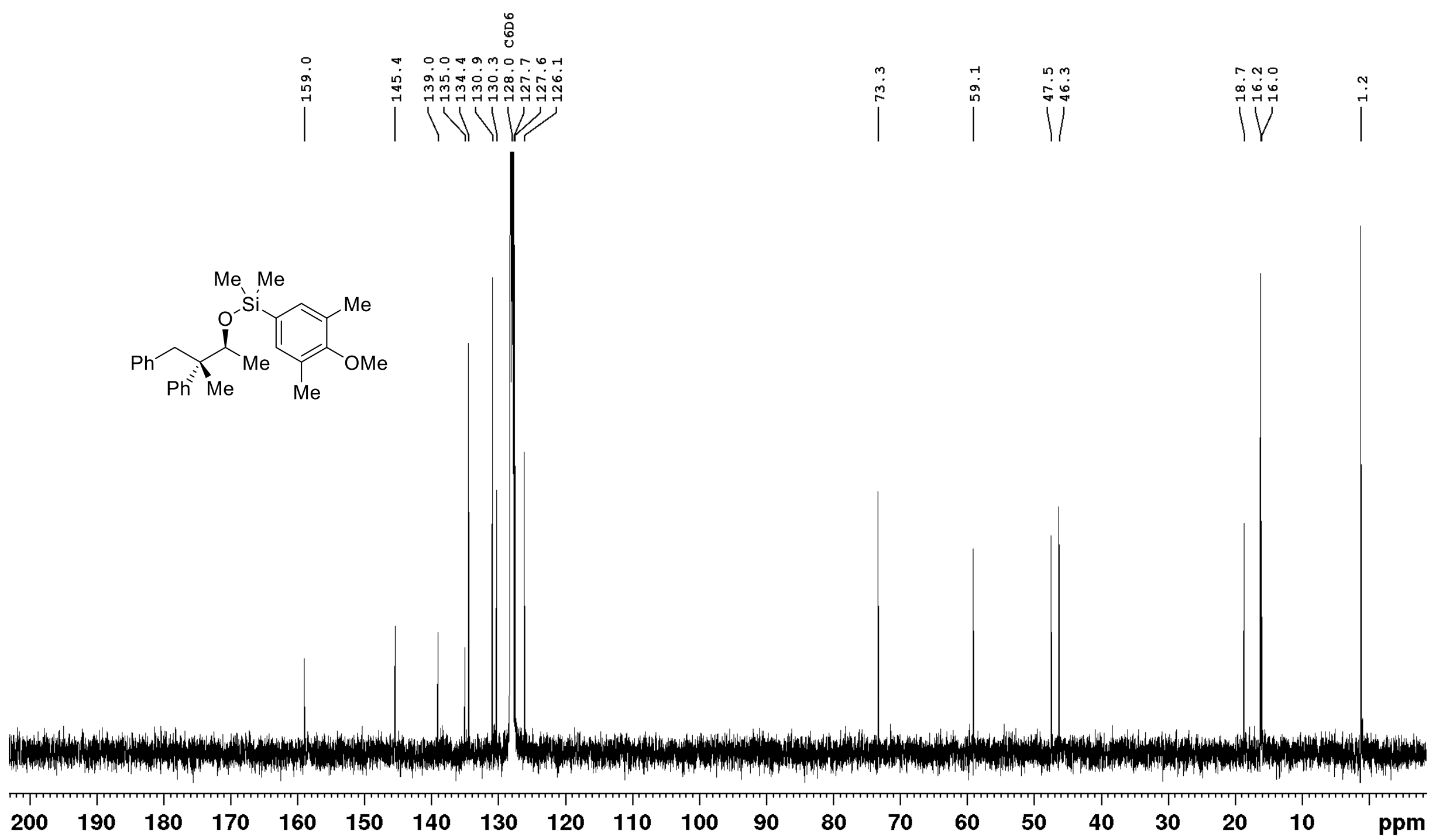


Figure 8.37 ${ }^{29} \mathrm{Si}$ DEPT NMR (99 MHz, $\left.\mathrm{C}_{6} \mathrm{D}_{6}, 298 \mathrm{~K}\right)$ of $(2 S, 3 S)-(4-M e t h o x y-3,5-d i m e t h y l p h e n y l)$ dimethyl((3-methyl-3,4diphenylbutan-2-yl)oxy)silane [(2S,3S)-3aj].

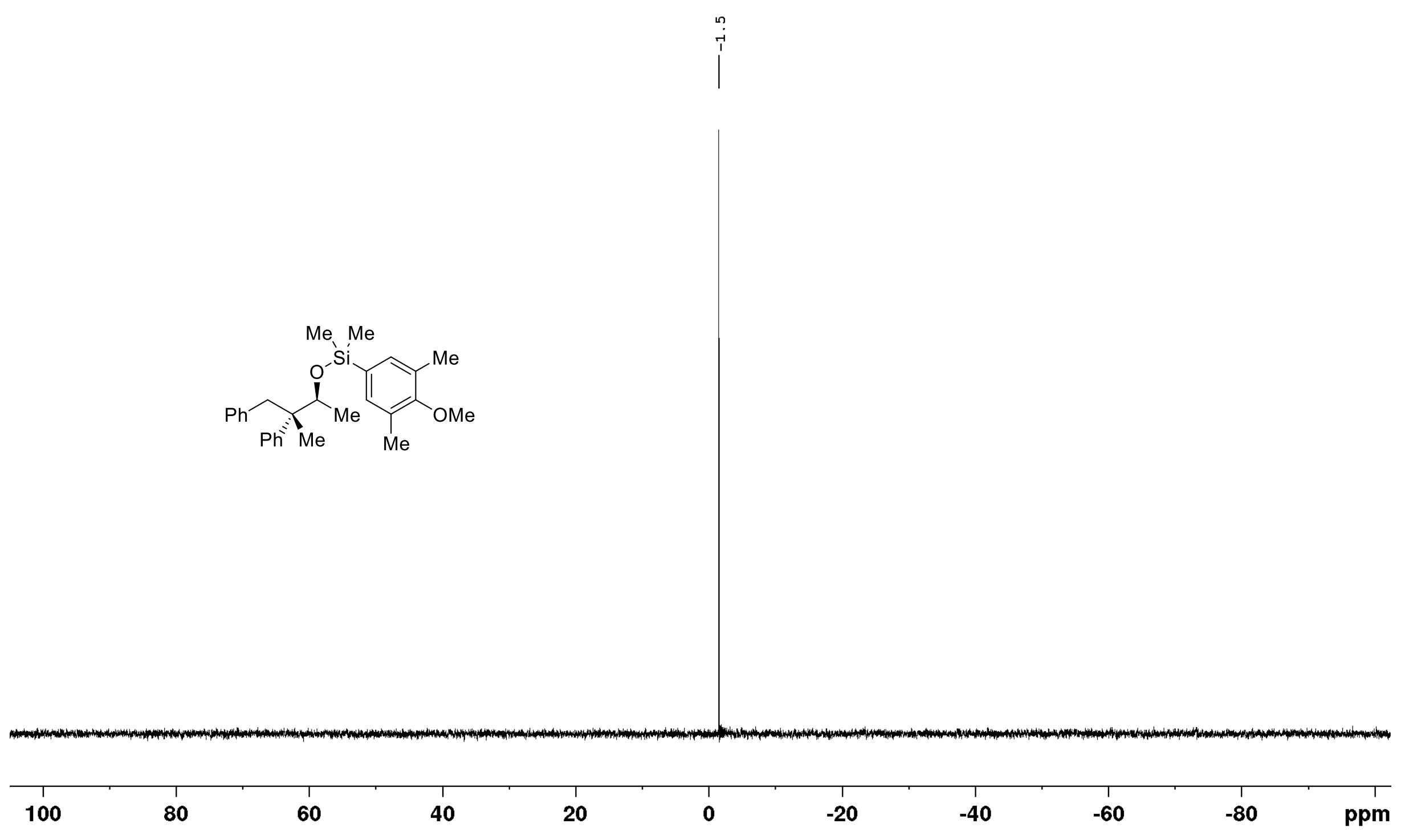


Figure 8.38 ${ }^{1} \mathrm{H}$ NMR (500 MHz, $\left.\mathrm{C}_{6} \mathrm{D}_{6}, 298 \mathrm{~K}\right)$ of (2S,3S)-Tribenzyl((3-methyl-3,4-diphenylbutan-2-yl)oxy)silane [(2S,3S)-3al]. :

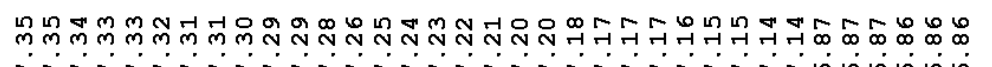

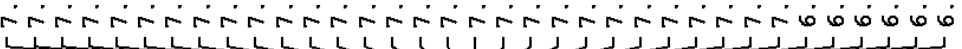

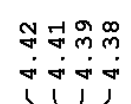

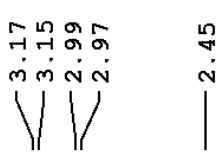<smiles>C[C@H](O[Si](Cc1ccccc1)(Cc1ccccc1)Cc1ccccc1)C(C)(C)c1ccccc1</smiles>
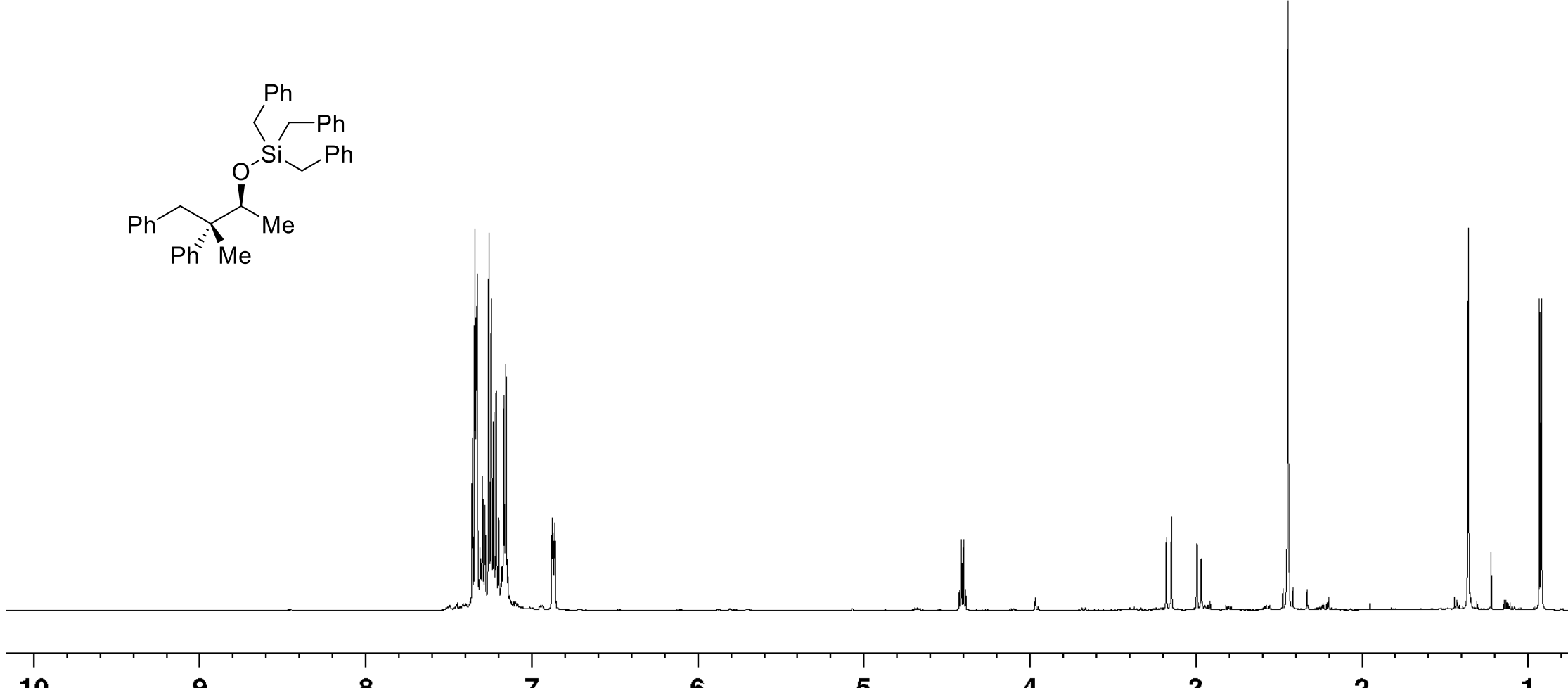

,

.

6

5

4

\begin{tabular}{c}
3 \\
\hdashline$|=|$ \\
\hdashline \\
\hdashline
\end{tabular}

2

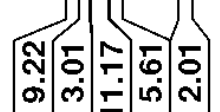

$\left|\begin{array}{l}0 \\ \hdashline\end{array}\right|$

ppm $\left|\begin{array}{ll}n \\ \bar{n} \\ m\end{array}\right|$ 
Figure 8.39 ${ }^{13} \mathrm{C}$ NMR (126 MHz, $\left.\mathrm{C}_{6} \mathrm{D}_{6}, 298 \mathrm{~K}\right)$ of (2S,3S)-Tribenzyl((3-methyl-3,4-diphenylbutan-2-yl)oxy)silane [(2S,3S)-3al].

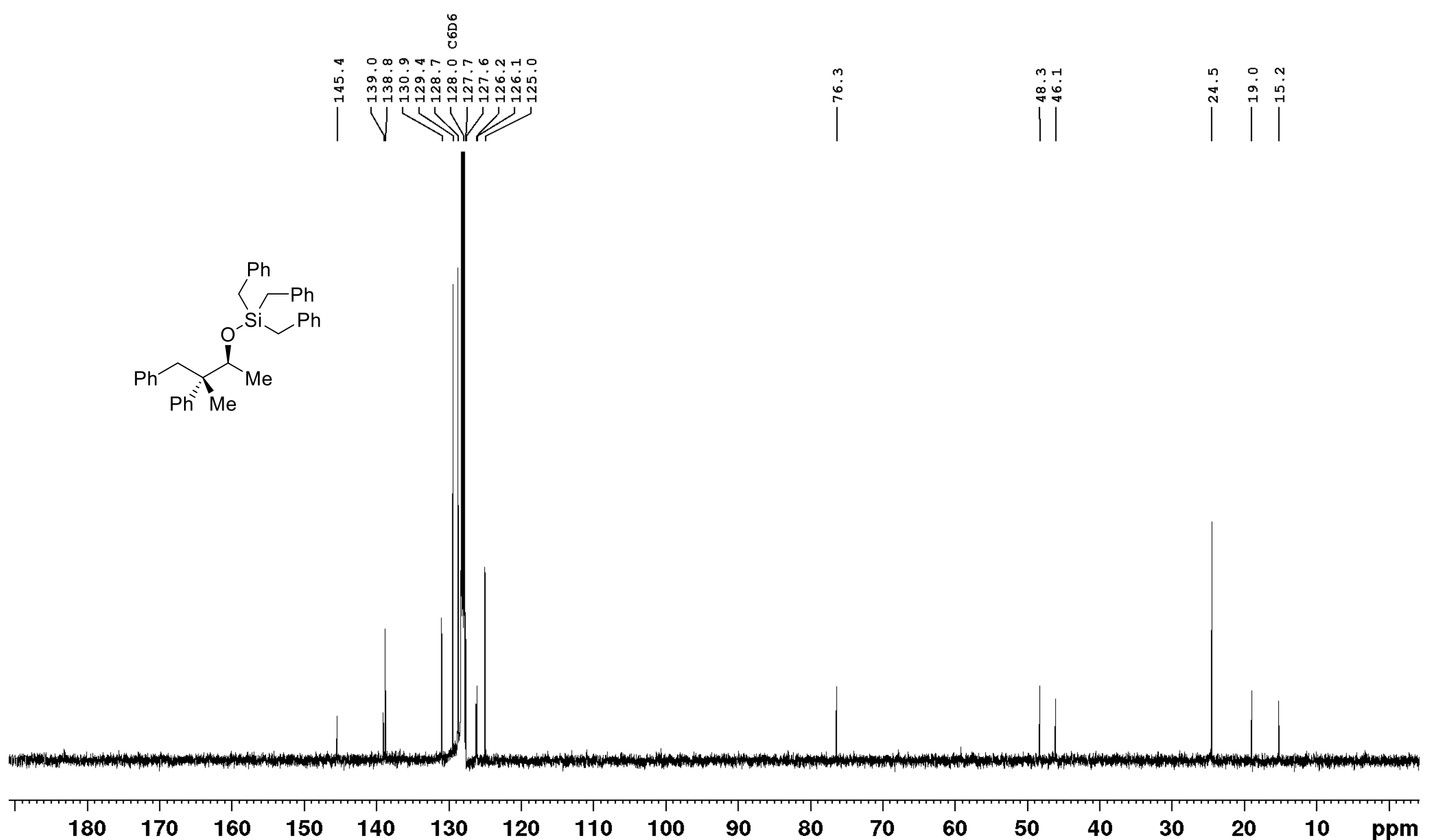


Figure 8.40 ${ }^{29} \mathrm{Si}$ DEPT NMR (99 MHz, $\left.\mathrm{C}_{6} \mathrm{D}_{6}, 298 \mathrm{~K}\right)$ of (2S,3S)-Tribenzyl((3-methyl-3,4-diphenylbutan-2-yl)oxy)silane [(2S,3S)3al].

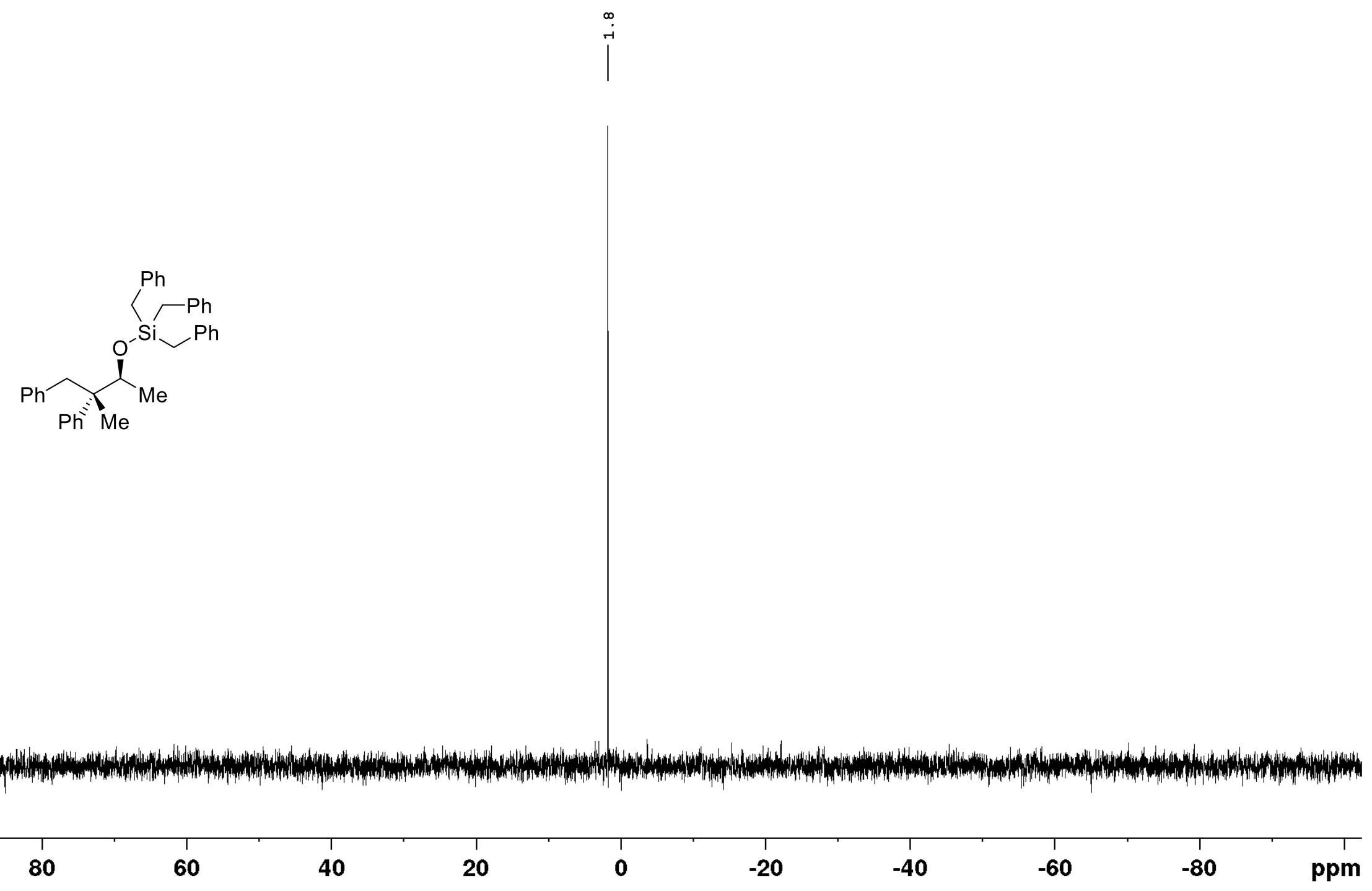

$60 \quad 40 \quad 20$


Figure 8.41 ${ }^{1} \mathrm{H}$ NMR (500 MHz, $\mathrm{CDCl}_{3}, 298 \mathrm{~K}$ ) of rac-anti/syn-3-Methyl-3,4-diphenylbutan-2-ol (rac-anti/syn-1a).

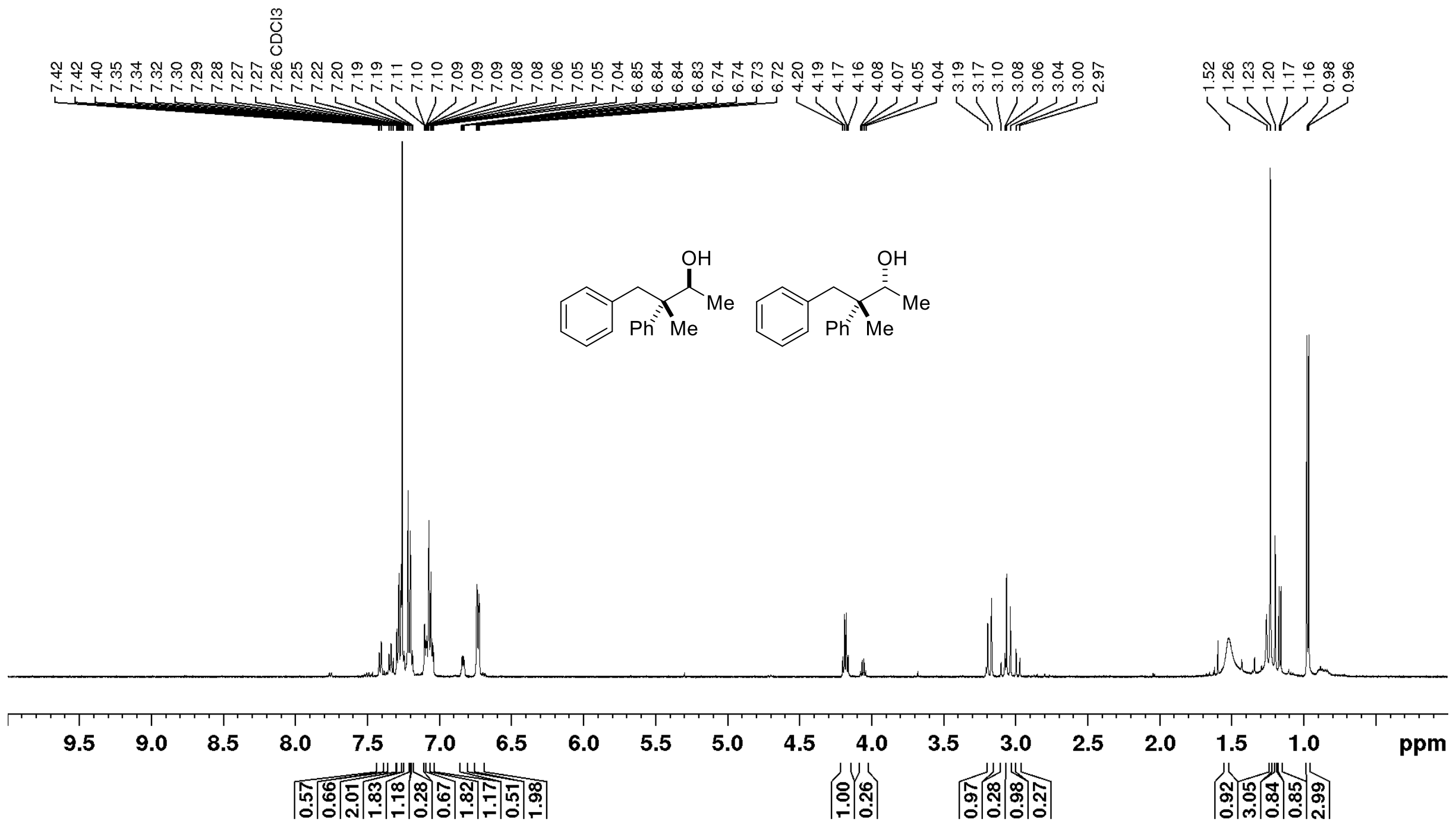


Figure 8.42 ${ }^{13} \mathrm{C} \mathrm{NMR} \mathrm{(126} \mathrm{MHz,} \mathrm{CDCl}_{3}, 298 \mathrm{~K}$ ) of rac-anti/syn-3-Methyl-3,4-diphenylbutan-2-ol (rac-anti/syn-1a).

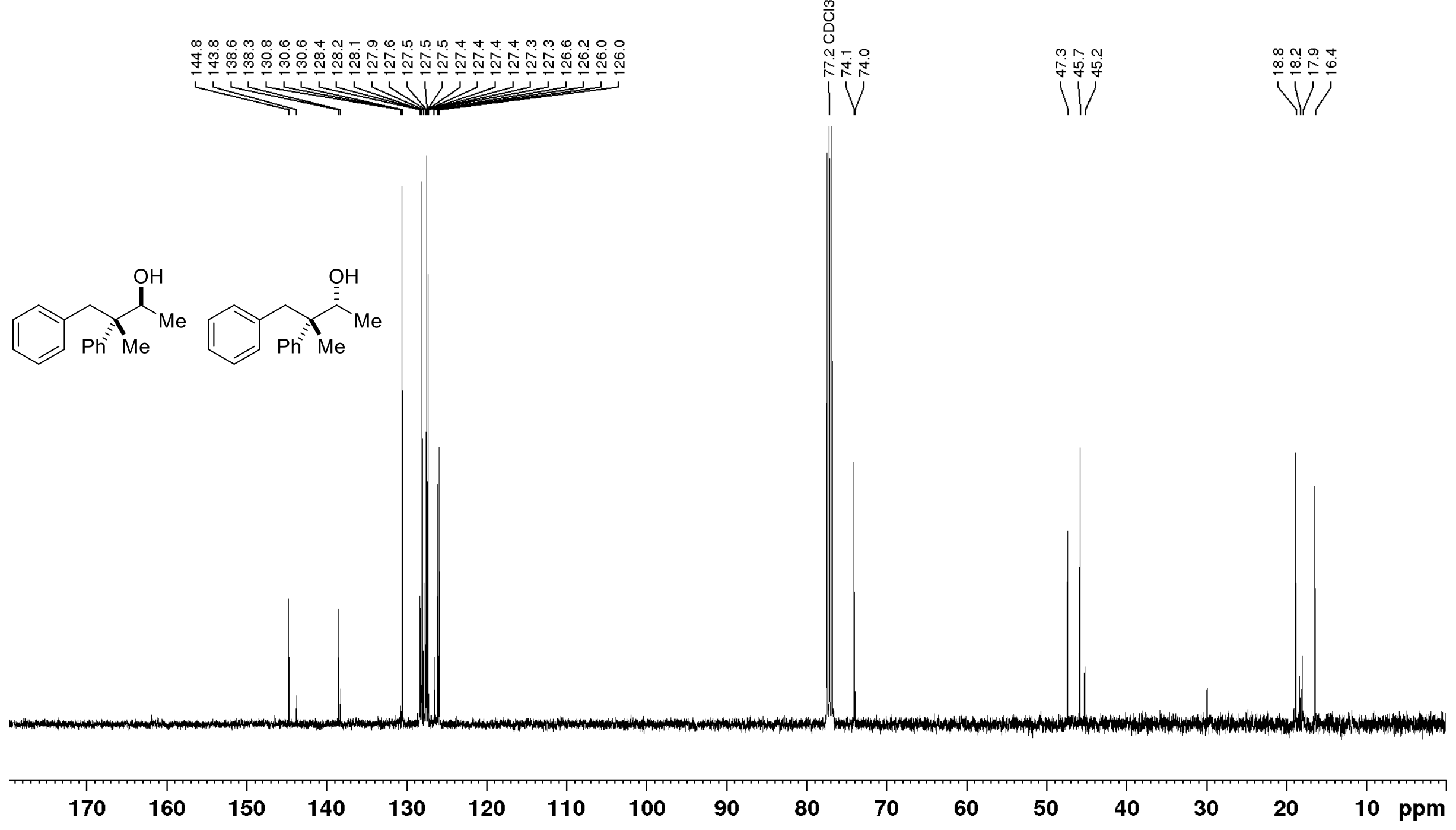


Figure 8.43 ${ }^{1} \mathrm{H}$ NMR (500 MHz, $\left.\mathrm{C}_{6} \mathrm{D}_{6}, 298 \mathrm{~K}\right)$ of $(2 S, 3 S)$-(3,5-Dimethylphenyl)dimethyl((3-methyl-3,4-diphenylbutan-2yl)oxy)silane $[(2 S, 3 S)-3 a e]$.

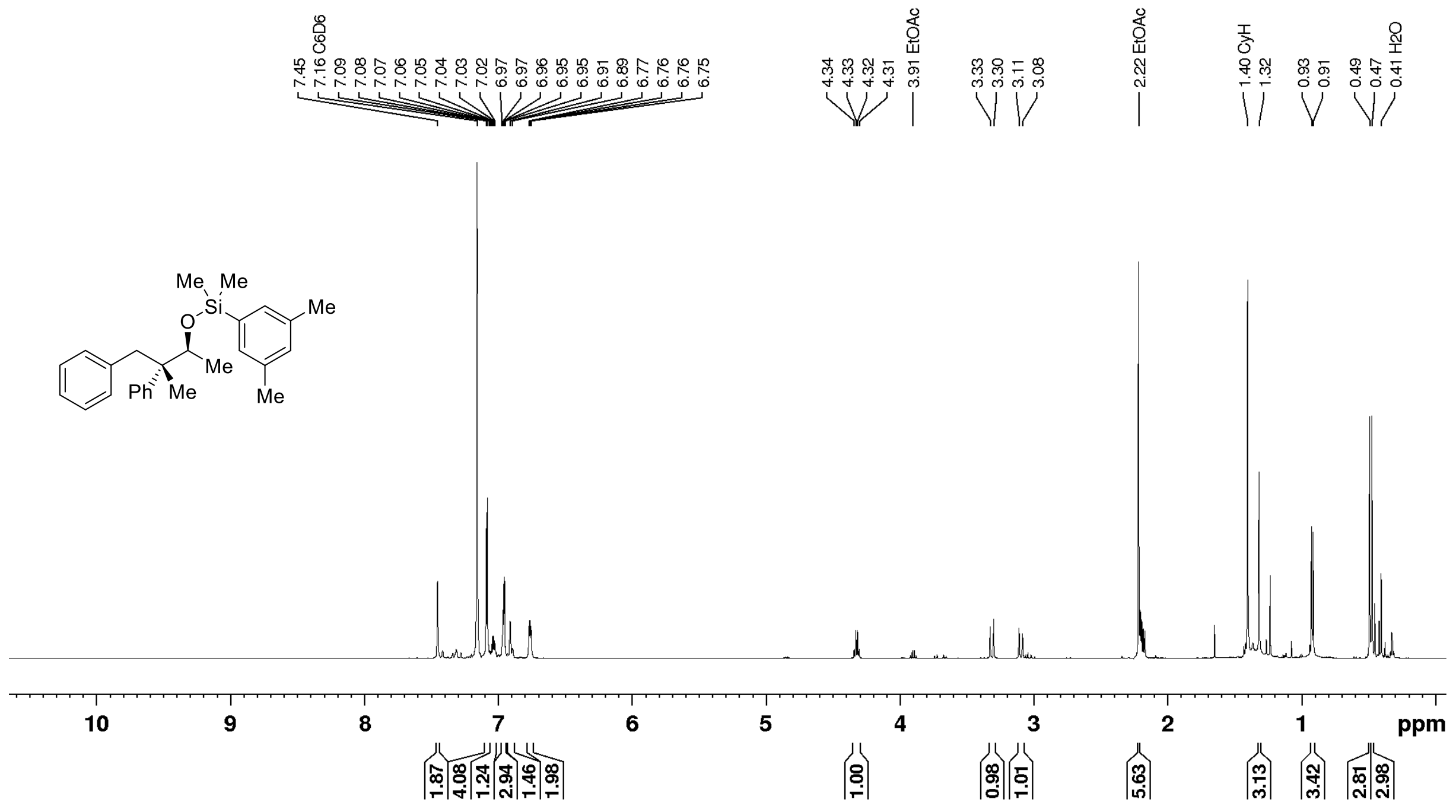


Figure 8.44 ${ }^{13} \mathrm{C}$ NMR (126 MHz, $\left.\mathrm{C}_{6} \mathrm{D}_{6}, 298 \mathrm{~K}\right)$ of $(2 S, 3 S)-(3,5-$ Dimethylphenyl)dimethyl((3-methyl-3,4-diphenylbutan-2yl)oxy)silane $[(2 S, 3 S)-3 a e]$.

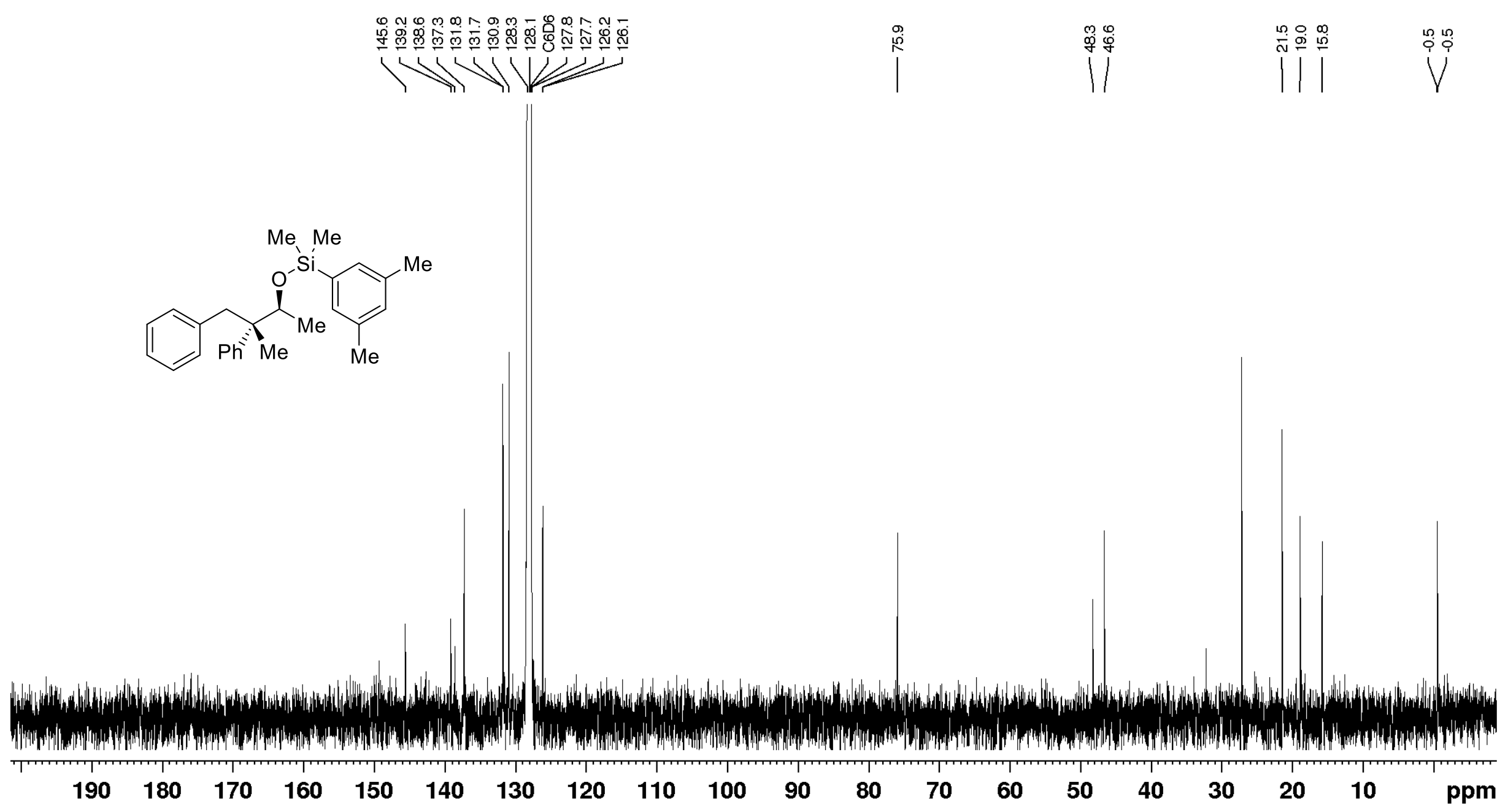


Figure 8.45 ${ }^{29} \mathrm{Si}$ DEPT NMR (99 MHz, $\left.\mathrm{C}_{6} \mathrm{D}_{6}, 298 \mathrm{~K}\right)$ of $(2 S, 3 S)-(3,5-$ Dimethylphenyl)dimethyl((3-methyl-3,4-diphenylbutan-2yl)oxy)silane $[(2 S, 3 S)-3 a e]$.
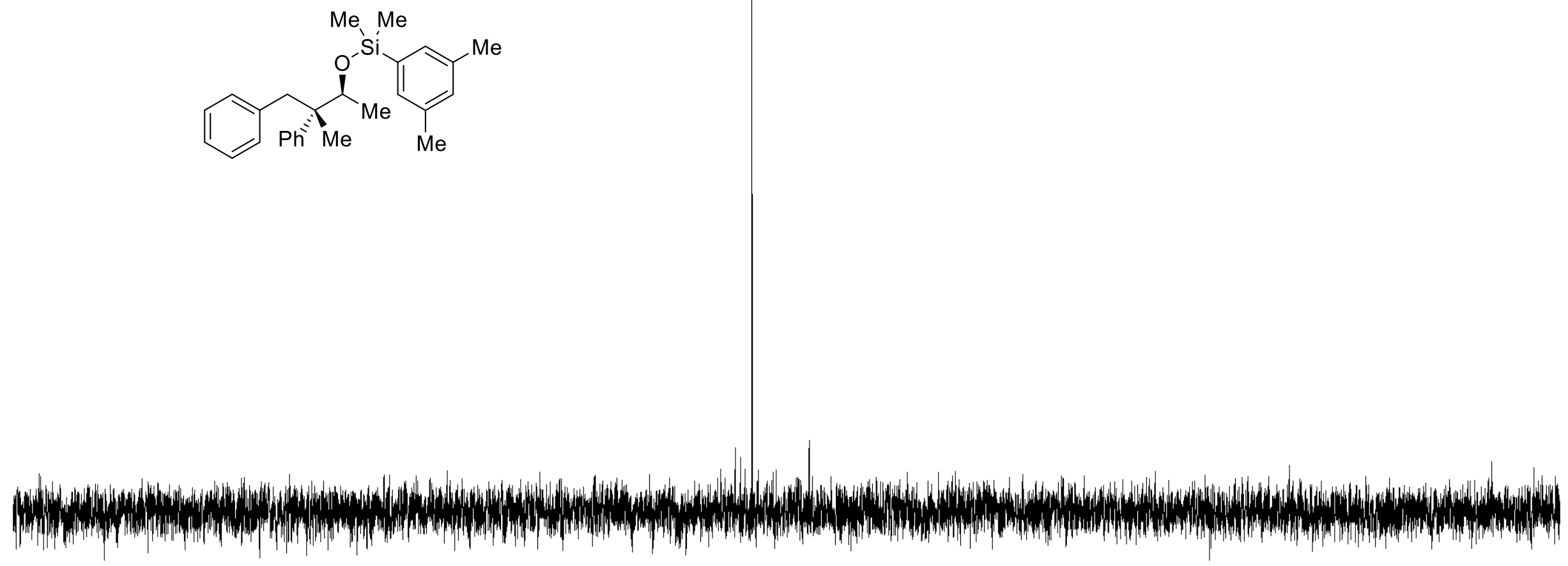
Figure 8.46 ${ }^{1} \mathrm{H}$ NMR (500 MHz, $\mathrm{CDCl}_{3}, 298 \mathrm{~K}$ ) of rac-anti/syn-3-Benzyl-3-phenylpentan-2-ol (rac-anti/syn-1b).

$\frac{m}{0}$

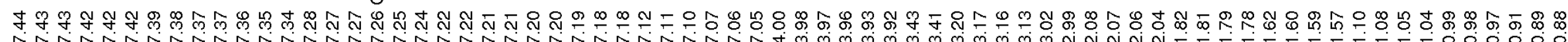

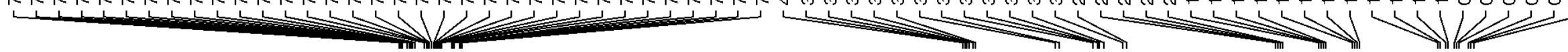

$1+C_{P h}^{O H}$
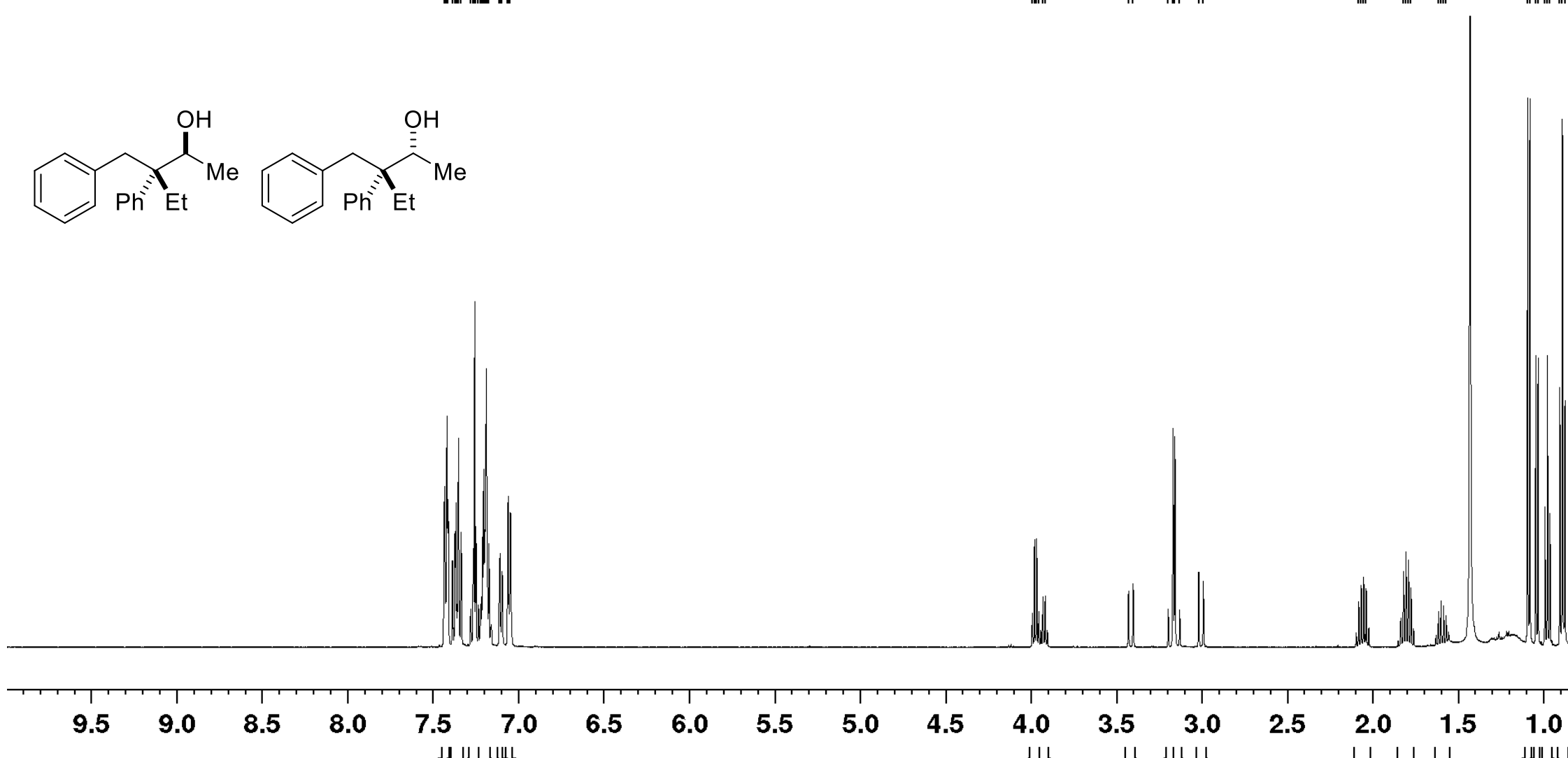

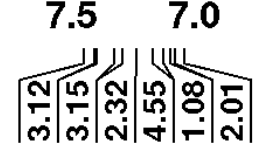
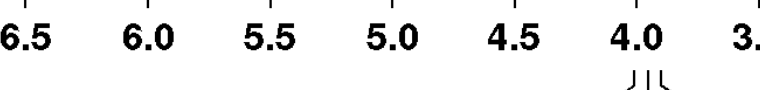

53.0

2.5
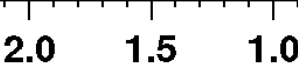

ppm

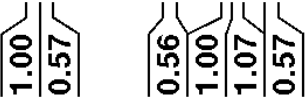

| 
Figure 8.47 ${ }^{13} \mathrm{C} \mathrm{NMR}\left(126 \mathrm{MHz}, \mathrm{CDCl}_{3}, 298 \mathrm{~K}\right)$ of rac-anti/syn-3-Benzyl-3-phenylpentan-2-ol (rac-anti/syn-1b).

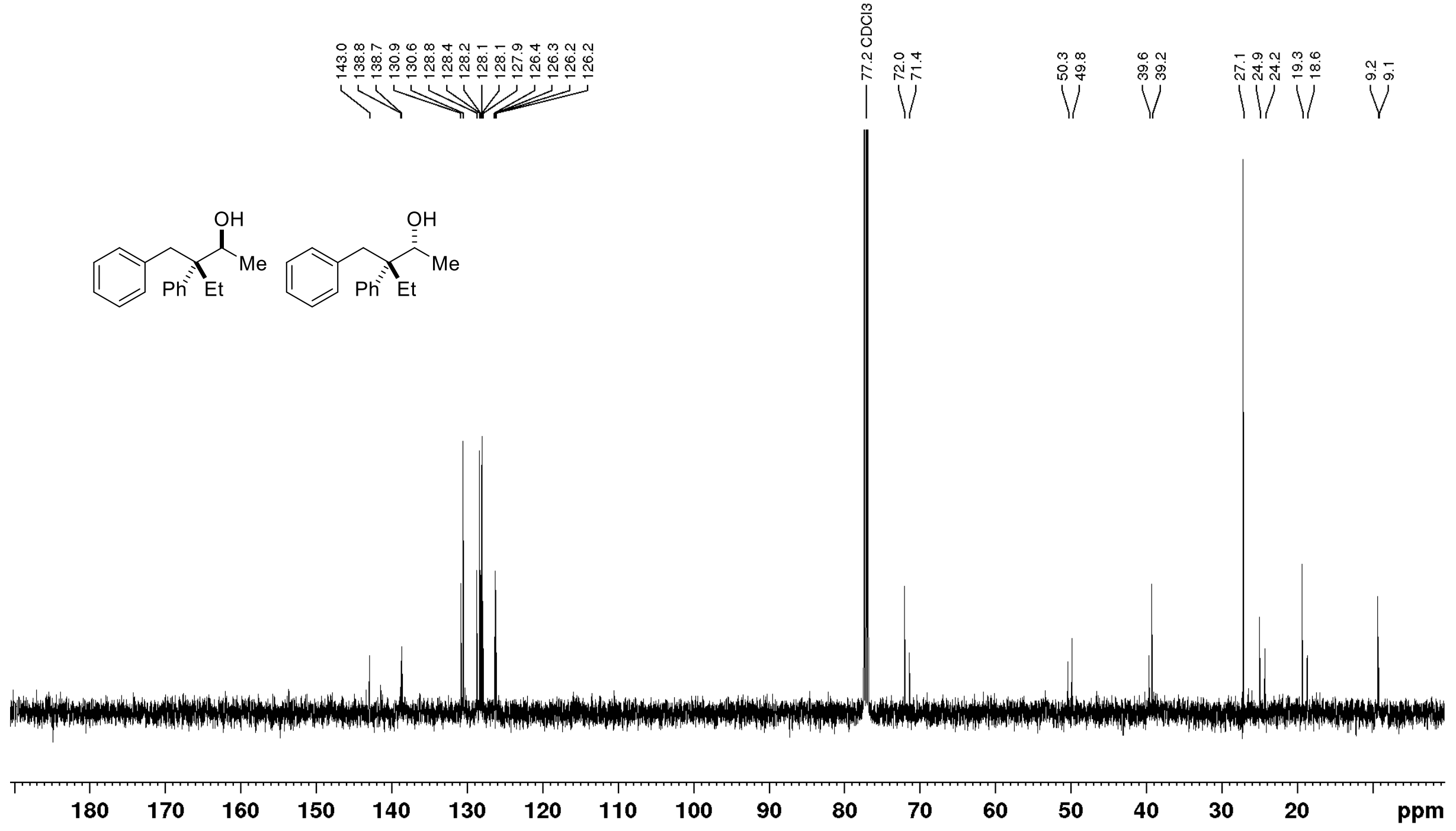


Figure 8.48 ${ }^{1} \mathrm{H}$ NMR (500 MHz, $\left.\mathrm{C}_{6} \mathrm{D}_{6}, 298 \mathrm{~K}\right)$ of $(2 S, 3 S)-(3,5-$ Dimethylphenyl)dimethyl((3-methyl-3,4-diphenylbutan-2yl)oxy)silane $[(2 S, 3 S)-3 b e]$.
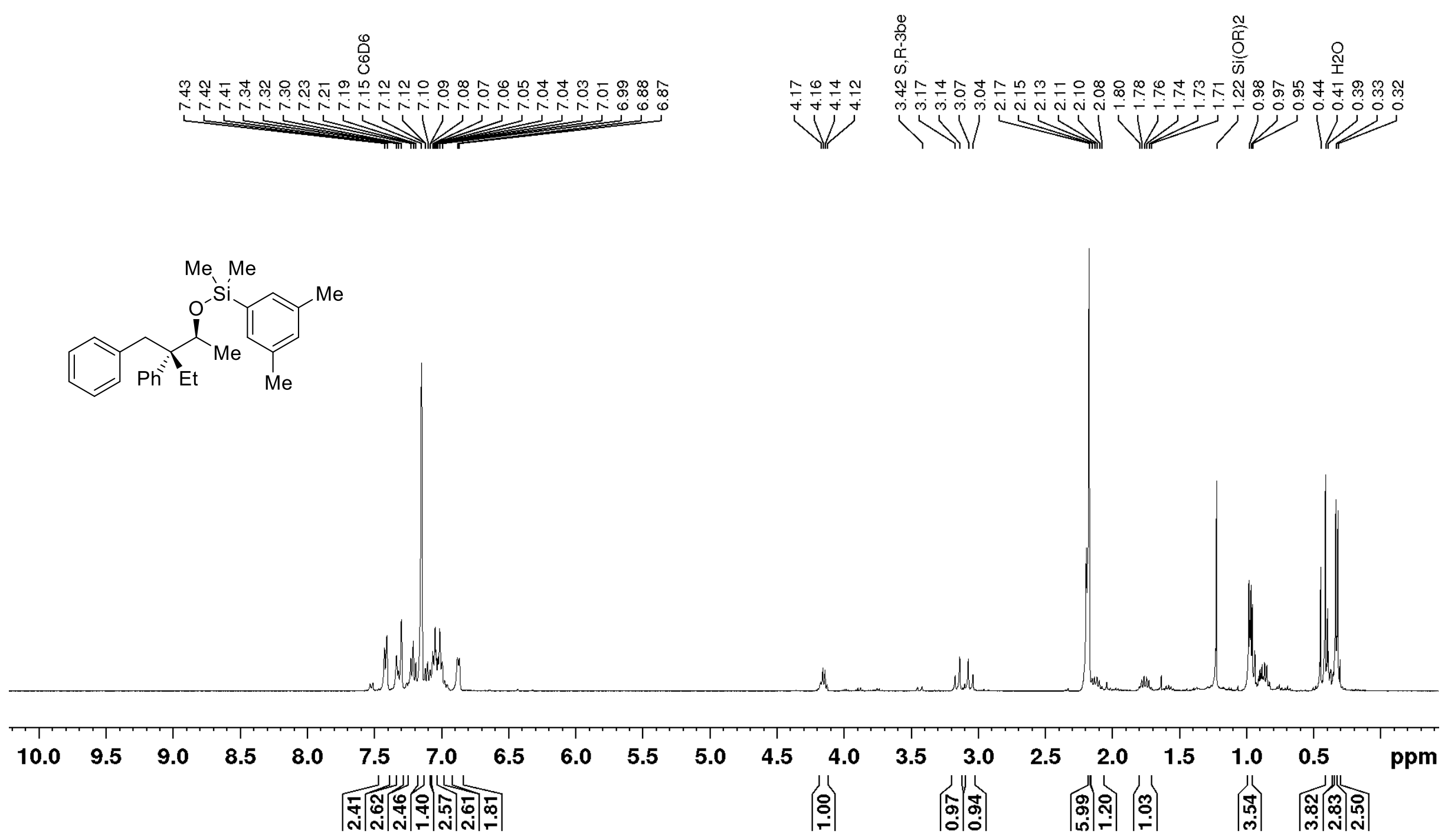
Figure 8.49 ${ }^{13} \mathrm{C}$ NMR (126 MHz, $\left.\mathrm{C}_{6} \mathrm{D}_{6}, 298 \mathrm{~K}\right)$ of $(2 S, 3 S)-(3,5-$ Dimethylphenyl)dimethyl((3-methyl-3,4-diphenylbutan-2yl)oxy)silane $[(2 S, 3 S)-3 b e]$.

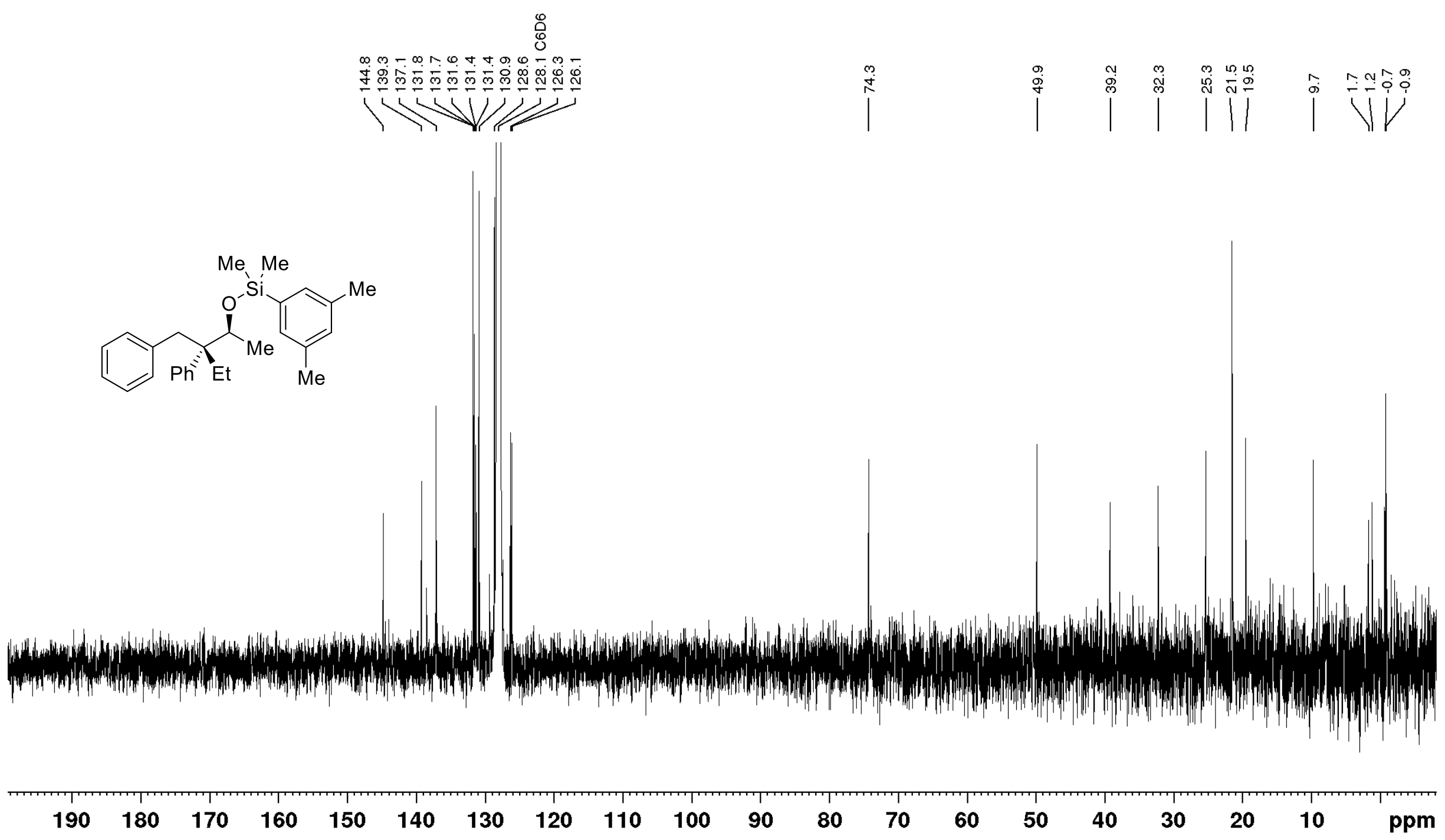


Figure 8.50 ${ }^{29} \mathrm{Si}$ DEPT NMR (99 MHz, $\left.\mathrm{C}_{6} \mathrm{D}_{6}, 298 \mathrm{~K}\right)$ of $(2 S, 3 S)-($ 3,5-Dimethylphenyl)dimethyl((3-methyl-3,4-diphenylbutan-2yl)oxy)silane $[(2 S, 3 S)-3 b e]$.

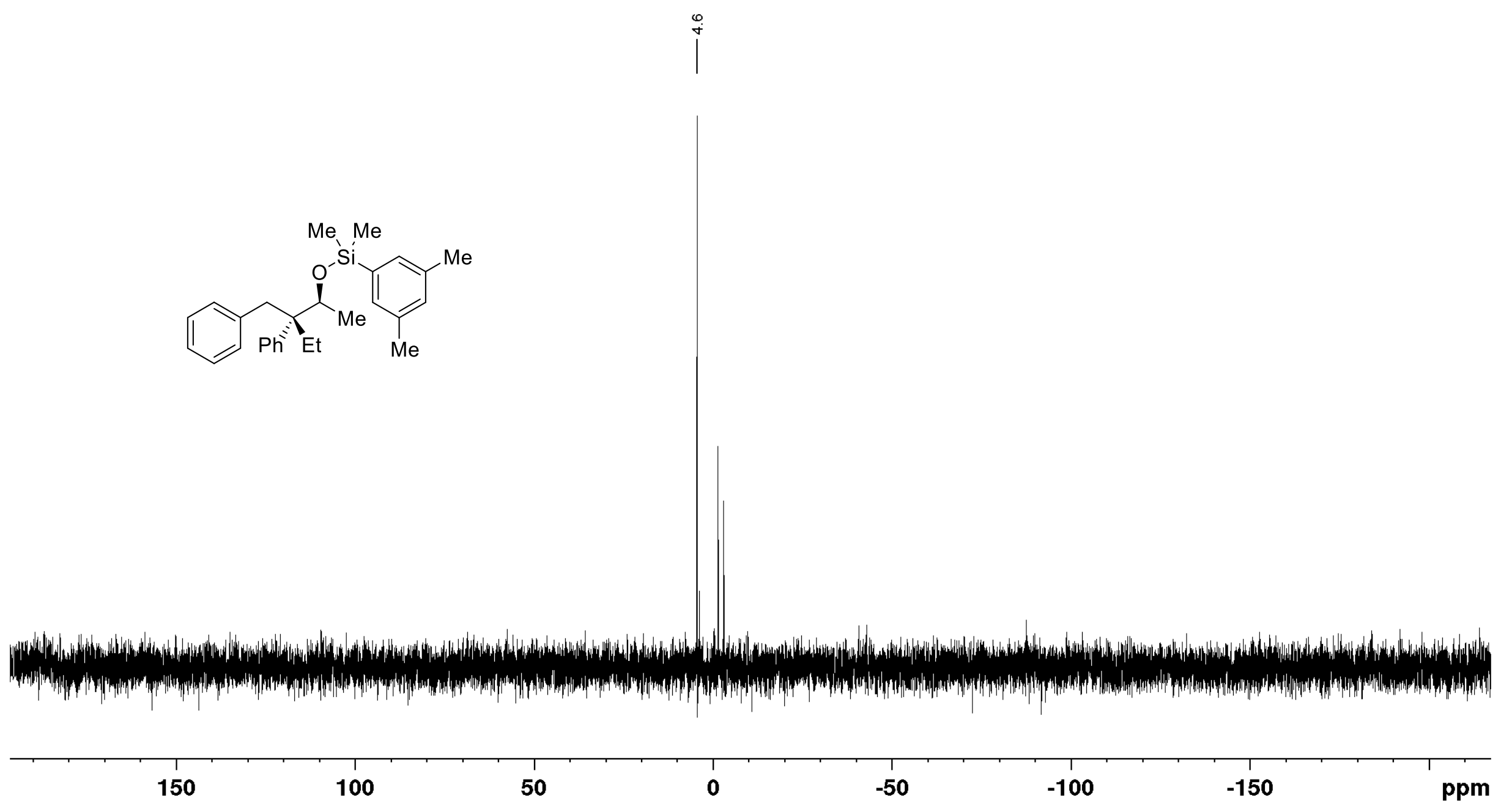


Figure 8.51 ${ }^{1} \mathrm{H}$ NMR $\left(500 \mathrm{MHz}, \mathrm{CDCl}_{3}, 298 \mathrm{~K}\right)$ of rac-anti/syn-3-Benzyl-3-methylpentan-2-ol (rac-anti/syn-1c).

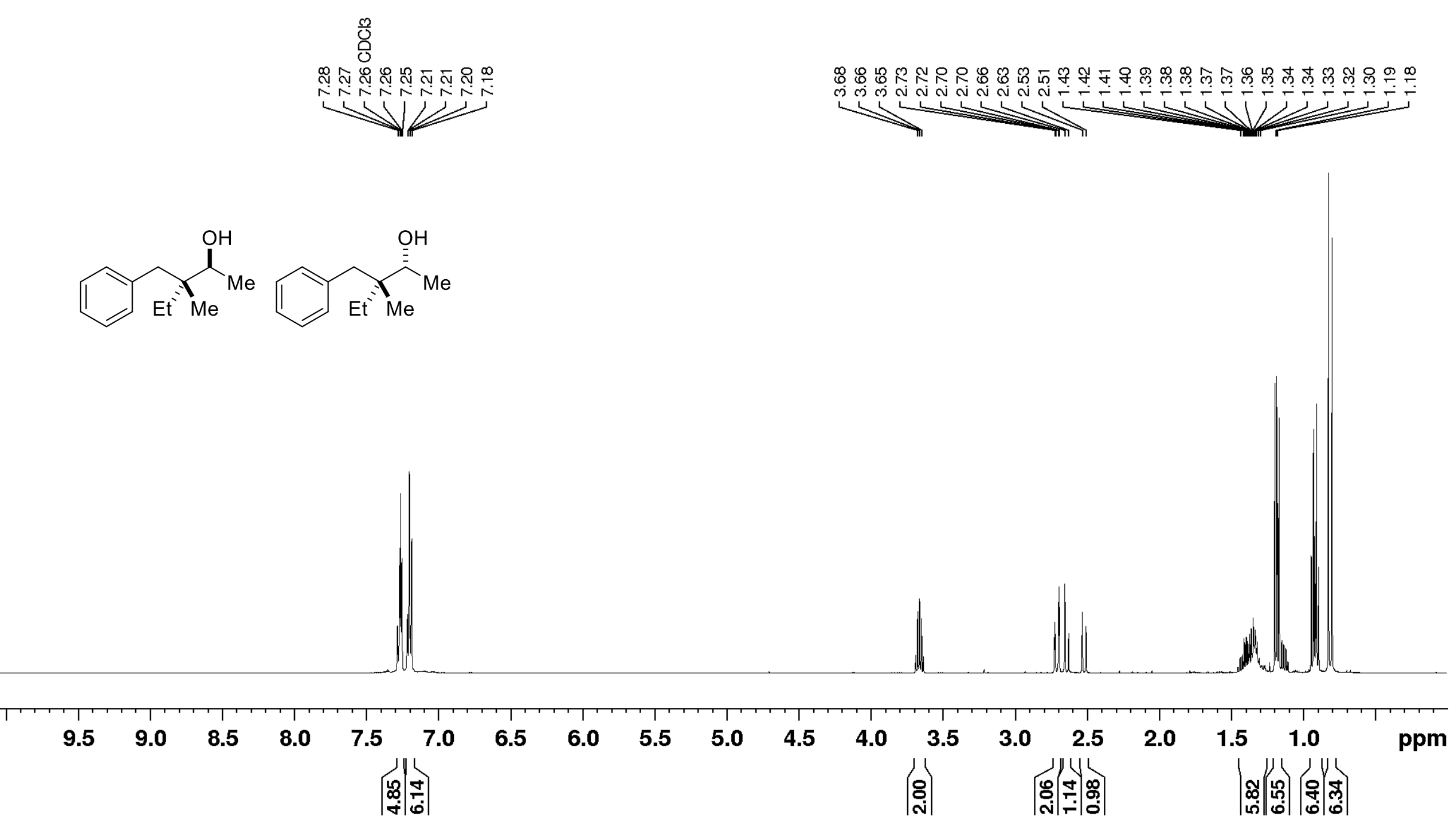


Figure 8.52 ${ }^{13} \mathrm{C}$ NMR (126 MHz, $\mathrm{CDCl}_{3}, 298 \mathrm{~K}$ ) of rac-anti/syn-3-Benzyl-3-methylpentan-2-ol (rac-anti/syn-1c).
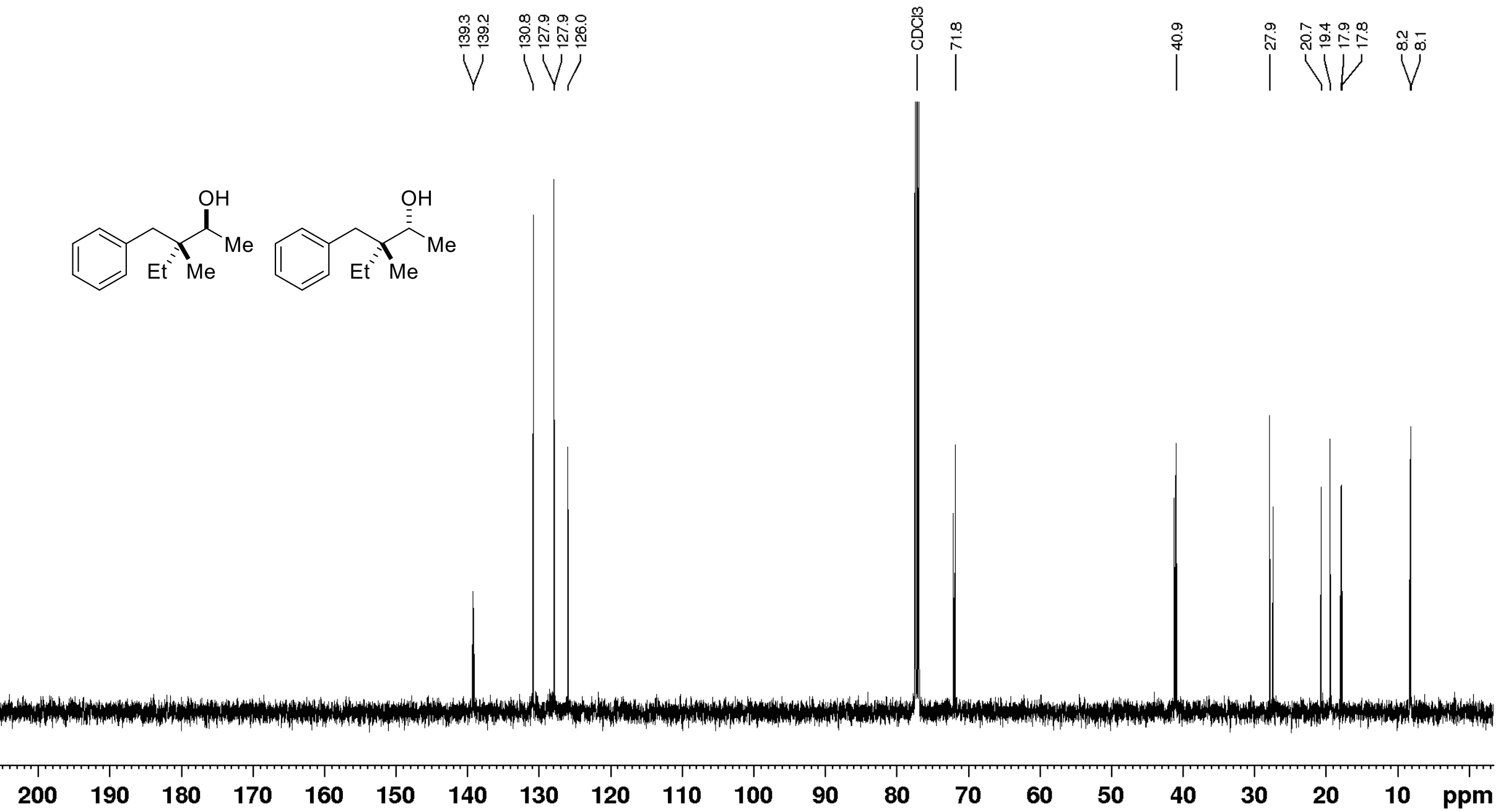
Figure 8.53 ${ }^{1} \mathrm{H}$ NMR $\left(500 \mathrm{MHz}, \mathrm{C}_{6} \mathrm{D}_{6}, 298 \mathrm{~K}\right)$ of $\left.(2 S, 3 S) /\left(2 S^{*}, 3 R^{\star}\right)-3-B e n z y l-3-m e t h y l p e n t a n-2-y l\right)$ oxy $)(3,5-$ dimethylphenyl)dimethylsilane $[(2 S, 3 S)-3 c e]$.

\section{萝}

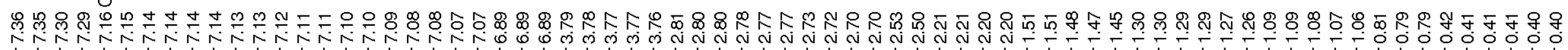

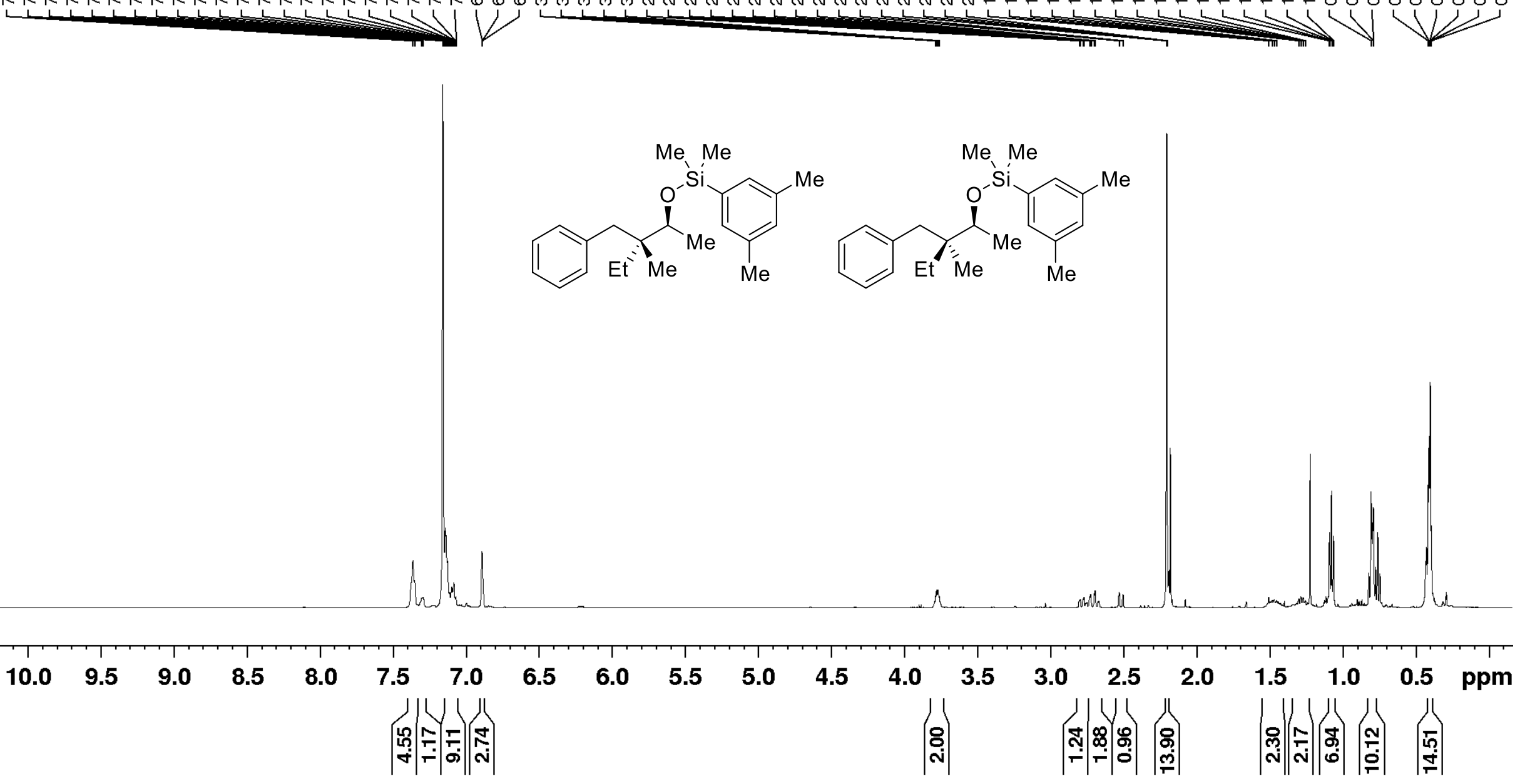


Figure 8.54 ${ }^{13} \mathrm{C}$ NMR (126 MHz, $\left.\mathrm{C}_{6} \mathrm{D}_{6}, 298 \mathrm{~K}\right)$ of $\left.\left.(2 S, 3 S) /\left(2 S^{\star}, 3 R^{\star}\right)-3-B e n z y l-3-m e t h y l p e n t a n-2-y l\right) o x y\right)(3,5-$ dimethylphenyl)dimethylsilane $[(2 S, 3 S)-3 c e]$.

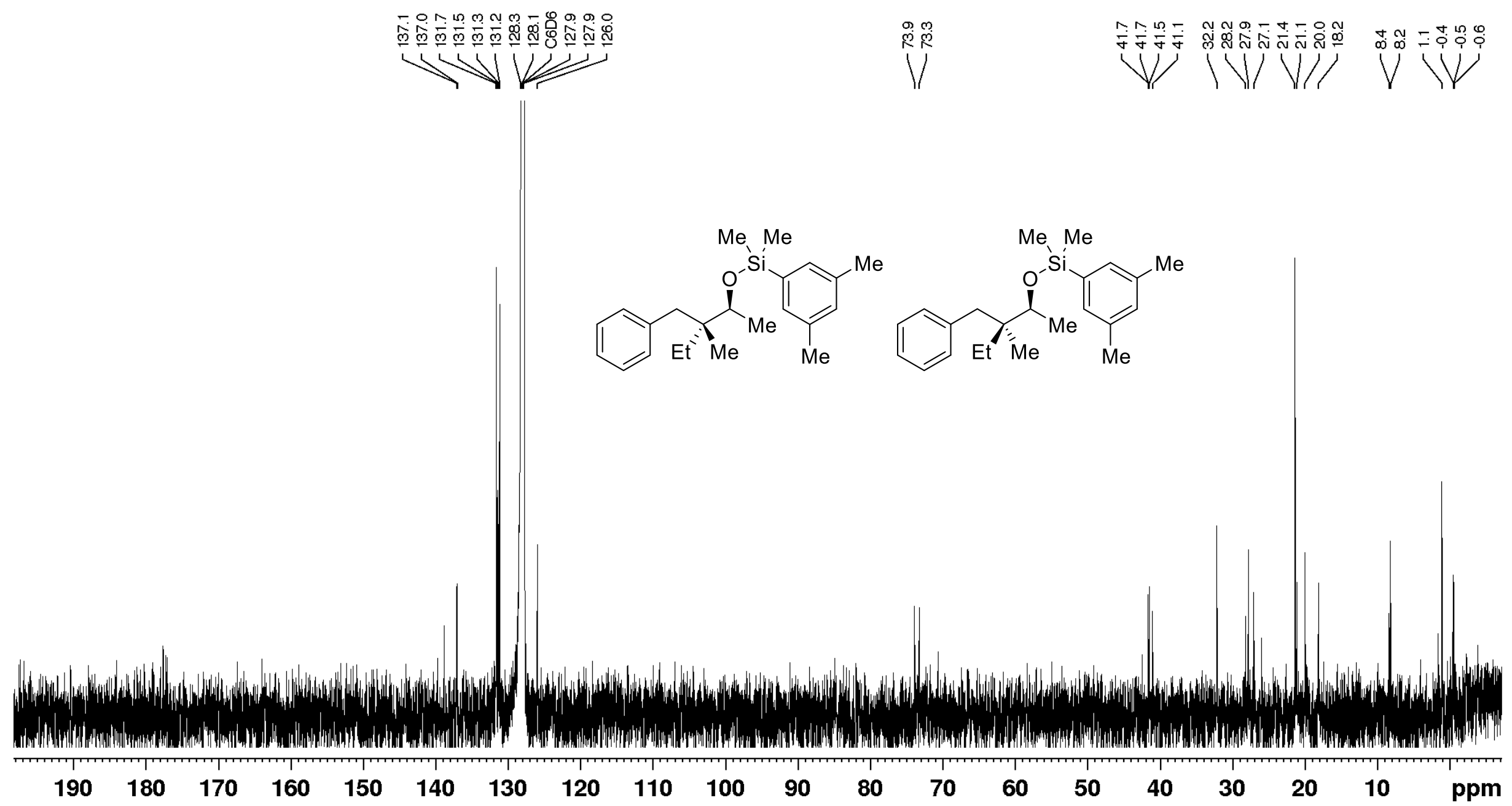


Figure 8.55 ${ }^{29} \mathrm{Si}$ DEPT NMR (99 MHz, $\left.\mathrm{C}_{6} \mathrm{D}_{6}, 298 \mathrm{~K}\right)$ of $(2 S, 3 S) /\left(2 S^{\star}, 3 R^{\star}\right)$-3-Benzyl-3-methylpentan-2-yl)oxy)(3,5dimethylphenyl)dimethylsilane $[(2 S, 3 S)-3 c e]$.

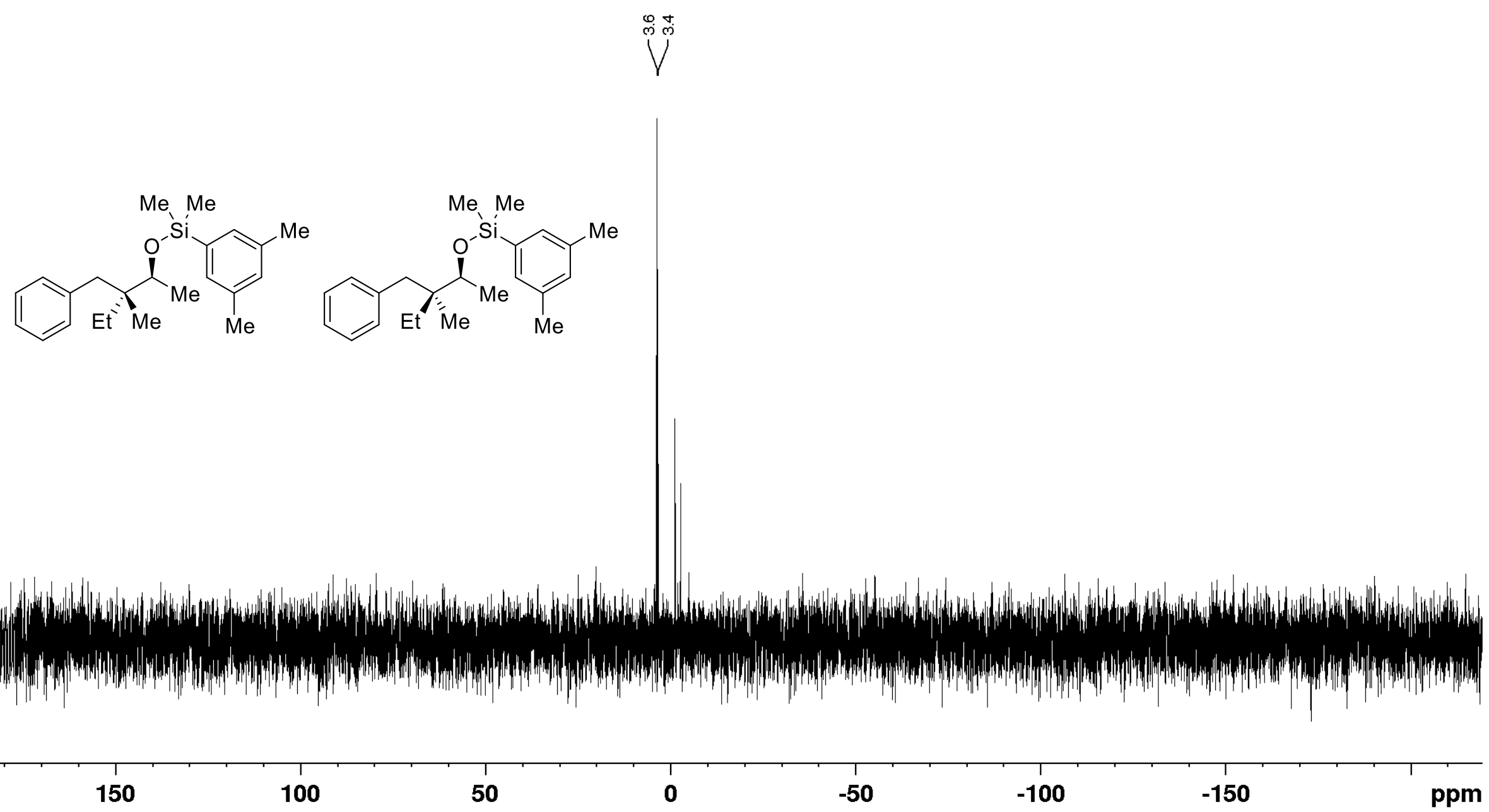


Figure 8.56 ${ }^{1} \mathrm{H}$ NMR (500 MHz, $\mathrm{CDCl}_{3}, 298 \mathrm{~K}$ ) of rac-anti/syn-4-(4-Fluorophenyl)-3-methyl-3-phenylbutan-2-ol (rac-anti/syn-1d).
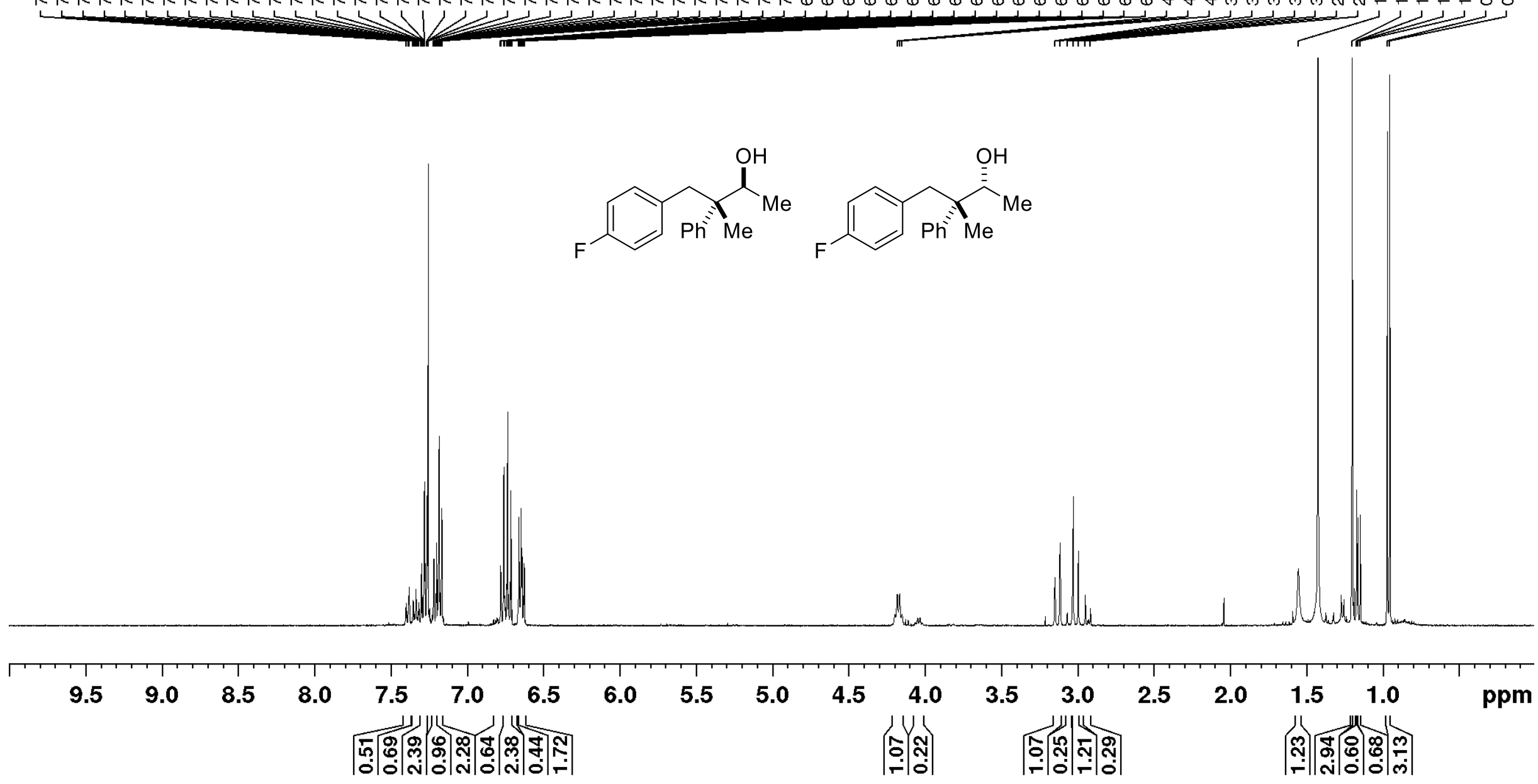
Figure 8.57 ${ }^{13} \mathrm{C} \mathrm{NMR} \mathrm{(126} \mathrm{MHz,} \mathrm{CDCl}_{3}, 298 \mathrm{~K}$ ) of rac-anti/syn-4-(4-Fluorophenyl)-3-methyl-3-phenylbutan-2-ol (rac-anti/syn-1d).

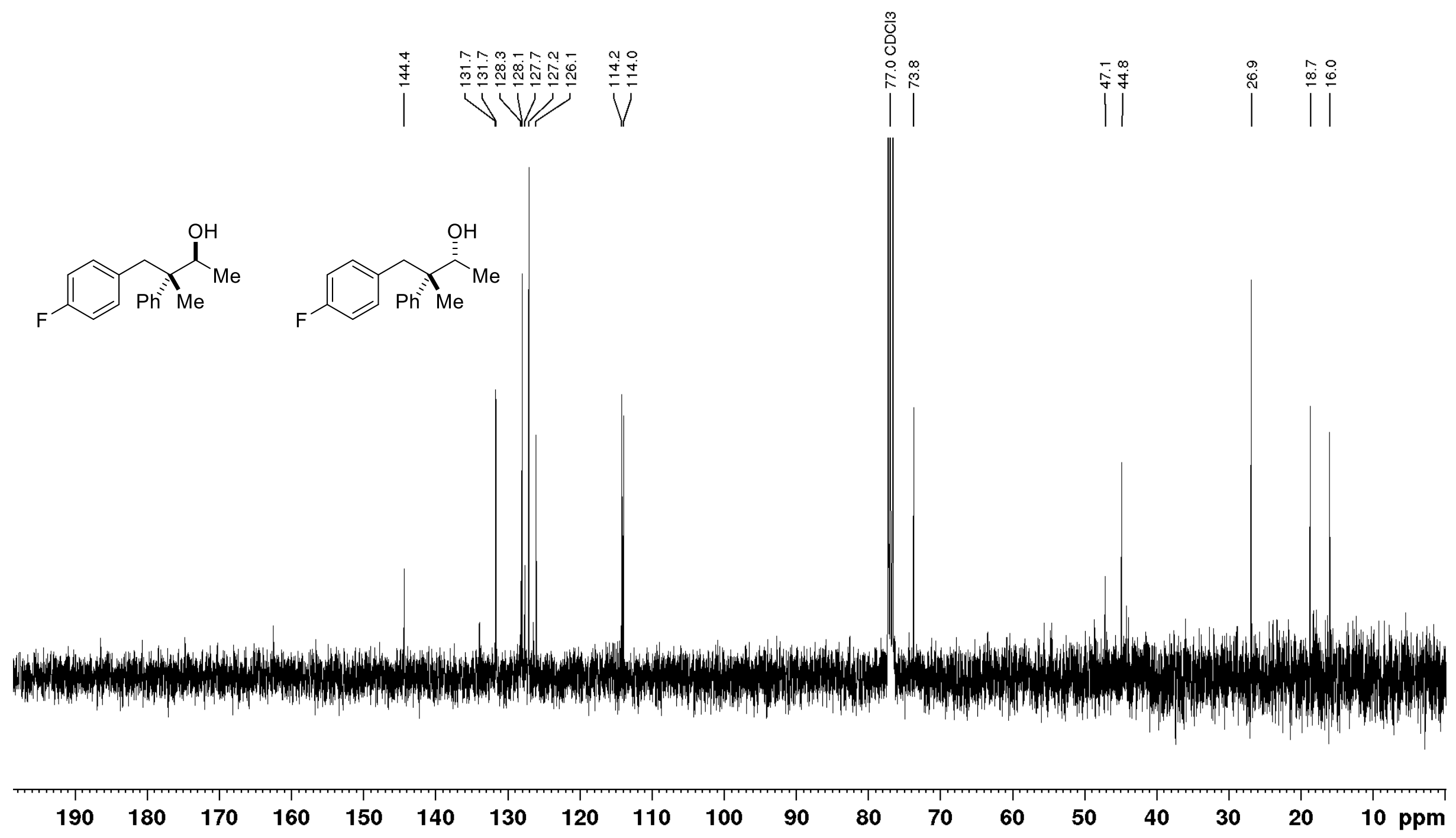


Figure 8.58 ${ }^{1} \mathrm{H}$ NMR (500 MHz, $\left.\mathrm{C}_{6} \mathrm{D}_{6}, 298 \mathrm{~K}\right)$ of (2S,3S)-(3,5-Dimethylphenyl)((4-(4-fluorophenyl)-3-methyl-3-phenylbutan-2yl)oxy)dimethylsilane [(2S,3S)-3de].

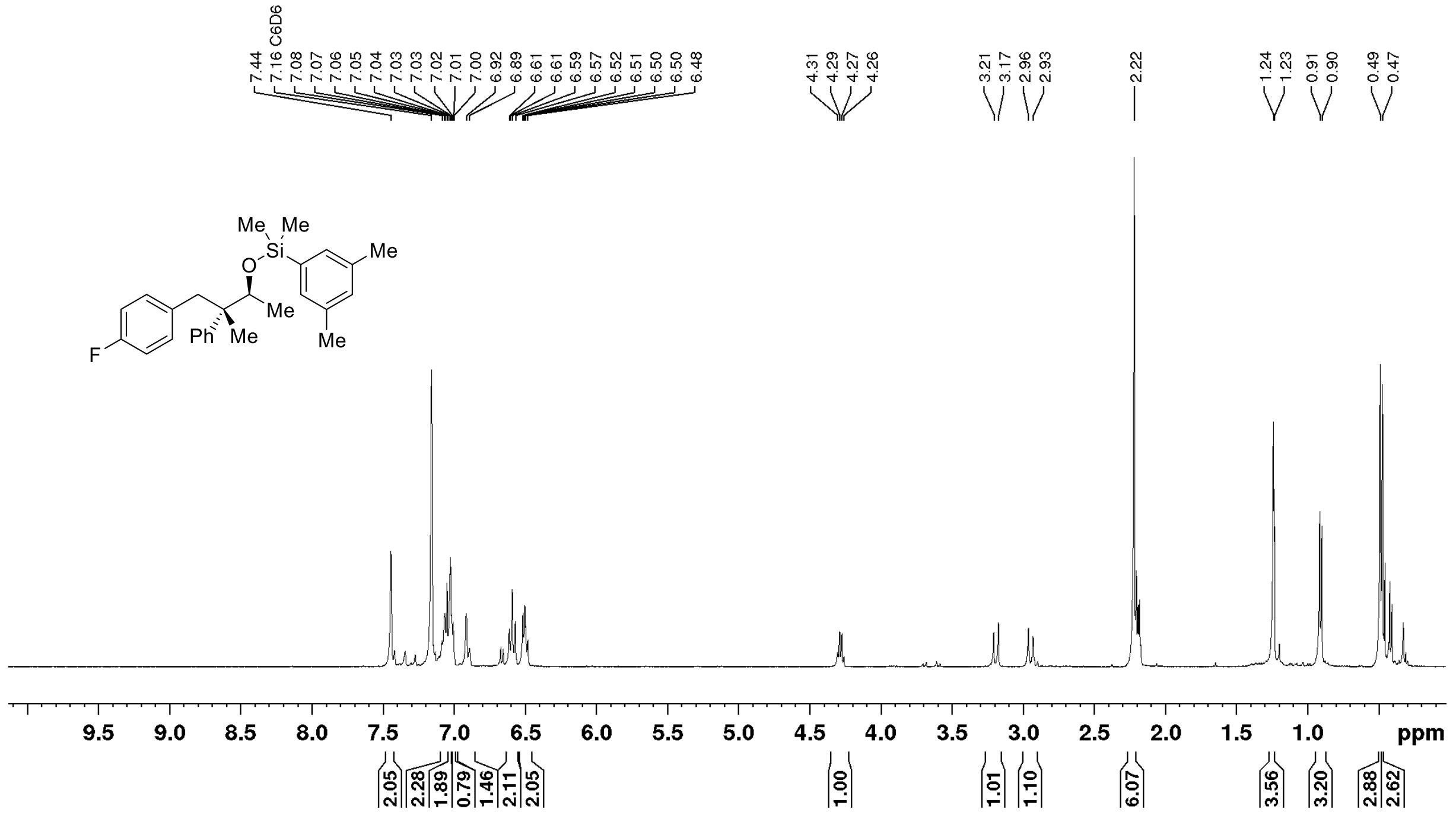


Figure 8.59 ${ }^{13} \mathrm{C}$ NMR (126 MHz, $\left.\mathrm{C}_{6} \mathrm{D}_{6}, 298 \mathrm{~K}\right)$ of (2S,3S)-(3,5-Dimethylphenyl)((4-(4-fluorophenyl)-3-methyl-3-phenylbutan-2yl)oxy)dimethylsilane [(2S,3S)-3de].

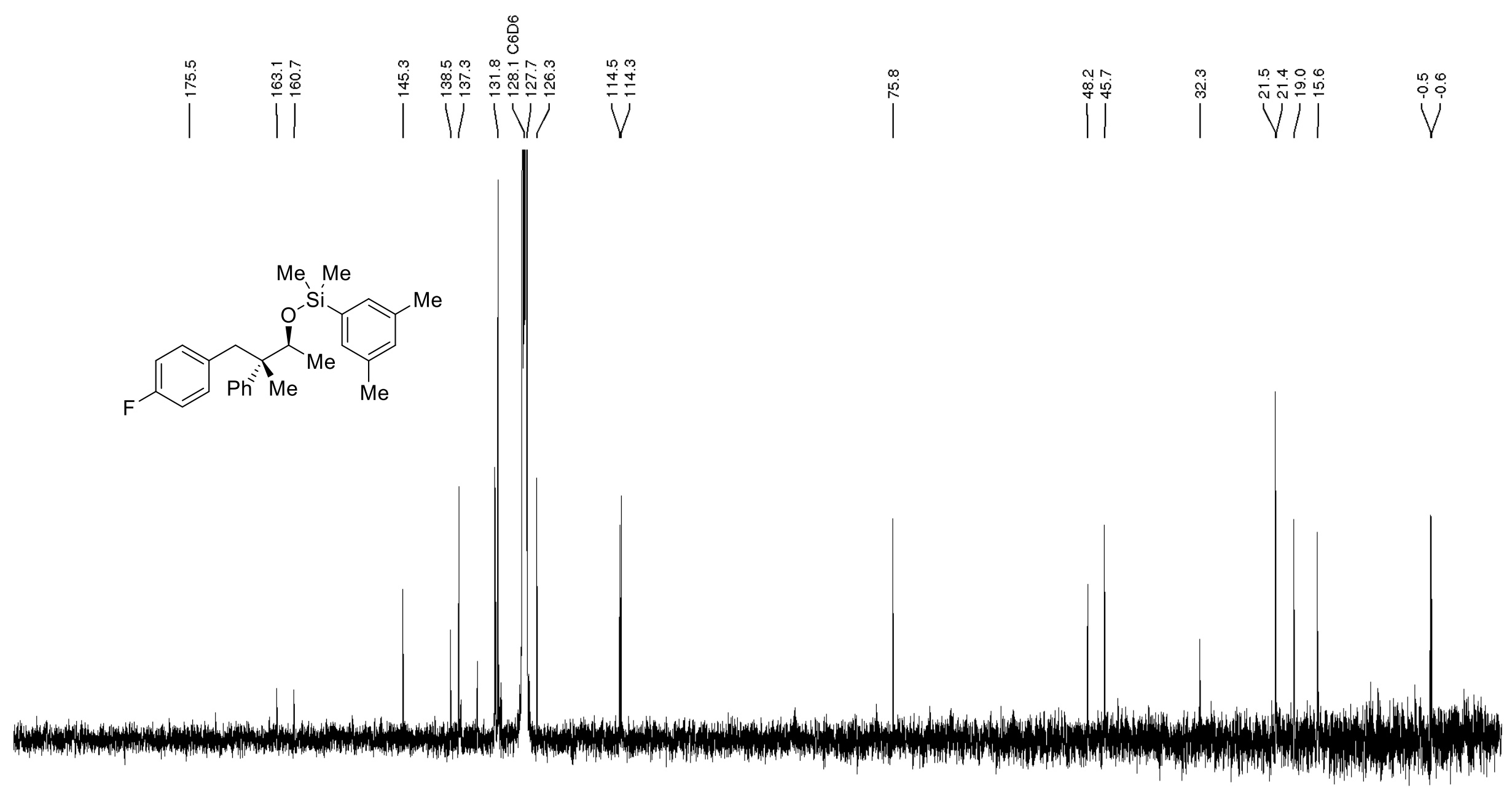

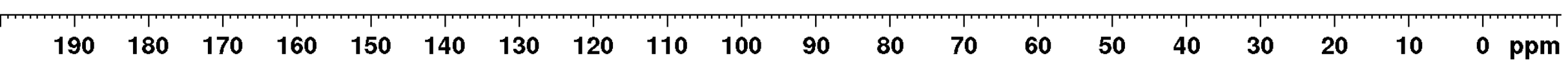


Figure 8.60 ${ }^{29} \mathrm{Si}$ DEPT NMR (99 MHz, $\left.\mathrm{C}_{6} \mathrm{D}_{6}, 298 \mathrm{~K}\right)$ of (2S,3S)-(3,5-Dimethylphenyl)((4-(4-fluorophenyl)-3-methyl-3phenylbutan-2-yl)oxy)dimethylsilane [(2S,3S)-3de].

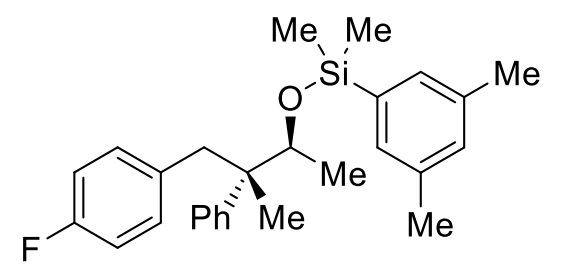

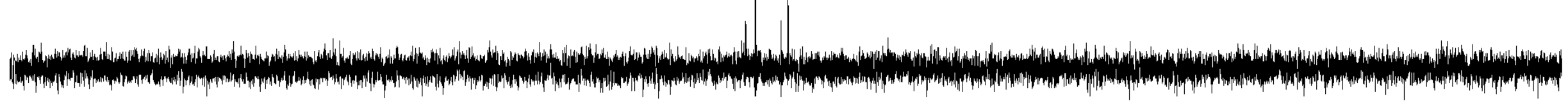


Figure 8.61 ${ }^{1} \mathrm{H}$ NMR (500 MHz, $\mathrm{CDCl}_{3}, 298 \mathrm{~K}$ ) of rac-anti/syn-3-Methyl-3-phenyl-4-(p-tolyl)butan-2-ol (rac-anti/syn-1i).

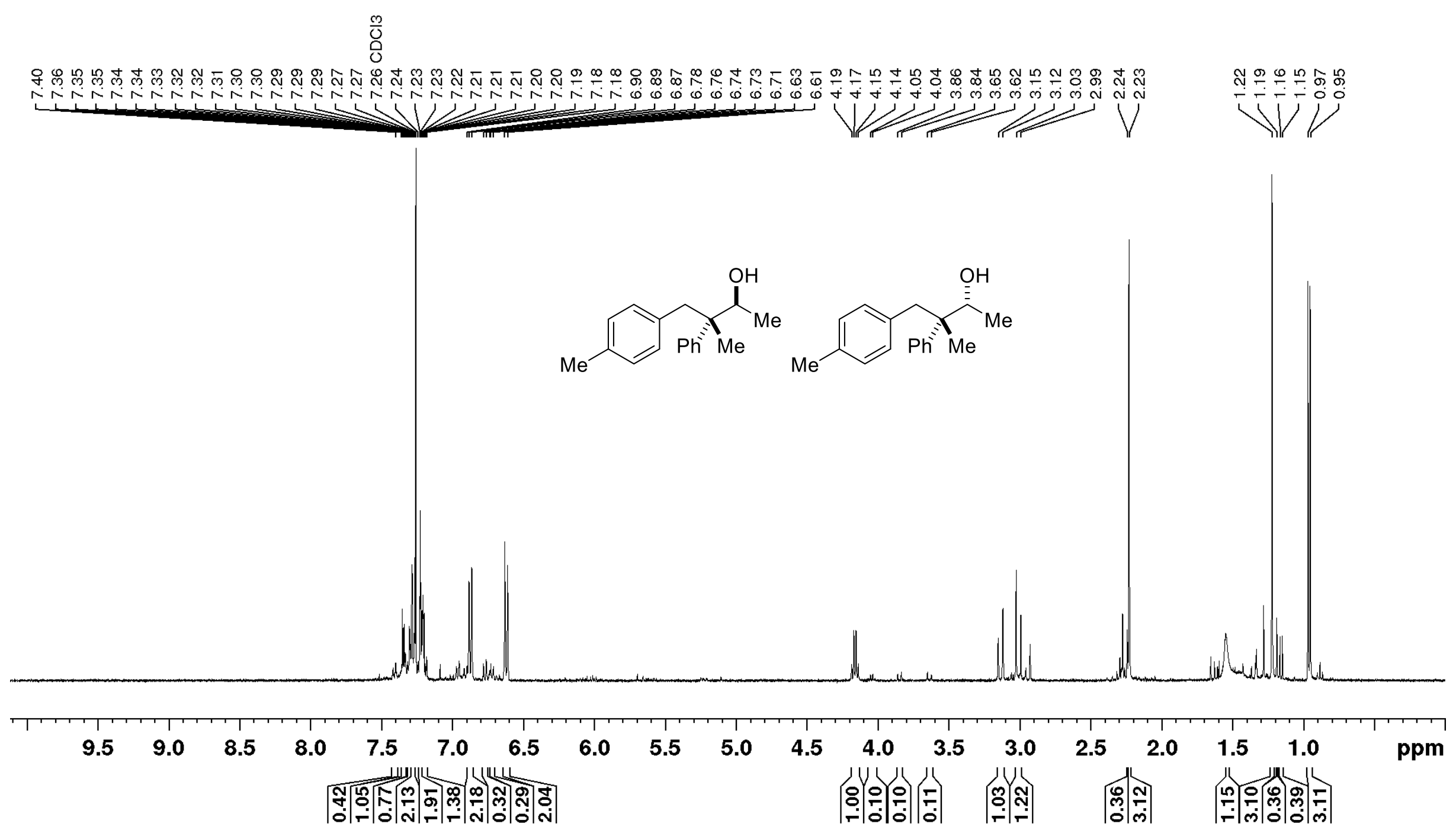


Figure $8.62{ }^{13} \mathrm{C}$ NMR (126 MHz, $\mathrm{CDCl}_{3}, 298 \mathrm{~K}$ ) of rac-anti/syn-3-Methyl-3-phenyl-4-(p-tolyl)butan-2-ol (rac-anti/syn-1i).

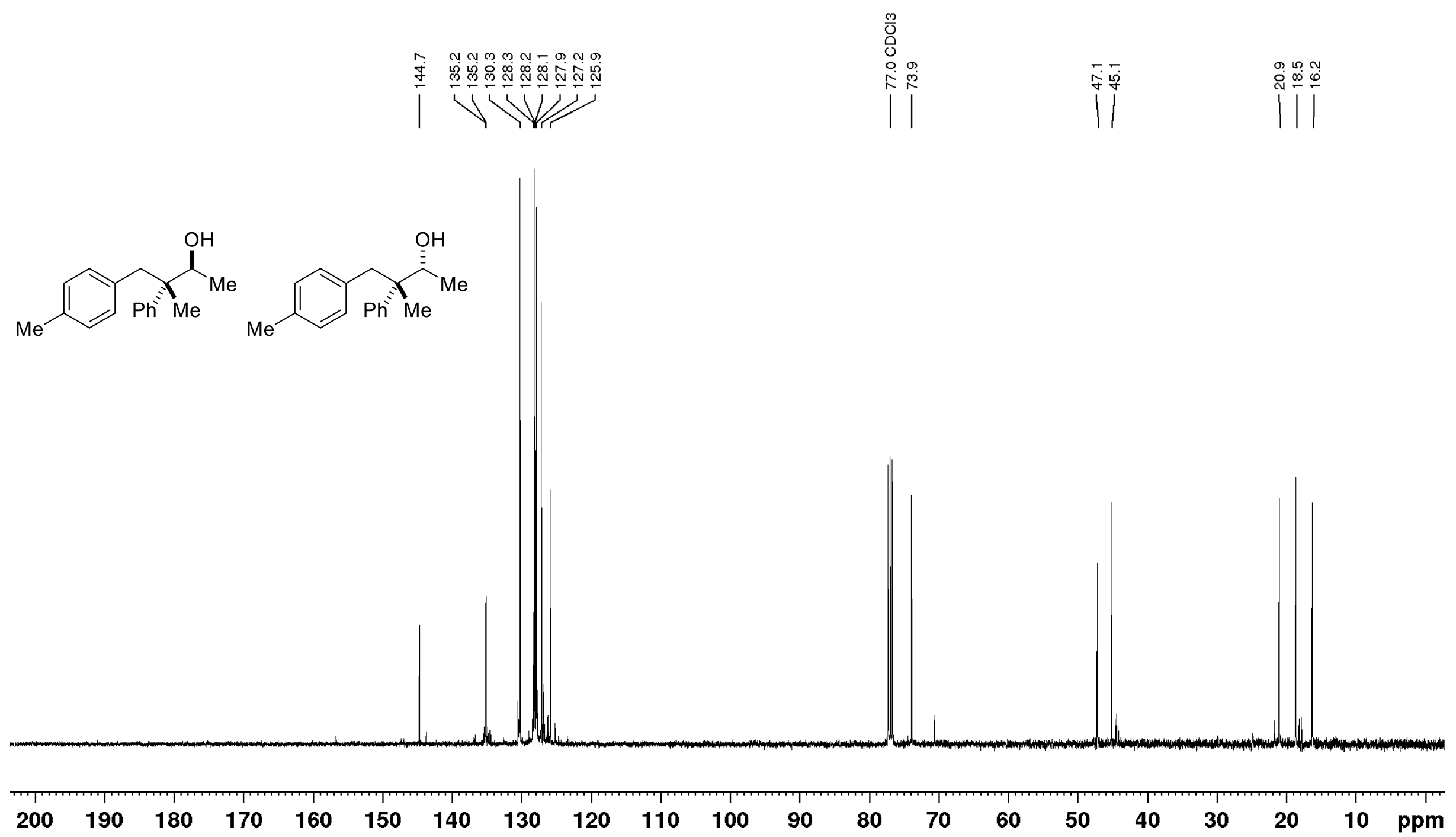


Figure 8.63 ${ }^{1} \mathrm{H}$ NMR (500 MHz, $\left.\mathrm{C}_{6} \mathrm{D}_{6}, 298 \mathrm{~K}\right)$ of (2S,3S)-(3,5-Dimethylphenyl)dimethyl((3-methyl-3-phenyl-4-( $p$-tolyl)butan-2yl)oxy)silane $[(2 S, 3 S)-3 i e]$.

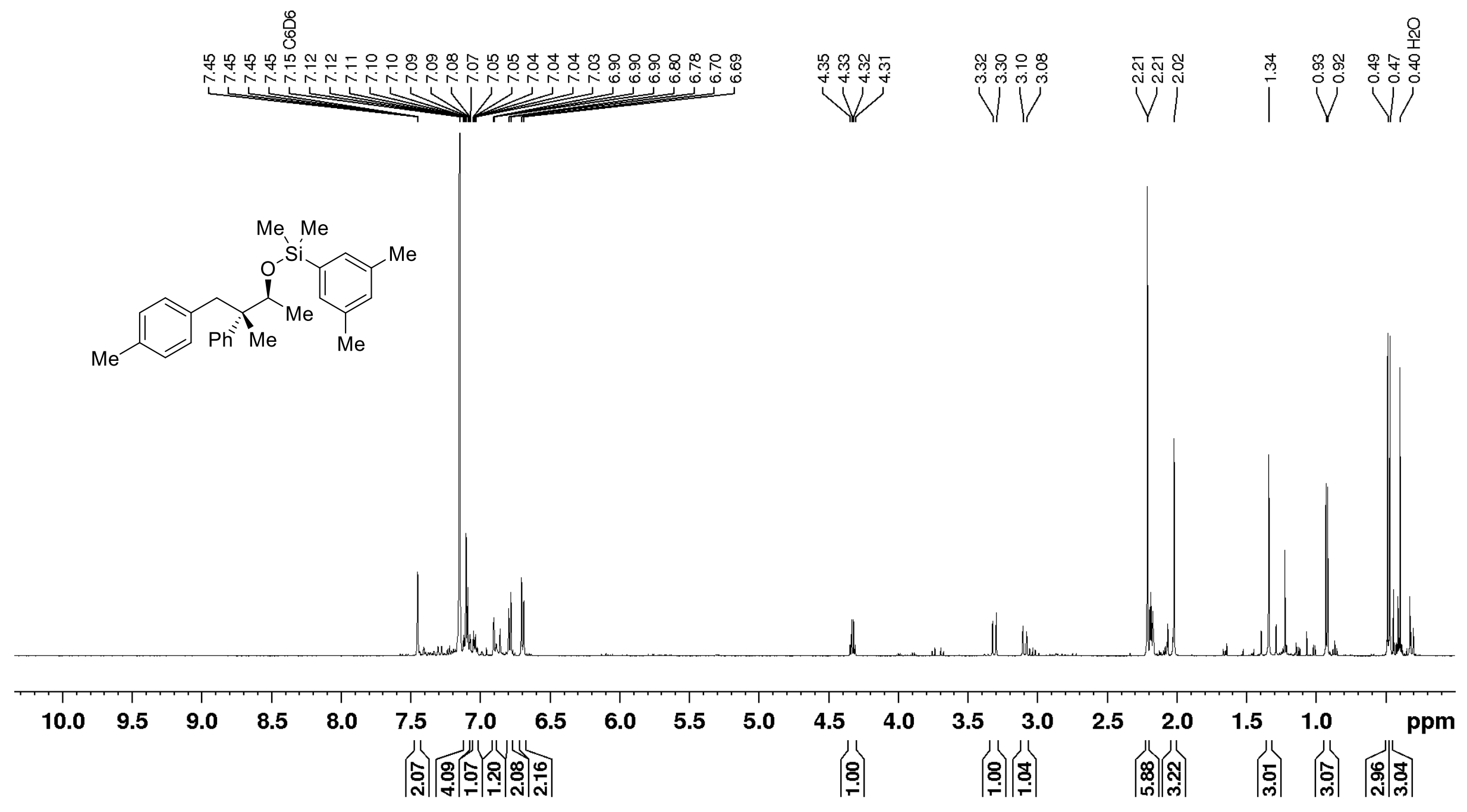


Figure 8.64 ${ }^{13} \mathrm{C}$ NMR (126 MHz, $\left.\mathrm{C}_{6} \mathrm{D}_{6}, 298 \mathrm{~K}\right)$ of (2S,3S)-(3,5-Dimethylphenyl)dimethyl((3-methyl-3-phenyl-4-(p-tolyl)butan-2yl)oxy)silane $[(2 S, 3 S)-3 i e]$.

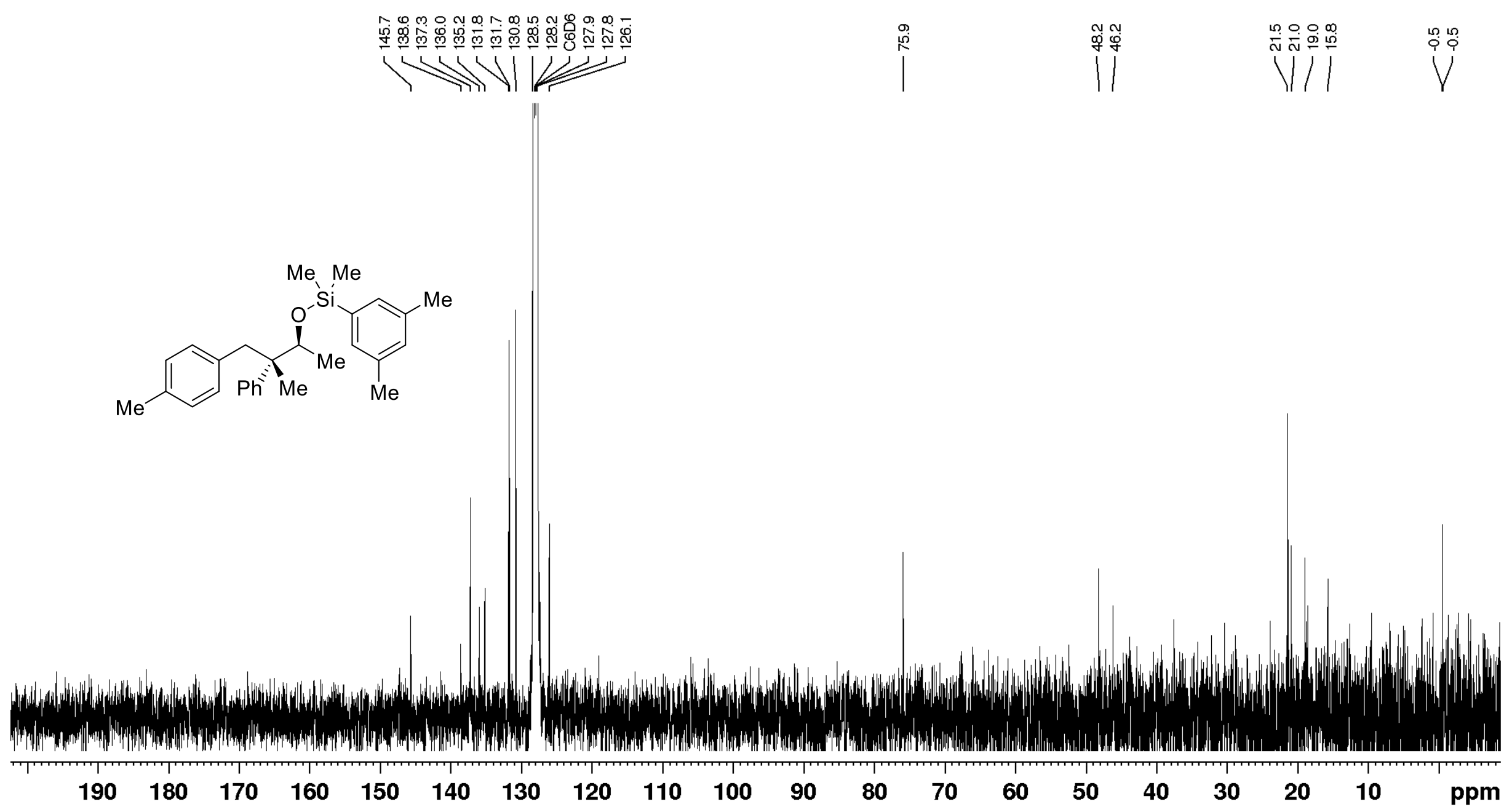


Figure 8.65 ${ }^{29} \mathrm{Si}$ DEPT NMR (99 MHz, $\left.\mathrm{C}_{6} \mathrm{D}_{6}, 298 \mathrm{~K}\right)$ of $(2 S, 3 S)-(3,5-$ Dimethylphenyl)dimethyl((3-methyl-3-phenyl-4-( $p$ tolyl)butan-2-yl)oxy)silane [(2S,3S)-3ie].

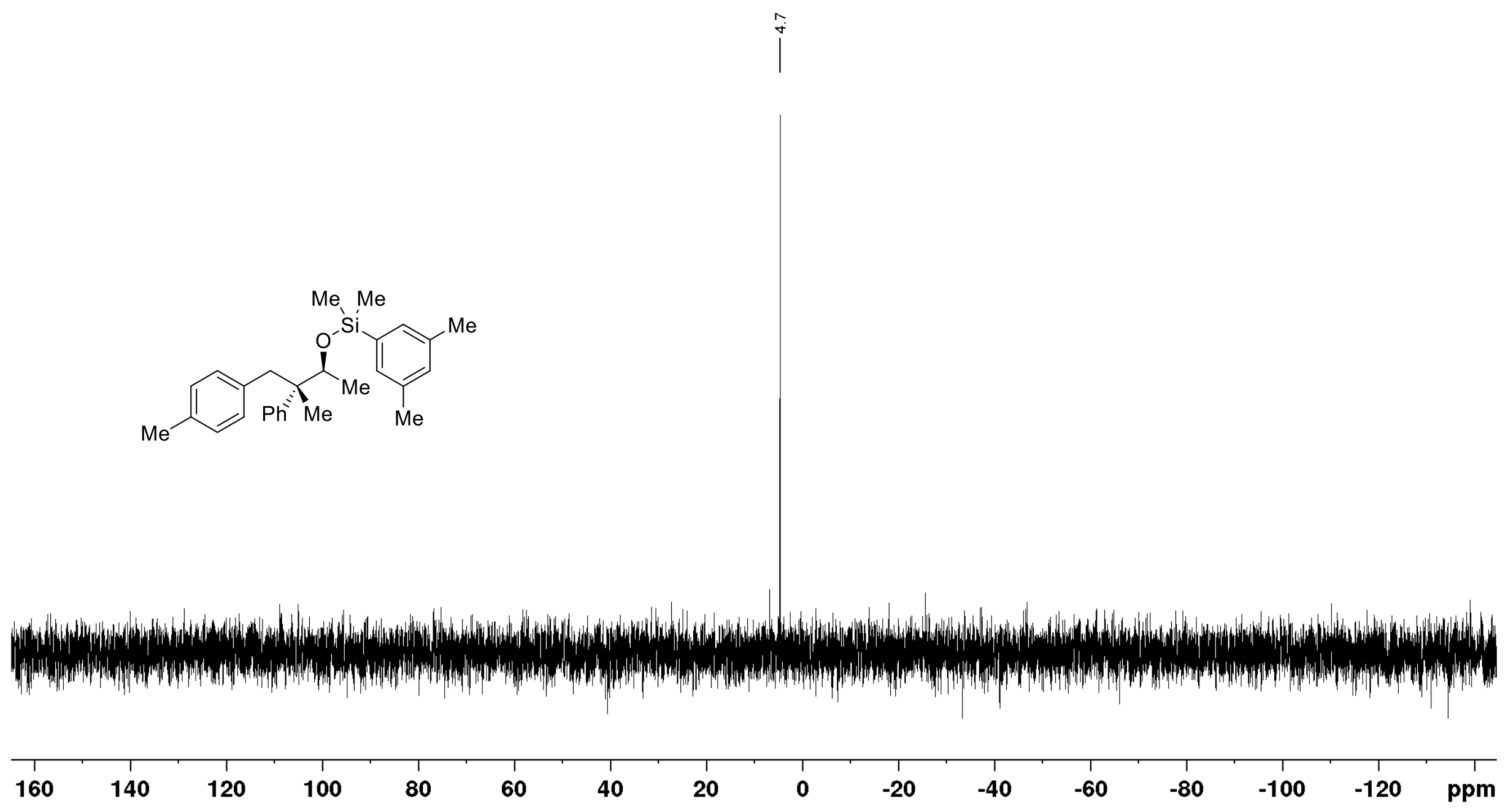


Figure 8.66 ${ }^{1} \mathrm{H}$ NMR $\left(500 \mathrm{MHz}, \mathrm{CDCl}_{3}, 298 \mathrm{~K}\right)$ of rac-anti/syn-4-(3,5-Dimethylphenyl)-3-methyl-3-phenylbutan-2-ol (rac-anti/syn1j).

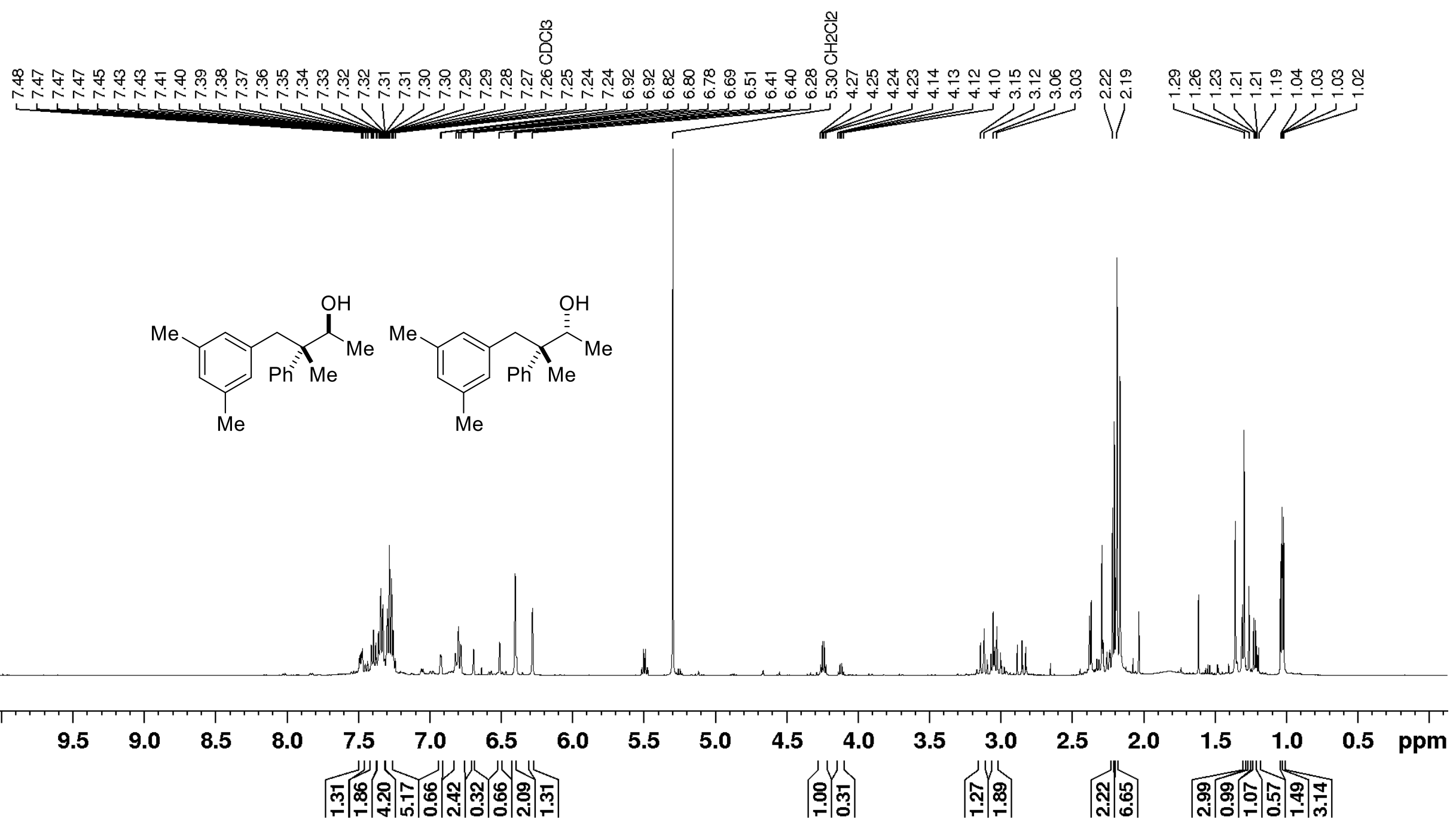


Figure 8.67 ${ }^{13} \mathrm{C} \mathrm{NMR}\left(126 \mathrm{MHz}, \mathrm{CDCl}_{3}, 298 \mathrm{~K}\right.$ ) of rac-anti/syn-4-(3,5-Dimethylphenyl)-3-methyl-3-phenylbutan-2-ol (rac-anti/syn1j).

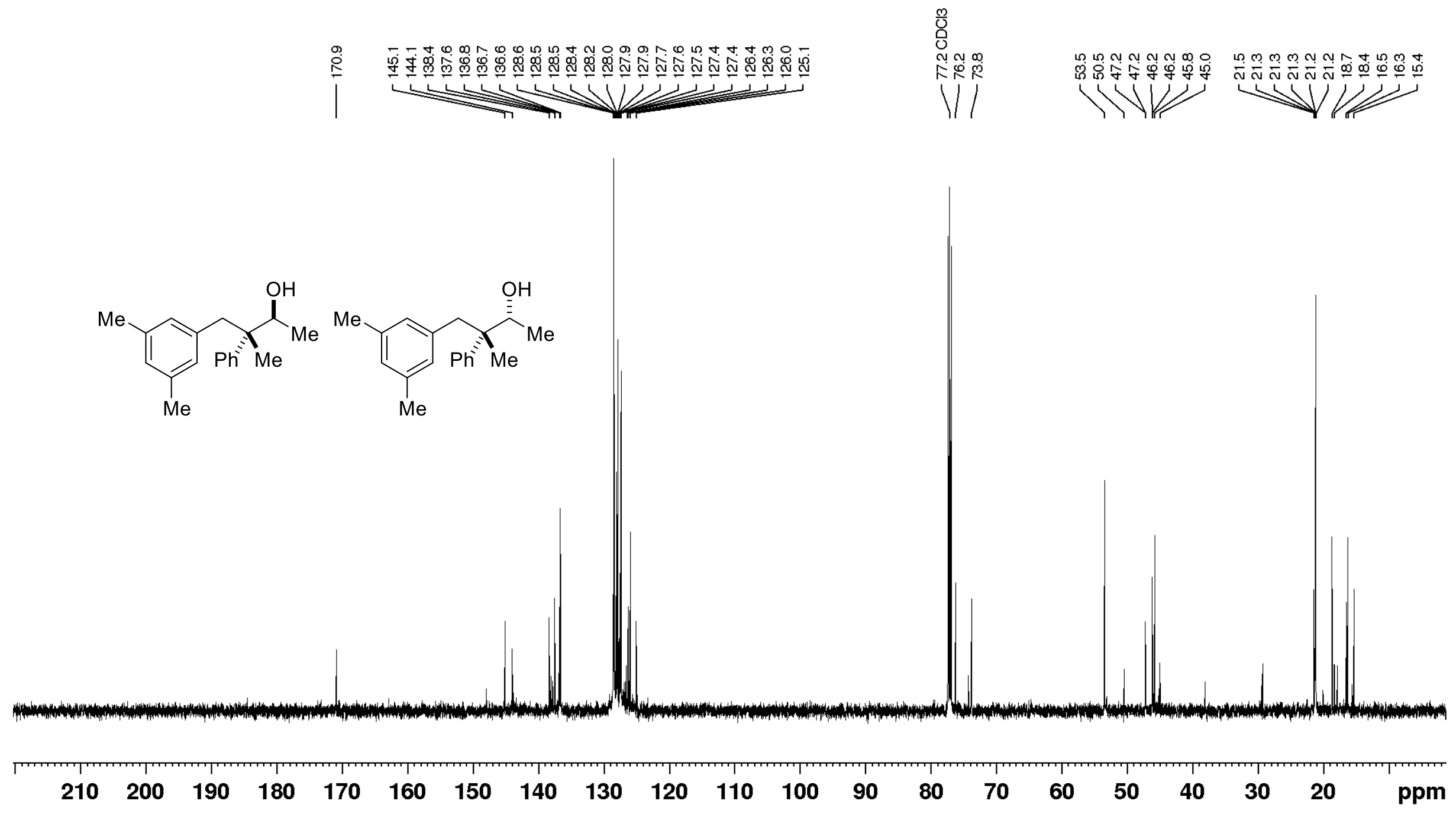


Figure 8.68 ${ }^{1} \mathrm{H}$ NMR (500 MHz, $\left.\mathrm{C}_{6} \mathrm{D}_{6}, 298 \mathrm{~K}\right)$ of (2S,3S)-(3,5-Dimethylphenyl)((4-(3,5-dimethylphenyl)-3-methyl-3phenylbutan-2-yl)oxy)dimethylsilane [(2S,3S)-3je]
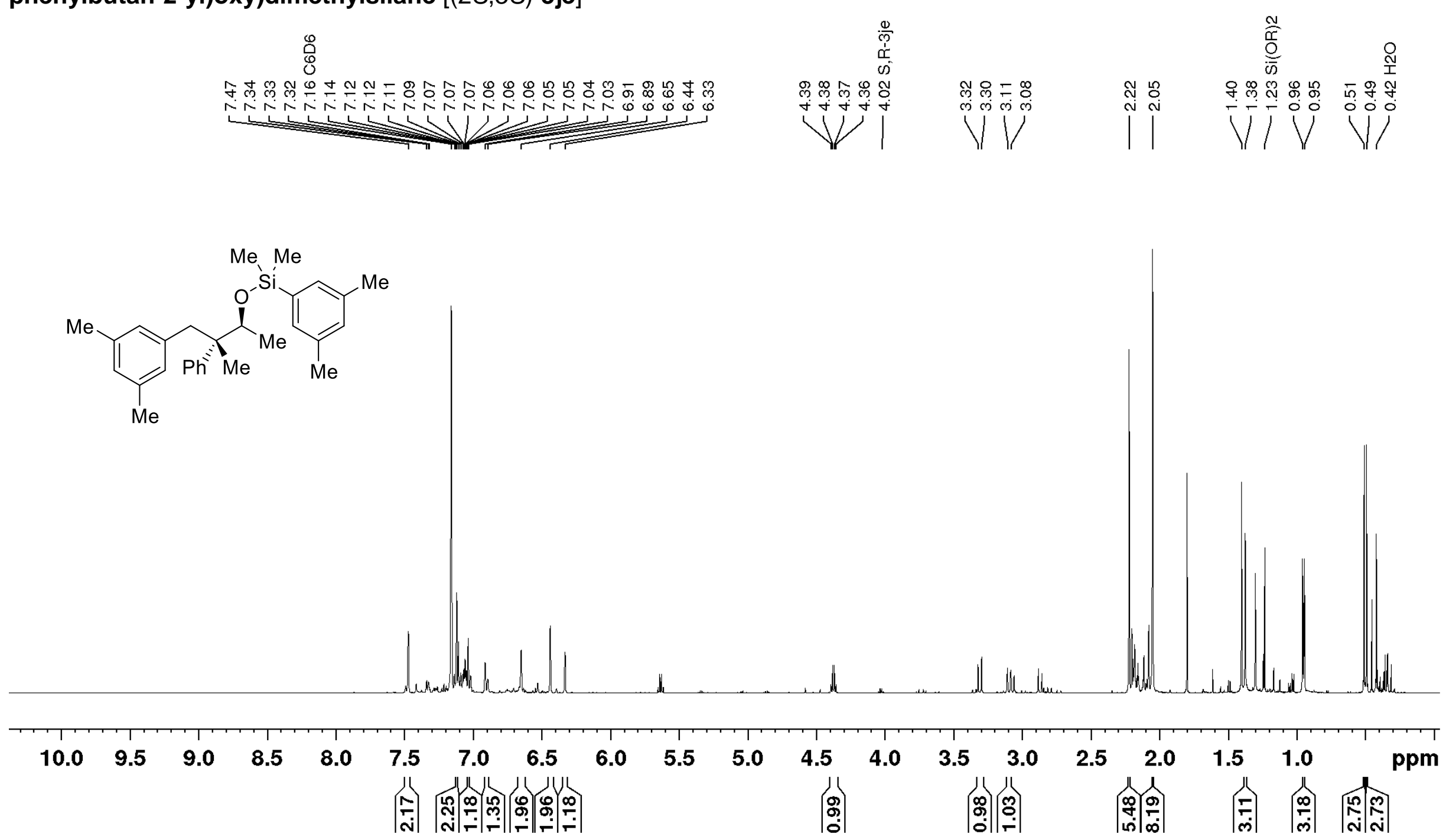
Figure 8.69 ${ }^{13} \mathrm{C}$ NMR (126 MHz, $\left.\mathrm{C}_{6} \mathrm{D}_{6}, 298 \mathrm{~K}\right)$ of (2S,3S)-(3,5-Dimethylphenyl)((4-(3,5-dimethylphenyl)-3-methyl-3phenylbutan-2-yl)oxy)dimethylsilane [(2S,3S)-3je].

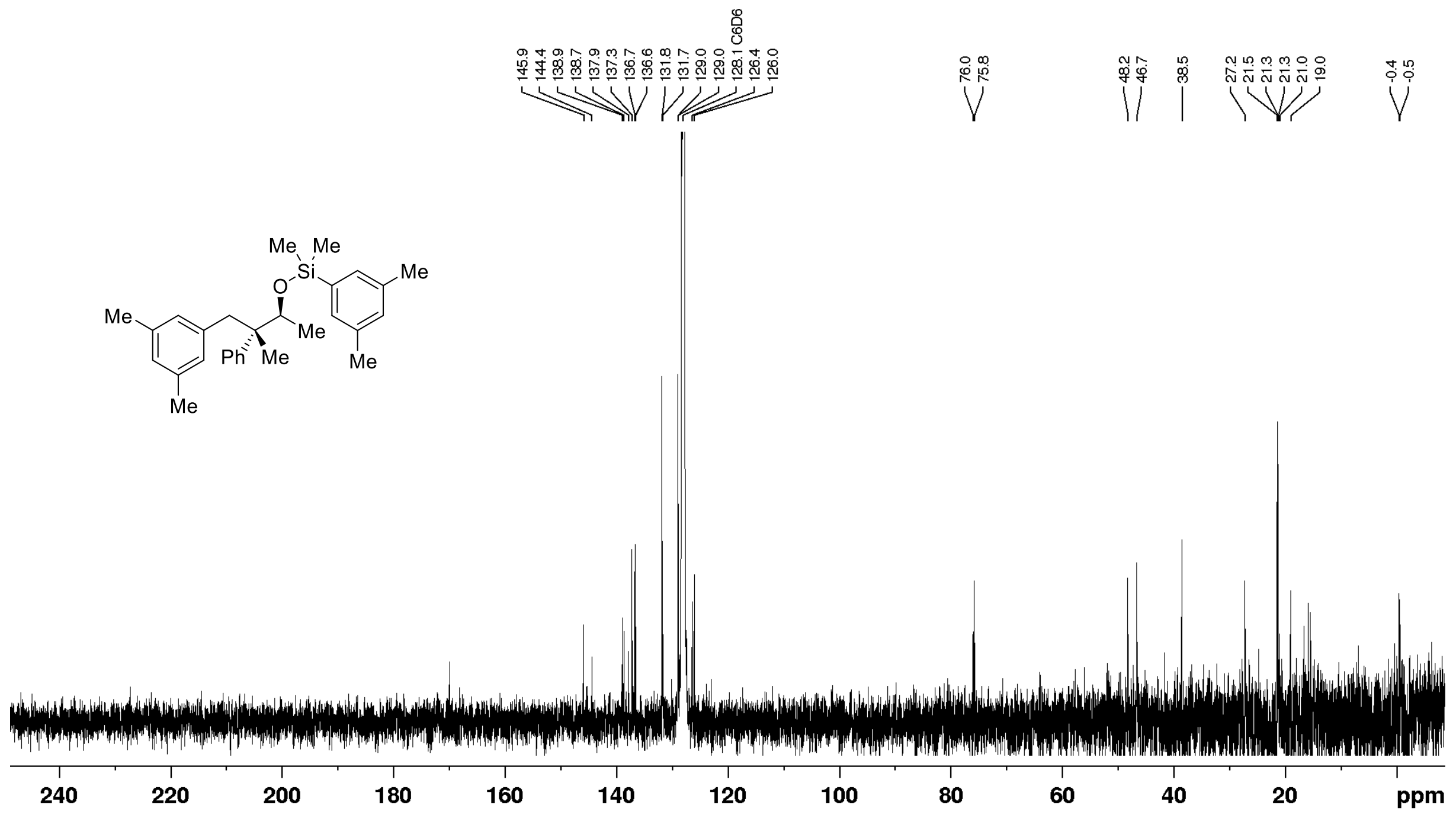


Figure 8.70 ${ }^{29} \mathrm{Si}$ DEPT NMR (99 MHz, $\left.\mathrm{C}_{6} \mathrm{D}_{6}, 298 \mathrm{~K}\right)$ of $(2 S, 3 S)-(3,5-$ Dimethylphenyl)((4-(3,5-dimethylphenyl)-3-methyl-3phenylbutan-2-yl)oxy)dimethylsilane [(2S,3S)-3je].

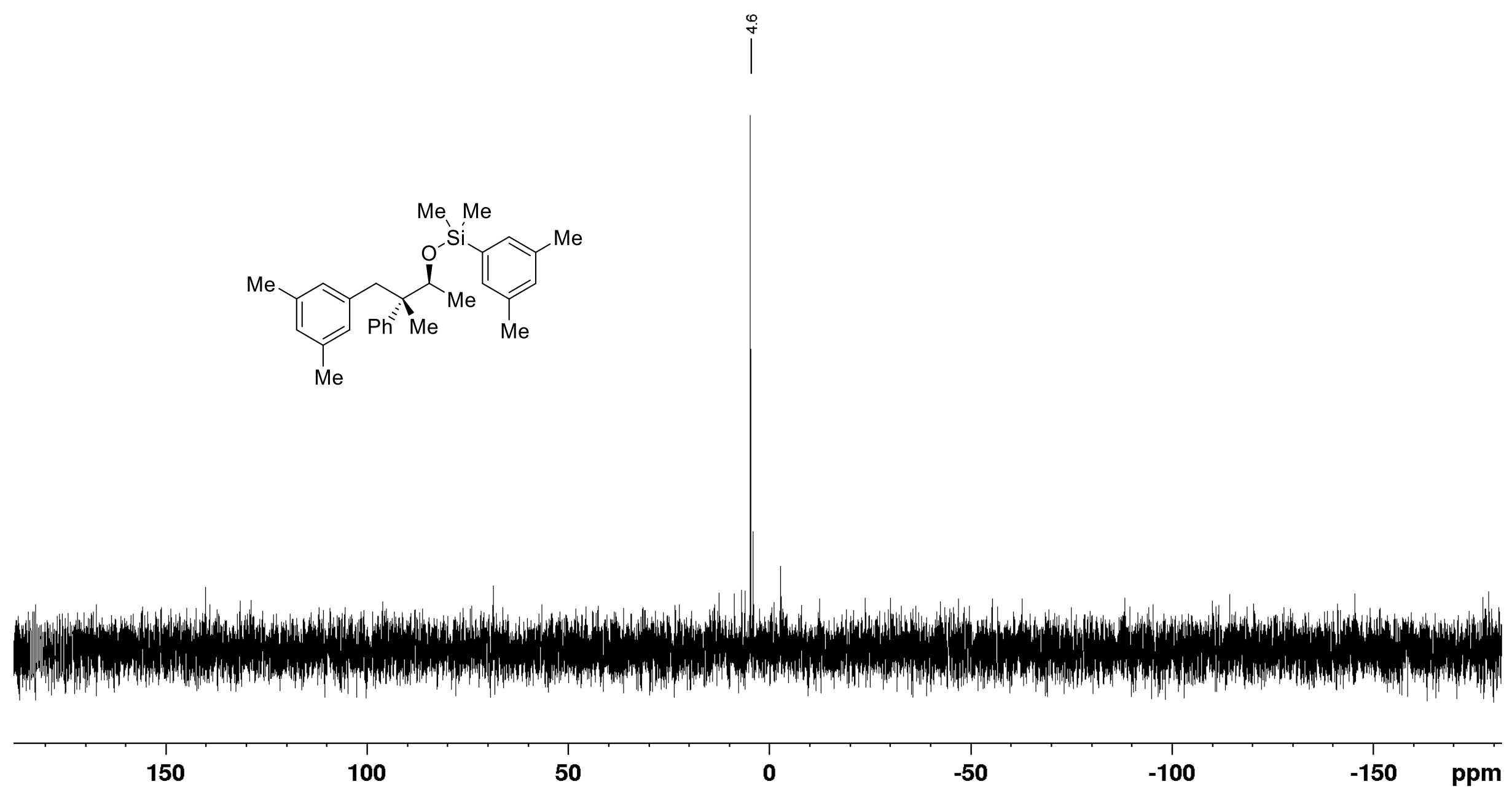


Figure 8.71 ${ }^{1} \mathrm{H}$ NMR (500 MHz, $\mathrm{C}_{6} \mathrm{D}_{6}, 298 \mathrm{~K}$ ) of rac-anti/syn-4-(3,5-Dimethoxyphenyl)-3-methyl-3-phenylbutan-2-ol (rac-anti/syn1I).

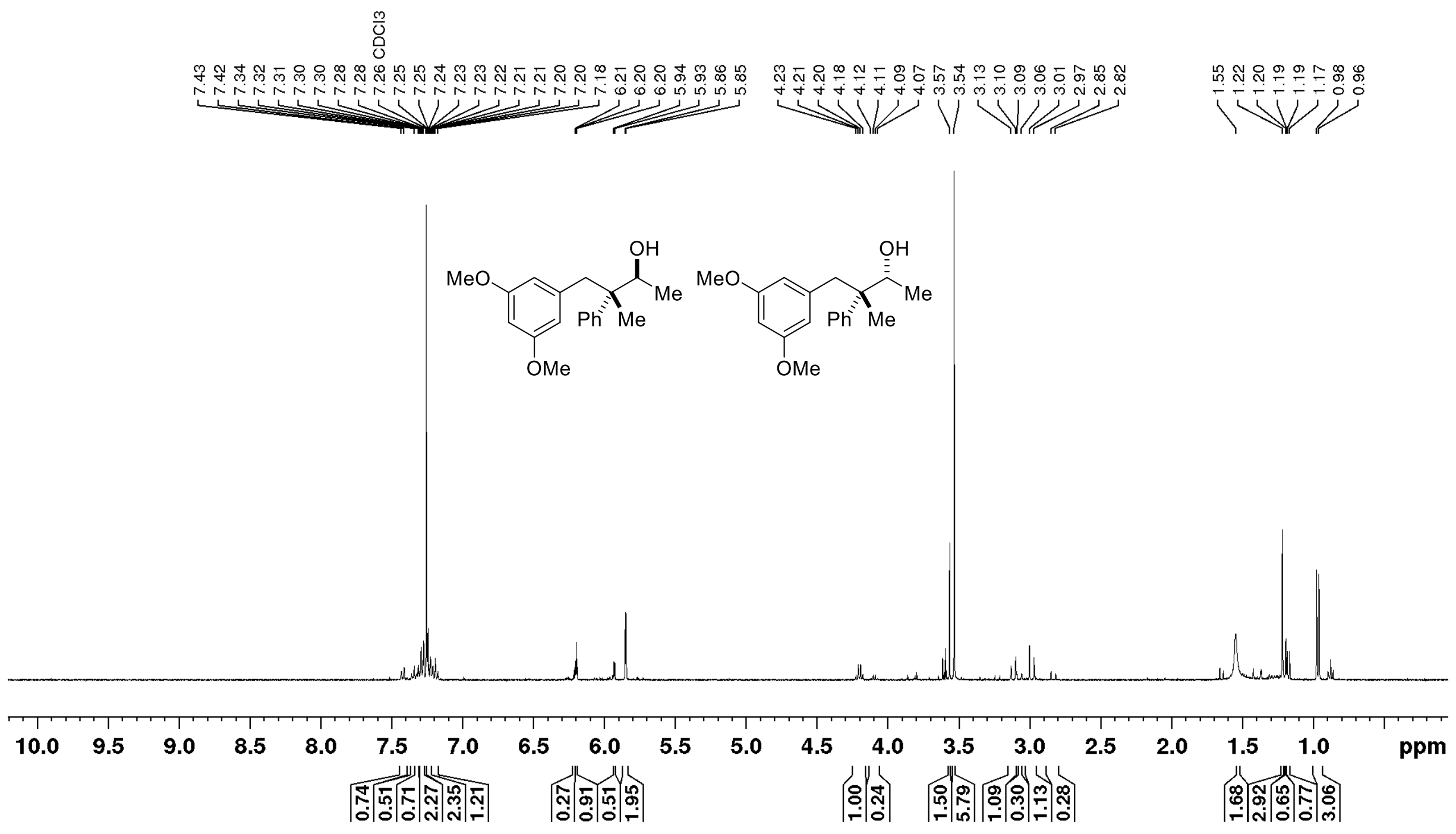


Figure 8.72 ${ }^{13} \mathrm{C}$ NMR (126 MHz, $\left.\mathrm{C}_{6} \mathrm{D}_{6}, 298 \mathrm{~K}\right)$ of rac-anti/syn-4-(3,5-Dimethoxyphenyl)-3-methyl-3-phenylbutan-2-ol (rac-anti/syn1I).

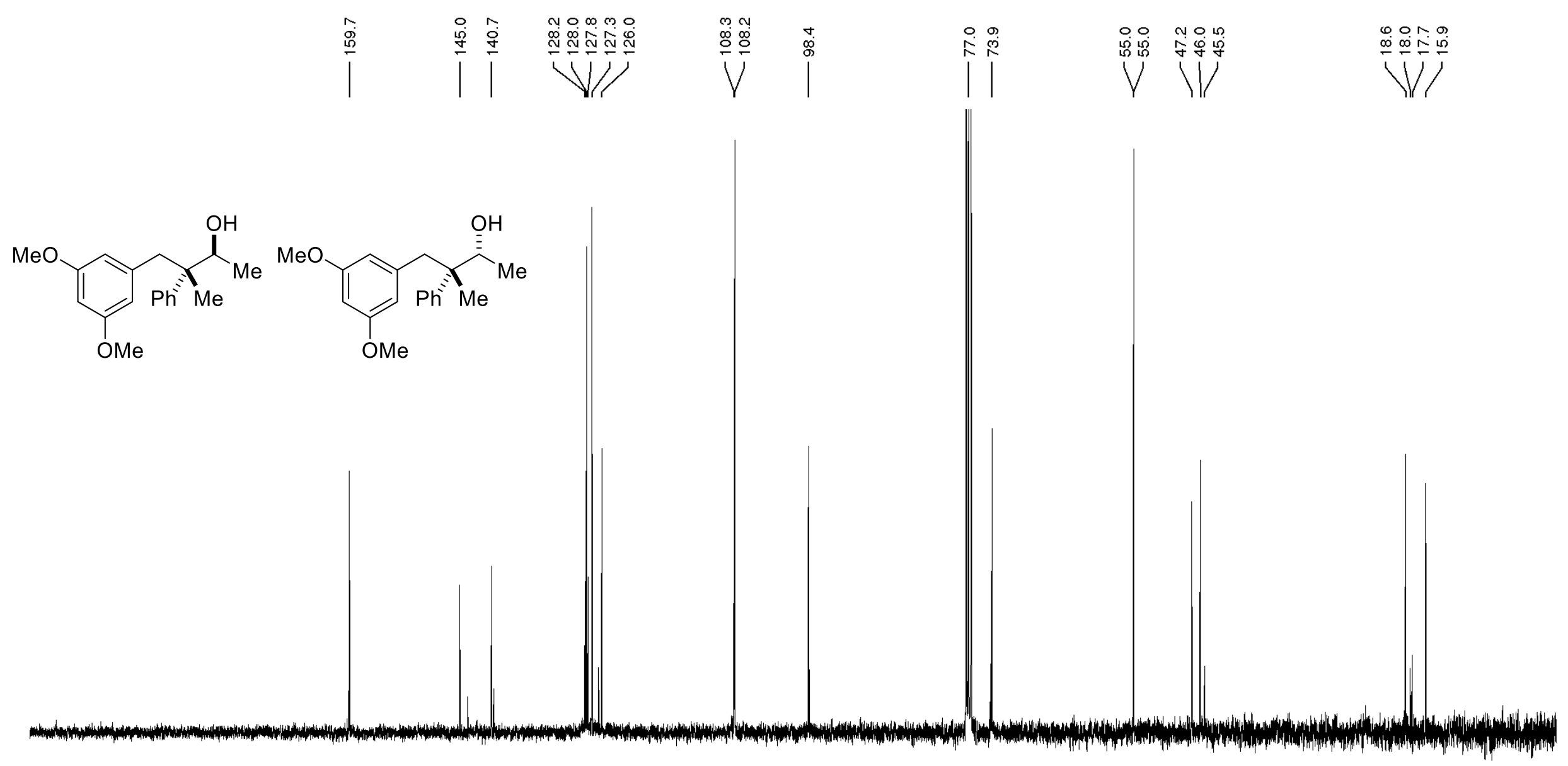


Figure 8.73 ${ }^{1} \mathrm{H}$ NMR (500 MHz, $\left.\mathrm{C}_{6} \mathrm{D}_{6}, 298 \mathrm{~K}\right)$ of (2S,3S)-((4-(3,5-Dimethoxyphenyl)-3-methyl-3-phenylbutan-2-yl)oxy)(3,5dimethylphenyl)dimethylsilane [(2S,3S)-3le].
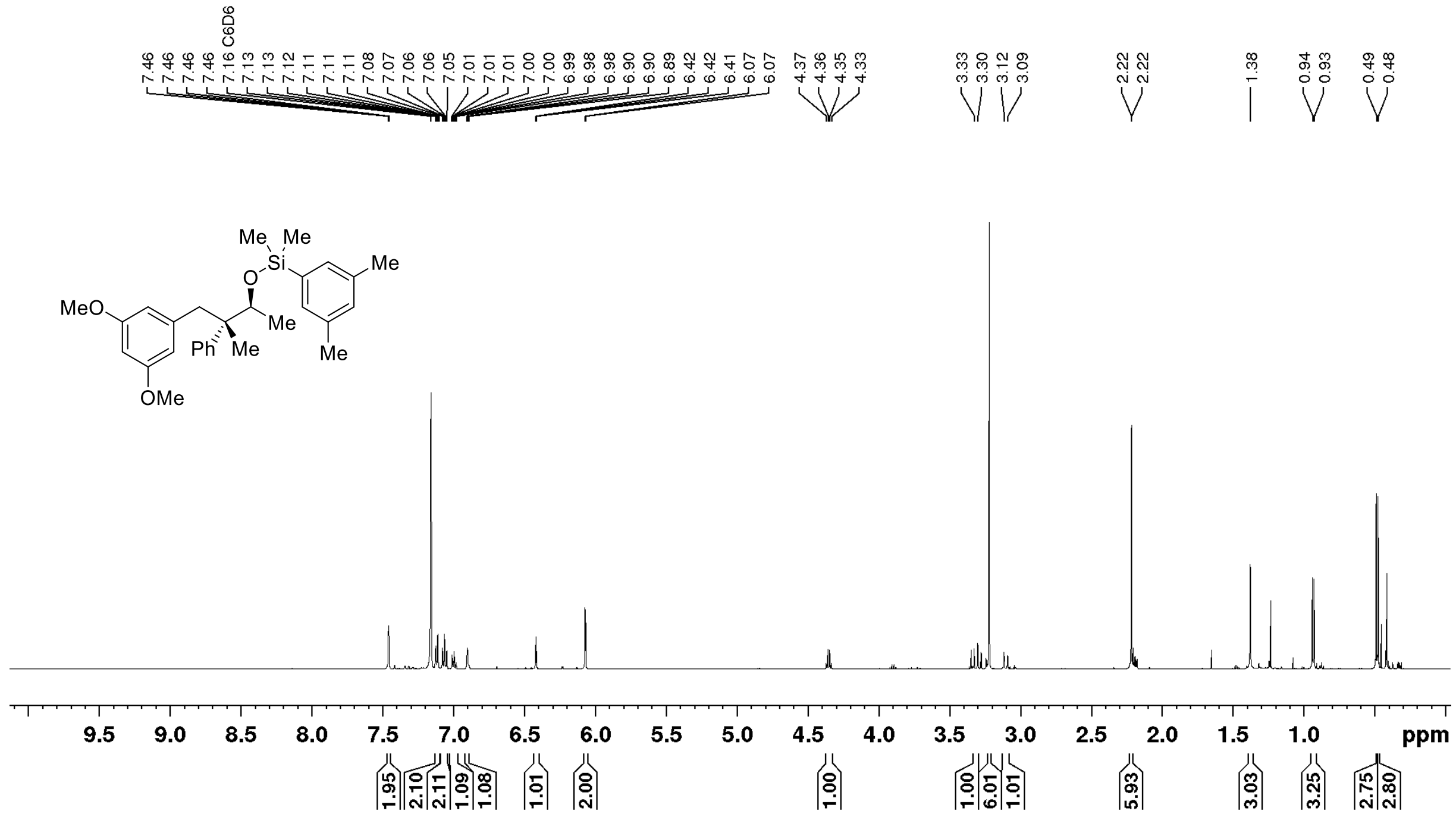
Figure 8.74 ${ }^{13} \mathrm{C}$ NMR (126 MHz, $\left.\mathrm{C}_{6} \mathrm{D}_{6}, 298 \mathrm{~K}\right)$ of $(2 S, 3 S)-((4-(3,5-D i m e t h o x y p h e n y l)-3-m e t h y l-3-p h e n y l b u t a n-2-y l) o x y)(3,5-$ dimethylphenyl)dimethylsilane $[(2 S, 3 S)-3 l e]$.

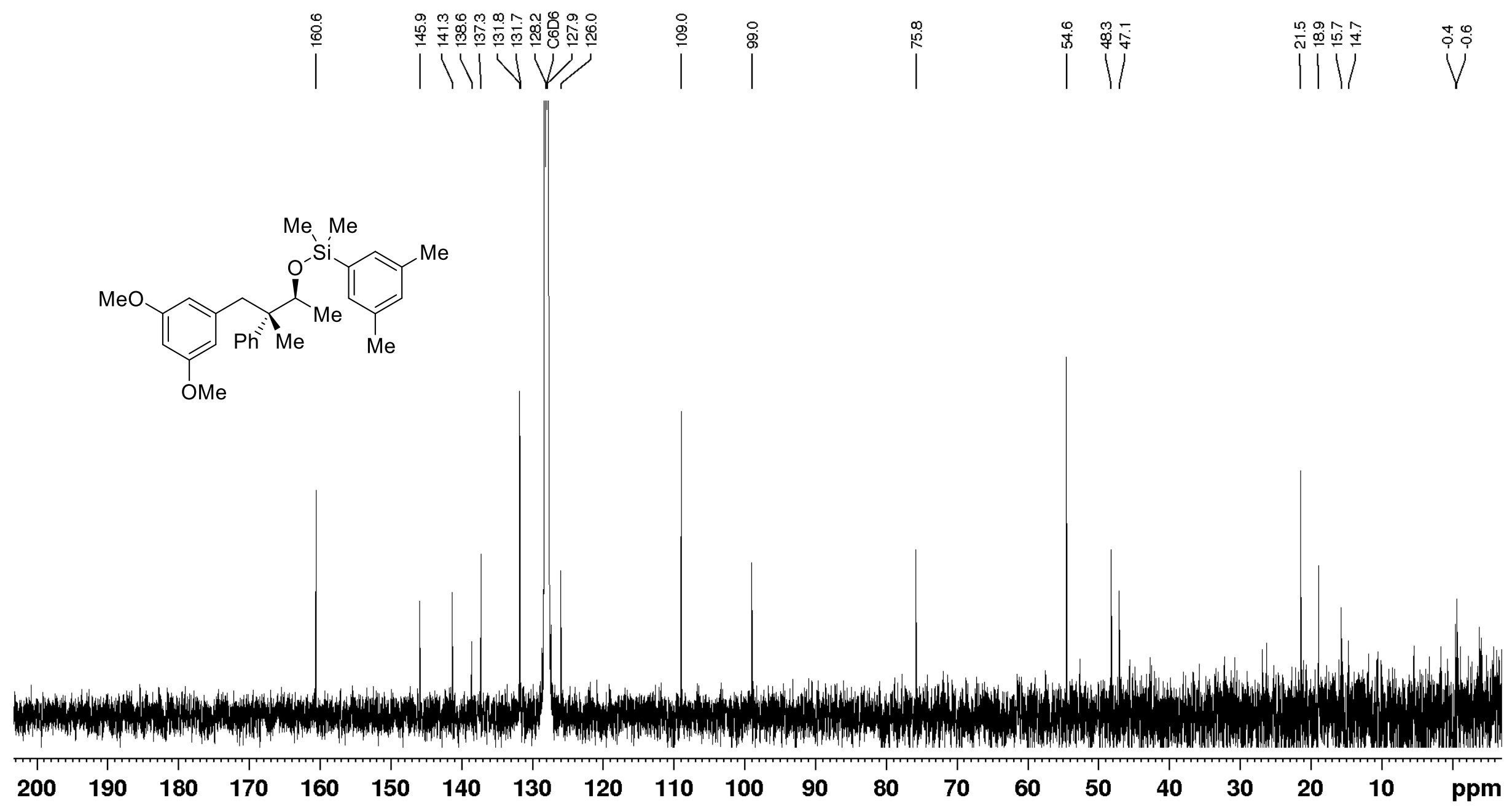


Figure 8.75 ${ }^{29} \mathrm{Si}$ DEPT NMR (99 MHz, CDCl $\left.3,298 \mathrm{~K}\right)$ of (2S,3S)-((4-(3,5-Dimethoxyphenyl)-3-methyl-3-phenylbutan-2yl)oxy)(3,5-dimethylphenyl)dimethylsilane [(2S,3S)-3le].

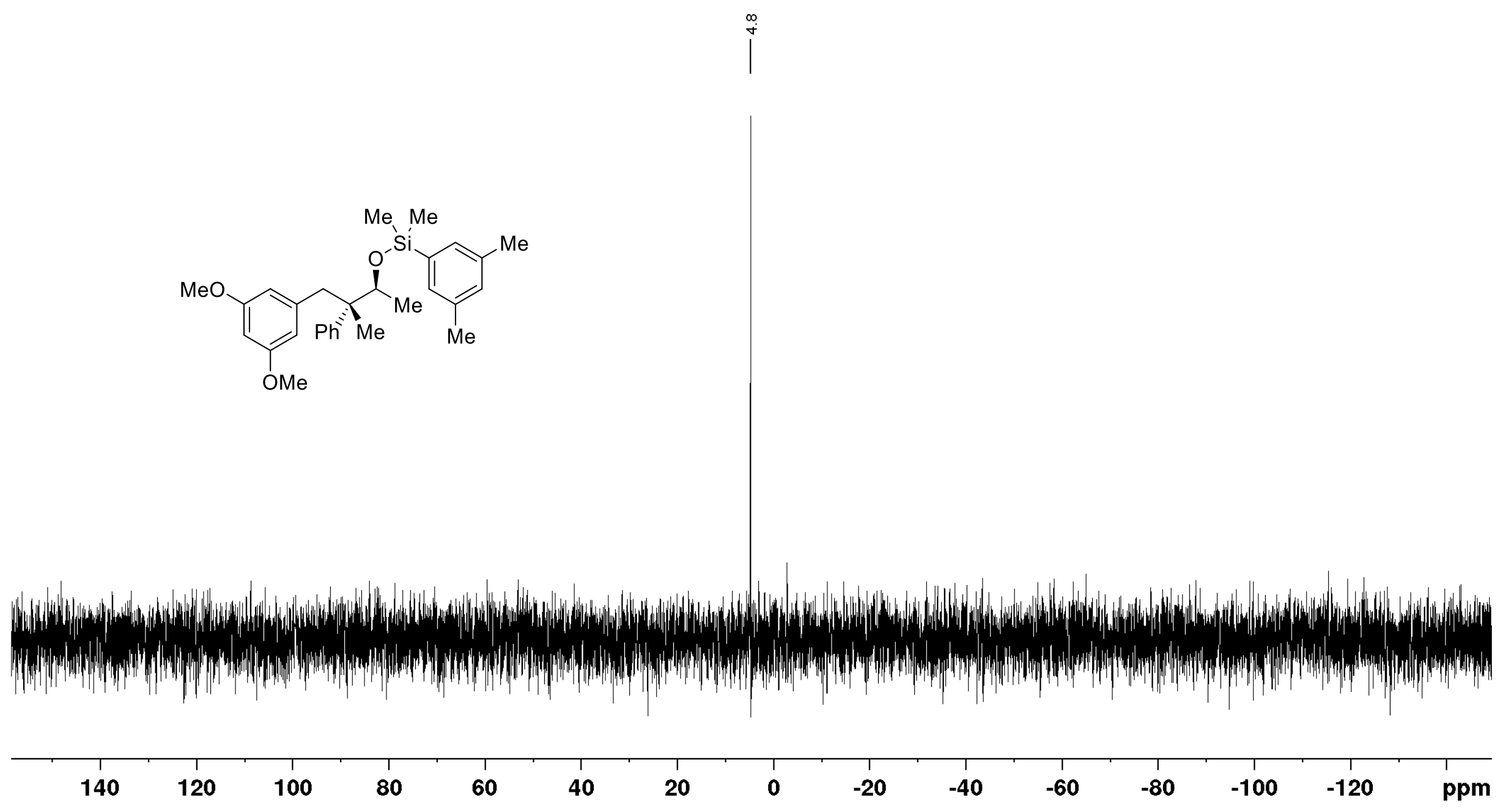


Figure 8.76 ${ }^{1} \mathrm{H}$ NMR (500 MHz, $\left.\mathrm{C}_{6} \mathrm{D}_{6}, 298 \mathrm{~K}\right)$ of rac-anti/syn-4-(4-Bromophenyl)-3-methyl-3-phenylbutan-2-ol (rac-anti/syn-1f).

$\frac{m}{0}$

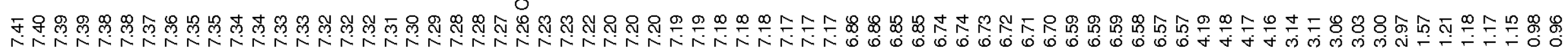

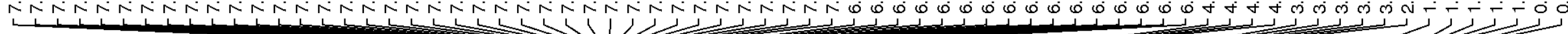

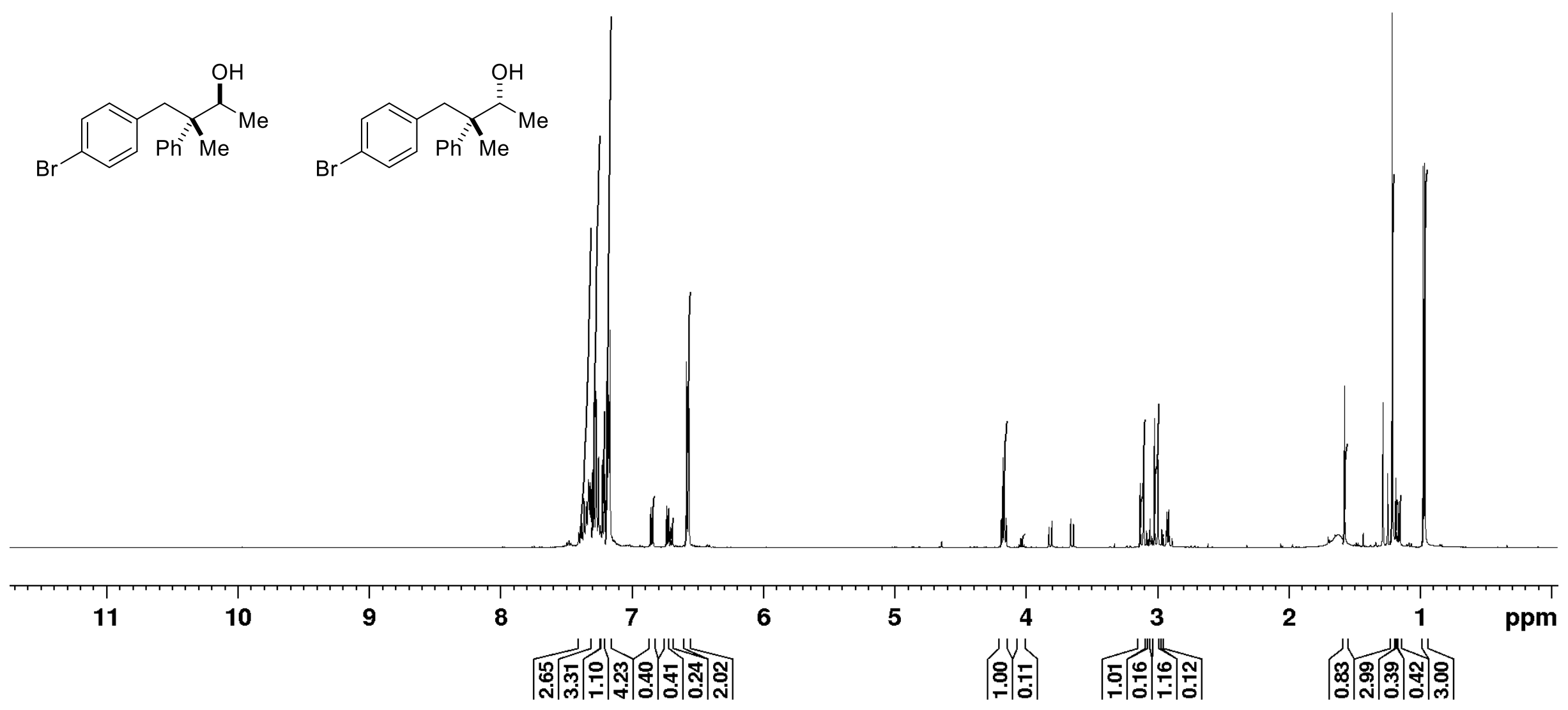


Figure 8.77 ${ }^{13} \mathrm{C}$ NMR (126 MHz, $\mathrm{C}_{6} \mathrm{D}_{6}, 298 \mathrm{~K}$ ) of rac-anti/syn-4-(4-Bromophenyl)-3-methyl-3-phenylbutan-2-ol (rac-anti/syn-1f).

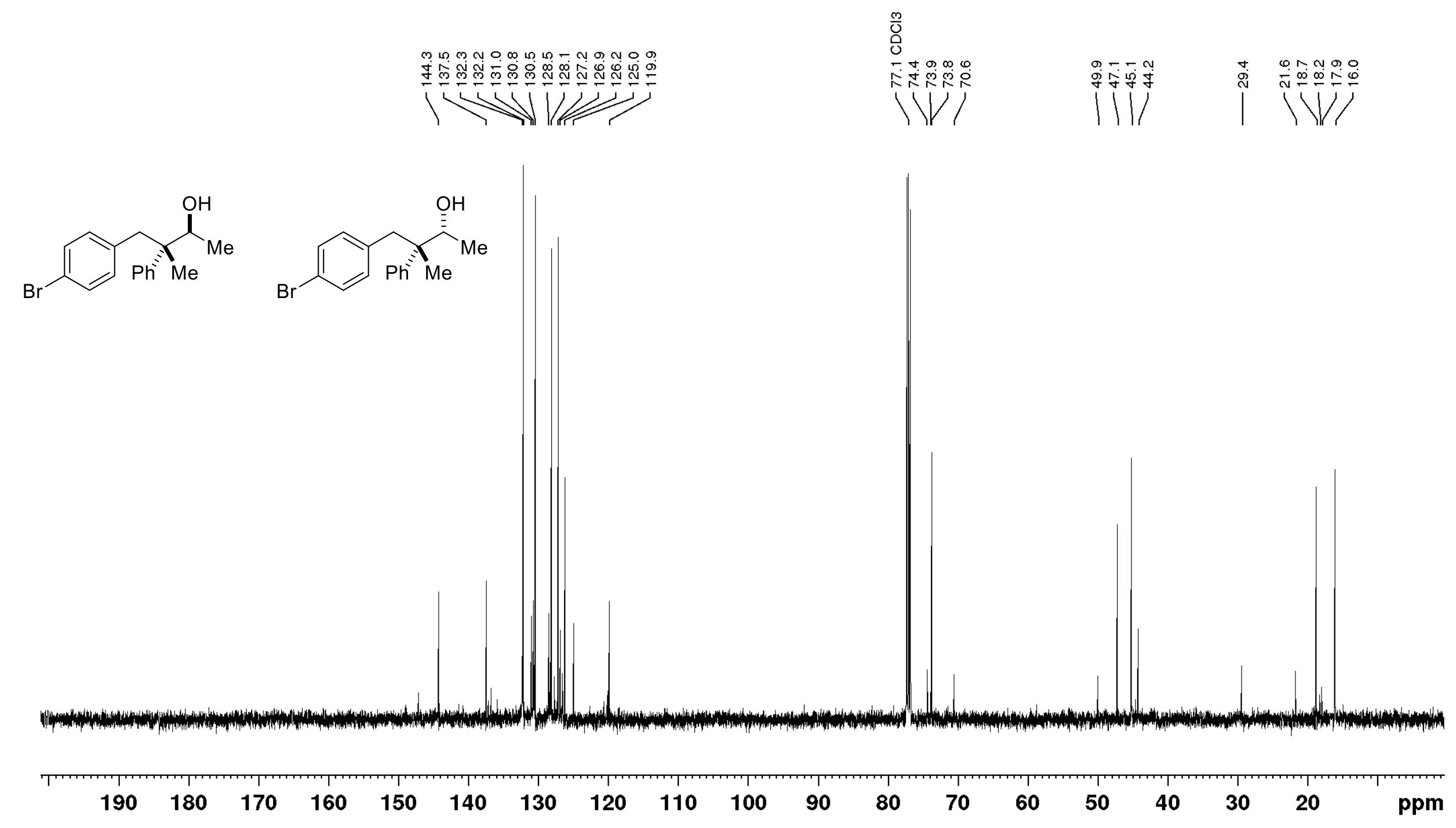


Figure 8.78 ${ }^{1} \mathrm{H} \quad \mathrm{NMR}\left(500 \mathrm{MHz}, \mathrm{C}_{6} \mathrm{D}_{6}, 298 \mathrm{~K}\right)$ of $(2 S, 3 S)-((4-(4-B r o m o p h e n y l)-3-m e t h y l-3-p h e n y l b u t a n-2-y l)$ oxy)(3,5dimethylphenyl)dimethylsilane $[(2 S, 3 S)-3 f e]$.

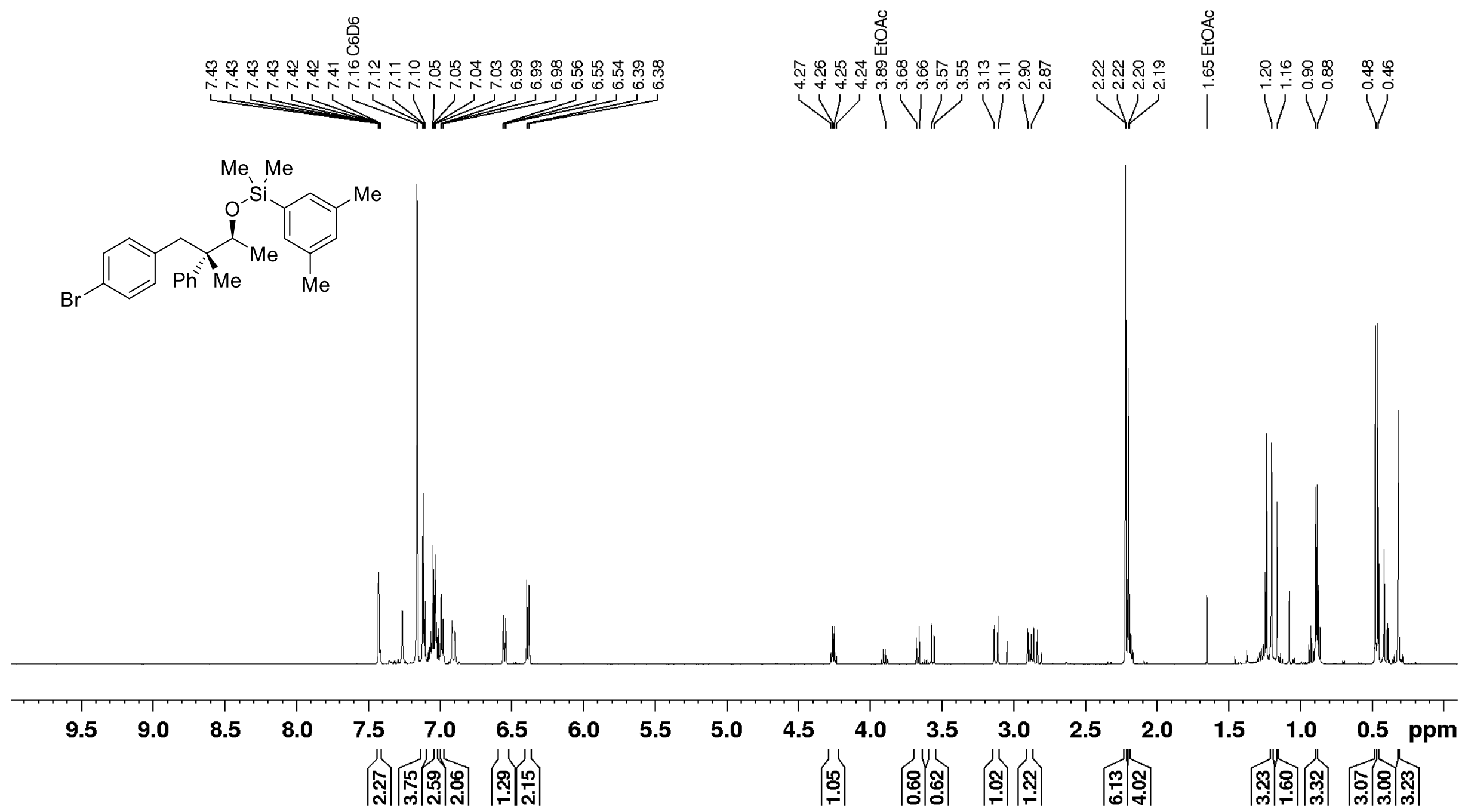


Figure 8.79 ${ }^{13} \mathrm{C}$ NMR (126 MHz, $\left.\mathrm{C}_{6} \mathrm{D}_{6}, 298 \mathrm{~K}\right)$ of $(2 S, 3 S)-((4-(4-B r o m o p h e n y l)-3-m e t h y l-3-p h e n y l b u t a n-2-y l) o x y)(3,5-$ dimethylphenyl)dimethylsilane [(2S,3S)-3fe].

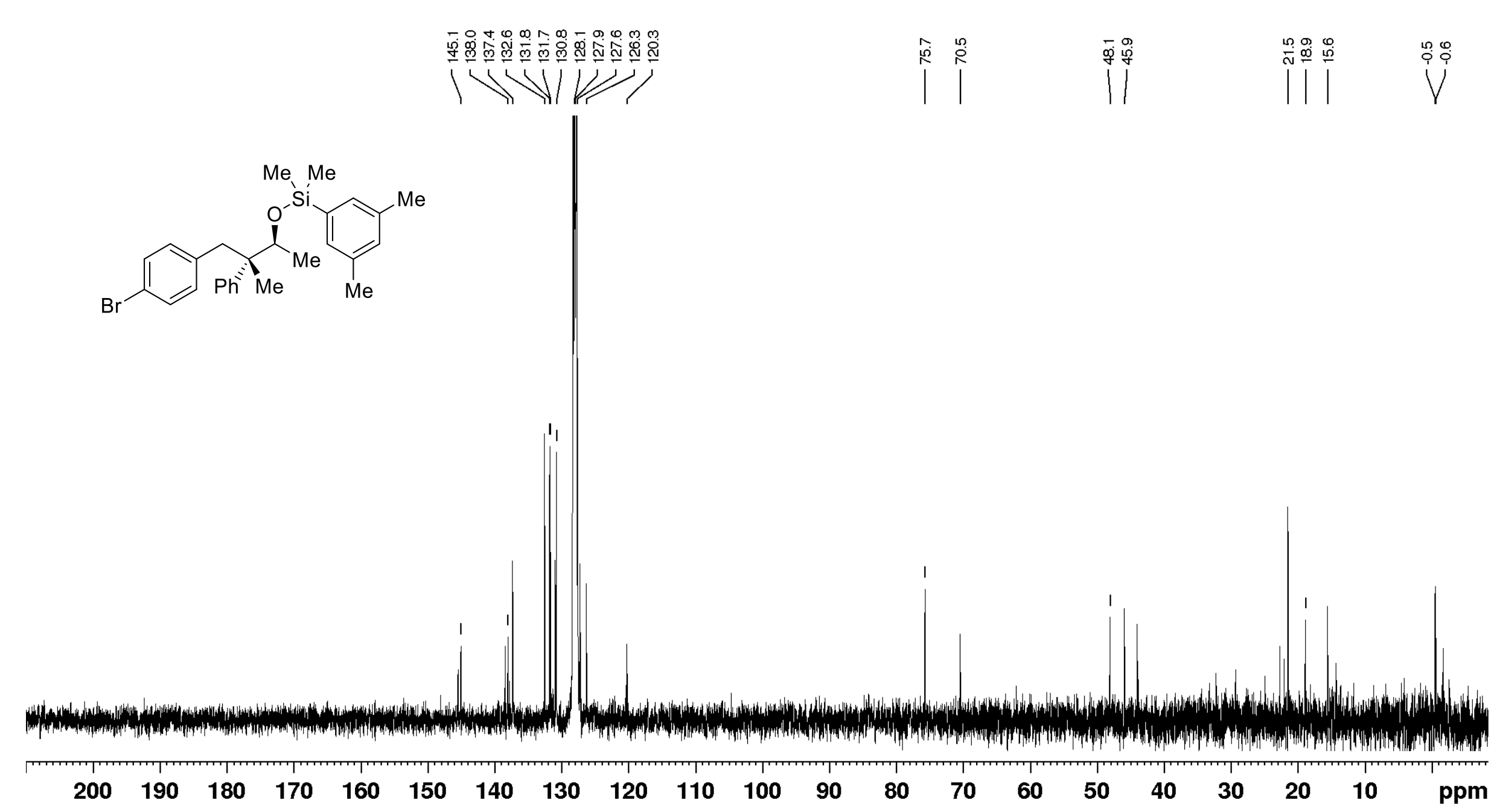


Figure 8.80 ${ }^{29} \mathrm{Si}$ DEPT NMR (99 MHz, $\left.\mathrm{CDCl}_{3}, 298 \mathrm{~K}\right)$ of $(2 S, 3 S)-((4-(4-B r o m o p h e n y l)-3-m e t h y l-3-p h e n y l b u t a n-2-y l) o x y)(3,5-$ dimethylphenyl)dimethylsilane [(2S,3S)-3fe].

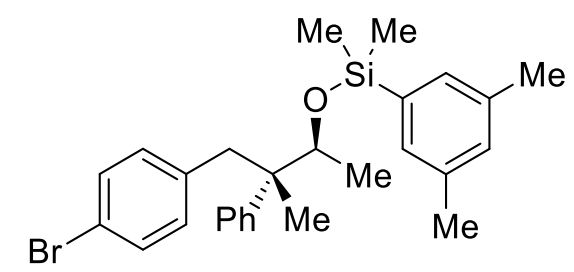

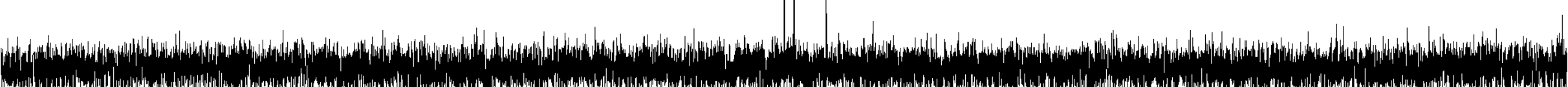
等 
Figure 8.81 ${ }^{1} \mathrm{H}$ NMR (500 MHz, $\mathrm{CDCl}_{3}, 298 \mathrm{~K}$ ) of rac-anti/syn-4-(4-Chlorophenyl)-3-methyl-3-phenylbutan-2-ol (rac-anti/syn-1e). $\frac{0}{0}$

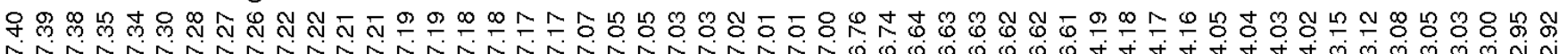

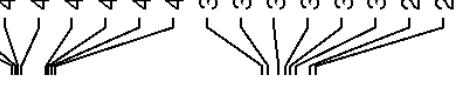<smiles>CC(O)C(C)(Cc1ccc(Cl)cc1)c1ccccc1</smiles><smiles>C[C@H](O)[C@H](Cc1ccc(Cl)cc1)c1ccccc1</smiles>

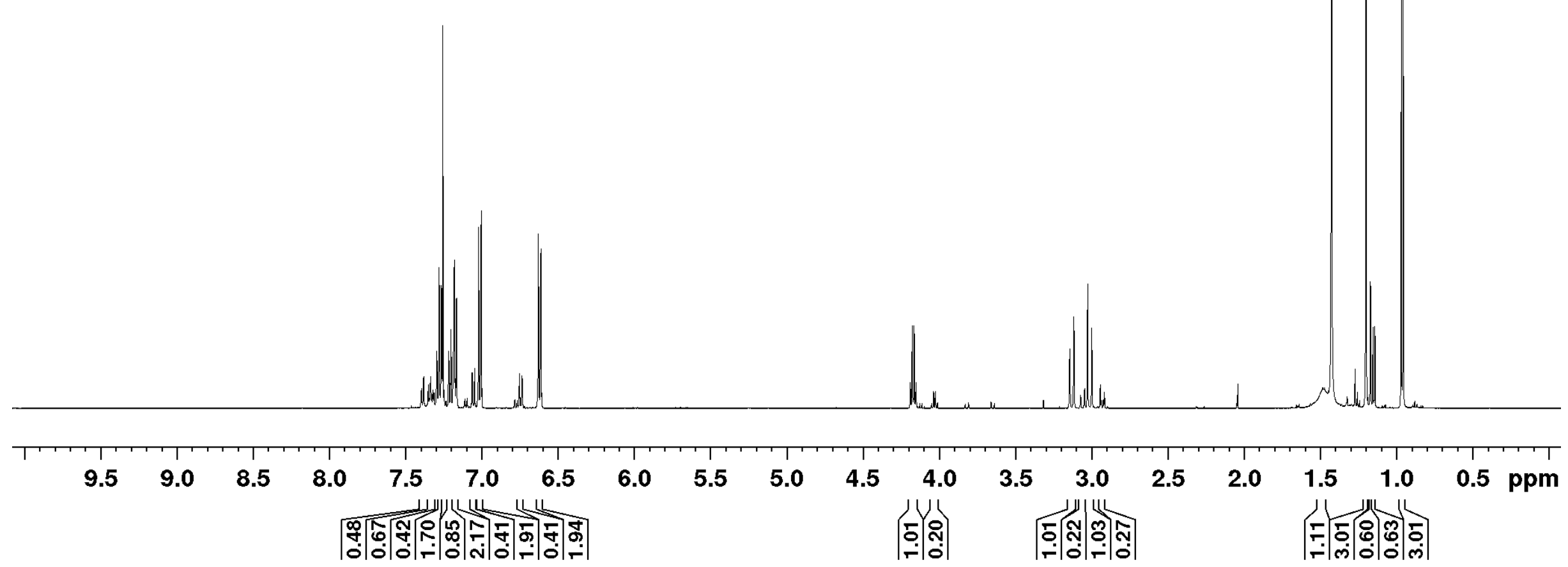


Figure 8.82 ${ }^{13} \mathrm{C} \mathrm{NMR} \mathrm{(126} \mathrm{MHz,} \mathrm{CDCl}_{3}, 298 \mathrm{~K}$ ) of rac-anti/syn-4-(4-Chlorophenyl)-3-methyl-3-phenylbutan-2-ol (rac-anti/syn-1e).
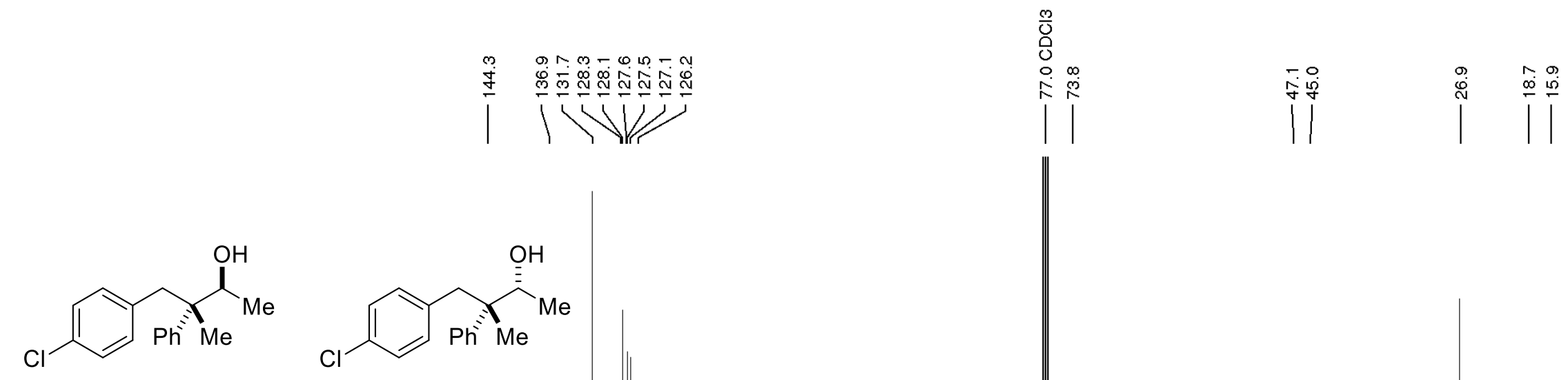

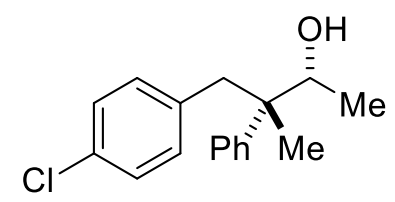
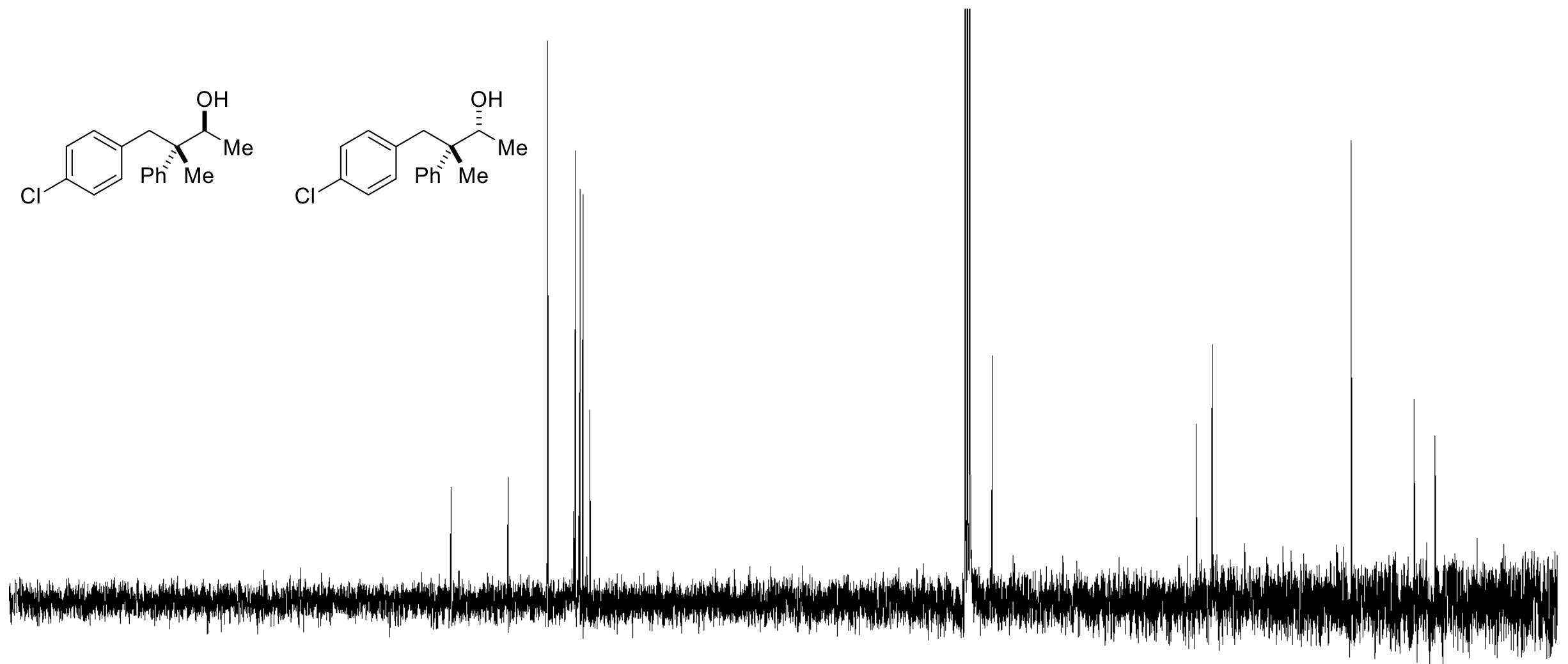

190180
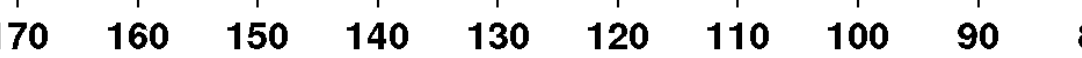

80

70

50

40

30

20

ppm 
Figure 8.83 ${ }^{1} \mathrm{H} \quad \mathrm{NMR}\left(500 \mathrm{MHz}, \mathrm{C}_{6} \mathrm{D}_{6}, 298 \mathrm{~K}\right)$ of $(2 S, 3 S)-((4-(4-B r o m o p h e n y l)-3-m e t h y l-3-p h e n y l b u t a n-2-y l) o x y)(3,5-$ dimethylphenyl)dimethylsilane $[(2 S, 3 S)-3 e e]$.

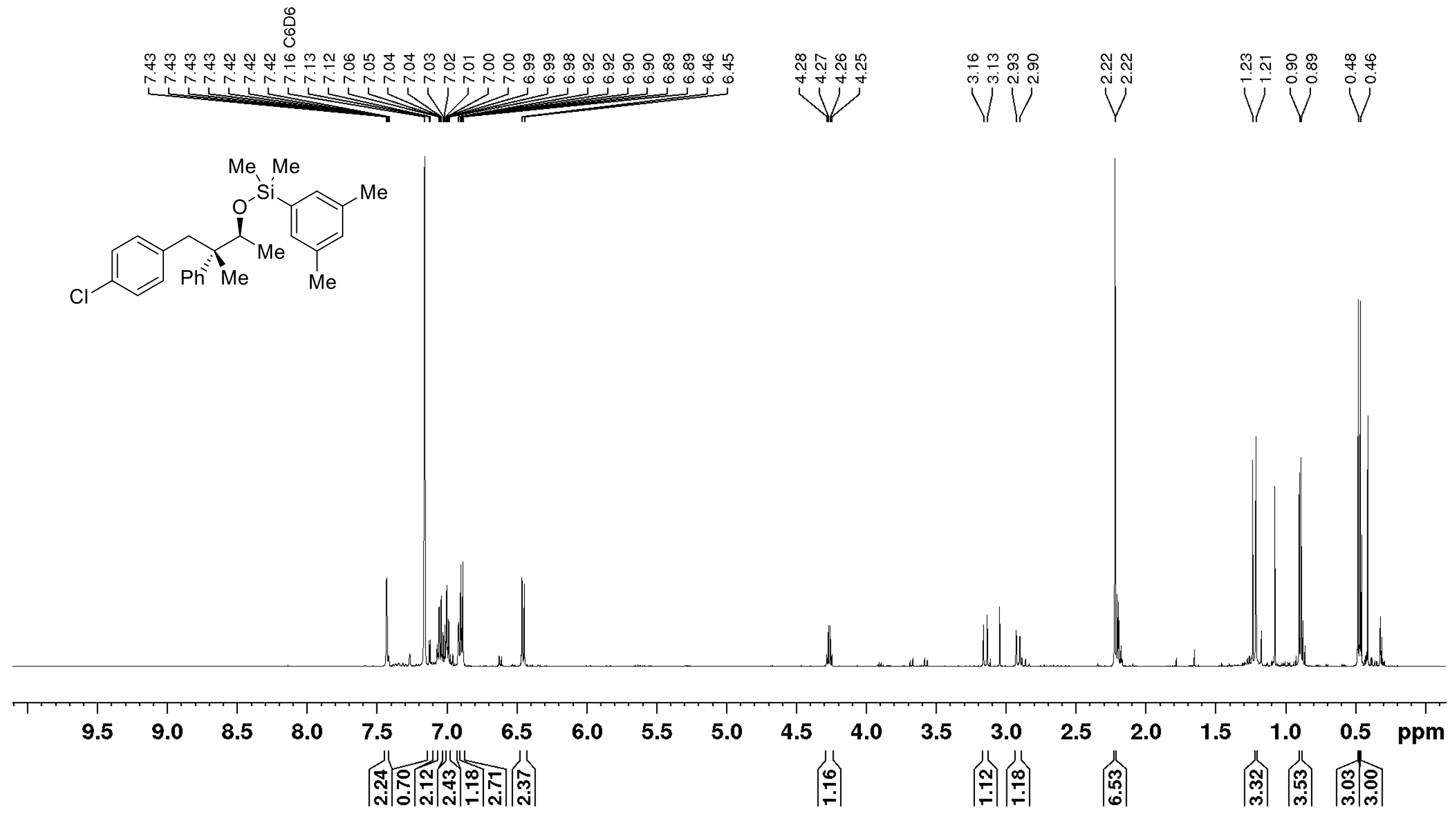


Figure $8.84{ }^{13} \mathrm{C}$ NMR (126 MHz, $\left.\mathrm{C}_{6} \mathrm{D}_{6}, 298 \mathrm{~K}\right)$ of $(2 S, 3 S)-((4-(4-B r o m o p h e n y l)-3-m e t h y l-3-p h e n y l b u t a n-2-y l)$ oxy)(3,5dimethylphenyl)dimethylsilane $[(2 S, 3 S)-3 e e]$.

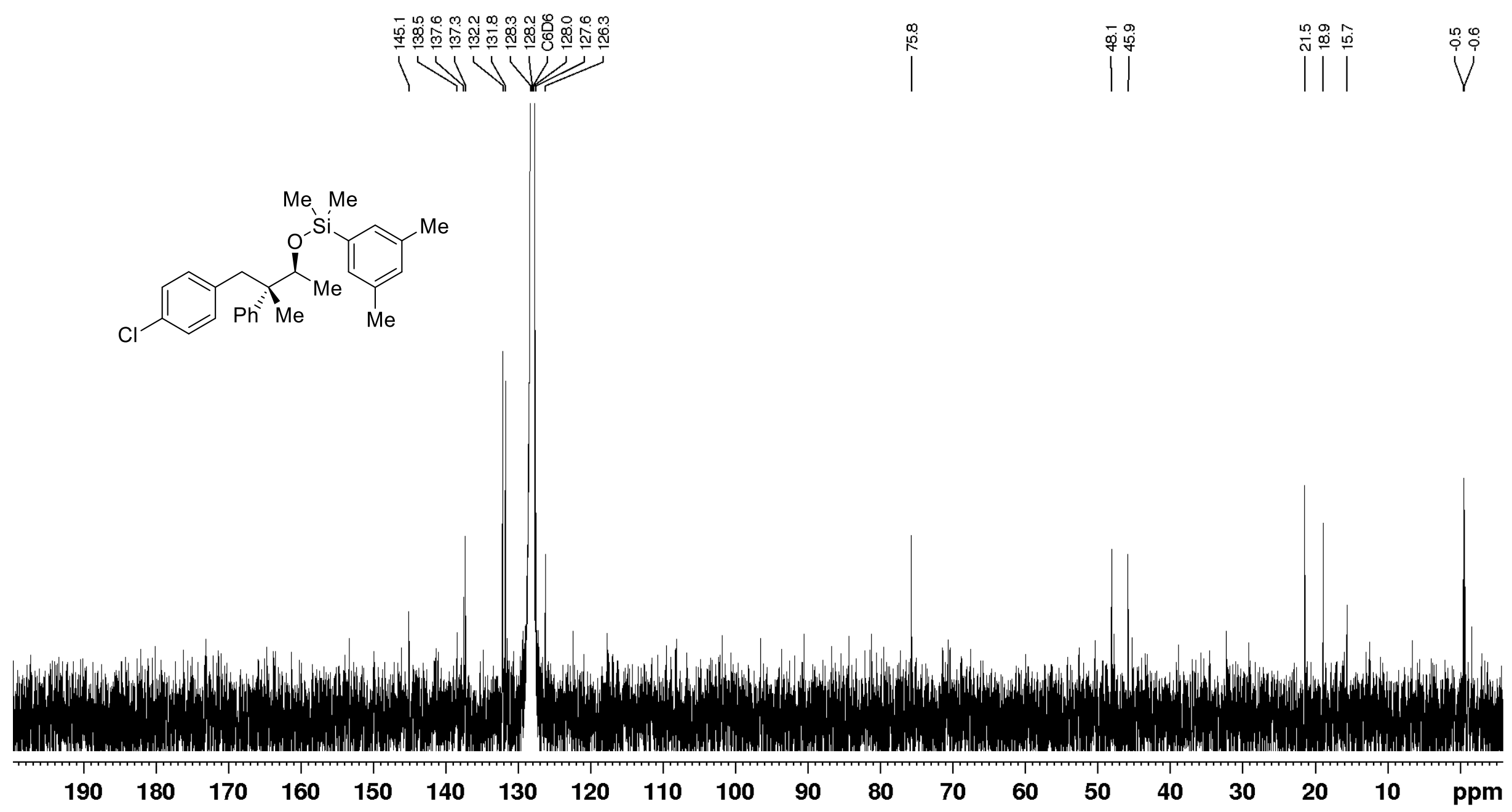


Figure 8.85 ${ }^{29} \mathrm{Si}$ DEPT NMR (99 MHz, $\left.\mathrm{C}_{6} \mathrm{D}_{6}, 298 \mathrm{~K}\right)$ of (2S,3S)-((4-(4-Bromophenyl)-3-methyl-3-phenylbutan-2-yl)oxy)(3,5dimethylphenyl)dimethylsilane $[(2 S, 3 S)-3 e e]$.

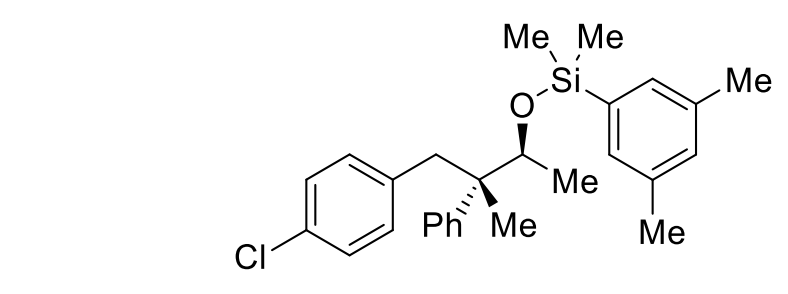

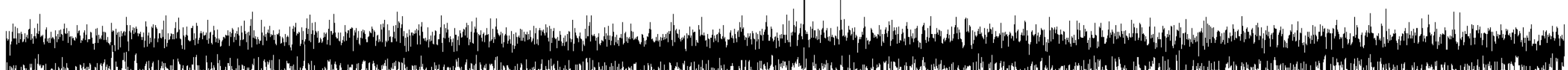

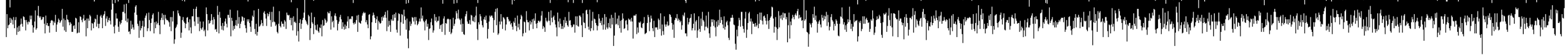


Figure 8.86 ${ }^{1} \mathrm{H}$ NMR $\left(500 \mathrm{MHz}, \mathrm{CDCl}_{3}, 298 \mathrm{~K}\right)$ of rac-anti/syn-4-(4-Methoxyphenyl)-3-methyl-3-phenylbutan-2-ol (rac-anti/syn$1 \mathbf{k})$.

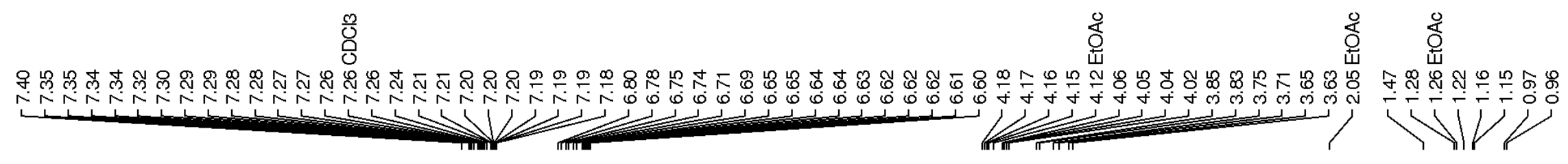<smiles>COc1ccc(C[C@](C)(C(C)O)[C@](C)(Cc2ccc(OC)cc2)c2ccccc2)cc1</smiles>
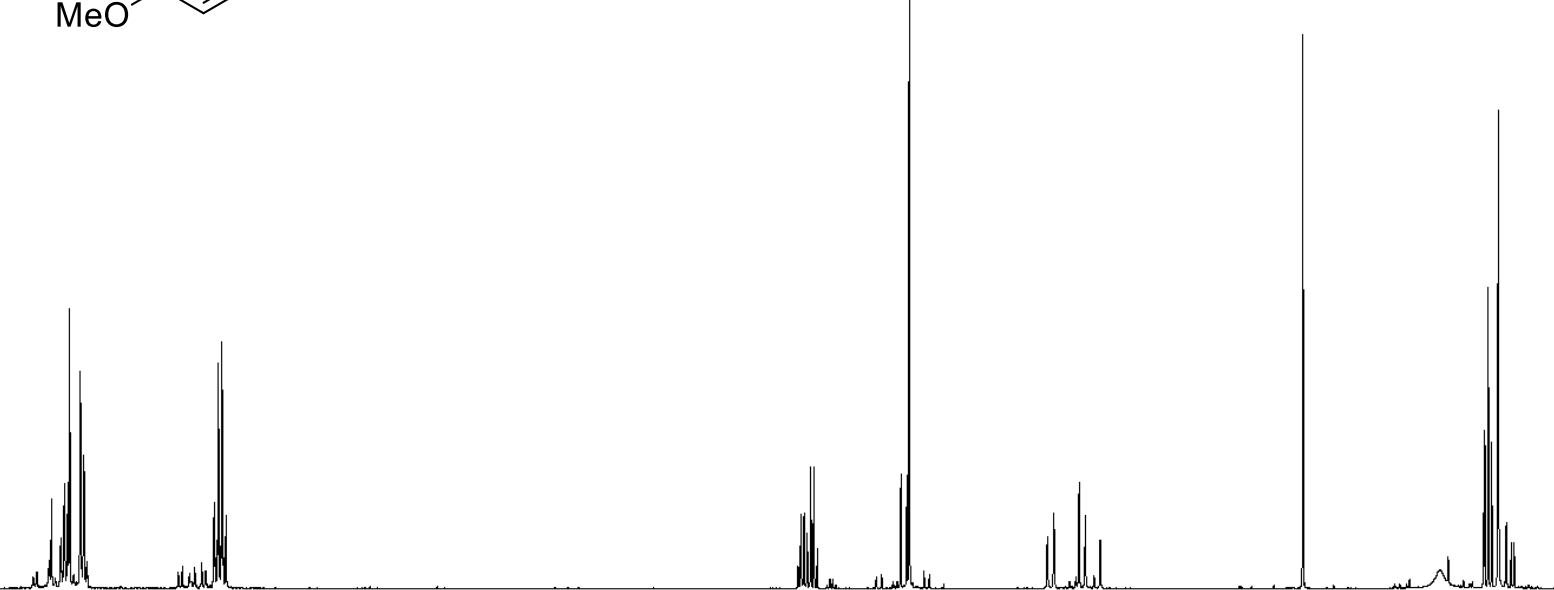

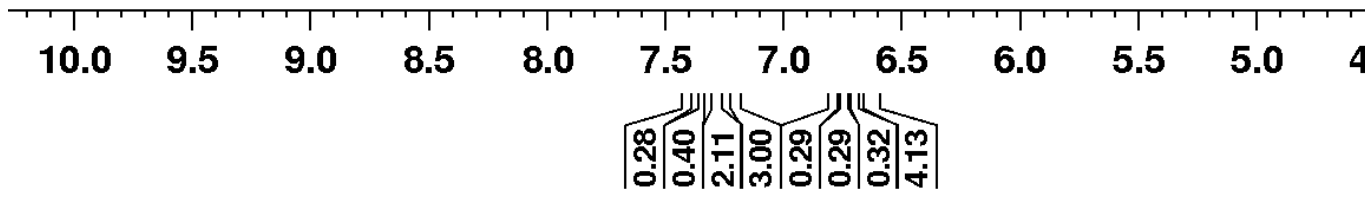

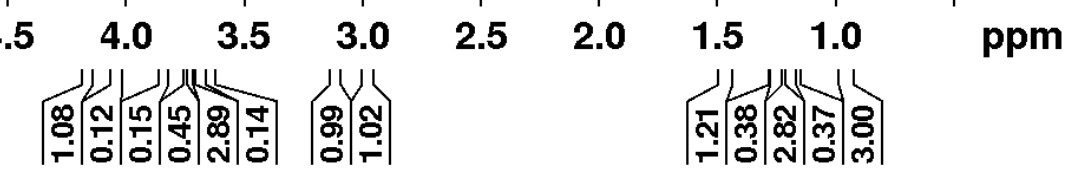


Figure $8.87{ }^{13} \mathrm{C} \mathrm{NMR} \mathrm{(126} \mathrm{MHz,} \mathrm{CDCl}_{3}, 298 \mathrm{~K}$ ) of rac-anti/syn-4-(4-Methoxyphenyl)-3-methyl-3-phenylbutan-2-ol (rac-anti/syn1k).

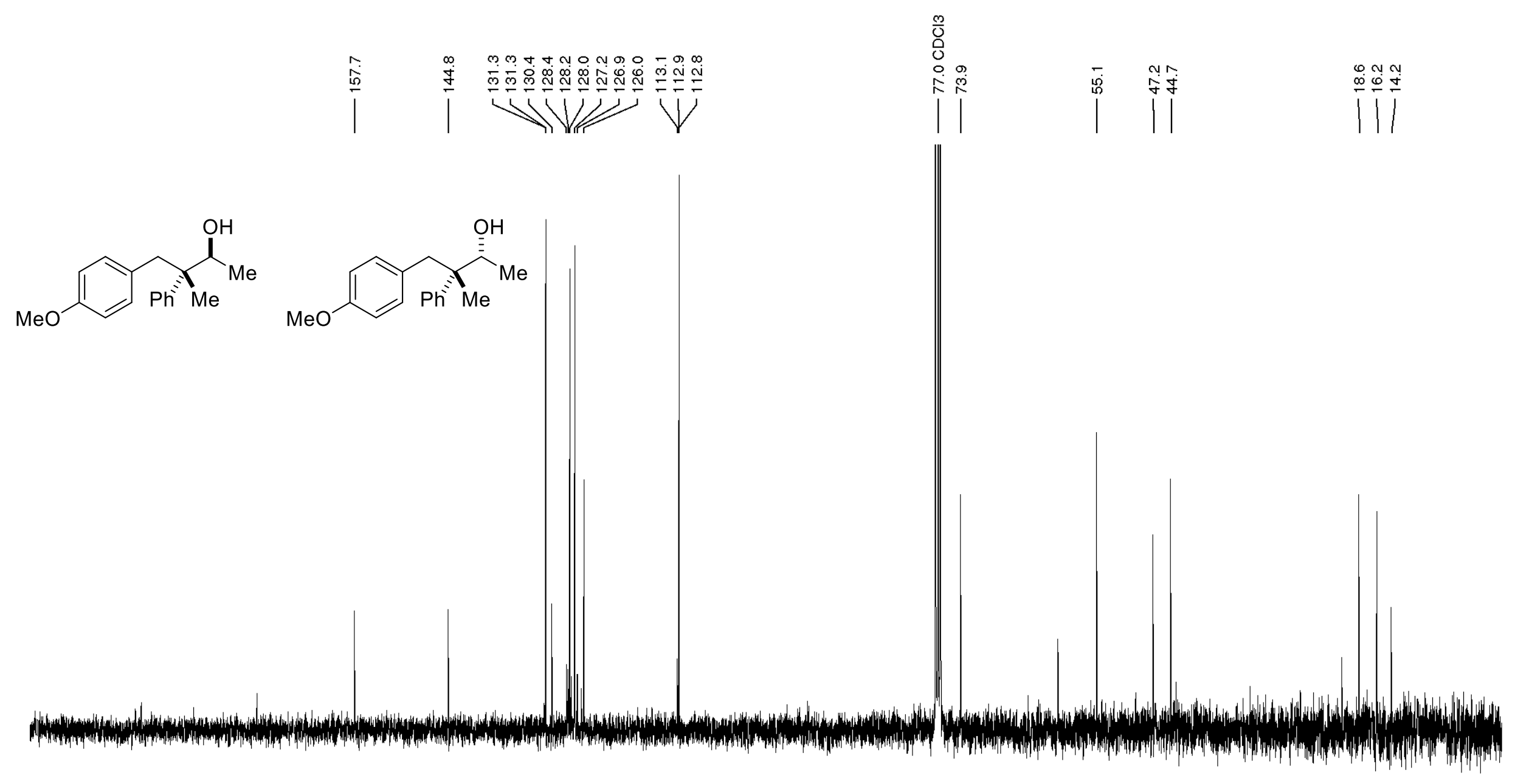

$\begin{array}{lllllllll}190 & 180 & 170 & 160 & 150 & 140 & 130 & 120 & 1\end{array}$

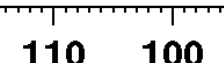

20

80

70

60

50

40

30

20

$10 \mathrm{ppm}$ 
Figure 8.88 ${ }^{1} \mathrm{H}$ NMR $\left(500 \mathrm{MHz}, \mathrm{C}_{6} \mathrm{D}_{6}, 298 \mathrm{~K}\right)$ of (2S,3S)-(3,5-Dimethylphenyl)((4-(4-methoxyphenyl)-3-methyl-3-phenylbutan-2yl)oxy)dimethylsilane [(2S,3S)-3ke].

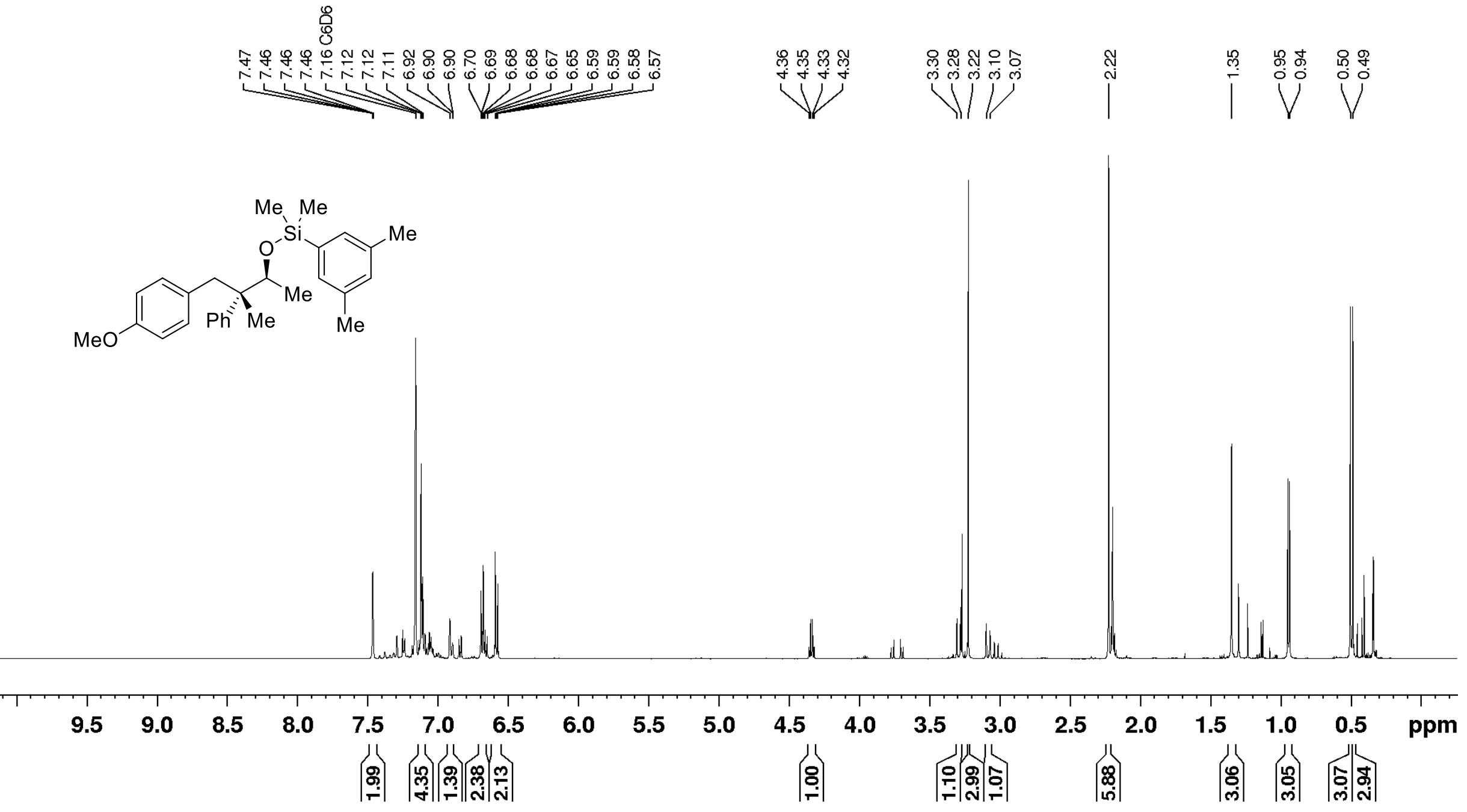


Figure 8.89 ${ }^{13} \mathrm{C}$ NMR (126 MHz, $\left.\mathrm{C}_{6} \mathrm{D}_{6}, 298 \mathrm{~K}\right)$ of (2S,3S)-(3,5-Dimethylphenyl)((4-(4-methoxyphenyl)-3-methyl-3-phenylbutan2-yl)oxy)dimethylsilane [(2S,3S)-3ke].

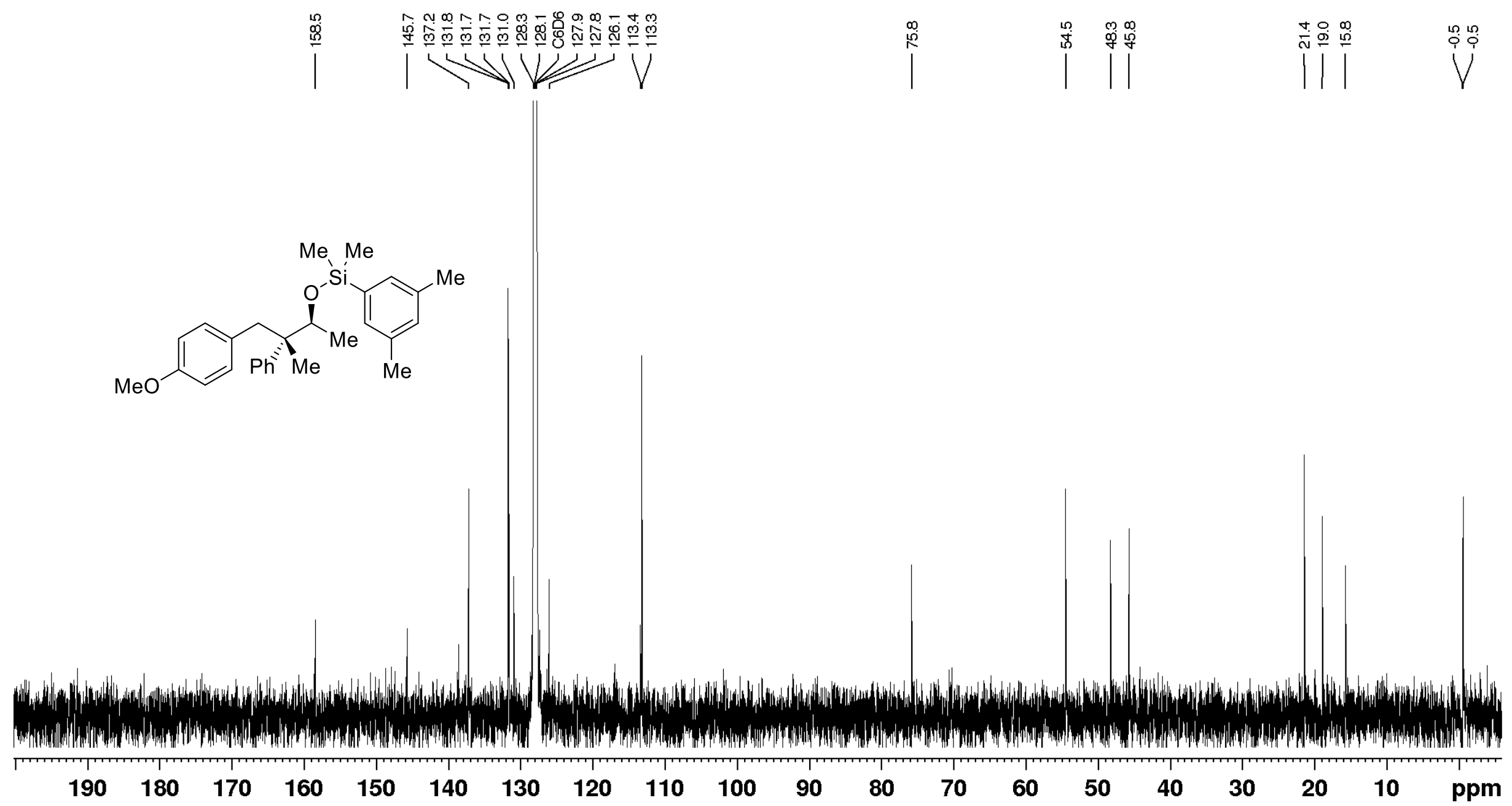


Figure 8.90 ${ }^{29} \mathrm{Si}$ DEPT NMR (99 MHz, $\left.\mathrm{C}_{6} \mathrm{D}_{6}, 298 \mathrm{~K}\right)$ of (2S,3S)-(3,5-Dimethylphenyl)((4-(4-methoxyphenyl)-3-methyl-3phenylbutan-2-yl)oxy)dimethylsilane [(2S,3S)-3ke].
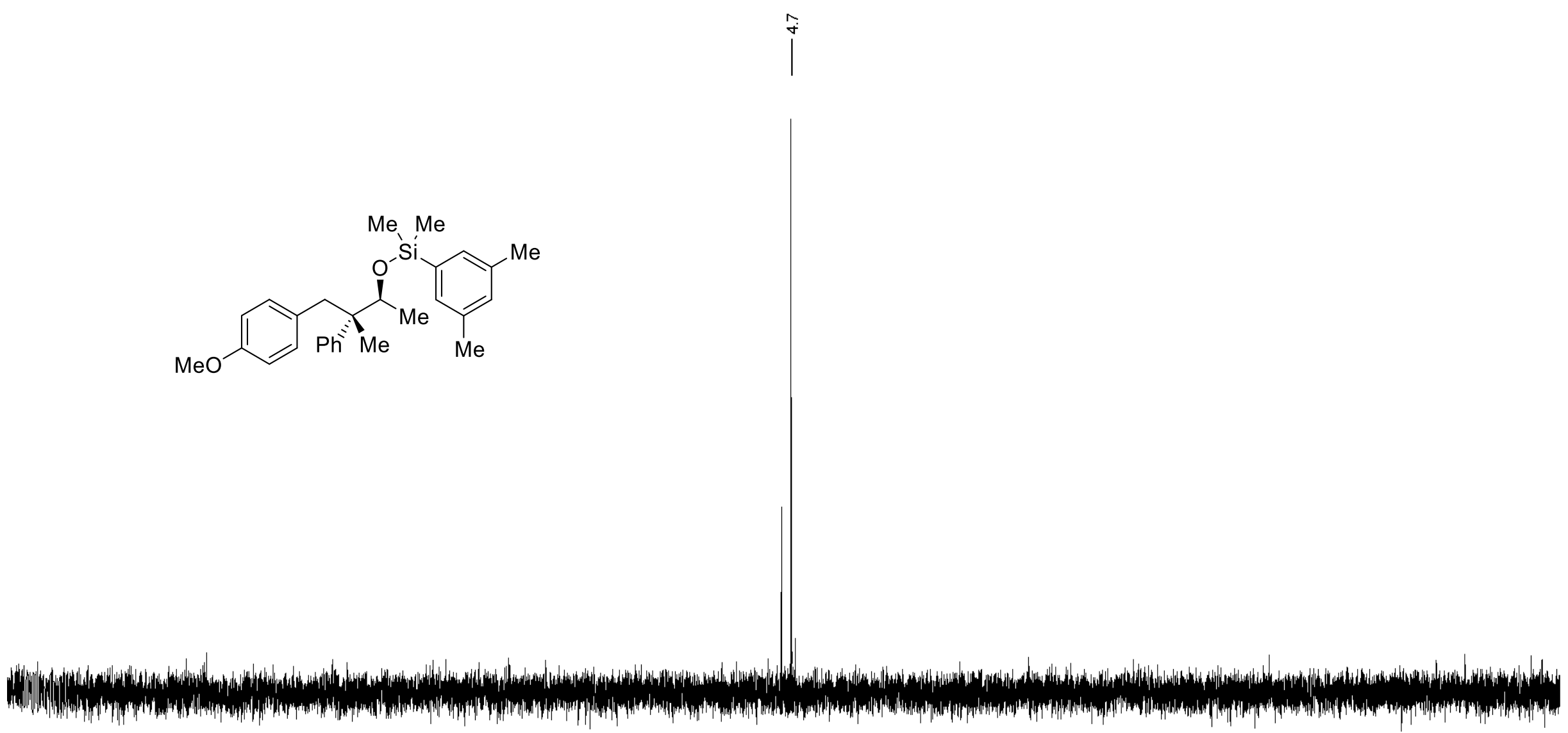
Figure 8.91 ${ }^{1} \mathrm{H}$ NMR (500 MHz, $\left.\mathrm{CDCl}_{3}, 298 \mathrm{~K}\right)$ of rac-anti/syn-4-(2-Bromophenyl)-2-methyl-2-phenylpropan-1-ol (rac-anti/syn-1h). $\frac{\mathrm{m}}{\mathrm{O}}$

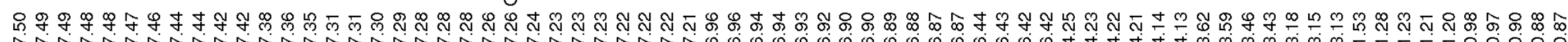
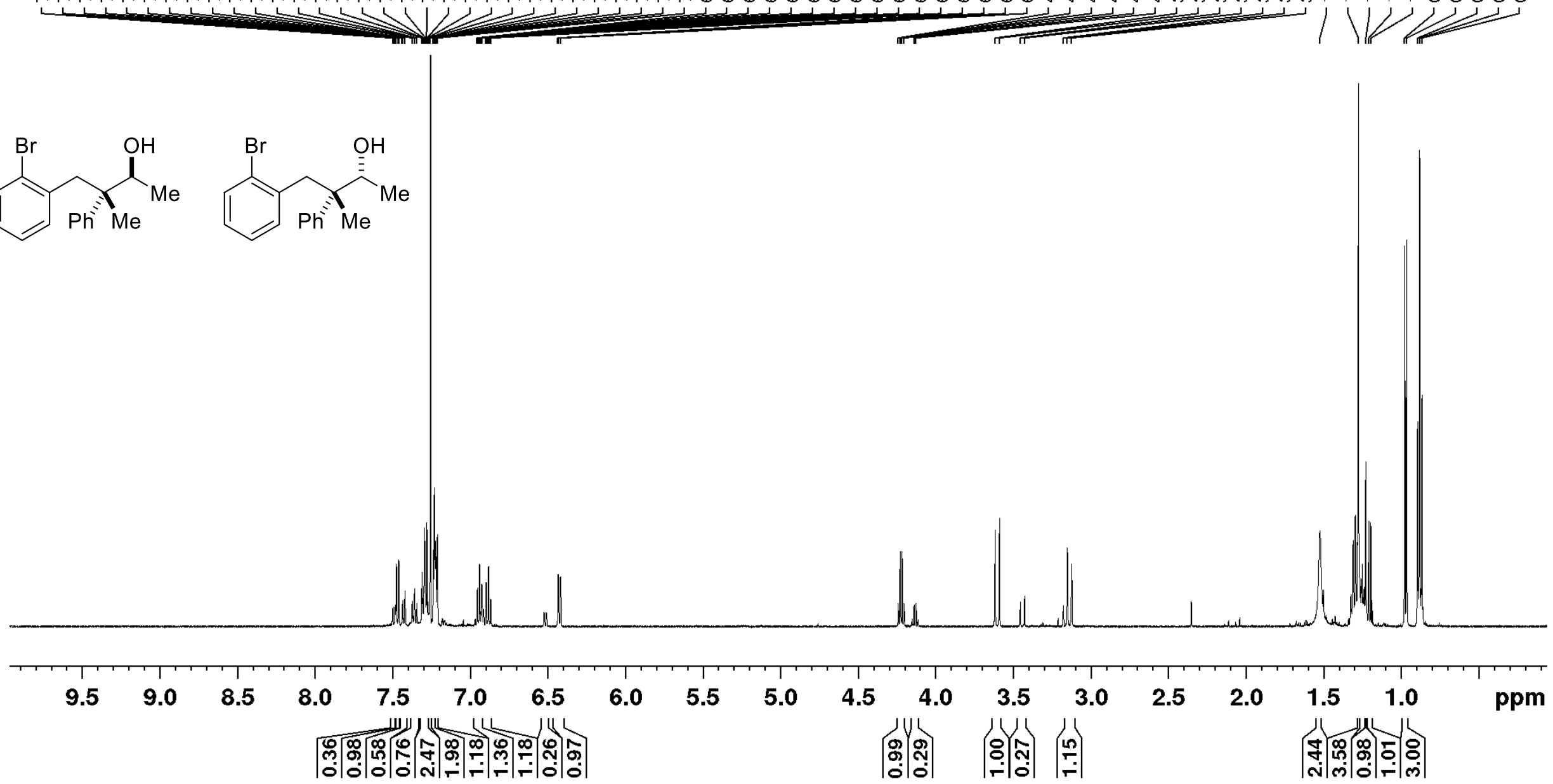
Figure $8.92{ }^{13} \mathrm{C}$ NMR (126 MHz, $\mathrm{CDCl}_{3}, 298 \mathrm{~K}$ ) of rac-anti/syn-4-(2-Bromophenyl)-2-methyl-2-phenylpropan-1-ol (rac-anti/syn1h).
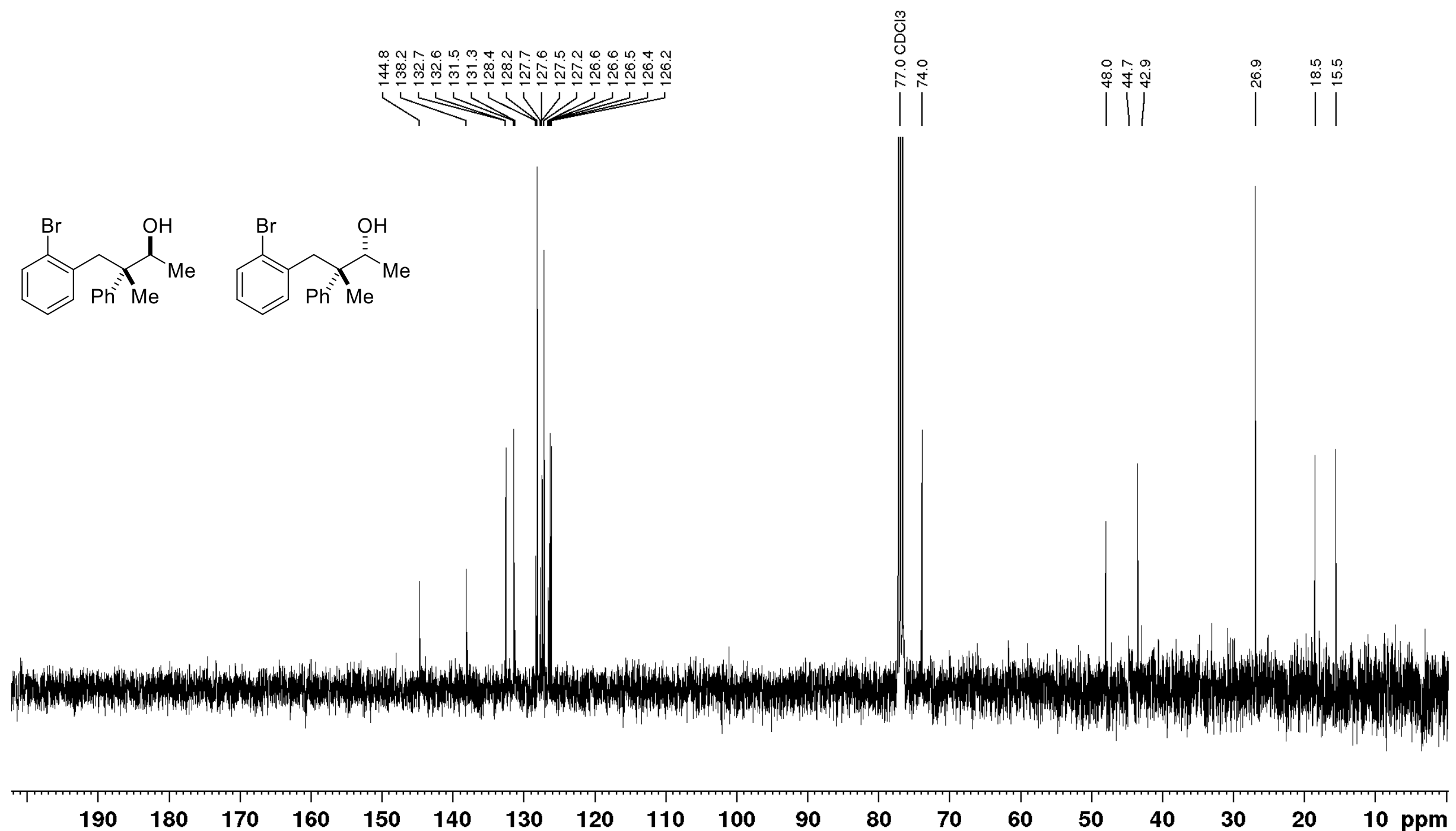
Figure 8.93 ${ }^{1} \mathrm{H} \quad \mathrm{NMR}\left(500 \mathrm{MHz}, \mathrm{C}_{6} \mathrm{D}_{6}, 298 \mathrm{~K}\right)$ of $(2 S, 3 S)-((4-(2-B r o m o p h e n y l)-3-m e t h y l-3-p h e n y l b u t a n-2-y l)$ oxy)(3,5dimethylphenyl)dimethylsilane $[(2 S, 3 S)-3 h e]$.
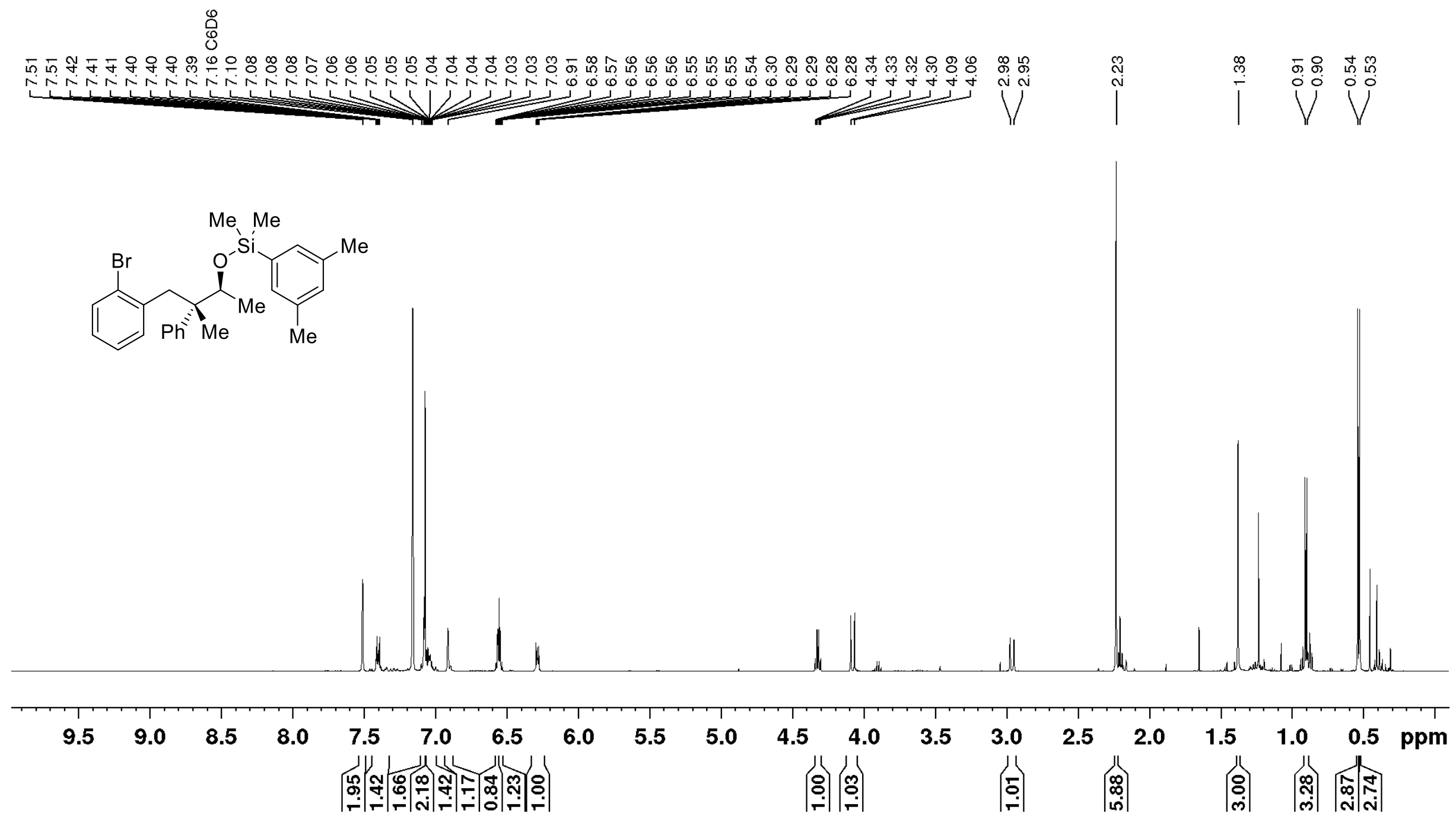
Figure $8.94{ }^{13} \mathrm{C}$ NMR (126 MHz, $\left.\mathrm{C}_{6} \mathrm{D}_{6}, 298 \mathrm{~K}\right)$ of $(2 S, 3 S)-((4-(2-B r o m o p h e n y l)-3-m e t h y l-3-p h e n y l b u t a n-2-y l)$ oxy)(3,5dimethylphenyl)dimethylsilane $[(2 S, 3 S)-3 h e]$.

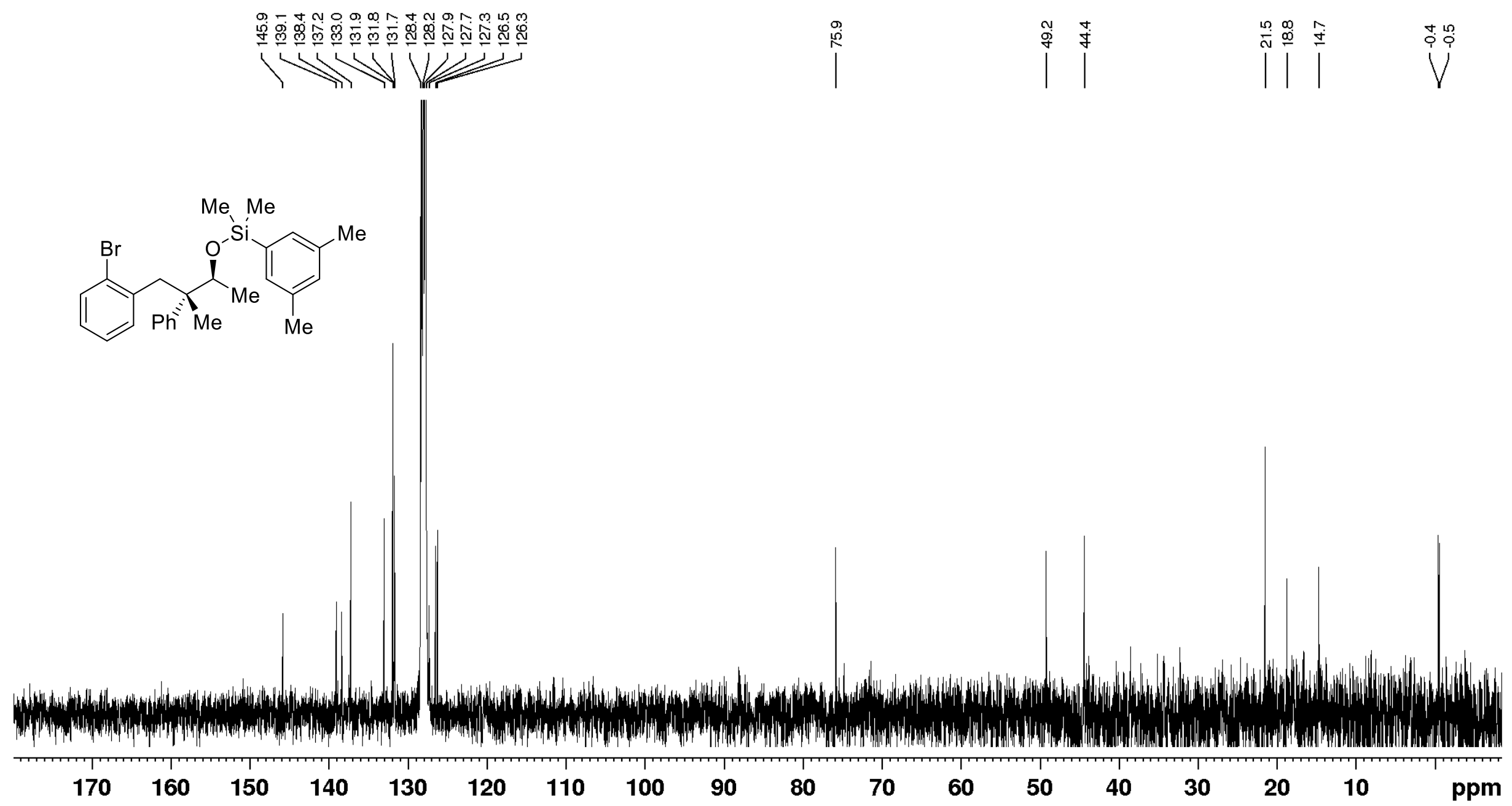


Figure 8.95 $\left.{ }^{29} \mathrm{Si} \mathrm{DEPT} \mathrm{NMR} \mathrm{(99} \mathrm{MHz,} \mathrm{C}_{6} \mathrm{D}_{6}, 298 \mathrm{~K}\right)$ of (2S,3S)-(4-(2-Bromophenyl)-3-methyl-3-phenylbutan-2-yl)oxy)(3,5dimethylphenyl)dimethylsilane $[(2 S, 3 S)-3 h e]$.

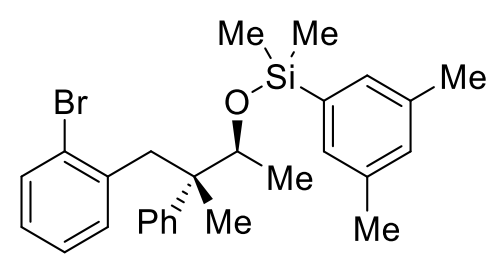

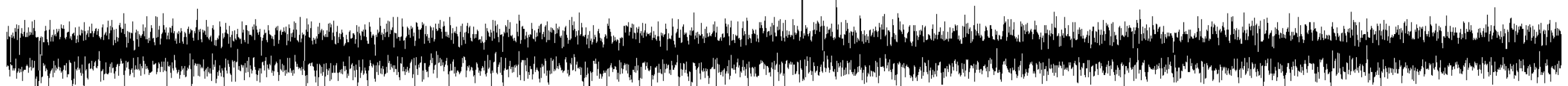


Figure 8.96 ${ }^{1} \mathrm{H}$ NMR (500 MHz, $\mathrm{CDCl}_{3}, 298 \mathrm{~K}$ ) of rac-anti/syn-4-(4-lodophenyl)-3-methyl-3-phenylbutan-2-ol (rac-anti/syn-1g).

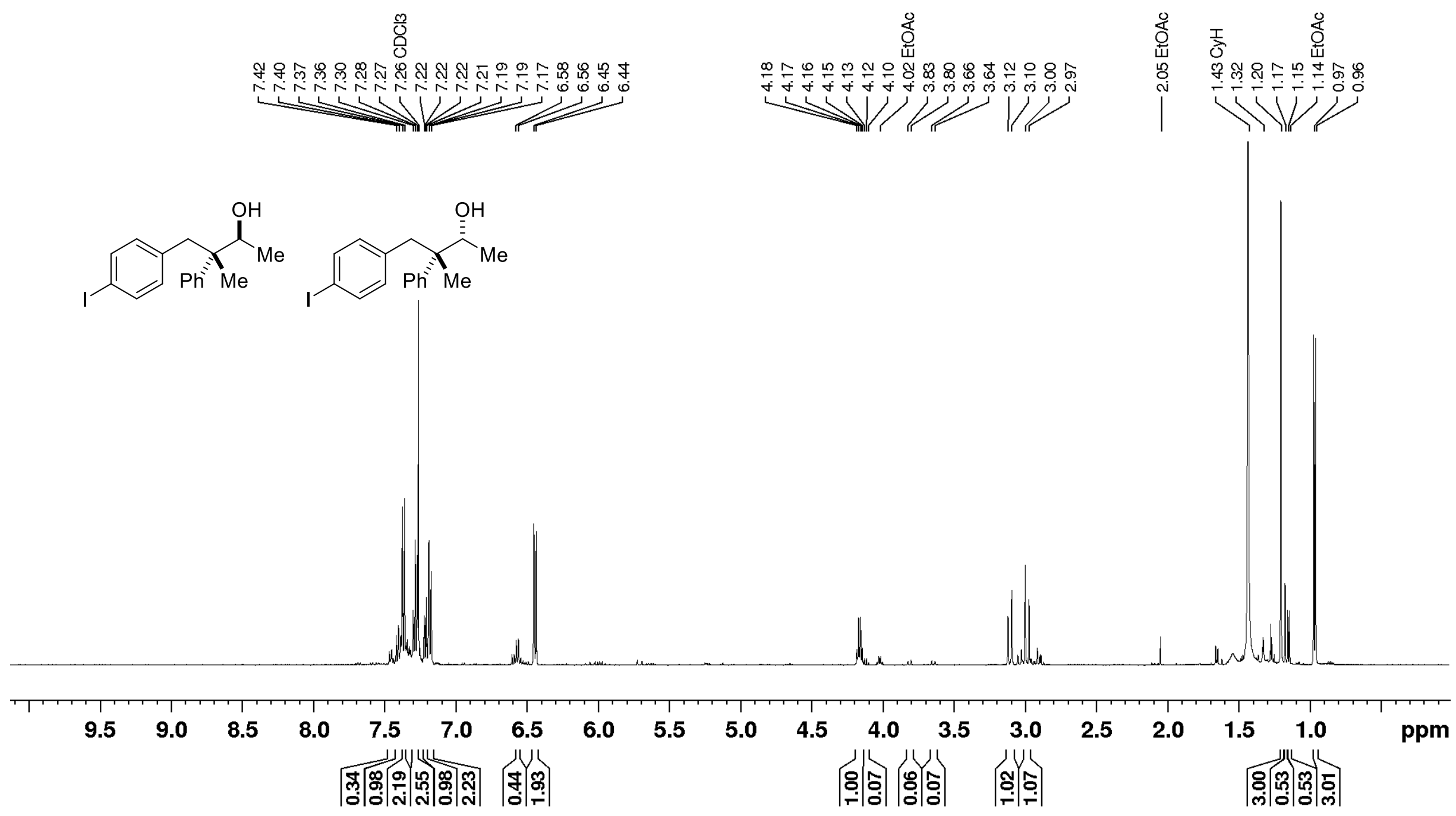


Figure $8.97{ }^{13} \mathrm{C} \mathrm{NMR} \mathrm{(126} \mathrm{MHz,} \mathrm{CDCl}_{3}, 298 \mathrm{~K}$ ) of rac-anti/syn-4-(4-lodophenyl)-3-methyl-3-phenylbutan-2-ol (rac-anti/syn-1g).

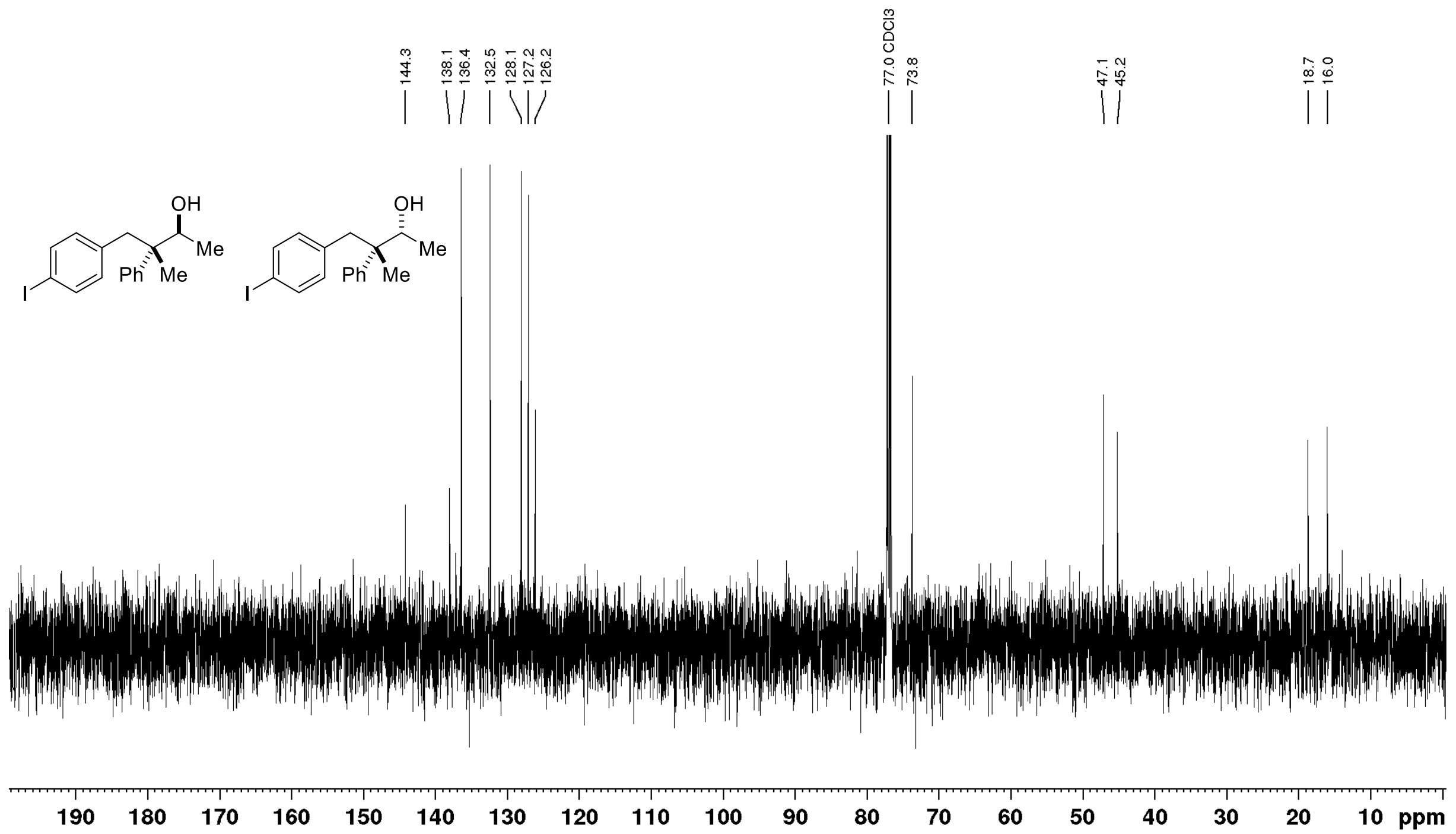


Figure 8.98 ${ }^{1} \mathrm{H}$ NMR (500 MHz, $\left.\mathrm{C}_{6} \mathrm{D}_{6}, 298 \mathrm{~K}\right)$ of (2S,3S)-(3,5-Dimethylphenyl)((4-(4-iodophenyl)-3-methyl-3-phenylbutan-2yl)oxy)dimethylsilane [(2S,3S)-3ge].
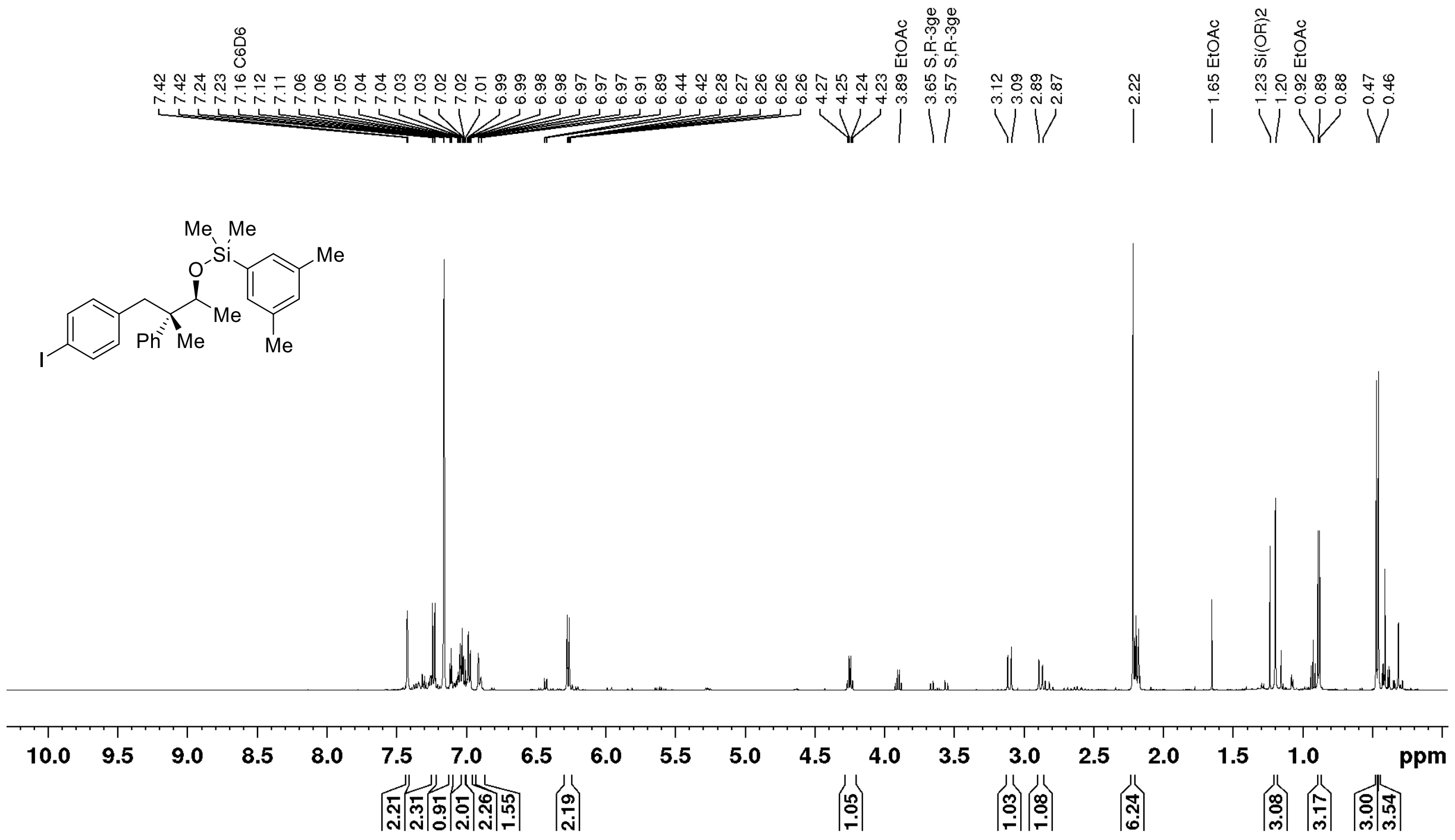
Figure 8.99 ${ }^{13} \mathrm{C}$ NMR (126 MHz, $\left.\mathrm{C}_{6} \mathrm{D}_{6}, 298 \mathrm{~K}\right)$ of (2S,3S)-(3,5-Dimethylphenyl)((4-(4-iodophenyl)-3-methyl-3-phenylbutan-2yl)oxy)dimethylsilane $[(2 S, 3 S)-3 g e]$.
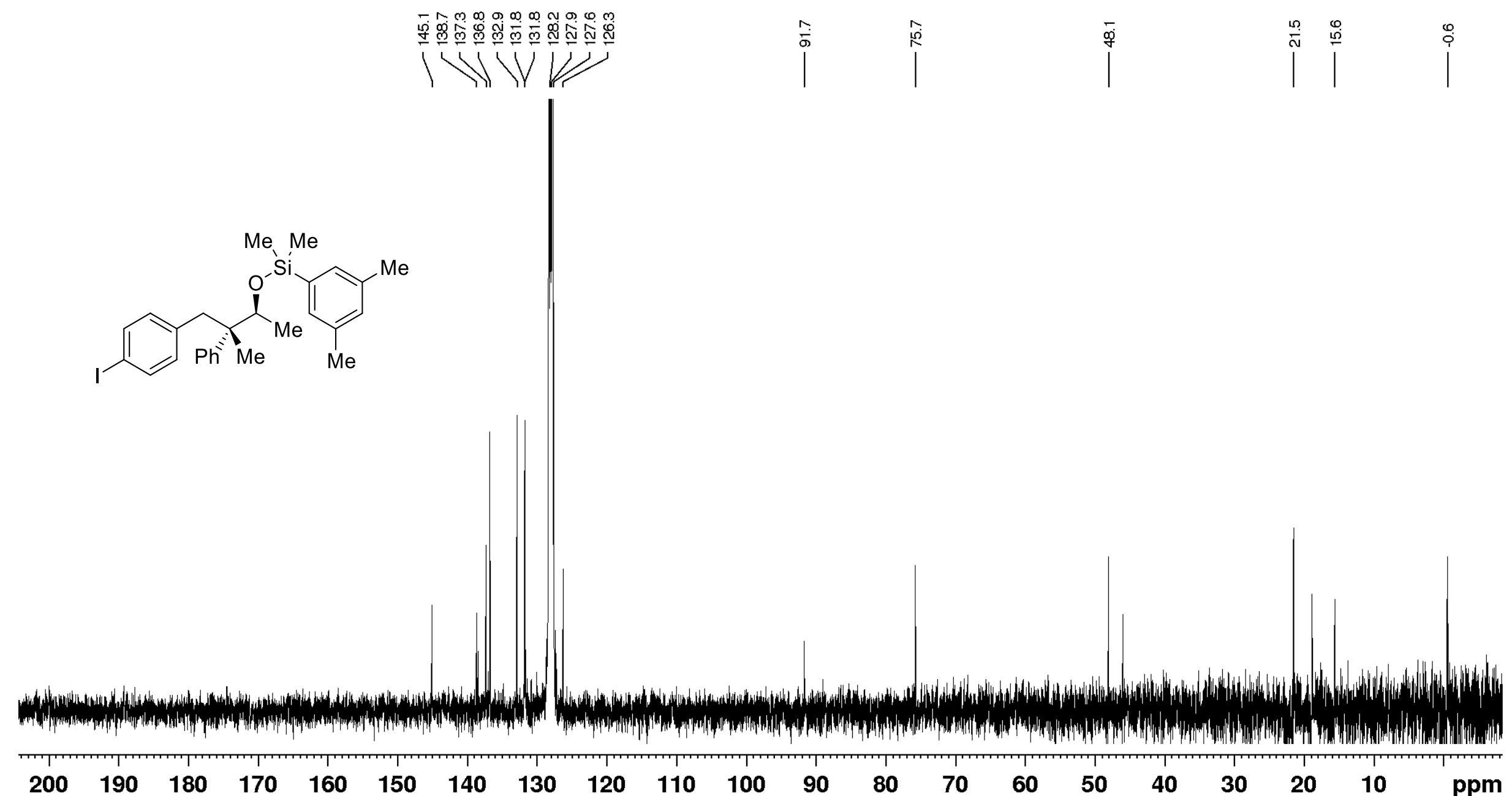
Figure 8.100 ${ }^{29} \mathrm{Si}$ DEPT NMR (99 MHz, $\left.\mathrm{C}_{6} \mathrm{D}_{6}, 298 \mathrm{~K}\right)$ of (2S,3S)-(3,5-Dimethylphenyl)((4-(4-iodophenyl)-3-methyl-3-phenylbutan2-yl)oxy)dimethylsilane [(2S,3S)-3ge].

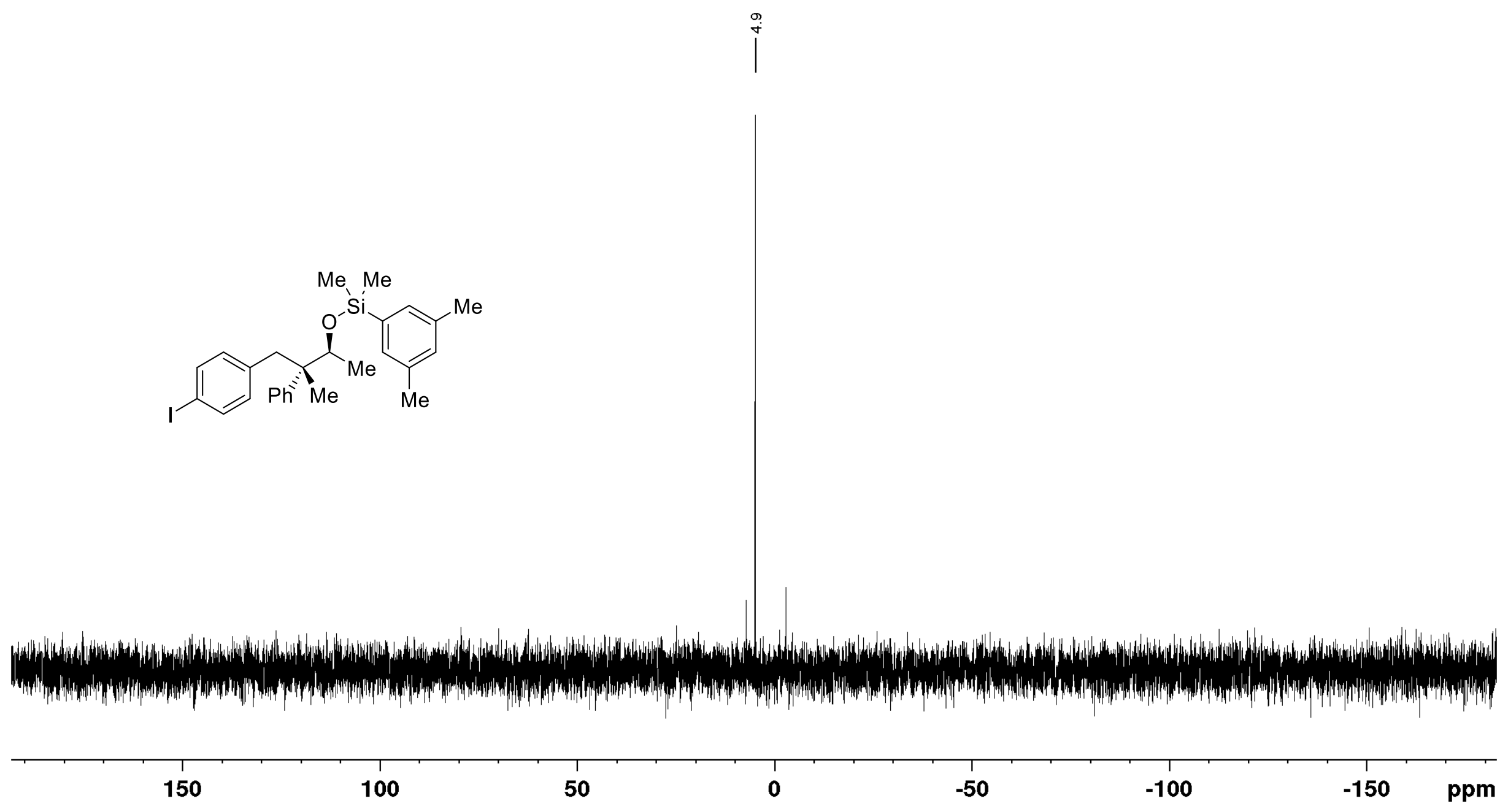


Figure 8.101 ${ }^{1} \mathrm{H}$ NMR (500 MHz, $\mathrm{CDCl}_{3}, 298 \mathrm{~K}$ ) of rac-2-Methyl-2,3-diphenylpropanal (rac-S2).

兽

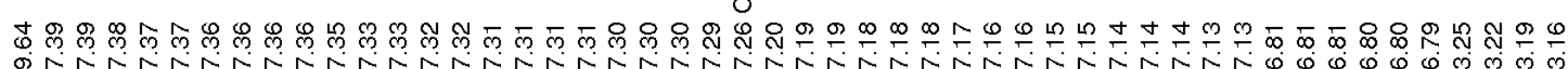

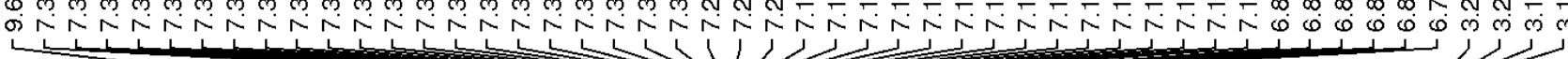

$\overbrace{\mathrm{Ph}}^{\mathrm{O}}$

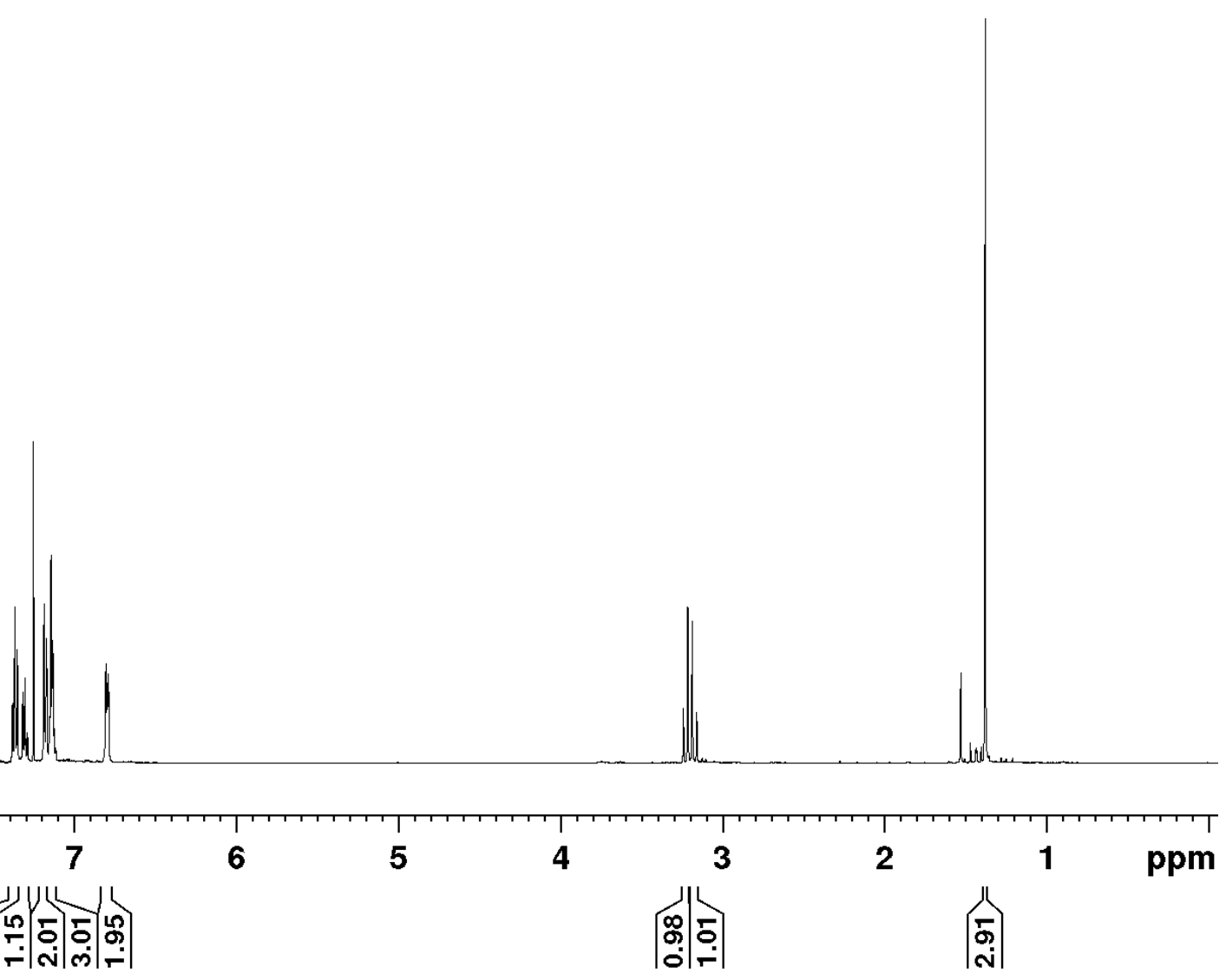

$12 \quad 11 \quad 10$

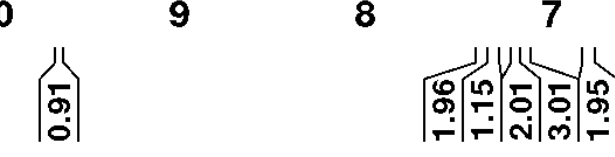

| 
Figure 8.102 ${ }^{13} \mathrm{C} \mathrm{NMR} \mathrm{(126} \mathrm{MHz,} \mathrm{CDCl}_{3}, 298 \mathrm{~K}$ ) of rac-2-Methyl-2,3-diphenylpropanal (rac-S2).

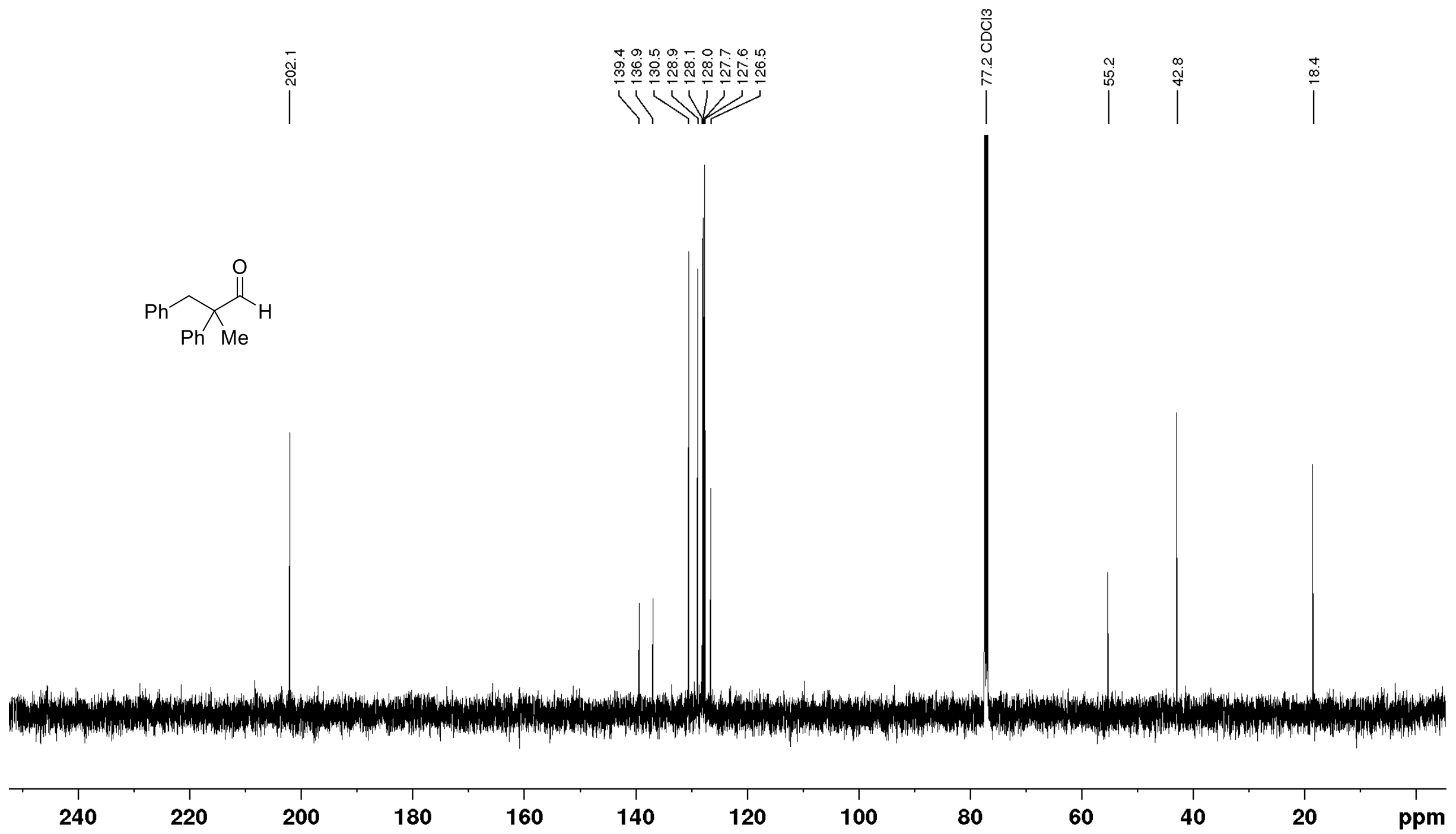


Figure 8.103 ${ }^{1} \mathrm{H}$ NMR (500 MHz, $\left.\mathrm{C}_{6} \mathrm{D}_{6}, 298 \mathrm{~K}\right)$ of (3,5-Dimethylphenyl)dimethylsilane.
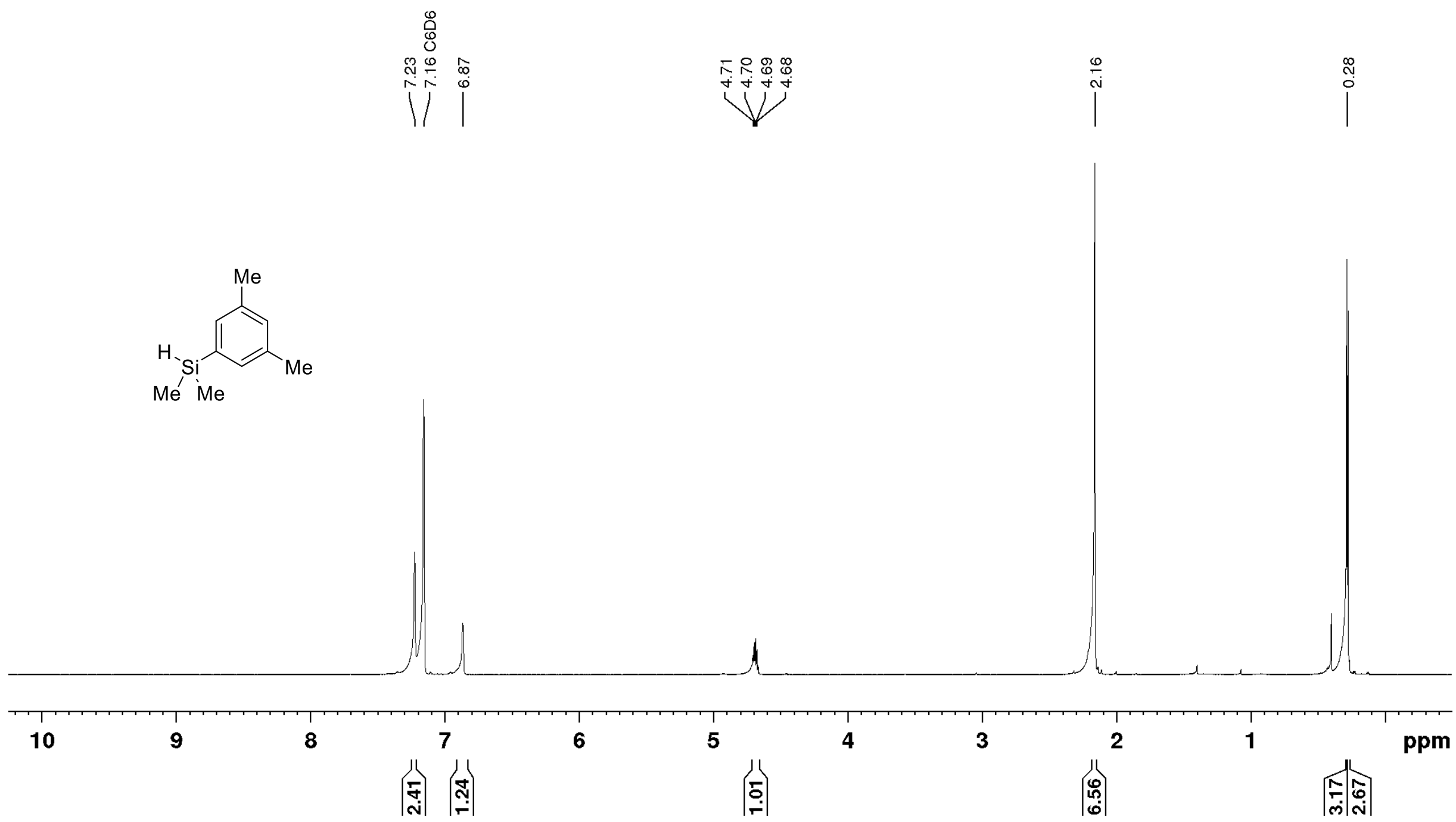
Figure 8.104 ${ }^{13} \mathrm{C}$ NMR $\left(126 \mathrm{MHz}, \mathrm{C}_{6} \mathrm{D}_{6}, 298 \mathrm{~K}\right)$ of (3,5-Dimethylphenyl)dimethylsilane.

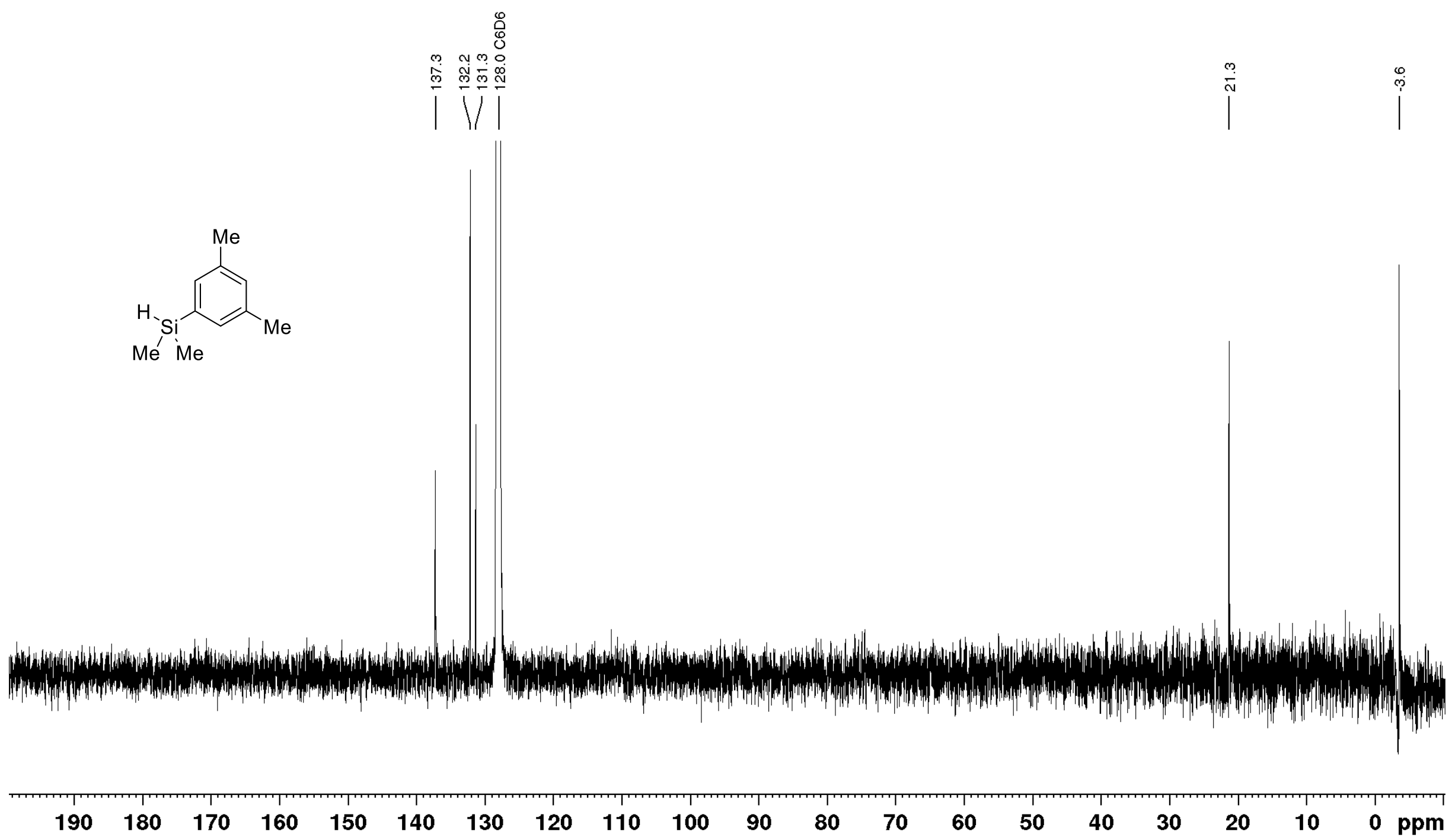


Figure 8.105 ${ }^{29} \mathrm{Si}$ DEPT NMR (99 MHz, $\left.\mathrm{C}_{6} \mathrm{D}_{6}, 298 \mathrm{~K}\right)$ of (3,5-Dimethylphenyl)dimethylsilane.

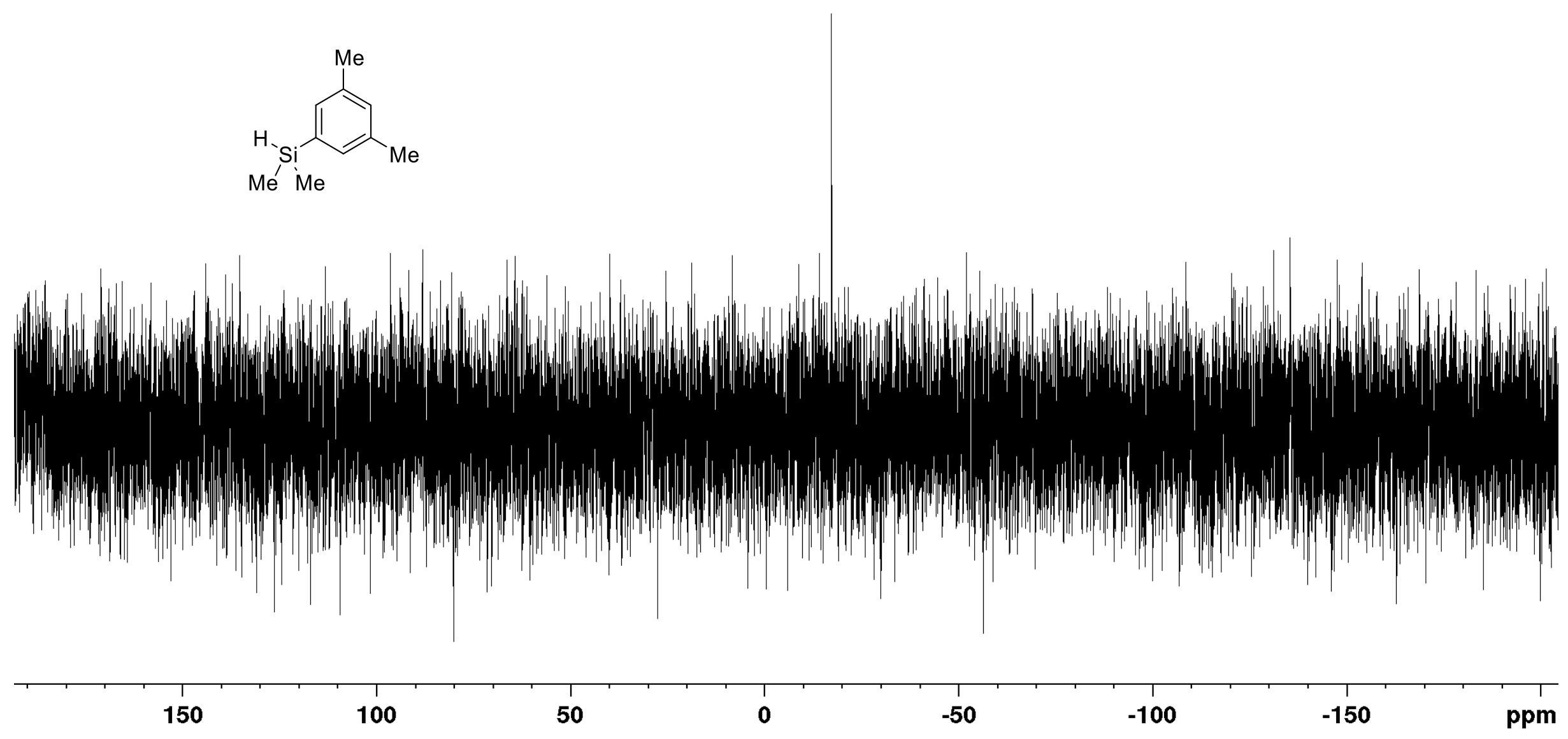


Figure 8.106 ${ }^{1} \mathrm{H}$ NMR (500 MHz, $\mathrm{CDCl}_{3}, 298 \mathrm{~K}$ ) of rac-2-Benzyl-2-phenylbutanenitrile (rac-S3).

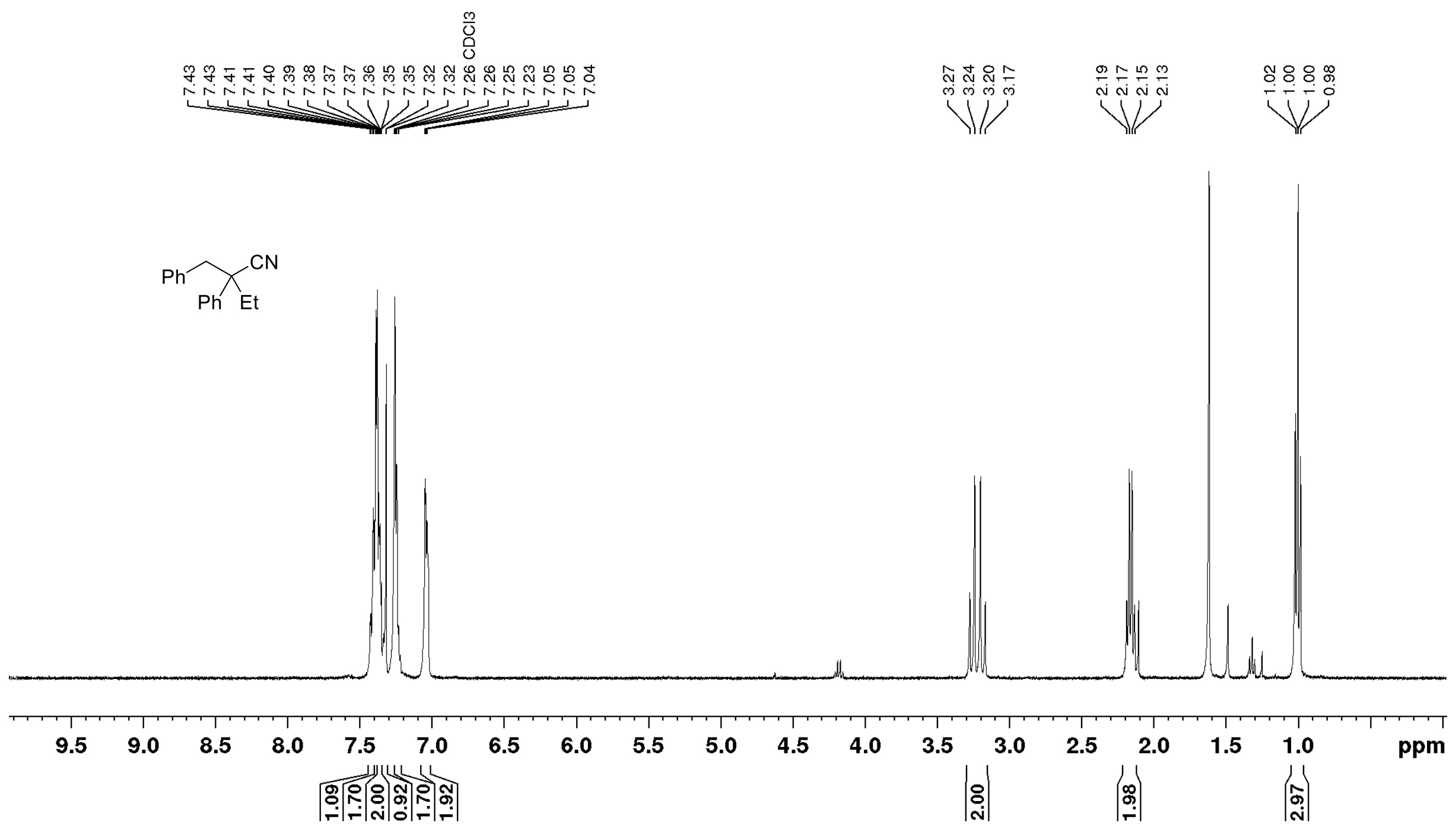


Figure 8.109 ${ }^{13} \mathrm{C} \mathrm{NMR} \mathrm{(126} \mathrm{MHz,} \mathrm{CDCl}_{3}, 298 \mathrm{~K}$ ) of rac-2-Benzyl-2-phenylbutanenitrile (rac-S3).

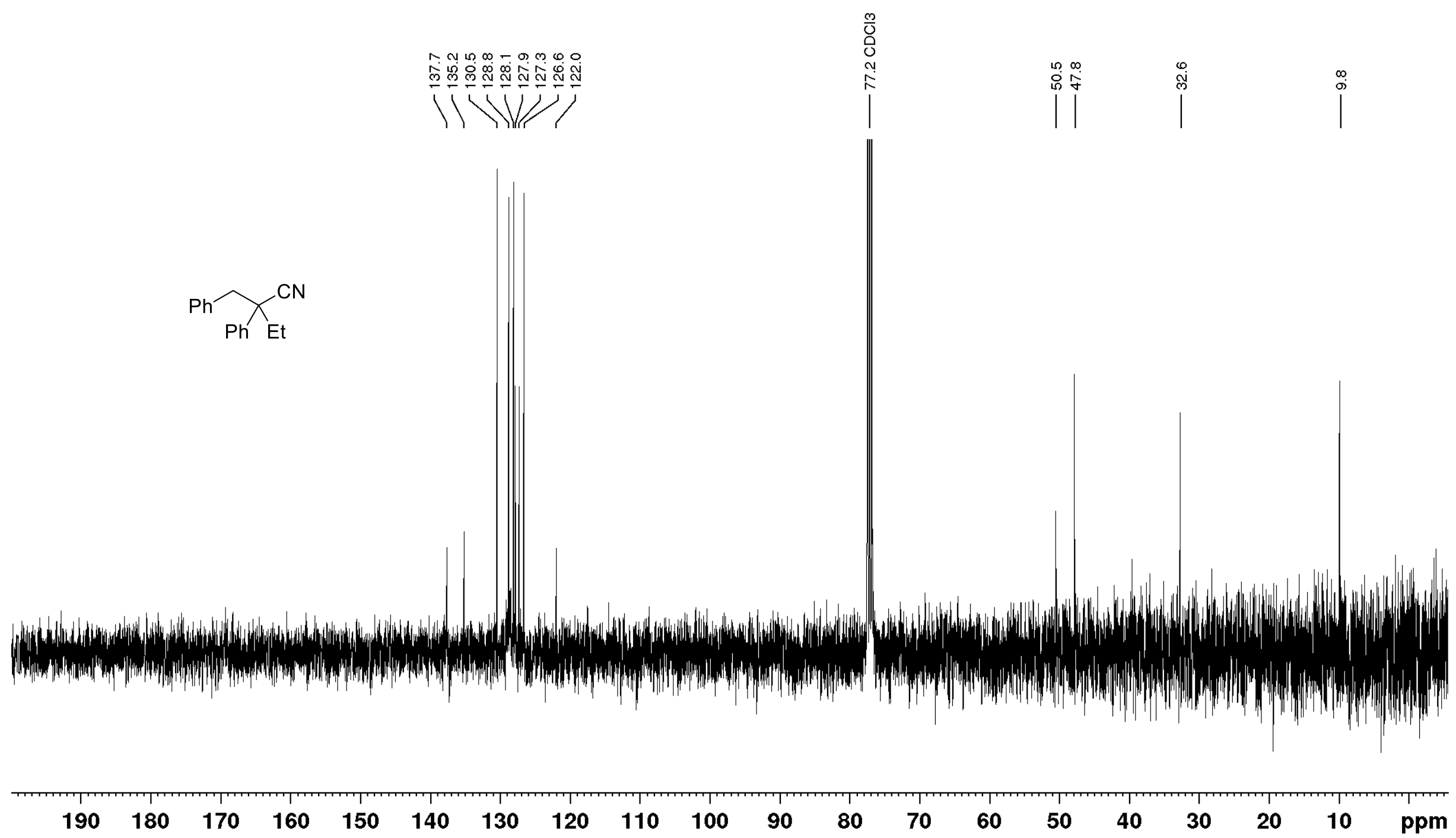


Figure 8.110 ${ }^{1} \mathrm{H}$ NMR (500 MHz, $\mathrm{CDCl}_{3}, 298 \mathrm{~K}$ ) of rac-2-Benzyl-2-phenylbutanal (rac-S4).

$\frac{9}{0}$

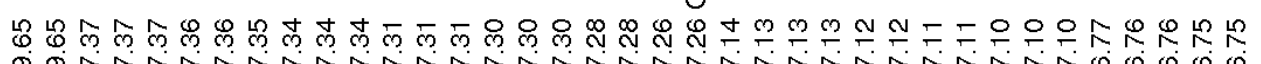

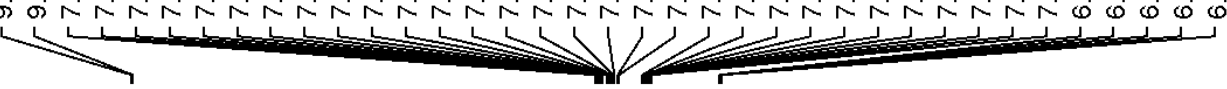

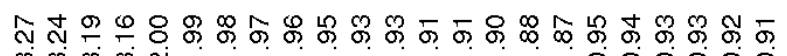
$\bigcup_{1}^{2}$<smiles>CCC(Cc1ccccc1)(C(C)=O)c1ccccc1</smiles>

10

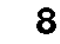

8

$$
\text { | }
$$

6

5

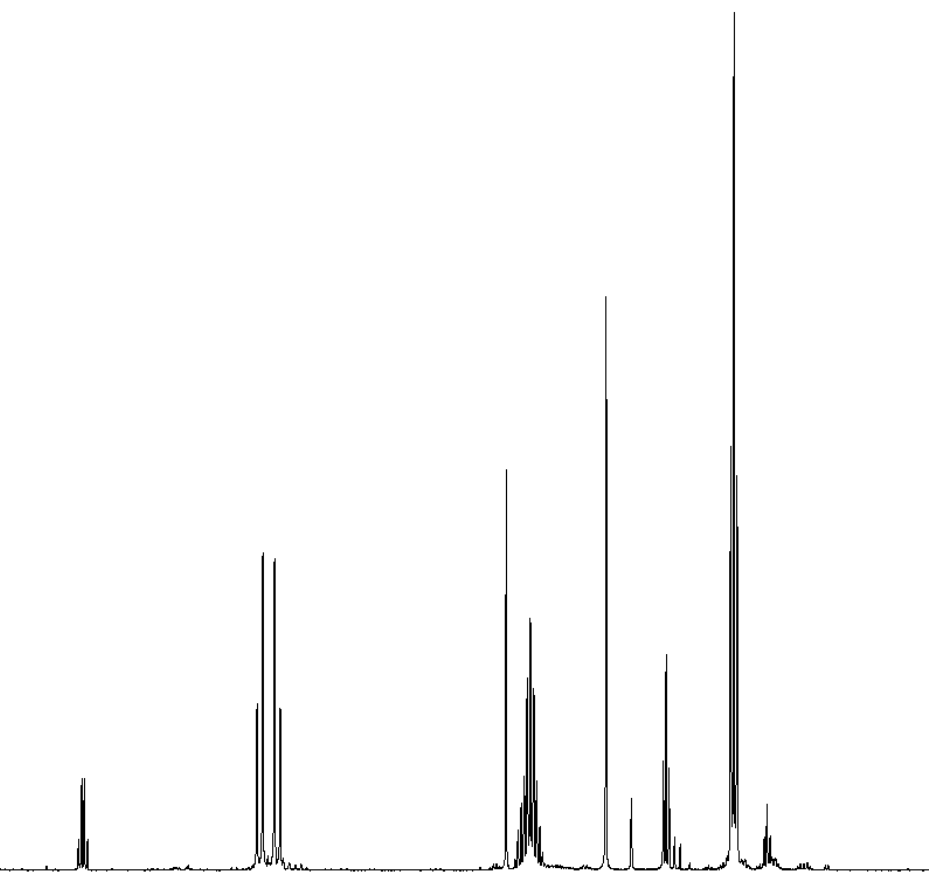

4

สุิ พฺุ

2

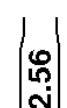

1

)क्ष 
Figure 8.111 ${ }^{1} \mathrm{H}$ NMR (500 MHz, $\mathrm{CDCl}_{3}, 298 \mathrm{~K}$ ) of rac-3-(4-Fluorophenyl)-2-methyl-2-phenylpropanal (rac-S5).

$\frac{m}{0}$

$\overline{0}$

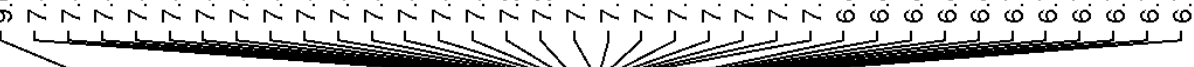
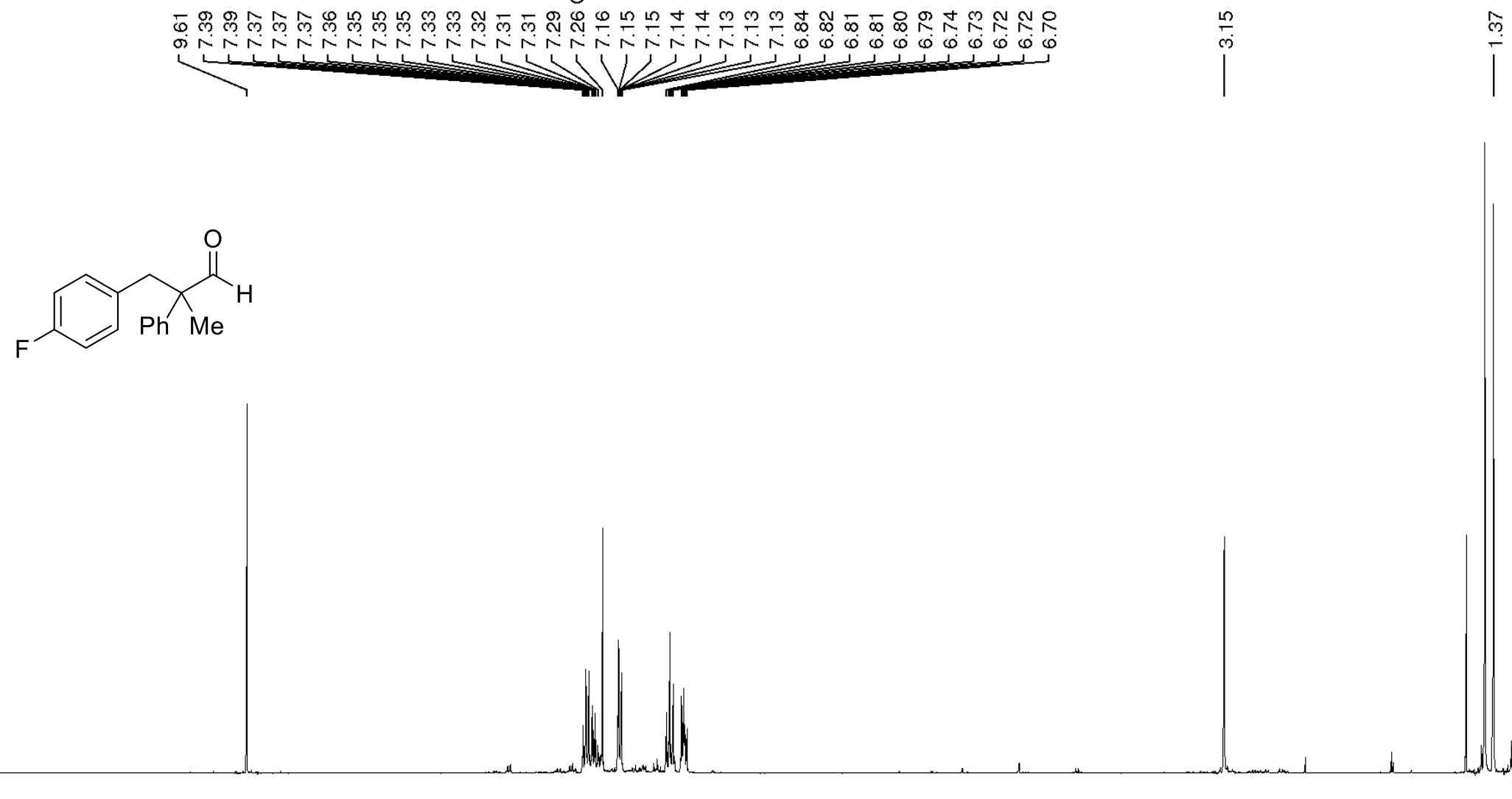

$$
3
$$

ฟึ่

2

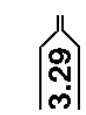


Figure 8.112 ${ }^{13} \mathrm{C} \mathrm{NMR} \mathrm{(126} \mathrm{MHz,} \mathrm{CDCl}_{3}, 298 \mathrm{~K}$ ) of rac-3-(4-Fluorophenyl)-2-methyl-2-phenylpropanal (rac-S5).

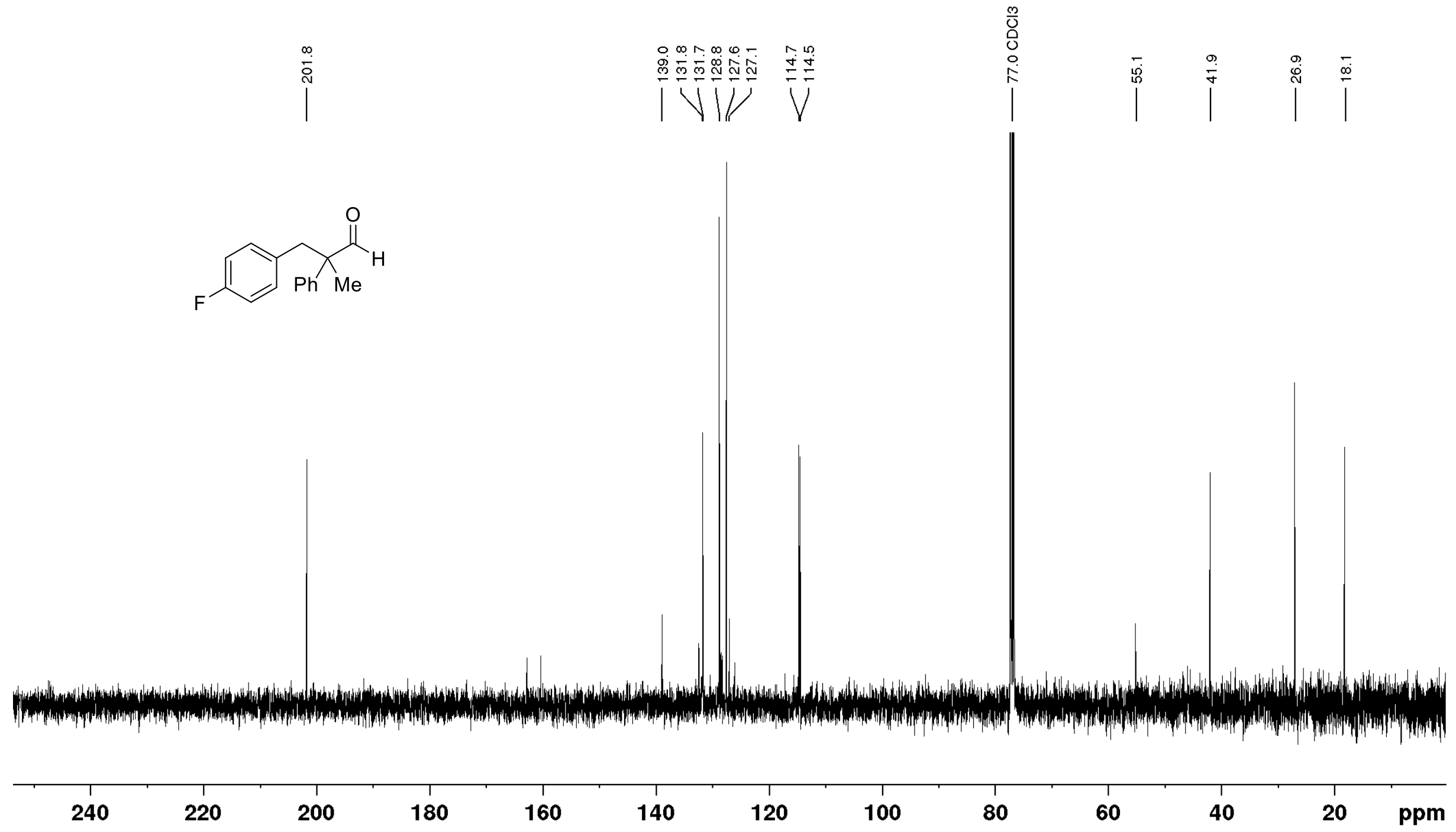


Figure 8.113 ${ }^{1} \mathrm{H} \mathrm{NMR}\left(500 \mathrm{MHz}, \mathrm{CDCl}_{3}, 298 \mathrm{~K}\right)$ of rac-2-Methyl-2-phenyl-3-(p-tolyl)propanal (rac-S6).

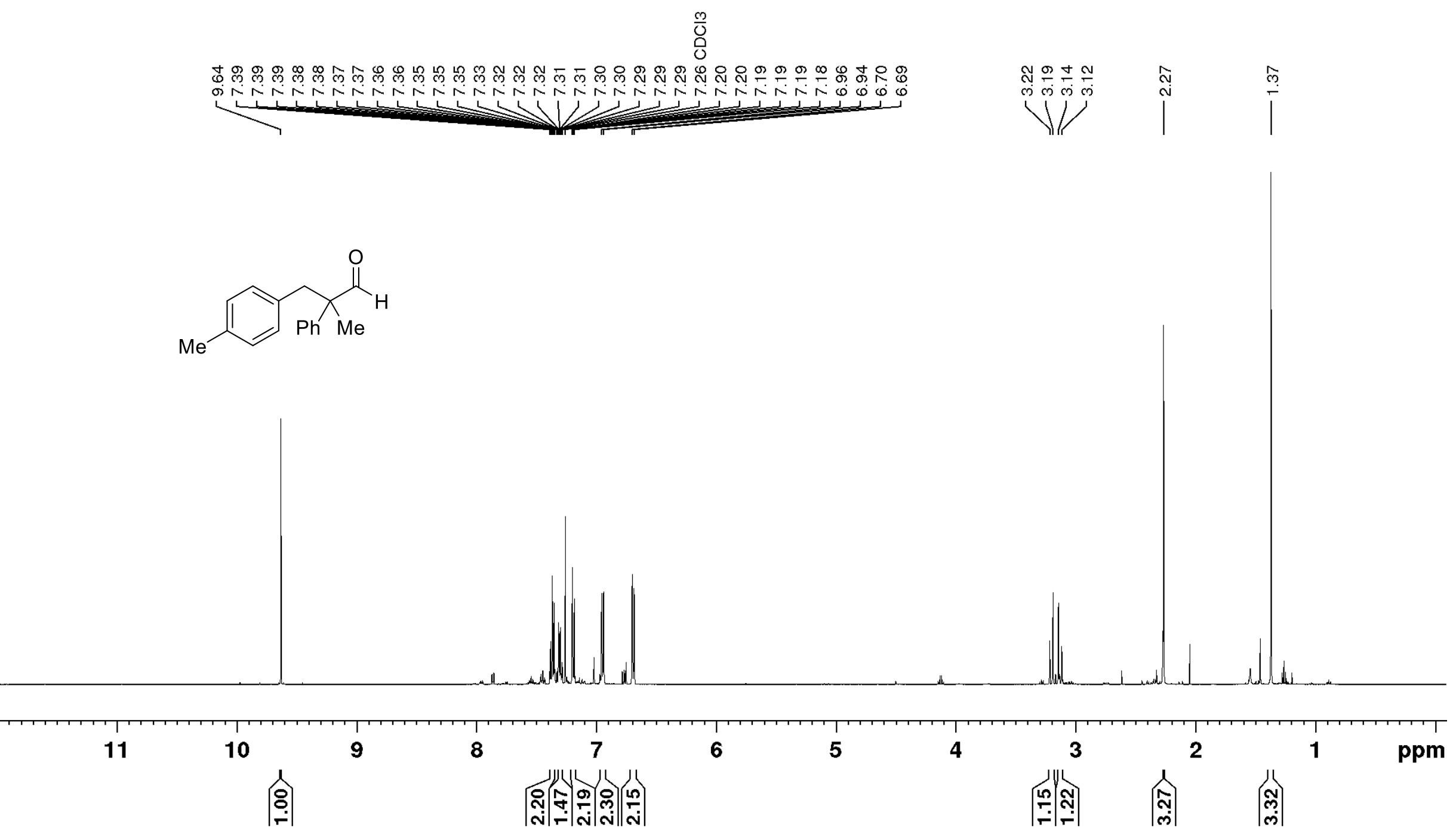


Figure 8.114 ${ }^{1} \mathrm{H}$ NMR (500 MHz, $\mathrm{CDCl}_{3}, 298 \mathrm{~K}$ ) of rac-3-(3,5-Dimethylphenyl)-2-methyl-2-phenylpropanal (rac-S7).

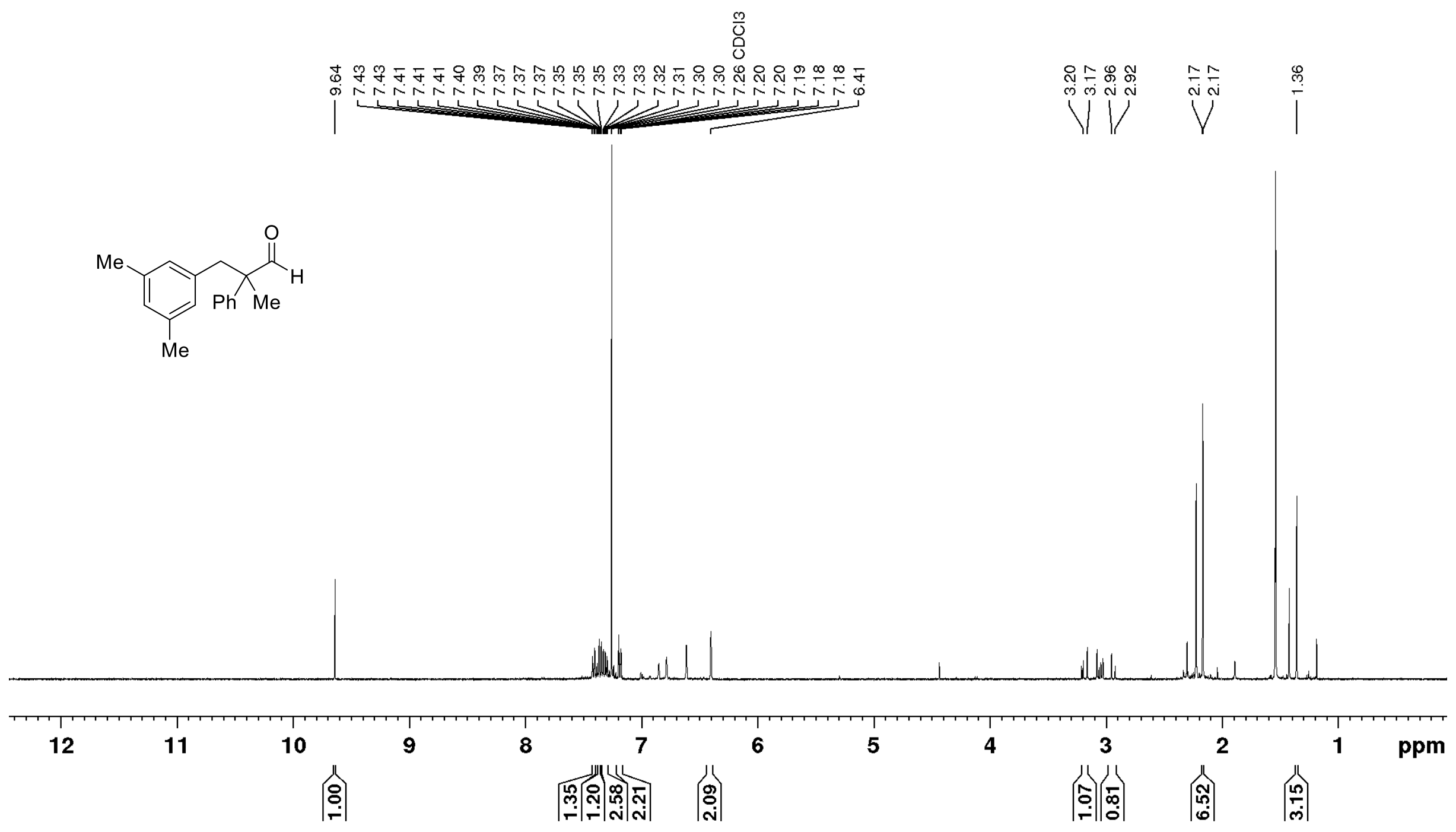


Figure 8.115 ${ }^{1} \mathrm{H}$ NMR $\left(500 \mathrm{MHz}, \mathrm{CDCl}_{3}, 298 \mathrm{~K}\right)$ of rac-3-(3,5-Dimethoxyphenyl)-2-methyl-2-phenylpropanal (rac-S8).<smiles>[Se]</smiles>

ڤ

$\longrightarrow$

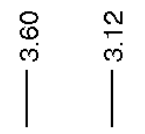

$\stackrel{\substack{+i}}{1}$<smiles>[2H]C(C)(Cc1cc(OC)cc(OC)c1)c1ccccc1</smiles>
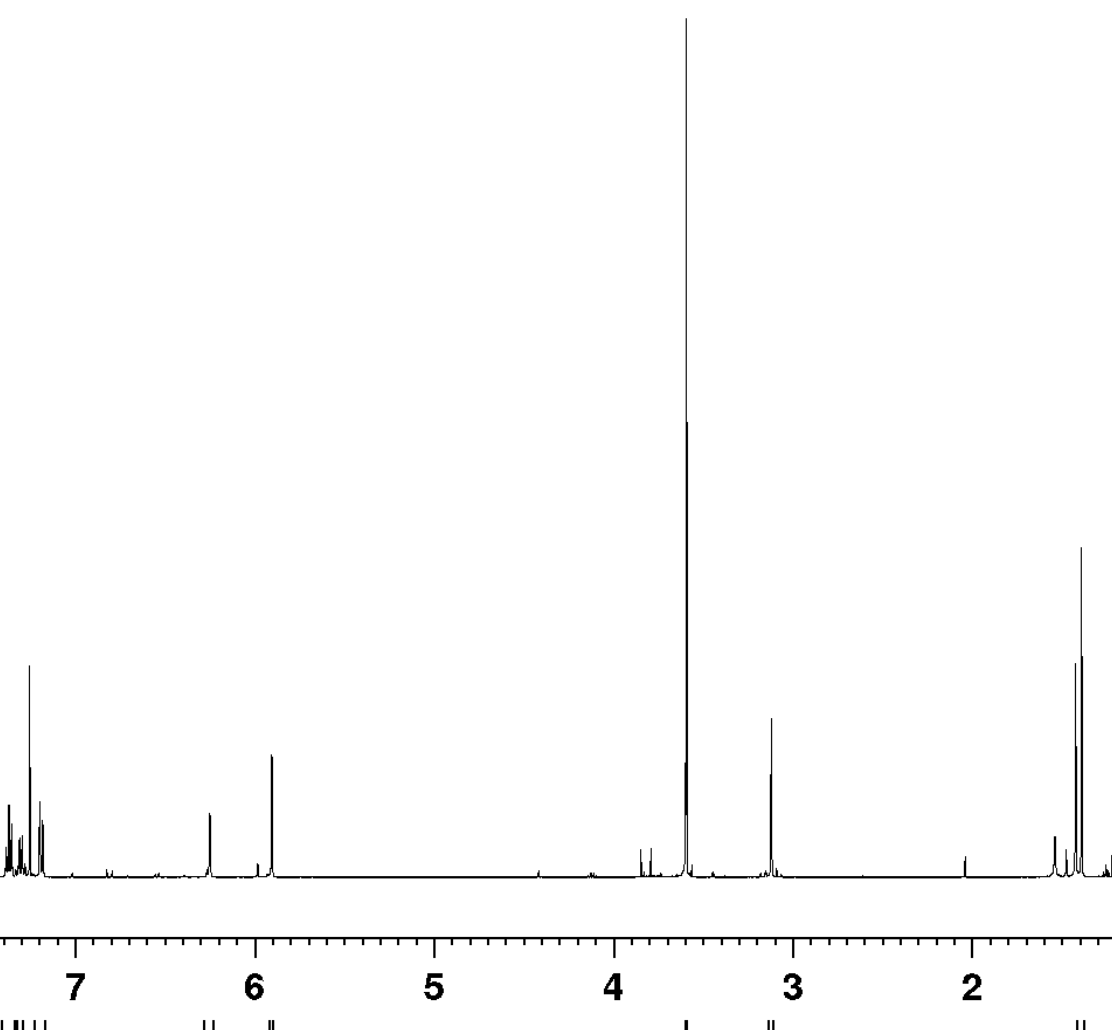

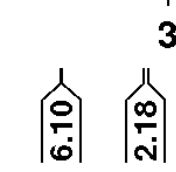
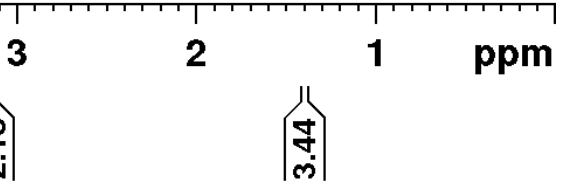
Figure 8.116 ${ }^{1} \mathrm{H}$ NMR (500 MHz, $\mathrm{CDCl}_{3}, 298 \mathrm{~K}$ ) of rac-3-(4-Bromophenyl)-2-methyl-2-phenylpropanal (rac-S9).

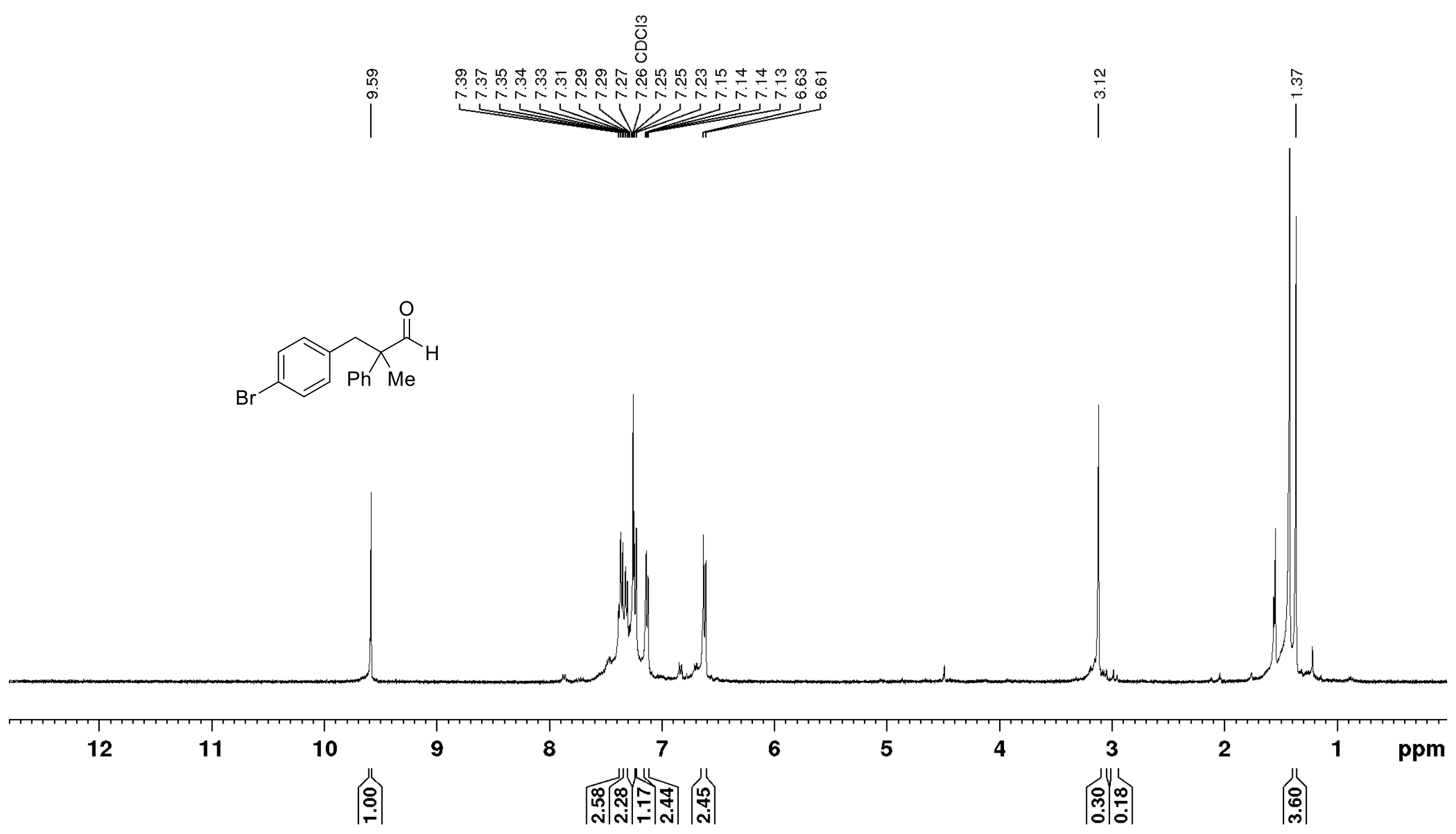


Figure 8.117 ${ }^{1} \mathrm{H}$ NMR $\left(500 \mathrm{MHz}, \mathrm{CDCl}_{3}, 298 \mathrm{~K}\right)$ of rac-3-(4-Chlorophenyl)-2-methyl-2-phenylpropanal (rac-S10).

$\frac{m}{0}$

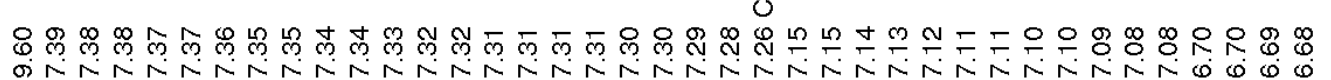

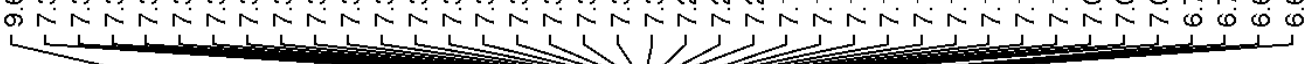
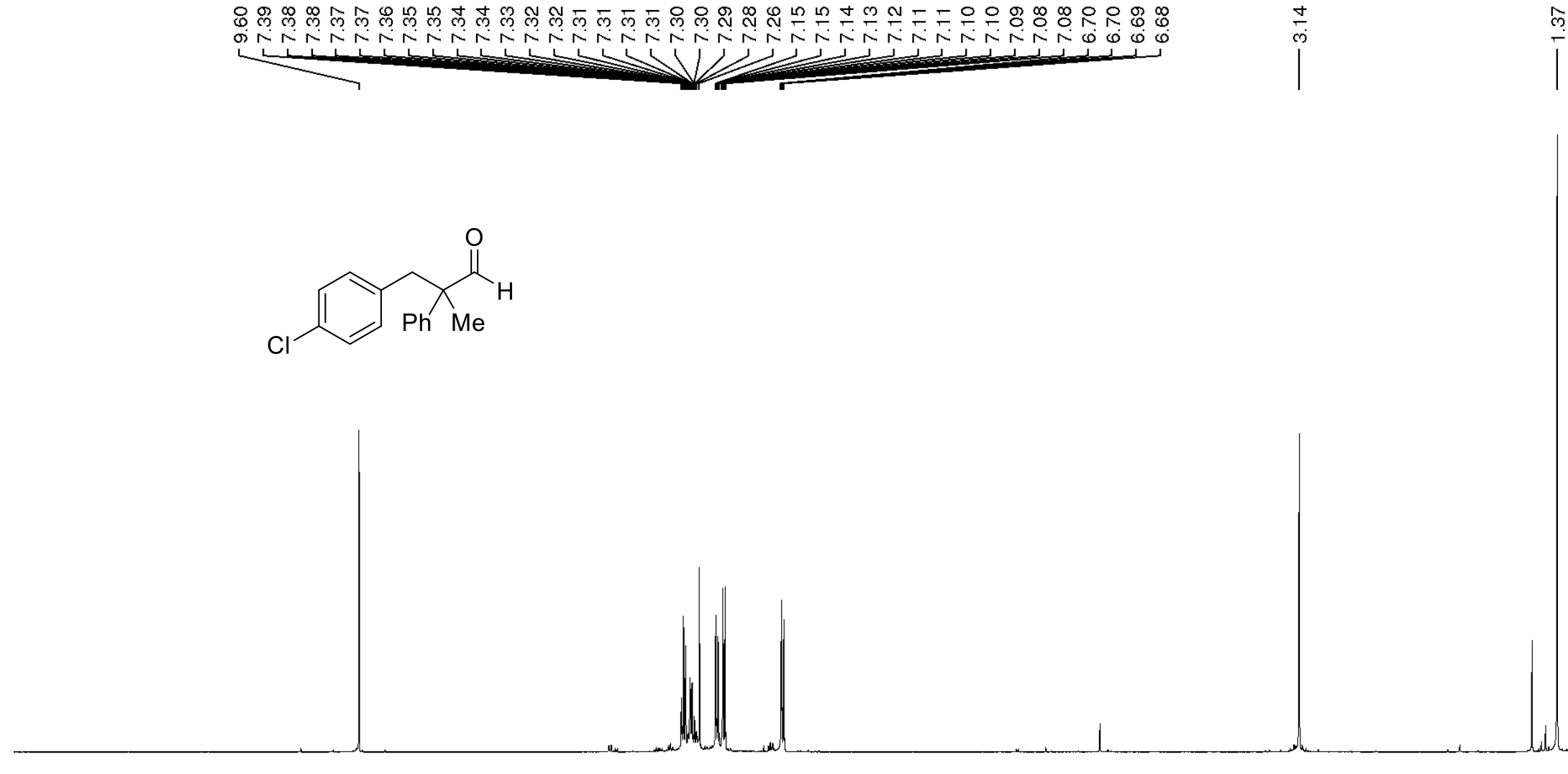

11

10

9

8

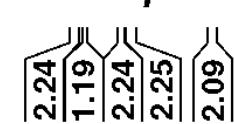

6

5

4

3

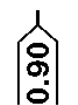

$\left|\begin{array}{l}\hat{m} \\ \text { ì }\end{array}\right|$

2

$\left|\begin{array}{c}N \\ \text { ले }\end{array}\right|$ 
Figure 8.118 ${ }^{1} \mathrm{H} \mathrm{NMR}\left(500 \mathrm{MHz}, \mathrm{CDCl}_{3}, 298 \mathrm{~K}\right)$ of rac-3-(4-Methoxyphenyl)-2-methyl-2-phenylpropanal (rac-S12).

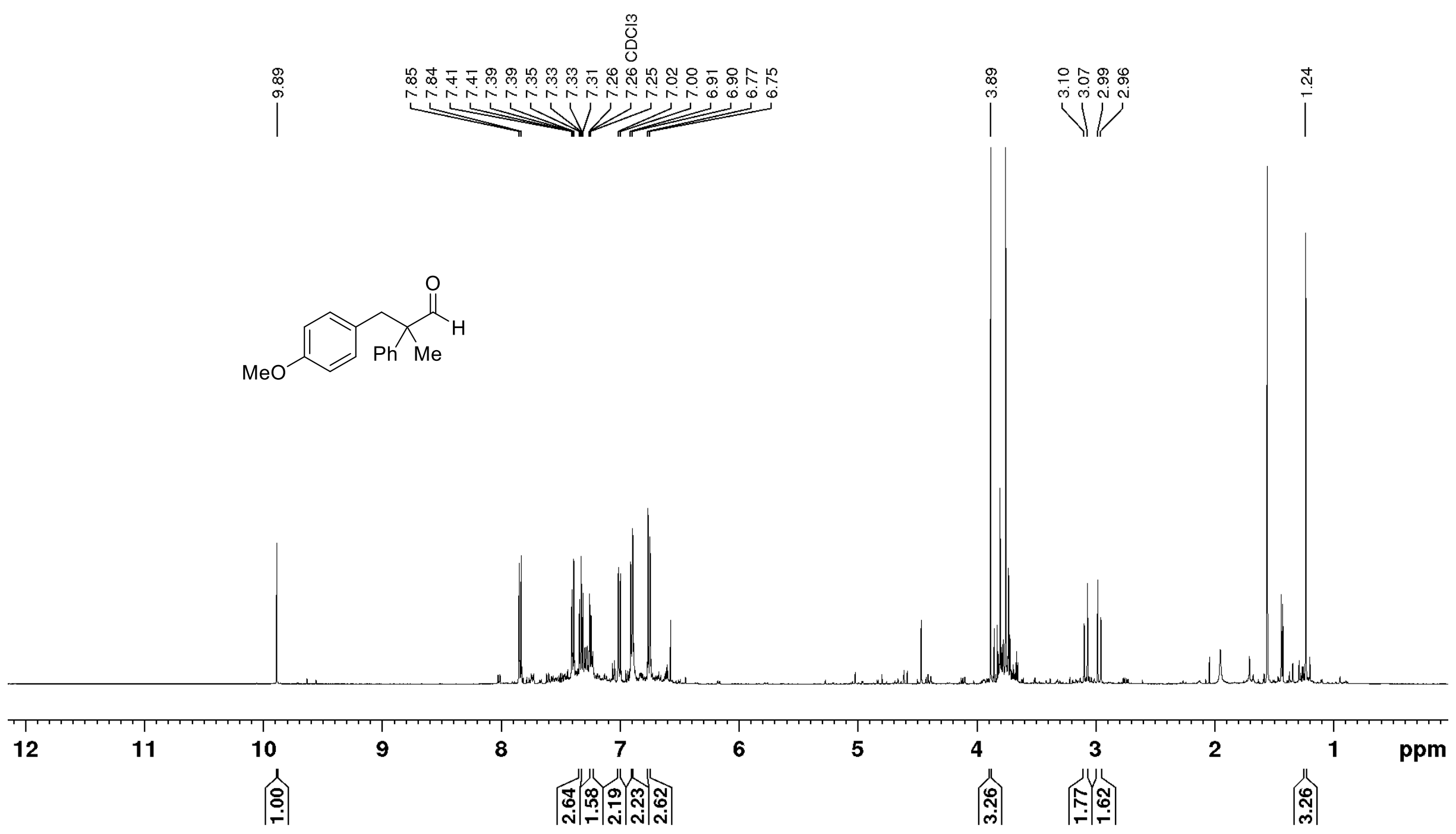


Figure 8.119 ${ }^{1} \mathrm{H}$ NMR (500 MHz, $\mathrm{C}_{6} \mathrm{D}_{6}, 298 \mathrm{~K}$ ) of rac-3-(2-Bromophenyl)-2-methyl-2-phenylpropanal (rac-S13).

$\frac{m}{0}$

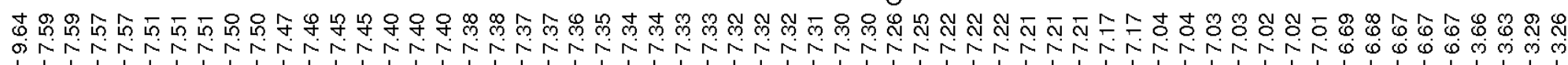
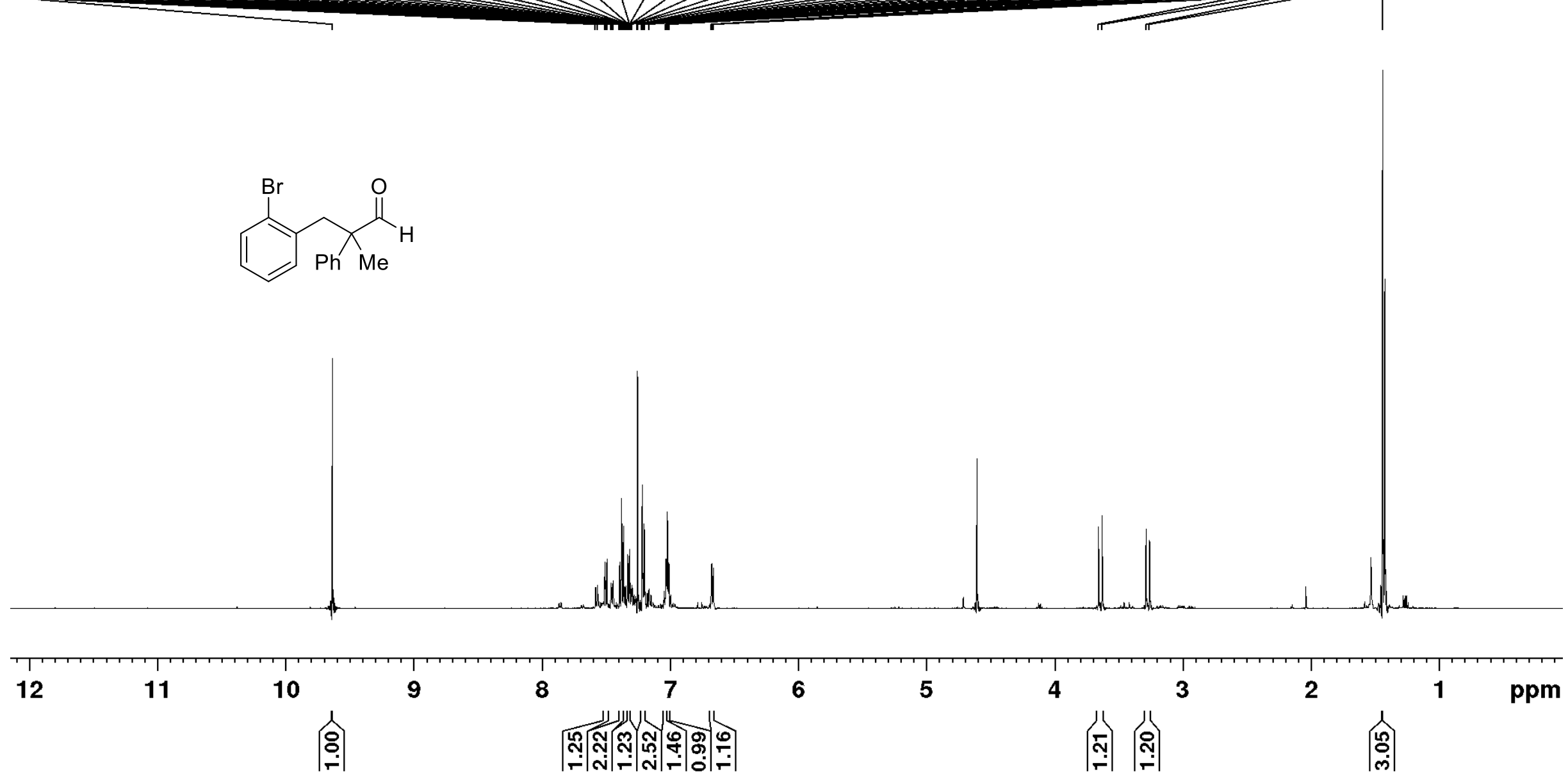
Figure 8.120 ${ }^{1} \mathrm{H}$ NMR (500 MHz, $\left.\mathrm{C}_{6} \mathrm{D}_{6}, 298 \mathrm{~K}\right)$ of rac-3-(4-lodophenyl)-2-methyl-2-phenylpropanal (rac-S14).
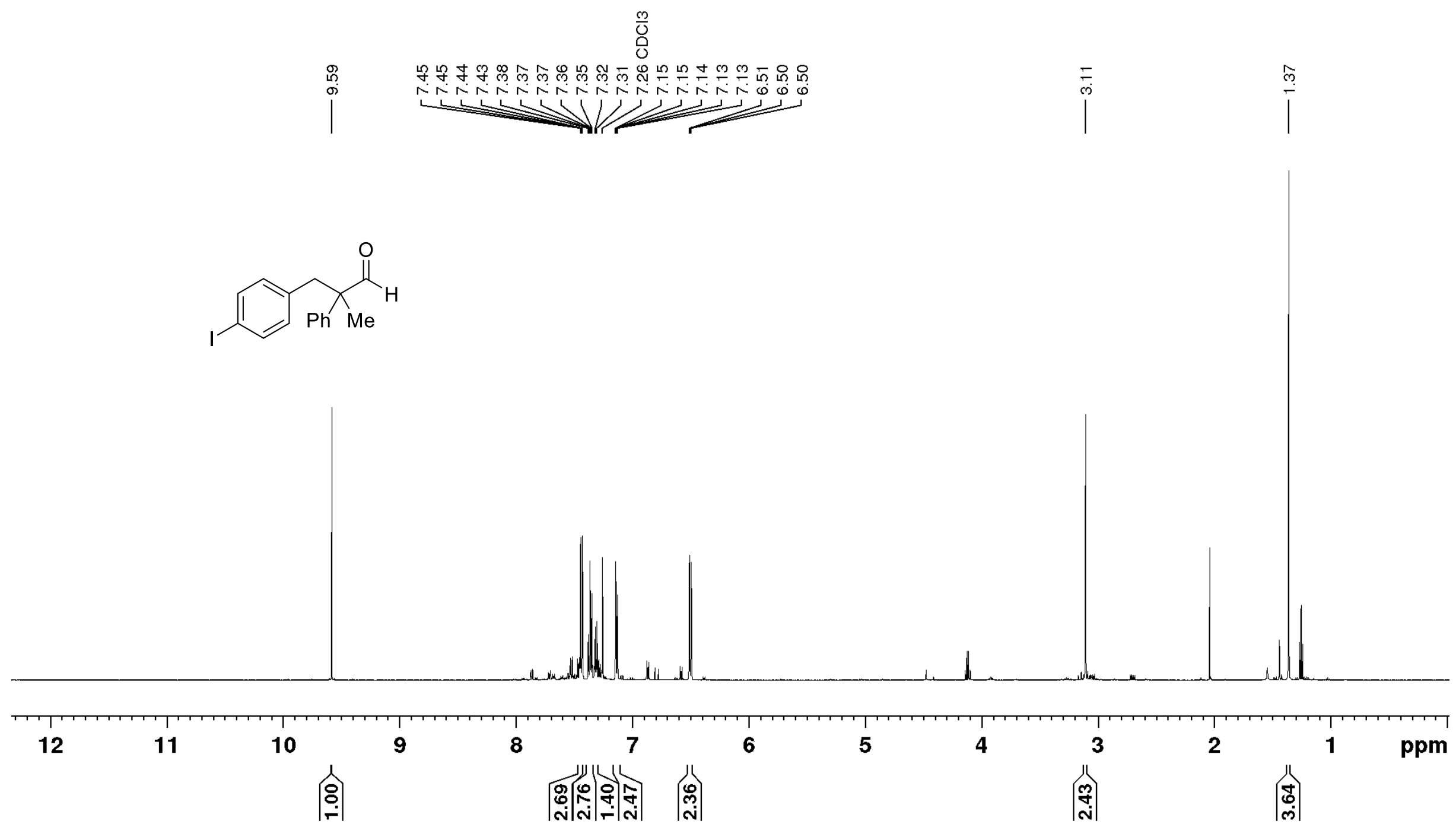
Figure 8.121 ${ }^{1} \mathrm{H}$ NMR (500 MHz, $\left.\mathrm{C}_{6} \mathrm{D}_{6}, 298 \mathrm{~K}\right)$ of rac-2-Benzyl-2-methylbutanal (rac-S15).

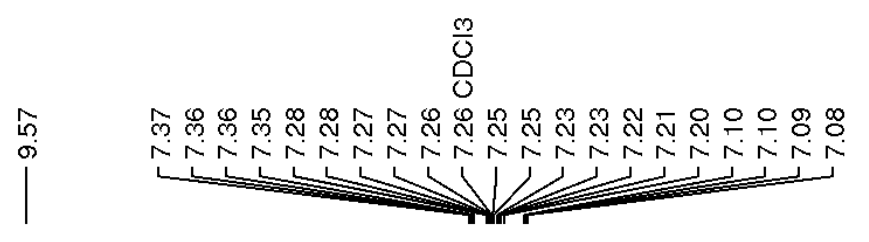

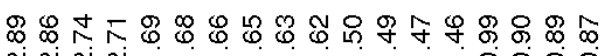
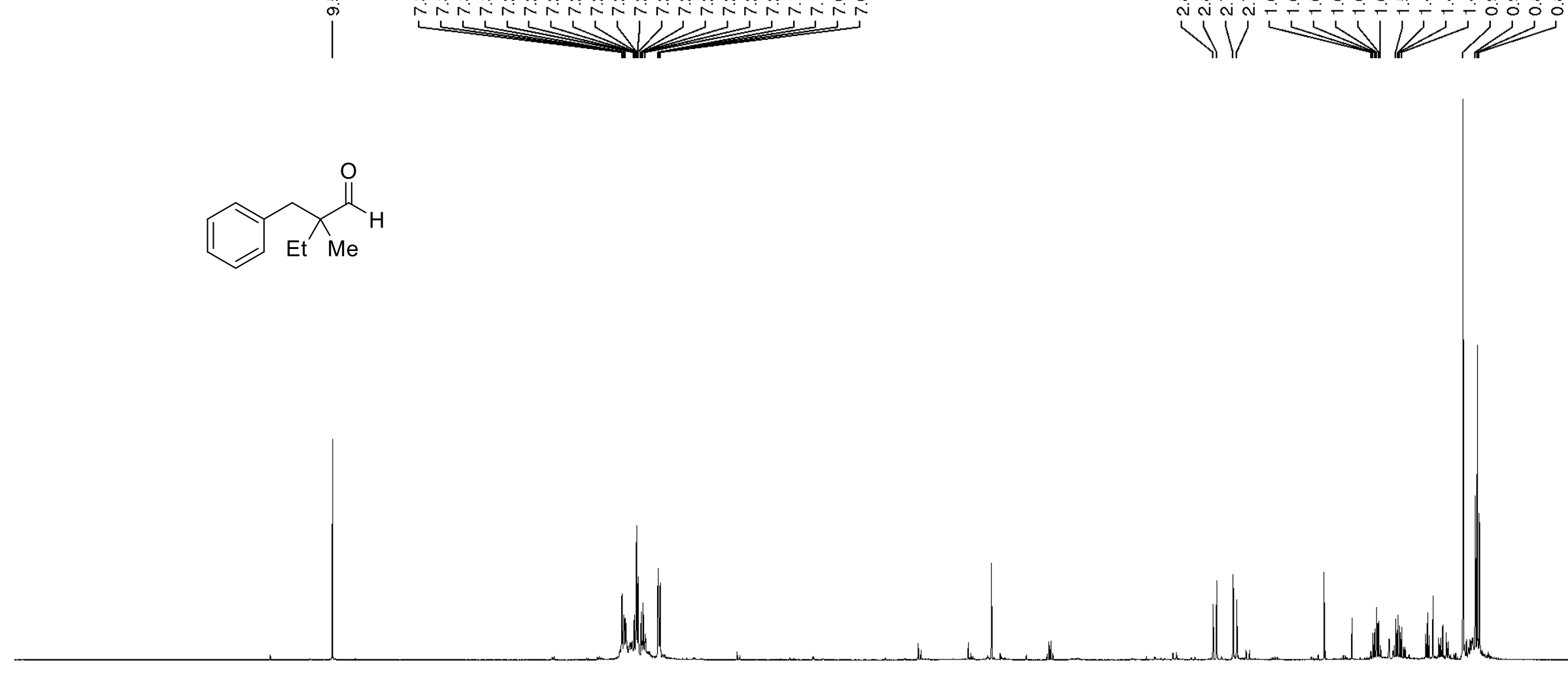

11

10

9

8

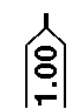

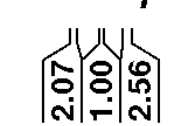

6

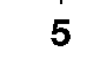

5

4
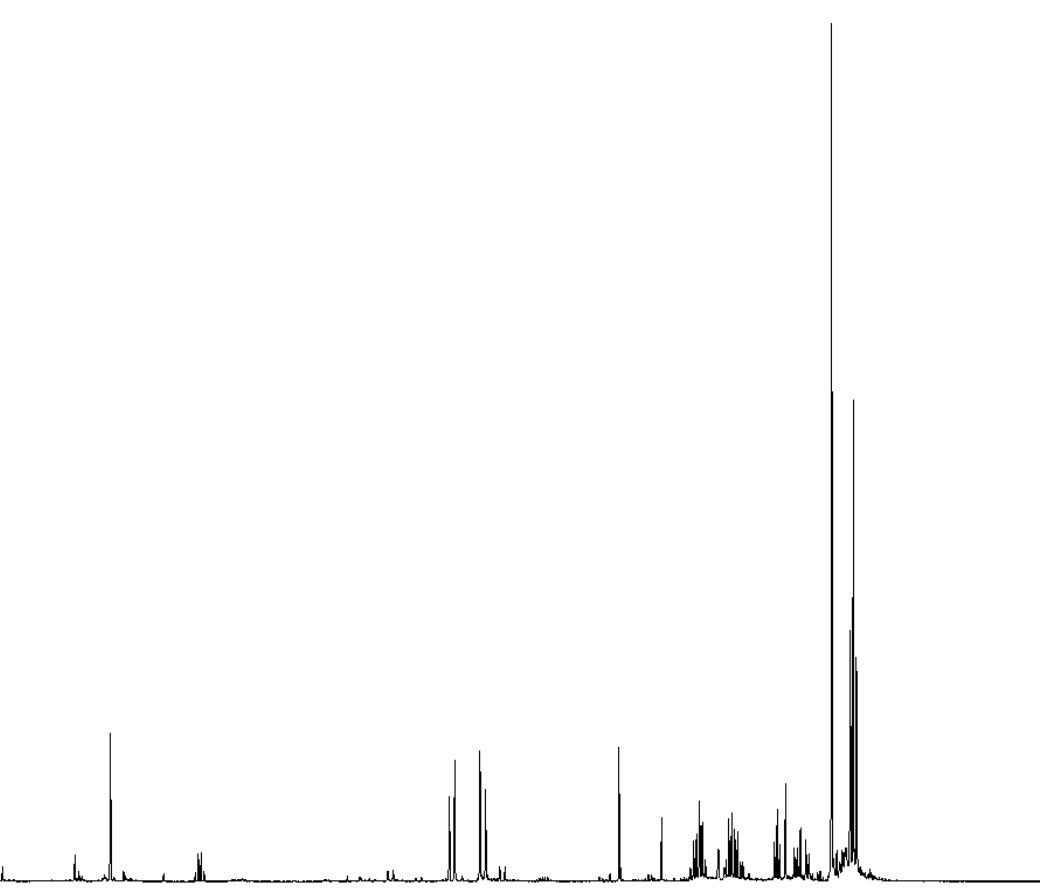

Hillad

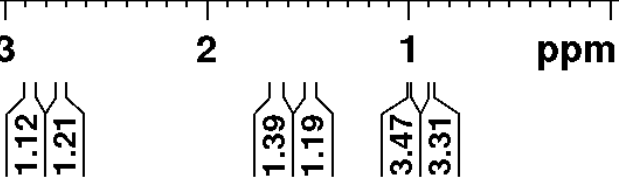




\section{References}

[S1] Dong, X.; Weickgenannt, A.; Oestreich, M. Nat. Commun. 2017, 8, 15547.

[S2] Qiu, G.; Mamboury, M.; Wang, Q.; Zhu, J. Angew. Chem., Int. Ed. 2016, 55, 15377.

[S3] Fuchibe, K.; Mayumi, Y.; Zhao, N.; Watanabe, S.; Yokota, M.; Ichikawa, J. Angew. Chem., Int. Ed., 2013, 52, 7825-7828.

[S4] Wang, K.; Zhou, J.; Jiang, Y.; Zhang, M.; Wang, C.; Xue, D.; Tang, W.; Sun, H.; Xiao, J.; Li, C. Angew. Chem., Int. Ed. 2019, 58, 6380.

[S5] Gou, B.-B.; Liu, H.-F.; Chen, J.; Zhou, L. Org. Lett. 2019, 21, 7084-7088.

[S6] M. A. Romero, J. A. González-Delgado, J. F. Arteaga, Nat. Prod. Commun. 2015, 10, 1257-1262.

[S7] Agilent CrysAlis PRO, 2012, Agilent Technologies, Yarnton, UK.

[S8] G. M. Sheldrick, Acta Crystallogr., Sect. A. 1990, 46, 467-473.

[S9] G. M. Sheldrick, Acta Crystallogr., Sect. A. 2008, 64, 112-122.

[S10] Mercury 3.9; Cambrige Crystallographic Data Center:

https://www.ccdc.cam.ac.uk/solutions/csd-system/components/mercury/. 